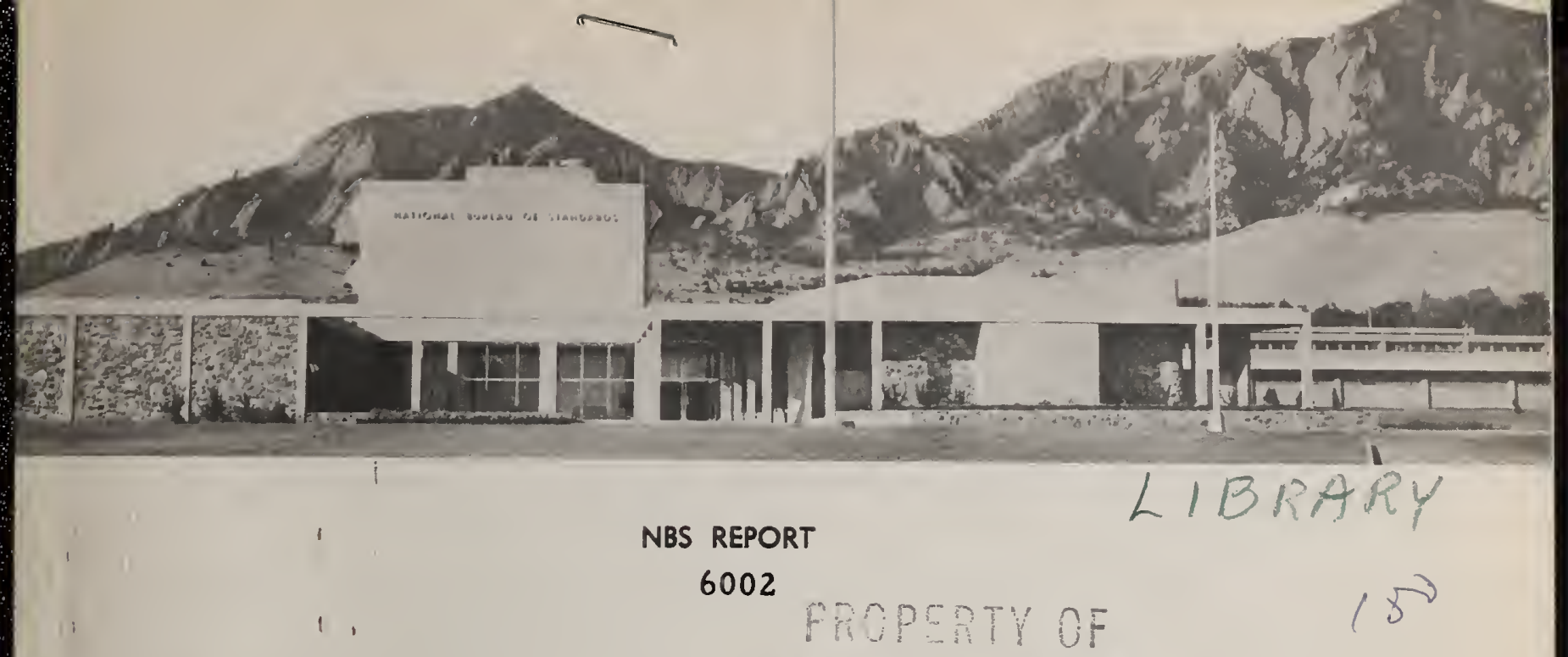

by
J. R. Johler
L. C. Walters
and
C. M. Lilley

TRANSFER CHARACTERISTICS OF RADIO WAVES PROPAGATED BETWEEN THE IONOSPHERE AND THE EARTH AT VERY LOW FREQUENCIES

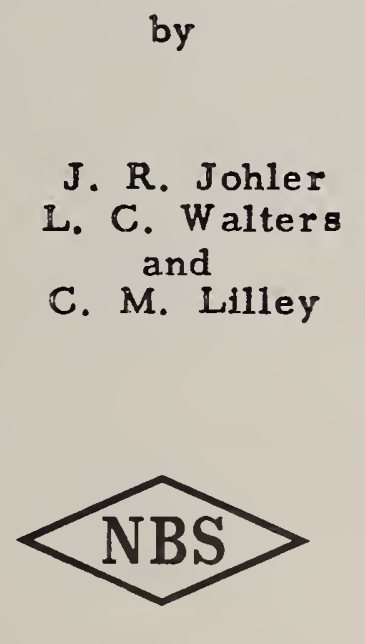

U. S. DEPARTMENT OF COMMERCE NATIONAL BUREAU OF STANDARDS BOULDER LABORATORIES Boulder, Colorado 


\section{THE NATIONAL BUREAU OF STANDARDS}

\section{Functions and Activities}

'lhe lunctions of the National Burcau of Standards are set forth in the Act of Congress, March 3, 1901, as amended by Congress in Public Law 619, 1950. These include the development and maintenance of the national standards of measurement and the provision of means and methods for making measurements consistent with these standards: the cletemination of physical constants and properties of materials; the development of methods and instruments for testing materials, devices, and structures; advisory services to Government Agencies on scientific and technical problems; invention and development of devices to serve special needs of the Government; and the development of standard practices, codes and specilications. The work includes basic and applied research, development, engineering, instrumentation, testing, evaluation, calibration services, and various consultation and information services. A major portion of the Bureau's work is performed for other Government Agencies, particularly the Department of Delense and the Atomic Energy Commission. The scope of activities is suggested by the listing of divisions and sections on the inside back cover.

\section{Reports and Publications}

The results of the Bureau's work take the form of either actual equipment and devices or published papers and reports. Reports are issued to the sponsoring agency of a particular project or program. Published papers appear either in the Bureau's own series of publications or in the journals of professional and scientific societies. The Bureau itself publishes three monthly periodicals, available from the Government Printing Office: The Journal of Research, which presents complete papers reporting technical investigations; the Technical News Bulletin, which presents summary and preliminary reports on work in progress; and Basic Radio Propagation Predictions, which provides clata for determining the best frequencies to use for radio communications throughout the world. There are also five series of nonperiodical publications: The Applied Mathematics Series, Circulars, Hanclbooks, Building Materials and Structures Reports, and Miscellaneous Publications. 


\section{NATIONAL BUREAU OF STANDARDS REPORT}

NBS PROJECT

$8330-11-8835$

$8330-11-8332$
September 15, 1958
NBS REPORT

6002

TRANSFER CHARACTERISTICS OF RADIO WAVES PROPAGATED BETWEEN THE IONOSPHERE AND THE EARTH AT VERY LOW FREQUENCIES

by

J. R. Johler

L. C. Walters and

C. M. Lilley

For The

Air Force Cambridge Research Center Air Research and Development Command

CSO \& A No. 58-28(AFCRC)

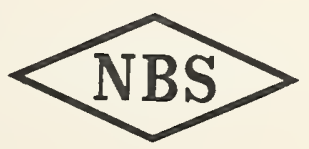

U. S. DEPARTMENT OF COMMERCE

NATIONAL BUREAU OF STANDARDS

BOULDER LABORATORIES

Boulder, Colorado 


\section{IMPORTANT NOTICE}

NATIONAL BUREAU OF STANDARDS REPORTS is subjected to odditionol evolu Approved for public releas institute of tion, or open-literoture listing mission is obtained in writing fro 25 , D. C. Such permission is $n$ been specificolly prepored if the Director of the Nationalins (NIST) on Stan October 9, 2015. rogress occounting docuis formolly published it on, reprinting, reproducit outhorized unless perP Stondords, Woshington or which the Report hos for its own use. 
Abstract

Introduction

1

Theory

3

Computations

Conclusions

Acknowledgements

Glossary

Lis $t$ of Figures

Additional Figures 31

Appendix: Púlses and Time-Modes

Appendix Figure

Preface to Tables

55

List of Tables

56

Tables

75 

TRANSFER CHARACTERISTICS OF RADIO WAVES PROPAGATED BETWEEN THE D-REGION AND THE E-REGION OF THE IONOSPHERE AND THE EARTH BASED ON THE QUASI-LONGITUDINAI THEORY

\section{ABSTRACT}

The transfer characteristics lamplitude and phase as a function of frequency) of the sky wave propagated between the D-region and the E-region of the ionosphere and the earth have been evaluated at low and very low frequencies by the geometrical-optical theory employing the quasi-longitudinal approximation of the ionosphere reflection coefficients. Distances, d/j, up to 1000 statute miles are considered and multiple hops or time-nodes $(j=1,2,3, \ldots)$ are evaluated for a vertical electric dipole source. The effects of the electron density, colision frequency, intensity of the eartir s magnetic field and the geometrical parameters are illustrated. The effect of the vertical lapse of the permittivity of tha earth ${ }^{8}$ atmosphere is introduced into the computation.

\section{Introduction}

A theory of geometrical-optical "ray-paths" has been developed to represent the propagation of radio waves between the earth and the ionosphere. The "ray-path" concept, implying infinite frequency, $f=\infty$, simplifies the physical picture of the propagation mechanism, Figs. 1, 2, 3, however, the numerical details of evaluating the field of the propagated wave remain quite involved. These numerical details are investigated in this paper for the particular case of propagation between the D-region and the E-region of the ionosphere and the earth. The formulation of the rigorous geometrical optics presented by Bremmer ${ }^{1}$ is followed

H. Bremmer, "Terrestrial Radio Waves - Theory of Propagation," Elsenier Pub. Co., Inc., New York, N. Y。, 1949. 
with a few ad hoc refinements in detail.

The theory of propagation of radio wave pulses or transients ${ }^{2}$ has emphasized the importance of a precise determination of the transfer characteristic of the propagation medium, $i_{0} e_{0}$, the description of the amplitude and phase of the continuous wave as a function of frequency, which task is the object of this paper.

The source of the radiation is assumed to be vertical electric polarization, although vertical magnetic components, Fig. 3, arise as a result of the ionosphexe reflection process. The local reflection coefficients of the ionosphere are evaluated by the quasilongitudinal ${ }^{3}$ approximation. 'lhis Eonfines the as:gument of this paper to frequencies in the neighborhood of 10 kilocycles, and a moderately wide $x$ ange of frequencies about 10 kilocycles were evaluated $\left(\omega / \omega_{x}=0.01\right.$ to 5). The local ground reflection coefficients have been determined by the Fresnel approximation. This confines the argument of this paper to distances ${ }^{4}, d / j$ up to 1000 statute miles from the source, Distances, $d / j$, in the neigliborhood of 1200 statute miles require corrections for diffraction and convergence, which

2

J. R. Johler and L. C. Walters, "Transmission of a Ground. Wave Pulse Around a. Finitely Conducting Spherical Earth, "National Bureau of Standards Report No. 5566, April 17, 1958.

3

K. G. Budden, "The Reflection of Very Low Frequency Radio Waves at the Surface of a Sharply Bounded Ionosphere with Superimposed Magnetic Field," Phil. Mag,42, 7 Series, LXXXV, 1951, pp.8334 850 .

$j=1,2,3 \ldots$ is an integer denoting the order of the time-modes or "hops" to and fro between the earth and the ionosphere. Thus $d / j=1000$ miles, for $j=2, d=2000$ miles. 
problems are beyond the scope of this paper.

The first, second, third and fourth time modes or "hops" are evaluated in considerable detail in this paper. The effect of the vertical lapse of the permittivity of the earth ${ }^{8} \mathrm{~s}$ atmosphere is considered by introducing into the computation the special geometrical parameters of the Norton ${ }^{5}$ type 301 atmosphere. These parameters have been determined by ray tracing techniques and Norton ${ }^{\circ} \mathrm{s}$ Lesults are presented in Table 16.

\section{Theory}

A vertically polarized Hertzian-type dipole oscillator source, s, is situated on the surface of the earth, Fig. 1. The oscillation is continuous, (undamped) and varies harmonically in time at each frequency under consideration. The vertical electric field strength, $\mathbb{E}(\omega, \mathrm{d})$, developed at a point, $0, F i g .1$, on the surface of the earth is determined at various frequencies $\omega$ and distances, $d$.

Physically, the sky-wave is characterized by time modes or "hops" which are clearly indicated in the geometrical-optical theory by the sum:

$$
E(\omega, d)=\sum_{j=0}^{n} E_{j}(\omega, d)
$$

For convergence and diffraction corrections see: J.R. Wait, "Diffractive Corrections to the Geometrical Optics of Low Frequency Propagation, "NBS Report No. 5572, June 2.1958. See also: J.R. Wait and A.M. Conda, "Pattern of An Antenna on a Curved Lossy Surface," NBS Report No. 5562, Feb. 15, 1958. 5

K. A. Norton, "Transmission Los in Radio Propagation, "NBS Report No. 5092, $25 \mathrm{July}$ 1957. See also: B. R. Bean and G. Thayer. "A Model Radio Refractivity Atmosphere," NBS Report No. 5576, June 1958. 


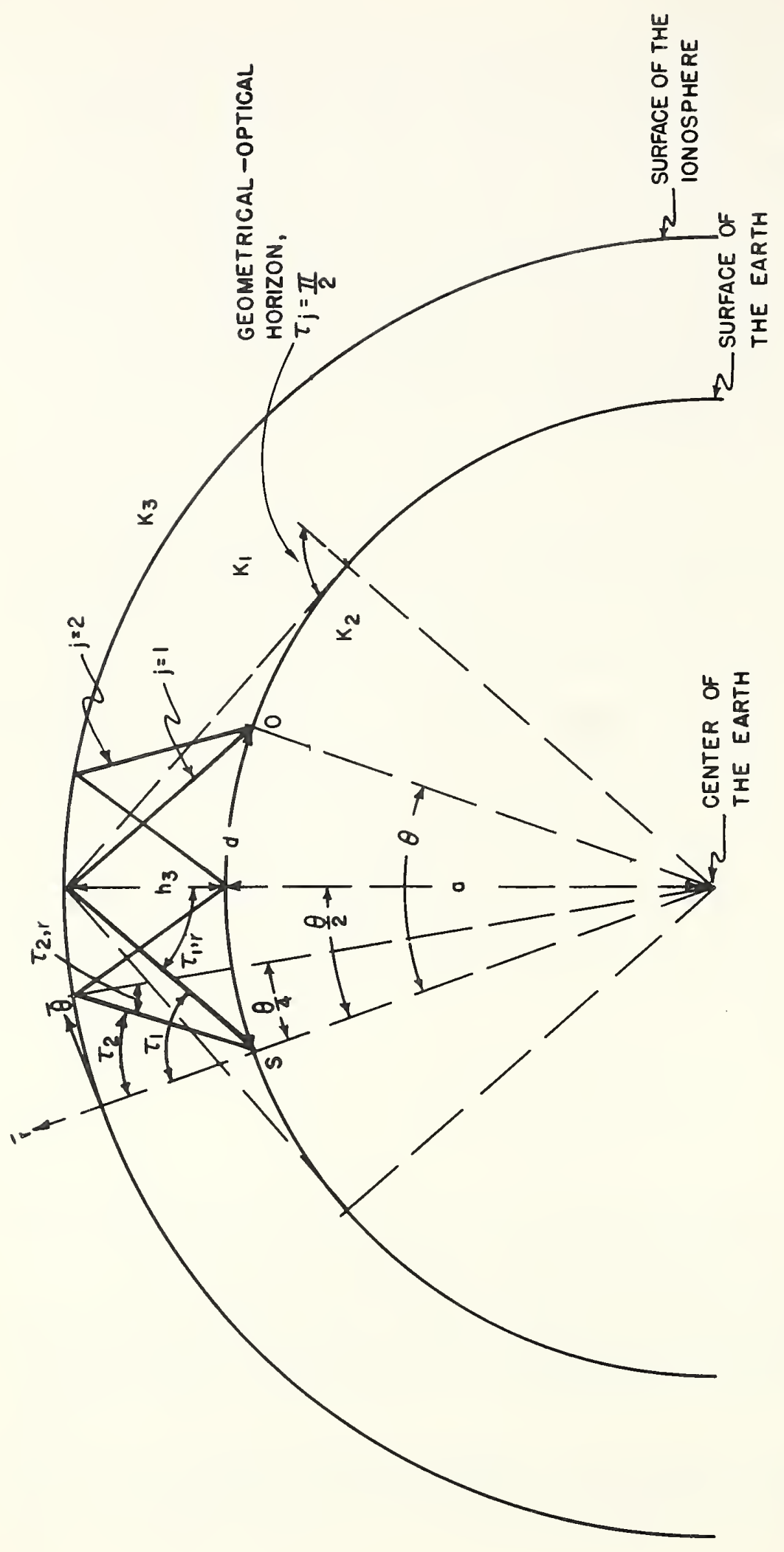

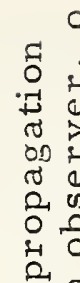

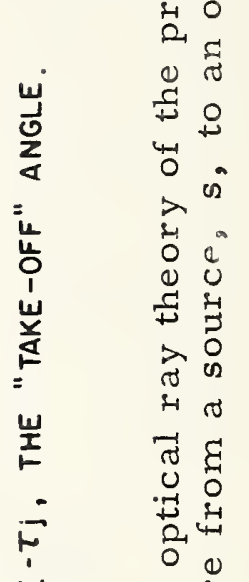

HN

जN

$=\quad \begin{gathered}1 \\ 0 \\ 0\end{gathered}$

क

$\rightarrow \quad+$

$\rightarrow$ द्वा

i⿱

㝴

का मे

.

茫

on

$\stackrel{2}{3}$

م

on $n$

के

$\stackrel{0}{*} 3$

$\stackrel{9}{7}:-7$

i

号出

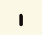

$-1$

$\underset{1}{\infty}$ 
The index, $j=1,2,3 \ldots:$, denotes the order of the time-modes or "hops" to and fro between the earth and the ionosphere, Figs. 1, 2, 3. Note that the time-mode, $E_{0}(\omega / d), j=0$, represents the ground wave ${ }^{6}$. This paper is primarily concerned with the calculation of the individual sky-wave time-modes, $E_{j}(\omega, d), j=1,2,3 \ldots$ 。 Such a time-mode can be represented as follows:

$E_{j}(\omega, d)=\frac{i \omega d}{D_{j}} C \exp \left(i \omega t^{\prime}{ }_{j}\right)\left(\sin ^{2} \tau_{j}\right) \propto_{j} F_{j} C_{j}$.

$$
C=\frac{2 I_{0} \ell a^{2}}{4 \pi k d^{3}}=\frac{2\left(10^{-7}\right)}{d}
$$

where, for purposes of this paper, the dipole momentum is assigned a value of unity,

$$
I_{0} l=1 \text { ampere-meter }
$$

The local time for the ground wave, $t^{\prime}$, is,

$$
\begin{gathered}
t^{\prime}=t-a, \\
a=\frac{\eta_{1} d}{c} .
\end{gathered}
$$

6

J. R. Johler, W. J. Kellar and L. C. Walters, "Phase of the Low Radio Frequency Ground Wave, "National Bureau of Standards Circular 573, 14 March 1956. 
Similarly, the local sky wave time, $t^{8}(j=1,2,3, \ldots)$ is,

$$
\begin{aligned}
& t^{\prime}{ }_{j}=t-\widehat{a}_{j} \\
& \hat{a}_{j}=\frac{\eta_{1} \widehat{D}_{j}}{c} .
\end{aligned}
$$

The local time is an important consideration in the transient problem since, $t^{\prime}{ }_{0}=0$, is the earliest time at which the first precursor of a ground wave pulse signal could be observed. Similarily, $t^{p}{ }_{j}=0$, is the earliest time a jth sky wave time-mode pulse signal could be observed, since the signal could not be expected to travel faster than the speed of light over the ray path. Thus, the parameter, $\widehat{a}_{j}-a$, has been computed, Table 123, as the "sky wave delay," i.e., the time delay introduced into the sky-wave propagation by the greater physical length, $\widehat{D}_{j}$, of the sky-wave ray as compared with the physical length, d, Fig. 1, of the ground wave "ray." Thus, the first signal to arrive could arrive no sooner than a signal travelling around the curvature of the earth, $d$, at the speed of light.

The physical length of the ray, $\bar{D}_{j}$, Fig. 1 , is,

$$
\widehat{D}_{j}=2 j\left[\left(\alpha+h_{3}\right) \cos \tau_{j, z}-a \cos T_{j}\right] \text {. }
$$

Thus, the radius of the earth, $a$, the height of the ionosphere reflecting region, $h_{3}$, the angle of incidence on the ionosphere, $\boldsymbol{T}_{\boldsymbol{j}, \boldsymbol{I}}$ ' and the angle of incidence of the ground reflection, $\boldsymbol{T}_{j}$, 
determine the geometry of the "ray path" if the vertical lapse of the permittivity of the atmosphere is neglected.

The effect of bending of rays by the atmosphere, as a result of this vertical lapse, Fig. 2, has been introduced by a modification of the parameters, $\tau_{j, r}$ and $\tau_{j}$, Table 16. The rigorous geometricaloptics can account for such bendirg at grazing incicience on the earth only $\left(\tau_{j}=\frac{\pi}{2}, \bar{\Psi}=0\right)$, and a value of effective earth radius factor, $\propto^{8}=0.75$, typifies an average atmosphere. This iactor cannot remain constant as $\tau_{j}$ decreases and at vertical incidence should approach unity, $\propto \alpha^{k}=1$. A value for $\alpha^{f}$ between the limits 0.75 and 1 can be determined from Norton's data, Table 16. As a consequence of the se considerations, the radian distance, $\theta$, Fig. I, for purposes of Tables 119-123, has been adjusted by a factor $\alpha_{1}$

$$
\begin{aligned}
& \theta=\frac{\gamma^{\prime} d}{\propto a} \\
& \sin \tau \frac{1}{\Delta !}\left(a+h_{3}\right) \sin \theta / 2 j \\
& \Delta_{j}^{g}=\left[2 a\left(a+h_{3}\right)\left(1-\cos \theta^{8} / 2 j\right)+h_{3}\right]^{\frac{1}{2}}
\end{aligned}
$$

and the value of $\alpha_{1}$ could be aeduced from Table 16, but can be represented to a close approximation by,

$$
\propto_{1}^{p} \sim 1-\left(1-\propto^{p}\right) \sin \tau_{1}^{p}
$$




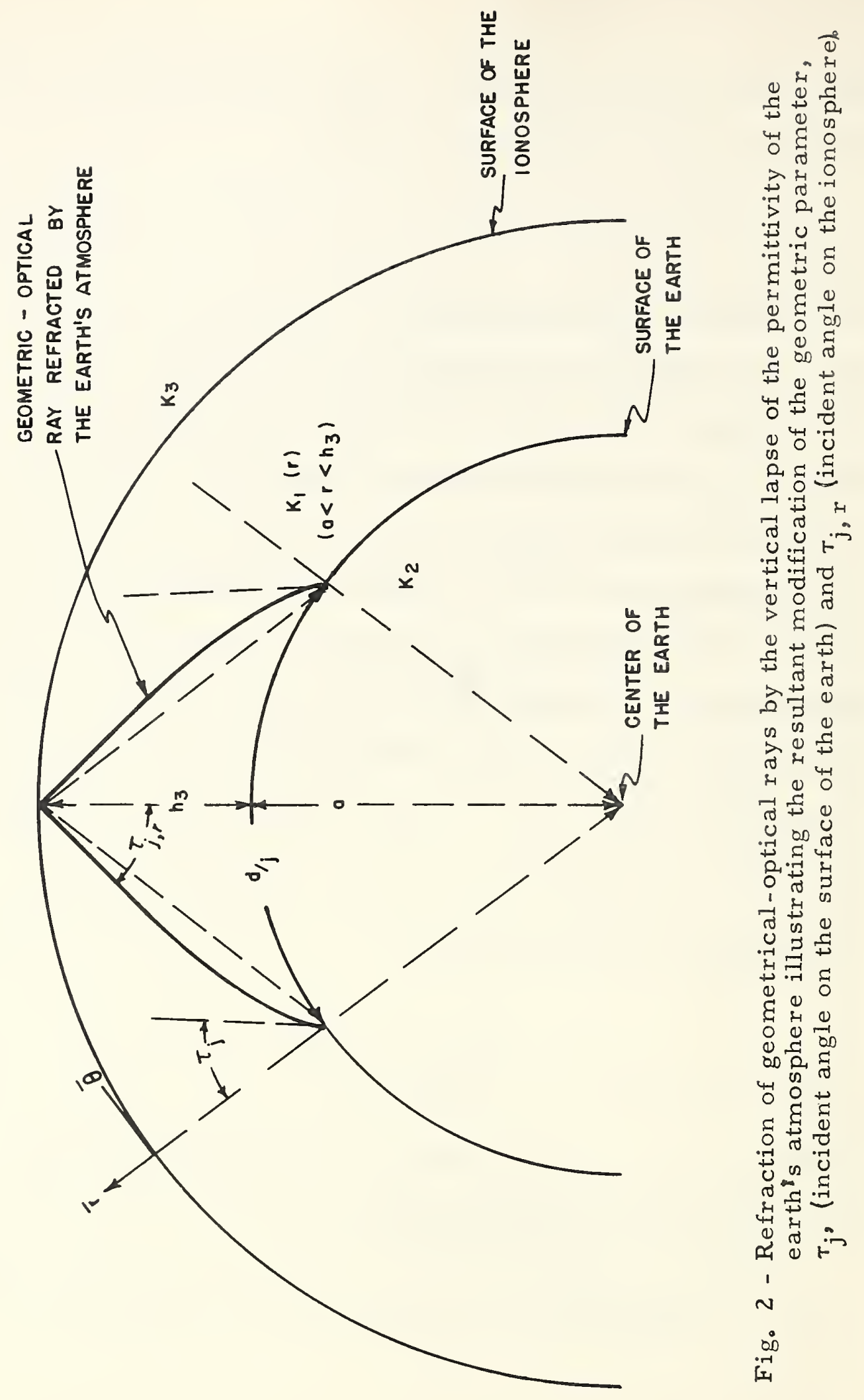




$$
\begin{aligned}
& \sin \tau_{j, r}=\frac{1}{\Delta_{j}} a \sin \frac{\theta}{2 j} \\
& \cos \tau_{j, r}=\frac{1}{\Delta_{j}}\left[a(1-\cos \theta / 2 j)+h_{3}\right]
\end{aligned}
$$

$$
\sin \tau_{j}=\frac{1}{\Delta_{j}}\left(\alpha+h_{3}\right) \cos \theta / 2 j
$$

$\cos \tau_{j}=\frac{1}{\Delta_{j}}\left[a(\cos \theta / 2 j-1)+h_{3} \cos \theta / 2 j\right]$

$$
\Delta_{j}=\left[2 a\left(a+h_{3}\right)(1-\cos \theta / 2 j)+h_{3}^{2}\right]^{\frac{1}{2}}
$$

It should be noted in passing that the validity of ray tracing at low and very low frequencies should be further investigated when the thickness of the earth's atmosphere is of the order of magnitude of a wavelength or less. This problem is beyond the scope of this paper.

The factor $\sin ^{2} \tau_{j}(2)$ accounts for the observer and source dipole radiation patterns. The factor, $\propto_{j}$ accounts for the convergence or divergence of the rays as a result of reflection from the ionosphere and the earth respectively,

$\alpha_{j}=\left[1+\frac{h_{3}}{a}\right]\left[\frac{2 j \sin \frac{\theta}{2 j}}{\sin \theta}\right]^{\frac{1}{2}}\left[\frac{a\left(1-\cos \frac{\theta}{2 j}\right)+h_{3}}{\left(a+h_{3}\right) \cos \frac{\theta}{2 j}-a}\right]^{\frac{1}{2}}$. 
The factor, $F_{j}$, accounts for the presence of the earth at the source and the observer. It is assumed that the ray is not too close to or beyond the geometrical optical horizon. Then the Fresnel approximation of the ground reflection coefficients determines $\cdot F_{j}$ '

$$
F_{j} \sim\left[1+R_{e T}\left(\tau_{j}\right)\right]\left[1+R_{e R}\left(\tau_{j}\right)\right]
$$

$\mathrm{R}_{\mathrm{eT}}$ is the plane reflection coefficient of Fresnel for the vertical electric source at an angle of incidence, $T_{j}$ and $R_{e} R$ refers to the observer. The Fresnel electric, $R_{e}$, and magnetic, $R_{m}$, reflection coefficients are,

$R_{e}\left(r_{j}\right)=\frac{\frac{k_{2}^{2}}{k_{1}^{2}} \cos \tau_{j}-\left[\frac{k_{2}^{2}}{k_{1}^{2}}-\sin ^{2} \tau_{j}\right]^{\frac{1}{2}}}{\frac{k_{2}^{2}}{k_{1}^{2}} \cos \tau_{j}+\left[\frac{k_{2}^{2}}{k_{1}^{2}}-\sin ^{2} \pi_{j}\right]^{\frac{1}{2}}}$,

$$
R_{m}\left(\tau_{j}\right)=\frac{\cos \tau_{j}-\left[\frac{k_{2}^{2}}{k_{1}^{2}}-\sin ^{2} \tau_{j}\right]^{\frac{1}{2}}}{\cos \tau_{j}+\left[\frac{k_{2}^{2}}{k_{1}^{2}}-\sin ^{2} \tau_{j}\right]^{\frac{1}{2}}},
$$




$$
\begin{aligned}
& k_{1}=\frac{\omega}{c} \eta_{1} \\
& k_{2}=\frac{\omega}{c}\left[\epsilon_{2}-i \frac{\sigma \mu_{0} c^{2}}{\omega}\right]^{\frac{1}{2}}
\end{aligned}
$$

The effective reflection coefficients, $C_{j}$, of primary interest in this paper, can be determined as follows ${ }^{7}$ :

$$
C_{j}=\frac{1}{j ! R_{e}} \frac{d^{j}}{d x^{j}}\left[\frac{1+A_{1} x}{1+A_{2} x+A_{3} x} 2\right]_{x=0}
$$

where,

$$
\begin{aligned}
& A_{1}=R_{m} T_{m m} \\
& A_{2}=R_{e} \cdot T_{e e}-R_{m} T_{m m} \\
& A_{3}=R_{e} R_{m}\left[T_{e e} T_{m m}+T_{e m} T_{m e}\right] .
\end{aligned}
$$

The T's refer to the ionosphere reflection coefficients, with subscripts e for the electric vector in the plane of incidence of the ray and $m$ for the magnetic vector in the plane of incidence of the ray and where the first subscript refers to the incident ray and the second subscript refers to the reflected ray. Thus, for example, Tem refers to the complex sky wave reflection coefficient. $T_{\text {em }}=\left|T_{\text {em }}\right| \exp \left[i A_{r} g T_{\text {em }}\right]$ with electric vector of incident ray

7 op. cit. 1. p. 296. 


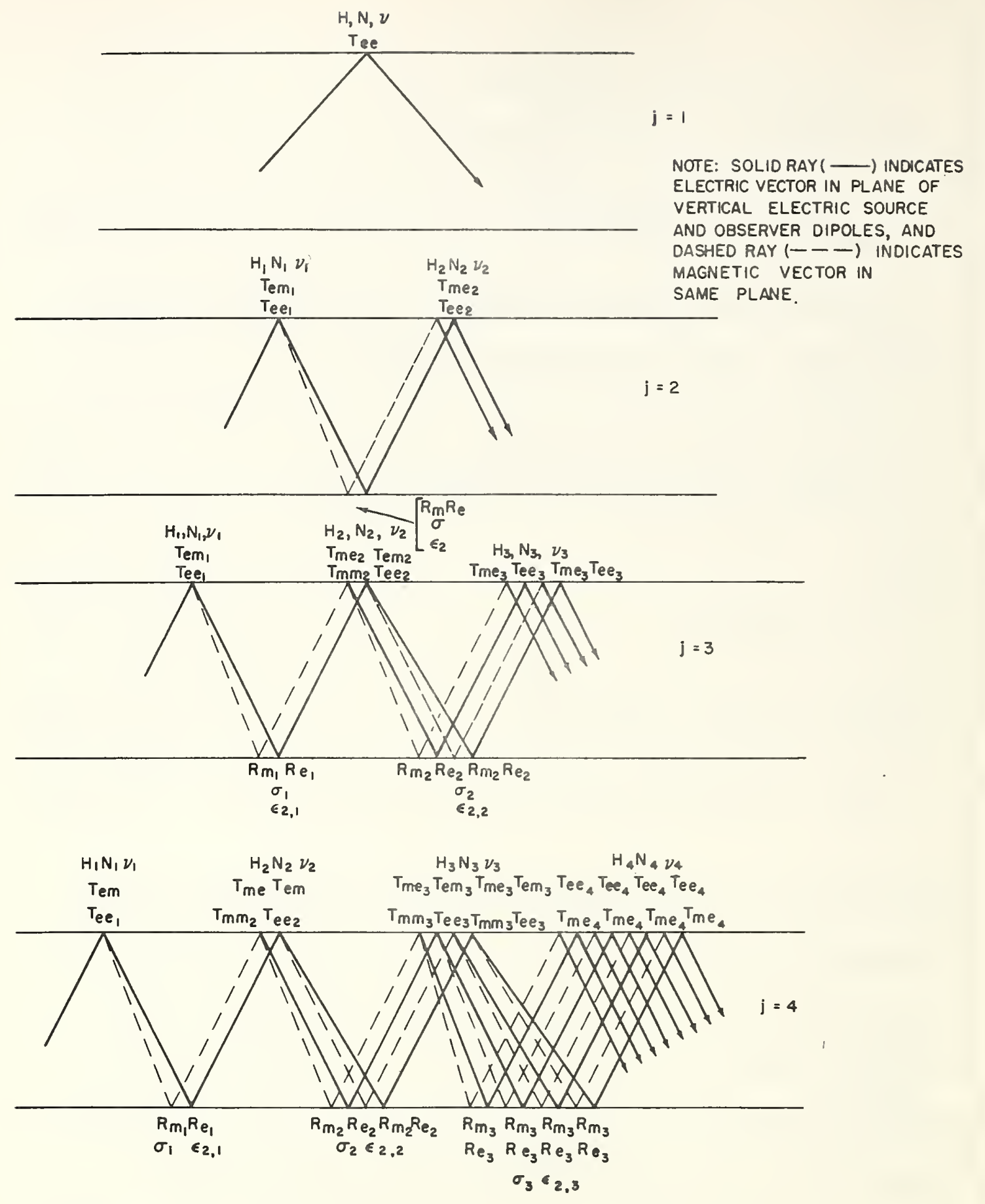

Fig. 3 - Geometrical-optical ray propagation mechanism illustrating the splitting of rays into vertical electric and vertical magnetic components applicable to vertical electric source, $\mathrm{s}$, and observer, o, dipoles. 
in plane of incidence and magnetic vector of reflected ray in plane of incidence. The effective reflection coefficients $(2), C_{j}$, are then, Fig. 4.,

$$
\begin{aligned}
& C_{1}=C_{1}\left(\tau_{1}\right)=T_{e e^{\prime}} \\
& C_{2}=C_{2}\left(T_{2}\right)=T_{e e_{1}} T_{e e_{2}} R_{e_{1}}+R_{m_{1}} T_{e m_{1}} T_{m e_{2}} \\
& C_{3}=C_{3}\left(T_{3}\right)=R_{e_{2}} R_{m_{1}} T_{e e_{3}} T_{e m_{1}} T_{m_{2}}+R_{e_{1}} R_{m_{2}} T_{e e_{1}} T_{e m_{2}} T_{m_{3}}
\end{aligned}
$$$$
+R_{e_{1}} R_{e_{2}} T_{e e_{1}} T_{e e_{2}} T_{e e_{3}}+R_{m_{1}} R_{m_{2}} T_{m m_{2}} T_{e m_{1}} T_{m e_{3}} \text {, }
$$$$
C_{4}=C_{4}\left(\tau_{4}\right)=R_{e_{1}} R_{e_{2}} R_{e_{3}} T_{e e_{4}} T_{e e_{3}} T_{e e_{2}} T_{e e_{1}}
$$

$+R_{e_{1}} R_{e_{2}} R_{m_{3}} T_{m^{2}} T_{e m_{3}} T_{e e_{2}} T_{e e_{1}}$

$+R_{e_{1}} R_{m_{2}} R_{e_{3}} T_{e e_{4}} T_{m e_{3}} T_{e m_{2}} T_{e_{e}}$

$+R_{e_{1}} R_{m_{2}} R_{m_{3}} T_{m_{4}} T_{m_{3}} T_{e m_{2}}{ }^{T} e_{1}$

$+\mathrm{R}_{\mathrm{m}_{1}} \mathrm{R}_{\mathrm{e}_{2}} \mathrm{R}_{\mathrm{e}_{3}} \mathrm{~T}_{\mathrm{ee}_{4}} \mathrm{~T}_{\mathrm{ee}_{3}} \mathrm{~T}_{\mathrm{me}_{2}} \mathrm{~T}_{\mathrm{em}}$

$+\mathrm{R}_{\mathrm{m}_{1}} \mathrm{R}_{\mathrm{e}_{2}} \mathrm{R}_{\mathrm{m}_{3}} \mathrm{~T}_{\mathrm{me}_{4}} \mathrm{~T}_{\mathrm{em}} \mathrm{T}_{\mathrm{me}_{2}} \mathrm{~T}_{\mathrm{em}}$

$+\mathrm{R}_{\mathrm{m}_{1}} \mathrm{R}_{\mathrm{m}_{2}} \mathrm{R}_{\mathrm{e}_{3}} \mathrm{~T}_{\mathrm{ee}_{4}} \mathrm{~T}_{\mathrm{me}_{3}} \mathrm{~T}_{\mathrm{mm}_{2}} \mathrm{~T}_{\mathrm{em}}$

$+\mathrm{R}_{\mathrm{m}_{1}} \mathrm{R}_{\mathrm{m}_{2}} \mathrm{R}_{\mathrm{m}_{3}} \mathrm{~T}_{\mathrm{me}_{4}} \mathrm{~T}_{\mathrm{mm}_{3}} \mathrm{~T}_{\mathrm{mm}_{2}} \mathrm{~T}_{\mathrm{em}}$ 。

The subscript for each $T$ or $R$ refers to the particular local conditions (constants $\sigma, \epsilon_{2}, \mathrm{~N}, v, H$ ) of the ionosphere or ground, Fig. 3. Thus, one of the most important advantages to the geometrical-optical theory is the convenience with which local 
conditions of a heterogeneous earth and ionosphere can be considered. For the particular case of the homogeneous earth and ionosphere, the coefficients, $C_{j}$, reduce as follows:

$C_{1}=C_{1}\left(\tau_{1}\right)=T_{e e}$

$C_{2}=C_{2}\left(T_{2}\right)=T_{e e}{ }^{2} R_{e}+R_{m} T_{e m} T_{m e}$,

$C_{3}=C_{3}\left(T_{3}\right)=2 R_{e} R_{m} T_{e e} T_{e m} T_{m e}+R_{e}^{2} T_{e e}{ }^{2}$

$+\mathrm{R}_{\mathrm{m}}^{2} \mathrm{~T}_{\mathrm{mm}} \mathrm{T}_{\text {em }} \mathrm{T}_{\mathrm{me}}$,

$C_{4}=C_{4}\left(T_{4}\right)=R_{e} R_{m}^{2} T_{e m}{ }^{2} T_{m e}{ }^{2}+3 R_{e}{ }^{2} R_{m} T_{e e}{ }^{2} T_{e m} T_{m e}$

$+2 R_{e} R_{m}^{2} T_{e e} T_{m m} T_{e m} T_{m e}+R_{m}{ }^{3} T_{m m}{ }^{2} T_{e m} T_{m e}$

$+R_{e}^{3} T_{e}^{4}$

These formulas can be derived intuitively, Fig. 3, by a consideration of each local reflection between the earth and the ionosphere and a concomitaint splitting of each ray into electric and magnetic components noting that the source radiates only vertical polarization (electric vector in the plane of incidence), and the observer can only receive vertical polarization.

Irrespective of the order of $j$, the effective reflection coefficient, $C_{j}$, obviously is dependent upon a complicated 
combination of only four basic lonosphere reflection coefficients, $\mathrm{T}_{\text {ee }}, \mathrm{T}_{\mathrm{em}}, \mathrm{T}_{\mathrm{me}}$ and $\mathrm{T} \mathrm{mm}$, the evaluation of which is the most difficult aspect of the geometrical-optical theory.

The argument of this paper will be confined to the region,about 10 kilocycles, hence, the numerical details of evaluating the ionosphere reflection coefficients can be simplified by the quasilongitudinal approximation formulated by Budden ${ }^{8}$. The wave number, $k_{3}$, of the ionosphere is represented in dual form,

$$
\begin{aligned}
k_{3} & =\frac{\omega}{c} \eta_{0} \\
& =\frac{\omega}{c} \eta_{e}
\end{aligned}
$$

where two waves are propagated upward with complex index of refraction $\eta_{0}$ for the ondinary wave and $\eta_{e}$ for the extraordinary wave. In terms of the two indexes, $\eta_{0}$ and $\eta_{e}$, and the corresponding transmitted wave normals determined by Snell's

$$
\sin \tau_{j, r}=\eta_{e} \sin \Theta_{\varrho}
$$

the four basic reflection coefficients can be determined,

$$
\begin{aligned}
T_{e e}=\Delta_{b}-1\left\{\left(\eta_{0}\right.\right. & \left.+\eta_{e}\right)\left(\cos ^{2} \tau_{j, r}-\cos \theta_{0} \cos \theta_{e}\right) \\
& \left.+\left(\eta_{0} \eta_{e}-1\right)\left(\cos \theta_{0}+\cos \theta_{e}\right) \cos \nabla_{j, r}\right\}
\end{aligned}
$$

K. G. Budden, op. cit. 3 . 


$$
\begin{aligned}
T_{m m}=\Delta_{b}-1\left\{\left(\eta_{0}+\eta_{e}\right)\left(\cos ^{2} T_{j, r}-\cos \theta_{o} \cos \theta_{e}\right)\right. \\
\left.-\left(\eta_{0} \eta_{e}-1\right)\left(\cos \theta_{0}+\cos \theta_{e}\right) \cos T_{j, r}\right\} \\
T_{e m}=\Delta_{b}{ }^{-1}\left\{2 i \cos \tau_{j, r}\left(\eta_{0} \cos \theta_{o}-\eta_{e} \cos \theta_{e}\right)\right\} \\
T_{m e}=\Delta_{b}{ }^{-1}\left\{2 i \cos \tau_{j, r}\left(\eta_{o} \cos \theta_{e}-\eta_{e} \cos \theta_{o}\right)\right\}
\end{aligned}
$$

where

$$
\begin{gathered}
\Delta_{b}=\left(\eta_{0}+\eta_{e}\right)\left(\cos ^{2} \tau_{j, r}+\cos \theta_{o}\right. \\
\left.\cos \theta_{e}\right)+\left(\eta_{0} \eta_{e}+1\right)\left(\cos \theta_{0}\right. \\
\left.+\cos \theta_{e}\right) \cos \gamma_{j, r} .
\end{gathered}
$$

The complex index of refraction " $\mathrm{C}$ is calculated by the quasilongitudinal approximation,

$$
\begin{aligned}
\eta_{\mathrm{e}}^{2} & =1-i \frac{\omega_{r}}{\omega} \exp \pm i \phi_{1} \\
\tan \phi_{1} & =\frac{\omega_{\mathcal{L}}}{v} \\
\frac{\omega_{r}}{\omega} & =\frac{\omega_{\mathrm{cr}}^{2}}{\omega_{v^{2}+\omega_{\mathcal{L}}^{2}}} \\
\omega_{\mathrm{cr}}{ }^{2} & =\frac{\mathrm{Ne}^{2}}{k \mathrm{~m}} \\
\omega_{\mathcal{L}} & =\frac{\mu_{0} \mathrm{H}}{\mathrm{m}},
\end{aligned}
$$

where $\omega_{c r}$ is the critical or plasma frequency of the electrons and $\omega_{\mathcal{L}}$ is the gyro frequency.

$\mp$ The value of $\eta$ should be selected in the fourth quadrant of the complex plane, i.e., $\operatorname{Im} \eta$ is negative. 
The ionosphere reflection coefficients, $T_{\text {ee, }} \mathrm{T}_{\mathrm{mm}} \mathrm{T}_{\mathrm{em}} \mathrm{I}_{\mathrm{me}}$ were calculated for various incident angles, $\tau_{j}, r$, earth ${ }^{8} s$ magnetic field intensity parameter, $\phi_{l}$, and frequencies, $\omega / \omega_{\mathrm{r}}$. Tables, 1 to 15. The ground reflection coefficients, $R_{e}\left\langle\tau_{j}\right\rangle, R_{m}\left(\tau_{j}\right)$ and the ionosphere reflection coefficients, $r$, were calculated at the various distances, $d / j$, for the type 301 atmosphere constants at various frequencies, $\omega / \omega_{r}$ and $f$, Tables $16-58$. The correspondirg effective reflection coefficients, $C_{j},\langle j=1,2,3,4)$ were then determined, Tables 59 - 118. The transfer characteristic of the sky wave $E_{j}(\omega, d)$, was then determined in the frequency range $1 \mathrm{kc}$ to $100 \mathrm{kc}$, Tables 119 to 122 . The corresponding sky wave delays $\widehat{a}_{j}-a$ and the convergence coefficient $\propto_{j}$ are presented in Table 123. A "cross section" of the data is presented graphically, Figs. 523. It is quite possible with the aid of Tables 59 - 118 or Figs. 5 23 and eq. 2. to determine field strengths, $E_{j}(\omega, d)$, for a wide variety of examples, and construct transfer characteristics similar to those presented in Tables $119-122$, or Figs. 20 to 23. Since, however, the form of the function is of primary interest and the precise variation with distance only of secondary interest in the transient problem, the approximation, eq. 12, was employed to facilitate the computation.

The results of the computation of the reflection coefficients, $T$, 
were checked where possible with previous calculations 9, 10, and reasonable agreement was obtained. Previous calculations of effective reflection coefficient, $C_{j}$, were not available. Conclusions

The simplicity of the quasi-longitudinal approximation permits the calculation of a wide variety of examples in the neighborhood of 10 kilocycles and the transfer characteristic can reasonably be extended over this region. However, the complete description of the D-region and the $\mathbb{E}-$ region as a transfer characteristic requires a more rigorous evaluation of the reflection coefficients at the higher frequencies. Yabroff. ${ }^{11}$ and Bremmer ${ }^{12}$ hävé proposed methods which are rigorous for a sharply bounded ionosphere at the higher frequencies. The effects of "non-sharply" and/or diffusely bounded ionosphere seem to be an appropriate enhancement of the theory at the higher frequencies.

Additional corrections in the vicinity of distances, $d / j$, of 1200 statute miles or greater to determine the value of the diffracted

9

K. G. Budden, op. cit. 3.

10

J. R. Wait and L. B. Perry, "Calculations of Ionosphere Reflection Coefficients at Very Low Frequencies, "Jour. of Geof. Res. Vol.1.62, No. 1, March 1957.

11

I. W. Yabroff, "Reflection at a sharply Bounded Ionosphere, proc. I.R.E., Vol. 45, No. 6, June 1957, p. 750-753.

12

H. Bremmer, op, cit. 1 . 
sky wave are also appropiriate to further enhance the range of

application of the theory.

Acknowiedgements

The authors wish to acknowledge the partial support of this work by E. A. Lewis, of Air Force Cambridge Research Center, Bedford, Massachusetts, under Air Research and Development Command CSO \& A No. 58-28(AFCRC) or National Bureau of Standards Project 8330-11-8835. It was the original intent of this work to calculate the amplitude and phase of the continuous wave as a function of frequency (transfer characteristic). The calculation of the reflection coefficients and the eifective reflection coefficients at 10 kilocycles and 20 kilocycles was suggested by $K$. A. Norton in connection with the sky wave investigations of National Bureau of Standards Project 8330-11-8332. J. R. Wait suggested that the extension of the computation of these reflection coefficients over considerable frequency range would provide useful data for other projects. The authors wish to thank $\mathrm{E}$. A. Norton for the use of the type 301 atmosphere data. The drawings were prepared by $R$. E. Whitaker (82.0), Figs. $1-11$ and D. G. Harrison (83.0), Figs. $12-24$.

Eileen Brackett typed the manuscript. The tables were calculated and printed by. the I. B. M. 650-407 electronic data processing machine. 


\section{GLOSSARY}

$\mathbb{E}=$

$j=$

$\omega=$

$d=$

$t=$

$t^{4}=$

$t_{j}=$

$\mathrm{D}_{\mathrm{j}}=$

$a_{j}=$

$\eta_{1}=$

$I_{0} l=$

$x=$

$c=$

$=$

$\sigma=$

$\mu_{0}=$
$\mathbb{E}(\omega, d)$, the electric field strength, volts/meter.

The order of the time-modes $(j=1,2,3, \ldots)$.

The frequency, $\omega=2 \pi f$, radians/second.

The distance, meters.

The time, seconds.

The local time $(4,5)$.

The local time for $j$ th time-mode $(4,5,6,7)$.

The ray distance for $j$ th time-mode (8).

The ray propagation time $(5,7)$.

Index of refraction of air at the surface of the earth. $\eta_{1} \sim 1.000338$.

The dipole momentum, ampere-meters.

The universal constant,

$$
\kappa=\frac{\varepsilon}{c^{2} \mu_{0}}
$$

The speed of light. c $\sim 2.997925\left(10^{8}\right)$ meters/second. A value of $2: 997951(108)$ is sometimes used.

The dielectric constant.

$E_{2} x$ 15, the dielectric constant of land.

$E^{2}$ 30, the dielectric constant of water.

The conductivity, mhos/meter.

$\sigma \sim 0.005$, the conductivity of land.

$\sigma \sim 5$, the conductivity of sea-water.

A universal constant, the permeability of space, $\mu_{0}=4 \pi(10-7)$. 
$\theta=\quad$ The effective radian distance aiong the surface of the earth, Fig. 1, $19 \%$.

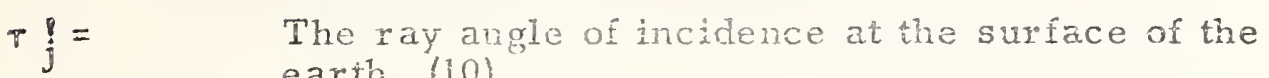
earth, (10).

$\tau_{j}=$

The effective ray angle of incidence at the surface of the earth, Fig. 1, (15, 16).

$\tau_{j, r}=$

$h_{3}=$

$\propto^{\nabla}=$

$\propto_{1}^{p}=$

$t_{0}^{0}=$

$t_{j}^{\prime}=$

$\bar{a}_{j}-a=$

$\propto_{j}=$

$I_{j}=$

$R_{e}(T)=$

The effective ray argle of incidence ar the surface of the ionospinere, Fig. $1,\langle 13,14\rangle$.

The height of the ionosphere, meters, Fig. 1.

Effective earth rodius factor at the surface of the earth for the tangentray, $\propto^{8}=0.75$.

The modified effective earth radius factor for the bending of rays passing through the atmosphere. (12).

The ground wave local time $\{4,5 \%$

The siky-wave local time $\{6,7\}$

Sky wave delay. $(5,7)$.

The convergence-divergence factor for rays reflecting from the ionosphere and the earth $\{18\}$.

The ground attenuation factor for the source and the observer (19).

$R_{m}(T)=$ The vertical magnetic ground reflection coefficient $\{21 \%$

$x_{1}=$

The wave number of air. $(22)$.

$k_{2}=$

The wave number of the earth. (22)。

$c_{j}=$

The effective refiection coefficient for propagation between the earth and the ionosphere (23).

$T_{\text {eé }}=$

Ionosphere reflection coefficient for vertical electric vector in plane of incidence for incident ray and vertical electric vector in plane of incidence for reflected ray. $(31)$. 


\begin{tabular}{|c|c|}
\hline $\mathrm{T}_{\mathrm{em}}=$ & $\begin{array}{l}\text { Ionosphere reflection coefficient for vertical electric } \\
\text { vector in plane of incidence of incident ray and } \\
\text { vertical magnetic vector in plane of incidence of } \\
\text { reflected ray (33). }\end{array}$ \\
\hline $\mathbb{T}_{\mathrm{me}}=$ & $\begin{array}{l}\text { Ionosphex reflection coefficient for vertical magnetic } \\
\text { vector in plane of incidence of incident ray and vertical } \\
\text { magnetic vector in plane of incidence of reflected } \\
\text { ray }(34) \text {. }\end{array}$ \\
\hline $\mathrm{T}_{\mathrm{mm}}=$ & $\begin{array}{l}\text { Ionosphere reflection coefficient for vertical magnetic } \\
\text { vector in plane of incidence of incident ray and } \\
\text { vertical magnetic vector in plane of incidence of } \\
\text { reflected ray (32). }\end{array}$ \\
\hline$k_{3}=$ & The wave number of the ionosphere $(29)$. \\
\hline$\Psi=$ & $\begin{array}{l}\text { The "take-off" angle for Norton's type } 301 \\
\text { atmosphere ray tracing lechniques. }\end{array}$ \\
\hline$a=$ & The radius of the earth, meters. a $\cdots 6.36739\left(10^{6}\right)$. \\
\hline$\eta_{0}=$ & $\begin{array}{l}\text { The complex index of refraction for the ordinary wave } \\
\text { in the ionosphere (29) (36). }\end{array}$ \\
\hline$\eta_{e}=$ & $\begin{array}{l}\text { The complex index of refraction for the extraordinary } \\
\text { wave in the ionosphere. }(29(36) \text {. }\end{array}$ \\
\hline$\theta_{0}=$ & $\begin{array}{l}\text { The direction of the wave normal of the ordinary wave } \\
\text { in the ionosphere }(30) \text {. }\end{array}$ \\
\hline$\theta_{\mathrm{e}}=$ & $\begin{array}{l}\text { The direction of the wave normal of the extraordinary } \\
\text { wave in the ionosphere }(30) \text {. }\end{array}$ \\
\hline$\phi_{1}=$ & The earth's magnetic field parameter (37). \\
\hline$\omega_{\mathrm{r}}=$ & A frequency parameter (38). \\
\hline$\omega_{c r}=$ & $\begin{array}{l}\text { The critical or plasma frequency of the electrons (39). } \\
\omega_{\mathrm{cr}} 2=3.1824858\left(10^{9}\right) \mathrm{N}\end{array}$ \\
\hline$\omega_{\mathscr{L}}=$ & The gyro frequency $(40) \omega_{\mathcal{L}}=1.75888\left(10^{7}\right) \mathrm{H}_{\mathrm{m}}$ \\
\hline $\mathbb{H}=$ & The earth's magnetic field intensity. $H \sim 0.535$ gauss. \\
\hline$N=$ & The number of electrons per cubic centimeter. \\
\hline
\end{tabular}


$v=$

$\mathrm{e} / \mathrm{m}=$
The collision frequency.

A universal constant, the ratio of charge to mass of the electron. 

Fig. 1 Coordinate system illustrating the geometry of the optical ray theory of the propagation of radio waves between the earth and the ionosphere from a source, $s$, to an observers, 0.

Fig. 2 Refraction of geometrical-optical rays by the vertical lapse of the permittivity of the earth's atmosphere illustrating the resultant modification of the geometric parameter, $T_{\dot{p}}$, (incident angle on the surface of the earth) and $\tau_{j, r}$ (incident angle on the ionosphere).

Fig. 3 Geometrical-optical ray propagation mechanism illustrating the splitting of rays into vertical electric arid vertical magnetic components applicable to vertical electric source, $s$, and observer, o, dipoles.

Fig. 4 Ionosphere reflection coefficients (amplitude, $|\mathrm{T}|$, and phase, Arg $\mathrm{T} \mid$ assuming the quasilongitudinal approximation, for various incident angles, $T_{j}, r$ and earth's magnetic field parameter, $\phi_{1}$. $\omega / \omega_{\mathrm{r}}=0.3002$

Fig. 5 Ionosphere reflection coefficients lamplitude, $|\mathrm{T}|$, and phase, Arg T) assuming the quasilongitudinal approximation, for various incident angles, $T_{j}, I$ and earth's magnetic field parameter, $\phi_{1}$. $\omega / \omega_{r}=0.467$

Fig. 6 Ionosphere reflection coefficients (amplitude, $\left|T_{e e}\right|$, and phase, Arg $\left.T_{e e}\right)$ assuming the quasi-longitudinal approximation for various frequencies, $\omega / \omega_{r}$ and incident angles, $\tau_{j, r}$.

Fig. I Ionosphere reflection coefficients lamplitude, $\left|T_{\mathrm{mm}}\right|$, and phase, Arg $\mathrm{T}_{\mathrm{mm}}$ ) assuming the quasi-longitudinal approximation, for various frequencies, $\omega / \omega_{r}$ and incident angles, $\tau_{j}, x^{\circ}$ 
Fig. 8 Ionosphere reflection coefficients (amplitude, $\left|\mathrm{T}_{\mathrm{em}}\right|$, and phase, $\operatorname{Arg} \mathrm{T}_{\mathrm{em}}$ ) assuming the quasi-longitudinal approximation, for various frequencies, $\omega / \omega_{\mathrm{r}}$ and incident angles, $\tau_{j}, x^{*}$

Fig. 9 Ionosphere reflection coefficients (amplitude, $\left|T_{m e}\right|$, and phase, $\operatorname{Arg} \mathrm{T}_{\mathrm{me}}$ ) assuming the quasi-longitudinal approximation, for various frequencies, $\omega / \omega_{r}$ and incident angles, $\tau_{j, r}$.

Fig 10. Ionosphere (quasi-longitudinal) and ground (Fresnel) reflection coefficients (amplitucle, $|T|,|R|$, and phase, $\operatorname{Arg} T, \operatorname{Arg} R)$ and effective reflection coefficients (amplitude, $\left|C_{j}\right|$, and phase, $A \mathrm{rg}_{\mathrm{j}}$ ) for the special ray configuration of the Norton type 301 atmosphere, applicable to the pro pagation of waves between the D-region (h3 $=70$ kilometers) and the E-region (h3 $=90$ kilometers) of the ionosphere and the earth. $\omega / \omega_{\mathrm{r}}=0.3002, \phi_{1}=60^{\circ}, \sigma=0.005, \epsilon_{2}=15$, $f=20$ kilocycles.

Fig. 11 Ionosphere (quasi-longitudinal) and ground (Fresnel) reflection coefficients (amplitude, $|T|,|R|$, and phase, $\operatorname{Arg} T, \operatorname{Arg} R)$ and effective reflection coefficients (amplitude, $\left|C_{j}\right|$, and phase, $\operatorname{Arg} C_{j}$ ) for the special ray configuration of the Norton type 301 atmosphere, applicable to the propagation of waves between the D-region ( $h_{3}=70$ kilometers) and the E-region ( $\mathrm{h}_{3}=90$ kilometers) of the ionosphere and the earth. $\cos / \omega_{x}=0.467, \phi_{1}=60^{\circ}, \sigma=0.005, \epsilon_{2}=15$, $\mathrm{f}=20$ kilocycles.

Fig. 12 Ionosphere (quasi-longitudinal) and ground (Fresnel) reflection coefficients (amplitude, $|T|,|R|$, and phase, $\operatorname{Arg} T, \operatorname{Arg} R)$ and effective reflection coefficients (amplitude, $\left|C_{j}\right|$, and phase, $A_{r} \mathrm{C}_{j}$ ) for the special ray configuration of the Norton type 301 atmosphere, applicable to the propagation of waves between the $D$-region ( $\mathrm{h}_{3}=70$ kilometers) and the E-Region ( $h_{3}=90$ kilometers) of the ionosphere and the earth. 
$\mathrm{d} / \mathrm{j}=124$ miles, $\phi_{\mathrm{j}}=60^{\circ}, \sigma=0.005, \epsilon_{2}=15$

Fig. 13 Ionosphere (quasi-.longitudinal) and ground (Fresnel) reflection coefficients (amplitude, $|T|,|R|$, and phase, Arg $\left.T, A_{1} g R\right)$ and effective reflection coefficients (amplitude, $\left|C_{j}\right|$, and phase, $\left.\Lambda x g C_{j}\right)$ for the special ray configuration of the Norton type 301 atmosphere, applicable to the propagation of waves between the D-region (h3 $=70$ kilometers) and the $\mathrm{E}$-region ( $\mathrm{h} 3=90$ kilometers) of the ionosphere and the earth.

$\mathrm{d} / \mathrm{j}=479$ miles, $\phi_{1}=60^{\circ}, \sigma=0.005, \epsilon_{2}=15$

Fig. 14 Ionosphere (quasi-longitudinal) and ground (Fresnel) reflection coefficients (amplitude, $|\mathrm{T}|,|\mathrm{R}|$, and phase, Arg $\mathrm{T}, \mathrm{Arg} \mathrm{R})$ and effective reflection coefficients (ampitude, $\left|C_{j}\right|$, and phase, $\left.A r^{\prime} C_{j}\right)$ for the special ray configuration of the Norton type 301 atmosphere, applicable to the propagation of waves between the $\mathrm{D}$-region $\left(\mathrm{h}_{3}=70\right.$ kilometers) and the E-region (h3 $=90$ kilometers) of the ionosphere and the earth.

$\mathrm{d} / \mathrm{j}=1026$ miles, $\phi_{1}=60^{\circ}, \sigma=0.005, \epsilon_{z}=15$

Fig. 15 Effective quasi-Iongitudinal-Fresnel reflection coefficients (amplitude, $\left|\mathrm{C}_{\mathrm{j}}\right|$ and phase, Arg $C_{j}$ ) for the special ray configuration of the Norton type 301 atmosphere, applicable to the propagation of waves between the D-region ( $\mathrm{h}_{3}=70$ kilometers) and the $\mathrm{E}$-region

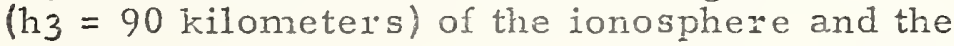
earth.

$\omega / \omega_{r}=0.3002, \phi_{1}=60^{\circ}, \sigma=0.005, \epsilon_{2}=15$, $f=20$ kilocycies.

Fig. 16 Effective quasi-longitudinal-Fresrel reflection coefficients (amplitude, $\left|\mathrm{C}_{\mathrm{j}}\right|$, and phase, Arg $C_{j}$ ) for the special ray configuration of the Norton type 301 atmosphere, applicable to the propagation of waves between the D-region ( $\mathrm{h}_{3}=70$ kilometers) and the E-region (h3 $=90$ kilometers) of the ionosphere and the earth.

$\omega / \omega_{r}=0.467, \phi_{1}=60^{\circ}, \sigma=0.005, \sigma_{2}=15$,

$f=20$ kilocycles. 
Fig. 17 Effective quasi-Jongitudinal-Fresnel reflection coefficients amplitude, $\left|C_{j}\right|$, and phase, $\operatorname{Arg} C_{j}$ ) for the special ray Configuration of the Norton type 301 atmosphere, applicable to the propagation of waves between the D-region ( $\mathrm{h}_{3}=70$ kilometers) and the E-region $\left(h_{3}=90\right.$ kilometers $)$ of the ionosphere and the earth.

$\mathrm{d} / \mathrm{j}=124$ miles, ...

Fig.18 Effective quasi-longitudinal-Fresnel reflection coefficients (amplitude, $\left|C_{j}\right\rangle_{\text {, and phase, }}$ Arg $C_{j}$ ) for the special ray configuration of the Norton type 301 atmosphere, applicable to the propagation of waves between the D.region $\left(h_{3}=70\right.$ kilometers $)$ and the $\mathrm{I}_{-}-\mathrm{region}$ (h3 $=90$ kilometers) of the ionosphere and the earth.

$\mathrm{d} / \mathrm{j}=479$ miles, $\ldots$

Fig. 19 teffective quasi-longitudinal- Eresnel reflection coefficients (amplitude, $\left|C_{j}\right|$, and phase, Arg $C_{j}$ ) for the special ray configuration of the Norton type 301 atmosphere, applicable to the propagation of waves between the D-region $\left(h_{3}=70\right.$ kilometers $)$ and the E-region ( $\mathrm{h}_{3}=90$ kilometers) of the ionosphere and the earth.

$\mathrm{d} / \mathrm{j}=1026$ miles, ...

Fig.20 Transfer.characteristic or "steady state" frequency characteristic, $\mathbb{E}_{0}(\omega, d)$, (amplitude $\left|E_{j}(\omega, d)\right|$, and phase, Arg $\left.E_{j}(\omega, d)\right)$ of the time-modes $(j=1,2)$ of the sky wave, assuming the quasi-longitudinal-Fresnel approximation. $d=100$ miles

Fig。21 Transfer characteristic or "steady state" frequency characteristic, $\mathbb{E}_{j}(\omega, d)$, (amplitude, $\left|E_{j}(\omega, d)\right|$, and phase, $\left.\operatorname{Arg} E_{j}(\omega, d)\right)$ of the time-modes $(j=1,2)$ of the sky wave, assuming the quasi-longitudinal-Fresnel approximation. $d=200$ miles 
Fig. 22 Transfer characteristic or "steady state" frequency characteristic, $E_{j}(\omega, d)$, (amplitude, $\left|E_{j}(\omega, d)\right|$, and phase, $\left.\operatorname{Arg} E_{j}(\omega, d)\right)$ of the time-modes $(j=1,2)$ of the sky wave, assuming the quasi-longitudinal-Fresnel approximation. $\mathrm{d}=500$ miles

Fig. 23 Transfer characteristic or "steady state" frequency characteristic, $\mathbb{E}_{j}(\omega, \mathrm{d})$, (amplitude, $\left|E_{j}(\omega, d)\right|$, and phase, $\left.\operatorname{Arg} E_{j}(\omega, d)\right)$ of the time-modes $(j=1,2)$ of the sky wave, assuming the quasi-longitudinal-Fresnei approximation. $\mathrm{d}=1000$ miles

Fig. 24 Observed oscillogramis (abscissa-time, ordinateamplitude) of sinusoidai radiofrequency pulse, illustrating the physical significance of the geometrical-optical time-modes for propagation between the earth and the ionosphere. 



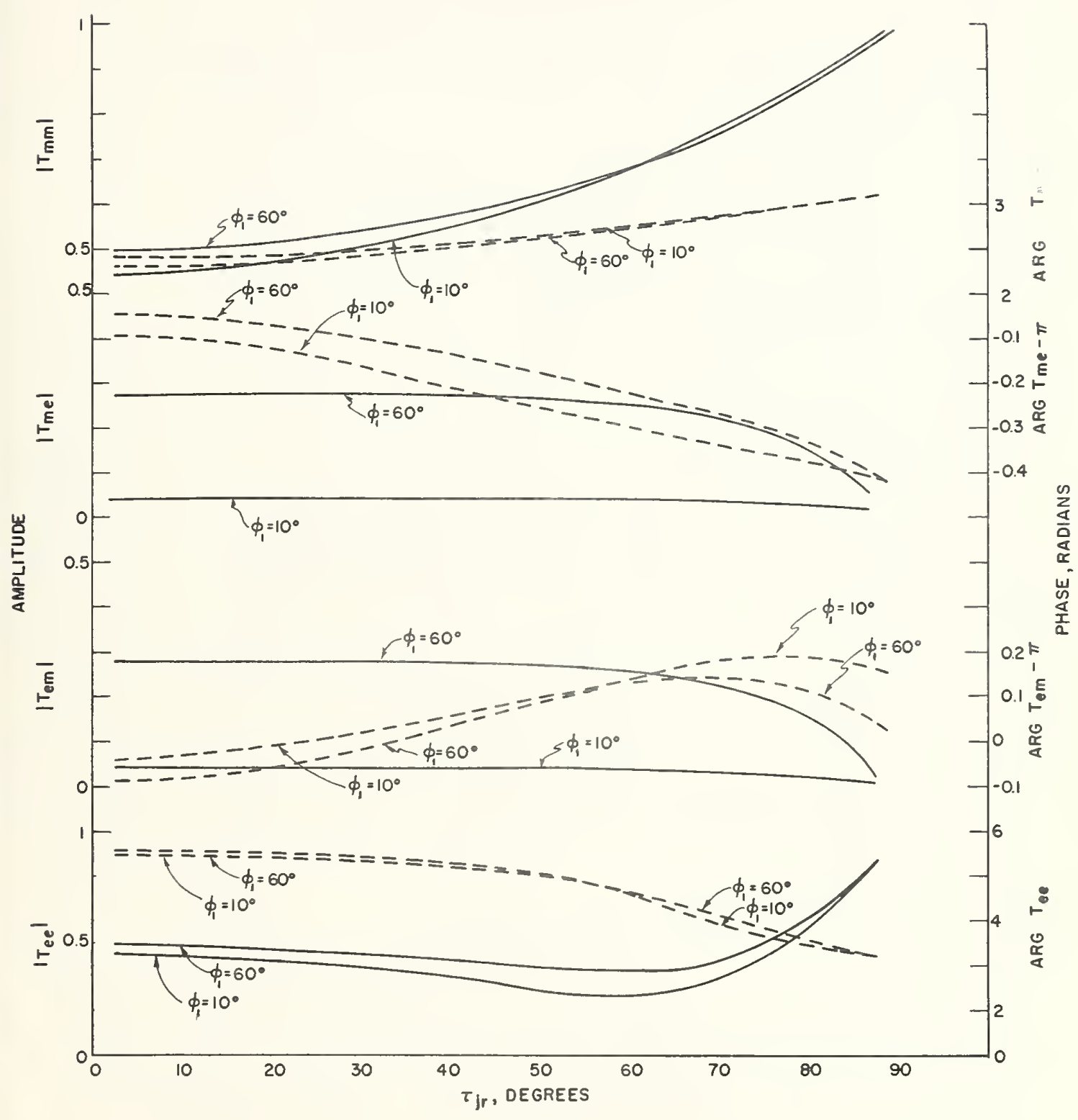

Fig. 4 - Ionosphere reflection coefficients (amplitude, $|\mathrm{T}|$, and phase, Arg T) assuming the quasi-longitudinal approximation for various incident angles, $T_{j}, r$ and earth's magnetic field parameter, $\phi_{1}$. 


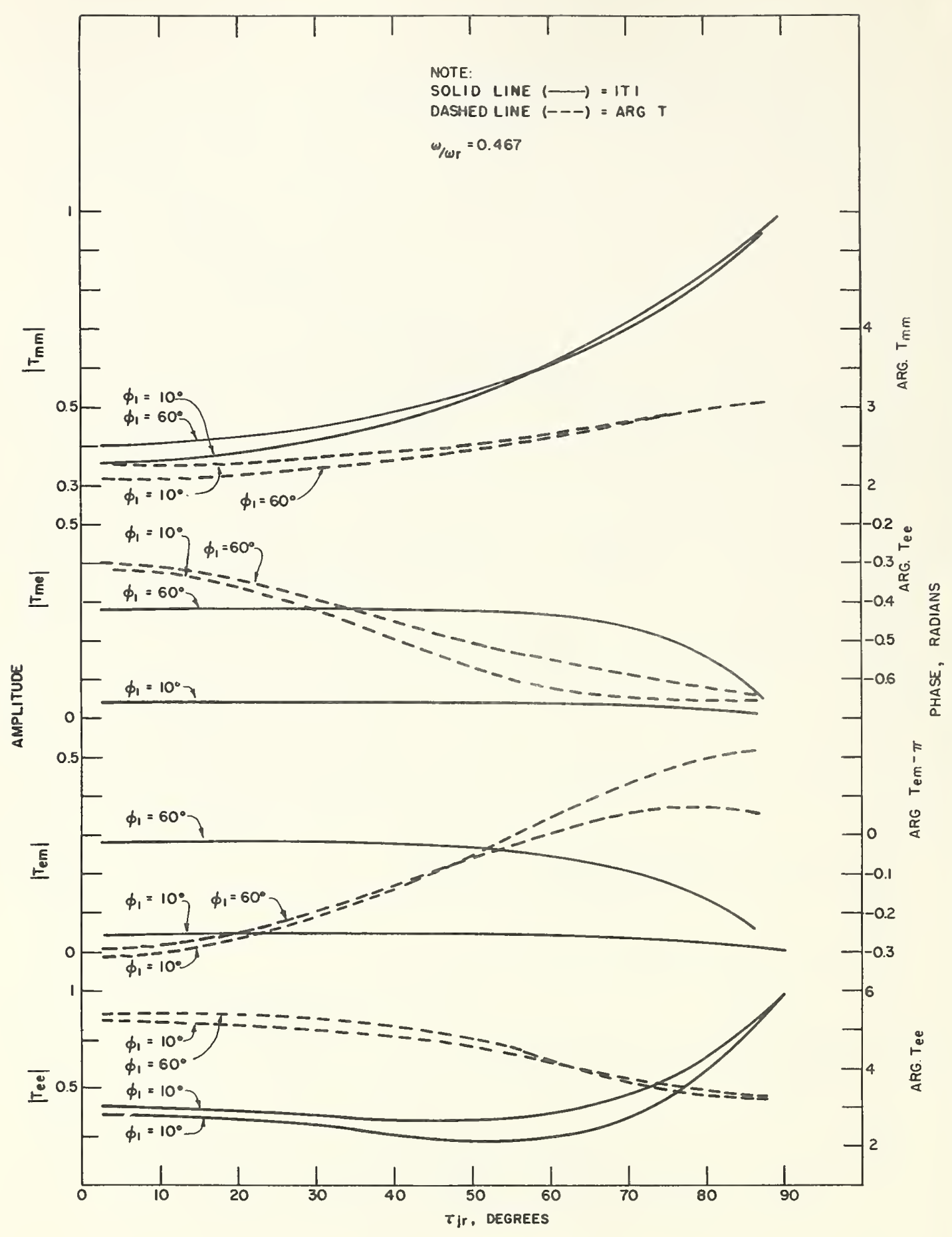

Fig. 5 - Ionosphere reflection coefficients (amplitude, $|\mathrm{T}|$, and phase, Arg T) assuming the quasi-longitudinal approximation for various incident angles, $\tau_{j, r}$ and earth's magnetic field parameter, $\phi_{1}$. 

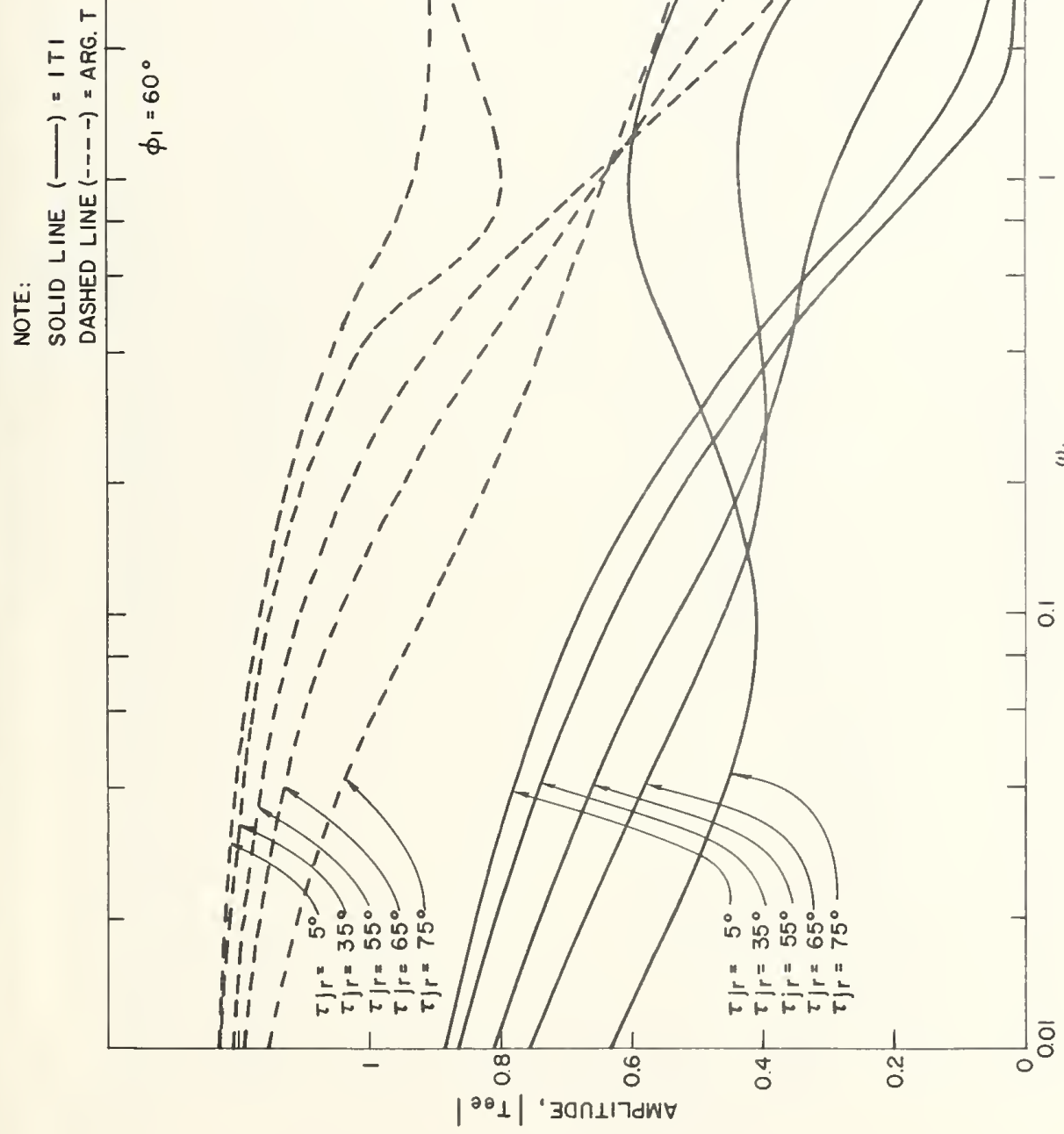

त.

\begin{tabular}{rl} 
& 0 \\
0 & 0 \\
0 & 0 \\
0 & 0 \\
$E$ & 0 \\
\hline
\end{tabular}

ฮี

3)

员䓪

ฮิ

$\stackrel{\infty}{\rightleftarrows}$

先

焉

$\circ$

प

.

पे

究害

$+3$

0.5

¿

동

in

0

in 


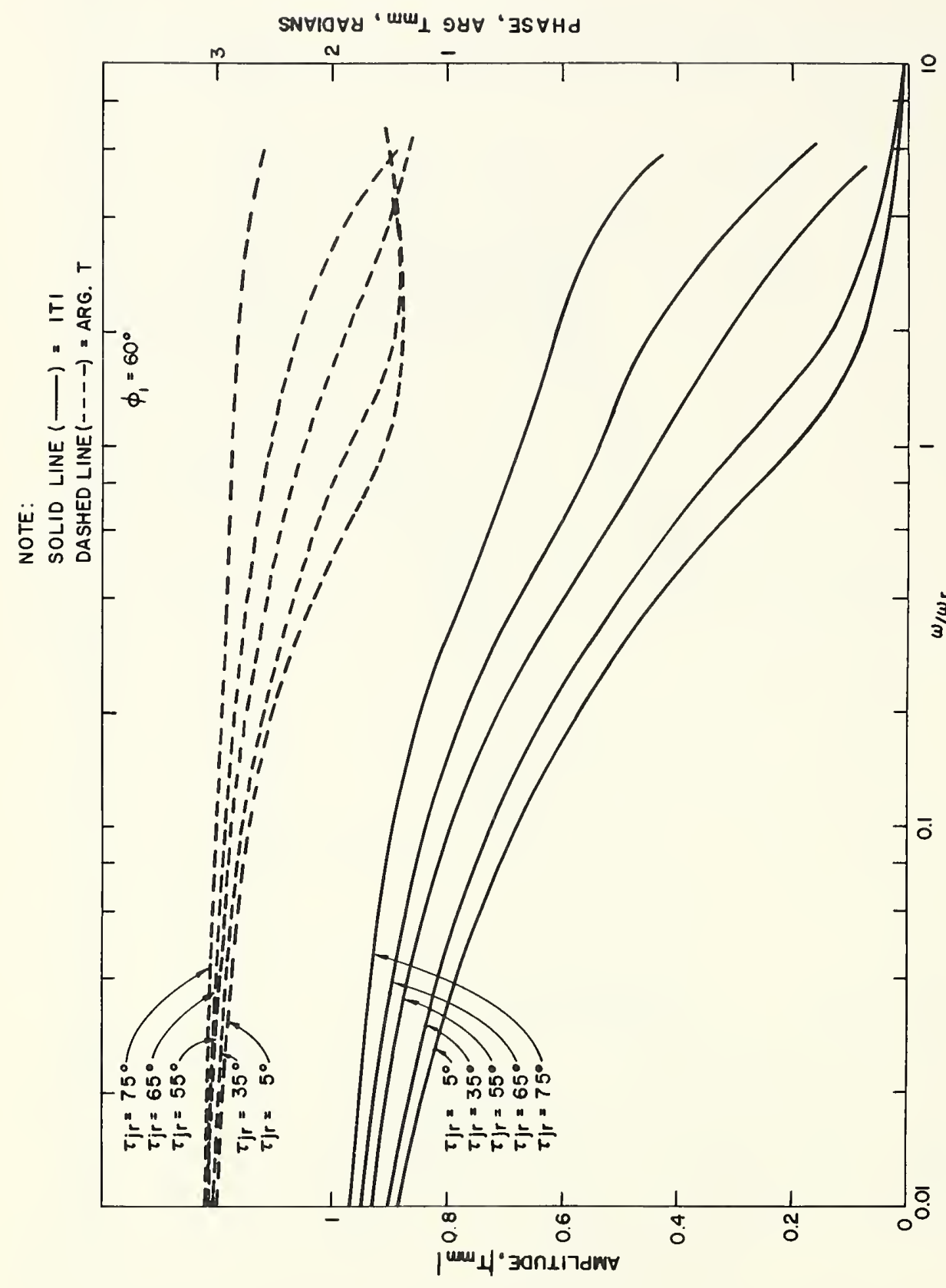

究

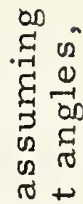

द्विष्ठ

द्विण

H.

की

$0^{2} 3^{2+1}$

究 3

ค

ชृ

त.

द्व

द्वि

स- H

i

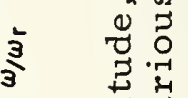

少

द्वे

तิ

$\stackrel{0}{+}$

.01

.

出.

๑

0

도용

耐

$\stackrel{1}{4}$

겅무

(1)

\&

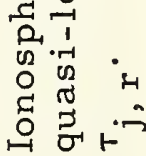

r

$\dot{\infty i n}_{1-1}^{-1}$ 


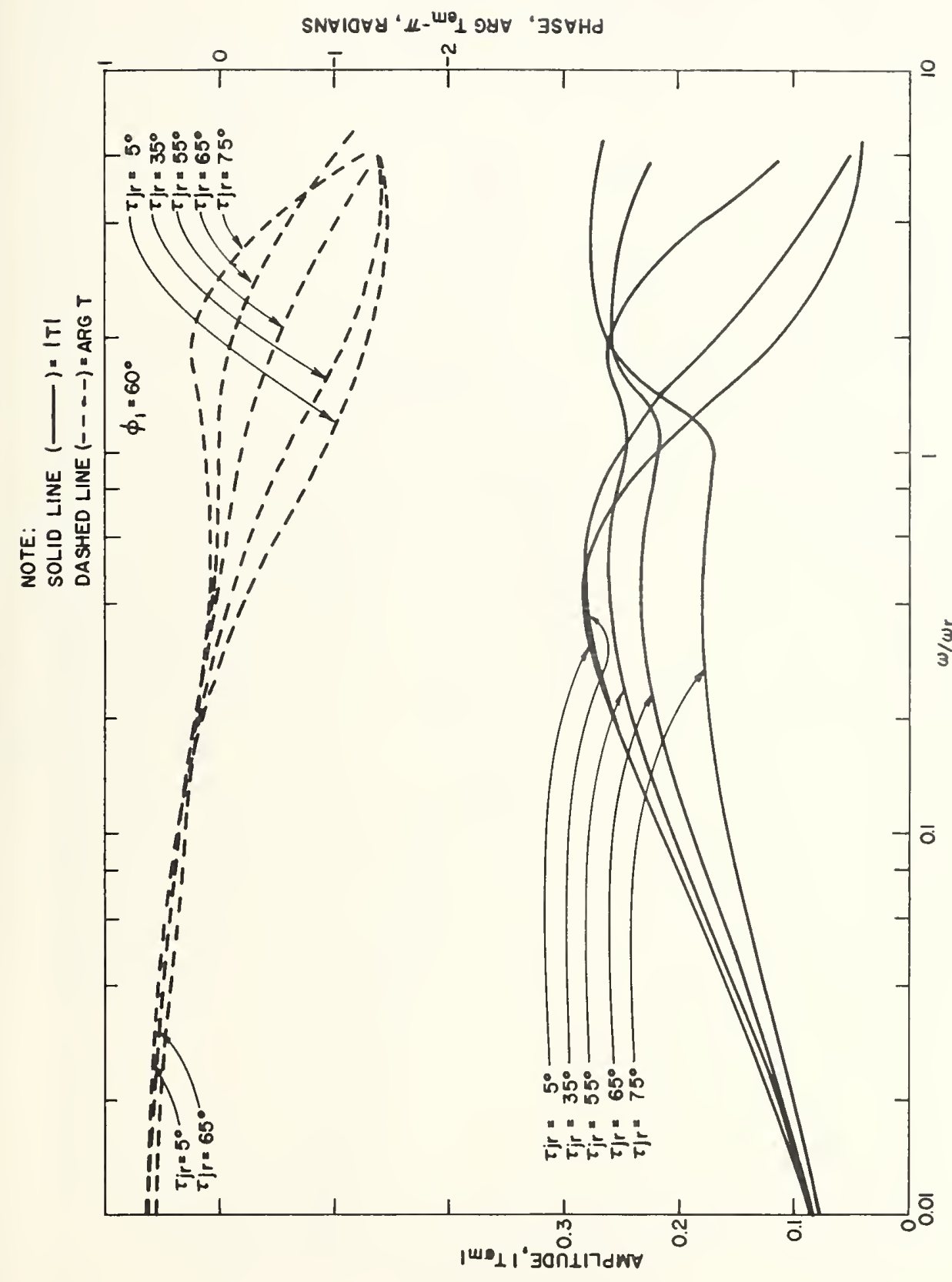

东

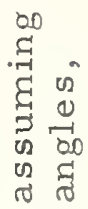

द्वि

$F-1$

or

से

iक

(n) $3^{i n}$

है. 3

प्व के

द्व

द्व

E-1

-

تृ

3 $\quad{ }_{3}^{3} \cdot 0$

$-1$

द्व

is

द

.

兽要

엉

दू مै

บ

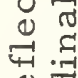

국

(1)

है

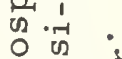

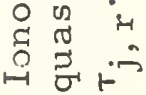

$\infty$

in 


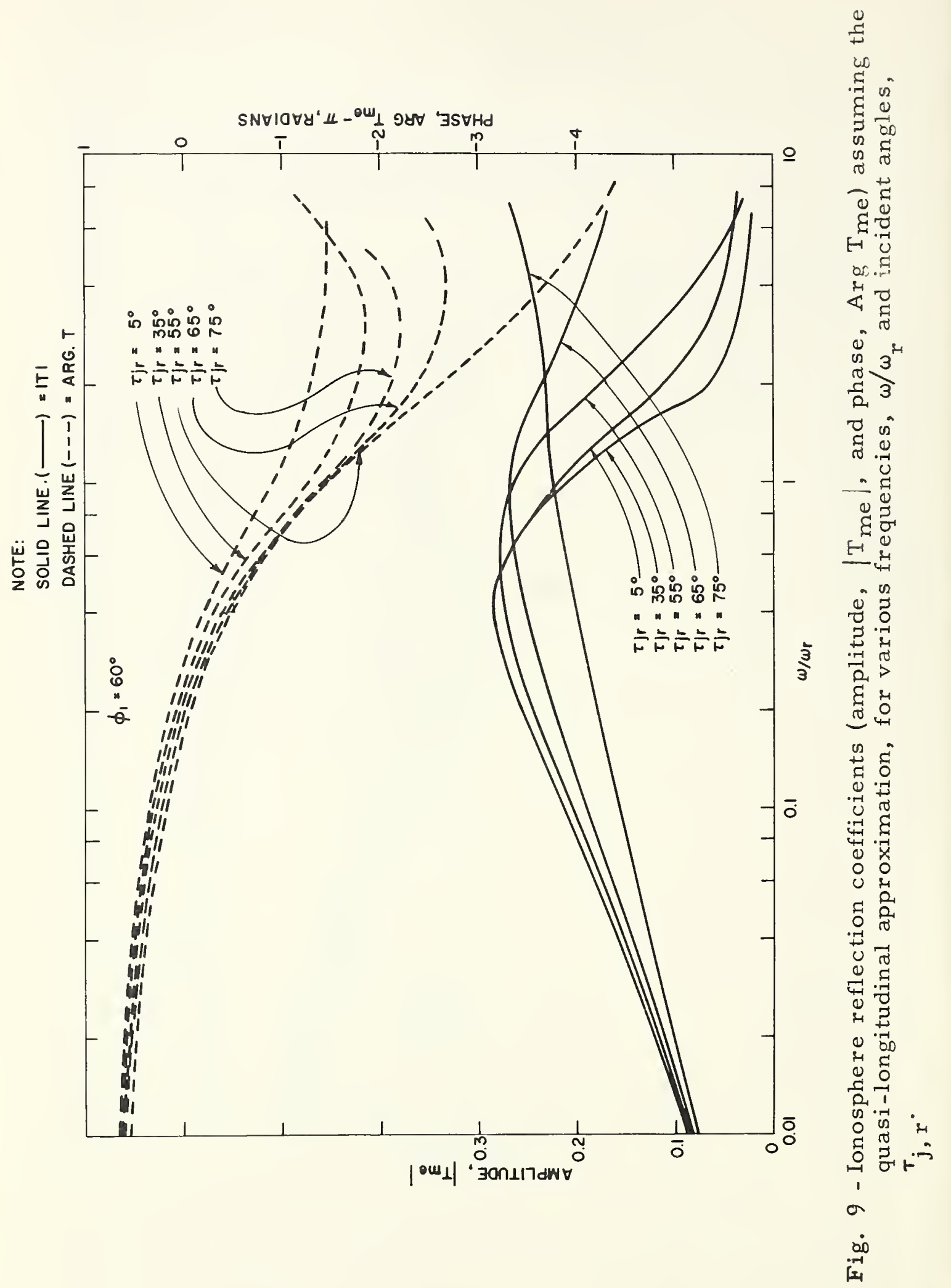




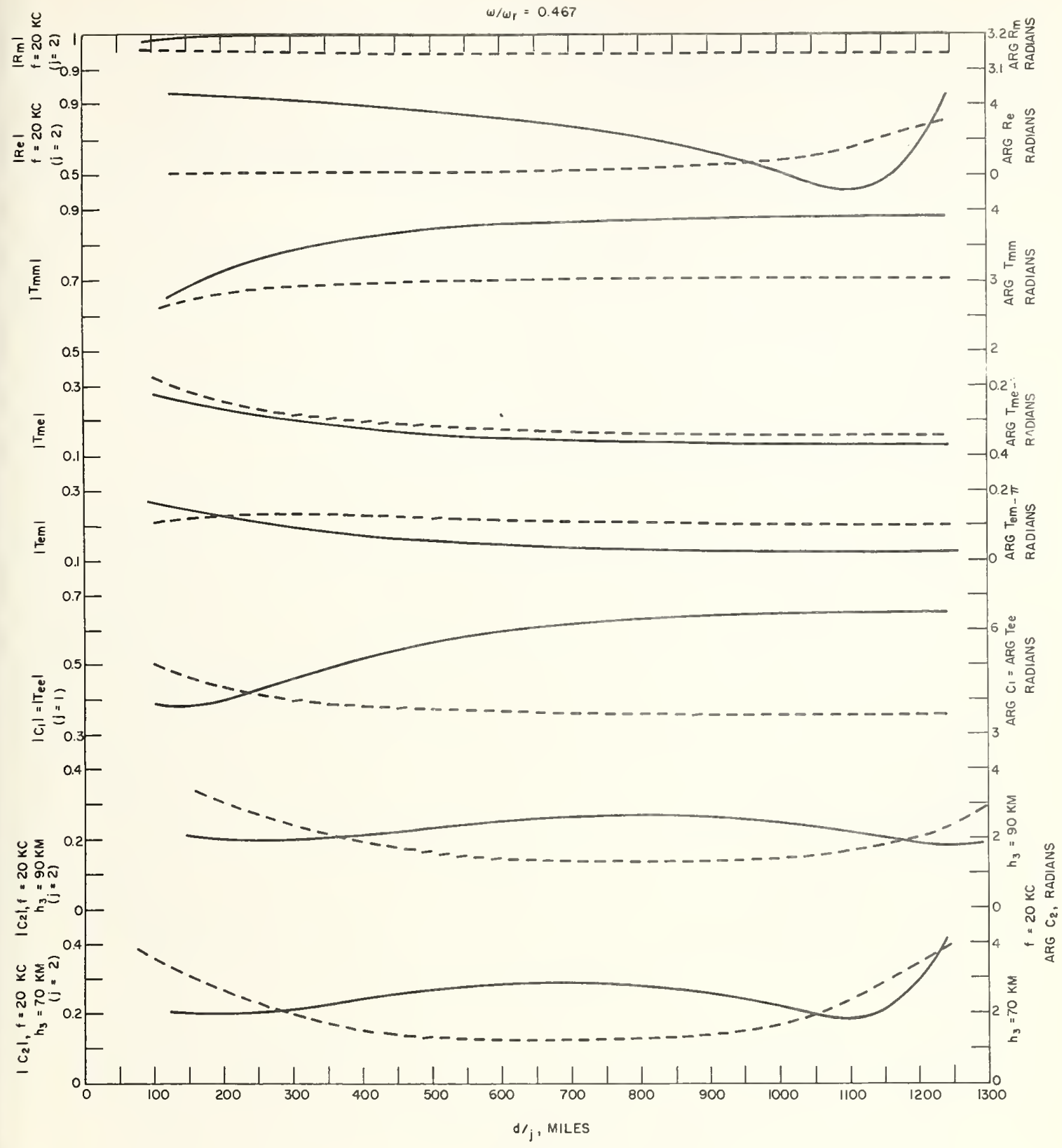

Fig. 10 - Ionosphere (quasi-longitudinal) and ground (Fresnel) reflection coefficients (amplitude, $|\mathrm{T}|,|\mathrm{R}|$, and phase, $\operatorname{Arg} T, \operatorname{Arg} R$ ) and effective reflection coefficients (amplitude, $\left|C_{j}\right|$, and phase, $\operatorname{Arg} C_{j}$ ) for the special ray configuration of the Norton type 301 atmosphere, applicable to the propagation of waves between the D-region ( $\mathrm{h}_{3}=70$ kilometers) and the E-region $\left(h_{3}=90\right.$ kilometers $)$ of the ionosphere and the earth. 


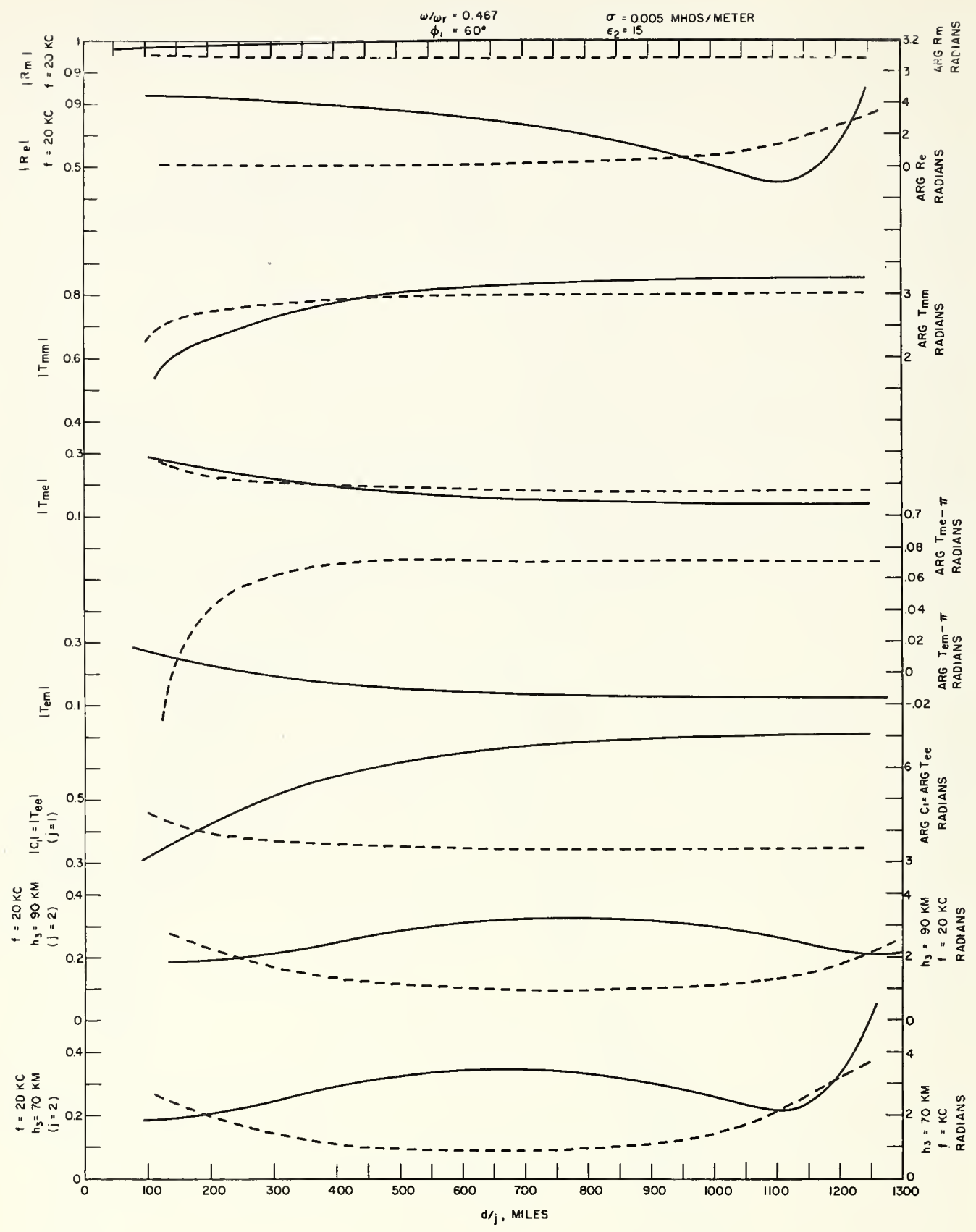

Fig. 11- Ionosphere (quasi-longitudinal) and ground (Fresnel) reflection coefficients (amplitude, $|\mathrm{T}|,|\mathrm{R}|$, and phase, Arg T, Arg R) and effective reflection coefficients (amplitude, $\left|C_{j}\right|$, and phase, Arg $C_{j}$ ) for the special ray configuration of the Norton type 301 atmosphere, applicable to the propagation of waves between the D-region $\left(h_{3}=70\right.$ kilometers) and the E-region $\left(h_{3}=90\right.$ kilometers $)$ of the ionosphere and the earth. 


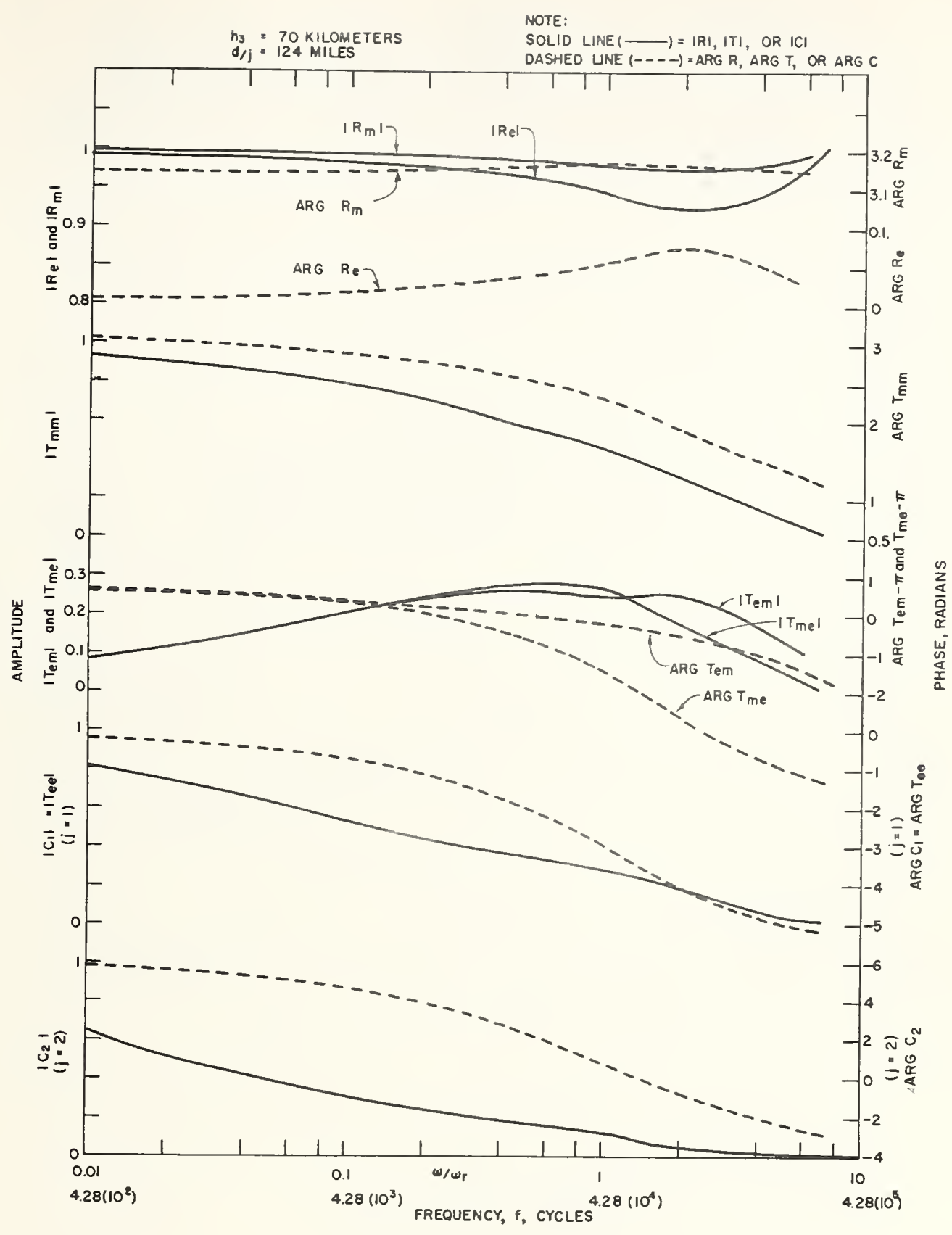

Fig. 12 - Ionosphere (quasi-longidutinal) and ground (Fresnel) reflection coefficients (amplitude, $|\mathrm{T}|,|\mathrm{R}|$, and phase, Arg T, Arg R) and effective reflection coefficients (amplitude, $\left|C_{j}\right|$, and phase, $\operatorname{Arg} C_{j}$ ) for the special ray configuration of the Norton type 301 atmosphere, applicable to the propagation of waves between the $\mathrm{D}$-region $\left(\mathrm{h}_{3}=70\right.$ kilometers and the E-region $\left(h_{3}=90\right.$ kilometers $)$ of the ionosphere and the earth. 


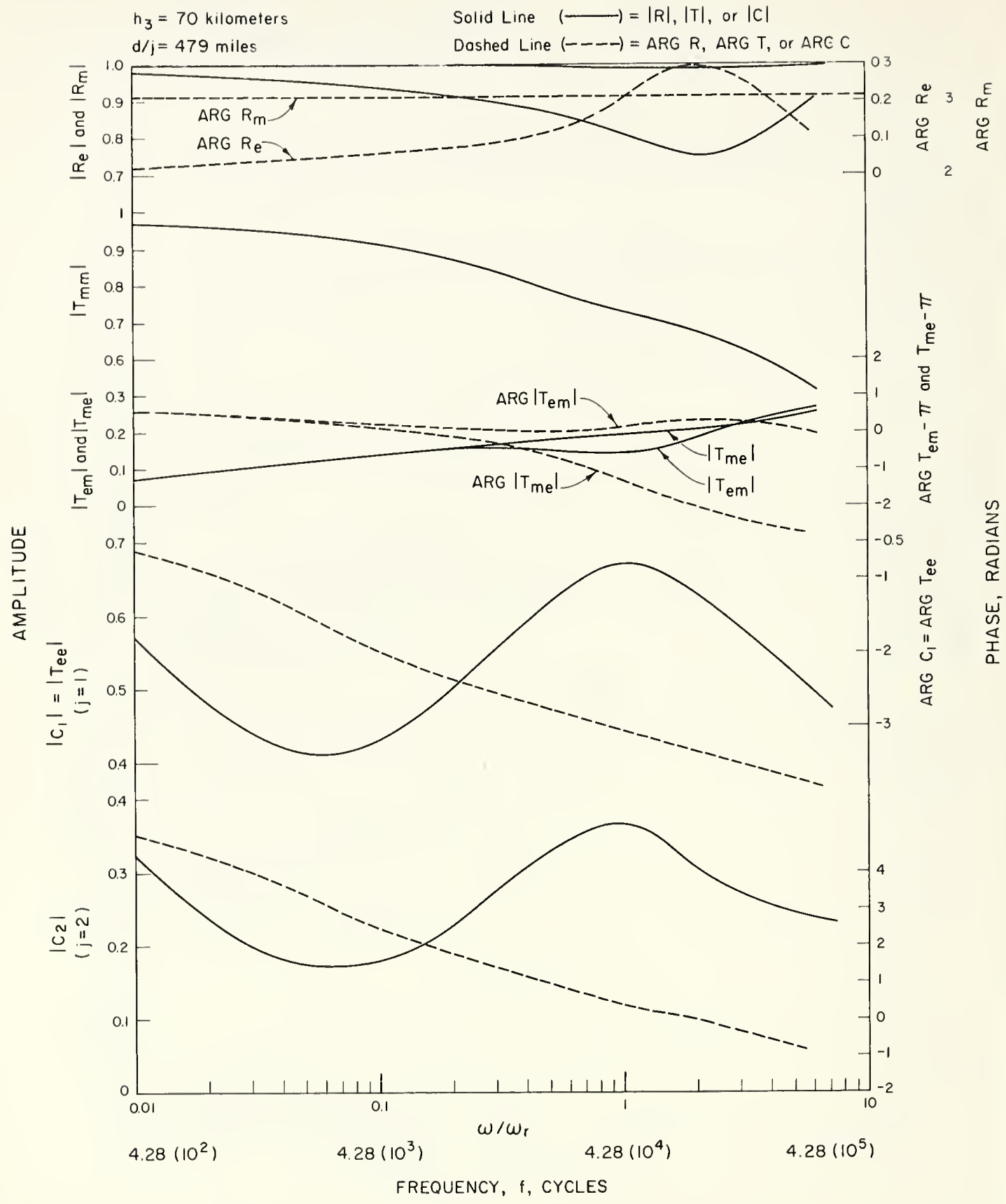

Fig. 13 - Ionosphere (quasi-longitudinal) and ground (Fresnel) rereflection coefficients (amplitude, $|\mathrm{T}|,|\mathrm{R}|$, and phase, $\operatorname{Arg} \mathrm{T}, \operatorname{Arg} \mathrm{R}$ ) and effective reflection coefficients (amplitude, $\left|C_{j}\right|$, and phase, Arg $C_{j}$ ) for the special ray configuration of the Norton type 301 atmosphere, applicable to the propagation of waves between the D-region ( $h_{3}=70$ kilometers and the E-region ( $h_{3}=90$ kilometers) of the ionosphere and the earth. 


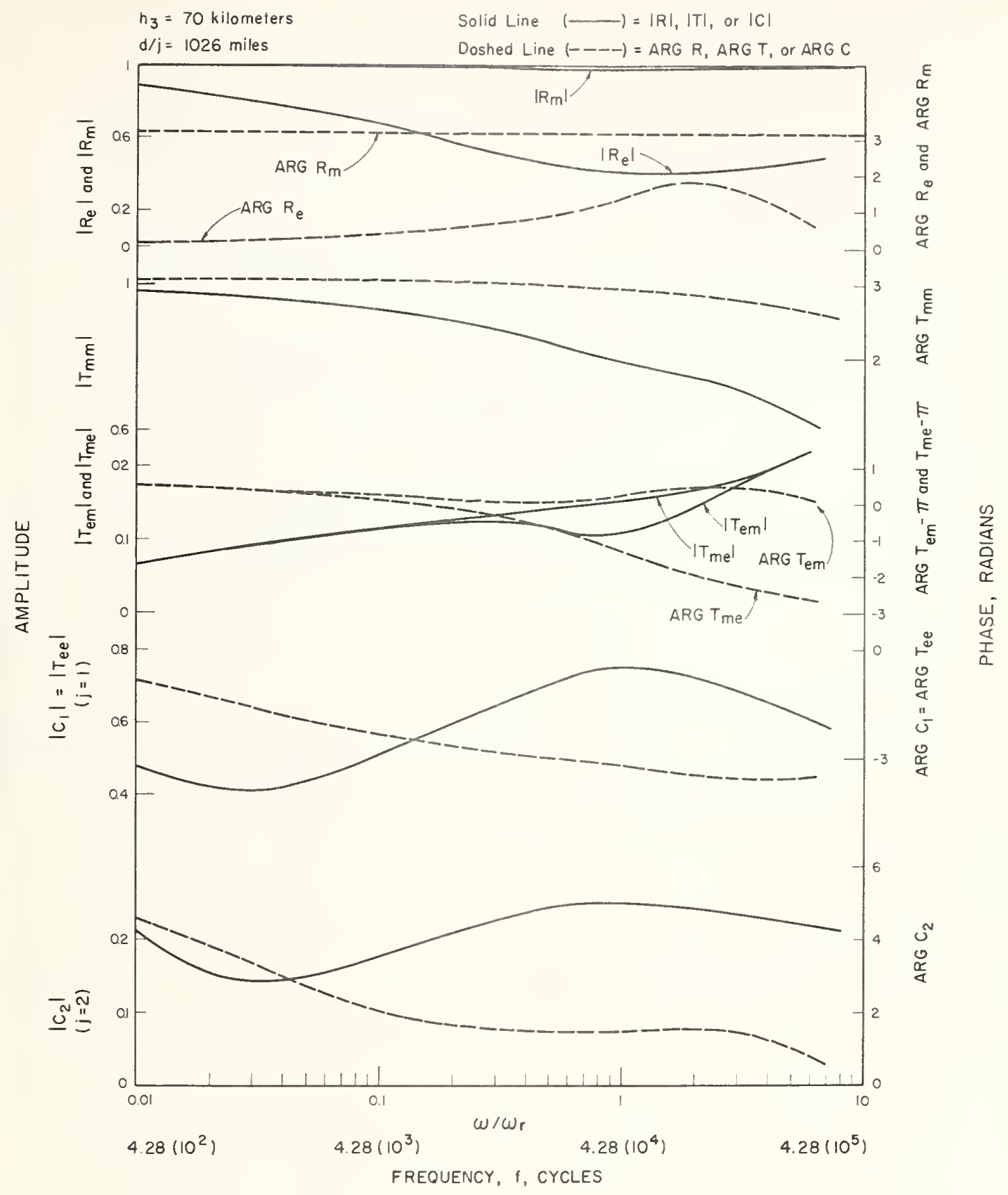

Fig. 14 - Ionosphere (quasi-longitudinal) and ground (Fresnel) reflection coefficients (amplitude, $|T|,|R|$, and phase, $\operatorname{Arg} T, \operatorname{Arg} R$ ) and effective reflection coefficients (amplitude, $\left|C_{j}\right|$, and phase, Arg $C_{j}$ ) for the special ray configuration of the Norton type 301 atmosphere, applicable to the propagation of waves between the D-region ( $h_{3}=70$ kilometers and the E-region ( $h_{3}=90$ kilometers) of the ionosphere and the earth. 


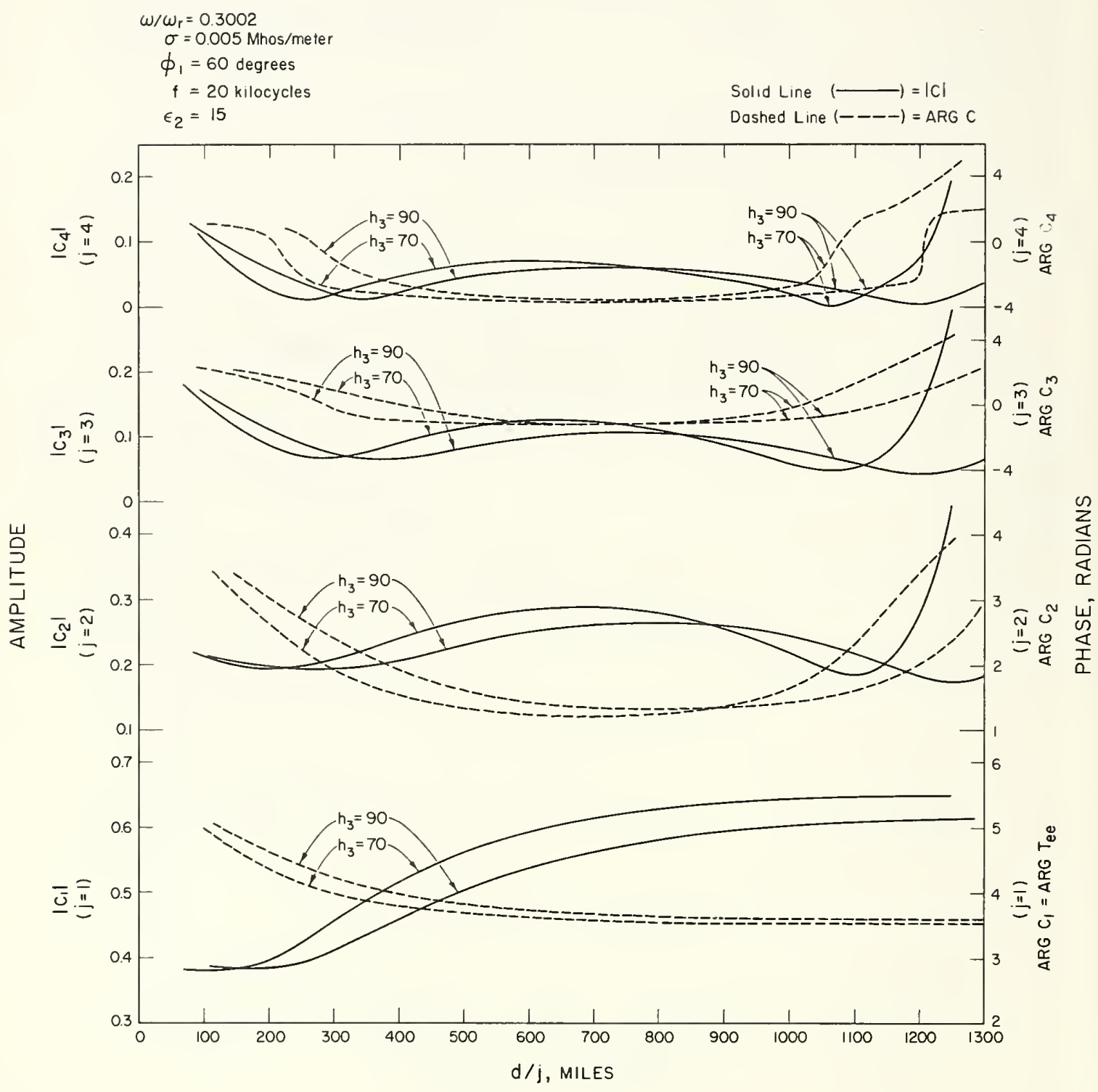

Fig. 15 - Effective quasi-longitudinal-Fresnel reflection coefficients (amplitude, $\left|C_{j}\right|$, and phase, $\operatorname{Arg~} C_{j}$ ) for the special ray configuration of the Norton type 301 atmosphere, applicable to the propagation of waves between the $\mathrm{D}$-region ( $\mathrm{h}_{3}=$ 70 kilometers) and the E-region ( $h_{3}=90$ kilometers) of the ionosphere and the earth. 


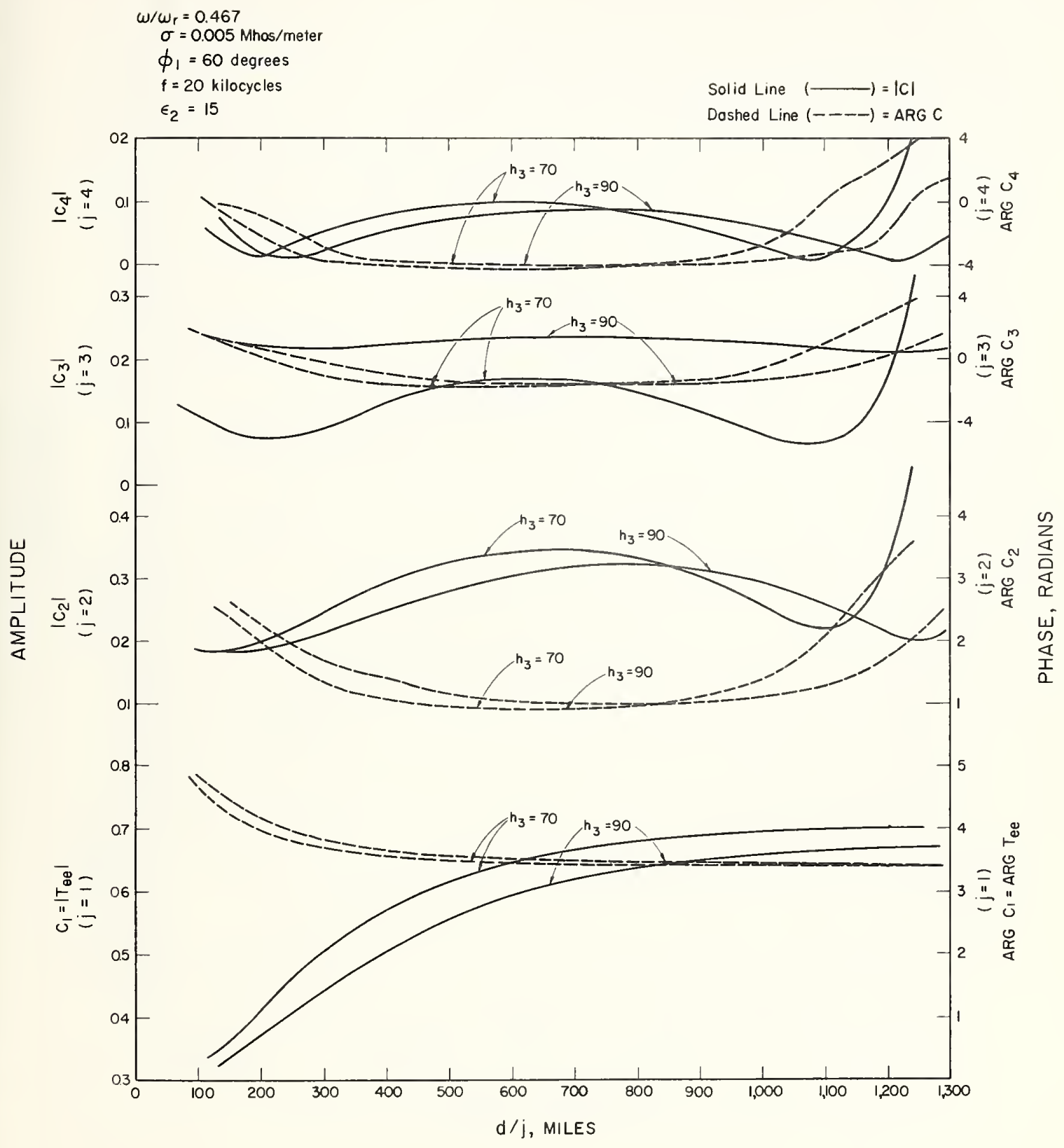

Fig. 16 - Effective quasi-longitudinal-Fresnel reflection coefficients (amplitude, $\left|C_{j}\right|$, and phase, $\operatorname{Arg~C}_{j}$ ) for the special ray configuration of the Norton type 301 atmosphere, applicable to the propagation of waves between the $\mathrm{D}$-region ( $\mathrm{h}_{3}=70$ kilometers) and the E-region $\left(h_{3}=90\right.$ kilometers) of the ionosphere and the earth. 


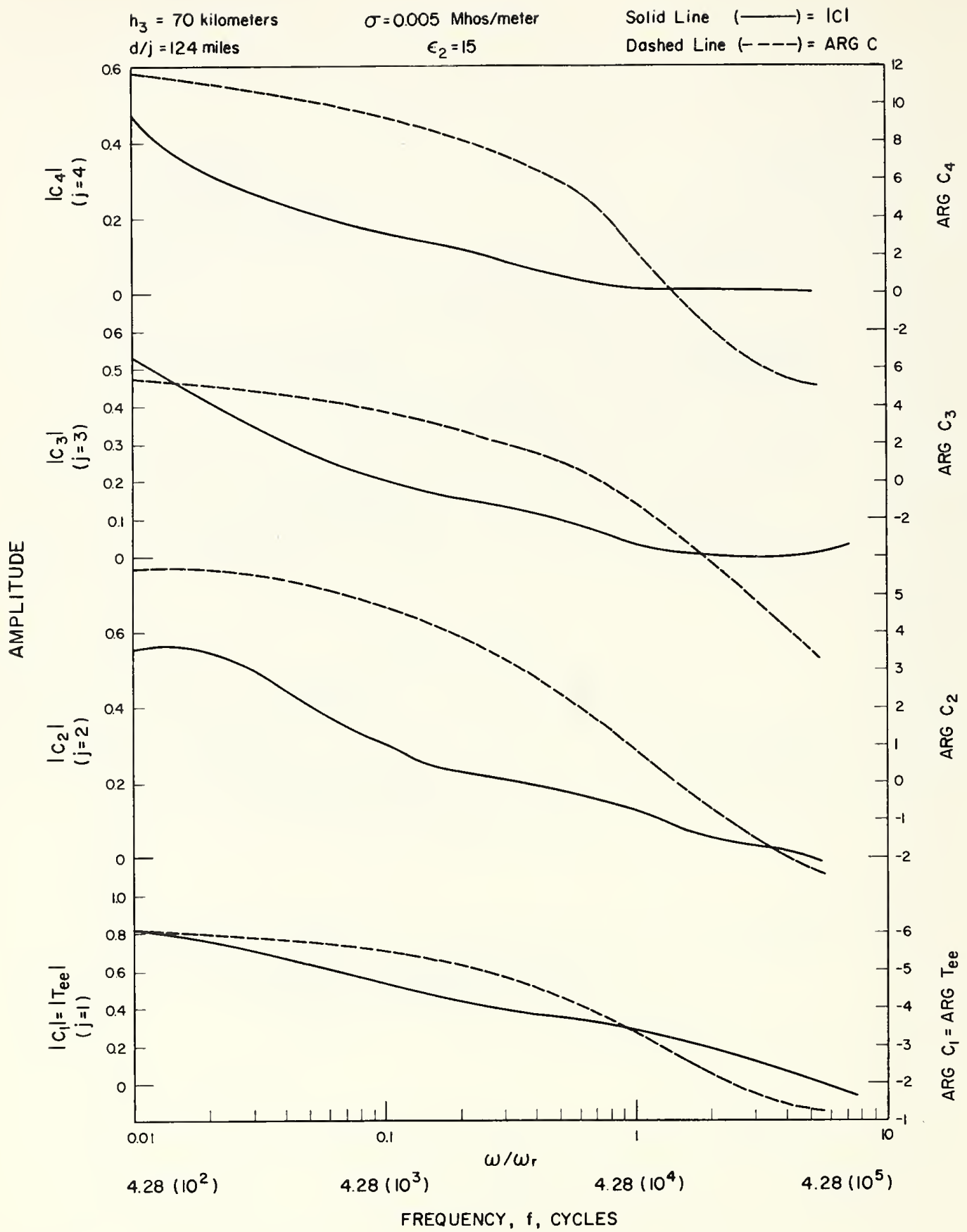

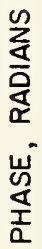

Fig. 17 -Effective quasi-longitudinal-Fresnel reflection coefficients (amplitude, $\left|C_{j}\right|$, and phase, Arg $C_{j}$ ) for the special ray configuration of the Norton type 301 atmosphere, applicable to the propagation of waves between the $\mathrm{D}-\mathrm{region}\left(\mathrm{h}_{3}=70\right.$ kilometers) and the E-region (h $3=90$ kilometers) of the ionosphere and the earth. 


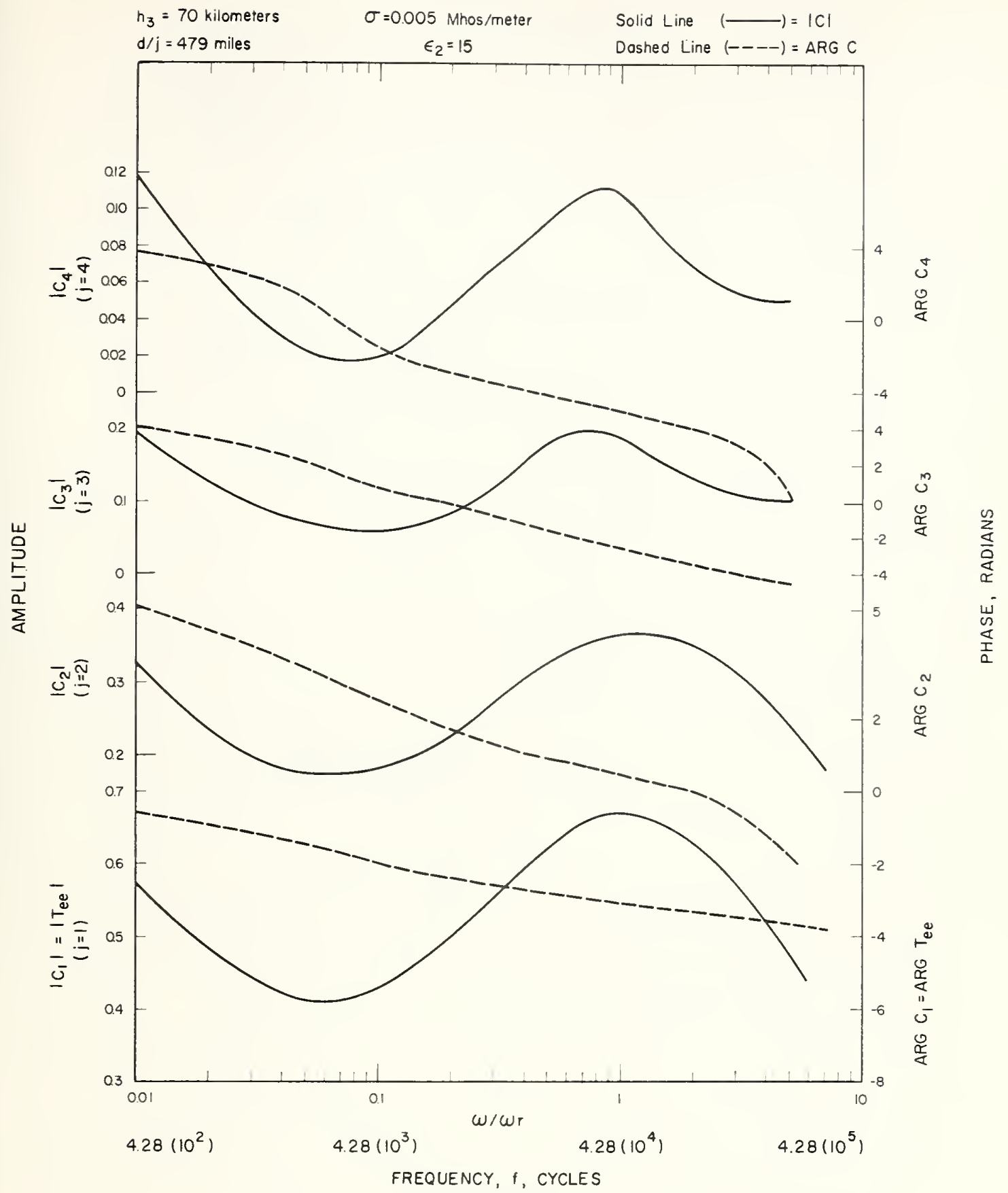

Fig. 18 -Effective quasi-longitudinal-Fresnel reflection coefficients (amplitude, $\left|C_{j}\right|$, and phase, $\operatorname{Arg~} C_{j}$ ) for the special ray configuration of the Norton type 301 atmosphere, applicable to the propagation of waves between the $\mathrm{D}$-region ( $\mathrm{h}_{3}=70$ kilometers) and the E-region ( $h_{3}=90$ kilometers) of the ionosphere and the earth. 


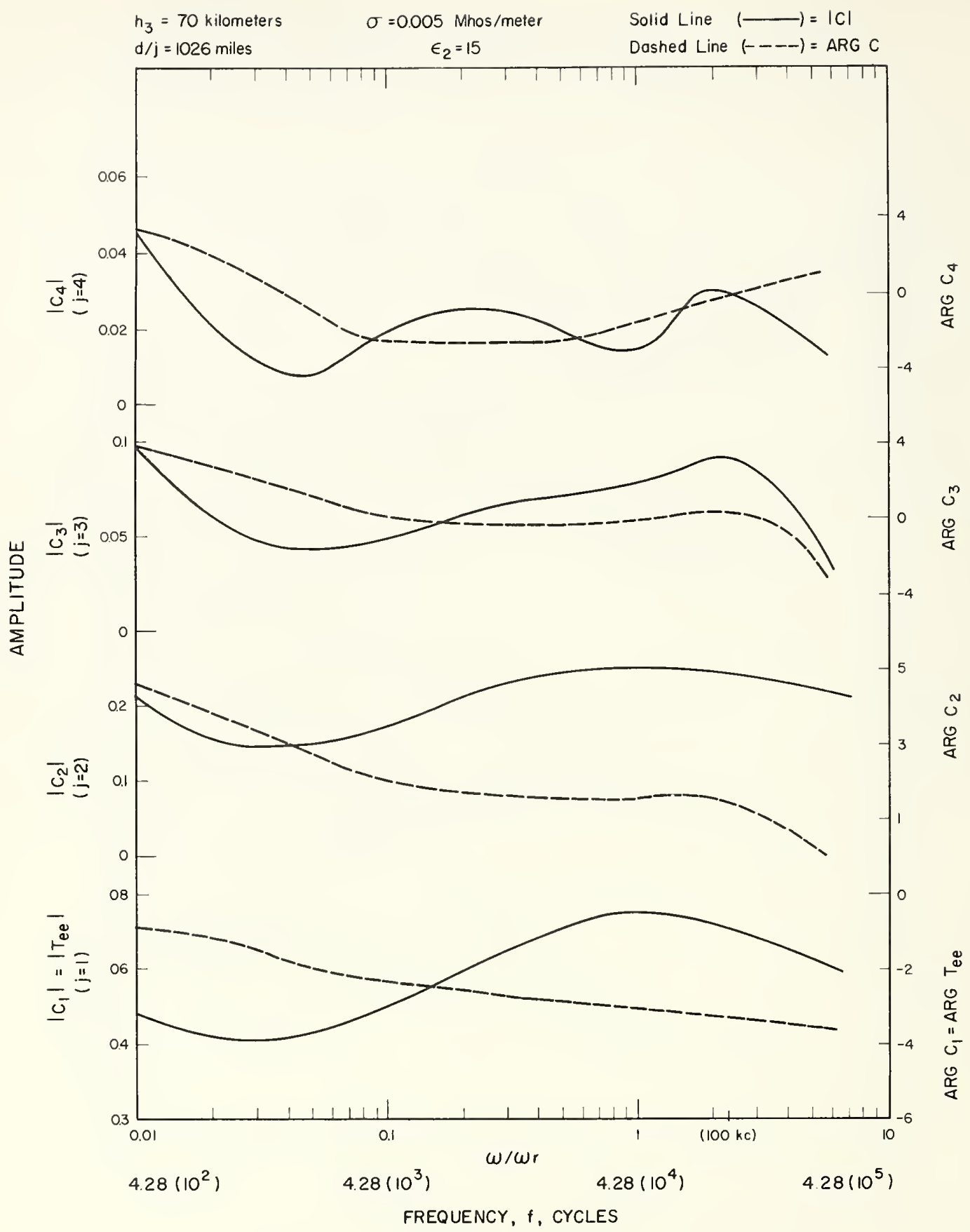

Fig. 19 - Effective quasi-longitudinal-Fresnel reflection coefficients (amplitude, $\left|C_{j}\right|$, and phase, $\operatorname{Arg} C_{j}$ ) for the special ray configuration of the Norton type 301 atmosphere, applicable to the propagation of waves between the $\mathrm{D}$-region $\left(\mathrm{h}_{3}=70\right.$ kilometers) and the E-region ( $\mathrm{h}_{3}=90$ kilometers) of the ionosphere and the earth. 


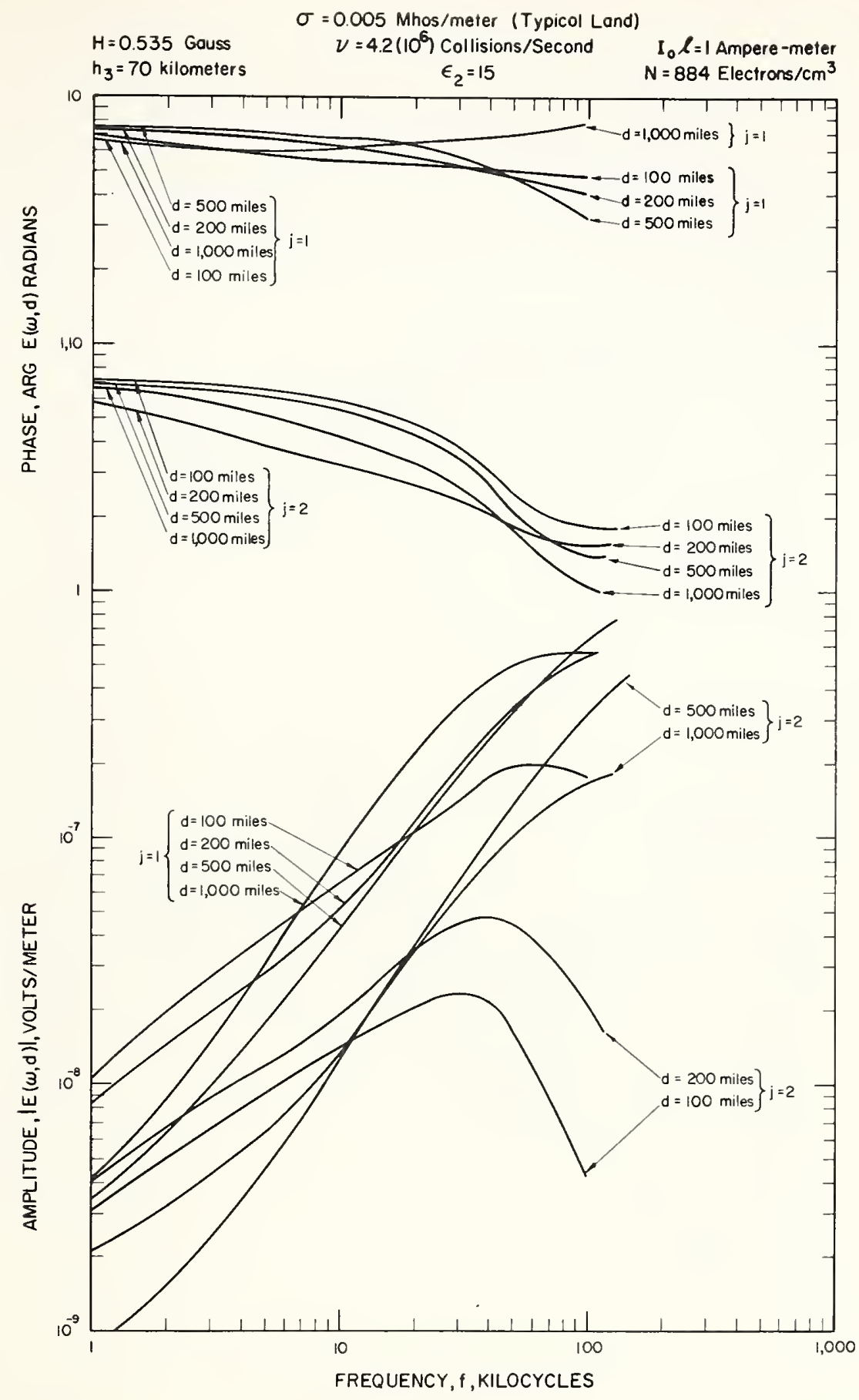

Fig. 20 - Transfer characteristic or "steady state" frequency characteristic, $E_{j}(\omega, d)$, (amplitude, $\left|E_{j}(\omega, d)\right|$, and phase, $\left.\operatorname{Arg} E_{j}(\omega, d)\right)$ of the time-modes $(j=1,2)$ of the sky wave, assuming the quasi-longitudinal-Fresnel approximation. 


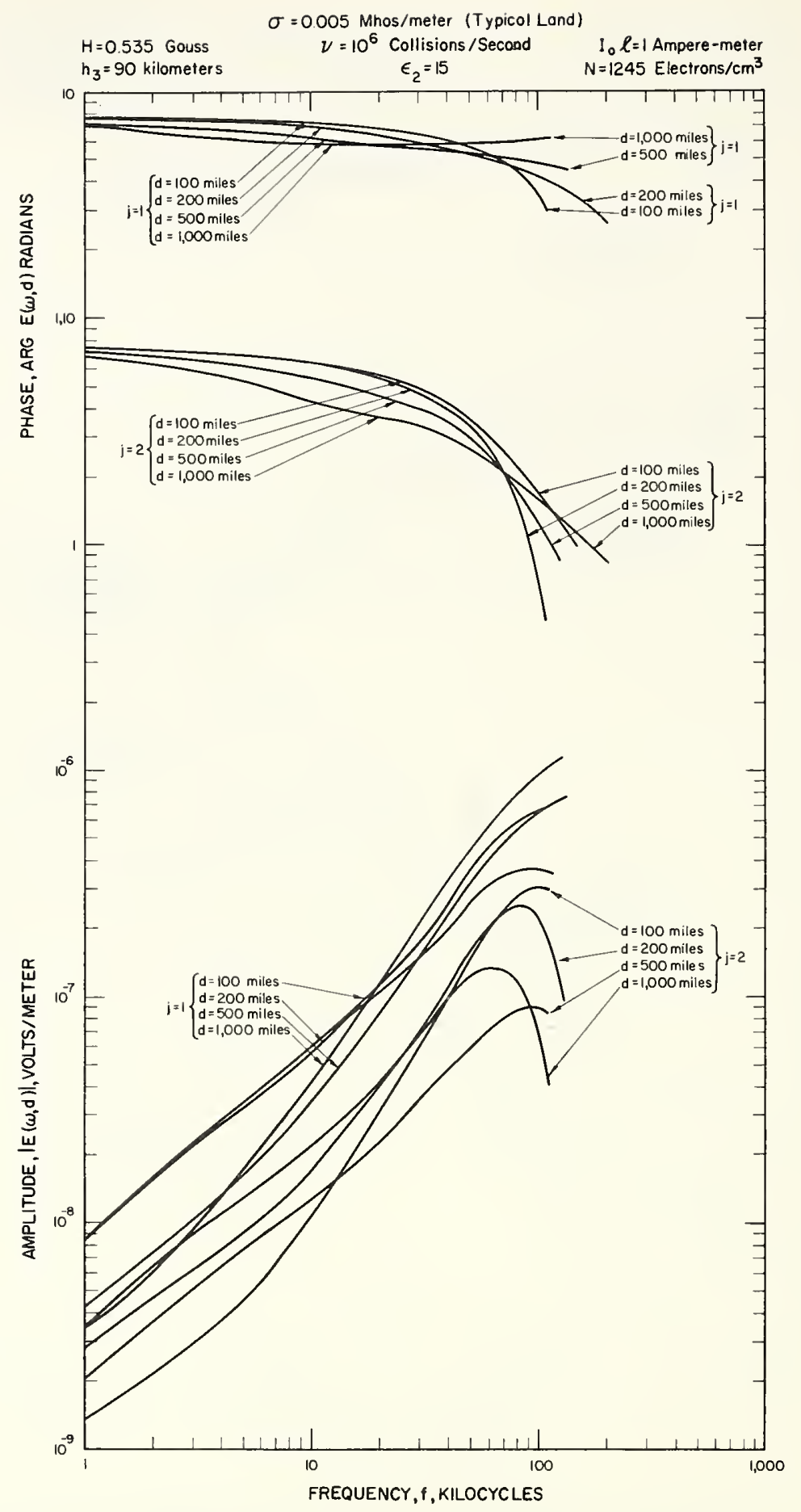

Fig. 21 - Transfer characteristic or "steady state" frequency characteristic, $E_{j}(\omega, d)$, (amplitude, $\left|E_{j}(\omega, d)\right|$, and phase, $\left.\operatorname{Arg} E_{j}(\omega, d)\right)$ of the time-modes $(j=1,2)$ of the sky wave, assuming the quasi-longitudinal-Fresnel approximation. 


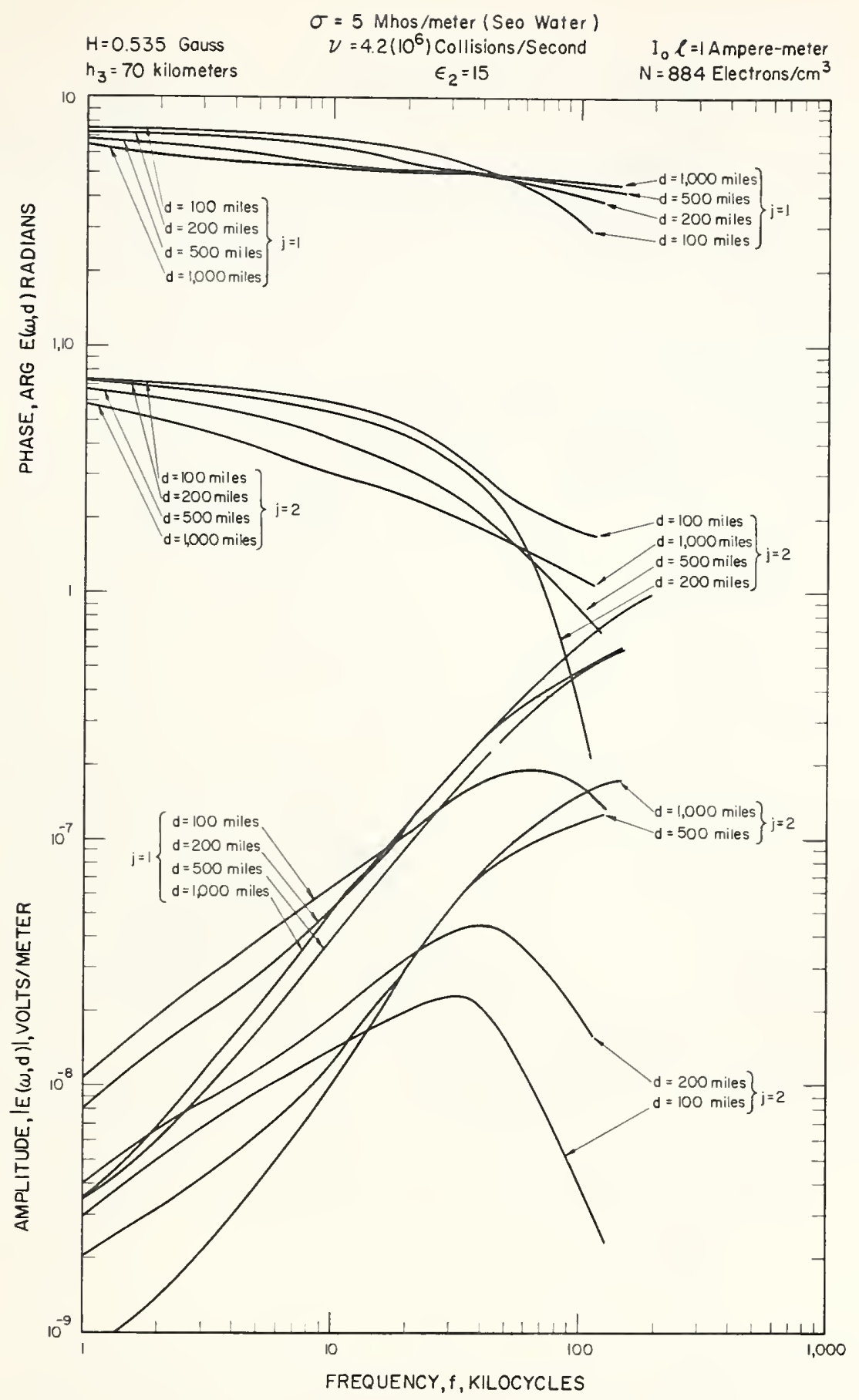

Fig. 22 -Transfer characteristic or "steady state" frequency characteristic, $E_{j}(\omega, d)$, (amplitude, $\left|E_{j}(\omega, d)\right|$, and phase, $\left.\operatorname{Arg} E_{j}(\omega, d)\right)$ of the time-modes $(j=1,2)$ of the sky wave, assuming the quasi-longitudinal-Fresnel approximation. 


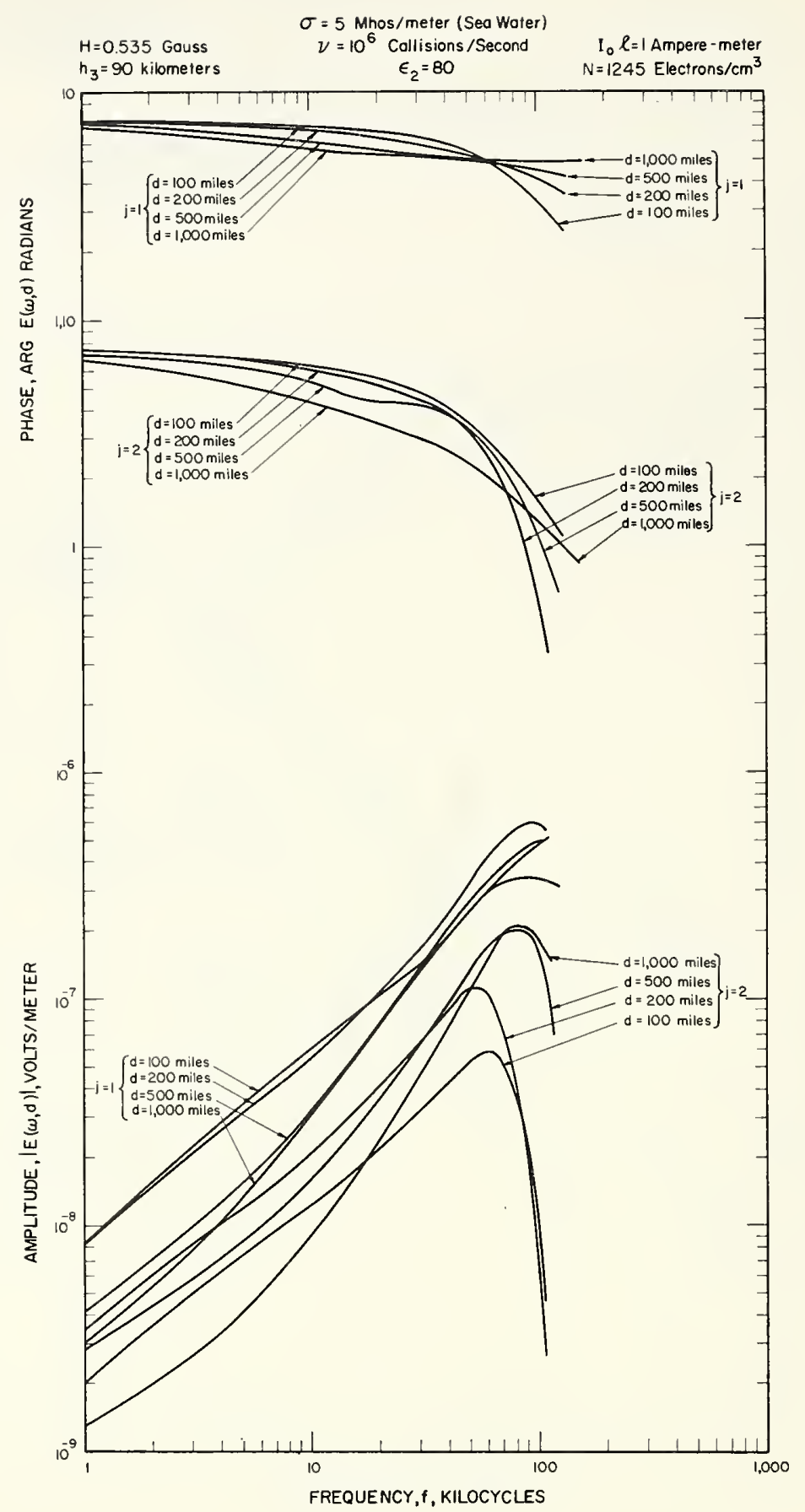

Fig. 23 -Transfer characteristic or "steady state" frequency characteristic, $E_{j}(\omega, d)$, (amplitude, $\left|E_{j}(\omega, d)\right|$, and phase, $\operatorname{Arg~} E_{j}\{(\omega, d))$ of the time-modes $(j=1,2)$ of the sky wave, assuming the quasi-longitudinal-Fresnel approximation. 


\section{APPENDIX}

Puises and Time-Modes

Data available in the form of oscillograms from the Loran-C $\left(C_{t a c}{ }^{13}\right)$ radio navigation system illustrates the physical aspects of time-mode's. Sinusoidal pulses radiated at a rate of approximately twenty per second were received on "broad-band" receivers at various distances from the transmitter. The changes in pulse form or shape as a result. of the propagation mechanism were observed at the radiofrequency output of the receivers. The envelope of the pulse was also observed at the output of the envelope detector.

In previous papexs ${ }^{14}$, the author has developed a mathematical representative of such puises as a Fourier integral,

$E\left(t^{i}, d\right)=\frac{1}{2 \pi} \int_{-\infty}^{\infty} \exp \left(i \omega t^{p}\right) E(\omega, d) \int_{0}^{\infty} \exp \left(-j \omega t^{p}\right) F_{S}(t) d t d \omega$,

or $E\left(t^{2}, d\right)=\frac{1}{2 \pi} \int_{-\infty}^{\infty} \exp \left(i \omega t^{2}\right) E(\omega, d\} \Psi_{S}(\omega) d \omega$,

The complex source function, $F_{S}(t)$, has been irtroduced to simplify the operational techniques employed in the analysis and also provide a description of the "ideal" amplitude envelope of the pulse, $\left|E\left(t^{p}, d\right)\right|$.

\section{3}

W. P. Frantz, W. N. Dean, R. L. Frank, "A Precision MultiPurpose Radio Navigation System," 1957 IRE National Convention Record, Part 8 (March 18-21, 1957) p.79-102。

14

J.R. Johler, op. cit, 2。 

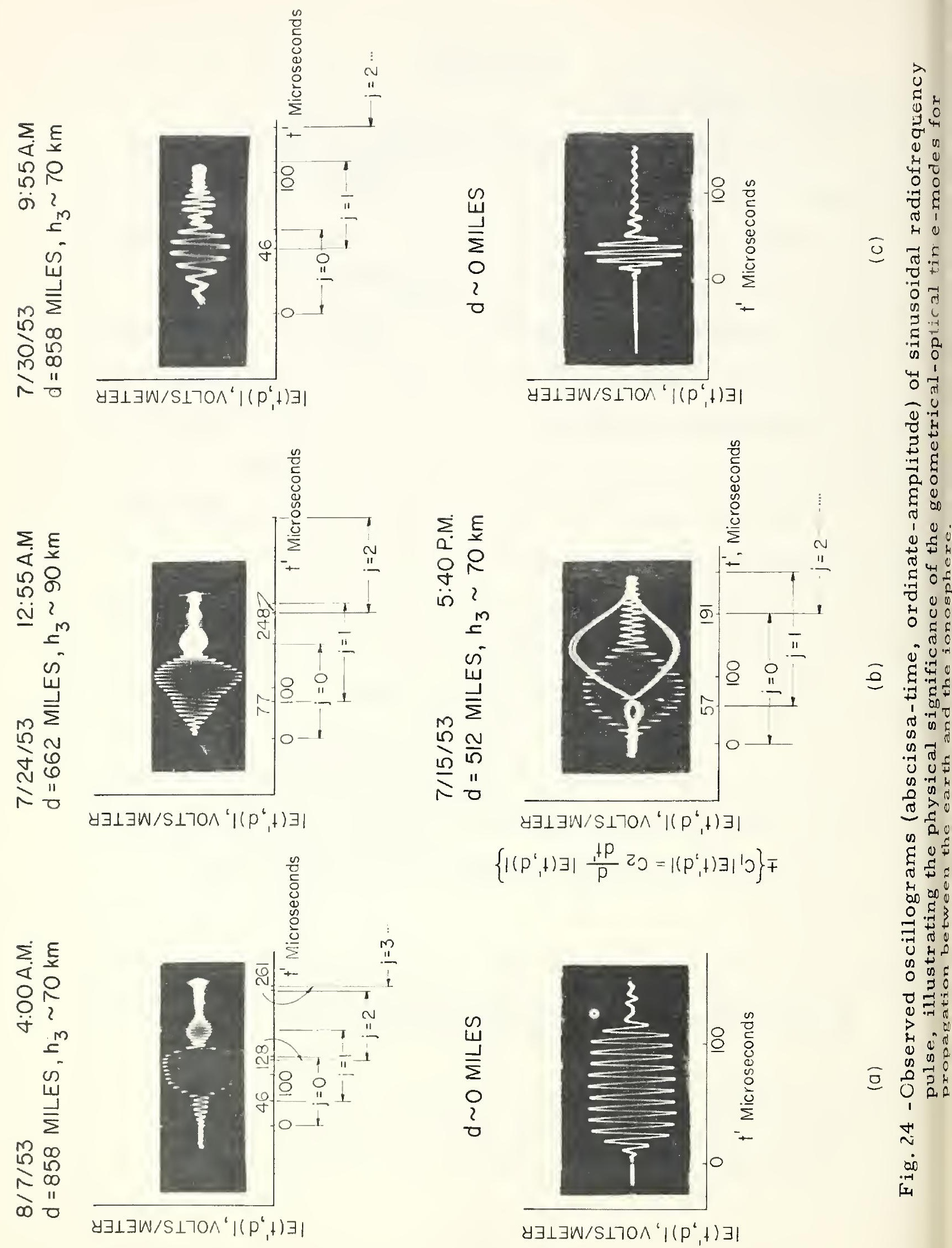
As a consequence of the time-modes eq. (2),

$E(t, d)=\sum_{j=0}^{n} E_{j}\left(t{ }_{j}, d\right)=\sum_{j=0}^{n} \frac{1}{2 \pi} \int_{-\infty}^{\infty} \exp \left(i \omega t{ }_{j}\right) E_{j}(\omega, d) F_{s}(\omega) d \omega$.

This merely represents the sum of separate Fourier integrals for each time-mode, separated in time by the sky wave time-mode delay, $\widehat{a}_{j}-a,(4,5,6,7)$.

The physical picture of the sum of $j$ time-modes or hops for the Cytac pulse is illustrated ${ }^{15}$ Fig. 24. The undisturbed form of the radiated pulse is shown by oscillograms at short distances. The action of the propagation medium is shown by the complicated multiple pulses observed at great distances. Note that at great distances the amplitude-time function, $\mathbb{E}(t, d)$, comprises the superimposed multiple time-modes or hops, $E_{j}(t, d)$ apparently added together according to the complex field strength, $E_{j}$, of each and separated in time in accord with the sky wave delay $\widehat{a}_{j}-a$, of each time-mode. The approximate area in time occupied by each time-mode of propagation is shown, Fig. 24. Note the areas of overlap in which both constructive and destructive interference between cycles of the multiple pulses produces "notches" and enhancements in the form of the composite pulse.

An interesting aspect of the equipment employed in the Cytac system is the method of "tagging" cycles or parts of cycles on the pulse. The envelope detector, in effect, forms the difference $\sqrt{5}$

From the author's (Johler's) personal notes on the 1953 preliminary testing of the Cytac System, RADC-WADC Contract 33(616)-54-?。 
between the envelope and its derivative,

$$
\pm\left\{a\left|E\left(t^{2}, d\right)\right|-b \frac{d}{d t}\left|E\left(t^{3}, d\right)\right|\right\} \text {, }
$$

where $a$ and $b$ are constants which move the null point or zero crossing on the oscillogram and which can be set by the operator to pick out a particular point on the pulse.

The technique for the evaluation of the amplitude-time function, $E\left(t^{9}, d\right)$, has been developed ${ }^{16}$. It should be noted that such a technique is not only applicable to radio navigation pulses but also to sferics. It is necessary, however, before calculating such pulses as described above, to provide this analysis with suitable transfer characteristics, $E_{j}(\omega, d)$, eq. 43 , which task was initiated in this paper.

op. cit. 2. 


\section{PREFACE TO TABLES}

Although the computation precision was held at eight significant figures, the physical significance of any such precision is open to question, especially as this implies more precise knowledge of the universal constants $(\mathrm{c}, \mathrm{e} / \mathrm{m})$ than is at present justified.

Consequently, the author recommends that the tables be rounded to at least five significant figures. This operation was not performed as it was concluded that the expense of the rounding operation could more profitably be applied to other more urgent problems. Also, the excess computation precision automatically available from the electronic computer is not detrimental if it is not misinterpreted.

The integer to the right of each table entry, if present, indicates a power of the factor 10 by which the number is multiplied, thus positioning the decimal point. For example, $8.8511589-1$ $=0.88511589$.

The phase of each entry (Arg $T$ or Arg C) can be ambiguous by a factor of $2 \pi(2 \pi=6.283185307$ radians $)$, hence any apparent discontinuities in the phase functions can be resolved merely by adding or subtracting $2 \pi$ from the argument or phase, (Arg). 
Table 1. Ionosphere reflection coefficients amplitude, $|\mathrm{T}|$, and phase, Arg T) assuming the quasi longitudinal approximation, for various incident angles, $\mathrm{T}_{j}, \mathrm{r}$, frequencies, $\omega / \omega_{r}$, and carth's magnetic field parameter, $\phi_{1}$. $T_{\text {ee }}{ } \mathrm{T}_{\mathrm{mm}}, \omega / \omega_{1}=0.3002,{ }_{1}=10,20,30$ degrees

Table 2. Ionosphere reflection coefficients (amplitude, $|\mathrm{T}|$, and phase, Arg T) assuming the quasi-longitudinal approximation, for various incident angles, ${ }^{T} j, r^{,}$frequencies, $\omega / \omega_{r}$, and earth ${ }^{8} \mathrm{~s}$ $\mathrm{T}_{\mathrm{ee}}, \mathrm{T}_{\mathrm{mm}}, \omega / \omega_{\mathrm{r}}=0.3002, \phi_{1}=40,50,60$ degrees

Table 3. Ionosphere reflection coefficient (amplitude, $|\mathrm{T}|$, and phase, $\Lambda \mathrm{r}$ T $\mathrm{T}$ ) assuming the quasi-longitudinal approximation, for various incident angles, $\tau_{j}, \ldots$, frequencies, $\omega / \omega_{\mathrm{r}}$, and earth"s magnetic field paramenter, $\phi_{1}$. ${ }_{\text {Tem, }} \mathrm{T}_{\text {me }} \omega / \omega_{x}=0.3002, \phi_{1}=10,20,30$ degrees

Table 4. Tonosphere reflection coefficients (amplitude, $|\mathrm{T}|$, and phase, $A_{1} \cdot \mathrm{g}$ ) assumiug the quasi-longitudinal approximation, for various incident angles, $\mathrm{T}_{\mathrm{j}_{2}}$, , frequencies, $\omega / \omega_{1}$, and earth ${ }^{\mathrm{S}}$ magnetic field parameter, $\phi_{1}$. ${ }_{\text {Tem }}{ }^{\mathrm{T}} \mathrm{me} \omega / \omega_{1}=0.3002, \mathrm{~d}_{1}=40,50,60$ degrees

Table 5. Ionosphere reflection coefficients (amplitude, $|\mathrm{T}|$, and phase, Arg T') assuming the quasi-longitudinal approximation, for various incident angles, $T_{j},{ }^{*}$, frequencies, $\omega / \omega_{1}$, and earth ${ }^{8} s$ magnetic field parameter, $\phi_{1}$. $T_{\text {ee }} \cdot T_{m^{\circ}} \omega / \omega_{x}=0.467, \phi_{1}^{1}=10,20,30$ degrees

Table 6. Ionosphere reflection coefficients (ampjitude, $|\mathrm{T}|$, and phase, Arg T) assuming the quasi-longitudinal approximation, for various incident angles, $\tau_{j}, x$, frequencies, $\omega / \omega_{r}$, and earth's magnetic field paramete $x^{\circ}, \phi_{1}$. $\mathrm{T}_{\mathrm{ee}}{ }^{\prime}{ }^{\mathrm{T}} \mathrm{mm} \mathrm{m} \omega / \omega_{\mathrm{r}^{\circ}}=0.167, \phi_{1}^{1}=40,50,60$ degrees

Table 7. Ionosphere reflection coefficients (amplitude, $|T|$, and phase, Arg T) assuming the quasi-longitudinal approximation, for various incident angles, $\tau_{j}, x^{\circ}$, frequencies, $\omega / \omega_{\mathrm{r}}$, and earth ${ }^{\circ} \mathrm{s}$ magnetic field parameter, $\phi_{1}$. $\mathrm{T}_{\mathrm{em}} \mathrm{T}_{\mathrm{me}} \omega / \omega_{\mathrm{T}}=0.467, \phi_{1}^{1}=10,20,30$ degrees 
Table 8. Ionosphere reflection coefficients (amplitude, $|\mathrm{T}|$, and phase, Arg T) assuming the quasi-longitudinal approximation, for various incident angles, $\tau_{j}, 1$, frequencies, $\omega / \omega_{r}$, and earth's magnetic field parameter, $\phi_{1}$. $\mathrm{T}_{\text {em }}, \mathrm{T}_{\mathrm{me}}, \omega / \omega_{\mathrm{r}}=0.467, \phi_{1}^{1}=40,50,60$ degrees

Table 9. Ionosphere reflection coefficients (amplitude, |T|, and phase, Arg T) assuming the quasi-longitudinal approximation, for various frequencies, $\omega / \omega_{r}$, earth's magnetic field parameter, $\phi_{1}$, and incident angle, $T_{j}$. $\mathrm{T}_{\mathrm{ee}}, \mathrm{T}_{\mathrm{mm}}, \phi_{1}=60$ degrees, $\tau_{j, \mathrm{r}}=5,15,25$ degrees

Table 10. Ionosphere reflection coefficients (amplitude, $|\mathrm{T}|$, and phase, Arg T) assuming the quasi-longitudinal approximation, for various frequencies, $\omega / \omega_{r}$, earth's magnetic field parameter, $\phi_{1}$ ' and incident angle, $\tau_{j}, r$. $\mathrm{T}_{\text {ee, }} \mathrm{T}_{\mathrm{mm}}, \phi_{1}=60$ degrees, $\mathrm{T}_{\mathrm{j}, \mathrm{r}}=35,45,55$ degrees

Table 11. Ionosphere reflection coefficients (amplitude, $|\mathrm{T}|$, and phase, Arg T) assuming the quasi-longitudinal approximation, for various frequencies, $\omega / \omega_{r}$, earth's magnetic field paramter, $\phi_{1}$, and incident angle, $T^{\top}$. $\mathrm{T}_{\text {ee, }} \mathrm{T}_{\mathrm{mm}}, \phi_{1}=60$ dègrees, $\mathrm{T}_{j, 1}=65,75,85$ degrees

Table 12. Ionosphere reflection coefficients (amplitude, $|\mathrm{T}|$, and phase, Arg T) assuming the quasi-longitudinal approximation, for various frequencies, $\omega / \omega_{1}$, earth's magnetic field parameter, $\phi_{1}$, and incident angle, $T$; $\mathrm{T}_{\text {em }}, \mathrm{T}_{\mathrm{me}}, \phi_{1}=60$ dég ${ }^{\mathrm{r}}$ ees, $\mathrm{T}_{\mathrm{j}, \mathrm{r}}=5,15,25$ degrees

Table 13. Ionosphere reflection coefficients (amplitude, $|\mathrm{T}|$, and phase, Arg T) assuming the quasi-longitudinal approximation, for various frequencies, $\omega / \omega_{\mathrm{r}}$, earth's magnetic field parameter, $\phi_{1}$. and incident angle, $\tau_{j}, \mathrm{r}$. $\mathrm{T}_{\text {em }}, \mathrm{T}_{\mathrm{me}}, \phi_{1}=60$ degrees, $\mathrm{T}_{\mathrm{j}, \mathrm{r}}=34,45,55$ degrees

Table 14. Ionosphere reflection coefficients (amplitude, $|\mathrm{T}|$, and phase, Arg T) assuming the quasi-longitudinal approximation, for various $f_{1}$ equencies, $\omega / \omega_{r}$, ear'th's magnetic field parameter, $\phi_{1}$, and incident angle, $\tau_{j}, \mathrm{r}$. $\mathrm{T}_{\text {em }} \mathrm{T}_{\mathrm{me}} \phi_{1}=60$ degrees, $\mathrm{T}_{\mathrm{j}, \mathrm{r}}=65,75,85$ degrees

Table 15. Ionosphere reflection coefficients (amplitude, $|\mathrm{T}|$, and phase, Arg T) assuming the quasi-longitudinal approximation, for

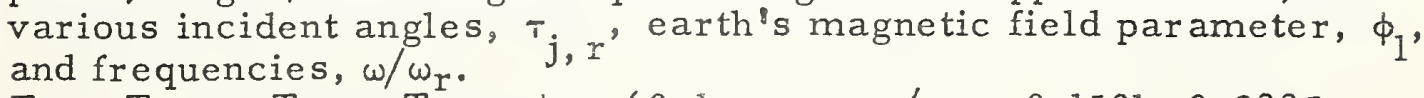
$\mathrm{T}_{\text {ee }} \mathrm{T}_{\mathrm{mm}}$, $\mathrm{T}_{\text {em }} \mathrm{T}_{\mathrm{me}^{\prime}} \phi_{1}=60$ degrees, $\omega / \omega_{\mathrm{r}}=0.1501,0.2335$ 
Table 16. Geomelric parancters, $\Psi, d / j$, and $\tau_{j}, r$, for the Norton type 301 atmosphere, applicable to the propagation of waves between the $D$-region $\left(h_{3}=70\right.$ kilometers $)$ or the $\mathrm{E}-\mathrm{region}\left(\mathrm{h}_{3}=90\right.$ kilometers) of the ionosphere and the earth.

Table 17. Ground reflection coefficients (amplitude, $|R|$, and phase. Arg $\mathrm{K}$ ) assuming the Fresnel approximation, for the special ray configuration of the Norton type 301 atmosphere, applicable to the propagation of waves between the $D$-region (h $3=70$ kilometers) or the E-region (h, $=90$ kilometers) of the jonosphere and the earth. $\mathrm{R}_{\mathrm{c}}, \mathrm{R}_{\mathrm{m}}, v=0.005 \mathrm{mbos} / \mathrm{mete}, \epsilon_{2}=15, \mathrm{f}=20 \mathrm{kc}$

Table 18. Ground reflection coefficients (amplitude, $|R|$, and phase. Arg K) assuming the Fresnel approximation, for the special ray configuration of the Norton type 301 atmosphexe, applicable to the propagation of waves between the D-region ( $13=70$ kilometers) or the $E$-region $\left(h_{3}=90\right.$ kilometers $)$ of the ionosphere and the earth. $\mathbb{R}_{\mathrm{e}}, \mathrm{R}_{\mathrm{m}}, \sigma=0.005$ nilios/meter, $\epsilon_{2}=15, \mathrm{f}=10 \mathrm{kc}$

Table 19. Ground reflection cocficients (amplitude, $|R|$, and phase. Arg (I) assuming the Fucsucl approximation, for the special ray configuration of the INorton type 301 atmosphere, applicable to the propagation of waves between the $\mathrm{D}$-region $\left(\mathrm{h}_{3}=70\right.$ kilometers) or the E-region (h, $=90$ lilometers) of the ionosphere and the earth. $R_{\mathrm{e}}, R_{\mathrm{m}}, \sigma=0.005 \mathrm{mhos} / \mathrm{meter},{ }_{2}=15, \stackrel{r}{\mathrm{r}}=0.42826552 \mathrm{kc}$

Table 20. Ground reflection coefficients (amplitude, $|R|$, and phase, Arg $R$ ) assuming the Fresnel approximation, for the special ray configuration of the IVorton type 301 atmosphere, applicable to the propagation of waves between the $\mathrm{D}$ - jegion (h3 $=70 \mathrm{kilomelers)}$ or the $E_{-r e g i o n ~}\left(h_{3}=90\right.$ kilometers $)$ of the ionosphere and the earth. $R_{\mathrm{e}}, \mathrm{R}_{\mathrm{m}}, \sigma=0.005 \mathrm{mhos} / \mathrm{meter}, \varepsilon_{2}=15, \mathrm{f}=0.85653105 \mathrm{kc}$

Table 21. Ground reflection coefficients (amplitude, $|R|$, and phase. Arg (R) assuming the Fresnel approximation, for the special $r$ ay configuration of the Norton type 301 atmosphere, applicable to the propagation of waves between the $\mathrm{D}-\mathrm{region}$ (h3 $=70$ kilometers) or the $E$-region (h3 $=90$ kilometers) of the ionosphere and the earth. $R_{e}, R_{m}, \sigma=0.005$ mlios $/$ meter, $\epsilon_{2}=15, f=2.1413276 \mathrm{kc}$

Table 22. Ground reflection coefficients (amplitude, $|R|$, and phase. Arg R) as suming the Fresnel approximation, for the special ray configuration of the Norton type 301 atmosphere, applicable to the propagation of waves between the D-region (h. $3=70$ kilometers) or the $E$-region (h3 = 90 lrilometers) of the ionosphere and the earth. $\mathbb{R}_{\mathrm{e}}, \mathbb{R}_{\mathrm{m}}, \sigma=0.005$ mhos/meter, $\epsilon_{2}=15, f=4.2826552 \mathrm{kc}$ 
Table 23. Ground reflection coefficients (amplitude, $|R|$, and phase, Arg R) assuming the Fresnel approximation, for the special ray configuration of the Norton type 301 atmosphere, applicable to the propagation of waves between the D-region (h3 $=70$ kilometers) or the E-region (h3 $=90$ kilometers) of the ionosphere and the earth. $R_{e}, R_{m}, \sigma=0.005$ mhos $/$ meter, $\epsilon_{2}=15, f=8.5653105 \mathrm{kc}$

Table 24. Ground reflection coefficients (amplitude, $|\mathbf{R}|$, and phase, Arg R/assuming the Fresnel approximation, for the special ray configuration of the Norton type 301 atmosphere, applicable to the propagation of waves between the D-region (h3 $=70$ kilometers) $0:$ the $E$-region ( $h_{3}=90$ kilometers) of the ionosphere and the earth. $\mathrm{R}_{\mathrm{e}}, \mathrm{R}_{\mathrm{m}}, \sigma=0.005 \mathrm{mhos} / \mathrm{meter} \epsilon_{2}=15, \mathrm{f}=42.826552 \mathrm{kc}$

Table 25. Ground reflection coefficients (amplitude, $|R|$, and phase, Arg IV) assuming the Fresiel approximation, for the special ray configuration of the Norton type 301 atmosphere, applicable to the propagation of waves between the $\mathrm{D}$-region ( $\mathrm{h}_{3}=70$ kilometers) or the E-region ( $\mathrm{h}_{3}=90$ kilometers) of the ionosphere and the earth. $R_{e}, R_{m}, \sigma=0.005$ mhos/meter, $\epsilon_{2}=15, f=85.653105 \mathrm{kc}$

Table 26. Ground reflection coefficients (amplitude, $|R|$, and phase, Arg $R$ ) assuming the Fresnel approximation, for the special ray configuration of the Norton type 301 atmosphere, applicable to the propagation of waves between the $\mathrm{D}$-region $\left(h_{3}=70\right.$ kilometers) or the $\mathrm{E}$-region (h3 = 90 kilometers) of the ionosphere and the earth. $R_{e}, R_{m}, \sigma=0.005$ mhos $/$ meter, $E_{2}=15, f=214.13276 \mathrm{kc}$

Table 27. Ground reflection coefficients (amplitude, $|R|$, and phase, ArgR/ as suming the Fresnel approximation, for the special ray configuration of the Norton type 301 atmosphere, applicable to the propagation of waves between the $\mathrm{D}$-region (h $3=70$ kilometers) or the $E$-region (h $3=90$ kilometers) of the ionosphere and the earth. $\mathrm{R}_{\mathrm{e}}, \mathrm{R}_{\mathrm{m}} \sigma=0.005 \mathrm{mhos} / \mathrm{meter}, \epsilon_{2}=15, \hat{f}=0.66622256 \mathrm{kc}$

Table 28. Ground reflection coefficients (amplitude, $|\mathrm{R}|$, and phase, Arg R/assuming the Fresnel approximation, for the special ray configuration of the Norton type 301 atmosphere, applicable to the propagation of waves between the D-region (h $3=70$ kilometers) or the E-region ( $h_{3}=90$ kilometers) of the ionosphere and the earth. $R_{e}, R_{\text {m, }} \sigma=0.005$ mhos $/$ meter, $\varepsilon_{2}=15, f=1.332425 \mathrm{kc}$

Table 29. Ground reflection coefficients (amplitude, |R|, and phase. Arg R) assuming the Fresnel approximation, for the special ray configuration of the Norton type 301 atmosphere, applicable to the propagation of waves between the D-region (h3 $=70 \mathrm{kilometers)} \mathrm{or}$ the $E$-region (h3 $=90$ kilometers) of the ionosphere and the earth. $R_{e}, R_{m}, \sigma=0.005$ mhos/meter, $\varepsilon_{2}=15, f=3.3311126 \mathrm{kc}$ 
Table 30. Ground reflection coefficients (amplitude, $|R|$, and phase, Arg R) assuming the Fresnel approximation, for the special ray configuration of the Norton type 301 atmosphere, applicable to the propagation of waves between the $\mathrm{D}$-region ( $\mathrm{h} 3=70$ kilometers) or the $E$-region ( $h_{3}=90$ kilometers) of the ionosphere and the earth. $\mathrm{R}_{\mathrm{e}}, \mathrm{R}_{\mathrm{m}}, \sigma=0.005 \mathrm{mhos} / \mathrm{meter}, \epsilon_{2}=15, \mathrm{f}=6.6622252 \mathrm{kc}$

Table 31. Ground reflection coefficients (amplitude, $|R|$, and phase, Arg R) as suming the Fresnel approximation, for the special ray configuration of the Norton type 301 atmosphere, applicable to the propagation of waves between the $\mathrm{D}$-region $\left(\mathrm{h}_{3}=70\right.$ kilometers) or the $E$-region ( $h_{3}=90$ kilometers) of the ionosphere and the earth. $R_{e}, R_{m}, \sigma=0.005$ mhos $/$ meter, ${ }_{2}=15, f=13.324450 \mathrm{kc}$

Table 32. Ground reflection coefficients (amplitude, $|R|$, and phase, Arg R) assuming the Fresnel approximation, for the special ray configuration of the Norton type 301 atmosphere, applicable to the propagation of waves between the $D$-region ( $h_{3}=70$ kilometers) or the E-region ( $\mathrm{h}_{3}=90$ kilometers) of the ionosphere and the earth. $R_{e}, R_{m}, \sigma=0.005$ mhos $/$ meter, $\epsilon_{2}=15, f=66.622252 \mathrm{kc}$

Table 33. Ground reflection coefficients (amplitude, $|R|$, and phase, Arg R) assuming the Fresnel approximation, for the special ray configuration of the Norton type 301 atmosphere, applicable to the propagation of waves between the $D$-region ( $h_{3}=70$ kilometers) or the $E$-region ( $h_{3}=90$ kilometers) of the ionosphere and the earth. $R_{e}, R_{m}, \sigma=0.005$ mhos $/$ meter, ${ }_{2}=15, f=133.24450 \mathrm{kc}$

Table 34. Ground reflection coefficients (amplitude, $|R|$, and phase, Arg R) assuming the Fresnel approximation, for the special ray configuration of the Norton type 301 atmosphere, applicable to the propagation of waves between the $\mathrm{D}$-region $\left(\mathrm{h}_{3}=70\right.$ kilometers) or theE-region ( $h_{3}=90$ kilometers) of the ionosphere and the earth. $\mathrm{R}_{\mathrm{e}}, \mathrm{R}_{\mathrm{m}}, \sigma=0.005 \mathrm{mhos} / \mathrm{meter}, \epsilon_{2}=15, f=333.11126 \mathrm{kc}$

Table 35. Ionosphere reflection coefficients (amplitude, $\left|T^{\prime}\right|$, and phase, Arg T) assuming the quasi-longitudinal approximation, for the special ray configuration of the Norton type 301 atmosphere, applicable to the propagation of waves between the $\mathrm{D}-\mathrm{region}\left(\mathrm{h}_{3}=70\right.$ kilometers) or the E-region $\left(\mathrm{h}_{3}=90\right.$ kilometers $)$ of the ionosphere and the earth.

$\mathrm{T}_{\text {ee }}, \mathrm{T}_{\mathrm{mm}}, \phi_{1}=60$ degrees, $\omega / \omega_{\mathrm{r}}=0.3002$ 
Table 36. Ionosphere reflection coefficients (amplitude, $|\mathrm{T}|$, and phase, Arg T) assuming the quasi-longitudinal approximation, for the special ray configuration of the Norton type 301 atmosphere, applicable to the propagation of waves between the D-region ( $h_{3}=$ 70 kilometers) or the E-region ( $h_{3}=90$ kilometers) of the ionosphere and the earth.

$\mathrm{T}_{\text {ee, }} \mathrm{T}_{\mathrm{mm}}, \phi_{1}=60$ degrees, $\omega / \omega_{\mathrm{r}}=0.467$

Table 37. Ionosphere reflection coefficients (amplitude, $|\mathrm{T}|$, and phase, Arg T) assuming the quasi-longitudinal approximation, for the special ray configuration of the Norton type 301 atmosphere, applicable to the propagation of waves between the $D$-region $\left(h_{3}=70\right.$ kilometers) or the E-region $\left(h_{3}=90\right.$ kilometers) of the ionosphere and the earth.

$\mathrm{T}_{\mathrm{ee}}, \mathrm{T}_{\mathrm{mm}}, \phi_{\mathrm{I}}=60$ degrees, $\omega / \omega_{\mathrm{r}}=0.1501$

Table 38. Ionosphere reflection coefficients (amplitude, $|\mathrm{T}|$, and phase, Arg T) assuming the quasi-longitudinal approximation, for the special ray configuration of the Norton type 301 atmosphere, applicable to the propagation of waves between the D-region $\left(h_{3}=70\right.$ kilometers) or the E-region $\left(\mathrm{h}_{3}=90\right.$ kilometers $)$ of the ionosphere and the earth.

$\mathrm{T}_{\text {ee, }} \mathrm{T}_{\mathrm{mm}}$ ' $\phi_{1}=60$ degrees, $\omega / \omega_{\mathrm{r}}=0.2335$

Table 39. Ionosphere reflection coefficients (amplitude, $|T|$, and phase, Arg T) assuming the quasi-longitudinal approximation, for the special ray configuration of the Norton type 301 atmosphere, applicable to the propagation of waves between the $D$-region $\left(h_{3}=70\right.$ kilometers) or the $E$-region $\left(h_{3}=90\right.$ kilometers $)$ of the ionosphere and the earth.

$\mathrm{T}_{\text {ee, }} \mathrm{T}_{\mathrm{mm}} \phi_{\mathrm{I}}=60$ degrees, $\omega / \omega_{\mathrm{r}}=0.01$

Table 40. Ionosphere reflection ccefficients (amplitude, $|\mathrm{T}|$, and phase, Arg T) assuming the quasi-longitudinal approximation, for the special ray configuration of the Norton type 301 atmosphere, applicable to the propagation of waves between the $D$-region $\left(h_{3}=70\right.$ kilometers) or the $E$-region $\left(\mathrm{h}_{3}=90\right.$ kilometers) of the ionosphere and the earth.

$T_{\text {ee, }} \mathrm{T}_{\mathrm{mm}} \phi_{1}=60$ degrees, $\omega / \omega_{\mathrm{r}}=0.02$

Table 41. Ionosphere reflection coefficients (amplitude, $|\mathrm{T}|$, and phase, Arg T) assuming the quasi-longitudinal approximation, for the special ray configuration of the Norton type 301 atmosphere, applicable to the propagation of waves between the $D$-region $\left(h_{3}=70\right.$ kilometers) or the $E$-region ( $h_{3}=90$ kilometers) of the ionosphere and the earth.

$\mathrm{T}_{\text {ee, }} \mathrm{T}_{\mathrm{mm}}, \phi_{1}=60$ degrees, $\omega / \omega_{\mathrm{r}}=0.05$ 
Table 42. Ionosphere reflection coefficients (amplitude, $|\mathrm{T}|$, and phase, Arg T) assuming the quasi-longitudinal approximation, for the special ray configuration of the Norton type 301 atmosphere, applicable to the propagation of waves between the $\mathrm{L}_{\mathrm{m}} \mathrm{region}\left(\mathrm{h}_{3}=70\right.$ kilometers $)$ or the $E-1$ egion $\left(h_{3}=90\right.$ kilometers $)$ of the ionosphere and the earth.

$\mathrm{T}_{\mathrm{ee}}, \mathrm{T}_{\mathrm{mm}}, \phi_{1}=60$ clegrees, $\omega / \omega_{1}=0.1$

Table 43. Ionosphere reflection coefficients famplitude, $\left|\mathrm{T}^{\prime}\right|$, and phase, Arg T) assuming the quasi-longiludinal approximation, for the special ray configuration of the Norton type 301 atmosphere, applicable to the propagation of waves between the D-region $\left(h_{3}=70\right.$ kilometers) or the II-region ( $h_{3}=90$ kilometers) of the ionosphere and the eaxth.

$\mathrm{T}_{\text {ee }}, \mathrm{T}_{\mathrm{mm}} \phi_{1}=60$ degrees, $\omega^{\prime} \omega_{\mathrm{r}}=0.2$

Table 44. Ionosplere reflection coefficients famplitude, $|\mathrm{T}|$, and phase, Axg T) assuming the quasi-longitudinal approximation, for the special ray configuration of the Noxton type 301 atmosphexe, applicable to the propagalion of waves belween the D-region $\left(h_{3}=70\right.$ kilometers) or the Emregion $\left(h_{3}=90\right.$ kilometers $)$ of the ionosphere and the earth.

$T_{\text {ee }}, T_{\text {mm }}, \phi_{1}=60$ deglees, $\omega / \omega_{1}=1$

Table 45. Ionosphexe reflection coefficients famplitude, $|\mathrm{T}|$, and phase, Arg T) assuming the quasj-Iongitudinal approximation, for the special ray configuration of the Norton type 301 atmosphere, applicable to the propagation of waves between the D-region ( $h_{3}=70$ kilometers) ox the $E_{-}-x e g i o n ~\left(h_{3}=90\right.$ kilometers $)$ of the ionospliere and the earth.

$T_{\text {ee }}, T_{\mathrm{mm}^{3}} \phi_{1}=60$ degrees, $\omega / \omega_{1^{\circ}}=2$

Table 46. Ionosphere xeflection coefficients famplitude, $|\mathrm{T}|$, and phase, Arg T) assuming the quasi-longitudinal approximation, for the special ray configuration of the Norton type 301 atmosphere, applicable to the propagation of waves between the $\mathrm{D}$-region $\left(\mathrm{h}_{3}=70\right.$ kilometers) or the E-region $\left(\mathrm{h}_{3}=90 \mathrm{kilomete} \mathrm{x}_{\mathrm{s}}\right)$ of the ionosphere and the earth.

$T_{\text {ee, }} T_{\mathrm{mm}}: \phi_{1}=60$ degrees, $\omega / \omega_{x}=5$

Table 47. Ionosphere reflection coefficients (amplitude, $|\mathrm{T}|$, and phase, Axg T) assuming the quasi-longitudinal approximation, for the special ray configuration of the Norton type 301 atmosphere, applicable to the propagation of waves between the D.r.egion $\left(h_{3}=70\right.$ kilometexs) ox the $E_{-10}$ egion $\left(h_{3}=90\right.$ kilometers $)$ of the ionosphere and the earth.

$\mathrm{T}_{\text {em, }} \mathrm{T}_{\mathrm{me}} \phi_{1}=60$ degrees, $\omega / \omega_{x^{*}}=0.3002$ 
Table 48. Ionosphere reflection coefficients (amplitude, $|T|$, and phase, Arg T) assuming the quasi-longitudinal approximation, for the special ray configuration of the Norton type 301 atmosphere. applicable to the propagation of waves between the $D$-region $\left(h_{3}=70\right.$ kilometers) or the E-region ( $h_{3}=90$ kilometers) of the ionosphere and the earth.

$\mathrm{T}_{\text {em }} \mathrm{T}_{\mathrm{me}}, \phi_{1}=60$ degrees, $\omega / \omega_{\mathrm{r}}=0.467$

Table 49. Ionosphere reflection coefficients (amplitude, $|T|$, and phase, Arg T) assuming the quasi-longitudinal approximation, for the special ray configuration of the Norton type 301 atmosphere, applicable to the propagation of waves between the $D$-region $\left(h_{3}=70\right.$ kilometers) or the $\mathbb{E}$-region $\left(h_{3}=90\right.$ kilometers) of the ionosphere and the earth.

$\mathrm{T}_{\text {em }} \mathrm{T}_{\mathrm{me}} \phi_{1}=60 \cdot$ degrees, $\omega / \omega_{r}=0.1501$

Table 50. Ionosphere reflection coefficients $\langle$ amplitude, $|T|$, and phase, Arg T) assuming the quasi-longitudinal approximation, for the special ray configuration of the Norton type 301 atmosphere, applicable to the propagation of waves between the $D$-region $\left(h_{3}=70\right.$ kilometers) or the E-region $\left(h_{3}=90\right.$ kilometers) of the ionosphere and the earth.

$\mathrm{T}_{\text {em }}, \mathrm{T}_{\mathrm{me}}, \phi_{1}=60$ degrees, $\omega / \omega_{x}=0.2335$

Table 51. Ionosphere reflection coefficients (amplitude, $|T|$, and phase, $\operatorname{Arg} T$ ) assuming the quasi-longitudinal approximation, for the special ray configuration of the Norton type 301 atmosphere, applicable to the propagation of waves between the $D$-region $\left(h_{3}=70\right.$ kilometers) or the $E$-region ( $h_{3}=90$ kilometers) of the ionosphere and the earth.

$\mathrm{T}_{\text {em }} \mathrm{T}_{\mathrm{me}} \dot{\phi}_{1}=60$ degrees, $\omega / \omega_{r}=0.01$

Table 52. Ionosphere reflection coefficients $\mid$ amplitude, $|T|$, and phase, Arg T) assuming the quasi-longitudinal approximation, for the special ray configuration of the Norton type 301 atmosphere, applicable to the propagation of waves between the $\mathrm{D}$-region $\left(\mathrm{h}_{3}=70\right.$ kilometers) or the $\mathrm{E}$-region $\left(\mathrm{h}_{3}=90\right.$ kilometers) of the ionosphere and the earth. $\mathrm{T}_{\text {em }} \mathrm{T}_{\mathrm{me}} \dot{\phi}_{1}=60$ degrees, $\omega / \omega_{\mathrm{r}}=0.02$

Table 53. Ionosphere reflection coefficients (amplitude, $|\mathrm{T}|$, and phase, $\operatorname{Arg}$ T) assuming the quasi-longitudinal approximation, for the special ray configuration of the Norton type 301 atmosphere, applicable to the propagation of waves between the $D$-region $\left(h_{3}=70\right.$ kilometers) or the $E_{-r e g i o n ~(h}=90$ kilometers) of the ionosphere and the earth.

$T_{\text {em, }} T_{m e}, \phi_{1}=60$ degrees, $\omega / \omega_{r}=0.05$ 
Table 54. Ionosphere reflection coefficients (amplitude, $|\mathrm{T}|$, and phase, Arg T) assuming the quasi-longitudinal approximation, for the special ray configuration of the Norton type 301 atmosphere, applicable to the propagation of waves between the D-region $\left(h_{3}=70\right.$ kilometers) or the E-region $\left(\mathrm{H}_{3}=90 \mathrm{kilometers}\right)$ of the ionosphere and the earth.

$$
\mathrm{T}_{\text {em, }} \mathrm{T}_{\mathrm{me}}, \phi_{1}=60 \text { degrees, } \omega_{\mathrm{x}}=0.1
$$

Table 55. Ionosphere reflection coefficients (amplitude, $|T|$, and phase, Arg T) assuming the quasi-longitudinal approximation, for the special ray configuration of the Norton type 301 atmosphere, applicable to the propagation of waves between the D-region $\left(\mathrm{h}_{3}=70\right.$ kilometers $)$ or the $\mathrm{E}$-region $\left(\mathrm{h}_{3}=90 \mathrm{kilometers)}\right.$ of the ionosphere and the earth.

$$
\mathrm{T}_{\mathrm{em}} \mathrm{T}_{\mathrm{me}}, \phi_{1}=60 \text { degrees, } \omega / \omega_{x^{*}}=0.2
$$

Table 56. Ionosphere reflection coefficients (amplitude, $|T|$, and phase, Arg T) assuming the quasj-longitudinal approximation, for. the special ray configuration of the Norton type 301 atmosphere, applicable to the propagation of waves between the $\mathrm{D}$-region $\left(\mathrm{h}_{3}=70\right.$ kilometers) or the $E_{-r}$ region $\left(h_{3}=90\right.$ kiloneters $)$ of the ionosphere and the earth.

$$
\mathrm{T}_{\mathrm{em}} \mathrm{T}_{\mathrm{me}} \phi_{1}=60 \text { degrees, } \omega_{\mathrm{r}}=1
$$

Table 57. Ionosphere reflection coefficients (amplitude, $|\mathrm{T}|$, and phase, $A_{1}$ g $T$ ) assuming the quasi-longitudinal approximation, for the special ray configuxation of the Norton type 301. atmosphere, applicable to the propagation of waves between the $D$-region $\left(h_{3}=70\right.$ kilometers) or the E-region $\left(h_{3}=90\right.$ kilometers $)$ of the ionosphere and the earth.

$$
\mathrm{T}_{\text {em }} \mathrm{T}_{\mathrm{me}^{2}} \phi_{1}=60 \text { degiees, } \omega / \omega_{\mathrm{r}}=2
$$

Table 58. Ionosphere reflection coefficients (amplitude, $|\mathrm{T}|$, and phase, Arg T) assuming the quasi-longitudinal approximation, for the special ray configuration of the Norton type 301 atmosphere, applicable to the propagation of waves between the $D$-region $\left(h_{3}=70\right.$ kilometers) or the $\mathrm{E}$-region $\left(\mathrm{h}_{3}=90\right.$ kilometers) of the ionosphere and the earth.

$\mathrm{T}_{\text {em }}, \mathrm{T}_{\mathrm{me}^{2}} \phi_{1}=60$ degrees, $\omega / \omega_{\mathrm{x}}=5$

Table 59. Effective reflection coefficients (amplitude, $\left|\mathrm{C}_{\mathrm{j}}\right|$, and phase, Arg $C_{j}$ ) for the special ray configuration of the Norton type 301 atmosphere, applicable to the propagation of waves between the $D$-region $\left(h_{3}=70\right.$ kilometers $)$ or the $F_{-1}$ egion $\left(h_{3}=90\right.$ kilometers $)$ of the ionosphere and the earth.

$\left|C_{2}\right|, \omega / \omega_{1}=0.3002, \phi_{1}=60$ degrees, $\sigma=0.005, \epsilon_{2}=15, f=20 \mathrm{kc}$ 
Table 60. Effective reflection coefficients amplitude, $\left|C_{j}\right|$, and phase, Arg $C_{j}$ ) for the special ray configuration of the Norton type 301 atmosphere, applicable to the propagation of waves between the $D$-region $\left(h_{3}=70\right.$ kilometers $)$ or the E-region $\left(h_{3}=90\right.$ kilometers $)$ of the ionosphere and the earth.

$\left|C_{2}\right|, \omega / \omega_{r}=0.467, \phi_{1}=60$ degrees, $\sigma=0.005, E_{2}=15, f=20 \mathrm{kc}$

Table 61. Effective reflection coefficients (amplitude, $\left|C_{j}\right|$, and phase, Arg $C_{j}$ ) for the special ray configuration of the Norton type 301 atmosphere, applicable to the propagation of waves between the $D$-region $\left(h_{3}=70\right.$ lilometers $)$ or the E-region $\left(h_{3}=90\right.$ kilometers $)$ of the ionosphere and the earth.

$\left|C_{2}\right|, \omega / \omega_{1:}=0.1501, \phi_{1}=60$ degrees, $\sigma=0.005, \varepsilon_{2}=15, f=10 \mathrm{kc}$

Table 62. Effective reflection coefficients (amplitude, $\left|C_{j}\right|$, and phase, Arg $C_{j}$ ) for the special ray configuration of the Norton type 301 atmosphere, applicable to the propagation of waves between the $D$-region $\left(h_{3}=70\right.$ kilometers $)$ or the $E$-region $\left\langle h_{3}=90\right.$ kilometers $\rangle$ of the ionosphere and the earth.

$\left|C_{2}\right|, \omega / \omega_{r}=0.2335, \phi_{1}=60$ degrees, $\sigma=0.005, E_{2}=15, f=10 \mathrm{kc}$

Table 63. Effective reflection coefficients \{amplitude, $\left|C_{j}\right|$, and phase, Arg $\mathrm{C}_{\mathbf{j}}$ ) for the special ray configuration of the Norton type 301 atmosphere, appicable to the propagation of waves between the D-region ( $h_{3}=70$ kilometers) or the s-region $\left(h_{3}=90\right.$ kilometers $)$ of the ionosphere and the earth.

$\left|C_{2}\right|, \omega / \omega_{1}=0.01, \phi_{1}=60$ degrees, $\sigma=0.005, \varepsilon_{2}=15, f=0.42826552$

Table 64. Effective reflection coefficients (amplitude, $\left|C_{j}\right|$, and phase, $A_{r} g_{j}$ ) for the special ray configuration of the Norton type 301 atmosphere, applicable to the propagation of waves between the $D$-region $\left(h_{3}=70\right.$ kilometers $)$ or the $E$-region $\left(h_{3}=90\right.$ kilometers $)$ of the ionosphere and the earth.

$\left|C_{2}\right|, \omega / \omega_{r}=0.02, \phi_{1}=60$ degrees, $\sigma=0.005, \epsilon_{2}=15$, $f=0.85653105 \mathrm{kc}$

Table 65. Effective reflection coefficients (amplitude, $\left|C_{j}\right|$, and phase, Arg $C_{j}$ ) for the special ray configuration of the Norton type 301 atmosphere, applicable to the propagation of waves between the D-region $\left(h_{3}=70\right.$ kilometers $)$ or the E-region $\left(h_{3}=90\right.$ kilometers $)$ of the ionosphere and the earth.

$\left|C_{2}\right|, \omega / \omega_{r}=0.05, \phi_{1}=60$ degrees, $\sigma=0.005, \varepsilon_{2}=15,1.1413276 \mathrm{kc}$ 
Table 66. Effective reflection coefficients (amplitude, $\left|\mathrm{C}_{j}\right|$, and phase, Arg $C_{j}$ ) for the special ray configuration of the Norton type 301 atmosphere, applicable to the propagation of waves between the $D$-region ( $h_{3}=70$ kilometers) or the E-region $\left(h_{3}=90\right.$ kilometers) of the ionosphere and the earth.

$\left|C_{2}\right|, \omega / \omega_{r}=0.1, \phi_{1}=60$ degrees, $\sigma=0.005, \epsilon_{2}=15$,

Table 67. Effective reflection coefficients (amplitude, $\mid \mathrm{C}_{j}$, and phase, $\operatorname{Arg} C_{j}$ ) for the special ray configuration of the Norton type 301 atmosphere, applicable to the propagation of waves between the $D$-region ( $h_{3}=70$ kilometers) or the $E_{-1} \cdot e g i o n\left(h_{3}=90\right.$ kilometers) of the ionosphere and the earth.

$\left|C_{2}\right|, \omega / \omega_{r}=0.2, \phi_{1}=60$ degrees, $\sigma=0.005, \epsilon_{2}=15$, $f=8.5653105 \mathrm{kc}$

Table 68. Effective reflection coefficients (amplitude, $\left|C_{j}\right|$, and phase, $\operatorname{Arg~} C_{j}$ ) for the special ray configuration of the Norton type 301 atmosphere, applicable to the propagation of waves between the D-region ( $h_{3}=70$ kilometers) or the E..region ( $h_{3}=90$ kilometers) of the ionosphere and the earth.

$\left|\mathrm{C}_{2}\right|, \omega_{\mathrm{r}}=1, \phi_{1}=60$ degiees, $\sigma=0.005, \epsilon_{2}=15,{ }_{\hat{1}}=42.826552 \mathrm{kc}$

Table 69. Effective reflection coefficients (amplitude, $\left|\mathrm{C}_{j}\right|$, and phase, Arg $C_{j}$ ) for the special ray configuration of the Norton type 301 atmosphere, applicable to the propagation of waves between the $D$-region ( $h_{3}=70$ kilometers) or the $E$-region (h $3=90$ kilometers) of the ionosphere and the earth.

$\left|\mathrm{C}_{2}\right|, \omega / \omega_{\mathrm{r}}=2, \phi_{1}=60$ degrees, $\sigma=0.005,{ }_{2}=15, f=85.653105 \mathrm{kc}$

Table 70. Effective reflection coefficients (amplitude, $\left|\mathrm{C}_{j}\right|$, and phase, Arg $C_{j}$ ) for the special ray configuration of the Norton type 301 atmosphere, applicabie to the propagation of waves between the $D$-region ( $h_{3}=70$ kilometers) or the $\mathrm{E}$-region ( $\mathrm{h}_{3}=90$ kilometers) of the ionosphere and the earth.

$\left|\mathrm{C}_{2}\right|, \omega / \omega_{\mathrm{r}}=5, \phi_{1}=60$ degrees, $\sigma=0.005, \epsilon_{2}=15, \mathrm{f}=214.132 .76 \mathrm{kc}$

Table 71. Effective reflection coefficients (amplitude, $\left|\mathrm{C}_{\mathrm{j}}\right|$, and phase, Arg $C_{j}$ ) for the special ray configuration of the Norton type 301 atmosphere, applicable to the propagation of waves between the D-region ( $h_{3}=70$ kilometers) or the $E$-region ( $h_{3}=90$ kilometers) of the ionosphere and the earth.

$\left|C_{2}\right|, \omega / \omega_{r}=0.01, \phi_{1}=60$ degrees, $\sigma=0.005, \epsilon_{2}=15$, $f=0.66622 .256 \mathrm{kc}$ 
Table 72 . Effective reflection coefficients (amplitude, $\left|C_{j}\right|$, and phase, $\operatorname{Arg} \mathrm{C}_{\mathrm{j}}$ ) for the special ray configuration of the Norton type 301 atmosphere, applicable to the propagation of waves between the D-region ( $\mathrm{h}_{3}=70$ kilometers) or the $\mathrm{E}$-region $\left(\mathrm{h}_{3}=90\right.$ kilometers $)$ of the ionosphere and the earth.

$\left|C_{2}\right|, \omega / \omega_{\mathrm{r}}=0.02, \phi_{1}=60$ degrees, $\sigma=0.005, \epsilon_{2}=15$, $\mathrm{f}=1.332425 \mathrm{kc}$

Table 73. Effective reflection coefficients amplitude, $\left|C_{j}\right|$, and phase, $\operatorname{Arg} C_{j}$ ) for the special ray configuration of the Norton type 301 atmosphere, applicable to the propagation of waves between the $D$-region ( $h_{3}=70$ kilometers) or the $\mathrm{E}$-region $\left(h_{3}=90\right.$ kilometers $)$ of the ionosphere and the earth.

$\left|C_{2}\right|, \omega / \omega_{r}=0.05, \phi_{1}=60$ degrees, $\sigma=0.005, \epsilon_{2}=15$, $\hat{f}=3.3311126 \mathrm{kc}$

Table 74. Effective reflection coefficients (amplitude, $\left|C_{j}\right|$, and phase, Arg $C_{j}$ ) for the special ray configuration of the Norton type 301 atmosphere, applicable to the propagation of waves between the D-region ( $h_{3}=70$ kilometers) or the E-region $\left(h_{3}=90\right.$ kilometers) of the ionosphere and the earth.

$\left|C_{2}\right|, \omega / \omega_{r}=0.1, \phi_{1}=60$ degrees, $\sigma=0.005, \epsilon_{2}=15$, $f=6.6622252 \mathrm{kc}$

Table 75. Effective reflection coefficients (amplitude, $\left|C_{j}\right|$, and phase, Ar $\mathrm{C}_{j}$ ) for the special ray configuration of the Norton type 301 atmosphere, applicable to the propagation of waves between the $D$-region ( $h_{3}=70$ kilometers) or the $\mathrm{E}$-region $\left(\mathrm{h}_{3}=90\right.$ kilometers) of the ionosphere and the earth.

$\left|C_{2}\right|, \omega / \omega_{r}=0.2, \phi_{1}=60$ degrees, $\sigma=0.005, c_{2}=15$, $f=13.324450 \mathrm{kc}$

Table 76. Effective reflection coefficients (amplitude, $\left|C_{j}\right|$, and phase, Arg $C_{j}$ ) for the special ray configuration of the Norton type 301 atmosphere, applicable to the propagation of waves between the D-region ( $h_{3}=70$ kilometers) or the E-region $\left(h_{3}=90\right.$ kilometers $)$ of the ionosphere and the earth.

$\left|C_{2}\right|, \omega / \omega_{r}=1, \phi_{1}=60$ degrees, $\sigma=0.005, \epsilon_{2}=15, \hat{f}=66.622252 \mathrm{kc}$

Table 77. Effective reflection coefficients (amplitude, $\left|C_{j}\right|$, and phase, Arg $\mathrm{C}_{j}$ ) for the special ray configuration of the Norton type 301 atmosphere, applicable to the propagation of waves between the D-region ( $h_{3}=70$ kilometers $)$ or the E-region $\left(h_{3}=90\right.$ kilometers $)$ of the ionosphere and the earth.

$\left|C_{2}\right|, \omega / \omega_{r}=2, \phi_{1}=60$ degrees, $\sigma=0.005, \varepsilon_{2}=15$, $f=133.24450 \mathrm{kc}$ 
Table 78. Effective reflection coefficients (amplitude, $\left|\mathrm{C}_{\mathbf{j}}\right|$, and phase, Arg $C_{j}$ ) for the special ray configuration of the Norton type 301 atmosphere, applicable to the propagation of waves between the $\mathrm{D}$-region $\left(\mathrm{h}_{3}=70\right.$ kilometers) or the $\mathrm{E}-\mathrm{region}\left(\mathrm{h}_{3}=90\right.$ kilometers) of the ionosphere and the earth.

$\left|\mathrm{C}_{2}\right|, \omega / \omega_{1}=5, \phi_{1}=60$ degrees, $\sigma=0.005, \varepsilon_{2}=15, f=333.11126 \mathrm{kc}$

Table 79. Effective reflection coefficients (amplitude, $\left|\mathrm{C}_{\mathbf{j}}\right|$, and phase, Arg $C_{j}$ ) for the special ray configuration of the Norton type 301 atmosphere, applicable to the propagation of waves between the $D$-region $\left(h_{3}=70\right.$ kilometers) or the E-region ( $h_{3}=90$ kilometers) of the ionosphere and the earth.

$\left|\mathrm{C}_{3}\right|, \omega / \omega_{\mathrm{r}}=0.3002, \phi_{1}=60$ degrees, $\sigma=0.005, \epsilon_{2}=15, f=20 \mathrm{kc}$

Table 80. Effective reflection coefficients (amplitude, $\left|\mathrm{C}_{\mathbf{j}}\right|$, and phase, Arg $C_{j}$ ) for the special ray configuration of the Norton type 301 atmosphere, applicable to the propagation of waves between the $\mathrm{D}$-region ( $\mathrm{h}_{3}=70$ kilometers) or the $\mathrm{E}$-region ( $\mathrm{h}_{3}=90$ kilometers) of the ionosphere and the earth.

$\left|C_{3}\right|, \omega / \omega_{r}=0.467, \phi_{1}=60$ degrees, $\sigma=0.005, \epsilon_{2}=15, f=20 \mathrm{kc}$

Table 81. Effective reflection coefficients (amplitude, $\left|\mathrm{C}_{\mathrm{j}}\right|$, and phase, Arg $C_{j}$ ) for the special ray configuration of the Norton type 301 atmosphere, applicable to the propagation of waves between the $D$-region ( $h_{3}=70$ kilometers) or the $\mathrm{E}_{-1}$ - egion ( $\mathrm{h}_{3}=90$ kilometers) of the ionosphere and the earth.

$\left|\mathrm{C}_{3}\right|, \omega / \omega_{\mathrm{r}}=0.1501, \phi_{1}=60$ degrees, $\sigma=0.005, \epsilon_{2}=15, \mathrm{f}=10 \mathrm{kc}$

Table 82. Effective reflection coefficients (amplitude, $\left|\mathrm{C}_{\mathbf{j}}\right|$, and phase, Arg $C_{j}$ ) for the special ray configuration of the Norton type 301 atmosphere, applicable to the propagation of waves between the D-region ( $h_{3}=70$ kilometers) or the $\mathbb{E}-1$ egion ( $h_{3}=90$ kilometers) of the ionosphere and the earth.

$\left|C_{3}\right|, \omega / \omega_{r}=0.2335, \phi_{1}=60$ degrees, $\sigma=0.005, \epsilon_{2}=15, f=10 \mathrm{kc}$

Table 83. Effective reflection coefficients (amplitude, $\left|C_{j}\right|$, and phase, Arg $C_{j}$ ) for the special ray configuration of the Norton type 301 atmosphere, applicable to the propagation of waves between the $\mathrm{D}$-region $\left(\mathrm{h}_{3}=70\right.$ kilometers) or the $\mathrm{E}$-region $\left(\mathrm{h}_{3}=90\right.$ kilometers) of the ionosphere and the earth.

$\left|\mathrm{C}_{3}\right|, \omega / \omega_{\mathrm{r}}=0.01, \phi_{1}=60$ degrees, $\sigma=0.005, \varepsilon_{2}=15$. $\mathrm{f}=0.42826532 \mathrm{kc}$

Table 84. Effective reflection coefficients (amplitude, $\left|\mathrm{C}_{\mathrm{j}}\right|$, and phase, Arg $C_{j}$ ) for the special ray configuration of the Norton type 301 atmosphere, applicable to the propagation of waves between the $\mathrm{D}$-region $\left(\mathrm{h}_{3}=70 \mathrm{kilometers}\right)$ or the $\mathrm{E}$-region $\left(\mathrm{h}_{3}=90\right.$ kilometers $)$ of the ionosphere and the earth.

$\left|C_{3}\right|, \omega / \omega_{10}=0.02, \phi_{1}=60$ degrees, $\sigma=0.005, \epsilon_{2}=15$, $2=15=0.85653105 \mathrm{kc}$ 
Table 85. Effective reflection coefficients (amplitude, $\left|C_{j}\right|$, and phase, Arg $C_{j}$ ) for the special ray configuration of the Norton type 301 atmosphere, applicable to the propagation of waves between the $D$-region $\left(h_{3}=70\right.$ kilometers $)$ or the $\mathrm{E}$-region $\left(\mathrm{h}_{3}=90\right.$ kilometers $)$ of the ionosphere and the earth.

$\left|C_{3}\right|, \omega / \omega_{r}=0.05, \phi_{1}=60$ degrees, $\sigma=0,005, \epsilon_{2}=15$, $f=2.1413276 \mathrm{kc}$

Table 86. Effective reflection coefficients lamplitude, $\left|\mathrm{C}_{i}\right|$, and phase, Arg $C_{j}$ ) for the special ray configuration of the Notton type 301 atmosphere, applicable to the propagation of waves between the D-region ( $h_{3}=70$ kilometers) or the 2 -region $\left(h_{3}=90\right.$ kilometers $)$ of the ionosphere and the earth.

$\left|C_{3}\right|, \omega / \omega_{r}=0.1, \phi_{1}=60$ degrees, $c=0,005, \epsilon_{2}=15$, $\frac{f}{i}=4.2826552 \mathrm{kc}$

Table 87. Effective reflection coefficients \{ampiitude, $\left|C_{j}\right|$, and phase, Arg $C_{j}$ ) for the special ray configuration of the Norton type 301 atmosphere, applicable to the propagation of waves between the $D$-region $\left(h_{3}=70\right.$ kilometers $)$ or the - region $\left(h_{3}=90\right.$ kilometers $)$ of the ionosphere and the earth.

$\left|C_{3}\right|, \omega / \omega_{\mathrm{r}}=0.2, \phi_{1}=60$ degrees, $\sigma=0.005, \varepsilon_{2}=15$, $f=8.5653105 \mathrm{kc}$

Table 88. Effective reflection coefficients (amplitude, $\left|C_{j}\right|$, and phase, $\operatorname{Arg} C_{j}$ ) for the special ray configuration of the Norton type 301 atmosphere, applicable to the propagation of waves between the $D$-region ( $h_{3}=70$ kilometers $)$ or the E-region $\left(h_{3}=90\right.$ kilometers) of the ionosphere and the earth.

$\left|C_{3}\right|, \omega / \omega_{r}=1, \phi_{1}=60$ degrees, $\sigma=0.005, \epsilon_{2}=15, \hat{i}=42.826552 \mathrm{kc}$

Table 89. Effective reflection coefficients (amplitude, $\left|C_{j}\right|$, and phase, Arg $C_{j}$ ) for the special ray configuration of the Norton type 301 atmosphere, applicable to the propagation of waves between the D-region ( $h_{3}=70$ kilometers) or the $E$-region $\left(h_{3}=90\right.$ kilometers $)$ of the ionosphere and the earth.

$\left|C_{3}\right|, \omega / \omega_{r}=2, \phi_{1}=60$ degrees, $\sigma=0.005, \epsilon_{2}=15, \hat{x}=85.653105 \mathrm{kc}$

Table 90. Effective reflection coefficients $\left\{\right.$ amplitude, $\left|C_{j}\right|$, and phase, Arg $C_{j}$ ) for the special ray configuration of the Norton type 301 atmosphere, applicable to the propagation of waves between the $D$-region $\left(h_{3}=70\right.$ kilometers) or the $E_{-r e g i o n ~}\left(h_{3}=90\right.$ kilometers) of the ionosphere and the earth.

$\left|C_{3}\right|, \omega / \omega_{r}=5, \phi_{1}=60$ degrees, $\sigma=0.005, \epsilon_{2}=15, \dot{x}=214.13276 \mathrm{kc}$ 
Table 91. Effective reflection coefficients (amplitude, $\left|\mathrm{C}_{\mathrm{j}}\right|$, and phase, $A_{r} \cdot C_{j}$ ) for the special ray configuration of the Norton type 301 atmosphere, applicable to the propagation of waves between the $\mathrm{D}$-region $\left(\mathrm{h}_{3}=70 \mathrm{kilometers}\right)$ ox the $\mathrm{I}$-region $\left(\mathrm{h}_{3}=90\right.$ kilometers) of the ionosphere and the earth.

$\left|C_{3}\right|, \omega / \omega_{x}=0.01, \phi_{1}=60$ degrees, $\sigma=0.005, \epsilon_{2}=15$,

$$
f=0.66622256 \mathrm{kc}
$$

Table 92. Effective reflection coeficients (amplitude, $\left|\mathrm{C}_{j}\right|$, and phase, Arg $C_{j}$ ) for the special ray configuration of the Norton type 301 atmosphere, applicable to the propagation of waves between the

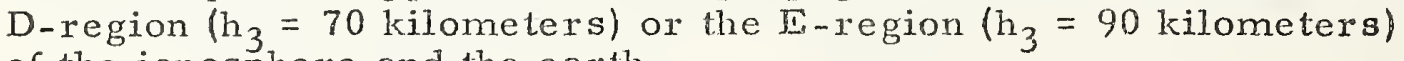
of the ionosphere and the earth.

$\left|C_{3}\right|, \omega / \omega_{r}=0.02, \phi_{1}=60$ degrees, $\sigma=0.005, \varepsilon_{2}=15$,

$$
f=1.332425 \mathrm{kc}
$$

Table 93. Effective reflection coefficients famplitude, $\left|\mathrm{C}_{j}\right|$, and phase, Arg $C_{j}$ ) for the special ray configuration of the Norton type 301 atmosphere, applicable to the propagation of waves between the D-region ( $h_{3}=70$ kjlometers) or the E-region (h $3=90$ kilometers) of the ionosphere and the earth.

$\left|\mathrm{C}_{3}\right|, \omega / \omega_{r}=0.005, \quad \phi_{1}=60$ degrees, $\sigma=0.005, \varepsilon_{t}=3.33$, $\tau_{i}=3,3311126 \mathrm{kc}$

Table 9.'. Effective reflection coefíicients (amplitude, $\left|\mathrm{C}_{\mathrm{j}}\right|$, and phase, Arg $\mathrm{C}_{j}$ ) for the special ray configuration of the Norton type 301 atmosphere, applicable to the propagation of waves between the $D$-region ( $h_{3}=70$ kilometers $)$ or the $\mathrm{s}_{-}-\mathrm{xegion}\left(\mathrm{h}_{3}=90\right.$ kilometers $)$ of the ionosphere and the earth.

$\left|C_{3}\right|, \omega / \omega_{1}=0.1, \phi_{1}=60$ degxees, $\sigma=0.005, \varepsilon_{2}=15$, $f=6.6622 .252 \mathrm{kc}$

Table 95. Effective reflection coefficients (amplitude, $\left|C_{j}\right|$, and phase, Arg $C_{j}$ ) for the special ray configuration of the Norton type 301 atmosphere, applicable to the propagation of waves between the $D$-region ( $h_{3}=70$ kilometers) or the $E-r e g i o n$ ( $h_{3}=90$ kilometers) of the ionosphere and the earth.

$\left|C_{3}\right|, \omega / \omega_{r}=0.2, \phi_{1}=60$ degrees, $\sigma=0.005, \epsilon_{2}=15$, $f=13.324450 \mathrm{kc}$

Table 96. Effective reflection coefficients (amplitude, $\left|C_{j}\right|$, and phase, $A_{1} \cdot \mathrm{g} \mathrm{C}_{j}$ ) for the special ray configuration of the Norton type 301 atmosphere, applicable to the propagation of waves between the $\mathrm{D}$-region $\left(\mathrm{h}_{3}=70\right.$ kilometers) or the $\mathrm{H}_{-r e g i o n}\left(\mathrm{~h}_{3}=90\right.$ kilometers) of the ionosphere and the earth.

$\left|C_{3}\right|, \omega / \omega_{1}=1, \phi_{1}=60$ degrees, $\sigma=0.005, \epsilon_{2}=15$, $f=66.622252 \mathrm{kc}$ 
Table 97. Efiective reflection coefficients (amplitude, $\left|C_{j}\right|$, and phase, $\operatorname{Arg~} \mathrm{C}_{\mathrm{j}}$ ) for the special ray configuration of the Norton type 301 atmosphere, applicable to the propagation of waves between the $\mathrm{D}$-region $\left(\mathrm{h}_{3}=70\right.$ kilometers) or the $\mathrm{E}$-region $\left(\mathrm{h}_{3}=90\right.$ kilometers $)$ of the ionosphere and the earth.

$\left|\mathrm{C}_{3}\right|, \omega / \omega_{\mathrm{r}}=2, \phi_{1}=60$ degrees, $\sigma=0.005, \epsilon_{2}=15, \mathrm{f}=133.24450 \mathrm{kc}$

Table 98. Effective reflection coefficients (amplitude, $\left|\mathrm{C}_{j}\right|$, and phase, Arg $C_{j}$ ) for the special ray configuration of the Notton type 301 atmosphere, applicable to the propagation of waves between the $D$-region $\left(h_{3}=70\right.$ kilometers $)$ or the E-region $\left(h_{3}=90\right.$ kilometers $)$ of the ionosphere and the earth.

$\left|C_{3}\right|, \omega / \omega_{\mathrm{r}}=5, \phi_{1}=60$ degrees, $\sigma=0.005, \epsilon_{2}=15, f=333.11126 \mathrm{kc}$

Table 99. Effective reflection coefficients (amplitude, $\left|C_{j}\right|$, and phase, $\operatorname{Arg~} C_{j}$ ) for the special ray configuration of the Norton type 301 atmosphere, applicable to the propagation of waves between the $D$-region $\left(h_{3}=70\right.$ kilometers) or the E-region $\left(h_{3}=90\right.$ kilometers) of the ionosphere and the earth.

$\left|C_{4}\right|, \omega / \omega_{\mathrm{r}}=0.3002, \phi_{1}=60$ degrees, $\sigma=0.005, \epsilon_{2}=15, \mathrm{f}=20 \mathrm{kc}$

Table 100. Effective reflection coefficients (amplitude, $\left|C_{j}\right|$, and phase, $\operatorname{Arg} C_{j}$ ) for the special ray configuration of the Norton type 301 atmosphere, applicable to the propagation of waves between the D-region $\left(h_{3}=70\right.$ kilometers) or the E-region $\left(h_{3}=90\right.$ kilometers) of the ionosphere and the earth.

$\left|C_{4}\right|, \omega / \omega_{r}=0.467, \phi_{1}=60$ degrees, $\sigma=0.005, \epsilon_{2}=15, f=20 \mathrm{kc}$

Table 101. Effective reflection coefficients (amplitude, $\left|C_{j}\right|$, and phase, Arg $C_{j}$ ) for the special ray configuration of the Norton type 301 atmosphere, applicable to the propagation of waves between the $D$-region $\left(h_{3}=70\right.$ kilometers) or the E-region $\left(h_{3}=90\right.$ kilometers $)$ of the ionosphere and the earth.

$\left|C_{4}\right|, \omega / \omega_{\mathrm{r}}=0.1501, \phi_{1}=60$ degrees, $\sigma=0.005, \epsilon_{2}=15, f=10 \mathrm{kc}$

Table 102. Effective reflection coefficients (amplitude, $\left|C_{j}\right|$, and phase, $A_{r} g C_{j}$ ) for the special ray configuration of the Norton type 301 atmosphere, applicable to the propagation of waves between the $D$-region $\left(h_{3}=70\right.$ kilometers $)$ or the E-region $\left(h_{3}=90\right.$ kilometers $)$ of the ionosphere and the earth.

$\left|C_{4}\right|, \omega / \omega_{r}=0.2335, \phi_{1}=60$ degrees, $\sigma=0.005, \epsilon_{2}=15, f=10 \mathrm{kc}$

Table 103. Effective reflection coefficients (amplitude, $\left|C_{j}\right|$, and phase, $\operatorname{Ar} g C_{j}$ ) for the special ray configuration of the Norton type 301 atmosphere, applicable to the propagation of waves between the $D$-region ( $h_{3}=70$ kilometers) or the E-region $\left(h_{3}=90\right.$ kilometers) of the ionosphere and the earth.

$\left|C_{4}\right|, \omega / \omega_{r}=0.01, \phi_{1}=60$ degrees, $\sigma=0.005, \epsilon_{2}=15, \quad f=0.42826552 \mathrm{kc}$ 
Table 104. Effective reflection coefficients (amplitude, $\left|C_{j}\right|$, and phase, Arg $C_{j}$ ) for the special ray configuration of the Norton type 301 atmosphere, applicable to the propagation of waves between the D-region $\left(h_{3}=70\right.$ kilometers $)$ or the E-region $\left(h_{3}=90\right.$ kilometers $)$ of the ionosphere and the earth.

$\left|\mathrm{C}_{4}\right|, \omega / \omega_{\mathrm{r}}=0.02, \phi_{1}=60$ degrees, $\sigma=0.005, \epsilon_{2}=15$,

Table 105. Effective reflection coefficients (amplitude, $\left|\mathrm{C}_{\dot{j}}\right|$, and phase, Arg $C_{j}$ ) for the special ray configuration of the Norton type 301 atmosphere, applicable to the propagation of waves between the $D$-region $\left(h_{3}=70\right.$ kilometers $)$ or the $E$-region $\left(h_{3}=90\right.$ kilometers $)$ of the ionosphere and the earth.

$\left|C_{4}\right|, \omega / \omega_{\mathrm{r}}=0.05, \phi_{1}=60$ degrees, $\sigma=0.005, \epsilon_{2}=15$, $f=2.1413276 \mathrm{kc}$

Table 106. Effective reflection coefficients (amplitude, $\left|C_{j}\right|$, and phase, $\mathrm{Ar} g \mathrm{C}_{j}$ ) for the special ray configuration of the Norton type 301 atmosphere, applicable to the propagation of waves between the $D$-region ( $h_{e}=70$ kilometers) or the $E$-region $\left(h_{3}=90\right.$ kilometers) of the ionosphere and the earth.

$\left|C_{4}\right|, \omega / \omega_{r}=0.1, \phi_{1}=60$ degxees, $\sigma=0.005, \epsilon_{2}=15$, $f=4.2826552 \mathrm{kc}$

Table 107. Effective reflection coefficients (amplitude, $\left|C_{j}\right|$, and phase, Arg $C_{j}$ ) for the special ray configuration of the Norton type 301 atmosphere, applicable to the propagation of waves between the $D$-region ( $h_{3}=70$ kilometers) or the $E-r e g i o n\left(h_{3}=90\right.$ kilometers $)$ of the ionosphere and the earth.

$\left|C_{4}\right|, \omega / \omega_{r}=0.2, \phi_{1}=60$ degrees, $\sigma=0.005, \epsilon_{2}=15$, $f=8.5653105 \mathrm{kc}$

Table 108. Effective reflection coefficients (amplitude, $\left|C_{j}\right|$, and phase, $\operatorname{Ar} g C_{j}$ ) for the special ray configuration of the Norton type 301 atmosphete, applicable to the propagation of waves between the D-region $\left(h_{3}=70\right.$ kilometers) or the $\mathrm{E}-$ region $\left(h_{3}=90\right.$ kilometers $)$ of the ionosphere and the earth.

$\left|C_{4}\right|, \omega / \omega_{r}=1, \phi_{1}=60$ degrees, $\sigma=0.005, \epsilon_{2}=15, f=42.826552 \mathrm{kc}$

Table 109. Effective reflection coefficients (amplitude, $\left|C_{j}\right|$, and phase, Arg $C_{j}$ ) for the special ray configuration of the Norton type 301 atmosphefe, applicable to the propagation of waves between the $\mathrm{D}$-region $\left(\mathrm{h}_{3}=70\right.$ kilometers $)$ or the $\mathrm{E}$-region $\left(\mathrm{h}_{3}=90\right.$ kilometers $)$ of the ionosphere and the earth.

$\left|C_{4}\right|, \omega / \omega_{r}=2, \phi_{1}=60$ degrees, $\sigma=0.005, \epsilon_{2}=15, f=85.653105 \mathrm{kc}$ 
Table 110. Effective reflection coefficients (amplitude, $\left|C_{j}\right|$, and phase, $\operatorname{Ar} g\left(C_{j}\right.$ ) for the special ray configuration of the Norton type 301 atmosphere, applicable to the propagation of waves between the $D$-region $\left(h_{3}=70\right.$ kilometers $)$ or the E-region $\left(h_{3}=90\right.$ kilometers $)$ of the ionosphere and the earth.

$\left|C_{4}\right|, \omega / \omega_{r}=5, \phi_{1}=60$ degree, $\sigma=0.005, \epsilon_{2}=15, f=214.13276 \mathrm{kc}$

Table 111. Effective reflection coefficients (amplitude, $\left|C_{j}\right|$, and phase, Arg $C_{j}$ ) for the special ray configuration of the Norton type 301 atmosphere, applicable to the propagation of waves between the $D$-region $\left(h_{3}=70\right.$ kilometers) or the E-region $\left(h_{3}=90\right.$ kilometers $)$ of the ionosphere and the earth.

$\left|C_{4}\right|, \omega / \omega_{\mathrm{r}}=0.01, \phi_{1}=60$ degrees, $\sigma=0.005, \epsilon_{2}=15$, $f=0.66622256 \mathrm{kc}$

Table 112. Effective reflection coefficients (amplitude, $\left|C_{j}\right|$, and phase, $\left.A_{1} g C_{j}\right)$ for the special ray configuxation of the Norton type 301 atmosphere, applicable to the propagation of waves between the $D$-region $\left(h_{3}=70\right.$ kilomelers) or the 1 - region $\left(h_{3}=90\right.$.kilometers $)$ of the ionosphere and the earth.

$\left|C_{4}\right|, \omega / \omega_{\mathrm{r}}=0.02, \phi_{1}=60$ degrees, $\sigma=0.005, \varepsilon_{2}=15$, $2 \mathrm{f}=1.332425 \mathrm{kc}$

Table I13. Effective reflection coefficients (amplitude, $\left|C_{j}\right|$, and phase, $\operatorname{Arg}_{j} C_{j}$ ) for the special ray configuration of the Norton type 301 atmosphere, applicable to the propagation of waves between the $D$-region ( $h_{3}=70$ kilometers) or the E-region $\left(h_{3}=90\right.$ kilometers $)$ of the ionosphere and the earth.

$\left|C_{4}\right|, \omega / \omega_{r}=0.05, \phi_{1}=60$ degrees, $\sigma=0.005, \epsilon_{2}=15$,

$$
f=3.3311126 \mathrm{kc}
$$

Table 114. Effective reflection coefficients (amplitude, $\left|C_{j}\right|$, and phase, $\overline{A r} g C_{j}$ ) for the special ray configuration of the Norton type 301 atmosphere, applicable to the propagation of waves between the $D$-region $\left(h_{3}=70\right.$ kilometers $)$ or the $\mathbb{E}$-region $\left(h_{3}=90\right.$ kilometers of the ionosphere and the earth.

$\left|C_{4}\right|, \omega / \omega_{r}=0.1, \phi_{1}=60$ degrees, $\sigma=0.005, \epsilon_{2}=15$, $f=6.6622262 \mathrm{kc}$

Table 115. Effective reflection coefficients (amplitude, $\left|C_{j}\right\rangle$, and phase, $\operatorname{Arg} C_{j}$ ) for the special ray configuration of the Norton type 301 atmosphere, applicable to the propagation of waves between the $\mathbb{D}$-region $\left(\mathrm{h}_{3}=70\right.$ kilometers) or the $\mathbb{E}$-region $\left(\mathrm{h}_{3}=90\right.$ kilometers $)$ of the ionosphere and the earth.

$\left|C_{4}\right|, \omega / \omega_{r}=0.2, \phi_{1}=60$ degrees, $\sigma=0.005, \varepsilon_{2}=15$. $f=13.324450 \mathrm{kc}$ 
Table 116. Effective reflection coefficients $\left\{\right.$ amplitude, $\left|\mathrm{C}_{j}\right|$, and phase, $\mathrm{Arg}_{j}$ ) for the special ray configuration of the Norton type 301 atmosplieie, applicable to the propagation of waves between the $D$-region ( $h_{3}=70$ kilometers) or the $E_{-}$-region $\left(h_{3}=90\right.$ kilometers) of the ionosphere and the earth.

$\left|\mathrm{C}_{4}\right|, \omega / \omega_{1}=1, \phi_{1}=60$ degrees, $\sigma=0.005, \epsilon_{2}=15, f=66.622252 \mathrm{kc}$

Table 117. Effective reflection coefficients lamplitude, $\left|\mathrm{C}_{j}\right|$, and phase, Arg $C_{j}$ ) for the special ray configuration of the Norton type 301 atmosphere, applicable to the propagation of waves between the D-region ( $h_{3}=70$ kilometers) or the E-region (h $h_{3}=90$ kilometerg) of the ionosphere and the earth.

$\left|\mathrm{C}_{4}\right|, \omega / \omega_{r}=2, \phi_{1}=60$ degrees, $\sigma=0.005, \epsilon_{2}=15, f=133.24450 \mathrm{kc}$

Table 118. Effective reflection coefficients (amplitude, $\left|\mathrm{C}_{\mathrm{j}}\right|$, and phase, Arg $C_{j}$ ) fox the special ray configuration of the Norton type 301 atmosphere, applicable to the propagation of waves between the $D$-region ( $\mathrm{h}_{3}=70$ kilometers) or the s-region ( $\mathrm{h}_{3}=90$ kilometers) of the ionosphere and the earth.

$\left|C_{4}\right|, \omega / \omega_{r}=5, \phi_{1}=60$ degrees, $\sigma=0.005, \varepsilon_{2}=15, f=333.11126 \mathrm{kc}$

Table 119. Very low frequency and low frequency part of sky wave transter characteristic (amplitude, $|\mathrm{E}(\omega, \mathrm{d})|$, and phase, Arg $E(\omega, d))$ assuming the geometrical-optical and quasi-longitudinalFresnel approximations for various distances, d, electron density, $N$, collision frequency, $v$, and eaxth ${ }^{8}$ s magnetic field strength, $H_{\text {. }}$

Table 120. Very low frequency and low frequency part of sky wave transfer characteristic (amplitude, $|E(\omega, d)|$, and phase, $A r g \mathbb{E}(\omega, d)$ assuming the geometrical-optical and quasi-longituclinal-Fresnel approximations for various distances, $d$, electron density, $N$, collision frequency, $\nu$, and earth's magnetic field strength, $H$.

Table 121. Very low frequency and low frequency part of sky wave transfer characteristic (amplitude, $|\mathrm{E}(\omega, \mathrm{d})|$, and phase, Arg $\mathrm{E}(\omega, \mathrm{d})$ assuming the geometrical-optical and quasi-longitudinal-Fresnel approximations for various distances, $d$, electron density, $N$, collision frequency, $v$, and earth ${ }^{2}$ magnetic field strength, $\mathbb{H}$.

Table 122. Very low frequency and low frequency part of sky wave transfer characteristic (amplitude, $|\mathrm{E}(\omega, \mathrm{d})|$, and phase, Arg $\mathbb{E}(\omega, d))$ assuming the geometrical-optical and quasi-longitudinal-Fresnel approximations for various distances, $d$, electron density, $N$, collision frequency, $v$, and earth ${ }^{8} \mathrm{~s}$ magnetic field strength, $\mathbb{H}$.

Table 123. Sky wave delay, $\bar{a} j-a$, and the geometrical-optical ray divergence-convergence coefficient, $\propto_{j}$, for various distances, d. 
$\omega / \omega_{r}=0.3002$

$\left|\mathrm{T}_{\text {ee }}\right|$

Arg $\mathrm{T}_{\text {ee }}$

$\left|\mathrm{T}_{\mathrm{mm}}\right|$

$\operatorname{Arg~T}$ mm

degrees

$\phi_{1}=10$ degrees

$\begin{array}{rl}5 & 4.4552333=1 \\ 15 & 4.3377702=1 \\ 25 & 4.0994560=1 \\ 35 & 3.7387817=1 \\ 45 & 3.2772442=1 \\ 55 & 2.8414416=1 \\ 65 & 2.9002620=1 \\ 75 & 4.2615247=1 \\ 85 & 7.4695888=1\end{array}$

$7.3725810-1$

$7.5812560-1$

$8.0616550-1$

$8.9784570-1$

1.0711519

1.4077680

1. 9831845

3. 7076184

3.3041685

$4.4844018=1$
$4.6018533=1$
$4.8407615=1$
$5.2085258=1$
$5.7148817=1$
$6.3707095=1$
$7.1871248=1$
$8.1749512=1$
$9.3443617=1$

2.4092733 2.4290032

$\phi_{1}=20$ degrees

$7.4582840-1$

$7.6728700=1$

$8.1650590-1$

$9.0977750-1$

1.0840055

1.4163042

1.9761741

3. 7209551

3.3094437

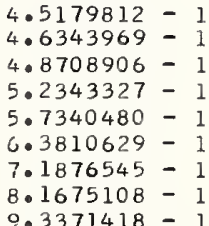

2.4008496 2.4211441 2.4617433 2. 5224739 2. 6026658 2.7008422 2.8145366 2. 9402798 $\phi_{1}=30$ degrees

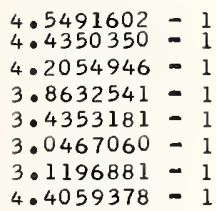

$7.5984010=1$
$7.8224050=1$

$8.3328980-1$

9.2887370 -

1. 1039507

1.4284345

1. 9637604

3. 7433517

3. 3184052

\section{$4.5775948-1$
$4.6919425-1$ \\ 4.9237872 - \\ $5.2792245=$ \\ $5.7670604-1$ \\ $6.3986929-1$ \\ 7. $1883874-1$ \\ $8.1546277-1$}

2.9413729

3.0739868

85

$0.3247053=$

$$
\begin{aligned}
& 2.3870732 \\
& 2 \cdot 4082737 \\
& 2.4505112 \\
& 2.5133055 \\
& 2.5956336 \\
& 2.6957487 \\
& 2.8110973 \\
& 2 \cdot 9383160 \\
& 3.0731267
\end{aligned}
$$

Table 1. Ionosphere reflection coefficients (amplitude, $|T|$, and phase, Arg $T$ ) assuming the quasi-Iongitudinal approximation, for various incident angles, $\tau_{j}, r$, frequencies, $\omega / \omega_{r}$ and earth's magnetic field parameter, $\phi_{1}$. 
$\omega / \omega_{\mathbf{r}}=0.3002$

$\boldsymbol{T}_{\mathbf{j}, \mathbf{r}}$

$\{\mathrm{T}$ ee $\mid$

Arg $T_{\text {ee }}$

$\left|\mathrm{T}_{\mathrm{mm}}\right|$

Arg $\mathrm{T}_{\mathrm{mm}}$

degrees

$\phi_{1}=40$ degrees

$\begin{array}{rl}5 & 4.6413032=1 \\ 15 & 4.5310073=1 \\ 25 & 4.3108063=1 \\ 35 & 3.9865326=1 \\ 45 & 3.5884212=1 \\ 55 & 3.2371482=1 \\ 65 & 3.3162964=1 \\ 75 & 4.5347526=1 \\ 85 & 7.5577027=1\end{array}$

$7.7875900-1$

$8.0236280-1$

8. $5564520-1$

$9.5372000-1$

1.1286349

1.4412371

1. 9449455

3. 7750788

3. 3313228

$4.6688630=1$
$4.7795975=1$
$5.0036206-1$
$5.3462121=1$
$5.8157659=1$
$6.4244335=1$
$7.1893282=1$
$8.1356762=1$
$9.3064388-1$

2. 3684597

2.3908321

2.4351729

2. 5005947

2. 5856404

2.6882642

2.8058611

2.9352472

$\phi_{1}=50$ degrees

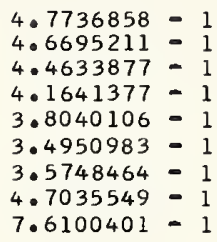

4. $7736858=1$

$4.6695211=1$

$4.4633877-1$

$4.1641377-1$

$3.8040106=1$

$3.4950983-1$

$3.5748464=1$

$4.7035549=1$

$7.6100401-1$

$8.0158970-1$

$8.2649000-1$

$8.8199550-1$

$9.8196580-1$

1. 1546418

1.4510906

1. 9185001

3. 8165426

3.3486045

$\phi_{1}=60$ degrees

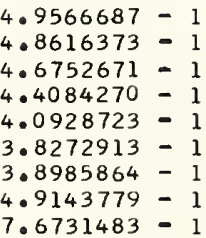

$8.2667620-1$

$8.5269800-1$

$9.0983470-1$

1.0102058

1. 1776945

1.4541628

1. 8830998

3. 8682997

3. 3708281

$4.7998056-1$
$4.9046849-1$
$5.1164960-1$
$5.4399257-1$
$5.8833248-1$
$6.4600998-1$
$7.1910180-1$
$8.1100422-1$
$9.2814664-1$

2.3459664

2.3696293 2.4162670 2.4845374 2. 5725453 2.6780017 2. 7983522 2.9307023 3. 0709719

$\begin{array}{ll}4.9805880-1 & 2.3211854 \\ 5.0766270-1 & 2.3460140 \\ 5.2705729-1 & 2.3947082 \\ 5.5670485-1 & 2.4655207 \\ 5.9749206-1 & 2.5562285 \\ 6.5093091-1 & 2.6644614 \\ 7.1952603-1 & 2.7879043 \\ 8.0774989-1 & 2.9241324 \\ 9.2486587-1 & 3.0690918\end{array}$

Table 2. Ionosphere reflection coefficients (amplitude, $|\mathrm{T}|$, and phase, Arg T) assuming the quasi-longitudinal approximation, for various incident angles, $\boldsymbol{T}_{j}, \mathbf{r}$, frequencies, $\omega / \omega_{\mathbf{r}}$ and earth's magnetic field parameter, $\phi_{1}$. 
$\omega / \omega_{r}=0.3002$

$T_{j, r}$

degrees

5
15
25
35
45
55
65
75
85

5
15
25
35
45
55
65
75
85

5
15
25
35
45
55
65
75
85

$$
\left|\mathrm{T}_{\mathrm{em}}\right|
$$

$4.1866723=2$ $4.2049756-2$ $4.2307904-2$ $4.2411299-2$ $4.1974015-2$ $4.0379401-2$ $3.6601813=2$ $2.8770215-2$ $1.3087681-2$

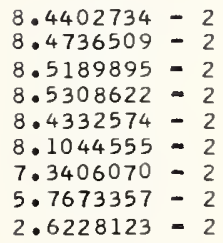

$1.2829470=1$ $1.2871358-1$ $1.2923595-1$ $1.2919694=1$ $1.2748740=1$ $1.2231971=1$ 1.1066333 - 1 $8.6891490=2$
$\operatorname{Arg~T}_{\text {em }}-\pi$

$\phi_{1}=10$ degrees

$8.9089000-2$ $7.2566600-2$ $4.0687100=2$

$3.9955000-3$

$5.7186400-2$

$1.1246580-1$

1.6074110 - 1

$1.8886360-1$

$1.7559910-1$

$\phi_{1}=20$ degrees

$8.5497800-2$ $6.9076100-2$ $3.7483200-2$

$6.5890000-3$

$5.8712900-2$

1.1238540 - 1

1.5848470 - 1

$1.8380010-1$

$1.6684570-1$

$\phi_{1}=30$ degrees

$7.9190800-2$ $6.2977900-2$

3. $1936100-2$

$1.1030000-2$

$6.1292200-2$

1.1223220 - 1

$1.5467520=1$

1.7528660 - 1

$1.5211500-1$

$$
\left|\mathrm{T}_{\mathrm{me}}\right|
$$

Art $T_{m e}-\pi$

$4 \cdot 1815986-2$ $4.1625768-2$ $4.1302304-2$ $4.0891971-2$ 4.0280757- 2 $3.8967306-2$ $3.5788995-2$ $2.8538821-2$ $1.3101547-2$

$8.4309365-2$ $8.3959841-2$ 8.3365922 - 2 $8.2599730-2$ $8.1402205-2$ $7.8742899-2$

$7.2285055-2$

$5.7607875-2$

$2.6434066-2$

$1.2817527-1$ $1.2772915-1$ $1.2697065-1$ 1.2595388 - 1 1.2421432 - 1 $1.2014213-1$ $1.1020646-1$ $8.7754783-2$ $4.0241265-2$
$9.3272300-2$ 1.0978920 - 1 $1.4144900-1$ $1.8467640-1$ $2.3317800-1$ $2.7946820-1$ $3.1880410-1$ $3.5390620=1$ $4.0001810-1$

$8.9660200-2$ $1.0607000-1$ $1.3741190-1$ $1.8001590-1$ $2.2769260-1$ $2.7335380-1$ $3.1280120-1$ $3.4921530-1$ $3.9825640-1$

$8.3307800-2$ $9.9498600-2$ $1.3025110-1$ $1.7177580-1$ 2.1809390 - 1 2.5278070 - 1 $3.0250170-1$ $3.4118260-1$ 3.9520350 -

Table 3. Ionosphere reflection coefficients (amplitude, $|T|$, and phase, Arg $T$ ) assuming the quasi-Iongitudinal approximation, for various incident angles, $T_{j}, r$, frequencies, $\omega / \omega_{r}$ and earth's magnetic field parameter, $\phi_{1}$. 


$$
\omega / \omega_{\mathrm{r}}=0.3002
$$

${ }^{\top} \mathbf{j , r}$

$$
\left|\mathrm{T}_{\mathrm{em}}\right|
$$

degrees

$\begin{array}{rl}5 & 1.7425748=1 \\ 15 & 1.7466179=1 \\ 25 & 1.7506600=1 \\ 35 & 1.7462134=1 \\ 45 & 1.7190915=1 \\ 55 & 1.6461240=1 \\ 65 & 1.4872778=1 \\ 75 & 1.1671209=1 \\ 85 & 5.3047658=2\end{array}$
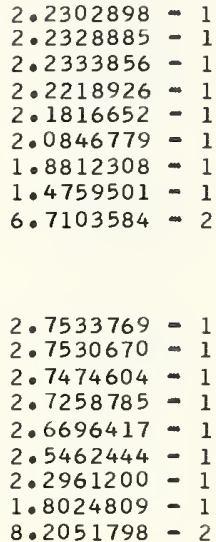

$\operatorname{Arg~T}_{\text {em }}-\pi$

$\left|\mathrm{T}_{\mathrm{me}}\right|$

$\operatorname{Arg} \mathrm{T}_{\mathrm{me}}-\pi$

$\phi_{1}=40$ degrees

$6.9677400-2$
$5.3841100-2$
$2.3722000-2$
$1.7518600-2$
$6.5022300-2$
$1.1204070=1$
$1.4932070-1$
$1.6330220-1$

$1.6330220=1$
$1.3128510-1$

$\phi_{1}=50$ degrees

$5.6265300-2$ $4.1054100-2$

$1.2361600-2$ $2.6393400-2$ $7.0137000-2$ 1. 1199840 - 1 $1.4260470-1$ $1.4801680-1$ $1.0439210-1$ $1.7413725=1$
$1.7368959=1$
$1.7292195=1$
$1.7180171=1$
$1.6957396=1$
$1.5398604=1$
$1.5028919=1$
$1.1956050=1$
$5.4793144=2$ $7.3708500-2$ $8.9512900=2$ 1.1932150 - 1 $1.5926150-1$ $2.0372260-1$ $2.4719560-1$ 2.8747410 - 1 $3.2948330-1$ $3.9067130-1$

$2.2294160=1$
$2.2261589=1$
$2.2203344=1$
$2.2098187=1$
$2.1831719=1$
$2.1108129=1$
$1.9328227=1$
$1.5365286=1$
$7.0401615=2$

$6.0149300-2$ $7.5325700-2$ 1. $0373900-1$ $1.4154970-1$ 1.8371950 - 1 $2.2587590-1$ 2.6713540 - 1 3.1365530 - 1 3.8438570 - 1

$\phi_{1}=60$ degrees

$3.8087000-2$

$2.3831800-2$

$2.8022000-3$

$3.8195500-2$

$7.7120700-2$

1. 1260460 - 1

1. 3508550 - 1

$1.3001740-1$

7. $1869400=2$

$\begin{array}{ll}2.7532146-1 & 4.1740100=2 \\ 2.7525674-1 & 5.5970900=2 \\ 2.7506843-1 & 8.2455300=2 \\ 2.7425708-1 & 1.1758670=1 \\ 2.7119537-1 & 1.5712740=1 \\ 2.6217212-1 & 1.9800700=1 \\ 2.3992588-1 & 2.4079150=1 \\ 1.9071364-1 & 2.9310820=1 \\ 8.7439262-2 & 3.7597460-1\end{array}$

Table 4. Ionosphere reflection coefficients (amplitude, $|\mathrm{T}|$, and phase, Arg $\mathrm{T}$ ) assuming the quasi-longitudinal approximation, for various incident angles, $\tau_{j}, r$, frequencies $\omega / \omega_{r}$ and earth's magnetic field parameter, $\phi_{1}$. 
$\omega / \omega_{r}=0.467$

${ }^{\top} \mathrm{j}, \mathrm{r}$

$\left|\mathrm{T}_{\text {ee }}\right|$

Arg $\mathrm{T}_{\mathrm{ee}}$

$\left|\mathrm{T}_{\mathrm{mm}}\right|$

Arg $T_{m m}$

degrees

$\phi_{1}=10$ degrees

$\begin{array}{ll}5 & 3.5859720=1 \\ 15 & 3.4649923=1 \\ 25 & 3.2215247=1 \\ 35 & 2.8631557=1 \\ 45 & 2.4480804=1 \\ 55 & 2.2223139=1 \\ 65 & 2.7684411=1 \\ 75 & 4.5112006=1 \\ 85 & 7.6796371=1\end{array}$

$8.8861870-1$
$9.1200190=1$

$9.6828490-1$

1.0834105

1. 3183237

1.7752773

3. 9118590

3.4913849

3.2413418

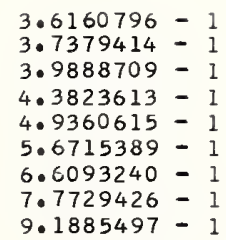

$3.6160796=1$

3.7379414 - 1
$3.9888709-1$

$3.9888709-1$
$4.3823613-1$

$4.3823613=1$
$4.9360615=1$

5.6715389 -

$5.6715389-1$
$6.6093240-1$

7.7729426 - 1

$9.1885497-1$

$\phi_{1}=20$ degrees

$3.6063967=1$

$3.4854021-1$

$3.2435763-1$

2.8926138 - 1

$2.4979005-1$

$2.3072269-1$

2.8678407 - 1

4.5860544 - 1

$7.7104995-1$

$9.0468970-1$

9.2927990 -

$9.8826100-1$

1. 1078125

1. 3468391

1.7951246

3. 9166809

3. 5024424

3.2459142

$\phi_{1}=30$ degrees

9.3183150 - 1

9.5850170 -

1. 0220002

1.1484745

1.3924189

1.8241110

3.9258094

3. 5210750

3. 2536837
2.2584106

2.2802339

2.3246079

2.3926055

2.4848622

2.6010015

2.7383809

2.8925639

3.0574058
$3.6365818=1 \quad 2.2426252$ 4.0093078 - $1 \quad 2.3120414$ 4.4008944 - $1 \quad 2.3828257$ $4.9497520-1 \quad 2.4780796$ $5.6769692-1 \quad 2.5969188$ 6.6045675 - $1 \quad 2.7363519$ 7.7603589 - $1 \quad 2.8918713$ $9.1787554-1 \quad 3.0573799$

Table 5. Ionosphere reflection coefficients (amplitude, $|\mathrm{T}|$, and phase, Arg $\mathrm{T}$ ) assuming the

$\begin{array}{ll}3.6760483=1 & 2.2159812 \\ 3.7979412=1 & 2.2408558 \\ 4.0471611=1 & 2.2909328 \\ 4.4340638=1 & 2.3664102 \\ 4.9731319=1 & 2.4666312 \\ 5.6851365=1 & 2.5899112 \\ 6.5949689=1 & 2.7327590 \\ 7.7377104=1 & 2.8905827 \\ 9.1616254-1 & 3.0573123\end{array}$
quasi-Tongitudinal approximation, for various incident angles, $\tau_{j}, r$, frequencies, $\omega / \omega_{r}$ and earth's magnetic field parameter, $\phi_{1}$. 
$\omega / \omega_{r}=0.467$

$\mathrm{T}_{\mathrm{j}, \mathrm{r}}$

$\left|\mathrm{T}_{\text {ee }}\right|$

Arg $T_{\text {ee }}$

$\left|\mathrm{T}_{\mathrm{mm}}\right|$

$\operatorname{Arg} \mathrm{T}_{\mathrm{mm}}$

degrees

$\phi_{1}=40$ degrees

$\begin{array}{rl}5 & 3.7136585=1 \\ 15 & 3.5952137=1 \\ 25 & 3.3662167=1 \\ 35 & 3.0551643=1 \\ 45 & 2.7502347=1 \\ 55 & 2.6871752=1 \\ 65 & 3.2845199=1 \\ 75 & 4.8967129=1 \\ 85 & 7.8374395=1\end{array}$

9.7031050 - 1

$9.9997500-1$

1.0696480

1. 2044650

1.4510982

1. 8564924

3. 9407840

3.5476436

3. 2648979

$3.7435592-1$
$3.8639813-1$
$4.1086687-1$
$4.4854962-1$
$5.0070749-1$
$5.6947043-1$
$6.5779700-1$
$7.7023675-1$
$9.1358567-1$

$\phi_{1}=50$ degrees

$3.8252637=1$
$3.7122823=1$
$3.5004740=1$
$3.2295383=1$
$2.9960588-1$
$3.0119048=1$
$3.6170243=1$
$5.1416956-1$
$7.9363533=1$

$4.0042726-1$

$3.9031007-1$

$3.7216247-1$

$3.5086620-1$

$3.3599495-1$

$3.4505296-1$

$4.0459400-1$

$5.4549435-1$

$8.0614568=1$
1.0198159

1. 0532709

1. 1300992

1. 2725075

1. 5157753

1. 8851576

3. 9636140

3. 5827632

3.2799582

$$
\phi_{1}=60 \text { degrees }
$$

1.0784740

1. 1159769

1. 1993008

1.3452587

1. 5761149

1. 9026259

3.9966999

3. 6274024

3. 2994773
$7.6504951-$

$9.0994395-1$
2. 1782307

2.2059206

2. 2611479

2. 3431855

2.4502136

2. 5795577

2. 7271897

2. 8884442

3.0571551

$2 \cdot 1296749$

2.1610052

2.2227539

2. 3128902

2.4281945

2. 5650011

$2 \cdot 7188417$

2. 8849748

3. 0568149

$\begin{array}{ll}4.0305332-1 & 2.0720831 \\ 4.1358040-1 & 2.1074695\end{array}$

$4.3471779-1 \quad 2.1763336$

4. $6693375-1 \quad 2.2751465$

$5.1152831-1 \quad 2.3993684$

$5.7129028-1 \quad 2.5446279$

$6.5079111-1 \quad 2.7062360$

$7.5772314-1 \quad 2.8792911$

$9.0495019-1 \quad 3.0561132$

Table 6. Ionosphere reflection coefficients (amplitude, $|\mathrm{T}|$, and phase, Arg $\mathrm{T}$ ) assuming the quasi-longitudinal approximation, for various incident angles, ${ }_{j}, r$, frequencies, $\omega / \omega_{r}$ and earth's magnetic field parameter, $\phi_{1}$. 
$\omega / \omega_{r}=0.467$

$\tau_{j, r}$
degrees

$\left|T_{e m}\right|$

Arg $\mathrm{T}_{\text {em }}-\pi$

$\left|\mathrm{T}_{\mathrm{me}}\right|$

$\operatorname{Arg~T}_{\mathrm{me}}{ }^{-\pi}$

$\phi_{1}=10$ degrees

$\begin{array}{rl}5 & 4 \cdot 1014007=2 \\ 15 & 4 \cdot 1425426=2 \\ 25 & 4 \cdot 2110740=2 \\ 35 & 4 \cdot 2769317=2 \\ 45 & 4 \cdot 28948802 \\ 55 & 4 \cdot 1712099=2 \\ 65 & 3.8038289=2 \\ 75 & 2.9893814=2 \\ 85 & 1.3503975=2\end{array}$

3.1067500 - 1

2.8734390 -

2.4143440 - 1

$1.7500980-1$

$9.2361500-2$

$4.3870000-4$

$9.0476200-2$

1.6720860 - 1

$2.1192300-1$

$4.0905189-2$
$4.0506081-2$
$3.9888653-2$
$3.9340642-2$
$3.9009531=2$
$3.8445266-2$
$3.6154969-2$
$2.9352558-2$
$1.3528105-2$

$3.1654130=1$ $3.4012080-1$ $3.8708880-1$ $4.5378400-1$ $5.2876750-1$ $5.9423210=1$ $6.3545820=1$ 5.6325296

5.6308749

$\phi_{1}=20$ degrees

$8.2929198=2$
$8.3708888=2$
$8.4985296=2$
$8.6150128=2$
$8.6200204=2$
$8.3618506-2$
$7.6086983-2$
$5.9687731-2$
$2.6918182-2$

$3.1031670-1$ 2.8672670 - 1 $2.4048070-1$ $1.7398190-1$ 9. $1912800-2$ $1.4927000-3$ $8.6967000-2$ $1.6041470-1$ $2.0109330-1$

$8.2721753-2$ $8.1966546-2$
$8.0821450-2$ $8.0821450-2$
$7.9845770-2$ $7.9271800-2$

$7.8113821-2$

$7.3347190-2$

5.9421200 -

$2.7330130-2$

$\phi_{1}=30$ degrees

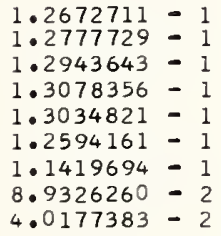

$3.1625520=$ $3.4006430=1$ $3.8716220-1$ $4.5331560-1$ $5.2679130-1$ $5.9045070-1$ $6.3086750-1$ 5.6361205 5.6315226

$\begin{array}{lll}3.0930870=1 & 1.2644482-1 & 3.1535710=1 \\ 2.8534100=1 & 1.2543048-1 & 3.3949060=1 \\ 2.3866120=1 & 1.2395289-1 & 3.8666250=1 \\ 1.7224290=1 & 1.2279589-1 & 4.5172190=1 \\ 9.1386400=2 & 1.2214079-1 & 5.2260460=1 \\ 3.6971000=3 & 1.2030553-1 & 5.8339100-1 \\ 8.0504900=2 & 1.1267231-1 & 6.2269810-1 \\ 1.4835960=1 & 9.0967319-2 & 5.6424141 \\ 1.8218240-1 & 4.1700853-2 & 5.6327431\end{array}$

4. $1700853-2$

$\begin{array}{rl}5 & 1.2672711=1 \\ 15 & 1.2777729=1 \\ 25 & 1.2943643=1 \\ 35 & 1.3078356=1 \\ 45 & 1.3034821=1 \\ 55 & 1.2594161=1 \\ 65 & 1.1419694=1 \\ 75 & 8.9326260=2 \\ 85 & 4.0177383=2\end{array}$

Table 7. Ionosphere reflection coefficients (amplitude, $|\mathrm{T}|$, and phase, Arg $\mathrm{T}$ ) as suming the quasi-longitudinal approximation, for various incident angles, $T_{j}, r$, frequencies, $\omega / \omega_{r}$ and earth's magnetic field parameter, $\phi_{1}$. 
$\omega / \omega_{r}=0.467$

$\tau_{j, r}$

$\left|\mathrm{T}_{\mathrm{em}}\right|$

degrees

$\begin{array}{rl}5 & 1.7355529=1 \\ 15 & 1.7469869=1 \\ 25 & 1.7637972=1 \\ 35 & 1.7738162=1 \\ 45 & 1.758353=1 \\ 55 & 1.6898833=1 \\ 65 & 1.5252982=1 \\ 75 & 1.1886775=1 \\ 85 & 5.3277129=2\end{array}$

$\operatorname{Arg} \mathrm{T}_{\mathrm{em}}-\pi$

$\phi_{1}=40$ degrees

$3.0694110=1$
$2.8258580=1$
$2.3560690=1$
$1.6977110=1$
$9.1147900=2$
$7.7477000=3$
$7.0161000=2$
$1.2993350=1$
$1.5387970-1$

$2.2481350=1$ $2.2481350=1$
$2.2575789=1$ $2.2689993-1$ 2.2680087 - 1 $2.2332073=1$ $2.1327631-1$ 1.9149933 - 1 $1.4861271-1$ $6.6337699-2$

1.7324176 1.7214268 1.7066131 - 1 1.6969793 - 1 1.6916209 1.6646497 - 1 1.5534069 - 1 1.2484504 $5.6979214-2$

3.1310890 $3.3754860=1$ $3.8449500-1$ 4.4763320 - 1 $5.1477590-1$ $5.7186850-1$ 6.1013240 - 1 6.3126370 - 1 5.6347853

$\phi_{1}=50$ degrees

$3.0201400=1$
$2.7747250=1$
$2.3074490=1$
$1.6654240=1$
$9.1717100=2$
$1.4589100=2$
$5.4697800=2$
$1.0365970=1$
$1.1440410=1$

$2.2454236-1$ 2.2354266 - 1 2.2264877 - 1 $2.2236453-1$ $2.2212354-1$ 2. 1822044 - 1 $2.0272208-1$ $1.6205079-1$ $7.3575899-2$

$3.0825920=$ $3.3277280-1$ $3.7880590-1$ $4.3886340-1$ $5.0115980-1$ $5.5415340-1$ 5.9194020 - ? $6.1772940-1$ 6.4510660 - 1

$\phi_{1}=60$ degrees

$2.8221185=1$
$2.8252031=1$
$2.8234237=1$
$2.8018850=1$
$2.7383554=1$
$2.5978873=1$
$2.3203969=1$
$1.7933872=1$
$7.9718413=2$

$2.9263990-1$

$2.6847900-1$

$2.2319770-1$

1.6241730 - 1

$9.3586300-2$

$2.5149900-2$

$3.2898400-2$

$6.8051700-2$

$6.1856100-2$

$2.9882540=1$ $3.2282800-7$ $3.6674240-1$ $4.2229080-1$ 4. $7888880-1$ $5.2796490-1$ $5.6643140-1$ $5.9896570-1$ $6.3986860-1$

Table 8. Ionosphere reflection coefficients (amplitude, $|\mathrm{T}|$, and phase, Arg $\mathrm{T}$ ) assuming the quasi-longitudinal approximation, for various incident angles, $\tau_{j}, r$, frequencies, $\omega / \omega_{r}$ and earth's magnetic field parameter, $\phi_{1}$. 
$\phi_{1}=60$ degrees

\begin{tabular}{|c|c|c|c|c|c|c|c|}
\hline$\omega / \omega_{r}$ & $\left|T_{e e}\right|$ & & $\operatorname{Arg}_{\mathrm{ee}}$ & & $\left|\mathrm{T}_{\mathrm{mm}}\right|$ & & $\operatorname{Arg} T_{m}$ \\
\hline & & & $\mathrm{T}_{\mathrm{j}, \mathrm{r}}=5 \mathrm{de}$ & gree & & & \\
\hline 0.0100 & 8.8429377 & -1 & 1.2794530 & -1 & 8.3511589 & -1 & 3.0145861 \\
\hline 0.0200 & 8.4032574 & -1 & 1.8387820 & -1 & 8.4142553 & -1 & 2.9590490 \\
\hline 0.0500 & 7.5914561 & -1 & 2.9998050 & -1 & 7.6069617 & -1 & 2.8437574 \\
\hline 0.1000 & 6.7602134 & -1 & 4.3909550 & -1 & 6.7793816 & -1 & 2.7056238 \\
\hline 0.2000 & 5.7028228 & -1 & 6.5118390 & -1 & 5.7251952 & -1 & 2.4951445 \\
\hline 0.3002 & 4.9566687 & -1 & 8.2667620 & -1 & 4.2805880 & -1 & 2.3211854 \\
\hline 0.4670 & 4.0042726 & -1 & 1.0784740 & & 4.0305332 & -1 & 2.072083 \\
\hline 1.0000 & 1.9488869 & -1 & 1.6214996 & & 1.9871032 & -1 & 1.5321267 \\
\hline $\begin{array}{l}2.0000 \\
5.0000\end{array}$ & $\begin{array}{l}7.3221492 \\
2.5538450\end{array}$ & $\begin{array}{l}-2 \\
-2\end{array}$ & $\begin{array}{l}1.7525490 \\
1.6655937\end{array}$ & & $\begin{array}{l}7.4749828 \\
2.5958680\end{array}$ & $\begin{array}{l}-2 \\
-2\end{array}$ & $\begin{array}{l}1.3888338 \\
1.4746904\end{array}$ \\
\hline & & & $T_{j, r}=15 d$ & gre & & & \\
\hline 0.0100 & 8.8091136 & -1 & 1.3181870 & -1 & 8.8838687 & -1 & 3.0183123 \\
\hline 0.0200 & 8.3580720 & -1 & 1.8938610 & -1 & 8.4580539 & -1 & 2.9643468 \\
\hline 0.0500 & 7.5279571 & -1 & 3.0884100 & -1 & 7.6688278 & -1 & 2.852274 \\
\hline 0.1000 & 6.6821019 & -1 & 4.5202300 & -1 & 6.8560286 & -1 & 2.718037 \\
\hline 0.2000 & 5.6126656 & -1 & 6.7080050 & -1 & 5.8149040 & -1 & 2.513931 \\
\hline 0.3002 & 4.8616373 & -1 & 8.5269800 & -1 & 5.0766270 & -1 & $2 \cdot 346014$ \\
\hline 0.4670 & 3.9031007 & -1 & 1.1159769 & & 4.1358040 & -1 & $2 \cdot 107469$ \\
\hline 1.0000 & 1.7999211 & -1 & 1.6810953 & & 2.1430646 & -1 & 1.581901 \\
\hline 2.0000 & 6.6671110 & -2 & 1.7525332 & & 8.1311070 & -2 & 1.388933 \\
\hline 5.0000 & 2.3767424 & -2 & 1.6592362 & & 2.7731443 & -2 & 1.469330 \\
\hline & & & ${ }^{\top} \mathrm{j,r}=25 \mathrm{~d}$ & gr & & & \\
\hline 0.0100 & 8.7363950 & -1 & 1.4021060 & -1 & 8.9486409 & -1 & 3.0256536 \\
\hline 0.0200 & 8.2612622 & -1 & 2.0132840 & -1 & 8.5449667 & -1 & 2.9747862 \\
\hline 0.0500 & 7.3930524 & -1 & 3.2809020 & -1 & 7.7921218 & -1 & 2.869065 \\
\hline 0.1000 & 6.5182805 & -1 & 4.8018610 & -1 & 7.0095125 & -1 & 2.742512 \\
\hline 0.2000 & 5.4290073 & -1 & 7.1371300 & -1 & 5.9956351 & -1 & 2.550911 \\
\hline 0.3002 & 4.6752671 & -1 & 9.0983470 & -1 & 5.2705729 & -1 & $2 \cdot 394708$ \\
\hline 0.4670 & 3.7216247 & -1 & 1.1993008 & & 4.3471779 & -1 & 2.176333 \\
\hline 1.0000 & 1.5529126 & -1 & 1.8702913 & & 2.4636618 & -1 & 1.690050 \\
\hline 2.0000 & 5.0817002 & -2 & 1.7639929 & & 9.7256825 & -2 & 1.395670 \\
\hline 5.0000 & 1.9678494 & -2 & 1.6375586 & & 3.1837793 & -2 & 1.457649 \\
\hline
\end{tabular}

Table 9. Ionosphere reflection coefficients (amplitude, |T |, and phase, Arg T) assuming the quasi-longitudinal approximation, for various $f \sim$ equencies, $\omega / \omega_{r}$, earth's magnetic field parameter, $\phi_{1}$, and incident angle, $\tau_{j, r}$. 


$$
\phi_{1}=60 \text { degrees }
$$

$\omega / \omega_{r}$

$\left|\mathrm{T}_{\mathrm{ee}}\right|$

Arg $\mathrm{T}_{\text {ee }}$

$\left|\mathrm{T}_{\mathrm{mm}}\right|$

Arg $\mathrm{T}_{\mathrm{mm}}$

$\tau_{j, r}=35$ degrees

$\begin{array}{ll}0.0100 & 8.6126604=1 \\ 0.0200 & 8.0976160=1 \\ 0.0500 & 7.1685422=1 \\ 0.1000 & 6.2520491=1 \\ 0.2000 & 5.1461541=1 \\ 0.3002 & 4.4084270=1 \\ 0.4670 & 3.5086620=1 \\ 1.0000 & 1.4671623=1 \\ 2.0000 & 1.8517306=2 \\ 5.0000 & 1.1779648=1\end{array}$

5.0000

$1.1779648-2$

$1.5469920=1$
$2.2197590=1$
$3.6150090=1$
$5.2931820=1$
$7.8900800=1$
1.0102058
1.3452587
2.2984695
2.0417237
1.5382695

$9.0441511-1$

$7.9758626-\mathrm{l} \quad 2.8936576$

$7.2401197-1 \quad 2.7783785$

$6.2702212-1 \quad 2.6049862$

$5.5670485-1 \quad 2.4655207$

$4.6693375-1 \quad 2.2751465$

2.9429755 - $1 \quad 1.8610742$

$1.3079280-1-1.4328543$

$\begin{array}{lll}1.5382695 & 3.9872770-2 & 1.4378299\end{array}$

$\tau_{j, r}=45$ degrees

$\begin{array}{ll}0.0100 & 8.4132262=1 \\ 0.0200 & 7.8367543=1 \\ 0.0500 & 6.8200219=1 \\ 0.1000 & 5.8550949=1 \\ 0.2000 & 4.7620897=1 \\ 0.3002 & 4.0928723=1 \\ 0.4670 & 3.3599112=1 \\ 1.0000 & 1.9496976=1 \\ 2.0000 & 5.8368942=2 \\ 5.0000 & 5.9278107=3\end{array}$

$1.7863980-1$

$2.5618390-1$

$4 \cdot 1723720$ - 1

6. $1194280-1$

$9.1612220-1$

1.1776945

1. 5762756

3.5362965

2.0124468

$9.1683749-1 \quad 3.0502071$

$8.8416515-1 \quad 3.0097320$

$8.2183102-1 \quad 2.9253824$

$7.5479993-1 \quad 2.8247127$

6.6433415 - $1 \quad 2.6747145$

$5.9749206-1 \quad 2.5562285$

5.1155793 - $1 \quad 2.3994461$

$3.5594609=1 \quad 2.0803039$

$1.9993596-1 \quad 1.5717297$

$7.8938600-1 \quad 5.6334561-2 \quad 1.4093987$

$\tau_{j, r}=55$ degrees

\begin{tabular}{|c|c|c|c|c|c|c|}
\hline 0.0100 & 8.0863492 & $=1$ & $2.1961060-1$ & 9.3185052 & $=1$ & 3.0667006 \\
\hline 0.0200 & $\begin{array}{r}7.4173589 \\
6.2852455\end{array}$ & -1 & $\begin{array}{l}3.1501950-1 \\
5.1426290-1\end{array}$ & $\begin{array}{l}9.0459466 \\
8.5166558\end{array}$ & $\begin{array}{l}-1 \\
-1\end{array}$ & $\begin{array}{l}3.0332495 \\
2.9634398\end{array}$ \\
\hline 0.0500 & $\begin{array}{l}6.2852455 \\
5.2891610\end{array}$ & $=1$ & 5.1426290 & 8.5166558 & $\begin{array}{l}-1 \\
-1\end{array}$ & 2.9634398 \\
\hline $\begin{array}{l}0.1000 \\
0.2000\end{array}$ & $\begin{array}{l}5.2891610 \\
4.3075096\end{array}$ & $=1$ & $\begin{array}{l}7.5729480 \\
1.1369939\end{array}$ & $\begin{array}{l}7.9329410 \\
7.1221475\end{array}$ & & $\begin{array}{l}2.8804583 \\
2.7585216\end{array}$ \\
\hline $\begin{array}{l}0.2000 \\
0.3002\end{array}$ & $\begin{array}{l}4.3075096 \\
3.8272913\end{array}$ & & 1.4541628 & $\begin{array}{l}6.1221413 \\
6.5093091\end{array}$ & & $\begin{array}{l}585216 \\
644614\end{array}$ \\
\hline 0.4670 & 3.4505296 & & 1.9026259 & 5.7129028 & & 2.5446279 \\
\hline 1.0000 & 2.9531957 & & 3.3229046 & 4.3279778 & - & 2.3224695 \\
\hline 2.0000 & 1.9041680 & -1 & 2.2372635 & 3.1326992 & - & 1.8923383 \\
\hline .0000 & 4.5816198 & - & 1.2991823 & 9.7430842 & - & 1.3955272 \\
\hline
\end{tabular}

Table 10. Ionosphere reflection coefficients (amplitude, $|\mathrm{T}|$, and phase, Arg T) assuming the quasi-longitudinal approximation, for various frequencies, $\omega / \omega_{r}$, earth's magnetic field parameter, $\phi_{1}$, and incident angle, $\tau_{j}, r^{\circ}$ 
$\phi_{1}=60$ degrees

$\omega / \omega_{\mathrm{r}}$

$\left|T_{\text {ee }}\right|$

Arg $\mathrm{T}_{\mathrm{ee}}$

$\left|\mathrm{T}_{\mathrm{mm}}\right|$

Arg $\mathrm{T}_{\mathrm{mm}}$

$\tau_{\mathrm{j}, \mathrm{r}}=65$ degrees

$\begin{array}{ll}0.0100 & 7.5104565=1 \\ 0.0200 & 6.7058220=1 \\ 0.0500 & 5.4613515=1 \\ 0.1000 & 4.5529763=1 \\ 0.2000 & 3.9712325=1 \\ 0.3002 & 3.8985864=1 \\ 0.4670 & 4.0459400=1 \\ 1.0000 & 4.3102292=1 \\ 2.0000 & 3.5976189=1 \\ 5.0000 & 1.7811782=1\end{array}$

2.9806110 - 1

$4.2884790-1$

7.0576340 -

1.0441259

1.5352191

1.8830998

3.9966999

3.2428149

2. 5673788

1.6531056

$9.4908796-1$

3.0854160

$8.8658326-1 \quad 3.0070336$

8.3946895 - $1 \quad 2.9446406$

$7.7182330-1 \quad 2.8549410$

$7.1952603-1 \quad 2.7879043$

$6.5079111-1 \quad 2.7062360$

$5.3315587-1 \quad 2.5691689$

4.4711049 - $1 \quad 2.2927606$

$2.3096071-1 \quad 1.6221571$

$\tau_{\mathrm{j}, \mathrm{r}}=75$ degrees

0.0100

0.0200

0.0500

0.1000

0.2000

0.3002

0.4670

1.0000

2.0000

5.0000

$6.3424738=1$
$5.4011480=1$
$4.3461680=1$
$4.0911405=1$
$4.4783115=1$
$4.9143779=1$
$5.4549435=1$
$6.0622348=1$
$5.5962446=1$
$4.3200936=1$

4.9352110 - 1

$7.1882960-1$

1. 1932647

1.6844665

4.1129799

3. 8682997

3.6274024

3. 2045786

2. 8436627

$2 \cdot 3577353$

$\begin{array}{ll}9.6808074-1 & 3.1059300 \\ 9.5443417-1 & 3.0895637 \\ 9.2645538-1 & 3.0557467 \\ 8.9365702-1 & 3.0168312 \\ 8.4552745-1 & 2.0628373 \\ 8.0774989-1 & 2.9241324 \\ 7.5772314-1 & 2.8792911 \\ 6.7174227-1 & 2.8101571 \\ 6.1111128-1 & 2.6678612 \\ 4.7177132-1 & 2.2913477\end{array}$

$\tau_{\mathrm{j}, \mathrm{r}}=85$ degrees

$\begin{array}{ll}0.0100 & 4.1377694=1 \\ 0.0200 & 4.4518437=1 \\ 0.0500 & 5.5262080=1 \\ 0.1000 & 6.4413768=1 \\ 0.2000 & 7.2598233=1 \\ 0.3002 & 7.6731469=1 \\ 0.4670 & 8.0614547=1 \\ 1.0000 & 8.4414668=1 \\ 2.0000 & 8.2382668=1 \\ 5.0000 & 7.6417887=1\end{array}$

1.5644026

2.0311422

3.8069332

3.5923234

3.4417584

3. 3708284

3. 2994775

3. 1673709

3.0570633

2. 9230076

\begin{tabular}{|c|c|c|}
\hline 9.8833903 & -1 & 3.1284032 \\
\hline 9.8297764 & & $3 \cdot 1224825$ \\
\hline 9.7187850 & -1 & 3.1107662 \\
\hline 9.5888449 & -1 & 3.0978933 \\
\hline 9.3984484 & -1 & 3.0308153 \\
\hline 9.2486583 & -1 & 3.0690917 \\
\hline 9.0425003 & - & 3.0561130 \\
\hline 8.6988574 & -1 & 3.0369789 \\
\hline 8.4280337 & -1 & 2.9966627 \\
\hline 7.8000075 & -1 & 2.8947042 \\
\hline
\end{tabular}

Table 11. Ionosphere reflection coefficients (amplitude, $|\mathrm{T}|$, and phase, Arg $\mathrm{T}$ ) assuming the quasi-longitudinal approximation, for various frequencies, $\omega / \omega_{r}$, earth's magnetic field parameter, $\phi_{1}$, and incident angle, $\tau_{j, r}$ 
$\phi_{1}=60$ degrees

\begin{tabular}{|c|c|c|c|c|c|c|c|}
\hline$\omega / \omega_{r}$ & $\left|\mathrm{~T}_{\mathrm{em}}\right|$ & & $\begin{array}{l}\text { Arg } T_{\text {em }}-\pi \\
T_{j, r}=5 \text { degrees }\end{array}$ & $\left|\mathrm{T}_{\mathrm{me}}\right|$ & & $\mathrm{Arg}_{\mathrm{me}}$ & $-\pi$ \\
\hline $\begin{array}{l}0.0100 \\
0.0200 \\
0.0500 \\
0.1000 \\
0.2000 \\
0.3002 \\
0.4670 \\
1.0000 \\
2.0000 \\
5.0000\end{array}$ & $\begin{array}{l}8.8471589 \\
1.1892370 \\
1.6997653 \\
2.1434754 \\
2.5682270 \\
2.7533769 \\
2.8221185 \\
2.1878662 \\
1.1196951 \\
4.3850293\end{array}$ & $\begin{array}{l}=2 \\
=1 \\
=1 \\
=1 \\
=1 \\
=1 \\
=1 \\
=1 \\
=1 \\
=2\end{array}$ & $\begin{array}{l}6.5797890=1 \\
6.0230080=1 \\
4.8676870=1 \\
3.4837900=1 \\
1.3724860=1 \\
3.8087000=2 \\
2.9263990=1 \\
9.0847230-1 \\
1.2952804 \\
1.4685586\end{array}$ & $\begin{array}{l}8.8471589 \\
1.1892371 \\
1.6997651 \\
2.1434750 \\
2.5682015 \\
2.7532146 \\
2.8209639 \\
2.1680383 \\
1.1019744 \\
4.3176223\end{array}$ & $\begin{array}{l}-2 \\
=1 \\
=1 \\
=1 \\
=1 \\
=1 \\
=1 \\
=1 \\
=1 \\
=2\end{array}$ & $\begin{array}{l}6.5786500 \\
6.0207270 \\
4.8619800 \\
3.4723100 \\
1.3490140 \\
4.1740100 \\
2.9882540 \\
9.2155850 \\
1.3006109 \\
1.4701922\end{array}$ & $\begin{array}{l}-1 \\
=1 \\
=1 \\
=1 \\
=1 \\
=2 \\
=1 \\
=1\end{array}$ \\
\hline & & & $\mathbf{T}_{\mathbf{j}, \mathbf{r}}=15$ degrees & & & & \\
\hline $\begin{array}{l}0.0100 \\
0.0200 \\
0.0500 \\
0.1000 \\
0.2000 \\
0.3002 \\
0.4670 \\
1.0000 \\
2.0000 \\
5.0000\end{array}$ & $\begin{array}{l}8.8465232 \\
1.1891170 \\
1.6995009 \\
2.1430252 \\
2.5676022 \\
2.7530670 \\
2.8252031 \\
2.2635439 \\
1.1940686 \\
4.6677960\end{array}$ & $\begin{array}{l}=2 \\
=1 \\
=1 \\
=1 \\
=1 \\
=1 \\
=1 \\
=1 \\
=1 \\
=2\end{array}$ & $\begin{array}{l}6.5836860=1 \\
6.0312230=1 \\
4.8891770-1 \\
3.5278900=1 \\
1.4637570=1 \\
2.3831800=2 \\
2.6847900=1 \\
8.5526510=1 \\
1.2727546 \\
1.4617444\end{array}$ & $\begin{array}{l}8.8465264 \\
1.1891187 \\
1.6995147 \\
2.1430857 \\
2.5677156 \\
2.7525674 \\
2.8181469 \\
2.1004073 \\
1.0285694 \\
4.0352590\end{array}$ & $\begin{array}{l}-2 \\
=1 \\
=1 \\
=1 \\
=1 \\
=1 \\
=1 \\
=1 \\
=1 \\
=2\end{array}$ & $\begin{array}{l}6.5736370 \\
6.0111220 \\
4.8388470 \\
3.4266890 \\
1.2569970 \\
5.5970900 \\
3.2282800 \\
9.7868560 \\
1.3270316 \\
1.4781829\end{array}$ & $\begin{array}{l}=1 \\
=1 \\
=1 \\
=1 \\
=1 \\
=2 \\
=1 \\
=1\end{array}$ \\
\hline & & & $\boldsymbol{T}_{\mathbf{j}, \mathbf{r}}=25$ degree & & & & \\
\hline $\begin{array}{l}0.0100 \\
0.0200 \\
0.0500 \\
0.1000 \\
0.2000 \\
0.3002 \\
0.4670 \\
1.0000 \\
2.0000 \\
5.0000\end{array}$ & $\begin{array}{l}8.8419763 \\
1.1882608 \\
1.6976177 \\
2.1398010 \\
2.5625945 \\
2.7474604 \\
2.8234237 \\
2.3888186 \\
1.3628532 \\
5.3121295\end{array}$ & $\begin{array}{l}=2 \\
=1 \\
=1 \\
=1 \\
=1 \\
=1 \\
=1 \\
=1 \\
=1 \\
=2\end{array}$ & $\begin{array}{l}6.5880810=1 \\
6.0430110=1 \\
4.9252080=1 \\
3.6066010=1 \\
1.6325250=1 \\
2.8022000=3 \\
2.2319770=1 \\
7.4557740=1 \\
1.2191262 \\
1.4461081\end{array}$ & $\begin{array}{l}8.8419972 \\
1.1882724 \\
1.6977185 \\
2.1402889 \\
2.5645782 \\
2.7506843 \\
2.8195705 \\
2.0363346 \\
8.7098022 \\
3.3952251\end{array}$ & $\begin{array}{l}=2 \\
=1 \\
=1 \\
=1 \\
=1 \\
=1 \\
=1 \\
=1 \\
=2 \\
=2\end{array}$ & $\begin{array}{l}6.5612890 \\
5.9894150 \\
4.7910340 \\
3.3369380 \\
1.0825720 \\
8.2455300 \\
3.6674240 \\
1.1083027 \\
4.8631428 \\
1.5056971\end{array}$ & $\begin{array}{l}=1 \\
=1 \\
=1 \\
=1 \\
=1 \\
=1 \\
=1\end{array}$ \\
\hline
\end{tabular}

Table 12. Ionosphere reflection coefficients (amplitude, $|\mathrm{T}|$, and phase, Arg $\mathrm{T}$ ) assuming the quasi-1ongitudinal approximation, for various frequencies, $\omega / \omega_{\mathbf{r}}$, earth's magnetic field parameter, $\phi_{1}$, and incident angle, $\tau_{j}, r^{*}$ 
$\phi_{1}=60$ degrees

$\omega / \omega_{\mathbf{r}}$

$\left|T_{\text {er }}\right|$

$\operatorname{Arg~} \mathrm{T}_{\mathrm{em}}-\pi$

$\left|\mathrm{T}_{\mathrm{me}}\right|$

Arg $\mathrm{T}_{\text {me }}$

$\tau_{j, r}=35$ degrees

$\begin{array}{ll}0.0100 & 8.8258062=2 \\ 0.0200 & 1.1852227=1 \\ 0.0500 & 1.6909616=1 \\ 0.1000 & 2.1284521=1 \\ 0.2000 & 2.5447915=1 \\ 0.3002 & 2.7258785=1 \\ 0.4670 & 2.8018850=1 \\ 1.0000 & 2.5022284=1 \\ 2.0000 & 1.6712486=1 \\ 5.0000 & 6.5283838=2\end{array}$

$6.5851980-1$

$6.0478990-1$

4.9605790 - 1

$3.6998750-1$

1.8511060 - 1

3.8195500 - 2

1.6241730 - 1

5.8385060 - 1

1.1095470

1.4161202

$8.8258784=2$

$6.5358460=1$
$5.9491800=1$

1.6913032 - 1

$2.1301516=1$

2.5524936 -

$2.7425708-1$

2. $8287938-1$

$2.1135165-1$

$6.616 \leq 386-2$

$2.2159298-2$

$4.7134910=1$

$3.2036240-1$

$8.4183600-2$

$1.1758670-1$

4. $2229080-1$

4. 9964004

4. 5044707

4.6559989

$\tau_{j, r}=45$ degrees

$\begin{array}{ll}0.0100 & 8.7825407=2 \\ 0.0200 & 1.1771197=1 \\ 0.0500 & 1.6733502=1 \\ 0.1000 & 2.0987214=1 \\ 0.2000 & 2.4986968=1 \\ 0.3002 & 2.6696417=1 \\ 0.4670 & 2.7383017=1 \\ 1.0000 & 2.5317992=1 \\ 2.0000 & 2.1530498=1 \\ 5.0000 & 8.8354368=2\end{array}$

$6.5601030-1$

$6.0262680=1$

4.9681850 - I

$3.7739170-1$

$2.0766070-1$

$7.7120700-2$

9.3546100 - 2

3.9149970 - 1

8.8708970 - 1

1. 3570827

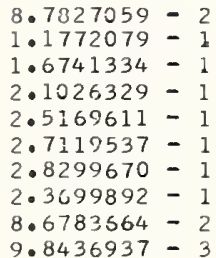

$6.4850980=1$ $5.8762400-2$ $4.5927750-1$ $3.0206250-1$ $5.5005100-2$ $1.5712740=1$ $4.7891960=1$

4. 8636866

3.7266013

3.1865873

$\boldsymbol{T}_{\mathrm{j}, \mathrm{r}}=55$ degrees

$\begin{array}{ll}0.0100 & 8.6798418=2 \\ 0.0200 & 1.1580205=1 \\ 0.0500 & 1.6324615=1 \\ 0.1000 & 2.0309029=1 \\ 0.2000 & 2.3960543=1 \\ 0.3002 & 2.5462444=1 \\ 0.4670 & 2.5978873=1 \\ 1.0000 & 2.4342074=1 \\ 2.0000 & 2.5809795=1 \\ 5.0000 & 1.3653684=1\end{array}$

$6.4831080-1$

$5.9404170-1$

4.8994500 - 1

$3.7728770-1$

2.2460060 - 1

1.1260460 - 1

2. $5149900-2$

$1.9509770-1$

5.1898050 - 1

1. 2201349

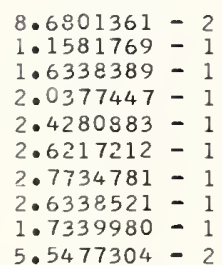

$6.3824510-1$ $5.7390890-1$

$4.3958110-1$

$2.7633130-1$

$2.0834300-2$

$1.9800700-1$

$5.2796490-1$

4. 8227249

3.6429653

2.3146106

Table 13. Ionosphere reflection coefficients (amplitude, $|\mathrm{T}|$, and phase, $\operatorname{Arg} \mathrm{T}$ ) assuming the quasi-longitudinal approximation, for various frequencies, $\omega / \omega_{r}$, earth's magnetic field parameter, $\phi_{1}$, and incident angle, $T_{j}, r^{*}$ 
$\phi_{1}=60$ degrees

$\omega / \omega_{r}$

$\left|\mathrm{T}_{\text {em }}\right|$

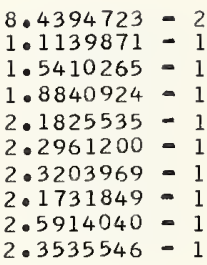

0.0100
0.0200

0.0500

0.1000

0.2000

0.3002

0.4670

1.0000

2.0000

5.0000

$\begin{array}{ll}0.0100 & 7.8118412=2 \\ 0.0200 & 1.0032222=1 \\ 0.0500 & 1.3263996=1 \\ 0.1000 & 1.5612352=1 \\ 0.2000 & 1.7444598=1 \\ 0.3002 & 1.8024809=1 \\ 0.4670 & 1.7933872=1 \\ 1.0000 & 1.6669467=1 \\ 2.0000 & 2.1213769=1 \\ 5.0000 & 2.7245669=1\end{array}$

Arg T

$T_{\mathrm{j}, \mathrm{r}}=65$ degrees

$6.2859580=1$
$5.7076430=1$

5.7076430 -

4.6573980 - 1

$3.5965390-1$

$2.2620650-1$

$1.3508550-1$

$3.2898400-2$

1. $2704900-2$

1.2113800 - 1

$8.0347670-1$

$\tau_{j, r}=75$ degrees

5.7631240 - 1

$5.0968140-1$

4. $0064880-1$

$3.0344200-1$

$1.9554400-1$

$1.3001740-1$

$6.8051700-2$

1.4430030 -

$2.2560790-1$

$8.7015000-2$

$T_{j, r}=85$ degrees

$3.8613220-1$

$3.1137430-1$

$2.1764470-1$

$1.5393820-1$

$9.8066500-2$

7. $1869500-2$

$6.1856100-2$

$2.6512520-1$

$5.0920310-1$

$4.9990490-1$
$8.4399007-2$

$1.1142125-1$

$1.5429754-1$

$1.8936140-1$

$2.2264779-1$

$2.3992588-1$

$2.5635185-1$

$2.6710263-1$

$2.3738048-1$

$1.7057965-1$

$7.8123513-2$

$1.0034344-1$

$1.3285640-1$

$1.5714166-1$

$1.7897845-1$

$1.9071364-1$

$2.0376340-1$

$2.2399638-1$

$2.2954383-1$

$2.5265442-1$

$5.3949551-2$

$6.2895003-2$

$7.3089985-2$

$7.9189023-2$

$8.4285470-2$

$8.7439347-2$

$9.2005430-2$

$1.0306341-1$

1.1480474 -

$1.5254824-1$
$\operatorname{Arg} T_{m e}-\pi$

$6.1627470-1$ $5.4612090-1$ $4.0410890-1$ $2.3623330-1$ $2.1936600-2$ $2.4079150-1$ $5.6643140-1$ 4. 8481129

3.8940635

2.6507151

$5.6231690-1$ 4. $8169010-1$ $3.3066130-1$ $1.6339570-1$ $8.5164800-2$ $2.9310820-1$ $5.9896570-1$ 4. 9087319

4. 1657744

3.3675378

$3.7124590-1$ $2.8160220=1$ $1.4321280=1$ $5.0634000-3$ $1.9983480=1$ $3.7597460-1$ $6.3986860-1$ 4. 9832327

4.4130092

3. 9437012

Table 14. Ionosphere reflection coefficients (amplitude, $|\mathrm{T}|$, and phase, Arg T) assuming the quasi-longitudinal approximation, for various frequencies, $\omega / \omega_{r}$, earth's magnetic field parameter, $\phi_{1}$, and incident angle, ${ }^{\top} \mathbf{j}, \mathbf{r}^{*}$ 
$\phi_{1}=60$ degrees

$\tau_{j, r}$

$\left|T_{\text {ee }}\right|$

Arg $\mathrm{T}_{\text {ee }}$

$\left|\mathrm{T}_{\mathrm{mm}}\right|$

$\operatorname{Arg} \mathrm{T}_{\mathrm{mm}}$

degrees

$\begin{array}{ll}5 & 6.1708365=1 \\ 15 & 6.0851277=1 \\ 25 & 5.9078300=1 \\ 35 & 5.6268790=1 \\ 45 & 5.2257613=1 \\ 55 & 4.699553=1 \\ 65 & 4.1511358=1 \\ 75 & 4.2493087=1 \\ 85 & 6.9373172=1\end{array}$

$\omega / \omega_{r}=0.1501$

$5.5211680-1$

$5.6850330-1$

$6.0428240-1$

$6.6692290-1$

$7.7267900-1$

9.5855490 -

1. 3132018

1. 9780930

3.4984252

$\omega / \omega_{\mathrm{r}}=0.2335$

$5.4307379-1$

$5.3385517-1$

$5.1529162-1$

$4.8731537-1$

$4.5079761-1$

4. $1118155-1$

$3.9175858=1$

$4.6323662-1$

7.4234630 - 1

$\left|\mathrm{T}_{\text {em }}\right|$

${ }_{\text {degrees }}$ j,r

$\begin{array}{ll}5 & 2.4016093-1\end{array}$

15

25

$2.4010331=1$

2.3967815 - 1

$2.3818118-1$

$2.3428815-1$

2.2552587 - 1

$2.0698161-1$

1.6782517 - I

$7.1269510-1$

$7.3444240-1$

7.8207370 - 1

8.6571710 - I

1.0066154

1. 2482384

1.6641085

4.0160879

3.4136795

Arg T

$\omega / \omega_{r}={ }^{2} .1501$

2.3591980 - I

$2.4265450-1$

$2.5496030-1$

$2.7044490-1$

$2.8525100-1$

$2.9339600-1$

2.8498950 - 1

$2.4130960-1$

$6.1919803=1$
$6.2766588=1$

$6.4468255-1$

6.7040938 - 1

$7.0508668-1$

7.4904368 - I

8.0280942 - I

$8.0280942=1$

9.4861936 - I

2. 5934354

2.6091515

2. 6401212

2.6854774

2.7440580

2.8145687

2.8958288

2.9871536

3.0884031

$5.4537141-1$

$5.7318464-1 \quad 2.4958597$

$6.0150967-1 \quad 2.5555904$

6.4018084 - $1 \quad 2.6324770$

$6.9018036-1 \quad 2.7247172$

7.5311194 - I 2.8306019

$8.3205390-1 \quad 2.9486102$

9.3450779 - I 3.0764542

$\left|T_{\text {me }}\right|$

Art $\mathrm{T}_{\mathrm{me}}$

$2.4016031-1$ $2.4011476-1$

2.3979373 - 1

$2.3860048-1$

$2.3526410=1$

$2.2723228-1$

$2.0333415-1$

$1.7028703-1$

$8.2226955-2$

$2.3418060-1$ $2.2732790-1$

$2.1415240-1$

$1.9543460-1$

$1.7155910-1$

$1.4128270-1$

$9.9331400-2$

$3.0913800-2$

1.0343640 - I

$\omega / \omega_{r}=0.2335$

$2.6478391=1$
$2.6472430=1$
$2.6418831=1$
$2.6225437=1$
$2.5725007=1$
$2.4617293=1$
$2.2335502=1$
$1.7723676=1$
$8.2144294=2$

$7.5896400-2$

$8.6684000-2$

$1.0672800-1$

$1.3299590-1$

$1.6090230-1$

$1.8393380-1$

$1.9245150-1$

$1.7047040-1$

$2.6477868-1$

$7.3126600-2$ .6472768 -

2.6444167 - 1

$2.6330565-1$

$2.5978668-1$

$2.5063994-1$

$2.2946698-1$

$1.8350008-1$

$8.5427275-2$

$6.2296600-2$

$4.1914400-2$

$1.4204500-2$

$1.8455300-2$

$5.5025200-2$

. $8010500-2$

$1.5763570-1$

$2.6075960-1$

Table 15. Ionosphere reflection coefficients (amplitude, $|T|$, and phase, $\operatorname{Arg} T$ ) assuming the quasi-longitudinal approximation, for various incident angles, $\tau, \mathrm{r}$, earth's magnetic field parameter, $\phi_{1}$ and frequencies, $\omega / \omega_{\mathrm{r}}$. 
$\Psi$

radians $d / j$

miles
${ }_{j}, r$

degrees
$T_{j, r}$

radians

$h_{3}=70$ kilometers

0.0005

0.0010

0.0020

0.0030

0.0040

0.0050

0.0060

0.0070

0.0080

0.0090

0.0100

0.0120

0.0150

0.0200

0.0250

0.0300

0.0400

0.0500

0.0650

0.0800

0.1000

0.1500

0.2000

0.3000

0.4000

0.6000

1.2433895
1.2381675
1.2278386
1.2176623
1.2076369
1.1977599
1.1880278
1.1784374
1.1689853
1.1596669
1.1504788
1.1324770
1.1063547
1.0649462
1.0259098
9.8899038
9.2076906
8.5914709
7.7737068
7.0657786
6.2644905
4.7915900
3.8174547
2.6471062
1.9802951
1.2445355

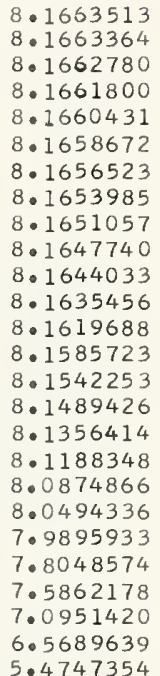

1. 4252972

1.4252972
1.4252946

1. 4252844

1.4252673

1. 4252434

1.4252127

1.4251752

1. 4251309

1. 4250798

1. 4250219

1.4249572

1.4248075

1.4245323

1.4239395

1. 4231808

1.4222588

1.4199373

1. 4170040

1.4115327

1.4048912

1. 3944471

1. 3622046

1. 3240448

1. 2383359

1. 1465005

$9.5552159-1$

$h_{3}=90$ kilometers

0.0005

0.0010

0.0020

0.0030

0.0040

0.0050

0.0060

0.0070

0.0080

0.0090

0.0100

0.0120

0.0150

0.0200

0.0250

0.0300

0.0400

0.0500

0.0650

0.0800

0.1000

0.1500

0.2000

0.3000

0.4000

0.6000

$\begin{array}{lll}1.4000780 & 3 & 8.0530037 \\ 1.3948535 & 3 & 8.0529911 \\ 1.3845147 & 3 & 8.0529395 \\ 1.3743220 & 3 & 8.0528536 \\ 1.3642736 & 3 & 8.0527338 \\ 1.3543670 & 3 & 8.0525791 \\ 1.3445989 & 3 & 8.0523900 \\ 1.3349661 & 3 & 8.0521666 \\ 1.3254647 & 3 & 8.0519099 \\ 1.3160909 & 3 & 8.0516177 \\ 1.3068406 & 3 & 8.0512922 \\ 1.2886954 & 3 & 8.0505382 \\ 1.2623103 & 3 & 8.0491517 \\ 1.2203378 & 3 & 8.0461631 \\ 1.1805854 & 3 & 8.0423341 \\ 1.1428039 & 3 & 8.0376753 \\ 1.0724503 & 3 & 8.0259159 \\ 1.0082107 & 3 & 8.0110030 \\ 9.2176798 & 2 & 7.9830306 \\ 8.4566114 & 2 & 7.9488216 \\ 7.5787071 & 2 & 7.8945241 \\ 5.9120543 & 2 & 7.7238039 \\ 4.7678510 & 2 & 7.5174669 \\ 3.3479623 & 2 & 7.0444427 \\ 2.5187906 & 2 & 6.5300097 \\ 1.5902204 & 2 & 5.4497290\end{array}$

1.4055143

1.4055121

I. 4055031

1.4054881

1.4054672

1. 4054402

1.4054072

1.4053682

1.4053234

1.4052724

1. 4052156

1.4050840

1. 4048420

1.4043204

1.4036521

1.4028390

1. 4007866

1. 3981838

1. 3933017

1. 3873311

1. 3778544

1. 3480581

1. 3120455

1. 2294872

1.1397017

$9.5115716-1$

Table 16. Geometric parameters, $\Psi, d / j$ and $\tau_{j}, r$ for the Norton type 301 atmosphere, applicable to the propagation of waves between the D-region $\left(h_{3}=70\right.$ kilometers) or the E-region ( $h_{3}=90$ kilometers) of the ionosphere and the earth. 
$\mathrm{f}=20$ kilocycles

$\sigma=0.005 \mathrm{mhos} /$ meter

$\epsilon_{2}=15$

$\Psi$

radians
$\mathrm{Arg} \mathrm{R}_{\mathrm{e}}$

$\mathrm{h}_{3}=70$ kilometers

$\begin{array}{ll}0.0005 & 9.5372371=1 \\ 0.0010 & 9.0968602=1 \\ 0.0020 & 8.2824224=1 \\ 0.0030 & 7.5542424=1 \\ 0.0040 & 6.9090857=1 \\ 0.0050 & 6.3433629=1 \\ 0.0060 & 5.8533576=1 \\ 0.0070 & 5.4352842=1 \\ 0.0080 & 5.0852359=1 \\ 0.0090 & 4.7990589=1 \\ 0.0100 & 4.5722208=1 \\ 0.0120 & 4.2761382=1 \\ 0.0150 & 4.1405843=1 \\ 0.0200 & 4.3803429=1 \\ 0.0250 & 4.8228099=1 \\ 0.0300 & 5.2788869=1 \\ 0.0400 & 6.0557853=1 \\ 0.0500 & 6.6418283=1 \\ 0.0650 & 7.2680204=1 \\ 0.0800 & 7.7026714=1 \\ 0.1000 & 8.1070517=1 \\ 0.1500 & 8.6867576=1 \\ 0.2000 & 8.9937743=1 \\ 0.3000 & 9.3111783=1 \\ 0.4000 & 9.4726187=1 \\ 0.6000 & 9.6331471=1\end{array}$

$\begin{array}{ll}3.0942024 & 9.9998941=1 \\ 3.0467061 & 9.9997889=1 \\ 2.9509792 & 9.9995777=1 \\ 2.8536195 & 9.9993673=1 \\ 2.7539360 & 9.9991554=1 \\ 2.6514007 & 9.9989450=1 \\ 2.5457212 & 9.9987339=1 \\ 2.4369124 & 9.9985228=1 \\ 2.3253590 & 9.9983117=1 \\ 2.2118403 & 9.9981006=1 \\ 2.0975011 & 9.9978902=1 \\ 1.8721316 & 9.9974673=1 \\ 1.5635072 & 9.9968347=1 \\ 1.1730416-1 & 9.9957801=1 \\ 9.1926660-1 & 9.9947256=1 \\ 7.5164990-1 & 9.9936719=1 \\ 5.4987050-1 & 9.9915642=1 \\ 4.3397390-1 & 9.9894577=1 \\ 3.3033940-1 & 9.9863010=1 \\ 2.6700680-1 & 9.9831490=1 \\ 2.1288160-1 & 9.9789526=1 \\ 1.4164630-1 & 9.9685113=1 \\ 1.0639390-1 & 9.9581597=1 \\ 7.1452500-2 & 9.9378255=1 \\ 5.4204500-2 & 9.9181515=1 \\ 3.7374400-2 & 9.8815410=1\end{array}$

$\mathrm{h}_{3}=90$ kilometers

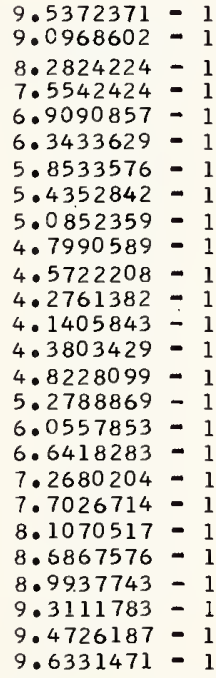

$$
\left|\mathrm{R}_{\mathrm{m}}\right|
$$

Arg $\mathrm{R}$

$\mathrm{d} / \mathrm{j}$

miles

$\begin{array}{lll}3.1416033 & 1.2433895 & 3 \\ 3.1416138 & 1.2381675 & 3 \\ 3.1416349 & 1.2278386 & 3 \\ 3.1416561 & 1.2176623 & 3 \\ 3.1416771 & 1.2076369 & 3 \\ 3.1416983 & 1.1977599 & 3 \\ 3.1417193 & 1.1880278 & 3 \\ 3.1417405 & 1.1784374 & 3 \\ 3.1417615 & 1.1689853 & 3 \\ 3.1417827 & 1.1596669 & 3 \\ 3.1418037 & 1.1504788 & 3 \\ 3.1418460 & 1.1324770 & 3 \\ 3.1419092 & 1.1063547 & 3 \\ 3.1420148 & 1.0649462 & 3 \\ 3.1421201 & 1.0259098 & 3 \\ 3.1422257 & 9.8899038 & 2 \\ 3.1424365 & 9.2076906 & 2 \\ 3.1426473 & 8.5914709 & 2 \\ 3.1429633 & 7.7737068 & 2 \\ 3.1432791 & 7.0657786 & 2 \\ 3.1436994 & 6.2644905 & 2 \\ 3.1447462 & 4.7915900 & 2 \\ 3.1457851 & 3.8174547 & 2 \\ 3.1478288 & 2.6471062 & 2 \\ 3.1498102 & 1.9802951 & 2 \\ 3.1535078 & 1.2445355 & 2\end{array}$

$3 \cdot 1416033$ 3. 1416138 3. 1416349 3.1416561 3.1416771 3.1416983 $3 \cdot 1417193$ 3. 1417405 3.1417615 $3 \cdot 1417827$ 3.1418037 3.1418460 3.1419092 3. 1420148 3. 1421201 3. 1422257 3. 1424365 3. 1426473 3.1429633 3. 1432791 3. 1436994 $3 \cdot 1447462$ 3.1457851 3. 1478288 3. 1498102 3. 1535078 $\begin{array}{ll}1.4000780 & 3 \\ 1.3948535 & 3 \\ 1.3845147 & 3 \\ 1.3743220 & 2 \\ 1.3642736 & 3 \\ 1.3543670 & 3 \\ 1.3445989 & 3 \\ 1.3349661 & 3 \\ 1.3254647 & 3 \\ 1.3160909 & 3 \\ 1.3068406 & 3 \\ 1.2886954 & 3 \\ 1.2623103 & 3 \\ 1.2203378 & 3 \\ 1.1805854 & 3 \\ 1.1428039 & 3 \\ 1.0724503 & 3 \\ 1.0082107 & 3 \\ 9.2176798 & 2 \\ 8.4566114 & 2 \\ 7.5787071 & 2 \\ 5.9120543 & 2 \\ 4.7678510 & 2 \\ 3.3479623 & 2 \\ 2.5187906 & 2 \\ 1.5902204 & 2 \\ & \end{array}$

Table 17. Ground reflection coefficients (amplitude, $|R|$, and phase, Arg R) assuming the Fresnel approximation for the special ray configuration of the Norton type 301 atmosphere, applicable to the propagation of waves between the D-region ( $h_{3}=70$ kilometers) or the E-region ( $h_{3}=90$ kilometers) of the ionosphere and the earth. 
$\mathrm{f}=10$ kilocycles

$\sigma=0.005 \mathrm{mhos} / \mathrm{meter}$

$\epsilon_{2}=15$
$\Psi$

radians

$\left|R_{e}\right|$
$\operatorname{Arg~} R_{e}$

$\mathrm{h}_{3}=70$ kilometers

$\begin{array}{ll}0.0005 & 9.3522075-1 \\ 0.0010 & 8.7490274=1 \\ 0.0020 & 7.6734772-1 \\ 0.0030 & 6.7650622-1 \\ 0.0040 & 6.0136974=1 \\ 0.0050 & 5.4087857-1 \\ 0.0060 & 4.9393008=1 \\ 0.0070 & 4.5931835-1 \\ 0.0080 & 4.3567140-1 \\ 0.0090 & 4.2144176=1 \\ 0.0100 & 4.1497609=1 \\ 0.0120 & 4.1893220=1 \\ 0.0150 & 4.4806613=1 \\ 0.0200 & 5.1265606=1 \\ 0.0250 & 5.7215580=1 \\ 0.0300 & 6.2147185=1 \\ 0.0400 & 6.9474504=1 \\ 0.0500 & 7.4522894=1 \\ 0.0650 & 7.9634980=1 \\ 0.0800 & 8.3055720=1 \\ 0.1000 & 8.6162674=1 \\ 0.1500 & 9.0510552-1 \\ 0.2000 & 9.2769612=1 \\ 0.3000 & 9.5077248=1 \\ 0.4000 & 9.6240947-1 \\ 0.6000 & 9.7391802=1 \\ 0 & \end{array}$

0.0005

0.0010

0.0020

0.0030

0.0040

0.0050

0.0060

0.0070

0.0080

0.0090

0.0100

0.0120

0.0150

0.0200

0.0250

0.0300

0.0400

0.0500

0.0650

0.0800

0.1000

0.1500

0.2000

0.3000

0.4000

0.6000

$9.3522075-1$
$8.7490274-1$
$7.6734772-1$
$6.7650622-1$
$6.0136974-1$
$5.4087857=1$
$4.9393008-1$
$4.5931835=1$
$4.3567140-1$
$4.2144176-1$
$4.1497609-1$
$4.1893220-1$
$4.4806613-1$
$5.1265606-1$
$5.7215580-1$
$6.2147185=1$
$6.9474504-1$
$7.4522894-1$
$7.9634980-1$
$8.3055720-1$
$8.6162674-1$
$9.0510552-1$
$9.2769612-1$
$9.5077248-1$
$9.6240947-1$
$9.7391802-1$

3.0745383
3.0071851
2.8704353
2.7292825
2.5822810
2.4289960
2.2703786
2.1089205
1.9483760
1.7930128
1.6466663
1.3903898
1.1009232
$8.0205290-1$
$6.2814710-1$
$5.1630310-1$
$3.8139170-1$
$3.0284560-1$
$2.3169360-1$
$1.8777660-1$
$1.5000480-1$
$1.0000810-1$
$7.5171900-2$
$5.0510000-2$
$3.8323900-2$
$2.6427900-2$
1

$9.9999256-1$ 9.9998507 $9.9997014-1$ $9.9995526-1$ $9.9994033-1$ $9.9992540-1$ $9.9991047=1$ $9.9989554-1$ $9.9988061-1$ $9.9986569-1$ 9.9985081 - 1 $9.9982095-1$ $9.9977617-$ $9.9970159-1$ $9.9962701-1$ $9.9955250-1$ $9.9940343-1$ 9.9903119 9.9880816 - 1 $9.9851137-1$ $9.9777249-1$ $9.9703968-1$ $9.9559974-1$ $9.9420562=$ $9.9160932-1$ $9.9925443-1$
$\operatorname{Arg~R}_{\mathrm{m}}$

$\mathrm{d} / \mathrm{j}$

miles $\mathrm{h}_{3}=90$ kilometers

$3 \cdot 1416002$
$3 \cdot 1416076$
$3 \cdot 1416225$
$3 \cdot 1416375$
$3 \cdot 1416524$
$3 \cdot 1416673$
$3 \cdot 1416823$
$3 \cdot 1416972$
$3 \cdot 1417121$
$3 \cdot 1417271$
$3 \cdot 1417419$
$3 \cdot 1417718$
$3 \cdot 1418165$
$3 \cdot 1418912$
$3 \cdot 1419656$
$3 \cdot 1420404$
$3 \cdot 1421894$
$3 \cdot 1423385$
$3 \cdot 1425620$
$3 \cdot 1427852$
$3 \cdot 1430824$
$3 \cdot 1438226$
$3 \cdot 1445573$
$3 \cdot 1460025$
$3 \cdot 1474036$
$3 \cdot 1500182$

$1 \cdot 2433895$ 1.2381675 1. 2278386 1. 2176623 1. 2076369 1. 1977599 $1 \cdot 1880278$ 1.1784374 1.1689853 $1 \cdot 1596669$ 1. 1504788 1. 1324770 1. 1063547 1.0649462 1.0259098 9. 8899038 9.2076906 8. 5914709 7. 7737068 7.0657786 6.2644905 4. 7915900 3. 8174547 2. 6471062 1. 9802951 1. 2445355
1.4000780 1. 3948535 1. 3845147 1. 3743220 1. 3642736 1. 3543670 1. 3445989 1. 3349661 1.3254647 1. 3160909 1. 3068406 1. 2886954 1. 2623103 1. 2203378 1. 1805854 1.1428039 1.0724503 1.0082107 9. 2176798 8.4566114 7.5787071 5. 9120543 4. 7678510 3. 3479623 2. 5187906 1. 5902204

Table 18. Ground reflection coefficients (amplitude, $|R|$, and phase, Arg $R$ ) assuming the Fresnel approximation for the special ray configuration of the Norton type 301 atmosphere, applicable to the propagation of waves between the D-region $\left(h_{3}=70\right.$ kilometers) or the E-region $\left(h_{3}=90\right.$ kilometers $)$ of the ionosphere and the earth. 
$f=0.42826552$ kilocycles

$\sigma=0.005 \mathrm{mhos} /$ meter

$\epsilon_{2}=15$

$\Psi$

radians
$\left|R_{e}\right|$

$7.2760752-1$
$5.5010743-1$
$4.1646035-1$
$4.4220005-1$
$5.0407376-1$
$5.6295438-1$
$6.1241260-1$
$6.5306259-1$
$6.8658189-1$
$7.1450144-1$
$7.3802716-1$
$7.7533889-1$
$8.1512753-1$
$8.5744432-1$
$8.8406682-1$
$9.0233375-1$
$9.2575371-1$
$9.4011862-1$
$9.5358624-1$
$9.6210341-1$
$9.6954535-1$
$9.7954847-1$
$9.8457671=1$
$9.8960483-1$
$9.9454581-1$
$9.9210129-1$

Arg $R_{e}$

$\mathrm{h}_{3}=70$ kilometers
Arg $R_{m}$

$\left|R_{m}\right|$

$9.9999845-1$

2. 8122977

2.4550692

1.6945478

1. 1423184

8.3288090 -

$6.5188650-1$

$5.3545320-1$

4.5458210 -

$3.9515360-1$

3.4961280 - 1

3.1357870 -

$2.6013070-1$

$2.0731660-1$

1.5502440 -

$1.2384960-1$

1.0313350 -

7.7299800 -

$6.1827100-2$

$4.7560600=$

$3.8652100-2$

$3.0937400-2$

$2.0666200-2$

$1.5544500=$

$1.0449800-2$

$5.4692000-3$

$7.9301000-3$

$\mathrm{h}_{3}=90$ kilometers

$\begin{array}{lll}2.8122977 & 9.9999845-1 & 3.1415942 \\ 2.4550692 & 9.9999690-1 & 3.1415958 \\ 1.6945478 & 9.9999384-1 & 3.1415988 \\ 1.1423184-1 & 9.9999074-1 & 3.1416020 \\ 8.3288090-1 & 9.9998766-1 & 3.1416050 \\ 6.5188650-1 & 9.9998456-1 & 3.1416082 \\ 5.3545320-1 & 9.9998148-1 & 3.1416112 \\ 4.5458210-1 & 9.9997838-1 & 3.1416143 \\ 3.9515360-1 & 9.9997530-1 & 3.1416174 \\ 3.4961280-1 & 9.9997220-1 & 3.1416205 \\ 3.1357870-1 & 9.9996912-1 & 3.1416236 \\ 2.6013070-1 & 9.9996294-1 & 3.1416298 \\ 2.0731660-1 & 9.9995366-1 & 3.1416390 \\ 1.5502440-1 & 9.9993827-1 & 3.1416544 \\ 1.2384960-1 & 9.9992281-1 & 3.1416699 \\ 1.0313350-1 & 9.9990735-1 & 3.1416854 \\ 7.7299800-2 & 9.9987652-1 & 3.1417162 \\ 6.1827100-2 & 9.9984565-1 & 3.1417471 \\ 4.7560600-2 & 9.9979945-1 & 3.1417933 \\ 3.8652100-2 & 9.9975326-1 & 3.1418395 \\ 3.0937400-2 & 9.9969174-1 & 3.1419010 \\ 2.0666200-2 & 9.9953864-1 & 3.1420541 \\ 1.5544500-2 & 9.9938668-1 & 3.1422063 \\ 1.0449800-2 & 9.9908781-1 & 3.1425053 \\ 7.9301000-3 & 9.9879816-1 & 3.1427953 \\ 5.4692000-3 & 9.9825785-1 & 3.1433364 \\ & & \end{array}$

9.9999690 -

$9.9999384-1$

9.9999074 -

9.9998766 -

$9.9998456-1$

9.9998148 -

.9997838 -

$9.9997530-1$

9.9997220 -

.9996912 -

$9.9996294-1$

9.9995366 -

9.9993827 -

$9.9992281-1$

$9.9990735-1$

9.9987652 -

$9.9984565-1$

$9.9979945-1$

9.9975326 -

$9.9969174-1$

$9.9953864-1$

9.9938668 -

$9.9908781-1$

9.9825785 - 1

9.9879816 -
3.1415942

$3 \cdot 1415958$

3. 1415988

3.1416020

3.1416050

3. 1416082

3. 1416112

3. 1416143

3. 1416174

3. 1416205

$3 \cdot 1416236$

3.1416298

3.1416390

3. 1416544

3. 1416699

$3 \cdot 1416854$

3. 1417162

3. 1417471

$3 \cdot 1417933$

3. 1418395

3. 1419010

3.1420541

3. 1422063

3.1425053

3. 1433364

3.1427953 $\mathrm{d} / \mathrm{j}$

miles

$\begin{array}{ll}0.0005 & 7.2760752-1 \\ 0.0010 & 5.5010743-1 \\ 0.0020 & 4.1646035-1 \\ 0.0030 & 4.4220005-1 \\ 0.0040 & 5.0407376-1 \\ 0.0050 & 5.6295438=1 \\ 0.0060 & 6.1241260-1 \\ 0.0070 & 6.5306259-1 \\ 0.0080 & 6.8658189-1 \\ 0.0090 & 7.1450144-1 \\ 0.0100 & 7.3802716-1 \\ 0.0120 & 7.7533889-1 \\ 0.0150 & 8.1512753-1 \\ 0.0200 & 8.5744432-1 \\ 0.0250 & 8.8406682-1 \\ 0.0300 & 9.0233375=1 \\ 0.0400 & 9.2575371-1 \\ 0.0500 & 9.4011862-1 \\ 0.0650 & 9.5358624-1 \\ 0.0800 & 9.6210341-1 \\ 0.1000 & 9.6954535-1 \\ 0.1500 & 9.7954847-1 \\ 0.2000 & 9.8457671-1 \\ 0.3000 & 9.8960483-1 \\ 0.4000 & 9.9210129-1 \\ 0.6000 & 9.9454581-1 \\ & \end{array}$

$\begin{array}{ll}1.2433895 & 3 \\ 1.2381675 & 3 \\ 1.2278386 & 3 \\ 1.2176623 & 3 \\ 1.2076369 & 3 \\ 1.1977599 & 3 \\ 1.1880278 & 3 \\ 1.1784374 & 3 \\ 1.1689853 & 3 \\ 1.1596669 & 3 \\ 1.1504788 & 3 \\ 1.1324770 & 3 \\ 1.1063547 & 3 \\ 1.0649462 & 3 \\ 1.0259098 & 3 \\ 9.8899038 & 2 \\ 9.2076906 & 2 \\ 8.5914709 & 2 \\ 7.7737068 & 2 \\ 7.0657786 & 2 \\ 6.2644905 & 2 \\ 4.7915900 & 2 \\ 3.8174547 & 2 \\ 2.6471062 & 2 \\ 1.9802951 & 2 \\ 1.2445355 & 2\end{array}$

1.4000780 1.3948535 1. 3845147 1.3743220 1.3642736 1.3543670 1. 3445989 1. 3349661 1. 3254647 1.3160909 1.3068406 1.2886954 1. 2623103 1.2203378 1.1805854 1.1428039 1.0724503 1.0082107 9. 2176798 8.4566114 7.5787071 5. 9120543 4. 7678510 3.3479623 2. 5187906 1. 5902204
3
3
3
3
3
3
3
3
3
3
3
3
3
3
3
2
2
2
2
2
2
2
2
2
2
2

Table 19. Ground reflection coefficients (amplitude, |R|, and phase, Arg R) assuming the Fresnel approximation, for the special ray configuration of the Norton type 301 atmosphere, applicable to the propagation of waves between the D-region ( $h_{3}=70$ kilometers) or the E-region ( $h_{3}=90$ kilometers) of the ionosphere and the earth. 
$f=0.85653105$ kilocycles

$\sigma=0.005$ mhos $/$ meter

$\epsilon_{2}=15$

$\Psi$

radians
$\operatorname{Arg} R_{e}$

$\mathrm{h}_{3}=70$ kilometers

\begin{tabular}{|c|c|c|}
\hline $\begin{array}{l}0.0005 \\
0.0010\end{array}$ & $\begin{array}{l}7.9696248 \\
6.4337594\end{array}$ & \\
\hline 0.0020 & 4.6433743 & \\
\hline 0.0030 & 4.1445199 & \\
\hline 0.0040 & 4.3311065 & \\
\hline 0.0050 & 4.7463973 & . \\
\hline 0.0060 & 5.1911354 & 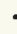 \\
\hline 0.0070 & 5.6020975 & 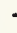 \\
\hline 0.0080 & 5.9650704 & \\
\hline 0.0090 & 6.2813736 & 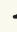 \\
\hline 0.0100 & 6.5565634 & 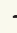 \\
\hline 0.0120 & 7.0074354 & 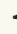 \\
\hline 0.0150 & 7.5054946 & 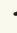 \\
\hline 0.0200 & 8.0523596 & . \\
\hline 0.0250 & 8.4045343 & \\
\hline 0.0300 & 8.6495214 & . \\
\hline 0.0400 & 8.9673583 & . \\
\hline 0.0500 & 9.1642860 & \\
\hline 0.0650 & 9.3502136 & \\
\hline 0.0800 & 9.4684264 & \\
\hline 0.1000 & 9.5721048 & \\
\hline 0.1500 & 9.7120215 & \\
\hline 0.2000 & 9.7825880 & \\
\hline 0.3000 & 9.8533083 & \\
\hline 0.4000 & 9.8884795 & \\
\hline 0.6000 & 9.9229527 & \\
\hline
\end{tabular}

2.9106499

2. 6687131

2.1357482

1.6117222

1. 2164162

$9.5461510-1$

$7.8046080-1$

$6.5909740-1$

$5.7037390-1$

$5.0284940-1$

$4.4976750-1$

$3.7164380-1$

$2.9516430-1$

$2.2008540-1$

I. $7558700-1$

$1.4610650-1$

$1.0942570-1$

$8.7491600-2$

$6.7285800-2$

$5.4675600-2$

$4.3758900-2$

$2.9228300-2$

2. $1984100-2$

$1.4778500-2$

1. $1215000-2$

$7.7346000-3$
$\left|\mathrm{R}_{\mathrm{m}}\right|$

$9.9999781-1$

9.9999561 -

$9.9999123-1$

$9.9998694-1$

$9.9998255-1$

9.9997817 -

$9.9997378-1$

$9.9996940=$

$9.9996507-1$

$9.9996069-1$

$9.9995630-1$

$9.9994753-1$

$9.9993450-1$

9.9991264 -

$9.9989081-1$

$9.9986898-1$

9.9982541 -

$9.9978172-1$

9.9971637 -

$9.9965107-1$

$9.9956408-1$

$9.9934754-1$

$9.9913277-1$

$9.9871022-1$

9.9830070 -

9.9753712 -
Arg $R_{m}$

3. 1415971

$3 \cdot 1416014$

3. 1416058

3. 1416102

3. 1416145

3.1416189

$3 \cdot 1416233$

3.1416276

3.1416320

3. 1416364

$3 \cdot 1416451$

3. 1416582

3. 1416800

$3 \cdot 1417019$

3. 1417237

$3 \cdot 1417674$

3. 1418110

3.1418763

$3 \cdot 1419416$

3. 1420287

3.1422454

3. 1424603

$3 \cdot 1428834$

3.1432934

3. 1440586 $\mathrm{d} / \mathrm{j}$

miles

$\mathrm{h}_{3}=90$ kilometers

$\begin{array}{ll}7.9696248-1 & 2.9106499 \\ 6.4337594-1 & 2.6687131 \\ 4.6433743-1 & 2.1357482 \\ 4.1445199-1 & 1.6117222 \\ 4.3311065-1 & 1.2164162 \\ 4.7463973-1 & 9.5461510-1 \\ 5.1911354-1 & 7.8046080-1 \\ 5.6020975-1 & 6.5909740-1 \\ 5.9650704-1 & 5.7037390-1 \\ 6.2813736-1 & 5.0284940-1 \\ 6.5565634-1 & 4.4976750-1 \\ 7.0074354-1 & 3.7164380-1 \\ 7.5054946-1 & 2.9516430-1 \\ 8.0523596-1 & 2.2008540-1 \\ 8.4045343-1 & 1.7558700-1 \\ 8.6495214-1 & 1.4610650-1 \\ 8.9673583-1 & 1.0942570-1 \\ 9.1642860-1 & 8.7491600-2 \\ 9.3502136-1 & 6.7285800-2 \\ 9.4684264=1 & 5.4675600-2 \\ 9.5721048-1 & 4.3758900-2 \\ 9.7120215-1 & 2.9228300-2 \\ 9.7825880-1 & 2.1984100-2 \\ 9.8533083-1 & 1.4778500-2 \\ 9.8884795-1 & 1.1215000-2 \\ 9.9229527-1 & 7.7346000-3\end{array}$

?. 2433895

1. 2381675

1.2278386

1.2176623

1. 2076369

1. 1977599

1. 1880278

1. 1784374

1. 1689853

1. 1596669

1. 1504788

1. 1324770

1. 1063547

1.0649462

1. 0259098

9.8899038

9. 2076906

8. 5914709

7.7737068

7.0657786

6. 2644905

4. 7915900

3. 8174547

2.6471062

1. 9802951

1. 2445355
0.0010

0.0020

0.0030

0.0040

0.0050

0.0060

0.0070

0.0080

0.0090

0.0100

0.0120

0.0150

0.0200

0.0250

0.0300

0.0400

0.0500

0.0650

0.0800

0.1000

0.1500

0.2000

0.3000

0.4000

0.6000

\begin{tabular}{|c|c|c|}
\hline 9.9999781 & -1 & 3.141594 \\
\hline 9.9999561 & & 3.1415971 \\
\hline 9.9999123 & & 3.1416014 \\
\hline 9.9998694 & & $3 \cdot 141605$ \\
\hline 9.9998255 & & 3.141610 \\
\hline 9.9997817 & -1 & 3.141614 \\
\hline 9.9997378 & & 3.141618 \\
\hline 9.9996940 & -1 & 3.141623 \\
\hline 9.9996507 & -1 & 3.14162 \\
\hline 9.9996069 & & 3.141632 \\
\hline 9.9995630 & -1 & 3.141636 \\
\hline 9.9994753 & -1 & 3.141645 \\
\hline 9.9993450 & -1 & $3 \cdot 141658$ \\
\hline 9.9991264 & & 3.141680 \\
\hline 9.9989081 & -1 & 3.141701 \\
\hline 9.9986898 & & 3.14172 \\
\hline 9.9982541 & -1 & 3.14176 \\
\hline 9.9978172 & -1 & 3.141811 \\
\hline 9.9971637 & -1 & 3.141876 \\
\hline 9.9965107 & & 3.141941 \\
\hline 9.9956408 & -1 & 3.142028 \\
\hline 9.9934754 & -1 & $3 \cdot 142245$ \\
\hline 9.9913277 & -1 & 3.142460 \\
\hline 9.9871022 & -1 & $3 \cdot 142883$ \\
\hline 9.9830070 & -1 & 3.143293 \\
\hline 9.9753712 & -1 & $3 \cdot 144058$ \\
\hline
\end{tabular}

$\begin{array}{ll}1.4000780 & 3 \\ 1.3948535 & 3 \\ 1 \cdot 3845147 & 3 \\ 1.3743220 & 3 \\ 1.3642736 & 3 \\ 1.3543670 & 3 \\ 1.3445989 & 3 \\ 1.3349661 & 3 \\ 1.3254647 & 3 \\ 1.3160909 & 3 \\ 1.3068406 & 3 \\ 1.2886954 & 3 \\ 1.2623103 & 3 \\ 1.2203378 & 3 \\ 1.1805854 & 3 \\ 1.1428039 & 3 \\ 1.0724503 & 3 \\ 1.0082107 & 3 \\ 9.2176798 & 2 \\ 8.4566114 & 2 \\ 7.5787071 & 2 \\ 5.9120543 & 2 \\ 4.7678510 & 2 \\ 3.3479623 & 2 \\ 2.5187906 & 2 \\ 1.5902204 & 2 \\ 1.59\end{array}$

Table 20. Ground reflection coefficients (amplitude, $|R|$, and phase, Arg $R$ ) assuming the Fresnel approximation, for the special ray configuration of the Norton type 301 atmosphere, applicable to the propagation of waves between the D-region $\left(h_{3}=70\right.$ kilometers) or the E-region ( $h_{3}=90$ kilometers) of the ionosphere and the earth. 
$f=2.1413276$ kilocycles

$\sigma=0.005 \mathrm{mhos} / \mathrm{meter}$

$\epsilon_{2}=15$

$\Psi$

radians
$\left|R_{e}\right|$

Arg $\mathrm{R}_{\mathrm{e}}$

$\left|\mathrm{R}_{\mathrm{m}}\right|$

$h_{3}=70$ kilometers

\begin{tabular}{|c|c|c|}
\hline $\begin{array}{l}.9962765 \\
2.8480213\end{array}$ & & $\begin{array}{l}9.9999653 \\
9.9999312\end{array}$ \\
\hline 2.5335359 & & 9.9998618 \\
\hline 2.1924738 & & 9.9997930 \\
\hline $\begin{array}{l}1.8474151 \\
1.5372500\end{array}$ & & $\begin{array}{l}9.9997236 \\
9.99965188\end{array}$ \\
\hline $\begin{array}{l}1.2853422 \\
1.0908877\end{array}$ & & $\begin{array}{l}9.9995860 \\
9.9995156\end{array}$ \\
\hline 9.4219960 & -1 & 9.9994478 \\
\hline 8.2718250 & -1 & 9.9993784 \\
\hline 7.3649720 & -1 & 9.9993096 \\
\hline 6.0372130 & -1 & 9.9991714 \\
\hline 4.7551070 & -1 & 9.9989644 \\
\hline 3.5188100 & -1 & 9.9986188 \\
\hline 2.7966120 & -1 & 9.9982735 \\
\hline 2.3220300 & & 9.9979285 \\
\hline 1.7352230 & -1 & 9.9972392 \\
\hline 1.3859530 & -1 & 9.9965490 \\
\hline 1.0650580 & & 9.9955157 \\
\hline 8.6512500 & -2 & 9.9944830 \\
\hline 6.9220700 & -2 & 9.9931084 \\
\hline 4.6223200 & & $9.989686 !$ \\
\hline 3.4763500 & -2 & 9.9862903 \\
\hline 2.3367800 & & 9.9796144 \\
\hline 1.7732600 & -2 & 9.9731454 \\
\hline 1.2229400 & -2 & 9.9610858 \\
\hline
\end{tabular}

$h_{3}=90$ kilometers
9.99993

$9.9998618-1$

$9.9997930-1$

9.9997235 -

$9.9996548-1$

$9.9995860-1$

$9.9995166-1$

$9.9994478-1$

$9.9993784-1$

$9.999309 E-1$

$9.9991714-1$

$9.9989644-1$

$9.9986188-1$

$9.9982736-1$

$9.9979285-1$

9.9972392 -

$9.9965490-1$

9.9955157 -

$9.9944830-1$

$9.9931084-1$

9.9896861 - 1

9.9862909 - 1

$9.9796144-1$

$9.9731454-1$

9.9610858 -
Arg $R_{m}$

$\mathrm{d} / \mathrm{j}$

miles

$3 \cdot 1415962$
3.1415996
$3 \cdot 1416065$
3.1416134
$3 \cdot 1416203$
$3 \cdot 1416272$
$3 \cdot 1416342$
$3 \cdot 1416410$
$3 \cdot 1416480$
$3 \cdot 1416548$
$3 \cdot 1416618$
$3 \cdot 1416756$
$3 \cdot 1416962$
$3 \cdot 1417309$
$3 \cdot 1417654$
$3 \cdot 1417999$
$3 \cdot 1418688$
$3 \cdot 1419378$
$3 \cdot 1420412$
$3 \cdot 1421445$
$3 \cdot 1422820$
$3 \cdot 1426247$
$3 \cdot 1429646$
$3 \cdot 1436333$
$3 \cdot 1442817$
3.1454917

1.2433895 1.2391675 1.2278386 1.2176623 1.2076369 1.1977599 1.1880278 1.1784374 1. 1689853 1.1596669 I. 1504788 1.1324770 1.1063547 1.0649462 1.0259098 9.8899038 9.2076906 8.5914709 7.7737068 7.0657786 6.2644905 4.7915900 3.8174547 2.6471062 1. 9802951 1. 2445355
3. 1415962 3. 1415996

3.1416065 3.1416134

3. 1416203

3. 1416272

3.1416342

3. 1416410

3.1416480

3. 1416548

3. 1416618

3.1416756

3.1416962

3.1417309

3. 1417654

3.1417999

3. 1418688

3.1419378

3.1420412

3. 1421445

3.1422820

3. 1426247

3. $142964 t$

3. 1436333

3.1442817

3.1454917

$\begin{array}{ll}1.4000780 & 3 \\ 1.3948535 & 3 \\ 1.3845147 & 3 \\ 1.3743220 & 3 \\ 1.3642730 & 3 \\ 1.3543670 & 3 \\ 1.3445989 & 3 \\ 1.3349651 & 3 \\ 1.3254647 & 3 \\ 1.3160909 & 3 \\ 1.3068406 & 3 \\ 1.2886954 & 3 \\ 1.2623103 & 3 \\ 1.2203378 & 3 \\ 1.1805854 & 3 \\ 1.1428039 & 3 \\ 1.0724503 & 3 \\ 1.0032107 & 3 \\ 9.2176793 & 2 \\ 3.4566114 & 2 \\ 7.5787071 & 2 \\ 5.9120543 & 2 \\ 4.7678510 & 2 \\ 3.3479623 & 2 \\ 2.5187906 & 2 \\ 1.5902204 & 2 \\ 1.590011 & \end{array}$

0.6000

$1.7732600-$

Table 2l. Ground reflection coefficients (amplitude, |R|, and phase, Arg R) assuming the Fresnel approximation, for 301 atmosphere, applicable to the propagation of waves between the $\mathrm{D}$-region $\left(\mathrm{h}_{3}=70\right.$ kilometers) or the E-region $\left(\mathrm{h}_{3}=90\right.$ kilometers $)$ of the ionosphere and the earth. 
$\mathrm{f}=4.2826552$ kilocycles

$\sigma=0.005 \mathrm{mhos} / \mathrm{meter}$

$\epsilon_{2}=15$

$\operatorname{Arg} R_{e} \quad\left|R_{m}\right|$

$\mathrm{h}_{3}=70$ kilometers

0.0005

0.0010

0.0020

0.0030

0.0040

0.0050

0.0060

0.0070

0.0080

0.0090

0.0100

0.0120

0.0150

0.0200

0.0250

0.0300

0.0400

0.0500

0.0650

0.0800

0.1000

0.1500

0.2000

0.3000

0.4000

0.6000

$9.0282360=1$
$8.1597957=1$
$6.7190501=1$
$5.6435159-1$
$4.8949004=1$
$4.4304970-1$
$4.1989988-1$
$4.1423305=1$
$4.2044529=1$
$4.3396714=1$
$4.5153280=1$
$4.9107873-1$
$5.4866849-1$
$6.2645828=1$
$6.8364307=1$
$7.2639714=1$
$7.8525441=1$
$8.2353793=1$
$8.6089482=1$
$8.8523621=1$
$9.0695069-1$
$9.3678143=1$
$9.5205017=1$
$9.6749899=1$
$9.7523595-1$
$9.8285368-1$
9

3. 0390190

2.9353883

2.7211641

2. 4934275

2. 2522884

2. 0057043

1. 7676166

1. 5515332

1.3648903

$1 \cdot 2084453$

1.0789615

$8.8282430-1$

$6.9045370-1$

$5.0619260-1$

4. $0009730-1$

$3.3110840-1$

$2.4657100-1$

$1.9660850-1$

1. 5089770 - 1

1. $2249450-1$

$9.7967600-2$

$6.5390700-2$

4. $9171400-2$

3. $3049200-2$

$2.5078300-2$

1. $7294900-2$

$9.9999517-1$
$9.9999019-1$
$9.9998046-1$
$9.9997072-1$
$9.9996092-1$
$9.9995118-1$
$9.9994138-1$
$9.9993164-1$
$9.9992184-1$
$9.9991210-1$
$9.9990236-1$
$9.9988282-1$
$9.9985355-1$
$9.9980467-1$
$9.9975586-1$
$9.9970712-1$
$9.9960952-1$
$9.9951206-1$
$9.9936589-1$
$9.9921994-1$
$9.9902559-1$
$9.9854164-1$
$9.9806172-1$
$9.9711819-1$
$9.9620430-1$
$9.9450107-1$

3. 1415976

3. 1416024

3. 1416122

3. 1416220

3. 1416318

3. 1416415

3.1416513

3.1416610

3. 1416708

3. 1416806

3. 1416904

3. 1417099

3. 1417392

3. 1417880

$3 \cdot 1418369$

3.1418856

3. 1419832

3. 1420808

$3 \cdot 1422270$

3. 1423730

3. 1425676

3. 1430520

3. 1435328

3. 1444786

3. 1453955

3. 1471067

$\mathrm{h}_{3}=90$ kilometer $\mathrm{s}$

0.0005
0.0010
0.0020
0.0030
0.0040
0.0050
0.0060
0.0070
0.0080
0.0090
0.0100
0.0120
0.0150
0.0200
0.0250
0.0300
0.0400
0.0500
0.0650
0.0800
0.1000
0.1500
0.2000
0.3000
0.4000
0.6000
3.0390190

2.9353883

2. 7211641

2. 4934275

2. 2522884

2. 0057043

1. 7676166

1. 5515332

1. 3648903

1.2084453

1.0789615

$8.8282430-1$

$6.9045370-1$

$5.0619260-1$

4.0009730- 1

$3.3110840-1$

$2.4657100-1$

$1.9660850-1$

$1.5089770-1$

$1.2249450-1$

9.7967600-2

$6.5390700-2$

4. $9171400-2$

3. $3049200-2$

2. $5078300-2$

$1.7294900-2$
$9.9999517-1$ $9.9999019=$ $9.9998046-1$ $9.9997072-1$ $9.9996092-1$ $9.9995118-1$ $9.9994138-1$ $9.9993164-1$ $9.9992184-1$ $9.9991210-$ 9.9990236 $9.9988282-1$ $9.9985355-1$ $9.9980467-1$ $9.9975586-1$ 9.9970712 - 1 $9.9960952=$ $9.9951206=1$ $9.9936589-$ $9.9921994-1$ $9.9902559-1$ $9.9854164-1$ $9.9806172-1$ $9.9711819-1$ 9.9620430 $9.9450107=1$
3.1415976 3. 1416024 $3 \cdot 1416122$ 3. 1416220 3. 1416318 3. 1416415 3. 1416513 $3 \cdot 1416610$ 3. 1416708 3. 1416806 3. 1416904 3. 1417099 3. 1417392 3.1417880 3. 1418369 3. 1418856 $3 \cdot 1419832$ 3. 1420808 3. 1422270 $3 \cdot 1423730$ 3. 1425676 3.1430520 3. 1435328 3. 1444786 3. 1453955 3.1471067 $\mathrm{d} / \mathrm{j}$ miles

1.2433895 1. 2381675

1. 2278386

1. 2176623

1. 2076369

1. 1977599

1. 1880278

1. 1784374

1. 1689853

1.1596669

1. 1504788

1. 1324770

1.1063547

1.0649462

1.0259098

9.8899038

9.2076906

8.5914709

7.7737068

7.0657786

6.2644905

4. 7915900

3.8174547

2.6471062

1.9802951

1. 2445355

3
3
3
3
3
3
3
3
3
3
3
3
3
3
3
2
2
2
2
2
2
2
2
2
2
2

1.4000780

1.3948535

1. 3845147

1. 3743220

1.3642736

1. 3543670

1. 3445989

1.3349661

1. 3254647

1.3160909

1. 3068406

1. 2886954

1. 2623103

1.2203378

1.1805854

1.1428039

1.0724503

1.0082107

9.2176798

8.4566114

7.5787071

5. 9120543

4. 7678510

3.3479623

2. 5187906

1. 5902204

Table 22. Ground reflection coefficients (amplitude, $|R|$, and phase, Arg R) assuming the Fresnel approximation, for the special ray configuration of the Norton type 301 atmosphere, applicable to the propagation of waves between the D-region ( $h_{3}=70$ kilometers) or the E-region $\left(h_{3}=90\right.$ kilometers) of the ionosphere and the earth. 
$\Psi$

radians

$\left|\mathrm{R}_{\mathrm{e}}\right|$

$9.3020145=1$
$8.6560578=1$
$7.5162135=1$
$6.5696598=1$
$5.8034986=1$
$5.2042451=1$
$4.7576038=1$
$4.4474944=1$
$4.2554105=1$
$4.1608914=1$
$4.1430849=1$
$4.2629094=1$
$4.6318294=1$
$5.3288151=1$
$5.9322533=1$
$6.4193963=1$
$7.1298301=1$
$7.6127243=1$
$8.0975696=1$
$8.4200587=1$
$8.7117884=1$
$9.1183597=1$
$9.3289095=1$
$9.5435347=1$
$9.6516049=1$
$9.7583776=1$

0.0005

0.0010

0.0020

0.0030

0.0040

0.0050

0.0060

0.0070

0.0080

0.0090

0.0100

0.0120

0.0150

0.0200

0.0250

0.0300

0.0400

0.0500

0.0650

0.0800

0.1000

0.1500

0.2000

0.3000

0.4000

0.6000

$\begin{array}{ll}0.0005 & 9.3020145=1 \\ 0.0010 & 8.6560578=1 \\ 0.0020 & 7.5162135=1 \\ 0.0030 & 6.5696598=1 \\ 0.0040 & 5.8034986=1 \\ 0.0050 & 5.2042451=1 \\ 0.0060 & 4.7576038=1 \\ 0.0070 & 4.4474944=1 \\ 0.0080 & 4.2554105=1 \\ 0.0090 & 4.1608914=1 \\ 0.0100 & 4.1430849=1 \\ 0.0120 & 4.2629094=1 \\ 0.0150 & 4.6318294=1 \\ 0.0200 & 5.3288151=1 \\ 0.0250 & 5.9322533=1 \\ 0.0300 & 6.4193963=1 \\ 0.0400 & 7.1298301=1 \\ 0.0500 & 7.6127243=1 \\ 0.0650 & 8.0975696=1 \\ 0.0800 & 8.4200587=1 \\ 0.1000 & 8.7117884=1 \\ 0.1500 & 9.1183597=1 \\ 0.2000 & 9.3289095=1 \\ 0.3000 & 9.5435347=1 \\ 0.4000 & 9.6516049=1 \\ 0.6000 & 9.7583776=1\end{array}$

$f=8.5653105$ kilocycles

$\sigma=0.005 \mathrm{mhos} / \mathrm{meter}$

$\epsilon_{2}=15$

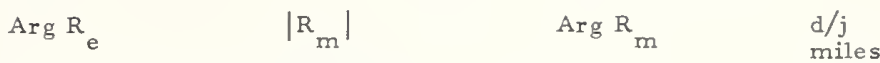

$h_{3}=70$ kilometers

$\begin{array}{ll}3.0691294 & 9.9999316=1 \\ 2.9962893 & 9.9998613=1 \\ 2.8480461 & 9.9997236=1 \\ 2.6943420 & 9.9995850=1 \\ 2.5335791 & 9.9994473=1 \\ 2.3656699 & 9.9993096=1 \\ 2.1925207 & 9.9991719=1 \\ 2.0180486 & 9.9990333=1 \\ 1.8474490 & 9.9988956=1 \\ 1.6858882 & 9.9987569=1 \\ 1.5372646 & 9.9986193=1 \\ 1.2853419 & 9.9983429=1 \\ 1.0116226 & 9.9979290-1 \\ 7.3649310-1 & 9.9972377-1 \\ 5.7771040-1 & 9.9965485-1 \\ 4.7553100-1 & 9.9958574=1 \\ 3.5192500-1 & 9.9944791-1 \\ 2.7972700-1 & 9.9931001=1 \\ 2.1417380-1 & 9.9910335=1 \\ 1.7364750-1 & 9.9889691=1 \\ 1.3875760-1 & 9.9862216=1 \\ 9.2536500-2 & 9.9793829=1 \\ 6.9562800-2 & 9.9725995-1 \\ 4.6744400-2 & 9.9592703=1 \\ 3.5467900-2 & 9.9463630=1 \\ 2.4458700-2 & 9.9223222-1\end{array}$

3.1415996

3.1416065

3. 1416203

3.1416341

3.1416480

3. 1416618

3.1416756

3. 1416894

3. 1417032

3.1417171

3. 1417309

3. 1417585

3. 1417999

3.1418690

3. 1419379

3.1420069

3. 1421450

3. 1422830

3.1424897

3. 1426963

3.1429715

3.1436564

3. 1443363

3. 1456739

3. 1469706

3.1493904

$\mathrm{h}_{3}=90$ kilometers

$\begin{array}{ll}3.0691294 & 9.9999316-1 \\ 2.9962893 & 9.9998613=1 \\ 2.8480461 & 9.9997236-1 \\ 2.6943420 & 9.9995850-1 \\ 2.5335791 & 9.9994473-1 \\ 2.3656699 & 9.9993096-1 \\ 2.1925207 & 9.9991719-1 \\ 2.0180486 & 9.9990333-1 \\ 1.8474490 & 9.9988956-1 \\ 1.6858882 & 9.9987569-1 \\ 1.5372646 & 9.9986193-1 \\ 1.2853419-1 & 9.9983429-1 \\ 1.0116226 & 9.9979290-1 \\ 7.3649310-1 & 9.9972377-1 \\ 5.7771040-1 & 9.9965485-1 \\ 4.7553100-1 & 9.9958574-1 \\ 3.5192500-1 & 9.9944791-1 \\ 2.7972700-1 & 9.9931001-1 \\ 2.1417380-1 & 9.9910335-1 \\ 1.7364750-1 & 9.9389691-1 \\ 1.3875760-1 & 9.9862216-1 \\ 9.2536500-2 & 9.9793829-1 \\ 6.9562800-2 & 9.9725995-1 \\ 4.6744400-2 & 9.9592703-1 \\ 3.5467900-2 & 9.9463630-1 \\ 2.4458700-2 & 9.9223222-1\end{array}$

3. 1415996

3.1416065

3. 1416203

3. 1416341

3. 1416480

3. 1416618

3. 1416756

3. 1416894

3. 1417032

3.1417171

3.1417309

3.1417585

3. 1417999

3.1418690

3. 1419379

3.1420069

3.1421450

3. 1422830

3.1424897

3. 1426963

3. 1429715

3. 1436564

3. 1443363

3. 1456739

3. 1469706

3. 1493904

$\begin{array}{ll}1.2433895 & 3 \\ 1.2381675 & 3 \\ 1.2278386 & 3 \\ 1.2176623 & 3 \\ 1.2076369 & 3 \\ 1.1977599 & 3 \\ 1.1880278 & 3 \\ 1.1784374 & 3 \\ 1.1689853 & 3 \\ 1.1596669 & 3 \\ 1.1504738 & 3 \\ 1.1324770 & 3 \\ 1.1063547 & 3 \\ 1.0549462 & 3 \\ 1.0259098 & 3 \\ 9.8899038 & 2 \\ 9.2076906 & 2 \\ 8.5914709 & 2 \\ 7.7737068 & 2 \\ 7.0657786 & 2 \\ 6.2644905 & 2 \\ 4.7915900 & 2 \\ 3.8174547 & 2 \\ 2.6471062 & 2 \\ 1.9802951 & 2 \\ 1.2445355 & 2\end{array}$

1.4000780 1.3948535 1. 3845147 1. 3743220 1.3642736 1. 3543670 1.3445989 1.3349661 1. 3254647 1. 3160909 1.3068406 1. 2886954 1. 2623103 1. 2203378 1. 1805854 1.1428039 1.0724503 1.0082107 9.2176798 8.4566114 7.5787071 5.9120543 4. 7678510 3. 3479623 2.5187906 1. 5902204

Table 23. Ground reflection coefficients (amplitude, |R|, and phase, Arg R) assuming the Fresnel approximation, for the special ray configuration of the Norton type 301 atmosphere, applicable to the propagation of waves between the D-region ( $h_{3}=70$ kilometers) or the E-region $\left(h_{3}=90\right.$ kilometers) of the ionosphere and the earth. 
$f=42.826552$ kilocycles

$\sigma=0.005 \mathrm{mhos} / \mathrm{meter}$

$\epsilon_{2}=15$

$\Psi$

radians

0.0005

0.0020

0.0030

0.0040

0.0050

0.0060

0.0070

0.0080

0.0090

0.0100

0.0120

0.0150

0.0200

0.0250

0.0300

0.0400

0.0500

0.0650

0.0800

0.1000

0.1500

0.2000

0.3000

0.4000

0.6000

0.0005

0.0020

0.0030

0.0040

0.0050

0.0060

0.0070

0.0080

0.0090

0.0100

0.0120

0.0150

0.0200

0.0250

0.0300

0.0400

0.0500

0.0650

0.0800

0.1000

0.1500

0.2000

0.3000

0.4000

0.6000
$\left|R_{e}\right|$

$9.6812326=1$
$9.3729451=1$
$8.7876084=1$
$8.2433792=1$
$7.7393938=1$
$7.2746259=1$
$6.8479583=1$
$6.4582235=1$
$6.1042355=1$
$5.7847942=1$
$5.4986840=1$
$5.0214339=1$
$4.5224533=1$
$4.1612034=1$
$4.1916904=1$
$4.4185474=1$
$5.0373414=1$
$5.6260703=1$
$6.3335699=1$
$6.8615753=1$
$7.3752938=1$
$8.1441622=1$
$8.5649933=1$
$9.0089443=1$
$9.2379786=1$
$9.4677600=1$

Arg $R_{e}$

$\left|\mathrm{R}_{\mathrm{m}}\right|$

$\mathrm{h}_{3}=70$ kilometers

3.1092242
3.0768216
3.0117802
2.9462050
2.8798470
2.8124795
2.7439077
2.6739782
2.6025908
2.5297090
2.4553707
2.3028952
2.0690368
1.6947121
1.3812038
1.1422778
$8.3283030-1$
$6.5189470-1$
$4.9178920-1$
$3.9538010-1$
$3.1393920-1$
$2.0798070-1$
$1.5597130-1$
$1.0462750-1$
$7.9339500-2$
$5.4690500-2$

9. $9998458-$

3. 1416082

3.1416236

3.1416545

3. 1416854

3. 1417162

3. 1417471

3. 1417780

3. 1418088

3. 1418398

3. 1418706

3. 1419015

3.1419632

3. 1420559

3. 1422103

3. 1423645

3.1425189

3. 1428275

3.1431359

3.1435983

3. 1440603

3.1446753

3. 1462070

3.1477271

3. 1507176

3. 1536168

3.1590271 $\mathrm{d} / \mathbf{j}$

miles

$\begin{array}{ll}1.2433895 & 3 \\ 1.2381675 & 3 \\ 1.2278386 & 3 \\ 1.2176623 & 3 \\ 1.2076369 & 3 \\ 1.1977599 & 3 \\ 1.1880278 & 3 \\ 1.1784374 & 3 \\ 1.1689853 & 3 \\ 1.1596669 & 3 \\ 1.1504788 & 3 \\ 1.1324770 & 3 \\ 1.1063547 & 3 \\ 1.0649462 & 3 \\ 1.0259098 & 3 \\ 9.8899038 & 2 \\ 9.2076906 & 2 \\ 8.5914709 & 2 \\ 7.7737068 & 2 \\ 7.0657786 & 2 \\ 6.2644905 & 2 \\ 4.7915900 & 2 \\ 3.8174547 & 2 \\ 2.6471062 & 2 \\ 1.9802951 & 2 \\ 1.2445355 & 2 \\ \end{array}$

$\mathrm{h}_{3}=90$ kilometers

3.1092242
3.0768216
3.0117802
2.9462050
2.8798470
2.8124795
2.7439077
2.6739782
2.6025908
2.5297090
2.4553707
2.3028952
2.0690368
1.6947121
1.3812038
1.1422778
$8.3283030-1$
$6.5189470-1$
$4.9178920=1$
$3.9538010=1$
$3.1393920-1$
$2.0798070=1$
$1.5597130=1$
$1.0462750=1$
$7.9339500-2$
$5.4690500-2$

9.9998458 9.9996912 9.9993823 - 1 $9.9990733-1$ 9.9987650 - 1 9.9984559 - 1 9.9981471 $9.9978382-1$ 9.9975293 9.9972208 9.9969117 - 1 $9.9962944=$ 9.9953685 $9.9938253-1$ 9.9922828 - 1 9.9907402 $9.9876568-1$ $9.9845761-1$ 9.9799593 9.9753487 - 1 9.9692142 - 1 $9.9539523=$ $9.9388289=$ 9.9091436 9.8804477 9.8271186 -
$3 \cdot 1416082$ 3. 1416236 3. 1416545 3. 1416854 3. 1417162 3. 1417471 3. 1417780 3. 1418088 3. 1418398 3. 1419015 3. 1419632 3. 1420559 3. 1422103 3. 1423645 3. 1425189 3. 1428275 3. 1431359 3. 1435983 3. 1440603 3. 1446753 3. 1462070 3. 1477271 3. 1507176 3. 1536168 3. 1590271 3. 1418706
1.4000780 1.3948535

1.3845147

1.3743220

1. 3642736

1. 3543670

1.3445989

$1 \cdot 3349661$

1. 3254647

1. 3160909

1. 3068406

1.2886954

1.2623103

1.2203378

1. 1805854

1. 1428039

1.0724503

1.0082107

9.2176798

8.4566114

7. 5787071

4. 7678510

3. 3479623

2.5187906

1.5902204
5.9120543

Table 24. Ground reflection coefficients (amplitude, $|R|$, and phase, Arg R) assuming the Fresnel approximation, for the special ray configuration of the Norton type 301 atmosphere, applicable to the propagation of waves between the D-region $\left(h_{3}=70\right.$ kilometers) or the $E$-region $\left(h_{3}=90\right.$ kilometers $)$ of the ionosphere and the earth. 
$\mathrm{f}=85.653105$ kilocycles

$\sigma=0.005 \mathrm{mhos} /$ meter

$\epsilon_{2}=15$

$\Psi$

radians

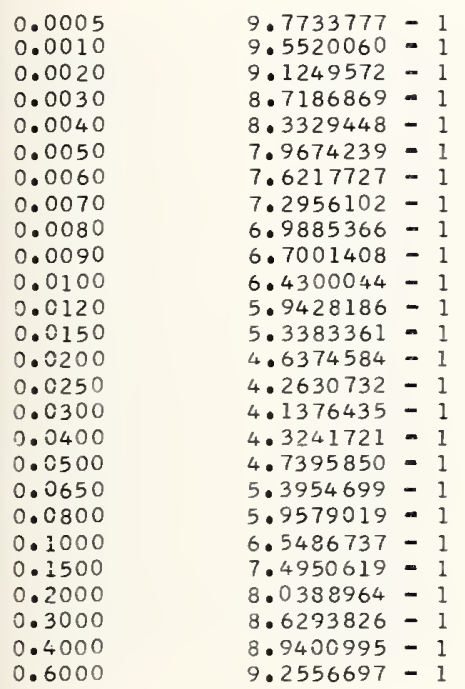

$\operatorname{Arg~} R_{e}$

$\mathrm{h}_{3}=70$ kilometers

3. 118720

3. 0958359

3. 0499830

3. 0039394

2. 9576124

2. 9109137

2. 8637598

2. 8160742

2. 7677888

2. 7188458

2.6691995

2. 5676877

2. 4097259

2. 1363543

1. 8636455

1. 6119791

1. 2163706

9. $5449250-1$

$7.1467080-1$

$5.7048640-1$

$4.5008930-1$

2. $9596530-1$

$2.2131790-1$

$1.4814800-1$

1. $1225750-1$

$7.7342300-2$

$\mathrm{h}_{3}=90$ kilometers

3. 1187203

3.0958359

3. 0499830

3. 0039394

2. 9576124

2. 9109137

2. 8637598

2.8160742

2. 7677888

2. 7188458

2.6691995

2. 5676877

2. 4099259

2. 1363543

1. 8636455

1. 6119791

1. 2163706

9. $5449250-1$

7. $1467080-1$

$5.7048640-1$

4. $5008930-1$

$2.9596530-1$

$2.2131790-1$

1. $4814800-1$

$1.1225750-1$

$7.7342300-2$
$9.9997814-1$

$9.9995627-1$

$9.9991261-1$

$9.9986892-1$

$9.9982526-1$

$9.9978160-1$

$9.9973795-1$

$9.9969432-1$

$9.9965061-1$

$9.9960693-1$

$9.9956328-1$

$9.9947590-1$

$9.9934501-1$

$9.9912678-1$

$9.9890867-1$

$9.9869054-1$

9.9825467 -

$9.9781915-1$

$9.9716663-1$

9.9651519 -

$9.9564849-1$

$9.9349336-$

$9.9135904-$

$9.8717365-1$

$9.8313254-1$

$9.7563519-1$
Arg R

3. 1416145

3.1416364

3. 1416801

3. 1417237

3. 1417674

3. 1418110

3.1418547

3. 1418984

3. 1419420

3. 1419856

3. 142.0294

3. 1421167

3. 1422475

3. 1424659

3. 1426841

3.1429023

3. 1433387

3. 1437749

3. 1444287

3. 1450820

3. 1459516

3. 1481174

3. 1502670

3. 1544956

3. 1585951

3. 1662449 $\mathrm{d} / \mathrm{j}$

miles

0.0500

0.1500

0.3000

0.4000

0.6000

8.6283

$8.9400995-1$

9.2556697 -

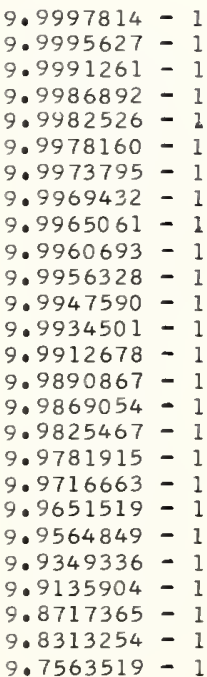

3. 1416145

3. 1416364

3. 1416801

3. 1417237

3. 1417674

3. 1418110

3. 1418547

3. 1418984

3.1419420

3. 1419856

3. 1420294

3.1421167

3. 1422476

3. 1424659

3. 1426841

3. 1429023

3. 1433387

3. 1437749

3. 1444287

3.1450820

3. 1459516

3. 1481174

$3 \cdot 1502670$

3. 1544956

3. 1585951

3. 1662449 $\begin{array}{ll}1.2433895 & 3 \\ 1.2381675 & 3 \\ 1.2278386 & 3 \\ 1.2176623 & 3 \\ 1.2076369 & 3 \\ 1.1977599 & 3 \\ 1.1880278 & 3 \\ 1.1784374 & 3 \\ 1.1689853 & 3 \\ 1.1596669 & 3 \\ 1.1504788 & 3 \\ 1.1324770 & 3 \\ 1.1063547 & 3 \\ 1.0649462 & 3 \\ 1.0259098 & 3 \\ 9.8899038 & 2 \\ 9.2076906 & 2 \\ 8.5914709 & 2 \\ 7.7737068 & 2 \\ 7.0657786 & 2 \\ 6.2644905 & 2 \\ 4.7915900 & 2 \\ 3.8174547 & 2 \\ 2.6471062 & 2 \\ 1.9802951 & 2 \\ 1.2445355 & 2 \\ & \end{array}$

1. 4000780

1. 3948535

1. 3845147

1. 3743220

1.3642736

1. 3543670

1. 3445989

1. 3349661

1. 3254647

1. 3160909

1. 3068406

1. 2886954

1. 2623103

1. 2203378

1.1805854

1. 1428039

1.0724503

1. 0082107

9.2176798

8.4566114

7.5787071

5.9120543

4. 7678510

3. 3479623

2. 5187906

1. 5902204

(

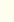

3

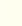

3

3

3

.

2

.

Table 25. Ground reflection coefficients (amplitude, $|R|$, and phase, Arg R) assuming the Fresnel approximation, for the special ray configuration of the Norton type 301 atmosphere, applicable to the propagation of waves between the D-region ( $h_{3}=70$ kilometers) or the E-region ( $h_{3}=90$ kilometers) of the ionosphere and the earth. 
$f=214.13276$ kilocycles

$\sigma=0.005 \mathrm{mhos} / \mathrm{meter}$

$\epsilon_{2}=15$

$\Psi$

radians

$\left|R_{e}\right|$

$9.5523975-1$

0.0005

0.0020

0.0030

0.0040

0.0050

0.0060

0.0070

0.0080

c.0090

0.0100

0.0120

0.0150

0.0200

0.0250

0.0300

0.0400

0.0500

c. 0650

0.0800

0.1000

0. 1500

0.2000

0.3000

0.4000

0.6000

0.0005

0.0010

0.0020

0.0030

0.0040

$\therefore .0050$

0.0060

0.0070

0.0080

0.0090

3.0100

0.0120

0.0150

0.0200

0.0250

0.0300

0.0400

0.0500

0.0650

0.0800

0.1000

0.1500

0.2000

0.3000

0.4000

0.6000
$\operatorname{Arg~R}$

$\left|\mathrm{R}_{\mathrm{m}}\right|$

$\mathrm{h}_{3}=70$ kilometers

3.0957951

3.0499015

2.9574512

2. 8635227

2.7674817

2.6688310

2.5572692

2.4627536

2.3555546

2.2462866

2.1358952

1.9167445

1.6117847

1.2164055

$9.5458550-1$

$7.8044180-1$

$5.7040100-1$

$4.4984530-1$

$3.4219490-1$

2.7648910 - 1

$2.2038250-1$

$1.4659650-1$

1.1010130 -

$7.3937100-2$

$5.6087900-2$

$3.8672400-2$

$\mathrm{h}_{3}=90$ kilometers

3.0957951

3.0499015

2.9574512

2.8635227

2.7674817

2. 6688310

2. 5672692

2.4627536

2.3555546

2.2462866

2.1358952

. 9167445

1.6117847

1.2164055

. 5458550 -

7. 8044180 - 1

$5.7040100=1$

$4.4984530-1$

$3.4219490-1$

2.7648910 - 1

2.2038250 -

$1.4659650-1$

1.1010130 -

$7.3937100-2$

$5.6087900-2$
$3.8672400-2$
$9.9998904-1$

$9.9997815-1$

9.9995630 - 1

$9.9993453-1$

9.9991269 -

9.9989084 - 1

$9.9986900-1$

9.9984715 -

$9.9982531-1$

$9.9980354-1$

$9.9978162-1$

9.9973801 -

9.9967248 - 1

9.9956336 - 1

9.9945425 -

9.9934522 -

9.9912714 - 1

9.9890910 -

9.9858256 -

$9.9825643-1$

$9.9782224-1$

9.9674188 - 1

$9.9557098-1$

$9.9356732-1$

9.9153203 -

9.8774517 -
Arg $R_{m}$

. 1416036

3.1416145

3.1416364

3.1416583

3.1416801

3. 1417019

3. 1417237

3. 1417456

3. 1417674

3. 1417892

3.1418111

3.1418548

3.1419202

3. 1420294

3.1421385

3. 1422476

3. 1424659

3.1426840

3.1430109

3. 1433377

3.1437726

3.1448557

3. 1459306

3.1480453

3.1500956

3.1539215 $\mathrm{d} / \mathrm{j}$

miles

Table 26. Ground reflection coefficients (amplitude, |R|, and phase, Arg R) assuming the Fresnel approximation, for the special ray configuration of the Norton type 301 atmosphere, applicable to the propagation of waves between the D-region $\left(h_{3}=70\right.$ kilometers) or the E-region $\left(h_{3}=90\right.$ kilometers $)$ of the ionosphere and the earth.

$9.9998904-1 \quad 3.1416036$ $9.9997815-1-3 \cdot 1416145$ $9.9995630-1 \quad 3.1416364$ $3.9993453-1 \quad 3 \cdot 1416583$ $9.9991269-1-3.1416801$

9.9989084 - $1 \quad 3.1417019$

$9.9986900-1 \quad 3.1417237$

$9.9984715-1$

$9.9982531-1 \quad 3.1417674$

$9.9980354-1 \quad 3.1417892$

$9.9978162-1-3.1418111$

9.9973801 - 13.1418548

$9.9967248-1 \quad 3.1419202$

$9.9956336-1-3.1420294$

$9.9945425-1 \quad 3.1421385$

$9.9934522-1 \quad 3.1422476$

$9.9912714-1 \quad 3.1424659$

$9.9890910-1 \quad 3.1426840$

$9.9858256-1 \quad 3.1430109$

$9.9825643-1 \quad 3.1433377$

$9.9782224-1 \quad 3.1437726$

$9.9674188-1 \quad 3.1448557$

$9.9567098-1-3.1459306$

9.9356732 - 13.1480453

$9.9153203-1 \quad 3.1500956$

3.1500956
$1.2433895 \quad 3$ 1. 2381675

1.2278386

1.2076369

$1 \cdot 1977599$

1.1880278

1.1784374

1.1689853

1.1596669

$1 \cdot 1504788$

1.1324770

1.1063547

1.0649462

1.0259098

9. 8899038

9.2076906

8.5914709

7.7737068

7.0657736

6.2644905

4. 7915900

3. 8174547

2.6471062

1. 9802951

1. 2445355

1.4000780

1. 3948535

1. 3845147

1. 3743220

1.3642736

I. 3543670

1. 3445989

1. 3349661

1.3254647

I. 3160909

I. 3068406

1. 2886954

1. 2623103

1.2203378

1.1805854

1. 1428039

1. 0724503

1.0082107

9. 2176798

8.4566114

7.5787071

5. 9120543

4.76785i0

3.3479623

2. 5187906

1. 5902204

3
3
3
2
3
3
3
3
3
3
3
3
3
3
3
2
2
2
2
2
2
2
2
2
2
2

3
3
3
3
3
3
3
3
3
3
3
3
3
3
3
3
3
3
2
2
2
2


$f=0.66622256$ kilocycles

$\sigma=0.005$ mhos $/$ meter

$\epsilon_{2}=15$

Arg R

$h_{3}=70$ kilometers
0.0005

0.0010

0.0020

0.0030

0.0040

0.0050

0.0060

0.0070

0.0080

0.0090

0.0100

0.0120

0.0150

0.0200

0.0250

0.0300

0.0400

0.0500

0.0650

0.0800

0.1000

0.1500

0.2000

0.3000

0.4000

0.6000

$\begin{array}{ll}0.0005 & 7.7363343-1 \\ 0.0010 & 6.1003451-1 \\ 0.0020 & 4.4073711-1 \\ 0.0030 & 4.1693168-1 \\ 0.0040 & 4.5424172=1 \\ 0.0050 & 5.0462510-1 \\ 0.0060 & 5.5249141-1 \\ 0.0070 & 5.9436666-1 \\ 0.0080 & 6.3021914-1 \\ 0.0090 & 6.6084278-1 \\ 0.0100 & 6.8711664=1 \\ 0.0120 & 7.2956543-1 \\ 0.0150 & 7.7575992-1 \\ 0.0200 & 8.2580763-1 \\ 0.0250 & 8.5772694=1 \\ 0.0300 & 8.7980568-1 \\ 0.0400 & 9.0831092-1 \\ 0.0500 & 9.2589969-1 \\ 0.0650 & 9.4245824=1 \\ 0.0800 & 9.5296356-1 \\ 0.1000 & 9.6216294=1 \\ 0.1500 & 9.7455769-1 \\ 0.2000 & 9.8080055=1 \\ 0.3000 & 9.8705140-1 \\ 0.4000 & 9.9015807=1 \\ 0.6000 & 9.9320180-1 \\ 0 & \end{array}$

$\begin{array}{ll}2.8791097 & 9.9999807-1 \\ 2.0010528 & 9.9999616-1 \\ 1.9883651 & 9.9999229-1 \\ 1.4346986 & 9.9998843-1 \\ 1.0625484-1 & 9.9998456-1 \\ 8.3085530-1 & 9.9998069-1 \\ 6.7992420-1 & 9.9997688-1 \\ 5.7524250-1 & 9.9997306-1 \\ 4.9866820-1 & 9.9996920-1 \\ 4.4026620-1 & 9.9996538-1 \\ 3.9424700-1 & 9.9996152-1 \\ 3.2631100-1 & 9.9995378-1 \\ 2.5954900-1 & 9.9994224-1 \\ 1.9377080-1 & 9.9992298-1 \\ 1.5468600-1 & 9.9990373-1 \\ 1.2875750-1 & 9.9988445-1 \\ 9.5464700-2 & 9.9984597-1 \\ 7.7140700-2 & 9.9980752-1 \\ 5.9332200-2 & 9.9974988-1 \\ 4.8215300-2 & 9.9969226-1 \\ 3.8590100-2 & 9.9961554-1 \\ 2.5776800-2 & 9.9942455-1 \\ 1.9388200-2 & 9.9923509-1 \\ 1.3033700-2 & 9.9886243-1 \\ 6.8215000-3 & 9.9782754-1 \\ 9.8908000-3 & 9.9850120-1 \\ .650301 & -1\end{array}$

$\mathrm{h}_{3}=90$ kilometers

$\begin{array}{lll}2.8791097 & 9.9999807-1 & 3.1415946 \\ 2.6010528 & 9.9999616-1 & 3.1415966 \\ 1.9883651 & 9.9999229-1 & 3.1416004 \\ 1.4346986 & 9.9998843-1 & 3.1416042 \\ 1.0625484-1 & 9.9998456-1 & 3.1416081 \\ 8.3085530-1 & 9.9998069-1 & 3.1416120 \\ 6.7992420-1 & 9.9997688-1 & 3.1416158 \\ 5.7524250-1 & 9.9997306-1 & 3.1416196 \\ 4.9866820-1 & 9.9996920-1 & 3.1416236 \\ 4.4026620-1 & 9.9996538-1 & 3.1416274 \\ 3.9424700-1 & 9.9996152-1 & 3.1416312 \\ 3.2631100-1 & 9.9995378-1 & 3.1416390 \\ 2.5954900-1 & 9.9994224-1 & 3.1416504 \\ 1.9377080-1 & 9.9992298-1 & 3.1416698 \\ 1.5468600-1 & 9.9990373-1 & 3.1416890 \\ 1.2875750-1 & 9.9988445-1 & 3.1417082 \\ 9.6464700-2 & 9.9984597-1 & 3.1417468 \\ 7.7140700-2 & 9.9980752-1 & 3.1417852 \\ 5.9332200-2 & 9.9974988-1 & 3.1418429 \\ 4.8215300-2 & 9.9969226-1 & 3.1419005 \\ 3.8590100-2 & 9.9961554-1 & 3.1419772 \\ 2.5776800-2 & 9.9942455-1 & 3.1421683 \\ 1.9388200-2 & 9.9923509-1 & 3.1423579 \\ 1.3033700-2 & 9.9886243-1 & 3.1427309 \\ 9.8908000-3 & 9.9850120-1 & 3.1430927 \\ 6.8215000-3 & 9.9782754-1 & 3.1437675\end{array}$

3.1416390 $\mathrm{d} / \mathrm{j}$

miles

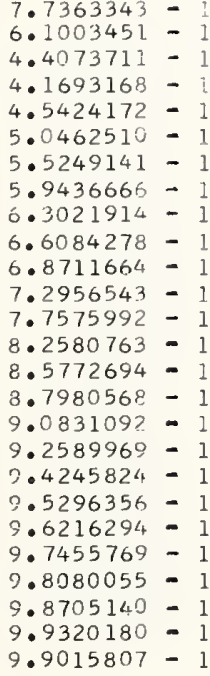

9.9320180 - 1
Arg $R_{m}$

3.1415946

3.1415966

3. 1416004

3.1416042

3. 1416081

3. 1416120

3.1416158

3. 1416196

$3 \cdot 1416274$

3. 1416312

$3 \cdot 1416504$

3. 1416698

$3 \cdot 1416890$

3. 1417082

3. 1417468

3. 1417852

3. 1418429

3. 1419005

3. 1419772

3. 1421683

3. 1423579

3. 1427309

3. 1437675

3. 1430927
3.1416236

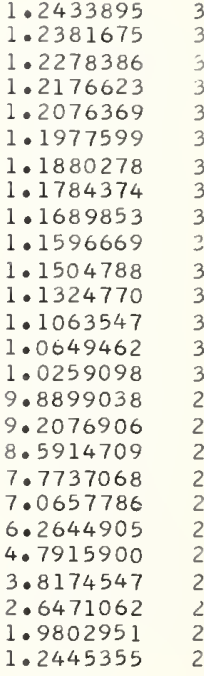

1.4000780 1.3948535 1. 3845147 1. 3743220 $1.3642736 \quad 3$ 1. 3543670 1. 3445989 1. 3349661 1. 3254647 1. 3160909 1. 3068406 1. 2886954 1. 2623103 1.2203378 1. $1805854 \quad 3$ 1. 14280393 $1.0724503 \quad 3$ 1. 0082107 9.2176798 2 8.45661142 7.57870712 5.91205432 4.7678510 2 3. $3479623 \quad 2$ 2.5187906 2 1.5902204

Table 27. Ground reflection coefficients (amplitude, $|R|$, and phase, Arg R) assuming the Fresnel approximation, for the special ray configuration of the Norton type 301 atmosphere, applicable to the propagation of waves between the D-region ( $h_{3}=70$ kilometers) or the E-region ( $h_{3}=90$ kilometers) of the ionosphere and the earth. 
$f=1.332425$ kilocycles

$\sigma=0.005$ mhos $/$ meter

$\epsilon_{2}=15$

$\Psi$

radians

0.0005

0.0010

0.0020

0.0030

0.0040

0.0050

0.0060

0.0070

0.0080

0.0090

0.0100

0.0120

0.0150

0.0200

0.0250

0.0300

0.0400

0.0500

0.0650

0.0800

0.1000

0.1500

0.2000

0.3000

0.4000

0.6000

0.0005

0.0010

0.0020

0.0030

0.0040

0.0050

0.0060

0.0075

0.0080

0.0090

0.0100

0.0120

0.0150

0.0200

0.0250

0.0300

0.0400

0.0500

0.0650

0.0800

0.1000

0.1500

0.2000

0.3000

0.4000

0.6000
$\left|R_{e}\right|$

$8.3314524=1$
$6.9864594-1$
$5.1682192-1$
$4.3187938-1$
$4.1462068-1$
$4.3341850-1$
$4.6620171-1$
$5.0209024-1$
$5.3667760-1$
$5.6845562-1$
$5.9710029-1$
$6.4567131-1$
$7.0126072-1$
$7.6419253-1$
$8.0560606-1$
$8.3477647-1$
$8.7302445-1$
$8.9693489-1$
$9.1964925-1$
$9.3415843-1$
$9.4692502-1$
$9.6421312-1$
$9.7295761-1$
$9.8173738-1$
$9.8610988-1$
$9.9039948-1$

Arg $R_{e}$

$\left|R_{m}\right|$

$h_{3}=70$ kilometers
9.9999727 9.9999457 $9.9998910-1$ $9.9998367-1$ 9.9997320 $9.9997277-1$ $9.9996730-1$ 9.9996187 $9.9995644-1$ $9.9995097-1$ 9.9994550 - 1 $9.9993464-1$ $9.9991831-1$ 9.9989104 $9.9986385-1$ $9.9983659-1$ $9.9978221-1$ $9.9972776-1$ 9.9964629 9.9956482 - 1 $9.9945637-1$ $9.9918632-1$ $9.9891843-1$ $9.9839157-1$ $9.9788107-1$ $9.9692911-1$
Arg $R_{m}$

$3 \cdot 1415954$ 3. 1415981 3. 1416036 3.1416091 3. 1416145 3. 1416199 3. 1416254 3.1416308 3.1416363 3. 1416417 3. 1416472 3. 1416581 3. 1416744 3. 1417016 3. 1417289 3. 1417561 3. 1418106 3. 1418649 3. 1419465 3.1420280 3. 1421364 3. 1424068 3. 1426748 3. 1432024 3. 1437139 3. 1446683 $\mathrm{d} / \mathrm{j}$

miles

$\mathrm{h}_{3}=90$ kilometers

2.9569901
2.7665271
2.3534935
1.9137780
1.5173903
1.2135851
$9.9611460-1$
$8.4016150-1$
$7.2521280-1$
$6.3767180-1$
$5.6902430-1$
$4.6848550-1$
$3.7079660-1$
$2.7565710-1$
$2.1960050-1$
$1.8258050-1$
$1.3662910-1$
$1.0919960-1$
$8.3956600-2$
$6.8212400-2$
$5.4587600-2$
$3.6457700-2$
$2.7420700-2$
$1.8432800-2$
$1.3987900-2$
$9.6469000-3$

\begin{tabular}{|c|c|c|}
\hline $\begin{array}{l}9.9999727 \\
9.9999457\end{array}$ & $\begin{array}{l}-1 \\
-1\end{array}$ & $\begin{array}{l}3.1415954 \\
3.1415981\end{array}$ \\
\hline 9.9998910 & -1 & 3.1416036 \\
\hline 9.9998367 & - & 3.1416091 \\
\hline 9.9997820 & - & 3.141614 \\
\hline 9.9997277 & - & 3.14161 \\
\hline 9.9996730 & $\begin{array}{lll}-1 & 1\end{array}$ & 3.14162 \\
\hline 9.9996187 & -1 & 3.14163 \\
\hline 9.9995644 & - & 3.141 \\
\hline .9995097 & - & $3 \cdot 1416$ \\
\hline 9.9994550 & -1 & 3.14164 \\
\hline 9.9993464 & -1 & 3.14165 \\
\hline 9.9991831 & - & $3 \cdot 14$ \\
\hline .9989104 & - & 3.14170 \\
\hline 9.9986385 & -1 & $3 \cdot 14172$ \\
\hline 9.9983659 & - & 3.14175 \\
\hline 9.9978221 & - & 3.14181 \\
\hline 9.9972776 & - & 3.14180 \\
\hline .9964629 & - & $3 \cdot 14194$ \\
\hline .9956482 & -1 & 3.14202 \\
\hline . 9945637 & - & 3.14213 \\
\hline .9918632 & - & 3.14240 \\
\hline .9891843 & -1 & 3.14267 \\
\hline .9839157 & -1 & $3 \cdot 14320$ \\
\hline 78810 & - & 3.14371 \\
\hline & -1 & \\
\hline
\end{tabular}

1. 4000780 1. 3948535 1.3845147 1. 3743220

1. 3642736 1. 3543670 1. 3445989

1. 3349661 1. 3254647 1. 3160909 1.3068406 1.2886954 1.2623103 1. 2203378

$1 \cdot 1805854$ 1.1428039 1.0724503 1.0082107 9. 2176798 8.4566114 7.5787071 5.9120543 4. 7678510 3.3479623 2. 5187906 1. 5902204

Table 28. Ground reflection coefficients (amplitude, $|R|$, and phase, Arg $R$ ) assuming the Fresnel approximation, for the special ray configuration of the Norton type 301 atmosphere, applicable to the propagation of waves between the D-region ( $h_{3}=70$ kilometers) or the E-region $\left(h_{3}=90\right.$ kilometers) of the ionosphere and the earth. 


$\begin{array}{ll}\Psi & {\left[\mathrm{R}_{\mathrm{e}}\right]} \\ \text { radians } & \\ 0.0005 & 8.9060610=1 \\ 0.0010 & 7.9444619=1 \\ 0.0020 & 6.3968844=1 \\ 0.0030 & 5.3058912=1 \\ 0.0040 & 4.6139894=1 \\ 0.0050 & 4.2533655=1 \\ 0.0060 & 4.1425140=1 \\ 0.0070 & 4.1980549=1 \\ 0.0080 & 4.3511845=1 \\ 0.0090 & 4.5542652=1 \\ 0.0100 & 4.7779409=1 \\ 0.0120 & 5.2275533=1 \\ 0.0150 & 5.8277324=1 \\ 0.0200 & 6.5923339=1 \\ 0.0250 & 7.1354748=1 \\ 0.0300 & 7.5344289=1 \\ 0.0400 & 8.0759576=1 \\ 0.0500 & 8.4242514=1 \\ 0.0650 & 8.7615724=1 \\ 0.0800 & 8.9801596=1 \\ 0.1000 & 9.1744195=1 \\ 0.1500 & 9.4402407=1 \\ 0.2000 & 9.5758621=1 \\ 0.3000 & 9.7127962=1 \\ 0.4000 & 9.7812716=1 \\ 0.6000 & 9.8486260-1 \\ 0 & \end{array}$

\begin{abstract}
0.0005
0.0010

0.0020

0.0030

0.0050

0.0060

0.0070

0.0080

0.0090

0.0120

0.0150

0.0200

0.0300

0.0400

0.0500

0.1000

0.3000

0.6000
\end{abstract}

\subsection{5 \\ 0.0020 \\ 0.0030 \\ 0.0040 \\ 0.0050 \\ 0.0060 \\ 0.0070 \\ 0.0080 \\ 0.0090 \\ 0.0100 \\ 0.0120 \\ 0.0150 \\ 0.0200 \\ 0.0250 \\ 0.0300 \\ 0.0400 \\ 0.0500 \\ 0.0650 \\ 0.0800 \\ 0.1000 \\ 0.1500 \\ 0.2000 \\ 0.3000 \\ 0.4000 \\ 0.6000}

$$
\operatorname{ArgR} \operatorname{er}_{\mathrm{h}_{3}=70 \text { kilometers }}\left|\mathrm{R}_{\mathrm{m}}\right|
$$

3. 0252287

2.9073313

2.6616329

2.3979255

2.1201113

1. 8444784

1. 5918577

1.3757265

1. 1984241

1.0553606

9.3990840 - 1

7.6845930 - I

$6.0228590-1$

4.4316440 -

$3.5108700-1$

$2.9096040-1$

$2.1700300-1$

$1.7316020-1$

1.3297450 - 1

$1.0797480-1$

$8.6372100-2$

$5.7662200-2$

4.3362800 - 2

$2.9146600-2$

$2.2117300-2$

I. $5253100-2$
Arg $R_{m}$

3.1415970

3.1416013

3.1416099

3.1416185

3.1416271

3. 1416357

3.1416444

3.1416530

3. 1416616

3. 1416702

3. 1416788

3. 1416960

3.1417219

3. 1417650

3. 1418081

3.1418511

3.1419371

3. 1420231

3. 1421521

3. 1422810

3. 1424525

3. 1428798

3.1433037

3. 1441378

3.1449465

3.1464556 $\mathrm{d} / \mathrm{j}$

1. 2433895

1. 2381675

1. 2278386

1.2176623

1.2076369

1.1977599

1.1880278

1.1784374

1. 1689853

1.1596669

1.1504788

I. 1324770

1. 1063547

1.0649462

1.0259098

9.8899038

9. 2076906

8.5914709

7.7737068

7.0657786

6.2644905

4.7915900

3.8174547

2.6471062

1. 9802951

I. 2445355

\begin{tabular}{|c|c|c|}
\hline \multicolumn{2}{|l|}{$\begin{array}{l}3.0252287 \\
2.9073313\end{array}$} & $\begin{array}{l}9.9999568 \\
9.9999141\end{array}$ \\
\hline \multicolumn{2}{|l|}{2.6616329} & 9.9998277 \\
\hline \multirow{2}{*}{\multicolumn{2}{|c|}{2.1201113}} & 9.9997412 \\
\hline & & 9.9996553 \\
\hline \multicolumn{2}{|l|}{1.8444784} & 9.9995694 \\
\hline \multicolumn{2}{|l|}{1.5918577} & 9.9994830 \\
\hline \multirow{2}{*}{\multicolumn{2}{|c|}{$\begin{array}{l}1.3757265 \\
1.1984241\end{array}$}} & 993977 \\
\hline & & 9.9993112 \\
\hline \multicolumn{2}{|c|}{1.0553606} & 9.9992248 \\
\hline 9.3990840 & -1 & 9.9991383 \\
\hline 7.6845930 & -1 & 9.9989666 \\
\hline 6.0228590 & & \\
\hline 4.4316440 & -1 & 9.9982773 \\
\hline .5108700 & -1 & 9.9978474 \\
\hline 2.9096040 & & 9.9974163 \\
\hline 2.1700300 & & 9.9965561 \\
\hline 1.7316020 & & 9.9956965 \\
\hline 1.3297450 & -1 & 9.9944073 \\
\hline 1.0797480 & -1 & 9.9931201 \\
\hline 3.6372100 & & 9.9914058 \\
\hline .7662200 & & 9.9871377 \\
\hline & & 9.9829036 \\
\hline & & 9.9745799 \\
\hline & & \\
\hline
\end{tabular}

$\mathrm{h}_{3}=90$ kilometers
3. 1415970

3. 1416013

3.1416099

3. 1416185

3.1416271

3. 1416357

3. 1416444

3.1416530

3. 1416616

3. 1416702

3.1416788

3.1416960

3.1417219

3.1417650

3.1418081

3. 1418511

3.1419371

3.1420231

3.1421521

3.1422810

3. 1424525

3.1428798

3.1433037

3. 1441378

3.1449465

3.1464556 $\begin{array}{ll}1.4000780 & 3 \\ 1.3948535 & 3 \\ 1.3845147 & 3 \\ 1.3743220 & 3 \\ 1.3642736 & 3 \\ 1.3543670 & 3 \\ 1.3445989 & 3 \\ 1.3349661 & 3 \\ 1.3254647 & 3 \\ 1.3160909 & 3 \\ 1.3068406 & 3 \\ 1.288695 & 3 \\ 1.2623103 & 3 \\ 1.2203378 & 3 \\ 1.1805854 & 3 \\ 1.1428039 & 3 \\ 1.0724503 & 3 \\ 1.0082107 & 3 \\ 9.2176798 & 2 \\ 8.4566114 & 2 \\ 7.5787071 & 2 \\ 5.9120543 & 2 \\ 4.7678510 & 2 \\ 3.3479623 & 2 \\ 2.5187906 & 2 \\ 1.5902204 & 2\end{array}$

Table 29. Ground reflection coefficients (amplitude, [R], and phase, Arg R) assuming the Fresnel approximation, for the special ray configuration of the Norton type 301 , atmosphere, applicable to the propagation of waves between the D-region ( $h_{3}=70$ kilometers) or the $E$-region $\left(h_{3}=90\right.$ kilometers $)$ of the ionosphere and the earth. 
$f=6.6622252$ kilocycles

$\sigma=0.005 \mathrm{mhos} / \mathrm{meter}$

$\epsilon_{2}=15$

$\Psi$

radians

$\left|R_{e}\right|$

$9.2125626=1$
$8.4918744=1$
$7.2444256=1$
$6.2411310=1$
$5.4628489=1$
$4.8894724=1$
$4.4987159=1$
$4.2645499=1$
$4.1574942=1$
$4.1472553=1$
$4.2061248-1$
$4.4454034=1$
$4.9159099-1$
$5.6672319=1$
$6.2702243=1$
$6.7406785=1$
$7.4098529=1$
$7.8562939=1$
$8.2992190=1$
$8.5913212=1$
$8.8540476-1$
$9.2180432=1$
$9.4056453-1$
$9.5963004=1$
$9.6920900=1$
$9.7865978-1$

0.0005

0.0010

0.0020

0.0030

0.0040

0.0050

0.0060

0.0070

0.0080

0.0090

0.0100

0.0120

0.0150

0.0200

0.0250

0.0300

0.0400

0.0500

0.0650

0.0800

0.1000

0.1500

0.2000

0.3000

0.4000

0.6000
Arg $R$

$\mathrm{h}_{3}=70$ kilometers

0.0005

0.0010

0.0020

0.0030

0.0040

0.0050

0.0060

0.0070

0.0080

0.0090

0.0100

0.0120

0.0150

0.0200

0.0250

0.0300

0.0400

0.0500

0.0650

0.0800

0.1000

0.1500

0.2000

0.3000

0.4000

0.6000

3.0594064
2.9766722
2.8075088
2.6306615
2.4444731
2.2501366
2.0522382
1.8579586
1.6748777
1.5086387
1.3618747
1.1251216
$8.8067270-1$
$6.4199250-1$
$5.0501300-1$
$4.1660280=1$
$3.0912810-1$
$2.4604790-1$
$1.8858660-1$
$1.5298440=1$
$1.2229330-1$
$8.1587900-2$
$6.1340700-2$
$4.1223500-2$
$3.1279800-2$
$2.1571000-2$

9.9999389 9.9998777 $9.9997563-1$ $9.9996348-1$ $9.9995134-1$ $9.9993911-1$ $9.9992688-1$ 9.9991474 $9.9990260-1$ 9.9989037 $9.9987814-1$ $9.9985386-1$ 9.9981734 - 1 9.9975638 $9.9969559-1$ 9.9963464 - 1 $9.9951308-1$ $9.9939145-1$ 9.9920916 - 1 $9.9902717-1$ 9.9878482 $9.9818148-1$ $9.9758306-1$ 9.9640694 9.9526796 $9.9314614-1$

$\mathrm{h}_{3}=90$ kilometers

3.0594064
2.9766722
2.8075088
2.6306616
2.4444731
2.2501366
2.0522382
1.8579586
1.6748777
1.5086387
1.3618747
1.1251216
$8.8067270-1$
$6.4199250-1$
$5.0501300-1$
$4.1660280-1$
$3.0912810-1$
$2.4604790-1$
$1.8858660-1$
$1.5298440-1$
$1.2229330-1$
$8.1587900-2$
$6.1340700-2$
$4.1223500-2$
$3.1279800-2$
$2.1571000-2$

Arg $R_{m}$

3.1416049

3. 1416171

3. 1416292

3. 1416414

3.1416536

3. 1416658

3.1416780

3.1416901

3. 1417023

3. 1417145

3.1417389

3.1417755

3.1418363

3.1418971

3. 1419580

3. 1420798

3. 1422014

3. 1423838

3. 1425660

3.1428087

3.1434128

3.1440124

3.1451920

3. 1463358
3. 1484698

$\mathrm{d} / \mathrm{j}$

miles

$\begin{array}{ll}1.2433895 & 3 \\ 1.2381675 & 3 \\ 1.2278386 & 3 \\ 1.2176623 & 3 \\ 1.2076369 & 3 \\ 1.1977599 & 3 \\ 1.1880278 & 3 \\ 1.1784374 & 3 \\ 1.1689853 & 3 \\ 1.1596669 & 3 \\ 1.1504788 & 3 \\ 1.1324770 & 3 \\ 1.1063547 & 3 \\ 1.0649462 & 3 \\ 1.0259098 & 3 \\ 9.8899038 & 2 \\ 9.2076906 & 2 \\ 8.5914709 & 2 \\ 7.7737068 & 2 \\ 7.0657786 & 2 \\ 6.2644905 & 2 \\ 4.7915900 & 2 \\ 3.8174547 & 2 \\ 2.6471062 & 2 \\ 1.9802951 & 2 \\ 1.2445355 & 2\end{array}$

3. 1415988

3. 1416049

3. 1416171

3. 1416292

3. 1416414

3. 1416536

3. 1416658

3. 1416780

3. 1416901

3.1417023

3. 1417145

3. 1417389

3.1417755

3.1418363

3.1418971

3. 1419580

3. 1420798

3.1422014

$3 \cdot 1423838$

3.1425650

3.1428087

3. 1434128

3. 1440124

3. 1451920

3.1463358

3.1484698
1.4000780 1.3948535 1. 3845147 1.3743220

1.3642736

1. 3543670

1.3445989

1.3349661

1. 3254647

1.3160909

1.3068406

1. 2886954

1.2623103

1. 2203378

1.1805854

1.1428039

1.0724503

1.0082107

9.2176798

8.4566114

7.5787071

5.9120543

4. 7678510

3.3479623

2.5187906

1. 5902204

Table 30. Ground reflection coefficients (amplitude, $|R|$, and phase, Arg R) assuming the Fresnel approximation, for the special $\mathrm{r}$ ay configuration of the Norton type 301 atmosphere, applicable to the propagation of waves between the D-region ( $h_{3}=70$ kilometers) or the E-region $\left(h_{3}=90\right.$ kilometers) of the ionosphere and the earth. 
$f=13.324450$ kilocycles

$\sigma=0.005 \mathrm{mhos} / \mathrm{meter}$

$\epsilon_{2}=15$

$\Psi$

radians

$\begin{array}{ll}0.0005 & 9.4361886=1 \\ 0.0010 & 8.9059159=1 \\ 0.0020 & 7.9441994=1 \\ 0.0030 & 7.1098222=1 \\ 0.0040 & 6.3964372=1 \\ 0.0050 & 5.7972522=1 \\ 0.0060 & 5.3053000=1 \\ 0.0070 & 4.9132943=1 \\ 0.0080 & 4.6132869=1 \\ 0.0090 & 4.3963627=1 \\ 0.0100 & 4.2525888=1 \\ 0.0120 & 4.1417015=1 \\ 0.0150 & 4.2650691=1 \\ 0.0200 & 4.7771377=1 \\ 0.0250 & 5.3342765=1 \\ 0.0300 & 5.8268994=1 \\ 0.0400 & 6.5913962=1 \\ 0.0500 & 7.1343946=1 \\ 0.0650 & 7.6945953=1 \\ 0.0800 & 8.0743520=1 \\ 0.1000 & 8.4222582=1 \\ 0.1500 & 8.9133855=1 \\ 0.2000 & 9.1703522=1 \\ 0.3000 & 9.4340009=1 \\ 0.4000 & 9.5673756=1 \\ 0.6000 & 9.6995418=1\end{array}$

Arg $R$

$\mathrm{h}_{3}=70$ kilometers

$\begin{array}{ll}3.0835161 & 9.9999135-1 \\ 3.0252447 & 9.9998277-1 \\ 2.9073629 & 9.9996553-1 \\ 2.7865479 & 9.9994836-1 \\ 2.6616909 & 9.9993106-1 \\ 2.5321596 & 9.9991389-1 \\ 2.3979984 & 9.9989665-1 \\ 2.2600886 & 9.9987943-1 \\ 2.1201820 & 9.9986219-1 \\ 1.9807343 & 9.9984495-1 \\ 1.8445312 & 9.9982779-1 \\ 1.5918860- & 9.9979333-1 \\ 1.2824132- & 9.9974163-1 \\ 9.3989400-1 & 9.9965555-1 \\ 7.3470980-1 & 9.9956947-1 \\ 6.0229420-1 & 9.9948346-1 \\ 4.4320380-1 & 9.9931140-1 \\ 3.5115580-1 & 9.9913943-1 \\ 2.6818210-1 & 9.9888171-1 \\ 2.1715010-1 & 9.9862437-1 \\ 1.7335530-1 & 9.9828179-1 \\ 1.1549900-1 & 9.9742913-1 \\ 8.6795100-2 & 9.9658364-1 \\ 5.8310100-2 & 9.9492238-1 \\ 4.4239600-2 & 9.9331439-1 \\ 3.0506000-2 & 9.9032068-1 \\ & \end{array}$

$\left|R_{m}\right|$

9.9032068 -
$\operatorname{Arg~} \mathrm{R}_{\mathrm{m}}$

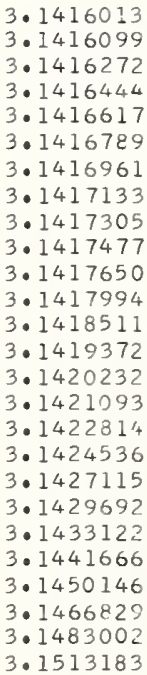

$\mathrm{d} / \mathrm{j}$

miles

$\mathrm{h}_{3}=90$ kilometers

$\begin{array}{ll}0.0005 & 9.4361886-1 \\ 0.0010 & 8.9059159-1 \\ 0.0020 & 7.9441994=1 \\ 0.0030 & 7.1098222=1 \\ 0.0040 & 6.3964372=1 \\ 0.0050 & 5.7972522-1 \\ 0.0060 & 5.3053000=1 \\ 0.0070 & 4.9132943-1 \\ 0.0080 & 4.6132869-1 \\ 0.0090 & 4.3963627=1 \\ 0.0100 & 4.2525888=1 \\ 0.0120 & 4.1417015=1 \\ 0.0150 & 4.2650691-1 \\ 0.0200 & 4.7771377=1 \\ 0.0250 & 5.3342765=1 \\ 0.0300 & 5.8268994=1 \\ 0.0400 & 6.5913962-1 \\ 0.0500 & 7.1343946=1 \\ 0.0650 & 7.6945953=1 \\ 0.0800 & 8.0743520=1 \\ 0.1000 & 8.4222582=1 \\ 0.1500 & 8.9133855=1 \\ 0.2000 & 9.1703522=1 \\ 0.3000 & 9.4340009-1 \\ 0.4000 & 9.5673756=1 \\ 0.6000 & 9.6995418=1\end{array}$

$\begin{array}{ll}3.0835161 & 9.9999135-1 \\ 3.0252447 & 9.9998277-1 \\ 2.9073620 & 9.9996553-1 \\ 2.7865479 & 9.9994336-1 \\ 2.6616909 & 9.9993106-1 \\ 2.5321596 & 9.9991389-1 \\ 2.3979984 & 9.9989666-1 \\ 2.2600886 & 9.9987943-1 \\ 2.1201820 & 9.9986219-1 \\ 1.9807343 & 9.9984496-1 \\ 1.8445312 & 9.9982779-1 \\ 1.5918860 & 9.9979333-1 \\ 1.2824132- & 9.9974163-1 \\ 9.3989400-1 & 9.9965555-1 \\ 7.3470980-1 & 9.9956947-1 \\ 6.0229420-1 & 9.9948346-1 \\ 4.4320380-1 & 9.9931140-1 \\ 3.5115580-1 & 9.9913943-1 \\ 2.6818210-1 & 9.9888171-1 \\ 2.1715010-1 & 9.9862437-1 \\ 1.7335530-1 & 9.9828179-1 \\ 1.1549900-1 & 9.9742913-1 \\ 8.6795100-2 & 9.9658364-1 \\ 5.8310100-2 & 9.9492238-1 \\ 4.4239600-2 & 9.9331439-1 \\ 3.0506000-2 & 9.9032068-1\end{array}$

$\begin{array}{ll}1.2433895 & 3 \\ 1.2381675 & 3 \\ 1.2278386 & 3 \\ 1.2176623 & 3 \\ 1.2076369 & 3 \\ 1.1977599 & 3 \\ 1.1880273 & 3 \\ 1.1784374 & 3 \\ 1.1689853 & 3 \\ 1.1596669 & 3 \\ 1.1504788 & 3 \\ 1.1324770 & 3 \\ 1.1063547 & 3 \\ 1.0649462 & 3 \\ 1.0259093 & 3 \\ 9.8899038 & 2 \\ 9.2076906 & 2 \\ 8.5914709 & 2 \\ 7.7737068 & 2 \\ 7.0657786 & 2 \\ 6.2644905 & 2 \\ 4.7915900 & 2 \\ 3.8174547 & 2 \\ 2.6471062 & 2 \\ 1.9802951 & 2 \\ 1.2445355 & 2\end{array}$

1.4000780 1. 3948535 1. 3845147 1.3743220 1.3642736 1.3543670 1.3445999 1. 3349661 1.3254647 1.3160909 1. $30684 \mathrm{Cb}$ 1.2886954 1. 2623103 1. 2203373 1. 1805854 I. 1428032 1. 0724503 1.0082107 9.2176798 8.4566114 7.5787071 5. 9120543 4.7678510 2.3479623 2. 5187906 1.5902204

Table 31. Ground reflection coefficients (amplitude, $|R|$, and phase, Arg R) assuming the Fresnel approximation, for the special ray configuration of the Norton type 301 atmosphere, applicable to the propagation of waves between the D-region $\left(h_{3}=70\right.$ kilometers) or the E-region $\left(h_{3}=90\right.$ kilometers $)$ of the ionosphere and the earth. 
$f=66.622252$ kilocycles

$\sigma=0.005 \mathrm{mhos} / \mathrm{meter}$

$\epsilon_{2}=15$

$\begin{array}{lll}\Psi & \left|R_{e}\right| & A r g R_{e} \\ \text { radians } & h_{3}=70 \text { kilometers }\end{array}$

$\begin{array}{ll}0.0005 & 9.7435063=1 \\ 0.0010 & 9.4937573=1 \\ 0.0020 & 9.0144068=1 \\ 0.0030 & 8.5616795=1 \\ 0.0040 & 8.1351833=1 \\ 0.0050 & 7.7344395=1 \\ 0.0060 & 7.3589094=1 \\ 0.0070 & 7.0080181=1 \\ 0.0080 & 6.6811678=1 \\ 0.0090 & 6.3777503=1 \\ 0.0100 & 6.0971503=1 \\ 0.0120 & 5.6019192=1 \\ 0.0150 & 5.0144205=1 \\ 0.0200 & 4.4024726=1 \\ 0.0250 & 4.1581840=1 \\ 0.0300 & 4.1639018=1 \\ 0.0400 & 4.5370777=1 \\ 0.0500 & 5.0409703=1 \\ 0.0650 & 5.7367001=1 \\ 0.0800 & 6.2963386=1 \\ 0.1000 & 6.8645584=1 \\ 0.1500 & 7.7485679=1 \\ 0.2000 & 8.2462685=1 \\ 0.3000 & 8.7802418=1 \\ 0.4000 & 9.0589451-1 \\ 0.6000 & 9.3405848=1 \\ 0 & \end{array}$

\begin{tabular}{|c|c|c|c|}
\hline $\begin{array}{l}3.1156509 \\
3.0896917\end{array}$ & & $\begin{array}{l}9.9998074 \\
9.9996146\end{array}$ & \\
\hline 3.0376509 & & 9.9992295 & - \\
\hline 2.9853328 & & 9.9988445 & \\
\hline 2.9326046 & & 9.9984591 & - \\
\hline 2.8793407 & & 9.9980738 & - \\
\hline 2.8254253 & & 9.9976891 & \\
\hline 2.7707561 & & 9.9973041 & \\
\hline $\begin{array}{l}2.7152469 \\
2.6588313\end{array}$ & & 9.9969185 & \\
\hline 2.6014671 & & 9.9961483 & \\
\hline 2.4838637 & & 9.9953784 & \\
\hline 2.3010263 & & 9.9942232 & - \\
\hline 1.9887867 & & 9.9922987 & - \\
\hline 1.6917208 & & 9.9903748 & \\
\hline 1.4347817 & & 9.9884513 & \\
\hline 1.0624639 & & 9.9846065 & - \\
\hline 8.3077680 & -1 & 9.9807645 & - \\
\hline 6.2323510 & -1 & 9.9750089 & - \\
\hline 4.9884330 & -1 & 9.9692614 & - \\
\hline 3.9460180 & $\rightarrow 1$ & 9.9616144 & - \\
\hline 2.6030880 & -1 & 9.9425961 & - \\
\hline 1.9489940 & -1 & 9.9237574 & - \\
\hline 1.3058600 & -1 & 9.8867995 & - \\
\hline 9.8982900 & -2 & 9.8510989 & - \\
\hline .821190 & -2 & 9.7848196 & - \\
\hline
\end{tabular}

3. 1416119

3. 1416312

3. 1416697

3. 1417083

3. 1417468

3. 1417853

3. 1418238

$3 \cdot 1418623$

3. 1419008

3. 1419393

3. 1419778

3. 1420548

3. 1421703

3.1423628

$3 \cdot 1425553$

3. 1427478

3. 1431327

3. 1435174

3. 1440940

3. 1446702

3. 1454371

3. 1473474

3. 1492432

3.1529729

3. 1565887

3. 1633359

$\mathrm{d} / \mathrm{j}$

miles

$\mathrm{h}_{3}=90$ kilometer $\mathrm{s}$

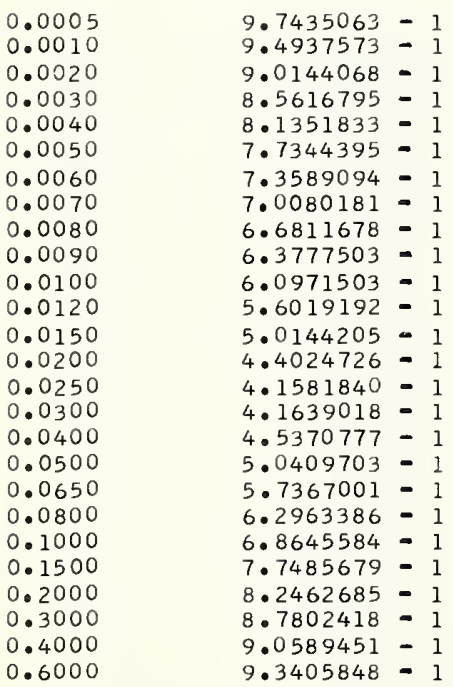

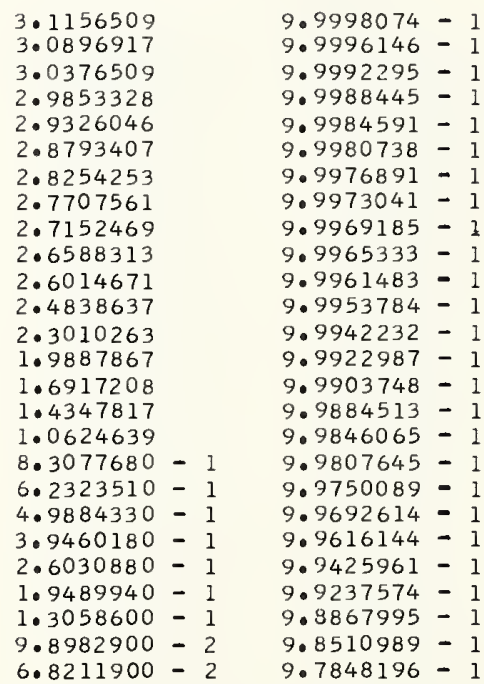

3. 1416119

3. 1416312

3. 1416697

3. 1417083

3. 1417468

3. 1417853

3. 1418238

3. 1418623

3. 1419008

3. 1419393

3. 1419778

3. 1420548

3. 1421703

3. 1423628

3. 1425553

3. 1427478

3. 1431327

3. 1435174

3. 1440940

3. 1446702

$3 \cdot 1454371$

3. 1473474

3. 1492432

3. 1529729

3. 1565887

3. 1633359
1. 2433895 1. 2381675 1. 2278386

1. 2176623 1. 2076369 1. 1977599

1. 1880278

1. 1784374

1. 1689853

1. 1596669

1. 1504788

1. 1324770

1.1063547

1. 0649462

1. 0259098

9. 8899038

9. 2076906

8.5914709

7.7737068

7.0657786

6. 2644905

4. 7915900

3. 8174547

2.6471062

1. 9802951

1. 2445355

1. 4000780 1. 3948535 1. 3845147 1. 3743220 1.3642736 I. 3543670 1. 3445989

1. 3349661

1. 3254647

1. 3160909

1.3068406

1. 2886954

1. 2623103

1. 2203378

1. 1805854

1.1428039

1.0724503

1. 0082107

9.2176798

8.4566114

7.5787071

5. 9120543

4. 7678510

3.3479623

2. 5187906

1. 5902204

Table 32. Ground reflection coefficients (amplitude, $|R|$, and phase, Arg R) assuming the Fresnel approximation, for the special ray configuration of the Norton type 301 atmosphere, applicable to the propagation of waves between the D-region ( $h_{3}=70$ kilometers) or the E-region $\left(h_{3}=90\right.$ kilometers) of the ionosphere and the earth. 
$f=133.24450$ kilocycles

$\sigma=0.005 \mathrm{mhos} /$ meter

$\epsilon_{2}=15$

$\Psi$

radians

$\begin{array}{ll}0.0005 & 9.8177657=1 \\ 0.0010 & 9.6389111=1 \\ 0.0020 & 9.2913196=1 \\ 0.0030 & 8.9571499=1 \\ 0.0040 & 8.6362920=1 \\ 0.0050 & 8.3286047=1 \\ 0.0060 & 8.0339250=1 \\ 0.0070 & 7.7520723=1 \\ 0.0080 & 7.4828534=1 \\ 0.0090 & 7.2260645=1 \\ 0.0100 & 6.9814967=1 \\ 0.0120 & 6.5281678=1 \\ 0.0150 & 5.9344318=1 \\ 0.0200 & 5.1601486=1 \\ 0.0250 & 4.6295104=1 \\ 0.0300 & 4.3087502=1 \\ 0.0400 & 4.1354226=1 \\ 0.0500 & 4.3234053=1 \\ 0.0650 & 4.8307519=1 \\ 0.0800 & 5.3561505=1 \\ 0.1000 & 5.9598538=1 \\ 0.1500 & 6.9988807=1 \\ 0.2000 & 7.6247635=1 \\ 0.3000 & 8.3226759=1 \\ 0.4000 & 8.6965100=1 \\ 0.6000 & 9.0803220=1\end{array}$

0.6000

0.0005
0.0010

0.0020

0.0030

0.0040

0.0050

0.0060

0.0070

0.0080

0.0090

0.0100

0.0120

0.0150

0.0200

0.0250

0.0300

0.0400

0.0500

0.0650

0.0800

0.1000

0.1500

0.2000

0.3000

0.4000

0.6000

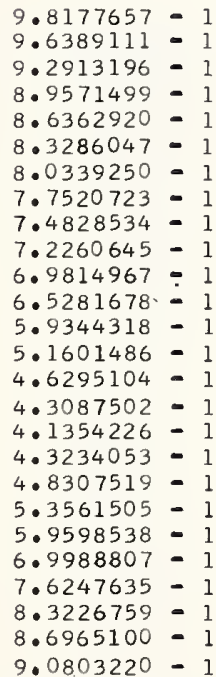

$\left|R_{e}\right|$

$\operatorname{Arg~R}_{\mathrm{e}}$

$\left|R_{m}\right|$

$\mathrm{h}_{3}=70$ kilometers

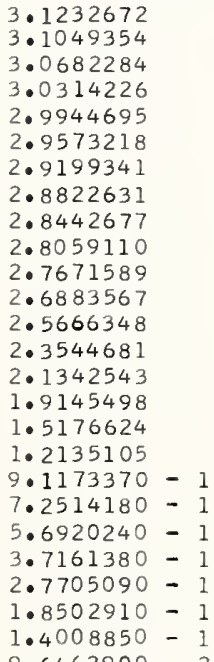

$9.9997273-1$
$9.9994550-1$
$9.9989100-1$
$9.9983655-1$
$9.9978206-1$
$9.9972761-1$
$9.9967309-1$
$9.9961864-1$
$9.9956417-1$
$9.9950973-1$
$9.9945522-1$
$9.9934632-1$
$9.9918301-1$
$9.9891081-1$
$9.9863883-1$
$9.9836685-1$
$9.9782334-1$
$9.9728032-1$
$9.9646686-1$
$9.9565483-1$
$9.9457478-1$
$9.9189005-1$
$9.8923265-1$
$9.8402555-1$
$9.7900311-1$
$9.9969839-1$

3. 1416199

3.1416471

3. 1417016

3. 1417560

3. 1418105

3. 1418650

3. 1419194

3. 1419739

$3 \cdot 1420282$

3. 1420828

$3 \cdot 1421372$

$3 \cdot 1422461$

3. 1424094

3. 1426816

3. 1429538

3. 1432260

3. 1437701

3. 1443140

3. 1451295

3. 1459441

3. 1470287

3. 1497296

3. 1524102

3.1576835

3.1627959

3.1723353

$\mathrm{h}_{3}=90$ kilometers

3. 1232672

3.1049354

3.0682284

3.0314226

2. 9944695

2.9573218

2. 9199341

2.8822631

2. 8442677

2. 8059110

2. 7671589

2. 6883567

2.5666348

2. 3544681

2.1342543

1. 9145498

1.5176624

1. 2135105

9. $1173370-1$

$7.2514180-1$

$5.6920240-1$

3.7161380- 1

$2.7705090-1$

$1.8502910-1$

$1.4008850-1$

$9.6969839-1$ $9.9994550-1 \quad 3.1416471$ 3. 1417016 3.1417560 3.1418105 3. 1418650 3.1419194 3.1419739 3. 1420282 $3 \cdot 1420828$ 3. 1421372 3. 1422461 3.1424094 3.1426816 3. 1429538 3.1432260 3.1437701 3. 1443140 $3 \cdot 1451295$ 3.1459441 3. 1470287 3.1497296 3.1524102 3.1576835 3.1627959 $3 \cdot 1627959$ $\mathrm{d} / \mathrm{j}$

miles

Table 33. Ground reflection coefficients (amplitude, $|R|$, and phase, Arg $R$ ) assuming the Fresnel approximation, for the special ray configuration of the Norton type 301 atmosphere, applicable to the propagation of waves between the D-region ( $h_{3}=70$ kilometers) or the E-region $\left(h_{3}=90\right.$ kilometers) of the ionosphere and the earth.

$\begin{array}{ll}1.2433895 & 3 \\ 1.2381675 & 3 \\ 1.2278386 & 3 \\ 1.2176623 & 3 \\ 1.2076369 & 3 \\ 1.1977599 & 3 \\ 1.1880278 & 3 \\ 1.1784374 & 3 \\ 1.1689853 & 3 \\ 1.1596669 & 3 \\ 1.1504788 & 3 \\ 1.1324770 & 3 \\ 1.1063547 & 3 \\ 1.0649462 & 3 \\ 1.0259098 & 3 \\ 9.8899038 & 2 \\ 9.2076906 & 2 \\ 8.5914709 & 2 \\ 7.7737068 & 2 \\ 7.0657786 & 2 \\ 6.2644905 & 2 \\ 4.7915900 & 2 \\ 3.8174547 & 2 \\ 2.6471062 & 2 \\ 1.9802951 & 2 \\ 1.2445355 & 2\end{array}$

$1.4000780 \quad 3$ $1.3948535 \quad 3$ 1.3845147 1.3743220 $1 \cdot 3642736$ 1.3543670 1.3445989 1.3349661 1.3254647 1.3160909 1.3068406 1. 2886954 1.2623103 1.2203378 1.1805854 1. 1428039 1.0724503 1.0082107 9.2176798 8.4566114 7.5787071 5.9120543 4. 7678510 3.3479623 2.5187906 1.5902204

$$
\text { 列 }
$$

年


$f=333.11126$ kilocycles

$\sigma=0.005 \mathrm{mhos} /$ meter

$\epsilon_{2}=15$
Arg $R_{e}$

$\mid \mathrm{R}_{\mathrm{m}}^{\mathrm{T}}$

$\mathrm{h}_{3}=70$ kilometers
$\Psi$

$\left\lceil R_{e}\lceil\right.$

$9.8840331-1$
$9.8840331-1$
$9.7694242-1$
$9.5442831-1$
$9.3245619-1$
$9.1102387-1$
$8.9012863-1$
$8.6976721-1$
$8.4993590-1$
$8.3063052-1$
$8.1184690-1$
$7.9358036-1$
$7.5857843-1$
$7.0982613-1$
$6.3821335-1$
$5.7805169-1$
$5.2863948-1$
$4.5908287-1$
$4.2277839-1$
$4.1272941-1$
$4.3243253-1$
$4.7515778-1$
$5.8004050-1$
$6.5615517-1$
$7.4934238-1$
$8.0226507-1$

\begin{tabular}{|c|c|c|c|c|}
\hline 3.1300353 & & 9.9995694 & & 3.1416357 \\
\hline 3.1300353 & & 9.9995694 & - & 3.1416357 \\
\hline 3. 1184762 & & 9.9991383 & - & 3.1416787 \\
\hline 3.0953471 & & 9.9982761 & - & 3.1417649 \\
\hline 3.0721926 & & 9.9974145 & - & 3.1418509 \\
\hline 3.0490004 & & 9.9965524 & - & 3.1419369 \\
\hline 3.0257578 & & 9.9956904 & - & 3.1420230 \\
\hline 3.0024528 & & 9.9948291 & - & 3.1421090 \\
\hline 2.9790732 & & 9.9939673 & - & 3.1421951 \\
\hline 2.9556072 & & 9.9931061 & - & 3.1422811 \\
\hline 2.9320433 & & 9.9922444 & - & 3.1423672 \\
\hline 2.9083704 & & 9.9913827 & - & 3.1424531 \\
\hline 2.8606547 & & 9.9896609 & & 3.1426253 \\
\hline 2.7880102 & & 9.9870781 & - & 3.1428834 \\
\hline 2.6635441 & & 9.9827747 & - & 3.1433136 \\
\hline 2.5343118 & & 9.9784744 & - & 3.1437436 \\
\hline 2.4003303 & & 9.9741759 & - & 3.1441737 \\
\hline 2.1224446 & & 9.9655870 & - & $3 \cdot 1450336$ \\
\hline 1.8462128 & & 9.9570079 & & 3.1458932 \\
\hline 1.4793958 & & 9.9441628 & - & 3.1471818 \\
\hline 1. 1981945 & & 9.9313457 & & 3.1484690 \\
\hline 9.3942210 & -1 & 9.9143076 & - & 3.1501829 \\
\hline 6.0256630 & -1 & 9.8720013 & - & 3.154451 \\
\hline 4.4448940 & -1 & 9.8301916 & - & 3.158686 \\
\hline 2.9420100 & -1 & 9.7484508 & - & 3.1670196 \\
\hline 2.2199660 & -1 & 9.6698436 & - & 3.1750972 \\
\hline
\end{tabular}

$\mathrm{h}_{3}=90$ kilometers

$\begin{array}{ll}0.0005 & 8.5851077-1 \\ 0.0010 & 9.7694242-1 \\ 0.0020 & 9.5442831-1 \\ 0.0030 & 9.3245619-1 \\ 0.0040 & 9.1102387-1 \\ 0.0050 & 8.9012863-1 \\ 0.0060 & 8.6976721-1 \\ 0.0070 & 8.4993590-1 \\ 0.0080 & 8.3063052-1 \\ 0.0090 & 8.1184690-1 \\ 0.0100 & 7.9358036-1 \\ 0.0120 & 7.5857843-1 \\ 0.0150 & 7.0982613-1 \\ 0.0200 & 6.3821335-1 \\ 0.0250 & 5.7805169-1 \\ 0.0300 & 5.2863948-1 \\ 0.0400 & 4.5908287-1 \\ 0.0500 & 4.2277839-1 \\ 0.0650 & 4.1272941-1 \\ 0.0800 & 4.3243253-1 \\ 0.1000 & 4.7515778-1 \\ 0.1500 & 5.8004050-1 \\ 0.2000 & 6.5615517-1 \\ 0.3000 & 7.4934238-1 \\ 0.4000 & 8.0226507-1 \\ 0.6000 & 8.5851077-1 \\ & \end{array}$

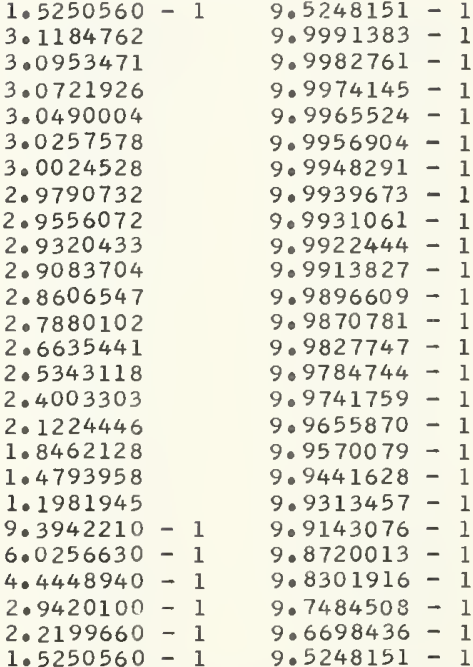

3. 1901681

3. 1416787

3. 1417649

3. 1418509

3. 1419369

3. 1420230

3. 1421090

3. 1421951

3. 1422811

3.1423672

3. 1424531

3. 1426253

$3 \cdot 1428834$

3. 1433136

3. 1437436

3. 1441737

3.1450336

3. 1458932

3. 1471818

3. 1484690

3. 1501829

3. 1544510

3. 1586869

3. 1670196

3. 1750972
3. 1901681 $\mathrm{d} / \mathrm{j}$

miles $\begin{array}{ll}1.2433895 & 3 \\ 1.2381675 & 3 \\ 1.2278386 & 3 \\ 1.2176623 & 3 \\ 1.2076369 & 3 \\ 1.1977599 & 3 \\ 1.1880278 & 3 \\ 1.1784374 & 3 \\ 1.1689853 & 3 \\ 1.1596669 & 3 \\ 1.1504788 & 3 \\ 1.1324770 & 3 \\ 1.1063547 & 3 \\ 1.0649462 & 3 \\ 1.0259098 & 3 \\ 9.8899038 & 2 \\ 9.2076906 & 2 \\ 8.5914709 & 2 \\ 7.7737068 & 2 \\ 7.0657786 & 2 \\ 6.2644905 & 2 \\ 4.7915900 & 2 \\ 3.8174547 & 2 \\ 2.6471062 & 2 \\ 1.9802951 & 2 \\ 1.2445355 & 2\end{array}$

$1.4000780 \quad 3$ 1.3948535 1. $3845147 \quad 3$ $1.3743220 \quad 3$ $1.3642736 \quad 3$ 1. $3543670 \quad 3$ 1. 34459893 $1.3349661 \quad 3$ $1.3254647 \quad 3$ $1.3160909-3$ 1. 30684063 1. 28869543 1. $2623103 \quad 3$ $1.2203378 \quad 3$ $1.1805854 \quad 3$ 1. 14280393 $1.0724503 \quad 3$ 1.00821073 9.21767982 $8.4566114 \quad 2$ $7.5787071 \quad 2$ 5. $9120543 \quad 2$ 4. 76785102 3. $3479623 \quad 2$ 2.5187906 2 1.5902204

Table 34. Ground reflection coefficients (amplitude, [R], and phase, Arg R) assuming the Fresnel approximation for the special ray configuration of the Norton type 301 atmosphere, applicable to the propagation of waves between the D-region $\left(h_{3}=70\right.$ kilometers) or the E-region $\left(h_{3}=90\right.$ kilometers) of the ionosphere and the earth. -108 - 
$\omega / \omega_{r}=0.3002$

$\phi_{1} r=60$ degrees

$\Psi$

radians

0.0010

0.0020

0.0030

0.0040

0.0050

0.0060

0.0070

0.0080

0.0090

0.0100

0.0120

0.0150

0.0200

0.0250

0.0300

0.0400

0.0500

0.0650

0.0800

0.1000

0.1500

0.2000

0.3000

0.4000

0.5000

0.0005

0.0010

0.0020

0.0030

0.0040

0.0050

0.0060

0.0070

0.0080

0.0090

0.0100

0.0120

0.0150

0.0200

0.0250

0.0300

0.0400

0.0500

0.0650

0.0800

0.1000

0.1500

0.2000

0.3000

0.4000

0.6000
$\left|\mathrm{T}_{\mathrm{ee}}\right|$

Arg T $\mathrm{T}_{\mathrm{e}}$

$\left|\mathrm{T}_{\mathrm{mm}}\right|$

Arg $\mathrm{T}_{\mathrm{mm}}$

d/ j

$\mathrm{h}_{3}=70$ kilometers

$6.5054409-1$

$6.5053942=1$

$6.5052144=1$

$6.5049114=1$

$6.5044874=1$

$6.5039432=1$

$6.5032801-1$

$6.5024944=1$

$6.5015910-1$

$6.5005663=1$

$6.4994214-1$

$6.4967747=1$

$6.4919117-1$

$6.4814564-1$

$6.4681162-1$

$6.4519519-1$

$6.4115719=1$

$6.3611182=1$

$6.2687250=1$

$6.1595333=1$

$5.9942706=1$

$5.5318278-1$

$5.0716718=1$

$4.3395658=1$

$3.9295984-1$

$3.8316778=$

$6.1696408-1$

$6.1696058-1$

$6.1694592-1$

$6.1692156=1$

$6.1688756-1$

$6.1684382-1$

$6.1679010-1$

$6.1672682-1$

$6.1665412-1$

$6.1657131-1$

$6.1647923=1$

$6.1626583=1$

$6.1587365-1$

$6.1502995=1$

$6.1325171-1$

$6.1264425-1$

$6.0936521=1$

$6.0525040-1$

$5.9766196=1$

$5.8860978-1$

$5.7474817-1$

$5.3506435-1$

$4.9451871-1$

$4.2849489=1$

$3.9115221-1$

$3.8362041-1$
3. 5290341

3.5290413

3. 5290695

3. 5291171

3. 5291833

3.5292688

3.5294960

3.5296378

3.5297987

3. 5299785

3. 5303943

3. 5311592

3.5328072

3.5349171

3.5374836

3.5439536

3. 5521476

3. 5574858

3. 5862016

3.6158525

3. 7091015

3.8226737

2.1968715

1.4454074

$\mathrm{h}_{3}=90$ kilometers

3.5844412

3. 5844727

3.5845151

3.5845742

3. 5846504

3.5847436

3.5848540

3. 5849804

3. 5851245

3. 5852850

3.5856568

3.5863406

3.5878150

3.5897054

3. 5920065

3.5978224

3.5052129

3. 5191206

3. 5362100

$3 \cdot 6635175$

3. 7508185

3. 8590227

$2 \cdot 1694486$

1. 8982300

1.4368417
3.5293730

1., 180198

3. 5844473
$8.8163764-1$

$8.8163573-1$

$8.8162861-1$

$8.8161662-1$

$8.8159976-1$

$8.8157817=1$

$8.8155200-1$

8.8152076 - 1

$8.8148500=1$

$8.8144427-1$

8.8139894 - 1

$8.8129403=1$

$8.8110087-1$

$8.8068543=1$

$8.8015446-1$

$8.7951000-1$

$8.7789240=1$

$8.7585825-1$

$8.7209290-1$

$8.6757205=1$

8.6057013 -

$8.3974363-1$

$8.1651765=1$

7.6923723 -

$7.2491069-1$

$6.4940751-1$
3.0202126

3.0202104

3.0202019

3. 0201876

3.0201675

3.0201418

3.0201105

3.0200733

3.0200305

3.0199820

3.0199279

3. 0198025

3. 0195721

3.0190757

3.0184404

3. 0176686

3.0157255

3.0132717

3.0086976

3. 0031513

2. 9944444

2.9677011

2.9363741

2.8676236

2.7969196

2.6615301
1. 2433895

1. 2381675

1.2278386

1. 2176623

1.2076369

1.1977599

1. 1880278

1. 1784374

1.1689853

1. 1596669

1. 1504788

1.1324770

1. 1063547

1.0649462

1.0259098

9.88990382

$9.2076906 \quad 2$

8.5914709

7. 7737068

7.0657786

6.2644905

4. 7915900

3.8174547

2.6471062

1. 9802951

1.2445355

1.4000780

1.3948535

1. 3845147

1.3743220

1. 3642736

1.3543670

1.3445989

1. 3349661

1. 3254647

1. 3160909

1. 3068406

1.2886954

1.2623103

1. 2203378

$1 \cdot 1805854$

1. 1428039

1. 0724503

1.0082107

9.2176798

8.4566114

7.5787071

5.9120543

4. 7678510

3.3479623

2.5187906

1. 5902204

3
3
2
2
2
2
3
3
3
3
3
3
3
3
3
3
3
3
3
3
3
3
3
3
3
3
3

Table 35. lonosphere reflection coefficients (amplitude, $|\mathrm{T}|$, and phase, $\operatorname{Arg} \mathrm{T}$ ) assuming the quasi-longi tudinal approximation, for the special ray configuration of the Norton type 301 atmosphere, applicable to the propagation of waves between the D-region ( $h_{3}=70$ kilometers) or the $E$-region $\left(h_{3}=90\right.$ kilometers) of the ionosphere and the earth. 
$\omega / \omega_{\mathrm{r}}=0.467$

$\phi_{1} r=60$ degrees

$\Psi$

radians

0.0005

0.0010

0.0020

0.0030

0.0040

0.0050

0.0060

0.0070

0.0080

0.0090

0.0100

0.0120

0.0150

0.0200

0.0250

0.0300

0.0400

C. 0500

0.0650

0.0800

0.1000

0.1500

0.2000

0.3000

0.4000

0.6000

\begin{abstract}
0.0005
0.0010

0.0020

0.0030

0.0040

0.0050

0.0060

0.0070

0.0080

0.0090

0.0100

0.0120

0.0150

0.0200

0.0250

0.0300

0.0400

0.0500

0.0650

0.0800

0.1000

0.1500

0.2000

0.3000

0.4000

0.6000
\end{abstract}

$\left|T_{\text {ee }}\right|$

$7.0264352=1$
$7.0253913=1$
$7.0262265=1$
$7.0259493=1$
$7.0255614=1$
$7.0250647=1$
$7.0244573=1$
$7.0237376=1$
$7.0229112=1$
$7.0219730=1$
$7.0209252=1$
$7.0185020=1$
$7.0140494=1$
$7.0044712=1$
$6.9922374=1$
$6.9774111=1$
$6.9402698=1$
$6.8937281=1$
$6.8080609=1$
$6.7060541=1$
$6.5499464=1$
$6.0999277-1$
$5.6256878=1$
$4.7653907=1$
$4.1133273=1$
$3.4432815=1$

$6.7155317=1$
$6.7154988=1$
$6.7153621=1$
$6.7151337=1$
$6.7148155=1$
$6.7144040=1$
$6.7139011=1$
$6.7133080=1$
$6.7126258=1$
$6.7118496=1$
$6.7109855=1$
$6.7089838=1$
$6.7053060=1$
$6.6973870=1$
$6.6872580=1$
$6.6749666=1$
$6.6440802=1$
$6.6052001=1$
$6.5331423=1$
$6.4465418=1$
$6.3124705=1$
$5.9170069=1$
$5.4888198=1$
$4.6912176-1$
$4.0747842=1$
$3.4364182=1$
Arg 'T

$\mathrm{h}_{3}=70$ kilometers

3.4055291
3.4055338
3.4055527
3.4055842
3.4056282
3.4056847
3.4057539
3.4058354
3.4059296
3.4060360
3.4061552
3.4064307
3.4069377
3.4080294
3.4094273
3.4111269
3.4154092
3.4208272
3.4309545
3.4432874
3.4627744
3.5237325
3.5976623
3.7717689
3.9700518
1.8933838

$\mathrm{h}_{3}=90$ kilometers

3.4421286
3.4421326
3.4421493
3.4421773
3.4422160
3.4422664
3.4423277
3.4424003
3.4424835
3.4425785
3.4426841
3.4429290
3.4433791
3.4443494
3.4455933
3.4471073
3.4509321
3.4557889
3.4649187
3.4761207
3.4939861
3.5508997
3.6213495
3.7903912
3.9850912
1.8842763

$8.5023379=1$
$8.5023131=1$
$8.5022233=1$
$8.5020716=1$
$8.5018588=1$
$8.5015874=1$
$8.5012556=1$
$8.5008614=1$
$8.5004093=1$
$8.4998964=1$
$8.4993225=1$
$8.4979967=1$
$8.4955596-1$
$8.4903138=1$
$8.4836074=1$
$8.4754725=1$
$8.4550529=1$
$8.4293831=1$
$8.3818900=1$
$8.3249129=1$
$8.2367679=1$
$7.9754539=1$
$7.6859183-1$
$7.1045671=1$
$6.5717344=1$
$5.6956239-1$

2.9974098 2. 9974071 2.9973968

2.9973795

2.9973553

2. 9973242

2.9972862

2.9972411

2.9971895

2.9971307

2. 9970551

2. 9969132

2.9966343

2.9960331

2.9952637

2.9943287

2. 9919735

2.9889971

2.9834426

2.9766966

2.9660815

2.9332922

2. 8945350

2.8082397

2.7178687

2. 5407388

8.3302267 -

2.9773296

2.9773274

2. 9773182

$2 \cdot 9773031$

2.9772817

2. 9772544

2.9772208

2.9771812

2.9771356

2.9770840

2.9770262

2.9768925

2.9766466

2.9761165

2.9754375

2.9746113

2. 9725257

2.9698801

2.9649169

2.9588461

2. 9492082

2.9189120

2.8823771

2.7994217

2.7112912

2. 5369000 $\mathrm{d} / \mathrm{j}$

miles

1.2433895

1.2381675

1. 2278386

1. 2176623

1.2076369

1.1977599

1.1880278

1. 1784374

1.1689853

1.1596669

1. 1504788

1.1324770

1.1063547

1.0649462

1.0259098

9.8899038

9.2076906

8.5914709

7.7737068

7.0657786

6.2644905

4. 7915900

3. 8174547

2.6471062

1.9802951

1. 2445355

3
3
3
3
3
3
3
3
3
3
3
3
3
3
3
2
2
2
2
2
2
2
2
2
2
2

1.4000780 . 3948535

1. 3845147

1.3743220

1.3642736

1.3543670

1. 3445989

1. 3349661

1. 3254647

1. 3160909

1.3068406

1.2886954

1.2623103

1.2203378

1.1805854

1.1428039

1.0724503

1.0082107

9.2176798

8.4566114

7. 5787071

5. 9120543

4. 7678510

3. 3479623

2. 5187906

1. 5902204

Table 36. Ionosphere reflection coefficients (amplitude, $|\mathrm{T}|$, and phase, Arg T) assuming the quasi-longitudinal approximation, for the special ray configuration of the Norton type 301 atmosphere, applicable to the propagation of waves between the D-region $\left(h_{3}=70\right.$ kilometers) or the E-region $\left(h_{3}=90\right.$ kilometers $)$ of the innosphere and the earth. 
$\omega / \omega_{r}=0.1501$

$\Phi_{1}=60$ degrees

$\Psi$

radians
Arg $\mathrm{T}_{\mathrm{ee}}$

$\mathrm{h}_{3}=70$ kilometers
$\left|\mathrm{T}_{\text {ee }}\right|$

$\begin{array}{ll}3.7550434 & 9.1915792=1 \\ 3.7550553 & 9.1915649=1 \\ 3.7551021 & 9.1915167=1 \\ 3.7551801 & 9.1914350=1 \\ 3.7552895 & 9.1913191=1 \\ 3.7554296 & 9.1911723=1 \\ 3.7556012 & 9.1909929=1 \\ 3.7558036 & 9.1907774=1 \\ 3.7560372 & 9.1905339=1 \\ 3.7563021 & 9.1902550=1 \\ 3.7565977 & 9.1899438=1 \\ 3.7572822 & 9.1892248=1 \\ 3.7585410 & 9.1879033=1 \\ 3.7612538 & 9.1850578=1 \\ 3.7647286 & 9.1814205=1 \\ 3.7689553 & 9.1770051=1 \\ 3.7796177 & 9.1659191=1 \\ 3.7931301 & 9.1519680=1 \\ 3.8184497 & 9.1261182=1 \\ 3.8493782 & 9.0950341=1 \\ 3.8984059 & 9.0467771=1 \\ 4.0519330 & 8.9023066=1 \\ 2.0488856 & 8.7392182=1 \\ 1.6681123 & 8.3992310=1 \\ 1.3478052 & 8.0690271=1 \\ 9.5232110-1 & 7.4781537=1\end{array}$

$\mathrm{h}_{3}=90$ kilometers

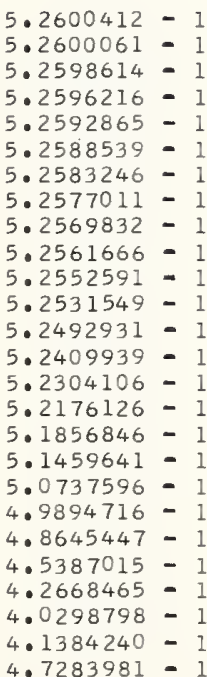

$\begin{array}{ll}0.0005 & 5.6030252=1 \\ 0.0010 & 5.6029750=1 \\ 0.0020 & 5.6027846=1 \\ 0.0030 & 5.6024660=1 \\ 0.0040 & 5.6020195=1 \\ 0.0050 & 5.6014468=1 \\ 0.0060 & 5.6007473=1 \\ 0.0070 & 5.5999203=1 \\ 0.0080 & 5.5989682=1 \\ 0.0090 & 5.5978896=1 \\ 0.0100 & 5.5966838=1 \\ 0.0120 & 5.5938982=1 \\ 0.0150 & 5.5887835=1 \\ 0.0200 & 5.5778020=1 \\ 0.0250 & 5.5638187=1 \\ 0.0300 & 5.5469300=1 \\ 0.0400 & 5.5049188=1 \\ 0.0500 & 5.4528778=1 \\ 0.0650 & 5.3588830=1 \\ 0.0800 & 5.2500775=1 \\ 0.1000 & 5.0904324=1 \\ 0.1500 & 4.6811506=1 \\ 0.2000 & 4.3438235=1 \\ 0.3000 & 4.0353424=1 \\ 0.4000 & 4.1226586=1 \\ 0.6000 & 4.7140703=1\end{array}$

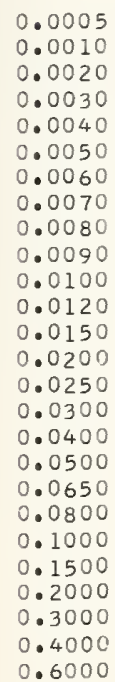

0.6000 $9.0979260=1$ $9.0978838-1$ $9.0978142-1$ $9.0977162-1$ 9.0975917 - 1 9.0974363 - 1 $9.0972556-1$ $9.0968076-1$ 9.0965446 - 1 $9.0959316-1$ $9.0948052-1$ 9.0923781 - 1 9.0892684 - 1 $9.0854916-1$ 9.0759777 - 1 $9.0415305-1$ 9.0143226 - 1 $8.9716113-1$ $8.8409057-1$ $8.6895352-1$ 8.3659724 - 1 $8.0458421-1$ 9.0970458 - 1 9.0639564 - 1
Arg $T_{\mathrm{mm}}$

$d / j$

miles
3.0535762 3.0535746 3.0535686 3.0535585 3.0535444 3.0535262 3.0535041 3.0534780 3.0534478 3.0534138 3.0533756 3.0532874 3.0531250 3.0527755 3.0523285 3.0517854 3.0504188 3.0486949 3.0454861 3.0416037 3.0355270 3.0169911 2.9954968 2.9489777 2.9018070 2.8126506

$\begin{array}{ll}1.2433895 & 3 \\ 1.2331675 & 3 \\ 1.2278386 & 3 \\ 1.2176623 & 3 \\ 1.2076369 & 3 \\ 1.1977599 & 3 \\ 1.1880278 & 3 \\ 1.1784374 & 3 \\ 1.1689853 & 3 \\ 1.1596669 & 3 \\ 1.1504788 & 3 \\ 1.1324770 & 3 \\ 1.1063547 & 3 \\ 1.0649462 & 3 \\ 1.0259098 & 3 \\ 9.8899038 & 2 \\ 9.2076906 & 2 \\ 8.5914709 & 2 \\ 7.7737068 & 2 \\ 7.0657786 & 2 \\ 6.2644905 & 2 \\ 4.7915900 & 2 \\ 3.8174547 & 2 \\ 2.6471062 & 2 \\ 1.9802951 & 2 \\ 1.2445355 & 2\end{array}$

3.0419675 3.0419662 3.0419609 3.0419522 3.0419399 3.0419242 3.0419050 3.0418822 3.0418560 3.0418261 3.0417932 3.0417163 3.0415751 3.0412708 3.0408811 3.0404071 3.0392115 3.0376972 3.0348627 3.0314069 3.0259455 3.0089664 2.9888379 2.9443127 $2 \cdot 8984244$ 2. 8107589

$\begin{array}{ll}1.4000780 & 3 \\ 1.3948535 & 3 \\ 1.3845147 & 3 \\ 1.3743220 & 3 \\ 1.3642736 & 3 \\ 1.3543670 & 3 \\ 1.3445989 & 3 \\ 1.3349661 & 3 \\ 1.3254647 & 3 \\ 1.3160909 & 3 \\ 1.3068406 & 3 \\ 1.2886954 & 3 \\ 1.2623103 & 3 \\ 1.2203378 & 3 \\ 1.1805854 & 3 \\ 1.1428035 & 3 \\ 1.0724503 & 3 \\ 1.0082107 & 3 \\ 9.2176798 & 2 \\ 8.4566114 & 2 \\ 7.5787071 & 2 \\ 5.9120543 & 2 \\ 4.7678510 & 2 \\ 3.3479623 & 2 \\ 2.5187906 & 2 \\ 1.5902204 & 2\end{array}$

Table 37. Ionosphere reflection coefficients (amplitude, $|\mathrm{T}|$, and phase, Arg T) assuming the quasi-longitudinal approximation, for the special ray configuration of the Norton type 301 atmosphere, applicable to the propagation of waves between the D-region ( $h_{3}=70$ kilometers) or the E-region $\left(h_{3}=90\right.$ kilometers) of the ionosphere and the earth. 


$$
\begin{aligned}
& \omega / \omega_{r}=0.2335 \\
& \phi_{1}=60 \text { degrees }
\end{aligned}
$$

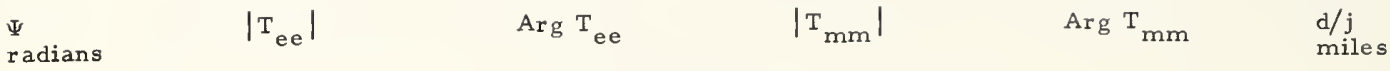

$\begin{array}{ll}0.0005 & 6.1853098=1 \\ 0.0010 & 6.1852613=1 \\ 0.0020 & 6.1850747=1 \\ 0.0030 & 6.1847615=1 \\ 0.0040 & 6.1843226=1 \\ 0.0050 & 6.1837589=1 \\ 0.0060 & 6.1830711=1 \\ 0.0070 & 6.1822587=1 \\ 0.0080 & 6.1813230=1 \\ 0.0090 & 6.1802611=1 \\ 0.0100 & 6.1790760=1 \\ 0.0120 & 6.1790760=1 \\ 0.0150 & 6.1713025=1 \\ 0.0200 & 6.1604845=1 \\ 0.0250 & 6.1466883=1 \\ 0.0300 & 6.1299941=1 \\ 0.0400 & 6.0883190=1 \\ 0.0500 & 6.0363807=1 \\ 0.0650 & 5.9416383=1 \\ 0.0800 & 5.8303171=1 \\ 0.1000 & 5.6632720=1 \\ 0.1500 & 5.2067374=1 \\ 0.2000 & 4.7737070=1 \\ 0.3000 & 4.1626484=1 \\ 0.4000 & 3.9258032=1 \\ 0.6000 & 4.1210073-1 \\ 0 & \end{array}$

$\mathrm{h}_{3}=70$ kilometers

$\begin{array}{lll}3.6042148 & 8.9686339-1 & 3.0330037 \\ 3.6042235 & 8.9686173-1 & 3.0330018 \\ 3.6042578 & 8.9685552-1 & 3.0329942 \\ 3.6043153 & 8.9684503-1 & 3.0329815 \\ 3.6043959 & 8.9683046=1 & 3.0329638 \\ 3.6044992 & 8.9681156-1 & 3.0329410 \\ 3.6046253 & 8.9678870-1 & 3.0329132 \\ 3.6047745 & 8.9676149-1 & 3.0328804 \\ 3.6049465 & 8.9673030-1 & 3.0328426 \\ 3.6051412 & 8.9669481-1 & 3.0327996 \\ 3.6053591 & 8.9665515-1 & 3.0327517 \\ 3.6053591 & 8.9665515-1 & 3.0327517 \\ 3.6067894 & 8.9639532-1 & 3.0324368 \\ 3.6087861 & 8.9603297-1 & 3.0319974 \\ 3.6113435 & 8.9556974-1 & 3.0314355 \\ 3.6144537 & 8.9500761-1 & 3.0307524 \\ 3.6222982 & 8.9359646-1 & 3.0290340 \\ 3.6322360 & 8.9182142-1 & 3.0268641 \\ 3.6508512 & 8.8853472-1 & 3.0228223 \\ 3.6735848 & 8.8458644-1 & 3.0179255 \\ 3.7096366 & 8.7846694-1 & 3.0102472 \\ 3.8231473 & 8.6022425-1 & 2.9867296 \\ 3.9610354 & 8.3979389-1 & 2.9592981 \\ 2.0095349 & 7.9784635-1 & 2.8994716 \\ 1.7007927 & 7.5799177-1 & 2.8383680 \\ 1.2403360 & 6.8876650-1 & 2.7222121\end{array}$

1. 2433895 1. 2381675 1. 2278386 1. 2176623 1. 2076369 1. 1977599 1. 1880278 1. 1784374 1. 1689853 1. 1596669 1. 1504788 1. 1324770 1. 1063547 1.0649462 1.0259098 9. 8899038 9. 2076906 8.5914709 7. 7737068 7.0657786 6.2644905 4. 7915900 3. 8174547 2. 6471062 1. 9802951 1. 2445355

$\mathrm{h}_{3}=90$ kilometers

\begin{tabular}{|c|c|c|}
\hline 0.0005 & 5.8405915 & 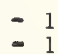 \\
\hline $\begin{array}{l}0.0010 \\
0.0020\end{array}$ & $\begin{array}{l}5.8405555 \\
5.8404064\end{array}$ & $\tilde{-}$ \\
\hline 0.0030 & 5.8401586 & - \\
\hline 0.0040 & 5.8398138 & - \\
\hline 0.0050 & 5.8393683 & - \\
\hline 0.0060 & 5.8388227 & - \\
\hline 0.0070 & 5.8381795 & - \\
\hline 0.0080 & 5.8374397 & - \\
\hline 0.0090 & 5.8365980 & - \\
\hline 0.0100 & 5.8356626 & - \\
\hline 0.0120 & 5.8334921 & - \\
\hline 0.0150 & 5.8295076 & - \\
\hline 0.0200 & 5.8209369 & - \\
\hline 0.0250 & 5.8099900 & - \\
\hline 0.0300 & 5.7967267 & - \\
\hline 0.0400 & 5.7635091 & - \\
\hline 0.0500 & 5.7219256 & - \\
\hline 0.0650 & 5.6455369 & - \\
\hline 0.0800 & 5.5549468 & - \\
\hline 0.1000 & 5.4174346 & - \\
\hline 0.1500 & 5.0332281 & - \\
\hline 0.2000 & 4.6597257 & - \\
\hline 0.3000 & 4.1237571 & - \\
\hline 0.4000 & 3.9206390 & - \\
\hline 0.6000 & 4.1302013 & - \\
\hline
\end{tabular}

3.6714456
3.6714531
3.6714841
3.6715353
3.6716072
3.6716999
3.6718132
3.6719470
3.6721007
3.6722759
3.6724709
3.6729228
3.6737538
3.6755457
3.6778427
3.6806398
3.6877095
3.6966960
3.7136128
3.7344082
3.7676514
3.8738915
4.0049168
1.9780068
1.6799471
1.2326235

\begin{tabular}{|c|c|c|}
\hline $\begin{array}{l}8.8495500 \\
8.8495375\end{array}$ & $\begin{array}{l}-1 \\
-1\end{array}$ & $\begin{array}{l}3.0183844 \\
3.0183828\end{array}$ \\
\hline 8.8494839 & -1 & 3.0183762 \\
\hline 8.8493946 & -1 & 3.0183651 \\
\hline 8.8492717 & -1 & 3.0183498 \\
\hline 8.8491119 & -1 & 3.0183299 \\
\hline $\begin{array}{l}8.8489157 \\
8.8486859\end{array}$ & $\begin{array}{l}-1 \\
-1\end{array}$ & $\begin{array}{l}3.0183056 \\
3.0182768\end{array}$ \\
\hline 8.8484199 & -1 & 3.0182437 \\
\hline 8.8481183 & -1 & 3.0182062 \\
\hline 8.8477833 & -1 & 3.0181644 \\
\hline 8.8470044 & -1 & 3.0180675 \\
\hline 8.8455748 & - & 3.0178892 \\
\hline 8.8424933 & - & 3.0175051 \\
\hline 8.8385479 & $=1$ & 3.0170130 \\
\hline 8.8337551 & -1 & 3.0164145 \\
\hline 8.8216860 & -1 & 3.0149044 \\
\hline 8.8064414 & -1 & 3.0129911 \\
\hline 8.7780221 & -1 & 3.0094068 \\
\hline 8.7435798 & -1 & 3.0050319 \\
\hline 8.6895999 & -1 & 2.9981080 \\
\hline 8.5251078 & -1 & 2.9765070 \\
\hline 8.3360828 & -1 & 2.9507693 \\
\hline 7.9379249 & $-i$ & 2.8934450 \\
\hline 7.5522611 & -1 & 2.8339743 \\
\hline
\end{tabular}

1.4000780 1.3948535 1. 3845147 1. 3743220 1. 3642736 1. 3543670 1. 3445989 1. 3349661 1. 3254647 1. 3160909 1. 3068406 1. 2886954 1. 2623103 1. 2203378 1. 1805854 1. 1428039 1.0724503 1.0082107 9. 2176798 8.4566114 7. 5787071 5. 9120543 4. 7678510 3. 3479623 2. 5187906 1. 5902204

Table 38. Ionosphere reflection coefficients (amplitude, |T|, and phase, Arg T) assuming the quasi-longitudinal approximation, for the special ray configuration of the Norton type 301 atmosphere, applicable to the propagation of waves between the D-region $\left(h_{3}=70\right.$ kilometers) or the E-region ( $h_{3}=90$ kilometers) of the ionosphere and the earth. 


$$
\begin{aligned}
& \omega / \omega_{r}=0.0100 \\
& \phi_{1}=60 \text { degrees }
\end{aligned}
$$

$\Psi$

radians

0.0005

0.0010

0.0020

0.0030

0.0040

0.0050

0.0060

0.0070

0.0080

0.0090

0.0100

0.0120

0.0150

0.0200

0.0250

0.0300

0.0400

0.0500

0.0650

0.0800

0.1000

0.1500

0.2000

0.3000

0.4000

0.6000

$\begin{array}{ll}0.0005 & 5.1280372=1 \\ 0.0010 & 5.1280711=1 \\ 0.0020 & 5.1282094=1 \\ 0.0030 & 5.1284401=1 \\ 0.0040 & 5.1287612=1 \\ 0.0050 & 5.1291764=1 \\ 0.0060 & 5.1296832=1 \\ 0.0070 & 5.1302829=1 \\ 0.0080 & 5.1309706=1 \\ 0.0090 & 5.1317548=1 \\ 0.0100 & 5.1326267=1 \\ 0.0120 & 5.1346474=1 \\ 0.0150 & 5.1383628=1 \\ 0.0200 & 5.1463607=1 \\ 0.0250 & 5.1565887=1 \\ 0.0300 & 5.1690062=1 \\ 0.0400 & 5.2002093=1 \\ 0.0500 & 5.2394794=1 \\ 0.0650 & 5.3121733=1 \\ 0.0800 & 5.3992572=1 \\ 0.1000 & 5.5331182=1 \\ 0.1500 & 5.9176473=1 \\ 0.2000 & 6.3122628=1 \\ 0.3000 & 6.9895559=1 \\ 0.4000 & 7.4867970=1 \\ 0.6000 & 8.1072268=1\end{array}$

$4.8176526=1$
$4.8176940=1$
$4.8178556=1$
$4.8181265=1$
$4.8185057=1$
$4.8189922=1$
$4.8195867=1$
$4.8202898=1$
$4.8210997=1$
$4.8220178=1$
$4.8230437=1$
$4.8254175=1$
$4.8297797=1$
$4.8391784=1$
$4.8512066=1$
$4.8658192=1$
$4.9025784=1$
$4.9489082=1$
$5.0347697=1$
$5.1376078=1$
$5.2952352=1$
$5.7419695=1$
$6.1887080=1$
$6.9290809=1$
$7.4553402=1$
$8.0969137=1$

Arg T

$\mathrm{h}_{3}=70$ kilometers

$9.2331030=1$
$9.2329230=1$
$9.2322170=1$
$9.2310330=1$
$9.2293780=1$
$9.2272510=1$
$9.2246580=1$
$9.2215910=1$
$9.2180600=1$
$9.2140630=1$
$9.2096000=1$
$9.1992850=1$
$9.1803850=1$
$9.1399230=1$
$9.0886320=1$
$9.0270410=1$
$8.8754530=1$
$8.6908170=1$
$8.3657000=1$
$8.0018190=1$
$7.4892430=1$
$6.2561410=1$
$5.2459340=1$
$3.8780730=1$
$3.0606190=1$
$2.1825000=1$

9.8144145 - 1

$9.8144114=1$

$9.8143998-1$

9.8143797 - 1

9.8143517 - 1

$9.8143157-1$

$9.8142723-1$

$9.8142202=1$

$9.8141605-1$

9.8140929 - 1

$9.8140184-1$

$9.8138439-1$

$9.8135198-1$

$9.8128271-1$

$9.8119414-1$

$9.8108640-1$

$9.8081531-1$

$9.8047301-1$

$9.7983553-1$

$9.7906309-1$

$9.7785163=1$

$9.7413474-1$

$9.6978238-1$

$9.6021480-1$

9.5034757 - 1

$9.3144209-1$

\section{$\mathrm{h}_{3}=90$ kilometers}

$8.0346200=1$
$8.0345040=1$
$8.0340290=1$
$8.0332360=1$
$8.0321310=1$
$8.0307060=1$
$8.0289640=1$
$8.0269070=1$
$8.0245440=1$
$8.0218550=1$
$8.0188640=1$
$8.0119430=1$
$7.9992400=1$
$7.9720030=1$
$7.9373750=1$
$7.8956420=1$
$7.7922260=1$
$7.6649040=1$
$7.4370060=1$
$7.1762120=1$
$6.7983430=1$
$5.8374190=1$
$4.9949890=1$
$3.7786830=1$
$3.0148380=1$
$2.1692440=1$

$9.7913557=$ $9.7913531-1$

$9.7913429-1$

$9.7913254-1$

$9.7913011-1$

$9.7912701-1$

$9.7912313-1$

$9.7911853-1$

$9.7911340-1$

$9.7910743-1$

$9.7910079=1$

$9.7908553-1$

9.79057140 - 1

$9.7899680-1$

$9.7891916-1$

$9.7882471-1$

$9.7858655-1$

$9.7828457-1$

$9.7771891-1$

$9.7702825-1$

$9.7593453-1$

$9.7251526-1$

$9.6842492-1$

$9.5924544-1$

9.4963508 - 1

$9.3103924-1$
Arg $\mathrm{T}_{\mathrm{mm}}$

$d / j$

miles

$\begin{array}{ll}3.1205808 & 1.2433895 \\ 3.1205804 & 1.2381675 \\ 3.1205790 & 1.2278386 \\ 3.1205768 & 1.2176623 \\ 3.1205737 & 1.2076369 \\ 3.1205697 & 1.1977599 \\ 3.1205648 & 1.1880278 \\ 3.1205590 & 1.1784374 \\ 3.1205524 & 1.1689853 \\ 3.1205448 & 1.1596669 \\ 3.1205363 & 1.1504788 \\ 3.1205168 & 1.1324770 \\ 3.1204810 & 1.1063547 \\ 3.1204036 & 1.0649462 \\ 3.1203046 & 1.0259098 \\ 3.1201845 & 9.8899038 \\ 3.1198823 & 9.2076906 \\ 3.1195014 & 8.5914709 \\ 3.1187929 & 7.7737068 \\ 3.1179366 & 7.0657786 \\ 3.1165976 & 6.2644905 \\ 3.1125168 & 4.7915900 \\ 3.1077759 & 3.8174547 \\ 3.0974269 & 2.6471062 \\ 3.0867770 & 1.9802951 \\ 3.0662545 & 1.2445355\end{array}$

3. 1180167

1.4000780

1. 3948535

.1180165

3. 1180153

3. 1180134

3.1180107

3. 1180072

3.1180029

3. 1179979

3.1179922

3. 1179856

3. 1179783

3.1179613

3.1179302

3. 1178632

3. 1177773

3. 1176728

3. 1174093

3.1170756

3. 1164513

3. 1156904

3. 1144882

3. 1107489

3.1063029

3.0963808

3.0860072

3.0658145

1. 3845147

1. 3743220

1. 3642736

1. 3543670

1. 3445989

1. 3349661

1. 3254647

1. 3160909

1. 3068406

1. 2886954

1. 2623103

1. 2203378

1. 1805854

1. 1428039

1.0724503

1.0082107

9. 2176798

8.4566114

7. 5787071

5. 9120543

4. 7678510

3.3479623

2. 5187906

1. 5902204

Table 39. Ionosphere reflection coefficients (amplitude, $|\mathrm{T}|$, and phase, Arg $\mathrm{T}$ ) assuming the quasi-longitudinal approximation, for the special ray configuration of the Norton type 301 atmosphere, applicable to the propagation of waves between the D-region ( $h_{3}=70$ kilometers) or the $E$-region $\left(h_{3}=90\right.$ kilometers) of the ionosphere and the earth. 
$\omega / \omega_{r}=0.0200$

$\phi_{1} r=60$ degrees
$\Psi$

radians
Arg $T_{\text {ee }}$

$\mathrm{h}_{3}=70$ kilometers
1.3413522

1.3413282

1.3412340

1.3410760

1.3408551

1. 3405719

1. 3402257

1.3398170

1. 3393459

1. 3388125

1.3382165

1. 3368394

1. 3343132

1.3288962

1. 3220116

1.3137173

1.2931835

1. 2679447

1.2229190

1.1717011

1. 0982327

9.1674580 -

$7.6544670-1$

5.6101980 - 1

4.4054760 -

$3.1305920-1$
$\left|\mathrm{T}_{\mathrm{mm}}\right|$

$9.7312330=1$
$9.7312277=1$
$9.7312119=1$
$9.7311833=1$
$9.7311438=1$
$9.7310925=1$
$9.7310308=1$
$9.7309569=1$
$9.7308741=1$
$9.7307773=1$
$9.7306705=1$
$9.7304237=1$
$9.7299682=1$
$9.7289897=1$
$9.7277349=1$
$9.7262142=1$
$9.7223847=1$
$9.7175540=1$
$9.7085647=1$
$9.6976893=1$
$9.6806637=1$
$9.6286278=1$
$9.5679866=1$
$9.4354019=1$
$9.2993835=1$
$9.0403725-1$

Arg $\mathrm{T}_{\mathrm{mm}}$

$d / j$

miles

\section{$\mathrm{h}_{3}=90$ kilometers}

$\begin{array}{ll}0.0005 & 4.3752559=1 \\ 0.0010 & 4.3752770=1 \\ 0.0020 & 4.3753616=1 \\ 0.0030 & 4.3755029=1 \\ 0.0040 & 4.3756990=1 \\ 0.0050 & 4.3759539=1 \\ 0.0060 & 4.3762642=1 \\ 0.0070 & 4.3766324=1 \\ 0.0080 & 4.3770556=1 \\ 0.0090 & 4.3775366=1 \\ 0.0100 & 4.3780740=1 \\ 0.0120 & 4.3793169=1 \\ 0.0150 & 4.3816095=1 \\ 0.0200 & 4.3865693=1 \\ 0.0250 & 4.3929629=1 \\ 0.0300 & 4.4007969=1 \\ 0.0400 & 4.4208263=1 \\ 0.0500 & 4.4467179=1 \\ 0.0650 & 4.4965658=1 \\ 0.0800 & 4.5593566=1 \\ 0.1000 & 4.6618640=1 \\ 0.1500 & 4.9906305=1 \\ 0.2000 & 5.3705769=1 \\ 0.3000 & 6.0975168=1 \\ 0.4000 & 6.6774142=1 \\ 0.6000 & 7.4438289=1 \\ 0 & 4\end{array}$

$3 \cdot 1109462$
$3 \cdot 1109456$
$3 \cdot 1109437$
$3 \cdot 1109404$
$3 \cdot 1109359$
$3 \cdot 1109300$
$3 \cdot 1109228$
$3 \cdot 1109143$
$3 \cdot 1109044$
$3 \cdot 1108933$
$3 \cdot 1108808$
$3 \cdot 1108521$
$3 \cdot 1107993$
$3 \cdot 1106856$
$3 \cdot 1105402$
$3 \cdot 1103634$
$3 \cdot 1099195$
$3 \cdot 1093596$
$3 \cdot 1083192$
$3 \cdot 1070629$
$3 \cdot 1051006$
$3 \cdot 0991379$
3.0922410
$3 \cdot 0772769$
3.0619679
$3 \cdot 0326128$

1.2433895 1. 2381675 1. 2278386 1.2176623 1.2076369 1. 1977599 1.1880278 1.1784374 1.1689853 1. 1596669 1. 1504783 1.1324770 1. 1063547 1.0649462 1.0259098 9.8899038 9. 2076906 8.5914709 7.7737068 7.0657786 6. 2644905 4. 7915900 3. 8174547 2.6471052 1. 9802951 I. 2445355
1. 1763519

1. 1763354

1. 1762680

1. 1761556

1. 1759994

1. 1757973

1. 1755504

1. 1752587

1. 1749236

$1 \cdot 1745424$

1. 1741184

1. 1731367

1. 1713351

1. 1674679

1. 1625447

$1 \cdot 1566028$

1. 1418346

1. 1235713

1.0906681

1.0527089

$9.9720450-1$

8. $5416210=$

7.2779420 -

$5.4628620-1$

$4.3385090=$

3. 1114980 - 1

\begin{tabular}{|c|c|c|}
\hline $\begin{array}{l}9.6987089 \\
9.6987061\end{array}$ & $\begin{array}{l}-1 \\
-1\end{array}$ & $\begin{array}{l}3.1071804 \\
3.1071800\end{array}$ \\
\hline 9.6986910 & -1 & 3.1071783 \\
\hline 9.6986665 & -1 & 3.1071754 \\
\hline 9.6986317 & & 3.1071715 \\
\hline 9.6985873 & -1 & 3.1071665 \\
\hline 9.6985331 & & 3.1071602 \\
\hline 9.6984687 & -1 & 3.1071529 \\
\hline 9.6983962 & & 3.1071444 \\
\hline 9.6983120 & -1 & 3.1071348 \\
\hline 9.6982206 & & 3.1071240 \\
\hline 9.6980038 & -1 & 3.1070993 \\
\hline 9.6976089 & -1 & 3.1070536 \\
\hline 9.6967560 & -1 & 3.1069552 \\
\hline 9.6956654 & -1 & 3.1068292 \\
\hline 9.6943380 & -1 & 3.1066760 \\
\hline 9.6909878 & -1 & 3.1062897 \\
\hline 9.6867456 & -1 & 3.1058008 \\
\hline 9.6788011 & -1 & 3.1048865 \\
\hline 9.6691111 & -1 & 3.1037728 \\
\hline 9.6537902 & -1 & 3.1020152 \\
\hline 9.5060320 & -1 & 3.0965622 \\
\hline 9.5491200 & -1 & 3.0901042 \\
\hline 9.422 & -1 & 3.075769 \\
\hline 9.2895851 & -1 & 3.060864 \\
\hline & & \\
\hline
\end{tabular}

1. 4000780 1. 3948535 1.3845147 1.3743220 1.3642736 1. 3543670 1.3445989 1. 3349661 1. 3254647 1. 3160903 1. 3068406 1. 2886954 1. 2623103 1. 2203378 1. 1805854 1.1428039 1.0724503 1.0082107 9.2176798 8.4566114 7. 5787071 5.9120543 4.7678510 3.3479623 2. 5187906

1. 5902204

Table 40. Ionosphere reflection coefficients (amplitude, $|\mathrm{T}|$, and phase, Arg $\mathrm{T}$ ) assuming the quasi-longitudinal approximation, for the special ray configuration of the Norton type 301 atmosphere, applicable to the propagation of waves between the $D$-region ( $h_{3}=70$ kilometers) or the E-region $\left(h_{3}=90\right.$ kilometers) of the ionosphere and the earth. 
$\omega / \omega_{r}=0.0500$

$\phi_{1}^{r}=60$ degrees

$\Psi$

radians
Arg $\mathrm{T}_{\mathrm{ee}}$

$\left|\mathrm{T}_{\mathrm{mm}}\right|$

$h_{3}=70$ kilometers
$\left|\mathrm{T}_{\mathrm{ee}}\right|$

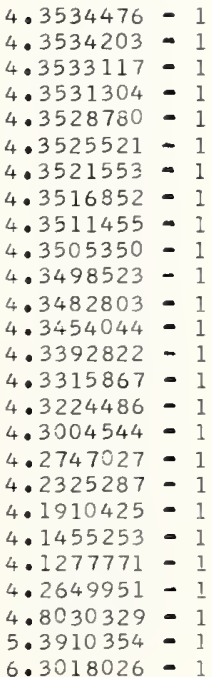

1. 9768188

1.9767965

1.9767097

1.9765642

1.9763606

1.9760991

1.9757795

1.9754023

1.9749670

1.9744740

1. 9739231

1.9726489

1.9703068

1.9652660

1.9588227

1.951 .0049

1.9313846

1.9067351

1.8612317

1.8069485

1.7240600

1.4916134

$1 \cdot 2672987$

$9.3099800-1$

$7.2565880=1$

5.1100650 -

$h_{3}=90$ kilometers

$\begin{array}{ll}0.0005 & 4.1945011=1 \\ 0.0010 & 4.1944885=1 \\ 0.0020 & 4.1944383=1 \\ 0.0030 & 4.1943539=1 \\ 0.0040 & 4.1942368=1 \\ 0.0050 & 4.1940868=1 \\ 0.0060 & 4.1939011=1 \\ 0.0070 & 4.1936822=1 \\ 0.0080 & 4.1934321=1 \\ 0.0090 & 4.1931478=1 \\ 0.0100 & 4.1928317=1 \\ 0.0120 & 4.1921038=1 \\ 0.0150 & 4.1907732=1 \\ 0.0200 & 4.1879494=1 \\ 0.0250 & 4.1844217=1 \\ 0.0300 & 4.1802618=1 \\ 0.0400 & 4.1704028=1 \\ 0.0500 & 4.1591896=1 \\ 0.0650 & 4.1418932=1 \\ 0.0800 & 4.1269830=1 \\ 0.1000 & 4.1161491=1 \\ 0.1500 & 4.1638133=1 \\ 0.2000 & 4.3288946=1 \\ 0.3000 & 4.8625424=1 \\ 0.4000 & 5.4309765=1 \\ 0.6000 & 6.3180104=1\end{array}$

$9.5580274=1$
$9.5580207=1$
$9.5579937=1$
$9.5579478=1$
$9.5578844=1$
$9.5578029=1$
$9.5577046-1$
$9.5575858=1$
$9.5574503=1$
$9.5572959=1$
$9.5571241=1$
$9.5567275=1$
$9.5559965=1$
$9.5544226-1$
$9.5524094=1$
$9.5499672=1$
$9.5438275-1$
$9.5360906-1$
$9.5217229=1$
$9.5043879=1$
$9.4773479=1$
$9.3954145=1$
$9.3010622=1$
$9.0979399=1$
$8.3927723=1$
$8.5084601-1$

3.0914950 3.0914943

3.0914909

3.0914854

3.0914778

3.0914679

3.0914558

3.0914414

3.0914251

3.0914064

3.0913857

3.0913375

3.0912490

3.0910582

3.0908144

3.0905181

3.0897735

3.0888348

3.0870902

3.0849834

3.0816942

3.0717116

3.0602021

3.0353936

3.0102285

2.9624070 d/

miles

$\begin{array}{lll}1.8119931 & 9.5060077-1 & 3.0851806 \\ 1.8119753 & 9.5060034=1 & 3.0851799 \\ 1.8119022 & 9.5059793-1 & 3.0851771 \\ 1.8117806 & 9.5059396-1 & 3.0851723 \\ 1.8116114 & 9.5058861-1 & 3.0851657 \\ 1.8113924 & 9.5058176-1 & 3.0851572 \\ 1.8111253 & 9.5057305-1 & 3.0851467 \\ 1.8108092 & 9.5056292-1 & 3.0851345 \\ 1.8104463 & 9.5055125-1 & 3.0851202 \\ 1.8100333 & 9.5053797-1 & 3.0851040 \\ 1.8095735 & 9.5052309-1 & 3.0850860 \\ 1.8085084 & 9.5048892-1 & 3.0850445 \\ 1.8065510 & 9.5042594=1 & 3.0849679 \\ 1.8023368 & 9.5029021-1 & 3.0848030 \\ 1.7969488 & 9.5011665=1 & 3.0845917 \\ 1.7904105 & 9.4990525-1 & 3.0843349 \\ 1.7739889 & 9.4937288-1 & 3.0836873 \\ 1.7533410 & 9.4869928-1 & 3.0828677 \\ 1.7151731 & 9.4743984=1 & 3.0813352 \\ 1.6695498 & 9.4590718=1 & 3.0794692 \\ 1.5996837 & 9.4349084-1 & 3.0765257 \\ 1.4020338 & 9.3601276-1 & 3.0674081 \\ 1.2076561 & 9.2719196-1 & 3.0566457 \\ 9.0594690-1 & 9.0776155-1 & 3.0329073 \\ 7.1426760-1 & 8.3780934-1 & 3.0084209 \\ 5.0783610-1 & 8.5003812-1 & 2.9613883\end{array}$

1.2381675

I. 2278386

1.2176623

1.2076369

1.1977599

1.1680278

1.1734374

1.1689853

1.1596669

1.1504788

1.1324770

1.1063547

1.0649462

1.0259098

9.8899038

9.2076906

8.5914709

7.7737068

7.0657786

6.2644905

4.7915900

3.8174547

2.5471062

1.9902951

1.2445355

1. 1.000780 1. 3948535

1. 3845147

1. 3743220

1. 3642736

1. 3543670

1. 3445989

1. 3349661

1. 3254647

1. 3160909

1. 3068406

1. 2886954

1. 2623103

1.2203378

1. 1805854

1. 1428039

1. 0724503

1.0082107

9. 2176798

8.4566114

7.5787071

5.9120543

4. 7678510

3.3479623

2.5187906

1.5902204

Table 41. Ionosphere reflection coefficients (amplitude, $|\mathrm{T}|$, and phase, Arg $T$ ) assuming the quasi-longitudinal approximation, for the special ray configuration of the Norton type 301 atmosphere, applicable to the propagation of waves between the D-region $\left(h_{3}=70\right.$ kilometers) or the E-region ( $h_{3}=90$ kilometers) of the ionosphere and the earth. 


$$
\begin{aligned}
& \omega / \omega_{r}=0.1000 \\
& \phi_{1}=60 \text { degrees }
\end{aligned}
$$

$\Psi$

$\mid \mathrm{T}$ ee $\mid$

0.0005
0.0010
0.0020
0.0030
0.0040
0.0050
0.0060
0.0070
0.0080
0.0090
0.0100
0.0120
0.0150
0.0200
0.0250
0.0300
0.0400
0.0500
0.0650
0.0800
0.1000
0.1500
0.2000
0.3000
0.4000
0.6000

0.0005

0.0010

0.0020

0.0030

0.0040

0.0050

0.0060

0.0070

0.0080

0.0090

0.0100

0.0120

0.0150

0.0200

0.0250

0.0300

0.0400

0.0500

0.0650

0.0800

0.1000

0.1500

0.2000

0.3000

0.4000

0.6000
Arg $\mathrm{T}_{\text {ee }}$

$\left|\mathrm{T}_{\mathrm{mm}}\right|$

$\mathrm{h}_{3}=70$ kilometers
3. 9245611

3. 9245764

3.9246371

3.9247380

3. 9248799

3.9250617

3. 9252840

3. 9255466

3. 9261928

3.9265762

3. 9274635

3. 9290952

3.9326112

3.9371141

3. 9425904

3. 9563977

3. 9738804

4. 0065840

4.0464110

4. 1091922

1.9815070

1. 7645282

1. 3628692

1. 0734235

7.5239610 -
3. 9258494

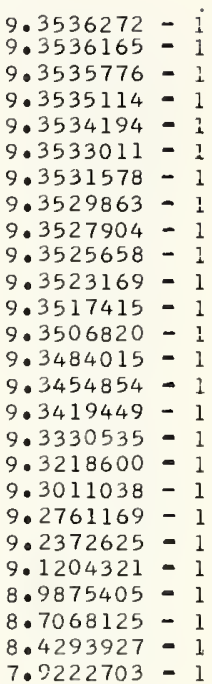

$\mathrm{h}_{3}=90$ kilometers

\begin{tabular}{|c|c|c|}
\hline $\begin{array}{l}4.0426697 \\
4.0426830\end{array}$ & $\begin{array}{l}9.2784521 \\
9.2784435\end{array}$ & \\
\hline 4.0427370 & 9.2784099 & \\
\hline 4.0428271 & 9.2783530 & \\
\hline 4.0429523 & 9.2782743 & \\
\hline 4.0431145 & 9.2781747 & 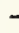 \\
\hline 4.0433125 & 9.2780501 & \\
\hline 4.0435467 & 9.2779044 & \\
\hline 4.0438155 & 9.2777359 & \\
\hline 4.0441220 & 9.2775441 & \\
\hline 4.0444628 & 9.2773324 & \\
\hline 4.0452531 & 9.2768397 & \\
\hline 4.0467061 & 9.2759321 & \\
\hline 4.0498386 & 9.2739798 & \\
\hline 4.0538526 & 9.2714797 & \\
\hline 4.0587373 & 9.2684401 & \\
\hline 4.0710716 & 9.2607830 & \\
\hline 4.0867205 & 9.2511032 & \\
\hline $\begin{array}{l}4.1160801 \\
4 \cdot 1519670\end{array}$ & $\begin{array}{l}9.2330332 \\
9.2110848\end{array}$ & \\
\hline 2.0744148 & 9.1765773 & 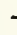 \\
\hline $\begin{array}{l}1.8991264 \\
1.7003879\end{array}$ & $\begin{array}{l}9.0705310 \\
8.9468340\end{array}$ & \\
\hline 1.3291056 & 8.6790810 & \\
\hline $\begin{array}{l}1.0566686 \\
7.4762770\end{array}$ & $\begin{array}{l}8.4097443 \\
7.9117563\end{array}$ & \\
\hline
\end{tabular}

Arg $\mathrm{T}_{\mathrm{mm}}$

$\mathrm{d} / \mathrm{j}$

miles
1.2433895
1.2381675
1.2278386
1.2176623
1.2076369
1.1977599
1.1880278
1.1784374
1.168985
1.1596669
1.1504788
1.1324770
1.1063547
1.0649462
1.0259098
9.889903
9.2076906
8.5914709
7.7737068
7.0657786
6.2644905
4.7915900
3.8174547
2.6471062
1.9802951
1.2445355
3.0604590

3.0604581

3.0604539

3.0604469

3.0604371

3.0604246

3.0604090

3.0603910

3.0603700

3. 0503460

3.0603197

3.0602582

3.0601452

3.0599017

3.0505898

3.0592105

3.0582542

3.0570434

3.0547780

3.0520181

3.0476608

3.0341438

3.0181663

2.9829333

2. 9466901

2.8774475
1.4000780 1.3948535

1. 3845147

1.3743220

1.3642736

1. 3543670

1.3445989

1.334966

1.3254647

1.3160909

1.3068406

1.2886954

1.2623103

1.2203378

1.1805854

1.142803 ?

1.0724503

1.0082107

9.2176798

8.4566114

7.5787071

5.9120543

4. 7678510

3.3479623

2.5187906

1.5902204

Table 42. Ionosphere reflection coefficients (amplitude, $|\mathrm{T}|$, and phase, $\operatorname{Arg} \mathrm{T}$ ) as suming the quasi-longitudinal approximation, for the special ray configuration of the Norton type 301 atmosphere, applicable to the propagation of waves between the D-region $\left(h_{3}=70\right.$ kilometers) or the E-region ( $h_{3}=90$ kilometers) of the ionosphere and the earth. 


$$
\begin{aligned}
& \omega / \omega_{r}=0.2000 \\
& \phi_{1}=60 \text { degrees }
\end{aligned}
$$

$\mathrm{d} / \mathrm{j}$

miles
$\Psi$

radians

$\left|T_{\text {ee }}\right|$

$5.9828483=1$
$5.9827984=1$
$5.9826089=1$
$5.9822914=1$
$5.9818467=1$
$5.9812750=1$
$5.9805795=1$
$5.9797552=1$
$5.9788067=1$
$5.9777306=1$
$5.9765294=1$
$5.9737530=1$
$5.9586527=1$
$5.9576949=1$
$5.9437257=1$
$5.9268340=1$
$5.8847054=1$
$5.8322990=1$
$5.7369789=1$
$5.6254703=1$
$5.4592322=1$
$5.0131577=1$
$4.6060762=1$
$4.0887688=1$
$3.9657297=1$
$4.3189926=1$

0.0005

0.0010

0.0020

0.0030

0.0040

0.0050

0.0060

0.0070

0.0080

0.0090

0.0100

0.0120

0.0150

0.0200

0.0250

0.0300

0.0400

0.0500

0.0650

0.0800

0.1000

0.1500

0.2000

0.3000

0.4000

0.6000
$\operatorname{Arg} \mathrm{T}_{\text {ee }}$

$\left|\mathrm{T}_{\mathrm{mm}}\right|$

$h_{3}=70$ kilometers

0.0005

0.0010

0.0020

0.0030

0.0040

0.0050

0.0060

0.0070

0.0080

0.0090

0.0100

0.0120

0.0150

0.0200

0.0250

0.0300

0.0400

0.0500

0.0650

0.0800

0.1000

0.1500

0.2000

0.4000

0.6000
3.6538869

3.6538967

3.6539351

3.6539994

3.6540892

3.6542047

3.6543456

3.6545122

3.6547043

3.6549219

3.6551651

3.6557281

3.6567632

3.6589941

3. 6618512

3.6653266

3.6740925

3.6852002

3.7060123

3.7314373

3. 7717679

3.8986847

4.0521371

1. 8908749

1. 5718465

1. 1296805 $\mathrm{h}_{3}=90$ kilometers

$5.6357378=1$
$5.6357015-1$
$5.6355534-1$
$5.6353047-1$
$5.6349599-1$
$5.6345155-1$
$5.6339703=1$
$5.6333267-1$
$5.6325882-1$
$5.6317460-1$
$5.6308108-1$
$5.6286434-1$
$5.6246609-1$
$5.6160988-1$
$5.6051689-1$
$5.5919324=1$
$5.5588209-1$
$5.5174447=1$
$5.4416673-1$
$5.3522089-1$
$5.2173359=1$
$4.8477056-1$
$4.5027877-1$
$4.0610025-1$
$3.9683704-1$
$4.3304015=1$

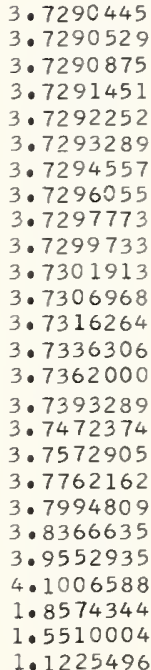

0.3000

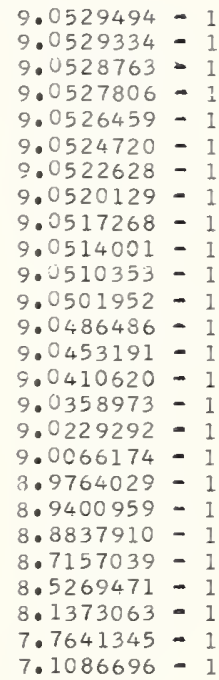

3.0405377

3.0405359

3.0405290

3.0405173

3. 0405009

3.0404798

3. 0404541

3. 0404237

3. 0403887

3. 0403490

3.0403047

3.0402022

3.0400135

3. 0396074

3. 0390879

3.0384568

3.0368683

3.0348632

3.0311296

3. 0266085

3.0195238

2. 9978588

2.9726459

2. 9178312

2. 8620243

2. 7562433

$8.9434850-1$
$8.9434734-1$
$8.9434247-1$
$8.9433425-1$
$8.9432289-1$
$8.9430825-1$
$8.9429022-1$
$8.9426903-1$
$8.9424462-1$
$8.9421678-1$
$8.9418605-1$
$8.9411446-1$
$8.9398288-1$
$8.9369942-1$
$8.9333659-1$
$8.9289568-1$
$8.9178550-1$
$8.9038273-1$
$8.8776738-1$
$8.8459654-1$
$8.7962442-1$
$8.6445089-1$
$8.4696740-1$
$8.0994856-1$
$7.7381257-1$
$7.0954020-1$
19

3.0270321

3.0270306

3. 0270245

3.0270142

3.0270001

3.0269818

3.0269594

3.0269328

3.0269023

3.0268676

3.0268290

3. 0267395

3.0265750

3.0262204

3. 0257662

3.0252139

3.0238204

3.0220550

3.0187488

3. 0147151

3.0083347

2. 9884562

2. 9648181

2. 9123201

2. 8580166

2.7539962 $\begin{array}{ll}1.2433895 & 3 \\ 1.2381675 & 3 \\ 1.2278386 & 3 \\ 1.2176623 & 3 \\ 1.2076369 & 3 \\ 1.1977599 & 3 \\ 1.1880278 & 3 \\ 1.1784374 & 3 \\ 1.1689853 & 3 \\ 1.1596669 & 3 \\ 1.1504788 & 3 \\ 1.1324770 & 3 \\ 1.1063547 & 3 \\ 1.0649462 & 3 \\ 1.0259098 & 3 \\ 9.8899038 & 2 \\ 9.2076906 & 2 \\ 8.5914709 & 2 \\ 7.7737068 & 2 \\ 7.0657736 & 2 \\ 6.2644905 & 2 \\ 4.7915900 & 2 \\ 3.8174547 & 2 \\ 2.6471062 & 2 \\ 1.9802951 & 2 \\ 1.2445355 & 2\end{array}$

1. 4000780 1. 3948535

1. 3845147

1. 3743220

I. 3642736

1. 3543670

1. 3445989

I. 3349661

1.3254647

1. 3160909

1. 3068406

1. 2886954

1. 2623103

I. 2203378

1. 1805854

1. 1428039

1.0724503

1.0082107

9.2176798

8.4566114

7. 5787071

5. 9120543

4. 7678510

3.3479623

2. 5187906

1.5902204

3
3
3
3
3
3
3
3
3
3
3
3
3
3
3
2
2
2
2
2
2
2
2
2
2
2

Table 43. Ionosphere reflection coefficients (amplitude, |T|, and phase, Arg T) assuming the quasi-longitudinal approximation, for the special ray configuration of the Norton type 301 atmosphere, applicable to the propagation of waves between the $\mathrm{D}$-region $\left(\mathrm{h}_{3}=70\right.$ kilometers) or the E-region (h $=90$ kilometers) of the ionosphere and the earth.

-117 - 
$\omega / \omega_{r}=1.0000$
$\phi_{1}=60$ degr

$\phi_{1}{ }^{r}=60$ degrees

$\Psi$

radians

$\left|T_{\text {ee }}\right|$

Arg $\mathrm{T}_{\text {ee }}$

$\left|\mathrm{T}_{\mathrm{mm}}\right|$

$\operatorname{Arg}_{\mathrm{mm}}$

d) $\mathrm{j}$

$\mathrm{h}_{3}=70$ kilometers

$\begin{array}{ll}0.0005 & 7.5550691=1 \\ 0.0010 & 7.5550318=1 \\ 0.0020 & 7.5548851=1 \\ 0.0030 & 7.5546408=1 \\ 0.0040 & 7.5542974=1 \\ 0.0050 & 7.5538581=1 \\ 0.0060 & 7.5533195=1 \\ 0.0070 & 7.5526856=1 \\ 0.0080 & 7.5519554=1 \\ 0.0090 & 7.5511255=1 \\ 0.0100 & 7.5501994=1 \\ 0.0120 & 7.5480560=1 \\ 0.0150 & 7.5441197=1 \\ 0.0200 & 7.5356465=1 \\ 0.0250 & 7.5248163=1 \\ 0.0300 & 7.5116766=1 \\ 0.0400 & 7.4787014=1 \\ 0.0500 & 7.4372530=1 \\ 0.0650 & 7.3605897=1 \\ 0.0800 & 7.2686398=1 \\ 0.1000 & 7.1264452=1 \\ 0.1500 & 6.7050502=1 \\ 0.2000 & 6.2378751=1 \\ 0.3000 & 5.2947874=1 \\ 0.4000 & 4.4167566=1 \\ 0.6000 & 2.9232239=1 \\ 0 & \end{array}$

3. 1812521

3. 1812548

3. 1312586

3.1812639

3.1812707

3. 1812791

3. 1812888

3.1813002

3. 1813128

3. 1813272

3. 1813603

3.1814210

3. 1815518

3.1817190

3. 1819217

3.1824301

3. 1830689

3. 1842492

3. 1856636

3. 1878497

3.1943516

3. 2017191

3. 2182845

3. 2394824

3. 3261082

$\mathrm{h}_{3}=90$ kilometers

0.0005
0.0010
0.0020
0.0030
0.0040
0.0050
0.0060
0.0070
0.0080
0.0090
0.0100
0.0120
0.0150
0.0200
0.0250
0.0300
0.0400
0.0500
0.0650
0.0800
0.1000
0.1500
0.2000
0.3000
0.4000
0.6000
0
3.1812525

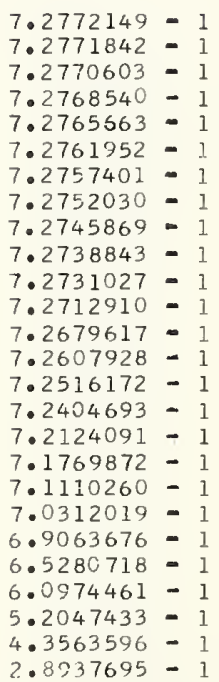

$7.9553533=1$
$7.9553224=1$
$7.9551995-1$
$7.9549950-1$
$7.9547074-1$
$7.9543395-1$
$7.9538879-1$
$7.9533574=1$
$7.9527463-1$
$7.9520514-1$
$7.9512751-1$
$7.9494801-1$
$7.9461845-1$
$7.9390887-1$
$7.9300200-1$
$7.9190190-1$
$7.8914151-1$
$7.8567277-1$
$7.7926023-1$
$7.7157495=1$
$7.5970477-1$
$7.2466082-1$
$6.8612185-1$
$6.0982271-1$
$5.4128068-1$
$4.3061552-1$

$4.3061552-1$
2.9634975
2.9634940
2.9634810
2.9634590
2.9634284
2.9633890
2.9633409
2.9632840
2.9632185
2.9631441
2.9630611
2.9628690
2.9625156
2.9617544
2.9607800
2.9595952
2.9566092
2.9528313
2.9457691
2.9371701
2.9235906
2.8812528
2.8304061
2.7137884
2.5860635
2.3162399 $\begin{array}{ll}1.2433895 & 3 \\ 1.2381675 & 3 \\ 1.2278386 & 3 \\ 1.2176623 & 3 \\ 1.2076369 & 3 \\ 1.1977599 & 3 \\ 1.1880278 & 3 \\ 1.1784374 & 3 \\ 1.1689853 & 3 \\ 1.1596669 & 3 \\ 1.1504788 & 3 \\ 1.1324770 & 3 \\ 1.1063547 & 3 \\ 1.0649462 & 3 \\ 1.0259098 & 3 \\ 9.8899038 & 2 \\ 9.2076906 & 2 \\ 8.5914709 & 2 \\ 7.7737068 & 2 \\ 7.0657786 & 2 \\ 6.2644905 & 2 \\ 4.7915900 & 2 \\ 3.8174547 & 2 \\ 2.6471062 & 2 \\ 1.9802951 & 2 \\ 1.2445355 & 2\end{array}$

1.4000780 1. 3948535

1. 3845147

I. 3743220

1.3642736

1. 3543670

1.3445989

1.3349661

1.3254647

1. 3160909

1. 3068406

1.2886954

1.2623103

1.2203378

1.1805854

1.0724503

1.0082107

9.2176798

8.4566114

7.5787071

5.9120543

4. 7678510

3.3479623

2. 5187906

1.5902204
3
3
3
3
3
3
3
3
3
3
3
3
3
3
3
3
3
3
2
2
2
2
2
2
2
2
1. 1428039

2. 7015889

2.576524

(1)

Table 44. Ionosphere reflection coefficients (amplitude, $|\mathrm{T}|$, and phase, Arg $\mathrm{T}$ ) assuming the quasi-longitudinal approximation, for the special ray configuration of the Norton type 301 atmosphere, applicable to the propagation of waves between the D-region ( $h_{3}=70$ kilometers) or the E-region $\left(h_{3}=90\right.$ kilometers) of the ionosphere and the earth. 


$$
\begin{array}{ll}
\omega / \omega_{\mathbf{r}} & =2.0000 \\
\phi_{1} & =60 \text { degrees }
\end{array}
$$

$\Psi$

radians

$\begin{array}{ll}0.0005 & 7.2509765=1 \\ 0.0010 & 7.2509356=1 \\ 0.0020 & 7.2507719=1 \\ 0.0030 & 7.2505005=1 \\ 0.0040 & 7.2501208=1 \\ 0.0050 & 7.2496315=1 \\ 0.0060 & 7.2490370=1 \\ 0.0070 & 7.2483329=1 \\ 0.0080 & 7.2475205=1 \\ 0.0090 & 7.2466009=1 \\ 0.0100 & 7.2455730=1 \\ 0.0120 & 7.2431964=1 \\ 0.0150 & 7.2388277=1 \\ 0.0200 & 7.2294256=1 \\ 0.0250 & 7.2174098=1 \\ 0.0300 & 7.2028350=1 \\ 0.0400 & 7.1662579=1 \\ 0.0500 & 7.1202974=1 \\ 0.0650 & 7.0353096=1 \\ 0.0800 & 6.9334169=1 \\ 0.1000 & 6.7759222=1 \\ 0.1500 & 6.3093948=1 \\ 0.2000 & 5.7914857=1 \\ 0.3000 & 4.7358871=1 \\ 0.4000 & 3.7236076=1 \\ 0.6000 & 1.8648557=1\end{array}$

$\left\lceil\mathrm{T}_{\text {ee }}\right\rfloor$

Arg $\mathrm{T}_{\text {ee }}$

$\left|\mathrm{I}_{\mathrm{mm}}\right|$

Arg $\mathrm{T}_{\mathrm{mm}}$

$\mathrm{d} / \mathrm{j}$

$h_{3}=70$ kilometers
$7.5497005-1$ $7.5496632-1$

$7.5495194-1$

$7.5492799-1$

$7.5489433-1$

7.5485111 - 1

$7.5479841-1$

$7.5473610-1$

7.5466428 - 1

$7.5458286-1$

$7.5449201-1$

$7.5428158-1$

7.5389520 - 1

$7.5306347-1$

$7.5200074-1$

$7.5071176-1$

$7.4747868-1$

7.4341867 - 1

$7.3591899-1$

$7.2694229-1$

$7.1310052-1$

6.7237573 - 1

$6.2774932-1$

5.3916486 - 1

4.5740439 - 1

3. $1007685-1$
2.8924725

2.8924678

2.8924490

2.8924176

2. 8923737

2.8923172

2.8922483

2.8921669

2.8920729

2.8919664

2. 8918475

2.8915723

2.8910662

2. 8899755

2.8885788

2. 8868804

2.8825973

2.8771729

2. 8670175

2. 8546246

2.8349914

2.7732779

2.6981201

2.5211855

2.3199268

1.8825428

$\begin{array}{ll}1.2433895 & 3 \\ 1.2381675 & 3 \\ 1.2278386 & 3 \\ 1.2176623 & 3 \\ 1.2076369 & 3 \\ 1.1977599 & 3 \\ 1.1880278 & 3 \\ 1.1784374 & 3 \\ 1.1689853 & 3 \\ 1.1596669 & 3 \\ 1.1504788 & 3 \\ 1.1324770 & 3 \\ 1.1063547 & 3 \\ 1.0649462 & 3 \\ 1.0259098 & 3 \\ 9.8899038 & 2 \\ 9.2076906 & 2 \\ 8.5914709 & 2 \\ 7.7737068 & 2 \\ 7.0657786 & 2 \\ 6.2644905 & 2 \\ 4.7915900 & 2 \\ 3.8174547 & 2 \\ 2.6471062 & 2 \\ 1.9802951 & 2 \\ 1.2445355 & 2\end{array}$

2.8557903

2.8557861

2. 8557694

2.8557413

2.8557022

2.8556517

2.8555901

2.8555171

2.8554332

2. 8553378

2.8552316

2.8549853

2.8545324

2. 8535560

2.8523045

2. 8507807

2. 8469297

2. 8420360

2. 8328274

2. 8215136

2.8034390

2.7456810

2. 6740194

2. 5022973

2.3046018

1. 8728959

\begin{tabular}{ll}
1.4000780 & 3 \\
1.3948535 & 3 \\
1.3845147 & 3 \\
1.3743220 & 3 \\
1.3642736 & 3 \\
1.3543670 & 3 \\
1.3445989 & 3 \\
1.3349661 & 3 \\
1.3254647 & 3 \\
1.3160909 & 3 \\
1.3068406 & 3 \\
1.2886954 & 3 \\
1.2623103 & 3 \\
1.2203378 & 3 \\
1.1805854 & 3 \\
1.1428039 & 3 \\
1.0724503 & 3 \\
1.0082107 & 3 \\
9.2176798 & 2 \\
8.4566114 & 2 \\
7.5787071 & 2 \\
5.9120543 & 2 \\
4.7678510 & 2 \\
3.3479623 & 2 \\
2.5187906 & 2 \\
1.5902204 & 2 \\
\hline
\end{tabular}

Table 45. Ionosphere reflection coefficients (amplitude, $[\mathrm{T}]$, and phase, Ar g $\mathrm{T}$ ) assuming the quasi-longitudinal approximation, for the special ray configuration of the Norton type 301 atmosphere, applicable to the propagation of waves between the D-region ( $h_{3}=70$ kilometers) or the E-region $\left(h_{3}=90\right.$ kilometers) of the ionosphere and the earth. 
$\omega / \omega_{r}=5.0000$
$\phi_{1}=60$ degrees

$\underset{\text { radians }}{\Psi}$

$|\mathrm{T} e \mathrm{e}|$

Arg $\mathrm{T}_{\text {ee }}$

$\left|\mathrm{T}_{\mathrm{mm}}\right|$

Arg $\mathrm{T}_{\mathrm{mm}}$

$\mathrm{d} / \mathrm{j}$

$\mathrm{h}_{3}=70$ kilometers

$\begin{array}{ll}0.0005 & 6.3820453=1 \\ 0.0010 & 6.3819940=1 \\ 0.0020 & 6.3817905=1 \\ 0.0030 & 6.3814491=1 \\ 0.0040 & 6.3809700=1 \\ 0.0050 & 6.3803564=1 \\ 0.0060 & 6.3796066=1 \\ 0.0070 & 6.3787227=1 \\ 0.0080 & 6.3777010=1 \\ 0.0090 & 6.3765451=1 \\ 0.0100 & 6.3752520=1 \\ 0.0120 & 6.3722615=1 \\ 0.0150 & 6.3667676=1 \\ 0.0200 & 6.3549466=1 \\ 0.0250 & 6.3398444=1 \\ 0.0300 & 6.3215284=1 \\ 0.0400 & 6.2755918=1 \\ 0.0500 & 6.2179251=1 \\ 0.0650 & 6.1114450=1 \\ 0.0800 & 5.9840256=1 \\ 0.1000 & 5.7875235=1 \\ 0.1500 & 5.2075765=1 \\ 0.2000 & 4.5638839=1 \\ 0.3000 & 3.2285550=1 \\ 0.4000 & 1.9323360-1 \\ 0.6000 & 4.4151811=2 \\ 0 & \end{array}$

2. 7546309

2. 7546232

2.7545920

2. 7545397

2. 7544666

2. 7543728

2. 7542582

2. 7541228

2. 7539667

2. 7537898

2. 7535917

2. 7531341

2. 7522921

2. 7504771

2. 7481514

2. 7453201

2. 7381698

2. 7290901

2. 7120192

2.6910584

2. 6575613

2. 5499170

2.4139570

2.0753448

1. 6972692

1. 2943800

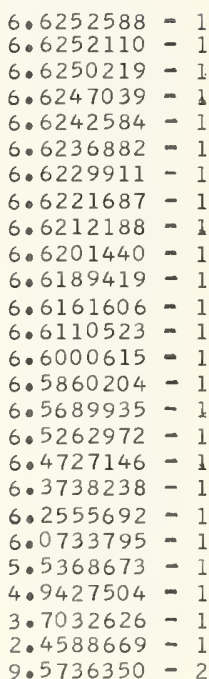

$9.5736350=$
2.7095843

2.7095759

2.7095420

2.7094853

2.7094059

2.7093040

2.7091795

2.7090325

2. 7088630

2.7086709

2.7084559

2.7079590

2. 7070448

2.7050747

2.7025508

2.6994796

2.6917290

2.6818989

2.6634530

2.6408682

2.6049228

2.4906422

2.3490138

2.0100038

1.6601140

1.3948472 $\begin{array}{ll}1.2433895 & 3 \\ 1.2381675 & 3 \\ 1.2278386 & 3 \\ 1.2176623 & 3 \\ 1.2076369 & 3 \\ 1.1977599 & 3 \\ 1.1880278 & 3 \\ 1.1784374 & 3 \\ 1.1689853 & 3 \\ 1.1596669 & 3 \\ 1.1504788 & 3 \\ 1.1324770 & 3 \\ 1.1063547 & 3 \\ 1.0649462 & 3 \\ 1.0259098 & 3 \\ 9.8899038 & 2 \\ 9.2076906 & 2 \\ 8.5914709 & 2 \\ 7.7737068 & 2 \\ 7.0657786 & 2 \\ 6.2644905 & 2 \\ 4.7915900 & 2 \\ 3.8174547 & 2 \\ 2.6471062 & 2 \\ 1.9802951 & 2 \\ 1.2445355 & 2\end{array}$

$\mathrm{h}_{3}=90$ kilometers

0.0005
0.0010
0.0020
0.0030
0.0040
0.0050
0.0060
0.0070
0.0080
0.0090
0.0100
0.0120
0.0150
0.0200
0.0250
0.0300
0.0400
0.0500
0.0650
0.0800
0.1000
0.1500
0.2000
0.3000
0.4000
0.6000

$5.9958955-1$

2. 6930361

2.6930291

2.6930007

2.6929530

2. 6928866

5.9956825 -

$5.9953959-1$

$5.9949969-1$

$5.9944832-1$

$5.9938535-1$

$5.9931103-1$

$5.9922582=1$

$5.9912850-1$

$5.9902018=1$

$5.9876964=1$

$5.9830876-1$

5.9731654 -

5.9604688 .

$5.9450475-1$

$5.9062479=1$

$5.8572994=1$

$5.7662489-1$

5.6562051 -

5.4843545 -

$4.9641952=1$

$4.3690886-1$

$3.0968186-1$

1. $8461446-1$

26928

2. 6926964

2.6925726

2.6924305

2.6922687

2.6920883

2.6916707

2.6909020

2.6892447

2.6871188

2.6845284

2.6779724

2.6696217

2.6538476

2.6343589

2. 6029748

2. 5006144

2. 3692444

2.0382825

I. 6720286

1. 2896447

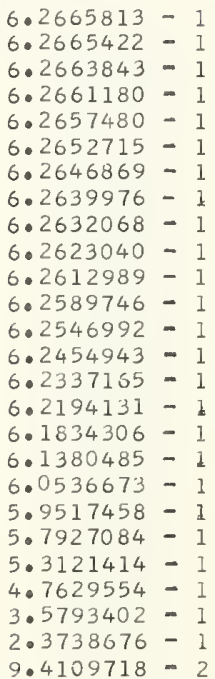

$2 \cdot 64$

2. 6429885

2.6429580

2.6429067

2.6428352

2.6427432

2.6426305

2.6424973

$2 \cdot 6423444$

2.6421703

2.6419762

2.6415269

2.6406999

2.6389172

2.6366311

2.6338407

2.6268042

2.6178438

2.6009488

2.5801308

2. 5467355

2.4389316

2.3031091

i. 9742511

1.6383142

1.3942786 jiles
I. 4000780

. 3948535

1. 3845147

1.3743220

1. 3642736

1. 3543670

1. 3445989

1. 3349661

1. 3254647

1. 3160909

1.3068406

1.2886954

1. 2623103

1. 2203378

I. 1805854

1. 1428039

1.0724503

1.0082107

9.2176798

8.4566114

7. 5787071

5. 9120543

4. 7678510

3.3479623

2. 5187906

2.5187906

(1)

Table 46. Ionosphere reflection coefficients (amplitude, $|\mathrm{T}|$, and phase, Arg $\mathrm{T}$ ) assuming the quasi-longitudinal approximation, for the special ray configuration of the Norton type 301 atmosphere, applicable to the propagation of waves between the D-region ( $h_{3}=70$ kilometers) or the E-region $\left(h_{3}=90\right.$ kilometers) of the ionosphere and the earth. 


$$
\begin{aligned}
& \omega / \omega_{r}=0.3002 \\
& \phi_{1}=60 \text { degrees }
\end{aligned}
$$

$\Psi$

radians

$\left|\mathrm{T}_{\text {em }}\right|$

$1.2267135=1$
$1.2267293=1$
$1.2267926=1$
$1.2268984=1$
$1.2270461=1$
$1.2272363=1$
$1.2274682=1$
$1.2277421=1$
$1.2280582=1$
$1.2284160=1$
$1.2288160=1$
$1.2297407=1$
$1.2314391=1$
$1.2350914=1$
$1.2397533=1$
$1.2453994=1$
$1.2595233=1$
$1.2771823=1$
$1.3095701=1$
$1.3479424=1$
$1.4062588=1$
$1.5717018=1$
$1.7420445=1$
$2.0431788=1$
$2.2717524=1$
$2.5505887-1$

0.0005

0.0010

0.0020

0.0030

0.0040

0.0050

0.0060

0.0070

0.0080

0.0090

0.0100

0.0120

0.0150

0.0200

0.0250

0.0300

0.0400

0.0500

0.0650

0.0800

0.1000

0.1500

0.2000

0.3000

C. 4000

0.6000
Arg $\mathrm{T}_{\text {em }}-\pi \quad\left|\mathrm{T}_{\text {me }}\right|$

$\mathrm{h}_{3}=70$ kilometers

0.0005

0.0010

0.0020

0.0030

0.0040

0.0050

0.0060

0.0070

0.0080

0.0090

0.0100

0.0120

0.0150

0.0200

0.0250

0.0300

0.0400

c. 0500

0.0650

0.0800

0.1000

0.1500

0.2000

0.3000

0.4000

0.6000

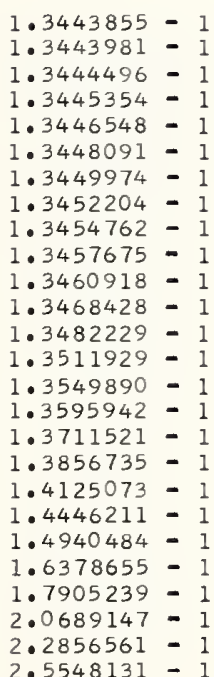

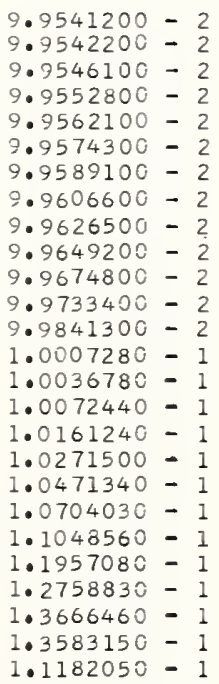

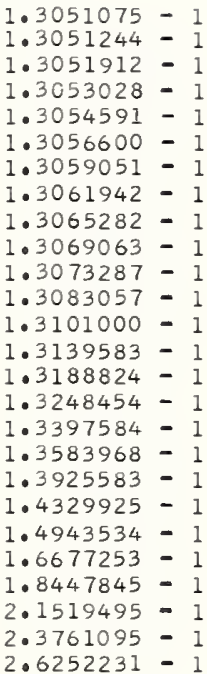

$\mathrm{h}_{3}=90$ kilometer $\mathrm{s}$

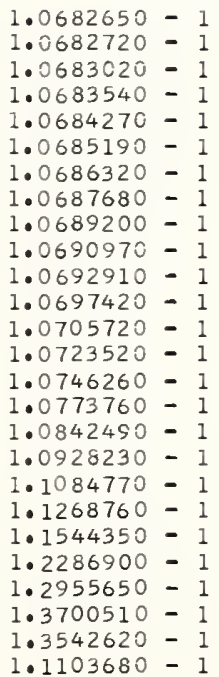
1. 4292600 $93140=$ 4294043 1.4295301 $1.4296928-1$ 1.4298910 $1.4301260-1$ $1.4303953-1$ $1.4307022=$ 1. $4310437=$ $1.4318348-1$ 1.4332882 1.4364159 $.4452618=$ 1.4574276 09215 $.5346535=$ 367021 8948232 2.1776912 $2.3893407=$
Arg $T_{\text {me }}-\pi$

$\mathrm{d} / \mathrm{j}$

miles

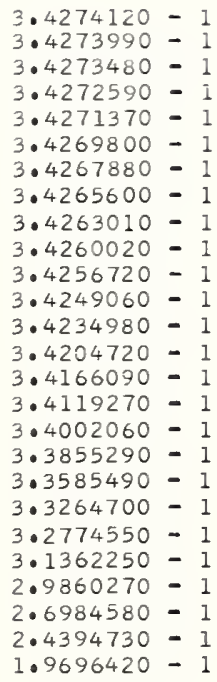

1. 2433895 1.2381675 1.2278386 1. 2176623 1.2076369 1. 1977599 1. 1880278 1. 1784374 1. 1689853 1.1596669 1. 1504788 1.1324770 1. 1063547 1.0649462 1.0259098 9.8899038 9.2076906 8.5914709 7.7737068 7.0657786 6.2644905 4. 7915900 3. 8174547 2.6471062 1.9802951 1.2445355

Table 47. Ionosphere reflection coefficients (amplitude, $|\mathrm{T}|$, and phase, $\mathrm{A}_{\mathrm{r}} \mathrm{g} \mathrm{T}$ ) assuming the quasi-longitudinal approximation, for the special ray configuration of the Norton type 301 atmosphere, applicable to the propagation of waves between the D-region ( $h_{3}=70$ kilometers) or the E-region ( $h_{3}=90$ kilometers) of the ionosphere and the earth.

$\begin{array}{lll}3.3294490-1 & 1.4000780 & 3 \\ 3.3294390-1 & 1.3948535 & 3 \\ 3.3293970-1 & 1.3845147 & 3 \\ 3.3293240-1 & 1.3743220 & 3 \\ 3.3292250-1 & 1.3642736 & 2 \\ 3.3290950-1 & 1.3543670 & 3 \\ 3.3289380-1 & 1.3445989 & 3 \\ 3.3287510-1 & 1.3349661 & 3 \\ 3.3285370-1 & 1.3254647 & 3 \\ 3.3282920-1 & 1.3160909 & 3 \\ 3.3280200-1 & 1.3068406 & 3 \\ 3.3273920-1 & 1.2886954 & 3 \\ 3.3262370-1 & 1.2623103 & 3 \\ 3.3237470=1 & 1.2203378 & 3 \\ 3.3205650-1 & 1.1805854 & 3 \\ 3.3167040-1 & 1.1428039 & 3 \\ 3.3070030-1 & 1.0724503 & 3 \\ 3.2947960-1 & 1.0082107 & 3 \\ 3.2721820-1 & 9.2176798 & 2 \\ 3.2450180-1 & 8.4566114 & 2 \\ 3.2029660-1 & 7.5787071 & 2 \\ 3.0785860-1 & 5.9120543 & 2 \\ 2.9420380-1 & 4.7678510 & 2 \\ 2.6717450-1 & 3.3479623 & 2 \\ 2.4215960-1 & 2.5187906 & 2 \\ 1.9593290-1 & 1.5902204 & 2\end{array}$

3
3
3
3
3
3
3
3
3
3
3
3
3
3
2
2
2
2
2
2
2
2
2
2
2




$$
\begin{aligned}
& \omega / \omega_{\mathbf{r}}=0.467 \\
& \phi_{1}=60 \text { degrees }
\end{aligned}
$$

$\Psi$ radians

$$
\mid \mathrm{T} \text { em } \mid
$$

$\operatorname{Arg} T_{\text {em }}-\pi$

\begin{tabular}{|c|c|c|}
\hline 0.0005 & 1.2025081 & - \\
\hline 0.0010 & 1.2025243 & $=$ \\
\hline 0.0020 & 1.2025879 & - \\
\hline 0.0030 & 1.2026947 & - \\
\hline 0.0040 & 1.2028439 & - \\
\hline 0.0050 & 1.2030354 & - \\
\hline 0.0060 & 1.2032695 & - \\
\hline 0.0070 & 1.2035457 & - \\
\hline 0.0080 & 1.2038641 & - \\
\hline 0.0090 & 1.2042251 & $=$ \\
\hline 0.0100 & 1.2046287 & - \\
\hline 0.0120 & 1.2055611 & - \\
\hline 0.0150 & 1.2072745 & - \\
\hline 0.0200 & 1.2109590 & - \\
\hline 0.0250 & 1.2156629 & - \\
\hline 0.0300 & 1.2213619 & - \\
\hline 0.0400 & 1.2356259 & - \\
\hline 0.0500 & 1.2534754 & - \\
\hline 0.0650 & 1.2862597 & - \\
\hline 0.0800 & 1.3251802 & - \\
\hline 0.1000 & 1.3844982 & - \\
\hline 0.1500 & 1.5539357 & - \\
\hline 0.2000 & 1.7303076 & - \\
\hline 0.3000 & 2.0475211 & - \\
\hline 0.4000 & 2.2938094 & - \\
\hline 0.6000 & 2.6027912 & $=$ \\
\hline
\end{tabular}

$\mathrm{h}_{3}=70$ kilometers

$\begin{array}{ll}7.0070700-2 & 1.3828884-1 \\ 7.0071000-2 & 1.3829067-1 \\ 7.0071800-2 & 1.3829790-1 \\ 7.0073300-2 & 1.3830997-1 \\ 7.0075100-2 & 1.3832689-1 \\ 7.0077700-2 & 1.3834857-1 \\ 7.0080800-2 & 1.3837512-1 \\ 7.0084400-2 & 1.3840640-1 \\ 7.0088500-2 & 1.3844249=1 \\ 7.0093300-2 & 1.3848340-1 \\ 7.0098500-2 & 1.3852910-1 \\ 7.0110400-2 & 1.3863476-1 \\ 7.0132700-2 & 1.3882884=1 \\ 7.0179700-2 & 1.3924620-1 \\ 7.0239200-2 & 1.3977893-1 \\ 7.0309800-2 & 1.4042415-1 \\ 7.0480600-2 & 1.4203828-1 \\ 7.0682200-2 & 1.4405646-1 \\ 7.1015000-2 & 1.4775811-1 \\ 7.1345100-2 & 1.5214344-1 \\ 7.1704500-2 & 1.5880620-1 \\ 7.1645000-2 & 1.7767290-1 \\ 6.9477400-2 & 1.9697145-1 \\ 5.7570600-2 & 2.3028312-1 \\ 3.6225800-2 & 2.5397696-1 \\ 2.6795800-2 & 2.7762778-1\end{array}$

$\mathrm{h}_{3}=90$ kilometers

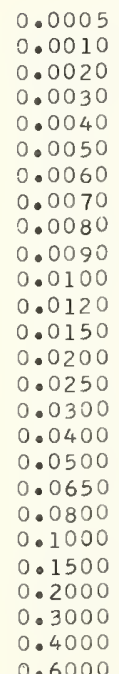

$1.3215692-1$
$1.3215821-1$
$1.3216341-1$
$1.3217211=1$
$1.3218422-1$
$1.3219990=1$
$1.3221905-1$
$1.3224165-1$
$1.3226763-1$
$1.3229720-1$
$1.3233015-1$
$1.3240641-1$
$1.3254653-1$
$1.3284813-1$
$1.3323374-1$
$1.3370159-1$
$1.3487642-1$
$1.3635362-1$
$1.3908664-1$
$1.4236323-1$
$1.4741895-1$
$1.6222002-1$
$1.7808855=1$
$2.0749913-1$
$2.3089716-1$
$2.6075628-1$
1.352

\section{$\left|\mathrm{T}_{\mathrm{me}}\right|$}

$\operatorname{Arg~} \mathrm{T}_{\mathrm{me}}{ }^{-\pi}$

6.2388590 6.2388530 $6.2388280=$ 6.2387840 - 1 $6.2387250-1$ 6.2386490 $6.2385560=1$ $6.2384470-1$ 6.2383190 $6.2381770-1$ $6.2380160-1$ $6.2376450=$ 6.2369620 - 1 $6.2354980=-1$ $6.2336260-1$ 6.2313570 $6.2256740=1$ $6.2185490-1$ $6.2054300-1$ $6.1897870-1$ $6.1657840=-1$ $6.0956570-1$ 6.0187000 5.8584860 $5.6876230=$ $5.2685820-1$ $d / j$

Miles

1.2433895 1.2381675

1. 2278386

1.2176623

1.2076369

1.1977599

1. 1880278

1. 1784374

1.1689853

1. 1596669

I. 1504788

1. 1324770

1. 1063547

1.0649462

1.0259098

9.8899038

9.2076906

8. 5914709

7.7737068

7.0657786

6.2644905

4. 7915900

3. 8174547

2.6471062

1.9802951

1.2445355
$6.1912420=$ 6.1912370 - 1 $6.1912160-1$ $6.1911810-?$ $6.1911330-1$ $6.1910700-1$ $6.1909940-1$ 6. $1909010-1$ 6.1907980 - 1 $6.1906780-1$ $6.1905440-1$ $6.1902380-1$ $6.1896750-1$ $6.1884580-1$ $6 \cdot 1869040=1$ $6.1850160-1$ $6.1802690-1$ $6.1742910=1$ $6.1631940-1$ $6.1498150-1$ $6.1290020-1$ $6.0664820-1$ 5.9954920 $5.8423030=1$ $5.6744950-1$ $5.2575580-1$
1. 4000780 1. 3948535

1. 3845147 1. 3743220 1.3642736 1. 3543670 1.3445989 I. 3349661 1.3254647 1. 3160909 1.3068406 1. 2886954 1.2623103 1. 2203378 1.1805854 1.1428039 1.0724503

1.0082107 9. 2176798 8.4566114 7.5787071

5.9120543 4. 7678510 3.3479623 2.5187906 1.5902204

3
3
3
3
3
3
3
3
3
3
3
3
3
3
3
2
2
2
2
2
2
2
2
2
2
2

Table 48. Ionosphere reflection coefficients (amplitude, $|\mathrm{T}|$, and phase, $\operatorname{Arg} \mathrm{T}$ ) assuming the quasi-longitudinal approximation, for the special ray configuration of the Norton type 301 atmosphere, applicable to the propagation of waves between the D-region ( $h_{3}=70$ kilometers) or the E-region $\left(h_{3}=90\right.$ kilometers) of the ionosphere and the earth. 
$\omega / \omega_{r}=0.1501$
$\phi_{1}=60$ degrees

radians

$\mid \mathrm{T}$ em $\mid$

$\operatorname{Arg} \mathrm{T}_{\mathrm{em}}-\pi \quad\left|\mathrm{T}_{\mathrm{me}}\right|$

$\mathrm{h}_{3}=70$ kilometers

$\begin{array}{ll}0.0005 & 1.1824714=1 \\ 0.0010 & 1.1824857=1 \\ 0.0020 & 1.1825425=1 \\ 0.0030 & 1.1826370=1 \\ 0.0040 & 1.1827695=1 \\ 0.0050 & 1.1829398=1 \\ 0.0060 & 1.1831477=1 \\ 0.0070 & 1.1833930=1 \\ 0.0080 & 1.1836762=1 \\ 0.0090 & 1.1839970=1 \\ 0.0100 & 1.1843551=1 \\ 0.0120 & 1.1851833=1 \\ 0.0150 & 1.1867046=1 \\ 0.0200 & 1.1899747=1 \\ 0.0250 & 1.1941461=1 \\ 0.0300 & 1.1991950=1 \\ 0.0400 & 1.2118084=1 \\ 0.0500 & 1.2275450=1 \\ 0.0650 & 1.2563099=1 \\ 0.0800 & 1.2902266=1 \\ 0.1000 & 1.3414304=1 \\ 0.1500 & 1.4844375=? \\ 0.2000 & 1.6281416=1 \\ 0.3000 & 1.8731917=1 \\ 0.4000 & 2.0512548=1 \\ 0.6000 & 2.2583920=1\end{array}$

0.0005

0.0010

0.0020

0.0030

0.0040

0.0050

0.0060

0.0070

0.0080

0.0090

0.0100

0.0120

0.0150

0.0200

0.0250

0.0300

0.0400

0.0500

0.0650

0.0800

0.1000

0.1500

0.2000

0.3000

0.4000

0.6000

$2.2583920-7$

$1.7370830-1$
$1.7371100-1$
$1.7371890-1$
$1.7373230-1$
$1.7375110-1$
$1.7377510-1$
$1.7380470-1$
$1.7383940-1$
$1.7387940-1$
$1.7392480-1$
$1.7397560-1$
$1.7409280-1$
$1.7430810-1$
$1.7477100-1$
$1.7535090-1$
$1.7607490-1$
$1.7785680-1$
$1.8007660-1$
$1.8412450-1$
$1.3888020-1$
$1.9602170-1$
$2.1567110-1$
$2.3482260-1$
$2.6511840-1$
$2.8340940-1$
$2.9334050-1$

$\mathrm{h}_{3}=90$ kilometers

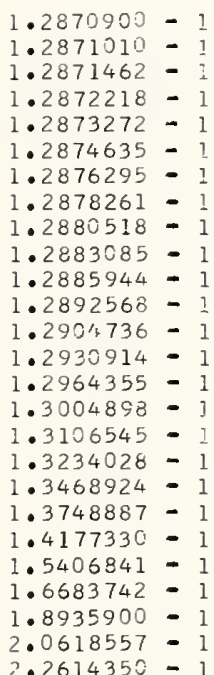

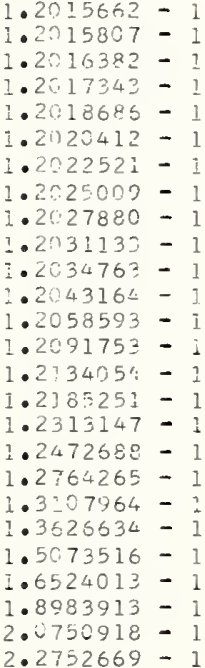

$2.2752669-$
$\operatorname{Arg~} \mathrm{T}_{\mathrm{me}}-\pi \quad \mathrm{d} / \mathrm{j}$

$4.6833202-2 \quad 1.2381673$

$4.6821500-2$ i. 2278380

$4.6807002-2-1.2176523$

$4.6786700-2 \quad 1.2076360$

$4.6760800-2 \quad i .1977593$

$4.6729002-2-1.1880273$

$4.6691500-? 1.1784374$

$4.6648100-2 \quad 2.1509053$

$4.6502000-2-1596569$

$4.6544200-2 \quad 1.1504788$

$4.6417400-2 \quad 1.1324770$

$4.6184600-21.1063547$

$4.5634100-2 \quad 2.0640462$

$4.5045700=2 \quad 1.0259073$

$4.4272400-2$

$4.2339600-2 \quad 3.2076506$

$3.9925700-2 \quad 3.5914709$

$3.5506300-27.7737058$

3.0282600 - 27.0557700

$2.2368200-2 \quad 5.2644905$

$4.8000000-5-4.7915900$

$2.2807800-2 \quad 3.8174547$

$6.3467100-2 ? .6471062$

$9.5723400-2 \quad 1.3802951$

$1.4215600-1 \quad 2.2445355$

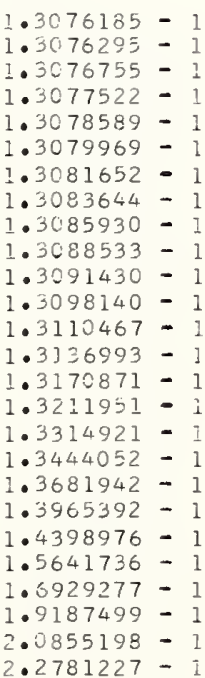

$\therefore .4000720$

. 3948535

i. 3845147

1. 3743220

. 1642736

$1 \cdot 35,43670$

1. 3445982

1. 3349661

$1.325464 ?$

1. 3160900

1. 3068400

1.2886954

1. 2623103

1. 2203378

1.1805854

1.1428039

1.0724503

1.0082107

9.2176798

8.4566114

7.5787071

5. 9120543

4. 7678510

3.3479623

2. 5137906

Table 49. Ionosphere reflection coefficients (amplitude, $|\mathrm{T}|$, and phase, Arg $\mathrm{T}$ ) assuming the quasi-longitudinal approximation, for the special ray configuration of the Norton type 301 atmosphere, applicable to the propagation of waves between the D-region ( $h_{3}=70$ kilometers) or the E-region $\left(h_{3}=90\right.$ kilometers) of the ionosphere and the earth. 


$$
\begin{aligned}
& \omega / \omega_{T}=0.2335 \\
& \phi_{1}=60 \text { degrees }
\end{aligned}
$$

$\Psi$

$\left|\mathrm{T}_{\mathrm{em}}\right|$

$\operatorname{Arg~T}_{\text {ern }}-\pi \quad\left|T_{\text {me }}\right|$

$\mathrm{h}_{3}=70$ kilometers

0.0005

0.0010

0.0020

0.0030

0.0040

0.0050

0.0060

0.0070

0.0080

0.0090

0.0100

0.0120

0.0150

0.0200

0.0250

0.0300

0.0400

0.0500

0.0650

0.0800

0.1000

0.1500

0.2000

0.3000

0.4000

0.6000

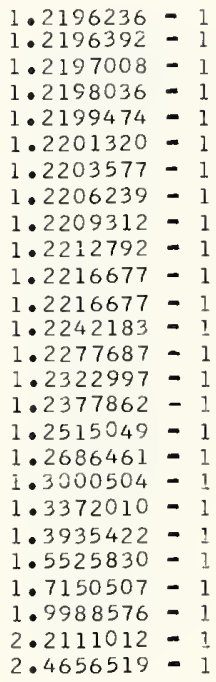

$1.2670633-1$
$1.2670795-1$
$1.2671428-1$
$1.2672493-1$
$1.2673981-1$
$1.2675889=1$
$1.2678224-1$
$1.2680979-1$
$1.2684156-1$
$1.2687755-1$
$1.2691776-1$
$1.2691776-1$
$1.2718151-1$
$1.2754871-1$
$1.2801726-1$
$1.2858460-1$
$1.3000298-1$
$1.3177474-1$
$1.3501943-1$
$1.3885541-1$
$1.4466757-1$
$1.6103154-1$
$1.7766125-1$
$2.0636326-1$
$2.2729688-1$
$1.5098285-1$

$2.5098285-1$ $\begin{array}{ll}\text { Arg } T_{m e}-\pi \quad & d / j \\ \text { miles }\end{array}$

2. $1865830-1$ $2.1865650-1$

2. $1865010-1$ $2.1863910-1$ $2.1862360-1$ 2. $1860390=$ 2. $1857980-1$ 2.1855130 2.1851860 2. 1848130 2.1843980 2.1843980 2. $1816710=$ 2.1778750 2.1730280 2. 1671560 $2.1524630=$ 2.1340880 2. 1003500 $2.0603200-$ 1.9993420 $1.8250330=$ $1.6423570=$ 1.3031190 $1.0140300-1$ $5.4044300-2$
1. 2433895 .. 2381675 1. 2278386 1.2176623 1.2076369 1. 1977590 1.1880278 1,1784374 1. 1639853 1.1596569 1.1504788 1.1324770 1. 1063547 1. 0649462 1.0259098 9.3899038 9. 2076906 8.5914709 7.7737058 7.0657786 6.2644905 4.7915900 3.2174547 $2 \cdot 5471052$ 1.9802951 I. 2445355

\section{$\mathrm{h}_{3}=90$ kilometer $\mathrm{s}$}

\begin{tabular}{|c|c|c|}
\hline $\begin{array}{l}0.0005 \\
0.0010\end{array}$ & $\begin{array}{l}1.3337600 \\
1.3337722\end{array}$ & - \\
\hline 0.0020 & 1.3338219 & - \\
\hline 0.0030 & 1.3339047 & - \\
\hline 0.0040 & 1.3340204 & - \\
\hline 0.0050 & 1.3341696 & - \\
\hline 0.0060 & 1.3343519 & - \\
\hline 0.0070 & 1.3345675 & - \\
\hline 0.0080 & 1.3348150 & - \\
\hline 0.0090 & 1.3350969 & - \\
\hline 0.0100 & 1.3354107 & - \\
\hline 0.0120 & 1.3361371 & - \\
\hline 0.0150 & 1.3374723 & - \\
\hline 0.0200 & 1.3403452 & - \\
\hline 0.0250 & 1.3440167 & - \\
\hline 0.0300 & 1.3484696 & - \\
\hline 0.0400 & 1.3596419 & - \\
\hline 0.0500 & 1.3736707 & - \\
\hline 0.0650 & 1.3995708 & - \\
\hline 0.0800 & 1.4305262 & 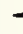 \\
\hline 0.1000 & 1.4780840 & - \\
\hline 0.1500 & 1.6158461 & - \\
\hline 0.2000 & $1.76: 0414$ & - \\
\hline 0.3000 & 2.0228984 & - \\
\hline 0.4000 & 2.2239145 & $=$ \\
\hline 0.6000 & 2.4694654 & 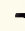 \\
\hline
\end{tabular}

$1.3504000-1$
$1.3504130-1$
$1.3504560-1$
$1.3505280-1$
$1.3506320-1$
$1.3507630-1$
$1.3509250-1$
$1.3511140-1$
$1.3513320-1$
$1.3515830-1$
$1.3518580-1$
$1.3525000-1$
$1.3536790-1$
$1.3562140-1$
$1.3594480-1$
$1.3633670-1$
$1.3731750-1$
$1.3854460-1$
$1.4079560-1$
$1.4346030-1$
$1.4749650-1$
$1.5873560-1$
$1.6966630-1$
$1.8572510-1$
$1.9234070-1$
$1.8302420-1$

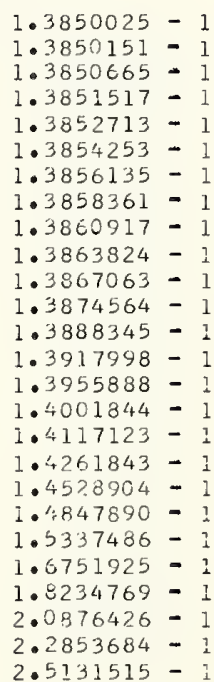

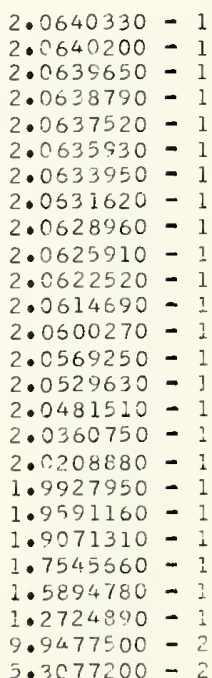

1. 4000780 1. 3948535 1. 3245147 ]. 3743220 1. 3642736 I. 3543670 $1.3445=39$ 1. 334966 ? 1.3254647 1. 3160909 1.3068406 1. 2836954 1. 2623103 1. 2203378 1.1805854 1.1428039 1.0724503 1.0032107 9. 2176798 $8.4506 i 14$ 7.5737071 5.9120543 4. 7678510 3.347062 2.5187905 .5002204

Table 50. Ionosphere reflection coefficients (amplitude, $|\mathrm{T}|$, and phase, Arg $\mathrm{T}$ ) assuming the quasi-longitudinal approximation, for the special ray configuration of the Norton type 301 atmosphere, applicable to the propagation of waves between the $D$-region $\left(h_{3}=70\right.$ kilometers $)$ or the E-region $\left(h_{3}=90\right.$ kilometers $)$ of the ionosphere and the earth. 


$$
\begin{aligned}
& \omega / \omega_{r}=0.0100 \\
& \phi_{1}=60 \text { degrees }
\end{aligned}
$$

$\Psi$

radians
$\operatorname{Arg~T}_{\text {em }}-\pi$

$\mathrm{h}_{3}=70$ kilometers

$4.8453870-1$
$4.8454190=1$
$4.8455450-1$
$4.8457570=1$
$4.8460520=1$
$4.8464320=1$
$4.8468970-1$
$4.8474440=1$
$4.8480760=1$
$4.8487920-1$
$4.8495900-1$
$4.8514370-1$
$4.8548250-1$
$4.8620940-1$
$4.8713390-1$
$4.8824830-1$
$4.9101290-1$
$4.9442230-1$
$5.0054290-1$
$5.0738070-?$
$5.1785350=1$
$5.4447770-1$
$5.6850700=1$
$6.0416510-1$
$6.4861030-1$
$5.2643880-1$

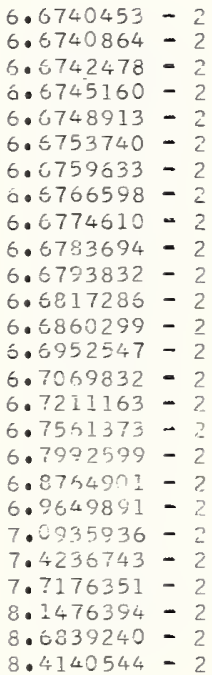

$\mathrm{h}_{3}=90$ kilometers

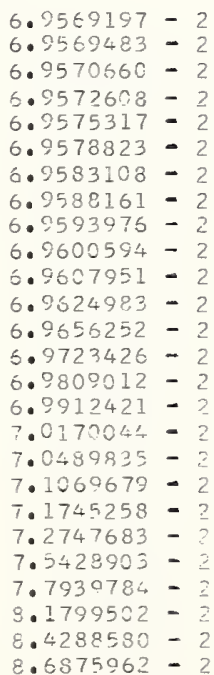

$\operatorname{Arg} \mathrm{T}_{\mathrm{me}}-\pi$

$d / j$

miles

$\begin{array}{ll}0.0005 & 6.6735661=2 \\ 0.0010 & 6.6736068=2 \\ 0.0020 & 6.6737673=2 \\ 0.0030 & 6.6740360=2 \\ 0.0040 & 6.6744118=2 \\ 0.0050 & 6.6748941=2 \\ 0.0060 & 6.6754835=2 \\ 0.0070 & 6.6761796=2 \\ 0.0080 & 6.6769814=2 \\ 0.0090 & 6.6778894=2 \\ 0.0100 & 6.6789039=2 \\ 0.0120 & 6.6812496=2 \\ 0.0150 & 6.6855492=2 \\ 0.0200 & 6.6947739=2 \\ 0.0250 & 6.7065017=2 \\ 0.0300 & 6.7206331=2 \\ 0.0400 & 6.7556518=2 \\ 0.0500 & 6.7987726=2 \\ 0.0650 & 6.8759997=2 \\ 0.0800 & 6.9644953=2 \\ 0.1000 & 7.0930931=2 \\ 0.1500 & 7.4231656=2 \\ 0.2000 & 7.7171242=2 \\ 0.3000 & 8.1471513=2 \\ 0.4000 & 8.6836337=2 \\ 0.6000 & 3.4136179=2\end{array}$

0.0005

0.0010

0.0020

0.0030

0.0040

0.0050

0.0060

0.0070

0.0080

0.0090

0.0100

0.0120

C. 0150

0.0200

0.0250

.0300

0.0400

0.3500

0.0650

0.0800

0.1000

.1500

3.2000

0.3000

.4000

0.6000

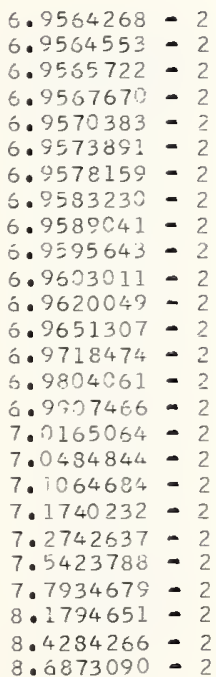

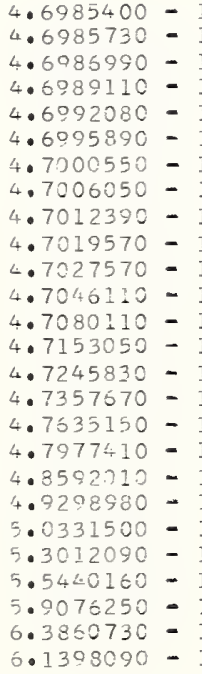

$\begin{array}{ll}1.2453695 & 3 \\ 1.2381675 & 3 \\ 1.2278386 & 3 \\ 1.2176623 & 3 \\ 1.2076369 & 3 \\ 1.1977599 & 3 \\ 1.1880278 & 3 \\ 1.1784374 & 3 \\ 1.1659853 & 3 \\ 1.1596669 & 3 \\ 1.1504788 & 3 \\ 1.1324770 & 3 \\ 1.1063547 & 3 \\ 1.0649462 & 3 \\ 1.0259098 & 3 \\ 0.8290038 & 2 \\ 9.2076906 & 2 \\ 8.5914709 & 2 \\ 7.7737068 & 2 \\ 7.0657736 & 2 \\ 6.2644905 & 2 \\ 4.7915900 & 2 \\ 3.3174547 & 2 \\ 2.6471062 & 2 \\ 1.9802951 & 2 \\ 1.2445355 & 2 \\ & \end{array}$

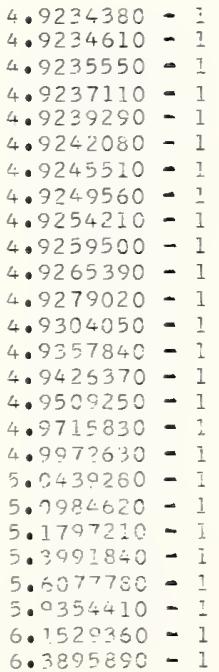

Table 51. Ionosphere reflection coefficients (amplitude, $|\mathrm{T}|$, and phase, Arg T) as suming the quasi-longitudinal approximation, for the special ray configuration of the Norton type 301 atmosphere, applicable to the propagation of waves between the D-region ( $h_{3}=70$ kilometers) or the E-region ( $h_{3}=90$ kilometers) of the ionosphere and the earth. 


$$
\begin{aligned}
& \omega / \omega_{r}=0.0200 \\
& \phi_{1}=60 \text { degrees }
\end{aligned}
$$

$\Psi$

radians
Arg $T_{\text {em }}-\pi\left|T_{m e}\right|$

$\mathrm{h}_{3}=70$ kilomete $\mathrm{s}$
Arg $T_{m e}-\pi$

d/ $\mathrm{j}$

miles
$\left|\mathrm{T}_{\mathrm{em}}\right|$ $8.1718017-2$ $8.1720525-2$ $8.1724710-2$ $8.1730537-2$ $8.1738048=2$ $8.1747206-2$ $8.1770502-2$ $8.1784631-2$ $8.1800409-2$ $8.1836884-2$ $8.1903804-2$ $8.2230156-2$ $8.2450632-2$ $8.2998127-$ ? $8.3674563=2$ $8.4892366-2$ $8.6297991-2$ $8.8360005-2$ $9.3760013-2$ $9.3702471-2$ $1.0616497-1$ 1. $1093051-1$ $1.1587227-1$ $8.1758033-2$ $8.2047422=2$

$4.0929260-1$
$4.0929590-1$
$4.0930910-1$
$4.0933110-1$
$4.0936220-1$
$4.0940190-1$
$4.0945040-1$
$4.0950760-1$
$4.0957350-1$
$4.0964830-1$
$4.0973170-1$
$4.0992480-1$
$4.1027900-1$
$4.1103930-1$
$4.1200680-1$
$4.1317490-1$
$4.1607760-1$
$4.1966830-1$
$4.2614580-1$
$4.3364320-1$
$4.4468220-1$
$4.7382450-1$
$5.0078830-1$
$5.4192640-1$
$5.6820500-1$
$5.9438880-1$

$3.7992300=1$
$3.7992630=1$
$3.7993950-1$
$3.7996190-1$
$3.7999300-1$
$3.8003290-1$
$3.8008180-1$
$3.8013940=1$
$3.8020570-1$
$3.8028090=1$
$3.8036500-1$
$3.8055930-1$
$3.8091580-1$
$3.8168120=1$
$3.8265560=1$
$3.8383160-1$
$3.8675490=1$
$3.9037200-1$
$3.9690000=1$
$4.0446100=1$
$4.1560520-1$
$4.4511040-1$
$4.7257750-1$
$5.1512070-1$
$5.4328770-1$
$5.7438050-1$
4

$5.7438050-1$
1. 2433895 1. 2381675 1. 2278386 1. 2176623 1. 2076369 1. 1977599 1. 1380278 1. 1784374 1. 1539853 1. 1596669 ]. 1504788 1. 1324770 1. 1063547 1. 0649462 1.0259098 $9.2076^{\circ} 06$ 8.5314700 7.7737068 7.0657780 6.2644905 4. 7915900 3.8174547 2.6471062 1. 9802951 1.2445355 9.8899038

\section{$\mathrm{h}_{3}=90$ kilometers}

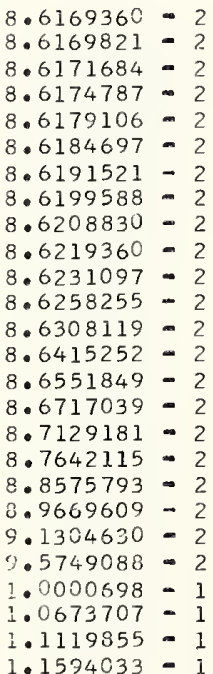

$4.3295610-1$
$4.3295860-1$
$4.3296860-1$
$4.3298520-1$
$4.3300820-1$
$4.3303800-1$
$4.3307440-1$
$4.3311760-1$
$4.3316700-1$
$4.3322340-1$
$4.3328600-1$
$4.3343100-1$
$4.3369730-1$
$4.3426970-1$
$4.3499960-1$
$4.3588260-1$
$4.3808720-1$
$4.4083340-1$
$4.4584040-1$
$4.5171880-1$
$4.6053140-1$
$4.8464340-1$
$5.0794860-1$
$5.4509040-1$
$5.6967110-1$
$5.9472390-1$
$8.6194293-2$ $8.6196154-2$ $8.6199264-2$ $8.6203581-2$ $8.6209170=2$ $8.6224054-2$ $8.6233314=2$ $8.6243354 \rightarrow 2$ $8.6255609-2$ $8.6282741=2$ $8.6332627-2$ $8.6439774-2$ $8.6576394-2$ $8.6741630-2$ $8.7153848-2$ $8.7666874-2$ $8.8600738-2$ $8.9694758-2$ 9. $1330038=2$ $9.5775067-2$ $1.0003318-1$ $1.0676232-1$ 1. $1122127-1$ 1. $1525559-1$ $8.6215990-2$

$4.0376790-1$
$4.0377040-1$
$4.0378040=1$
$4.0379710=1$
$4.0382040-1$
$4.0385050-1$
$4.0388730-1$
$4.0393070=1$
$4.0398060=1$
$4.0403740-1$
$4.0410060=1$
$4.0424710=1$
$4.0451570-1$
$4.0509320=1$
$4.0582960-1$
$4.0672070-1$
$4.0894540=1$
$4.1171800-1$
$4.1677510-1$
$4.2271680-1$
$4.3163410=1$
$4.5610680=1$
$4.7991190=1$
$5.1845030-1$
$5.4490780-1$
$5.7483920=1$

1. 4000780 1. 3948535 1. 3045147 1. 3743220 1. $3 \leqslant 42736$ 1. 3543670 ]. 3445989 1. 3349661 1. 3254647 1. 3160909 1.3068406 1. 2886954 1.2623103 1. 2203378 1. 1805854 1. 1428039 1.0724503 1. 0082107 9. 2176798 8.4566114 7.5787071 5.9120543 4. 7678510 3.3479623 2. 5187906 1. 5902204
Table 52. Ionosphere reflection coefficients (amplitude, |T|, and phase, Arg T) assuming the quasi-longitudinal approximation, for the special ray configuration of the Norton type 301 atmosphere, applicable to the propagation of waves between the D-region $\left(h_{3}=70\right.$ kilometers) or the $E-x e g i o n\left(h_{3}=90\right.$ kilometers $)$ of the ionosphere and the earth. 


$$
\begin{aligned}
& \omega / \omega_{r}=0.0500 \\
& \phi_{1}=60 \text { degrees }
\end{aligned}
$$

$\Psi$

radians

$\left|\mathrm{T}_{\text {em }}\right|$

$.0092407=1$
$.0082508=1$
$.0032904=1$
$.0083566=1$
$.0034493=1$
$.0085682=1$
$.0087136=1$
$.0038851=1$
$.0090829=1$
$.0093070=1$
$.0095573=1$
$.0101359=1$
$.0111982=1$
$.0134797=1$
$.0163861=1$
$.0198985=1$
$.0286461=1$
$.0395063=1$
$.0592041=1$
$.0821767=1$
$.1263465=1$
$.2085877=1$
$.2367094=1$
$.4370069=1$
$.5315207=1$
$.6339502=1$

0.0005

0.0010

0.0020

0.0030

0.0040

0.0050

0.0060

0.0070

0.0080

0.0090

0.0100

0.0120

0.0150

0.0200

0.0250

0.0300

0.0400

0.0500

0.0650

0.0800

0.1000

0.1500

0.2000

0.3000

0.4000

0.6000
$\operatorname{Arg} \mathrm{T}_{\mathrm{em}}-\pi\left|\mathrm{T}_{\mathrm{me}}\right|$

$\mathrm{h}_{3}=70$ kilometers

0.0005

0.0010

0.0020

0.0030

0.0040

0.0050

0.0060

0.0070

0.0080

0.0090

0.0100

0.0120

0.0150

0.0200

0.0250

0.0300

0.0400

0.0500

0.0650

0.0800

0.1000

0.1500

0.2000

0.3000

0.4000

0.6000

$3.0243240=1$
$3.0243560=1$
$3.0244770=1$
$3.0246790=1$
$3.0249630=1$
$3.0253280=1$
$3.0257730=1$
$3.0262990=1$
$3.0269060=1$
$3.0275930=1$
$3.0283610=1$
$3.0301340=1$
$3.0333940=1$
$3.0403900=1$
$3.0493070=1$
$3.0600810=1$
$3.0869270=1$
$3.1202740=1$
$3.1808100=1$
$3.2514890=1$
$3.3567700=1$
$3.6417520=1$
$3.9145000=1$
$4.3463460=1$
$4.6298490=1$
$4.9027480=1$

$\mathrm{h}_{3}=90$ kilometers

$1.0800639=1$
$1.0800714=1$
$1.0301020=1$
$1.0801529=1$
$1.0802237=1$
$1.0803158=1$
$1.0804277=1$
$1.0805600=1$
$1.0807122=1$
$1.0808853=1$
$1.0810779=1$
$1.0815240=1$
$1.0823433=1$
$1.0841048=1$
$1.0863531=1$
$1.0890752=1$
$1.0958828=1$
$1.1043862=1$
$1.1199555=1$
$1.1383450=1$
$1.1661397=1$
$1.2436129=1$
$1.3205888=1$
$1.4481431=1$
$1.5369577=1$
$1.6353948=1$

$1.0100520=1$
$1.0100621=1$
$1.0101017=1$
$1.0101681=1$
$1.0102509=1$
$1.0103801=1$
$1.0105257=1$
$1.0106973=1$
$1.0108955-1$
$1.0111200=1$
$1.0113706=1$
$1.0119501=1$
$1.0130139=1$
$1.0152989=1$
$1.0182096=1$
$1.0217274=1$
$1.0304881=1$
$1.0413643=1$
$1.0610908=1$
$1.0840960=1$
$1.1183122=1$
$1.2106629=1$
$1.2938587=1$
$1.4391572=1$
$1.5335008=1$
$1.6353119=1$

$2.2900500=1$ $2.2900820=1$ $2.2902050-1$

$2.2904120-1$

$2.2907010-1$

2.2910730 - 1

$2.2915260=1$

$2.2920610-1$

$2.2926800-1$

$2.2933790-1$

$2.2941600=1$

$2.2959670-1$

$2.2992840-1$

$2.3064090=1$

$2.3154900-1$

$2.3264650-1$

$2.3538250-1$

$2.3878310=1$

$2.4496270-1$

$2.5218930-1$

2.6297960 - 1

$2.9238380-1$

$3.2092420-1$

$3.6765590=1$

$4.0067060=1$

4.4022210 - 1

1.2433895 1.2381675 1. 2278386 1.2176623 1.2076369 1.1977599 1.1880278 1.1784374 1.1689853 1.1596669 1.1504788 1.1324770 1.1063547 1.0649462 1.0259098 9.8899038 9.2076906 8.5914709 7. 7737068 7.0657786 6.2644905 4.7915900 3.8174547 2. 6471062 1.9802951 1. 2445355

$1.0819803=1$
$1.0819878=1$
$1.0820185=1$
$1.0820695=1$
$1.0821405=1$
$1.0822325=1$
$1.0823446=1$
$1.0824772=1$
$1.0826295=1$
$1.0828027=1$
$1.0829957=1$
$1.0834423=1$
$1.0842628=1$
$1.0860268-1$
$1.0882784=1$
$1.0910040=1$
$1.0978210=1$
$1.1063359-1$
$1.1219261=1$
$1.1403391=1$
$1.1681678=1$
$1.2457221=1$
$1.3227508-1$
$1.4502833=1$
$1.5389203=1$
$1.6367407=1$

1.4000780 1.3948535 1.3845147 1. 3743220 1.3642736 1.3543670 1. 3445989 1. 3349661 1. 3254647 1. 3160909 1.3068406 1. 2886954 1.2623103 1. 2203378 1. 1805854 1.1428039 1.0724503 1.0082107 9.2176798 8.4566114 7.5787071 5.9120543 4.7678510 3. 3479623 2.5137906 1. 5902204

Table 53. Ionosphere reflection coefficients (amplitude, $|\mathrm{T}|$, and phase, Arg T) assuming the quasi-longitudinal approximation, for the special ray configuration of the Norton type 301 atmosphere, applicable to the propagation of waves between the D-region $\left(h_{3}=70\right.$ kilometers) or the E-region $\left(h_{3}=90\right.$ kilometers $)$ of the ionosphere and the earth. 


$$
\begin{aligned}
& \omega / \omega_{r}=0.1000 \\
& \phi_{1}=60 \text { degrees }
\end{aligned}
$$

$\Psi$ radians

$\left|\mathrm{T}_{\mathrm{em}}\right|$

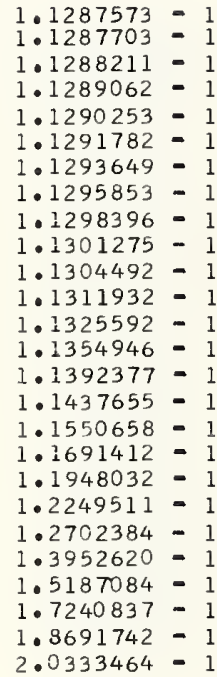

$\begin{array}{ll}0.0005 & 1.1287573=1 \\ 0.0010 & 1.1287703=1 \\ 0.0020 & 1.1288211=1 \\ 0.0030 & 1.1289062=1 \\ 0.0040 & 1.1290253=1 \\ 0.0050 & 1.1291782=1 \\ 0.0060 & 1.1293649=1 \\ 0.0070 & 1.1295853=1 \\ 0.0080 & 1.1298396=1 \\ 0.0090 & 1.1301275=1 \\ 0.0100 & 1.1304492=1 \\ 0.0120 & 1.1311932=1 \\ 0.0150 & 1.1325592=1 \\ 0.0200 & 1.1354946=1 \\ 0.0250 & 1.1392377=1 \\ 0.0300 & 1.1437655=1 \\ 0.0400 & 1.1550658=1 \\ 0.0500 & 1.1691412=1 \\ 0.0650 & 1.1948032=1 \\ 0.0800 & 1.2249511=1 \\ 0.1000 & 1.2702384=1 \\ 0.1500 & 1.3952620=1 \\ 0.2000 & 1.5187084=1 \\ 0.3000 & 1.7240837=1 \\ 0.4000 & 1.3691742=1 \\ 0.6000 & 2.0333464=1\end{array}$

$\operatorname{Arg~}_{\text {em }}-\pi \quad\left|\mathrm{T}_{\text {me }}\right|$

$\mathrm{h}_{3}=70$ kilometers

0.0005

0.0010

0.0020

0.0030

0.0040

0.0050

0.0060

0.0070

0.0080

0.0090

0.0100

0.0120

0.0150

0.0200

0.0250

0.0300

0.0400

0.0500

0.0650

0.0800

0.1000

0.1500

0.2000

0.3000

0.4000

0.6000

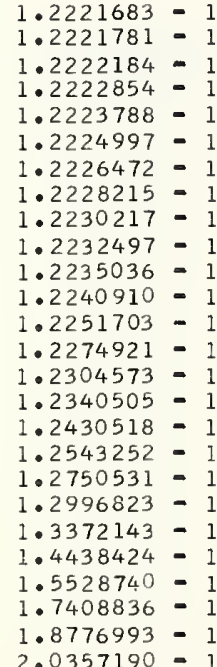

$2.2066070=1$
$2.2066310=1$
$2.2067300=1$
$2.2068930=1$
$2.2071260=1$
$2.2074220=1$
$2.2077860=1$
$2.2082120=1$
$2.2087070=1$
$2.2092650=1$
$2.2098880=1$
$2.2113330=1$
$2.2139840=1$
$2.2196800=1$
$2.2269420=1$
$2.2357280=1$
$2.2576430=1$
$2.2849330=1$
$2.3346490=1$
$2.3929880=1$
$2.4804640=1$
$2.7206340=1$
$2.9547950=1$
$3.3309580=1$
$3.5736920=1$
$3.7744980=1$

$1.1368611=1$
$1.1368740=1$
$1.1369252=1$
$1.1370105=1$
$1.1371305=1$
$1.1372843=1$
$1.1374722=1$
$1.1376941=1$
$1.1379499=1$
$1.1382397=1$
$1.1385633=1$
$1.1393119=1$
$1.1406364=1$
$1.1436400=1$
$1.1474061=1$
$1.1519620=1$
$1.1633317=1$
$1.1774926=1$
$1.2033087=1$
$1.2336334=1$
$1.2791769=1$
$1.4048369=1$
$1.5287687=1$
$1.7343942=1$
$1.8788316=1$
$2.0401119=1$

$\mathrm{h}_{3}=90$ kilometers

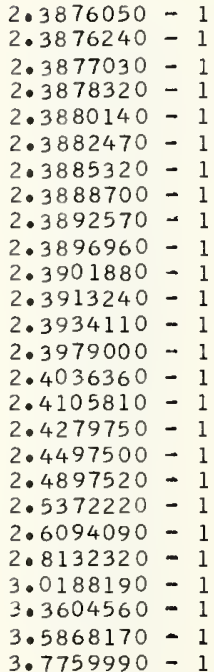

$\operatorname{Arg~T}_{\text {me }}-\pi \quad \frac{\mathrm{d} / \mathrm{j}}{\text { miles }}$

$7.3780200=2$
$7.3782900=2$
$7.3793400=2$
$7.3810400=2$
$7.3834600=2$
$7.3865500=2$
$7.3903300=2$
$7.3948000=2$
$7.3999600=2$
$7.4058100=2$
$7.4123100=2$
$7.4273800=2$
$7.4550700=2$
$7.5146000=2$
$7.5904800=2$
$7.6823200=2$
$7.9116700=2$
$8.1976400=2$
$8.7198300=2$
$9.3347700=2$
$1.0261630=1$
$1.2843230=1$
$1.5434350=1$
$1.9893260=1$
$2.3258760=1$
$2.7711430=1$

1.2433895

1.2381675

1. 2278386

1.2176623

1.2076369

1.1977599

1. 1880278

1.1784374

1.1689853

1.1596669

1.1504788

1. 1324770

1.1063547

1.0649462

1.0259098

9. 8899038

9.2076906

8.5914709

7. 7737068

7.0657786 6.2644905

4.7915900

3.8174547

2.6471062

1.9802951

1.2445355

Table 54. Ionosphere reflection coefficients (amplitude, $|\mathrm{T}|$, and phase, Arg $\mathrm{T}$ ) assuming the quasi-longitudinal approximation, for the special ray configuration of the Norton type 301 atmosphere, applicable to the propagation of waves between the D-region $\left(h_{3}=70\right.$ kilometers) or the E-region ( $h_{3}=90$ kilometers) of the ionosphere and the earth.

$\begin{array}{lll}9.2779100=2 & 1.4000780 & 3 \\ 9.2781300=2 & 1.3948535 & 3 \\ 9.2789500=2 & 1.3845147 & 3 \\ 9.2803200=2 & 1.3743220 & 3 \\ 9.2822300=2 & 1.3642736 & 3 \\ 9.2846900=2 & 1.3543670 & 3 \\ 9.2877000=2 & 1.3445989 & 3 \\ 9.2912700=2 & 1.3349661 & 3 \\ 9.2953500=2 & 1.3254647 & 3 \\ 9.3000000=2 & 1.3160909 & 3 \\ 9.3052000=2 & 1.3068406 & 3 \\ 9.3172000=2 & 1.2886954 & 3 \\ 9.3392300=2 & 1.2623103 & 3 \\ 9.3866700=2 & 1.2203378 & 3 \\ 9.4472300=2 & 1.1805854 & 3 \\ 9.5206800=2 & 1.1428039 & 3 \\ 9.7047400=2 & 1.0724503 & 3 \\ 9.9354900=2 & 1.0082107 & 3 \\ 1.0360400=1 & 9.2176798 & 2 \\ 1.0866430=1 & 8.4566114 & 2 \\ 1.1640070=1 & 7.5787071 & 2 \\ 1.3857160=1 & 5.9120543 & 2 \\ 1.6161010=1 & 4.7678510 & 2 \\ 2.0270440=1 & 3.3479623 & 2 \\ 2.3466520=1 & 2.5187906 & 2 \\ 2.7788100=1 & 1.5902204 & 2\end{array}$




$$
\begin{aligned}
& \omega / \omega_{r}=0.2000 \\
& \phi_{1}=60 \text { degrees }
\end{aligned}
$$

$\Psi$

radians
$\operatorname{Arg~T}_{\mathrm{em}}-\pi \quad\left|\mathrm{T}_{\mathrm{me}}\right|$

$\mathrm{h}_{3}=70$ kilometers
Arg $\mathrm{T}_{\mathrm{me}}-\pi$

$\mathrm{d} / \mathrm{j}$

miles
0.0005

0.0010

0.0020

0.0030

0.0040

0.0050

0.0060

0.0070

0.0080

0.0090

0.0100

0.0120

0.0150

0.0200

0.0250

0.0300

0.0400

0.0500

0.0650

0.0800

0.1000

0.1500

0.2000

0.3000

0.4000

0.6000

$\begin{array}{ll}0.0005 & 1.3209063=1 \\ 0.0010 & 1.3209181=1 \\ 0.0020 & 1.3209665=1 \\ 0.0030 & 1.3210470=1 \\ 0.0040 & 1.3211595=1 \\ 0.0050 & 1.3213046=1 \\ 0.0060 & 1.3214820=1 \\ 0.0070 & 1.3216917=1 \\ 0.0080 & 1.3219325=1 \\ 0.0090 & 1.3222064=1 \\ 0.0100 & 1.3225118=1 \\ 0.0120 & 1.3232183=1 \\ 0.0150 & 1.3245169=1 \\ 0.0200 & 1.3273105=1 \\ 0.0250 & 1.3308805=1 \\ 0.0300 & 1.3352104=1 \\ 0.0400 & 1.3460701=1 \\ 0.0500 & 1.3597012=1 \\ 0.0650 & 1.3848509=1 \\ 0.0800 & 1.4148820=1 \\ 0.1000 & 1.4609615=1 \\ 0.1500 & 1.5940319=1 \\ 0.2000 & 1.7336028=1 \\ 0.3000 & 1.9834485=1 \\ 0.4000 & 2.1734857=1 \\ 0.6000 & 2.4032468=1\end{array}$

$1.2096947=1$
$1.2097099=1$
$1.2097697=1$
$1.2098702=1$
$1.2100103=1$
$1.2101906=1$
$1.2104109=1$
$1.2106710=1$
$1.2109709=1$
$1.2113103=1$
$1.2116898=1$
$1.2125673=1$
$1.2141790=1$
$1.2176439=1$
$1.2220650=1$
$1.2274175=1$
$1.2407984=1$
$1.2575086=1$
$1.2881010=1$
$1.3242529=1$
$1.3789986=1$
$1.5329998=1$
$1.6894707=1$
$1.9606153=1$
$2.1614208=1$
$1.3997022=1$

$2.3997022=1$

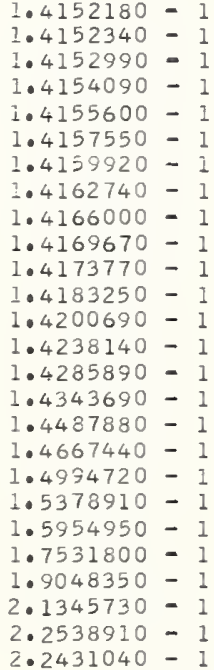

$1.2442911-1$
$1.2443066=1$
$1.2443682=1$
$1.2444711=1$
$1.2446148=1$
$1.2447997-1$
$1.2450256-1$
$1.2452920=1$
$1.2455995-1$
$1.2459476-1$
$1.2463363-1$
$1.2472360=1$
$1.2488884=1$
$1.2524400=1$
$1.2569717=1$
$1.2624580-1$
$1.2761711=1$
$1.2932936-1$
$1.3246307-1$
$1.3616448-1$
$1.4176574=1$
$1.5749114=1$
$1.7340579-1$
$2.0073298=1$
$2.2058969-1$
$2.4313827-1$

$\mathrm{h}_{3}=90$ kilometers

$1.5343460-1$
$1.5343590-1$
$1.5344100-1$
$1.5344960-1$
$1.5346160-1$
$1.5347700-1$
$1.5349560-1$
$1.5351780-1$
$1.5354330-1$
$1.5357250=1$
$1.5360470-1$
$1.5367950-1$
$1.5381710=1$
$1.5411270-1$
$1.5449020-1$
$1.5494780=1$
$1.5609320-1$
$1.5752740-1$
$1.6016100-1$
$1.6328460=1$
$1.6802940-1$
$1.8135280-1$
$1.9455780-1$
$2.1510490-1$
$2.2586940-1$
$1.2401400-1$

$1.3582193=1$ 1. 3582313 - 1 $1.3582812=$ $1.3583634-1$ 1.3584784 - 1 1.3586272 - I $1.3588085=1$ $1.3590235-1$ 1.3592697 1.3595502 - 1 1.3598627 1.3605861 - 1 1.3619152 - 1 $1.3647748-1$ 1.3684287 - 1 $1.3728598-1$ 1.3839728 $1.3979189-1$ 1.4236417 - 1 1.4543423 1.5014144 1.6370764 - 1 1.7787915 - 1 2.0301241 $2.2176562=1$
$1.5250170 \rightarrow 1$ $1.5249980=1$ 1.5249260 - 1 1.5248020 - 1 $1.5246320 \rightarrow 1$ $1.5244100-1$ $1.5241390=1$ 1.5238230 - 1 1.5234570 - I 1.5230410 1.5225780 - 1 $1.5215020-1$ $1.5195310=1$ 1.5152910 - 1 $1.5098770=1$ $1.5033210=1$ $1.4869250=1$ $1.4664230-1$ $1.4288220=$ ] $1.3842680-1$ $1.3165260=1$ 1. $1238000=1$ $9.2351500-2$ $5.5756000-2$ $2.5429000-2$ $2.1776500-2$

$\begin{array}{ll}1.2433895 & 3 \\ 1.2381675 & 3 \\ 1.2278386 & 3 \\ 1.2176623 & 3 \\ 1.2076369 & 3 \\ 1.1977599 & 3 \\ 1.1880278 & 3 \\ 1.1784374 & 3 \\ 1.1689853 & 3 \\ 1.1596669 & 3 \\ 1.1504788 & 3 \\ 1.1324770 & 3 \\ 1.1063547 & 3 \\ 1.0649462 & 3 \\ 1.0259098 & 3 \\ 9.8899038 & 2 \\ 9.2076906 & 2 \\ 8.5914709 & 2 \\ 7.7737068 & 2 \\ 7.0657786 & 2 \\ 6.2644905 & 2 \\ 4.7915900 & 2 \\ 3.8174547 & 2 \\ 2.6471062 & 2 \\ 1.9802951 & 2 \\ 1.2445355 & 2\end{array}$

$1.3884000-1$ $1.3883840-1$ 1.3883250 - 1 1.3882250 - 1 1.3880860 - 1 $1.3879090=1$ $1.3876880=1$ $1.3874300=1$ 1.3871340 = I.3867940 - 1 $1.3864180=1$ 1.3855450 - 1 $1.3839430-1$ $1.3804940-1$ $1.3760870-1$ $1.3707380=1$ 1.3573140 - 1 $1.3404440=1$ $1.3092630-1$ $1.2719260=1$ $1.2143950-1$ $1.0463190=1$ $8.6591100-2$ $5.2499300=2$ $2.3444700-2$ 2.2704100-2
1. 4000780 1. 3948535 1. 3845147 1. 3743220 1. 3642736 1. 3543670 1. 3445989 1.3349661 1. 3254647 1. 3160909 1. 3068406 1.2886954 1.2623103 1.22033 .78 1. 1805854 1. 1428039 1.0724503 1.0082107 9.2176798 8.4566114 7.5787071 5.9120543 4.7678510 3.3479623 2. 5187906 1.5902204
Table 55. Ionosphere reflection coefficients (amplitude, $|\mathrm{T}|$, and phase, $\mathrm{Arg} \mathrm{T}$ ) assuming the quasi-longitudinal approximation, for the special ray configuration of the Norton type 301 atmosphere, applicable to the propagation of waves between the D-region ( $h_{3}=70$ kilometers) or the E-region $\left(h_{3}=90\right.$ kilometers) of the ionosphere and the earth. 


$$
\begin{aligned}
& \omega / \omega_{r}=1.0000 \\
& \phi_{1}=60 \text { degrees }
\end{aligned}
$$

$\underset{\Psi}{\text { radians }}$

$\left|\mathrm{T}_{\mathrm{em}}\right|$

$\operatorname{Arg} \mathrm{T}_{\mathrm{em}}-\pi$

$\left|\mathrm{T}_{\mathrm{me}}\right|$

$\operatorname{Arg} \mathrm{T}_{\mathrm{me}}-\pi$

$\mathrm{d} / \mathrm{j}$

$\mathrm{h}_{3}=70$ kilometers

0.0005
0.0010
0.0020
0.0030
0.0040
0.0050
0.0060
0.0070
0.0080
0.0090
0.0100
0.0120
0.0150
0.0200
0.0250
0.0300
0.0400
0.0500
0.0650
0.0800
0.1000
0.1500
0.2000
0.3000
0.4000
0.6000

0.0005

0.0010

0.0020

0.0030

0.0040

0.0050

0.0060

0.0070

0.0080

0.0090

0.0100

0.0120

0.0150

0.0200

0.0250

0.0300

0.0400

0.0500

0.0650

0.0800

0.1000

0.1500

0.2000

0.3000

0.4000

0.6000

$1.1098227=1$
$1.1098378=1$
$1.1098971=1$
$1.1099969=1$
$1.1101362=1$
$1.1103152=1$
$1.1105338=1$
$1.1107921=1$
$1.1110900=1$
$1.1114272=1$
$1.1118040=1$
$1.1126756=1$
$1.1142768=1$
$1.1177206=1$
$1.1221175=1$
$1.1274454=1$
$1.1407839=1$
$1.1574828=1$
$1.1881752=1$
$1.2246496=1$
$1.2803181=1$
$1.4398900=1$
$1.6069452=1$
$1.9100933=1$
$2.1475325=1$
$2.4384811=1$

$1.2212635-1$ $1.2212755=1$ 1.2213244 - 1 $1.2214060-1$ $1.2215196-1$ 1.2216666 - 1 1.2220580 - 1 $1.2225789-1$ 1.2228877 1.2236026 - 1 $1.2249165-1$ 1.2277449 - 1 1.2313612 - 1 1.2357492 - 1 1.2467708 1.2363004 - 1 $1.3170985=1$ 1.3646810 - 1 1.5044280 1.6550437 $1.9365086-1$ $2.1621632-1$ $2.4426115-1$ 1.2218460 - 1 1.2223016 - 1 $1.2606343=1$

$2.2960840=1$
$2.2960650=1$
$2.2959990=1$
$2.2958850=1$
$2.2957300=1$
$2.2955290=1$
$2.2952840-1$
$2.2949940=1$
$2.2946610-1$
$2.2942810=1$
$2.2938600=1$
$2.2928770=1$
$2.2910730-1$
$2.2871870=1$
$2.2822050=1$
$2.2761370=1$
$2.2608090-1$
$2.2413280-1$
$2.2046610-1$
$2.1595760=1$
$2.0874230=1$
$1.8552940=1$
$1.5631350-1$
$8.4442200=2$
$9.4720000=4$
$1.9994350-1$
1.5448664 - 1 1.5449457 - 1 1.5450785 - 1 $1.5452645-1$ 1.5455033 - 1 1.5457950 - I 1.5461396 - 1 $1.5465369-1$ 1.5469868 - 1 1.5474896 - 1 $1.5486519-1$ $1.5507876-1$ $1.5553784-1$ $1.5612364-1$ $1.5683289-1$ 1.5860597 - 1 $1.6082026-1$ $1.6487359-1$ 1.6966102 - 1 $1.7690068-1$ $1.9711678-1$ $2.1716287-1$ $2.4883385-1$ $2.6593270=1$ $2.6290092-1$
1.5448463 - 1

$\mathrm{h}_{3}=90$ kilometers

$\begin{array}{ll}2.1638310-1 & 1.6921797-1 \\ 2.1638180-1 & 1.6921953-1 \\ 2.1637560-1 & 1.6922598-1 \\ 2.1636520-1 & 1.6923664-1 \\ 2.1635110-1 & 1.6925153-1 \\ 2.1633250-1 & 1.6927074-1 \\ 2.1631000-1 & 1.6929423-1 \\ 2.1628340-1 & 1.6932197-1 \\ 2.1625290-1 & 1.6935386-1 \\ 2.1621790-1 & 1.6939013-1 \\ 2.1617920-1 & 1.6943054-1 \\ 2.1608920-1 & 1.6952405-1 \\ 2.1592390-1 & 1.6969595-1 \\ 2.1556710-1 & 1.7006576-1 \\ 2.1510960-1 & 1.7053828-1 \\ 2.1455180-1 & 1.7111120-1 \\ 2.1314020-1 & 1.7254796-1 \\ 2.1134130-1 & 1.7435056-1 \\ 2.0794190-1 & 1.7767353-1 \\ 2.0373980-1 & 1.8163535-1 \\ 1.9697040-1 & 1.8769741-1 \\ 1.7491260-1 & 2.0501885-1 \\ 1.4675590-1 & 2.2265874-1 \\ 7.6569100-2 & 2.5117219-1 \\ 7.5735000-3 & 2.6662109-1 \\ 2.0474910-1 & 2.6240760-1\end{array}$

$4 \cdot 9579320$
$4 \cdot 9579309$
$4 \cdot 9579266$
$4 \cdot 9579190$
$4 \cdot 9579087$
$4 \cdot 9578954$
$4 \cdot 9578791$
$4 \cdot 9578598$
$4 \cdot 9578377$
$4 \cdot 9578125$
$4 \cdot 9577844$
$4 \cdot 9577195$
$4 \bullet 9575998$
$4 \cdot 9573425$
$4 \cdot 9570130$
$4 \cdot 9566126$
$4 \cdot 9556051$
$4 \cdot 9543327$
$4 \cdot 9519616$
$4 \cdot 9490877$
$4 \cdot 9445810$
$4 \cdot 9308007$
$4 \bullet 9148664$
$4 \cdot 8814721$
$4 \cdot 8514507$
$4 \cdot 8227901$

1.2433895 1.2381675 1.2278386 1. 2176623 1.2076369 1.1977599 1.1880278 1. 1784374 1.1689853 1.1596669 1.1504788 1.1324770 1.1063547

1.0649462 1.0259098 9.8899038 9.2076906 8.5914709 7. 7737068 7.0657786 6.2644905 4.7915900 3. 8174547 2.6471062 1.9802951 1.2445355

4.949357 4. 9493562 $4 \cdot 9493523$ 4.9493458 4.9493368 4. 9493251 4.9493109 4. 9492940 4. 9492746 4. 9492525 4.9492279 4. 9491711 4. 9490665 4. 9488411 4.9485523 4. 9482009 4. 9473146 4. 9461917 4.9440880 4. 9415209 4. 9374607 4. 9248376 4. 9099671 4.8782767 4. 8495459 4. 8228964
1.4000780 1.3948535 1. 3845147 1.3743220 1. 3642736 1. 3543670 1. 3445989 1. 3349661 1. 3254647 1. 3160909 1.3068406 1. 2886954 1. 2623103 1.2203378 1. 1805854 1. 1428039 1.0724503 1. 0082107 9. 2176798 8.4566114 7.5787071 5.9120543 4. 7678510 3.3479623 2. 51.87906 1. 5902204

3
3
3
3
3
3
3
3
3
3
3
3
3
3
3
2
2
2
2
2
2
2
2
2
2
2

Table 56. Ionosphere reflection coefficients (amplitude, |T, and phase, Arg T) assuming the quasi-longitudinal approximation, for the special ray configuration of the Norton type 301 atmosphere, applicable to the propagation of waves between the D-region ( $h_{3}=70$ kilometers) or the E-region ( $h_{3}=90$ kilometers) of the ionosphere and the earth. 


$$
\begin{aligned}
& \omega / \omega_{T}=2.0000 \\
& \phi_{1}=60 \text { degrees }
\end{aligned}
$$

radians
$\operatorname{Arg} \mathrm{T}_{\text {em }}-\pi \quad\left|\mathrm{T}_{\mathrm{me}}\right|$

$h_{3}=70$ kilometers
$\left|\mathrm{T}_{\text {em }}\right|$

$\begin{array}{ll}0.0005 & 1.4635752=1 \\ 0.0010 & 1.4635938=1 \\ 0.0020 & 1.4636677=1 \\ 0.0030 & 1.4637918=1 \\ 0.0040 & 1.4639648=1 \\ 0.0050 & 1.4641874=1 \\ 0.0060 & 1.4644592=1 \\ 0.0070 & 1.4647798=1 \\ 0.0080 & 1.4651499=1 \\ 0.0090 & 1.4655688=1 \\ 0.0100 & 1.4660372=1 \\ 0.0120 & 1.4671200=1 \\ 0.0150 & 1.4691088=1 \\ 0.0200 & 1.4733849=1 \\ 0.0250 & 1.4788414=1 \\ 0.0300 & 1.4854482=1 \\ 0.0400 & 1.5019669=1 \\ 0.0500 & 1.5226015=1 \\ 0.0650 & 1.5603910=1 \\ 0.0800 & 1.6050646=1 \\ 0.1000 & 1.6727328=1 \\ 0.1500 & 1.8628886=1 \\ 0.2000 & 2.0547115=1 \\ 0.3000 & 2.3745073=1 \\ 0.4000 & 2.5749317=1 \\ 0.6000 & 2.5746033=1\end{array}$

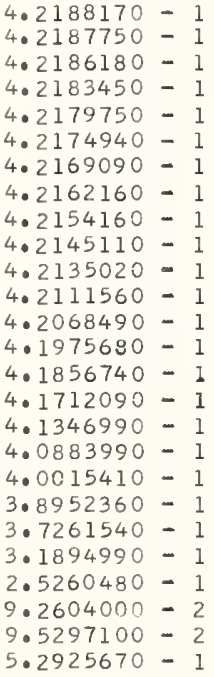

$1.6799323=1$
$1.6799522=1$
$1.6800310=1$
$1.6801627=1$
$1.6803474=1$
$1.6805844=1$
$1.6808740=1$
$1.6812159=1$
$1.6816099=1$
$1.6820560=1$
$1.6825551=1$
$1.6837079=1$
$1.6858246=1$
$1.6903733=1$
$1.6961706=1$
$1.7031809=1$
$1.7206598=1$
$1.7423951=1$
$1.7819019=1$
$1.8280734=1$
$1.8967976=1$
$2.0802470=1$
$2.2450001=1$
$2.4325864=1$
$2.3947913=1$
$1.7115844=1$

$\mathrm{h}_{3}=90$ kilometers

$\begin{array}{lll}0.0005 & 1.6009286=1 & 3.9052500= \\ 0.0010 & 1.6009432=1 & 3.9052140= \\ 0.0020 & 1.6010028=1 & 3.9050730= \\ 0.0030 & 1.6011027=1 & 3.9048300= \\ 0.0040 & 1.6012415=1 & 3.9044940= \\ 0.0050 & 1.6014210=1 & 3.9040590= \\ 0.0060 & 1.6016400=1 & 3.9035310= \\ 0.0070 & 1.6018993=1 & 3.9029030= \\ 0.0080 & 1.6021968=1 & 3.9021840= \\ 0.0090 & 1.6025355=1 & 3.9013630= \\ 0.0100 & 1.6029126=1 & 3.9004520= \\ 0.0120 & 1.6037862=1 & 3.8983340= \\ 0.0150 & 1.6053908=1 & 3.8944460- \\ 0.0200 & 1.6088438=1 & 3.8860570= \\ 0.0250 & 1.6132563=1 & 3.8753010= \\ 0.0300 & 1.6186076=1 & 3.8621990= \\ 0.0400 & 1.6320302=1 & 3.8290660= \\ 0.0500 & 1.6488792=1 & 3.7869170= \\ 0.0650 & 1.6799661=1 & 3.7074680= \\ 0.0800 & 1.7170832=1 & 3.6096120= \\ 0.1000 & 1.7740113=1 & 3.4527350= \\ 0.1500 & 1.9379771=1 & 2.9471040= \\ 0.2000 & 2.1082595=1 & 2.3111510= \\ 0.3000 & 2.3998080=1 & 7.5230900= \\ 0.4000 & 2.5845073=1 & 1.0985970- \\ 0.6000 & 2.5680026=1 & 5.3941250=\end{array}$

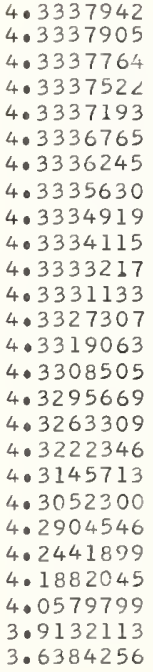

1.2433895 1.2381675 1. 2278386 1. 2176623 1.2076369 1.1977599 1. 1880278 1.1784374 1.1689853 1.1596669 1.1504788 1.1324770 1. 1063547 1.0649462 1.0259098 9.8899038 9.2076906 8.5914709 7.7737068 7.0657786 6. 2644905 4. 7915900 3.8174547 2.6471062 1.9802951 1. 2445355
4. 3061083 4.3061050 4. 3060927

4. 3060714

4. 3060419

4. 3060038

4. 3059574

4. 3059024

4. 3058393

4. 3057673

4.3056874

4. 3055016

4.3051608

4. 3044252

4. 3034827

4.3023350

4. 2994358

4. 2957529

4. 2888277

4. 2803277

4. 2667671

4. 2235876

4. 1703342

4. 0442199

3.9023950

3.6341094
1. 4000780 1.3948535 1. 3845147 1. 3743220 1.3642736 1.3543670 1.3445989 1. 3349661 1. 3254647 1.3160909 1. 3068406 1. 2886954 1. 2623103 1.2203378 1. 1805854 1. 1428039 1.0724503 1.0082107 9.2176798 8.4566114 7.5787071 5. 9120543 4. 7678510 3. 3479623 2. 5.187906 1. 5902204

Table 57. Ionosphere reflection coefficients (amplitude, $|\mathrm{T}|$, and phase, Arg $\mathrm{T}$ ) assuming the quasi-longitudinal approximation, for the special ray configuration of the Norton type 301 atmosphere, applicable to the propagation of waves between the D-region ( $h_{3}=70$ kilometers) or the E-region ( $h_{3}=90$ kilometers) of the ionosphere and the earth. 
$\omega / \omega_{r}=5.0000$
$\phi_{1}=60$ degrees

$\Psi$

radians

0.0005

0.0010

0.0020

0.0030

0.0040

0.0050

0.0060

0.0070

0.0080

0.0090

0.0100

0.0120

0.0150

0.0200

0.0250

0.0300

0.0400

0.0500

0.0650

0.0800

0.1000

0.1500

0.2000

0.3000

0.4000

0.6000

0.0005

0.0010

0.0020

0.0030

0.0040

0.0050

0.0060

0.0070

0.0080

0.0090

0.0100

0.0120

0.0150

0.0200

0.0250

0.0300

0.0400

0.0500

0.0650

0.0800

0.1000

C. 1500

0.2000

0.3000

0.4000

0.6000

$2.1184709-1$

$2.2724190=1$
$2.2724357=1$

$2.2725015=1$

$2.2729615-1$

$2.2746032-1$

$2.2755640-1$

$2.2811168=1$

$2.2859478-1$

$2.3063624=1$

2. $3244849=1$

$2.3958258-1$

$2.7448187-1$

$2.3849864-1$

$1.3306597=1$
$\left|\mathrm{T}_{\text {em }}\right|$

$2.1155666=1$

$\operatorname{Arg~T}_{\text {em }}-\pi \quad\left|\mathrm{T}_{\mathrm{me}}\right|$

$\mathrm{h}_{3}=70$ kilometers

$2.1155889-1$

$2.1156760-1$

$2.1158220-1$

$2.1162891=$

$2.1166091=$

$2.1169881-1$

$2.1174245-1$

$2.1179186-1$

$2.1197473-1$

$2.1271192=1$

$2.1335232=1$

$2.1412559-1$

$2.1604846-1$

$2.1842940-1$

$2.2769693=1$

$2.3498124-1$

$2.5358751-1$

$2.6855312=1$

$2.7583810-1$

$2.4245730-1$

1. $3477413-1$
$2.1160263-1$

$2.1220894=1$

$2.2272677-1$

$2 \cdot 1122241-1$

$2.1122440-1$

2. $1123219-1$

$2.1124513=1$

2. $1126339-1$

$2.1128685-1$

$2.1131533-1$

2. $1134916-1$

$2.1138805-1$

$2.1143206-1$

$2.1148126-1$

$2.1159489-1$

$2.1180337=1$

$2.1225045-1$

2. $1281874-1$

$2.1350323-1$

2. $1519754-1$

$2.1727917-1$

$2.2098711-1$

$2.2518743-1$

2.3114177 -

$2.4474592=1$

$2.5216149=1$

$2.3765366-1$

$1.8022902-1$

$5.3603473-2$

$\mathrm{h}_{3}=90$ kilometers

$2.2726112-1$

$2.2727638-1$

$2.2732031-1$

$2.2734881-1$

$2.2738158-1$

$2.2741886-1$

$2.2773272-1$

$2.2917902-1$

$2.3574141-1$

$2.4526845-1$

$2.5999800=1$

$2.7175560-1$

$2.5788750-1$
$2.5788080-1$
$2.5785140-1$
$2.5780090-1$
$2.5773230-1$
$2.5764270-1$
$2.5753320-1$
$2.5740440-1$
$2.5725660-1$
$2.5708730-1$
$2.5689890-1$
$2.5646400-1$
$2.5566280-1$
$2.5393510-1$
$2.5171920-1$
$2.4901930-1$
$2.4218980-1$
$2.3349490-1$
$2.1708850-1$
$1.9684940-1$
$1.6432460-1$
$5.8833200-2$
$7.5321500-2$
$4.0912800-1$
$7.8338550-1$
1.2308185

$2.2480715=$

3.7063471

3.7063405

3. 7063117

3.7062623

3.7061952

3.7061074

3.7060001

3.7058740

3.7057293

3.7055636

3.7053791

3.7049531

3.7041686

3.7024765

3. 7003067

3.6976626

3.6909746

3.6824596

3.6663911

3.6465660

3.6146964

3.5111927

3.3790856

3.0469226

2.6707218

2.3149455
1.2433895
1.2381675
1.2278386
1.2176623
1.2076369
1.1977599
1.1880275
1.1784374
1.1689852
1.1596659
1.1504785
1.1324770
1.1063547
1.0649462
1.0259028
9.8899038
9.2076906
8.5914709
7.7737068
7.0657786
5.2644905
4.7915900
3.8174547
2.6471062
1.9802951
1.2445355

3
3
3
3
3
3
3
3
3
3
3
3
3
3
3
2
2
2
2
2
2
2
2
2
2
2

1. 4000780 1.3948535

1.3845147

I. 3743220

1. 3642736

$1.354367 \mathrm{C}$

1. 3445989

1. 3349661

I. 3254647

1. 3160909

1. 3068406

]. 2886954

1. 2623103

1. 2203378

1. 1805854

1. 1428039

1. 0724503

1.0082107

9.2176798

8.4566114

7.5787071

5.9120543

4.7678510

3.3479623

2. 5187906

1. 5902204

Table 58. Ionosphere reflection coefficients (amplitude, $|\mathrm{T}|$, and phase, Arg $\mathrm{T}$ ) assuming the quasi-longitudinal approximation, for the special ray configuration of the Norton type 301 atmosphere, applicable to the propagation of waves between the D-region ( $h_{3}=70$ kilometers) or the E-region ( $h_{3}=90$ kilometers) of the ionosphere and the earth. 


$$
\begin{array}{ll}
\omega / \omega_{r}=0.3002 & f=20 \text { kilocycles } \\
\phi_{1}=60 \text { degrees } & \sigma=0.005 \text { mhos } / \text { meter } \\
& \epsilon_{2}=15
\end{array}
$$

$\Psi$

radians

0.0005

0.0010

0.0020

0.0030

0.0040

0.0050

0.0060

0.0070

0.0080

0.0090

0.0100

0.0120

0.0150

0.0200

0.0250

0.0300

0.0400

0.0500

0.0650

0.0800

0.1000

0.1500

0.2000

0.3000

0.4000

0.6000

0.0005

0.0010

0.0020

0.0030

0.0040

0.0050

0.0060

0.0070

0.0080

0.0090

0.0100

0.0120

0.0150

0.0200

0.0250

0.0300

0.0400

0.0500

0.0650

0.0800

0.1000

0.1500

0.2000

0.3000

0.4000

0.6000 $\left|c_{2}\right|$

Arg C 2

$\mathrm{h}_{3}=70$ kilometers

$4.1287588-1$

3. $9484557-1$

3.6152155 - 1

$3.3175230=$

$3.0539490-1$

$2.8228636-1$

$2.6225363-1$

2. $4511728-1$

$2.3069282=1$

$2.1878733-1$

$2.0919871-1$

$1.9611499-1$

$1.8838966-1$

$1.9396829-1$

$2.0832416-1$

$2.2380686-1$

$2.4987210-1$

$2.6791020=1$

$2.8330041-1$

$2.8920449-1$

$2.8798498=1$

2. $6441229-1$

$2.3557661-1$

$2.0057473-1$

$1.9414728=1$

2. $0466471=1$

3.8370798
3.7892607
3.6933205
$3 \cdot 5964664$
3.4981882
3.3981456
3.2962114
$3 \cdot 1925117$
3.0874503
2.9817008
2.8761644
2.6699763
2.3878953
2.0232842
1.7789499
1.6155136
1.4221042
1.3196062
1.2456422
1.2217408
1.2329101
1.3751166
1.6045393
2.1514172
2.6436885
3.3151912

$h_{3}=90$ kilometers

$3.7272809=1$

$3.5674435=1$

$3.2722804=1$

3.0089492 -

$2.7761326-1$

$2.5723206-1$

$2.3959016=1$

$2.2452061=1$

$2.1185055-1$

2.0140029 - 1

$1.9298361-1$

$1.8147147=1$

$1.7454721=$

$1.7907106-1$

$1.9139523-1$

$2.0488863=1$

$2.2798266-1$

2.4435543 - 1

$2.5898681-1$

$2.6547500-1$

$2.6621690=1$

$2.4949346=1$

2.2683867 - 1

1.9869372 - 1

$1.9416774=1$

2.0490606 - 1
3. 9348034

3. 8865920

3.7899603

3.6925811

3.5940069

3.4939641

3. 3923887

3.2894598

3. 1856077

3.0815053

2. 9780098

2. 7767484

2. 5024877

2. 1470698

1.9065443

1.7440262

1.5494620

1.4447329

1.3670313

1. 3392525

1. 3451457

1.4739480

1.6894615

2. 2067538

2. 6756027

3. 3269857 $\tau_{\mathrm{j}}$

$\mathrm{d} / \mathrm{j}$

miles

1. 2433895 1.2381675

1. 2278386

1. 2176623

1.2076369

1. 1977599

1.1880278

1. 1784374

1.1689853

1.1596669

1. 1504788

1. 1324770

1.1063547

1.0649462

1.0259098

9.8899038

9.2076906

8.5914709

7.7737068

7.0657786

6.2544905

4.7915900

3.8174547

2.6471062

1.9802951

1.2445355

1. 1707963

$9.7079630-1$

1.5702963

1.5702963
1.5697963

1. 5687963

1.5677963

1.5667963

1. 5657963

1. 5647963

1.5637963

1.5627963

1. 5617963

1. 5607963

1. 5587963

1.5557963

1.5507963

1. 5457963

1. 5407963

1. 5307963

1. 5207963

1.5057963

1.4907963

1.4707963

1.4207963

1.3707963

1. 2707963

1. 1707963

$9.7079630-1$
1.4000780

1.3948535

1. 3845147

1. 3743220

1.3642736

1.3543670

1.3445989

1.3349661

1. 3254647

1. 3160909

1.3068406

1.2886954

1. 2623103

1. 2203378

$1 \cdot 1805854$

1. 1428039

1. 0724503

1. 0082107

9.2176798

8.4566114

7. 5787071

5.9120543

4.7678510

3.3479623

2. 5187906

1. 5902204

Table 59. Effective reflection coefficient (amplitude, $\left|C_{j}\right|$, and phase, $A r g C_{j}$ ) for the specia ray configuration of the Norton type 301 atmosphere, applicable to the propagation of waves between the $\mathrm{D}$-region $\left(\mathrm{h}_{3}=70\right.$ kilometers) or the $\mathrm{E}$-region $\left(\mathrm{h}_{3}=90\right.$ kilometer $\left.\mathrm{s}\right)$ of the ionosphere and the earth. 


$$
\begin{array}{ll}
\omega / \omega_{r}=0.467 & f=20 \text { kilocycles } \\
\phi_{1}=60 \text { degrees } & \sigma=0.005 \text { mhos } / \text { meter } \\
& \epsilon_{2}=15
\end{array}
$$

$\Psi$

radians

$$
\begin{aligned}
& 0.0005 \\
& 0.0010 \\
& 0.0020 \\
& 0.0030 \\
& 0.0040 \\
& 0.0050 \\
& 0.0060 \\
& 0.0070 \\
& 0.0080 \\
& 0.0090 \\
& 0.0100 \\
& 0.0120 \\
& 0.0150 \\
& 0.0200 \\
& 0.0250 \\
& 0.0300 \\
& 0.0400 \\
& 0.0500 \\
& 0.0650 \\
& 0.0800 \\
& 0.1000 \\
& 0.1500 \\
& 0.2000 \\
& 0.3000 \\
& 0.4000 \\
& 0.6000
\end{aligned}
$$

0.0005

0.0010

0.0020

0.0030

0.0040

0.0050

0.0060

0.0070

0.0080

0.0090

0.0100

0.0120

0.0150

0.0200

0.0250

0.0300

0.0400

0.0500

0.0650

0.0800

0.1000

0.1500

0.2000

0.3000

0.4000

0.6000 $\left|\mathrm{c}_{2}\right|$

Arg C 2

$\mathrm{h}_{3}=70$ kilometers

$4.7957379=1$
$4.5849250=1$
$4.1953862=1$
$3.8475311=1$
$3.5396976=1$
$3.2699951=1$
$3.0364151=1$
$2.8368785=1$
$2.6692395=1$
$2.5312462=1$
$2.4205284=1$
$2.2708063=1$
$2.1859914=1$
$2.2599019=1$
$2.4346442=1$
$2.6212586=1$
$2.9357764=1$
$3.1564900=1$
$3.3525028=1$
$3.4387722=1$
$3.4471322=1$
$3.2081872=1$
$2.8584249=1$
$2.2899790=1$
$2.0111960=1$
$1.8552125=1$

3.5922680
3.5443255
3.4480640
3.3507743
3.2519350
3.1511953
3.0484263
2.9437603
2.8376205
2.7307096
2.6239715
2.4154434
2.1305581
1.7635606
1.5181879
1.3537428
1.1570890
$1.0495975-$
$9.6534106-1$
$9.2826372-1$
$9.1798690-1$
$9.9109167-1$
1.1375145
1.5295749
1.9392362
2.5592026

$\mathrm{h}_{3}=90$ kilometers

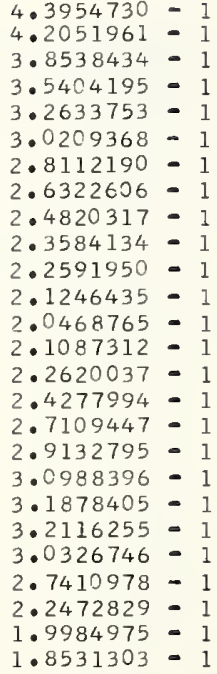

1.573434

2. 5702834
$T_{j}$

$d / j$

miles

1.5702963

1.2433895

1.2381675

1.2278386

1.2176623

1.2076369

1.1977599

1.1880278

1. 1794374

1.1689853

1. 1596669

1.1504788

1.1324770

1. $10 \leqslant 3547$

1. 0649462

1.0259098

9.8899038

9.2076906

8.5914709

7.7737068

7.0657786

6.2644905

4.7915900

3.8174547

2.6471062

1.9802951

1.2445355

1.17079

9.7079630

1.5702963

1. 5697963

1. 5687963

1. $5 \leqslant 77963$

1.5657963

1.5657963

1. 5647963

1.5637963

1. 5627963

1.5617963

1. 5607963

1.5537963

1.5557963

1. 5507963

1.5457963

1.5407963

1.5307963

1. 5207963

1.5057963

1.4907963

1.4707963

1.4207963

1. 3707963

1.2707963

1. 1707963

9.7079630

1.4000780 1.3948535

1.3845147

1.3743220

1.3642736

1. 3543670

1.3445989

1.3349661

1. 3254647

1.3160909

1.3068406

1. 2885954

1.2623103

1. 2203378

1.1805854

1.142803 ?

1.0724503

1.0082107

$9.217 \leqslant 798$

8.4566114

7.5787071

5.9120543

4.7678510

3.3479623

2.5187906

1.5902204

Table 60. Effective reflection coefficient (amplitude, $\left|C_{j}\right|$, and phase, Arg $C_{j}$ ) for the special ray configuration of the Norton type 301 atmosphere, applicable to the propagation of waves between the $D$-region $\left(h_{3}=70\right.$ kilometers) and the E-region $\left(h_{3}=90\right.$ kilometers $)$ of the ionosphere and the earth. 


$$
\begin{array}{ll}
\omega / \omega_{r}=0.1501 & f=10 \text { kilocycles } \\
\phi_{1}=60 \text { degrees } & \sigma=0.005 \text { mhos } / \text { meter } \\
& \epsilon_{2}=15
\end{array}
$$

$\Psi$

radians

$\left|\mathrm{C}_{2}\right|$

Arg $\mathrm{C}_{2}$

$\mathbf{h}_{3}=70$ kilometers

0.0005

0.0010

0.0020

0.0030

0.0040

0.0050

0.0060

0.0070

0.0080

0.0090

0.0100

0.0120

0.0150

0.0200

0.0250

0.0300

0.0400

0.0500

0.0650

0.0800

0.1000

0.1500

0.2000

0.3000

0.4000

0.6000

0.0005

0.0010

0.0020

0.0030

0.0040

0.0050

0.0060

0.0070

0.0080

0.0090

0.0100

0.0120

0.0150

0.0200

0.0250

0.0300

0.0400

0.0500

0.0650

0.0800

0.1000

0.1500

0.2000

0.3000

0.4000

0.6000

4.2609074
4.1928077
4.0556583
3.2160756
3.7732516
3.6272629
3.4792170
3.3311879
3.1859100
3.0462633
2.9147376
2.6821293
2.4122171
2.1236877
1.9536938
1.8466980
1.7283149
1.6749739
1.6553686
1.6770965
1.7454715
2.0302046
2.3908322
3.0845315
3.6054918
4.2578185

1.5702963

I. 5697963

1. 5687963

1.5677963

1.5667963

1.5657963

1.5647963

1.5637963

1.5627963

1.5617963

1. 5607963

1. 5587963

1. 5557963

1.5507963

1.5457963

1.5407963

1.5307963

1.5207963

1.5057963

1.4907963

1.4707963

1.4207963

1. 3707963

1.2707963

1.1707963

9.7079630 - I

$\mathbf{h}_{3}=90$ kilometers

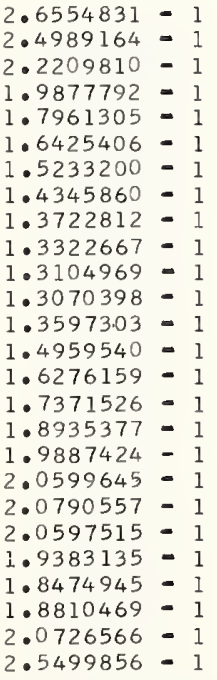

$4 \cdot 4255347$
$4 \cdot 3563666$
$4 \cdot 2172709$
$4 \cdot 0761824$
$3 \cdot 9325507$
$3 \cdot 7867085$
$3 \cdot 6399408$
$3 \cdot 4943530$
$3 \cdot 3525156$
$3 \cdot 2169942$
$3 \cdot 0898870$
$2 \cdot 8656663$
$2 \cdot 6048667$
$2 \cdot 3231609$
$2 \cdot 1548911$
$2 \cdot 0477399$
$1 \cdot 9271726$
$1 \cdot 8707255$
$1 \cdot 8456141$
$1 \cdot 8610599$
$1 \cdot 9200139$
$2 \cdot 1767689$
$2 \cdot 5055177$
$3 \cdot 1451657$
$3 \cdot 6374411$
$4 \cdot 2686239$

$d / j$

miles

\begin{tabular}{|c|c|c|}
\hline 1.5702963 & & 1.4000780 \\
\hline 1.5697963 & & 1.3948535 \\
\hline 1.5687963 & & 1.3845147 \\
\hline 1.5677963 & & 1.3743220 \\
\hline 1.5667963 & & 1.3642736 \\
\hline 1.5657963 & & 1.3543670 \\
\hline 1.5647963 & & 1.3445989 \\
\hline 1.5637963 & & 1.3349661 \\
\hline 1.5627963 & & 1.3254647 \\
\hline 1.5617963 & & 1.3160909 \\
\hline I. 5607963 & & 1.3068406 \\
\hline 1.5587963 & & $1 \cdot 2886954$ \\
\hline 1.5557963 & & 1.2623103 \\
\hline 1.5507963 & & 1.2203378 \\
\hline 1.5457963 & & 1.1805854 \\
\hline 1.5407963 & & 1.1428039 \\
\hline 1.5307963 & & 1.0724503 \\
\hline 1.5207963 & & 1.0082107 \\
\hline 1.5057963 & & 9.2176798 \\
\hline 1.4907963 & & 8.4566114 \\
\hline 1.4707963 & & 7.5787071 \\
\hline 1.4207963 & & 5.9120543 \\
\hline 1.3707963 & & 4.7678510 \\
\hline 1.270796 & & 3.3479623 \\
\hline 1.170796 & & 2.5187 \\
\hline 7079630 & -1 & 1.5902 \\
\hline
\end{tabular}

$\begin{array}{ll}1 \cdot 2433895 & 3 \\ 1 \cdot 2381675 & 3 \\ 1 \cdot 2278386 & 3 \\ 1 \cdot 2176623 & 3 \\ 1 \cdot 2076369 & 3 \\ 1 \cdot 1977599 & 3 \\ 1 \cdot 1880278 & 3 \\ 1 \cdot 1784374 & 3 \\ 1 \cdot 1589853 & 3 \\ 1 \cdot 1596669 & 3 \\ 1 \cdot 1504788 & 3 \\ 1 \cdot 1324770 & 3 \\ 1 \cdot 1063547 & 3 \\ 1.0649462 & 3 \\ 1.0259098 & 3 \\ 9.8899038 & 2 \\ 9 \cdot 2076906 & 2 \\ 8 \cdot 5914709 & 2 \\ 7 \cdot 7737068 & 2 \\ 7 \cdot 0657786 & 2 \\ 6.2644905 & 2 \\ 4 \cdot 7915900 & 2 \\ 3.8174547 & 2 \\ 2.6471062 & 2 \\ 1.9802951 & 2 \\ 1.2445355 & 2\end{array}$

Table 61. Effective reflection coefficient (amplitude, $\left|C_{j}\right|$, and phase, Arg $C_{j}$ ) for the special ray configuration of the Norton type 301 atmosphere, applicable to the propagation of waves between the $D$-region $\left(h_{3}=70\right.$ kilometers) and the E-region $\left(h_{3}=90\right.$ kilometers) of the ionosphere and the earth. 


$$
\begin{array}{ll}
\omega / \omega_{r}=0.2335 & f=10 \text { kilocycles } \\
\phi_{1}=60 \text { degrees } & \sigma=0.005 \text { mhos } / \text { meter } \\
& \epsilon_{2}=15
\end{array}
$$

$\Psi$

radians

0.0005

0.0010

0.0020

0.0030

0.0040

0.0050

0.0060

0.0070

0.0080

0.0090

0.0100

0.0120

0.0150

0.0200

0.0250

0.0300

0.0400

0.0500

0.0650

0.0800

0.1000

0.1500

0.2000

0.3000

0.4000

0.6000

0.0005
0.0010
0.0020
0.0030
0.0040
0.0050
0.0060
0.0070
0.0080
0.0090
0.0100
0.0120
0.0150
0.0200
0.0250
0.0300
0.0400
0.0500
0.0650
0.0800
0.1000
0.1500
0.2000
0.3000
0.4000
0.6000 $\left|c_{2}\right|$

Arg $C_{2}$

$\mathrm{h}_{3}=70$ kilometer $\mathrm{s}$

$3.6697651=1$
$3.4471322=1$
$3.0506054=1$
$2 \cdot 7161215=1$
$2.4394890=1$
$2 \cdot 2161763=1$
$2.0414449=1$
$1.9102890=1$
$1.8173759=1$
$1.7571007=1$
$3.4405661=1$
$1.7238030=1$
$1.7931498=1$
$1.9912556=1$
$2.1812029=1$
$2.3376968=1$
$2.5574704=1$
$2.6867258=1$
$2.7742924=1$
$2.7832876=1$
$2.7253217=1$
$2.4462187=1$
$2 \cdot 1768103=1$
$1.9336788=1$
$1.9640671=1$
$2.2007462=1$

3. 9654737

3. 8977541

3. 7613011

3.6222649

3.4797463

3. 3337261

3. 1852334

3. 0363176

2.8897536

2. 7485266

3. 8997900

2. 6152682

2.1052440

1.8125398

1.6397308

1.5301194

1. 4057926

1.3450668

1. 3115933

1. 3163743

1. 3581309

1. 5649354

1.8527309

2.4798191

2.9996079

3.6828005

$h_{3}=90$ kilometers

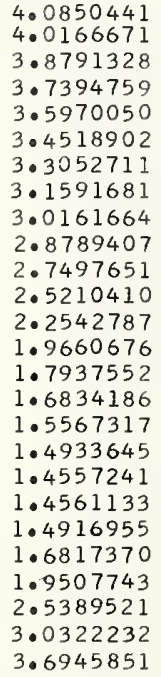

$\tau_{\mathbf{j}}$

$\mathrm{d} / \mathrm{j}$

miles

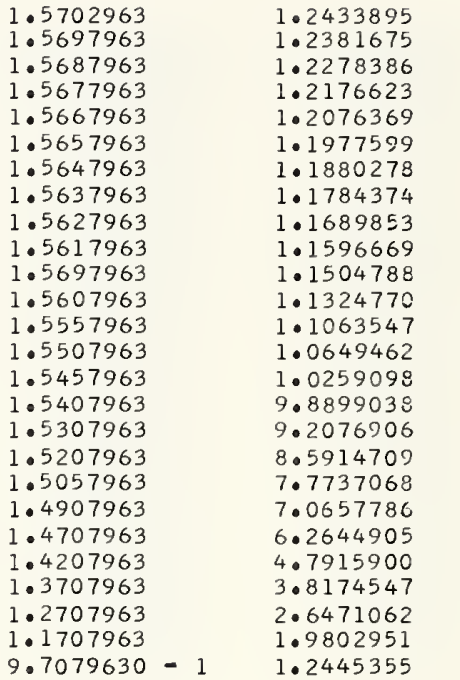

\begin{tabular}{|c|c|c|}
\hline $\begin{array}{l}1.5702963 \\
1.5697963\end{array}$ & & $\begin{array}{l}1.4000780 \\
1.3948535\end{array}$ \\
\hline 1.5687963 & & 1.3845147 \\
\hline 1.5677963 & & 1.3743220 \\
\hline 1.5667963 & & 1.3642736 \\
\hline 1.5657963 & & 1.3543670 \\
\hline 1.5647963 & & 1.3445980 \\
\hline 1.5637963 & & 1.3349661 \\
\hline 1.5627963 & & 1.3254647 \\
\hline 1.5617963 & & 1.3160909 \\
\hline 1.5607963 & & 1.3068406 \\
\hline 1.5587963 & & 1.2886954 \\
\hline 1.5557963 & & 1.2623103 \\
\hline 1.5507963 & & 1.2203378 \\
\hline 1.5457963 & & 1.1805854 \\
\hline 1.5407963 & & 1.1428039 \\
\hline 1.5307963 & & 1.0724503 \\
\hline 1.5207963 & & 1.0082107 \\
\hline 1.5057963 & & 9.2176798 \\
\hline 1.4907963 & & 8.4566114 \\
\hline 1.4707963 & & 7.5787071 \\
\hline 1.4207963 & & 5.9120543 \\
\hline 1.3707963 & & 4.7678510 \\
\hline .2707963 & & $3 \cdot 3479623$ \\
\hline 1.1707963 & & 2.5187906 \\
\hline .7079630 & -1 & 1.5902204 \\
\hline
\end{tabular}

Table 62. Effective reflection coefficient (amplitude, $\left|C_{j}\right|$, and phase, $A r g C_{j}$ ) for the special ray configuration of the Norton type 301 atmosphere, applicable to the propagation of waves between the D-region $\left(h_{3}=70\right.$ kilometers) or the E-region $\left(h_{3}=90\right.$ kilometers $)$ of the ionosphere and the earth. 


$$
\begin{array}{ll}
\omega / \omega_{\mathbf{r}}=0.0100 & f=0.42826552 \mathrm{kilocycles} \\
\phi_{1}=60 \text { degrees } & \sigma=0.005 \mathrm{mhos} / \mathrm{meter} \\
\epsilon_{2} & =15
\end{array}
$$

$\Psi$

radians

0.0005

0.0010

0.0020

0.0030

0.0040

0.0050

0.0060

0.0070

0.0080

0.0090

0.0100

0.0120

0.0150

0.0200

0.0250

0.0300

0.0400

0.0500

0.0650

0.0800

0.1000

0.1500

0.2000

0.3000

0.4000

0.6000

0.0010

0.0020

0.0030

0.0040

0.0050

0.0060

0.0070

0.0080

0.0090

0.0100

0.0120

0.0150

0.0200

0.0250

0.0300

0.0400

0.0500

0.0650

0.0800

0.1000

0.1500

0.2000

0.3000

0.4000

0.6000 $\left|c_{2}\right|$

$\operatorname{Arg~} \mathrm{C}_{2}$

$\mathrm{h}_{3}=70$ kilometers

$1.6442242=1$ $1.2349996-1$ $9.4756125=2$ $1.0313893=1$ $1.1882837-1$ $1.3321751=1$

$1.4514250=1$ $1.5488814=1$ $1.6290776=1$

$1.6958890-1$ $1.7522891=1$

1.8422114 - 1

$1.9395265=$ ? $2.0471487-1$ $2.1204119=1$ 2.1765981 $2.2657279-1$ 2.3433723 - 1 $2.4581561-1$ 2.5801995 - 1 $2.7586730=1$ $3.2667779-1$ $3.8041980=1$ $4.7769273=1$ $6.5318570=1$ 5.5339275 - 1

$9.6598276-1$
$5.9625614-1$
6.0892502
5.5362594
5.2356043
5.0615933
4.9500909
4.8728634
4.8163089
4.7731793
4.7392752
4.6896519
4.6422515
4.5995209
4.5793623
4.5713761
4.5761591
4.5975508
4.6478946
4.7111879
4.8051927
5.0401129
5.2365100
5.5051805
5.8407578
5.6666943

$h_{3}=90$ kilometers

$1.8660277=1$
$1.3987987=1$
$1.0662726=1$
$1.1588506=1$
$1.3364844=1$
$1.4998043=1$
$1.6352048=1$
$1.7458379=1$
$1.8368353=1$
$1.9126006=1$
$1.9765057=1$
$2.0782415=1$
$2.1879637=1$
$2.3083203=1$
$2.3890111=1$
$2.4496798=1$
$2.5427622=1$
$2.6203602=1$
$2.7304464=1$
$2.8439243=1$
$3.0067902=1$
$3.4658618=1$
$3.9550216=1$
$4.8595371=1$
$5.5801760=1$
$6.5483375=1$

1.2106809

5.7773188

5.4741532

5.2989873

5.1868468

5. 1091788

5.0522600

5.0087936

4. 9745511

4. 9242098

$4 \cdot 8755661$

4. 8302365

4. 8068178

4. 7949471

4.7903008

4.8004637

4. 8317413

4.8747929

4. 9423052

5.1235130

5. 2866371

5.5251024

5.6758869

5.8434246
$\boldsymbol{T}_{\mathrm{j}}$ $d / j$ miles

1.5702963

1.2433895 1.2381675 1.2278386 1.2176623 1.2076369 1.1977599 1.1880278 1. 1784374 1. 1689853 1.1596669 1. 1504788 1.1324770 1. 1063547 1.0649462 1.0259098 9.8899038 9.2076906 8.5914709 7.7737068 7.0657786 6.2644905 4. 7915900 3. 8174547 2.6471062

$\begin{array}{ll}9.7079630-1 & 1.9802951 \\ 1.1707963 & 1.2445355\end{array}$

$\begin{array}{ll}9.7079630-1 & 1.9802951 \\ 1.1707963 & 1.2445355\end{array}$
1.5702963

1.5687963

1. 5677963

1. 5667963

1. 5657963

1.5647963

1. 5637963

1. 5627963

1.5617963

1.5607963

1.5587963

1. 5557963

1. 5507963

1. 5457963

I. 5407963

1.5307963

1.5207963

1. 5057963

1.4907963

1.4707963

1.4207963

1.3707963

1. 2707963

1.1707963

9.7079630
$5.1855900-2$
1.4000780 .3948535 1. 3845147 1.3743220 1. 3642736 1.3543670 1.3445989 1.3349661 1.3254647 1.3160909 1.3068406 1.2886954 1.2623103 1.2203378 1.1805854 1.1428039 1.0724503 1.0082107 9.2176798 8.4566114 7.5787071 5.9120543 4.7678510 3.3479623 2. 5187906 1. 5902204

3
3
3
3
3
3
3
3
3
3
3
3
3
3
3
2
2
2
2
2
2
2
2
2
2
2

Table 63. Effective reflection coefficient (amplitude, $\left|C_{j}\right|$, and phase, $\mathrm{Arg}_{\mathrm{j}}$ ) for the special ray configuration of the Norton type 301 atmosphere, applicable to the propagation of waves between the $D$-region $\left(h_{3}=70\right.$ kilometers $)$ or the E-region $\left(h_{3}=90\right.$ kilometers $)$ of the ionosphere and the earth. 


$$
\begin{aligned}
& \omega / \omega_{r}=0.0200 \quad f=0.85653105 \text { kilocycles } \\
& \phi_{1}{ }^{\mathrm{r}}=60 \text { degrees } \quad \sigma=0.005 \mathrm{mhos} / \mathrm{meter} \\
& \epsilon_{2}=15
\end{aligned}
$$

$\Psi$

radians $\left|c_{2}\right|$

$\operatorname{Arg~} C_{2}$

$h_{3}=70$ kilometers

$1.3593754=1$
$1.0973550=1$
$8.1178885=2$
$7.5790885=2$
$8.1322760=2$
$8.9823755=2$
$9.8289220=2$
$1.0589294=1$
$1.1251750=1$
$1.1824864=1$
$1.2321574=1$
$1.3133783=1$
$1.4032962=1$
$1.5033620=1$
$1.5699465=1$
$1.6187154=1$
$1.6895078=1$
$1.7441648=1$
$1.8173421=1$
$1.8918704=1$
$2.0026705=1$
$2.3506708=1$
$2.7715315=1$
$3.6463290=1$
$4.4101344=1$
$5.5096915=1$

$2.0178840-1$

6.2254275

5. 6563494

5. 1281614

4. 7541613

4. 5130750

4. 3534846

4. 2421048

4. 1605380

4.0984557

4. 0497590

3.9786510

3.9108290

3. 8494557

3. 8199752

3. 8075885

3. 8118680

3.8394764

3.9070207

3. 9938914

4. 1254968

4.4637551

4.7527788

5. 1503463

5. 3877755

5. 6410671

$\mathrm{h}_{3}=90$ kilometers

0.0005

0.0010

0.0020

0.0030

0.0040

0.0050

0.0060

0.0070

0.0080

0.0090

0.0100

0.0120

0.0150

0.0200

0.0250

0.0300

0.0400

0.0500

0.0650

0.0800

0.1000

0.1500

0.2000

0.3000

0.4000

0.6000

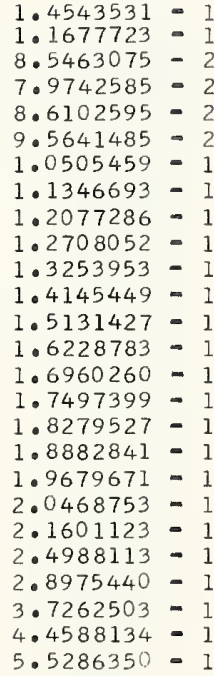

\section{$5.4389227-1$}

2.8439610 =

5. 9907848

5. 4493121

5. 0681060

4.8249459

4. 6651488

4. 5540668

4.4728599

4.4110732

4. 3625677

4. 2915445

4. 2232201

4. 1597653

4. 1270309

4. 1104391

4. 1039638

4. 1182941

4. 1626007

4. 2240219

4. 3211929

4. 5858305

4. 8268521

5. 1797052

5.4012125

5. 6449236 $\tau_{j}$

$d / j$

miles

1.5702963
1.5697963
1.5687963
1.5677963
1.5667963
1.5657963
1.5647963
1.5637963
1.5627963
1.5617963
1.5607963
1.5587963
1.5557963
1.5507963
1.5457963
1.5407963
1.5307963
1.5207963
1.5057963
1.4907963
1.4707963
1.4207963
1.3707963
1.2707963
1.1707963
$9.7079630-1$

1. 2433895

1. 2381675

1. 2278386

1. 2176623

1. 2076369

1. 1977599

1. 1880278

1. 1784374

1. 1689853

1. 1596669

I. 1504788

1. 1324770

I. 1063547

1. 0649462

1. 0259098

9.8899038

9.2076906

8. 5914709

7.7737068

7.0657786

6. 2644905

4.7915900

3.8174547

2.6471062

1.9802951

1.2445355

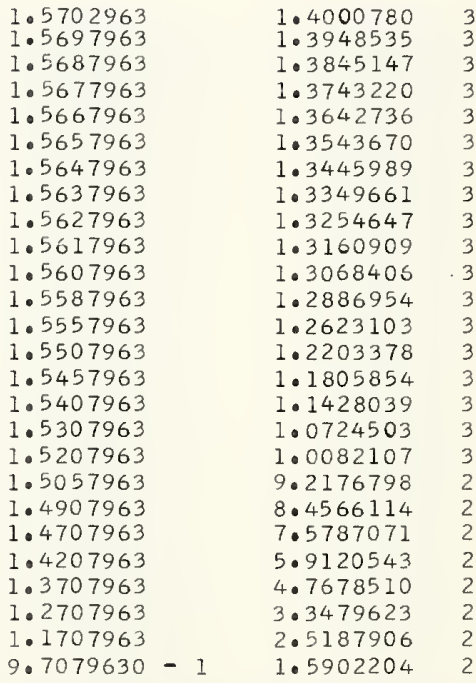

Table 64. Effective reflection coefficient (amplitude, $\left|C_{j}\right|$, and phase, $\operatorname{Arg} C_{j}$ ) for the special ray configuration of the Norton type 301 atmosphere, applicable to the propagation of waves between the D-region $\left(h_{3}=70\right.$ kilometers) or the E-region $\left(h_{3}=90\right.$ kilometers) of the ionosphere and the earth. 


$$
\begin{array}{ll}
\omega / \omega_{\mathrm{r}}=0.0500 & \mathrm{f}=2.1413276 \mathrm{kilocycles} \\
\phi_{1}=60 \text { degrees } & \sigma=0.005 \text { mhos } / \text { meter } \\
& \epsilon_{2}=15
\end{array}
$$

$\Psi$

radians

$\left|c_{2}\right|$

$\operatorname{Arg~C} 2$

$\mathrm{h}_{3}=70$ kilometers

0.0005

0.0010

0.0020

0.0030

0.0040

0.0050

0.0060

0.0070

0.0080

0.0090

0.0100

0.0120

0.0150

0.0200

0.0250

0.0300

0.0400

0.0500

0.0650

0.0800

0.1000

0.1500

0.2000

0.3000

0.4000

0.6000

0.0010

0.0020

0.0030

0.0040

0.0050

0.0060

0.0070

0.0080

0.0090

0.0100

0.0120

0.0150

0.0200

0.0250

0.0300

0.0400

0.0500

0.0650

0.0800

0.1000

0.1500

0.2000

0.3000

0.4000

0.6000

5.2637219
5.1067357
4.7803803
4.4437549
4.1229288
3.8459328
3.6232867
3.4498282
3.3150705
3.2092105
3.1247133
2.9996408
2.8785199
2.7651302
2.7051142
2.6726542
2.6524272
2.6664781
2.7251677
2.8131227
2.9591214
3.3825478
3.7896377
4.4040478
4.7916582
5.2118334

1.1707963

$\mathrm{h}_{3}=90$ kilometers

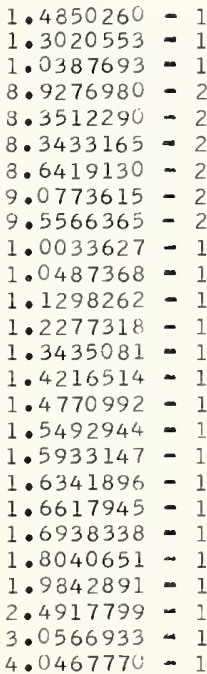

1.5697963

1.5687963

1.5677963

1.5667963

1.5657963

1.5647963

1.5637963

1.5627963

1.5617963

1.5607963

1.5587963

1.5557963

1.5507963

1.5457963

1. 5407963

1.5307963

1.5207963

1.5057963

1.4907963

1.4707963

1.4207963

1.3707963

1.2707963

9.7079630 -

$\mathrm{d} / \mathrm{j}$

miles

$\begin{array}{ll}1.2433895 & 3 \\ 1.2381675 & 3 \\ 1.2278386 & 3 \\ 1.2176623 & 3 \\ 1.2076369 & 3 \\ 1.1977599 & 3 \\ 1.1880278 & 3 \\ 1.1784374 & 3 \\ 1.1689853 & 3 \\ 1.1596669 & 3 \\ 1.1504788 & 3 \\ 1.1324770 & 3 \\ 1.1063547 & 3 \\ 1.0649462 & 3 \\ 1.0259098 & 3 \\ 9.8899038 & 2 \\ 9.2076906 & 2 \\ 8.5914709 & 2 \\ 7.7737068 & 2 \\ 7.0657786 & 2 \\ 6.2644905 & 2 \\ 4.7915900 & 2 \\ 3.8174547 & 2 \\ 2.6471062 & 2 \\ 1.9802951 & 2 \\ 1.2445355 & 2\end{array}$

1.5702963

1.5697963

1. 5687963

1. 5677963

1.5667963

1.5657963

1.5647963

1. 5637963

1.5627963

1.5617963

1.5607963

1.5587963

1.5557963

1.5507963

1.5457963

1. 5407963

1.5307963

1.5207963

1.5057963

1.4907963

1.4707963

1.4207963

1.3707963

1.2707963

1.1707963

$9.7079630-1$ $\begin{array}{ll}1.4000780 & 3 \\ 1.3948535 & 3 \\ 1.3845147 & 3 \\ 1.3743220 & 3 \\ 1.3642736 & 3 \\ 1.3543670 & 3 \\ 1.3445989 & 3 \\ 1.3349661 & 3 \\ 1.3254647 & 3 \\ 1.3160909 & 3 \\ 1.3068406 & 3 \\ 1.2886954 & 3 \\ 1.2623103 & 3 \\ 1.2203378 & 3 \\ 1.1805854 & 3 \\ 1.1428039 & 3 \\ 1.0724503 & 3 \\ 1.0082107 & 3 \\ 9.2176798 & 2 \\ 8.4566114 & 2 \\ 7.5787071 & 2 \\ 5.9120543 & 2 \\ 4.7678510 & 2 \\ 3.3479623 & 2 \\ 2.5187906 & 2 \\ 1.5902204 & 2\end{array}$

Table 65. Effective reflection coefficient (amplitude, $\left|C_{j}\right|$, and phase, $\operatorname{Arg} C_{j}$ ) for the special ray configuration of the Norton type 301 atmosphere, applicable to the propagation of waves between the $\mathrm{D}$-region $\left(\mathrm{h}_{3}=70\right.$ kilometers $)$ or the $\mathrm{E}$-region $\left(\mathrm{h}_{3}=90\right.$ kilometer $\left.\mathrm{s}\right)$ of the ionosphere and the earth. 


$$
\begin{array}{ll}
\omega / \omega_{\mathbf{r}}=0.1000 & f=4.2826552 \mathrm{kilocycles} \\
\phi_{1}=60 \text { degrees } & \sigma=0.005 \mathrm{mhos} / \text { meter } \\
& \epsilon_{2}=15
\end{array}
$$

$\Psi$

radians

$\left|c_{2}\right|$

Arg $\mathrm{C}_{2}$

$h_{3}=70$ kilometers

0.0005

0.0010

0.0020

0.0030

0.0040

0.0050

0.0060

0.0070

0.0080

0.0090

0.0100

0.0120

0.0150

0.0200

0.0250

0.0300

0.0400

0.0500

0.0650

0.0800

0.1000

0.1500

0.2000

0.3000

0.4000

0.6000

$4 \cdot 5553173$
4.4495495
$4 \cdot 2341636$
4.0116631
3.7841462
3.5588789
3.3457456
3.1531291
2.9852436
2.8422147
2.7216723
2.5348161
2.3469899
2.1658973
2.0650240
2.0043581
1.9448887
1.9296832
1.9516760
2.0071729
2.1154934
2.4837500
2.8954418
3.6035078
4.0984636
4.6806167

1.5702963

1.5697963

1.5687963

1.5677963

1.5667963

1.5657963

1.5647963

1. 5637963

1.5627963

1.5617963

1.5607963

1.5587963

1.5557963

1.5507963

1. 5457963

1.5407963

1. 5307963

1.5207963

1.5057963

1.4907963

1.4707963

1.4207963

1.3707963

1.2707963

1. 1707963

$9.7079630=$

$h_{3}=90$ kilometers $\mathrm{d} / \mathrm{j}$

miles
0.0005

0.0010

0.0020

0.0030

0.0040

0.0050

0.0060

0.0070

0.0080

0.0090

0.0100

0.0120

0.0150

0.0200

0.0250

0.0300

0.0400

0.0500

0.0650

0.0800

0.1000

0.1500

0.2000

0.3000

0.4000

\begin{tabular}{|c|c|}
\hline $\begin{array}{l}1.5702963 \\
1.5697963\end{array}$ & $\begin{array}{l}1.4000780 \\
1.3948535\end{array}$ \\
\hline 1.5687963 & 1.3845147 \\
\hline 1.5677963 & 1.3743220 \\
\hline 1.5667963 & $1 \cdot 3642736$ \\
\hline 1.5657963 & 1.3543670 \\
\hline 1.5647963 & $1 \cdot 3445989$ \\
\hline 1.5637963 & 1.3349661 \\
\hline 1.5627963 & 1.3254647 \\
\hline 1.5617963 & 1.3160909 \\
\hline 1.5607963 & 1.3068406 \\
\hline 1.5587963 & 1.2886954 \\
\hline 1.5557963 & 1.2623103 \\
\hline 1.5507963 & 1.2203378 \\
\hline 1.5457963 & 1.1805854 \\
\hline 1.5407963 & 1.1428039 \\
\hline 1.5307963 & 1.0724503 \\
\hline 1.5207963 & 1.0082107 \\
\hline 1.5057963 & 9.2176798 \\
\hline 1.4907963 & 8.4566114 \\
\hline $\begin{array}{l}1.4707963 \\
1.4207963\end{array}$ & 7.5787071 \\
\hline $\begin{array}{l}.42017963 \\
.3707963\end{array}$ & $\begin{array}{l}5.9120543 \\
4.7678510\end{array}$ \\
\hline .2707963 & 3.3479623 \\
\hline .1707963 & 2.5187906 \\
\hline & 1.5002304 \\
\hline
\end{tabular}

0.6000

$2.0861478=1$
$1.9041461=1$
$1.6061722=1$
$1.3883866=1$
$1.2399870=1$
$1.1490763=1$
$1.1030672=1$
$1.0897477=1$
$1.0985574=1$
$1.1213118=1$
$1.1521916=1$
$1.2241165=1$
$1.3321916=1$
$1.4811482=1$
$1.5904740=1$
$1.6702952=1$
$1.7725985=1$
$1.8282644=1$
$1.8635992=1$
$1.8671266=1$
$1.8485436=1$
$1.7893982=1$
$1.7926915=1$
$1.9955435=1$
$2.3234269=1$
$3.0265868=1$
10

Table 66. Effective reflection coefficient (amplitude, $\left|C_{j}\right|$, and phase, Arg $C_{j}$ ) for the special ray configuration of the Norton type 301 atmosphere, applicable to the propagation of waves between the D-region $\left(h_{3}=70\right.$ kilometers) or the E-region $\left(h_{3}=90\right.$ kilometers $)$ of the ionosphere and the earth. 


$$
\begin{array}{ll}
\omega / \omega_{\mathrm{r}}=0.2000 & f=8.5653105 \mathrm{kilocycles} \\
\phi_{1}=60 \text { degrees } & \sigma=0.005 \mathrm{mhos} / \mathrm{meter} \\
\epsilon_{2}=15
\end{array}
$$

$\begin{array}{ll}\Psi & \left|C_{2}\right| \\ \text { radians } & \\ & \\ 0.0005 & 3.4177859=1 \\ 0.0010 & 3.1951908=1 \\ 0.0020 & 2.8029758=1 \\ 0.0030 & 2.4777813=1 \\ 0.0040 & 2.2145360=1 \\ 0.0050 & 2.0077939=1 \\ 0.0060 & 1.8518290=1 \\ 0.0070 & 1.7405397=1 \\ 0.0080 & 1.6674416=1 \\ 0.0090 & 1.6258569=1 \\ 0.0100 & 1.6092922=1 \\ 0.0120 & 1.6283913=1 \\ 0.0150 & 1.7254543=1 \\ 0.0200 & 1.9288783=1 \\ 0.0250 & 2.1097916=1 \\ 0.0300 & 2.2538861=1 \\ 0.0400 & 2.4502983=1 \\ 0.0500 & 2.5614537=1 \\ 0.0650 & 2.6305579=1 \\ 0.0800 & 2.6286997=1 \\ 0.1000 & 2.5645680=1 \\ 0.1500 & 2.2972574=1 \\ 0.2000 & 2.0624947=1 \\ 0.3000 & 1.8999801=1 \\ 0.4000 & 1.9868018=1 \\ 0.6000 & 2.3071445=1 \\ & \end{array}$

Axg C,

$\mathbf{T}_{\mathbf{j}}$

$\mathrm{d} / \mathrm{j}$

$\mathrm{h}_{3}=70$ kilometers

4.0575547

3. 9842747

3. 8364279

3.6854210

3. 5303549

3. 3715984

3. 2109428

3. 0514176

2.8966955

2.7502803

2. 6148106

2. 3814205

2. 1199412

1. 8486882

1.6909638

1. 5917167

1.4808251

1.4291077

1.4060673

1.4200397

1.4737697

1. 7117493

2.0293678

2.6900566

3.2158171

3. 8952686

1. 5702963

1. 5697963

1.5687963

1.5677963

1.5667963

1.5657963

1.5647963

1.5637963

1.5627963

1.5617963

1. 5607963

1.5587963

1.5557963

1.5507963

1.5457963

1. 5407963

1.5307963

1.5207963

1.5057963

1.4907963

1.4707963

1.4207963

1.3707963

1.2707963

1.1707963

$9.7079630-1$

1.2433895

1.2381675

1. 2278386

1. 2176623

1.2076369

1. 1977599

1. 1880278

1. 1784374

1. 1689853

1.1596669

1. 1504788

1. 1324770

1. 1063547

1.0649462

1.0259098

9.8899036

9.2076906

8.5914709

7.7737068

7.0657786

6.2644905

4.7915900

3. 8174547

2.6471062

1.9802951

1.2445355

$\mathrm{h}_{3}=90$ kilometers

0.0005

0.0010

0.0020

0.0030

0.0040

0.0050

0.0060

0.0070

0.0080

0.0090

0.0100

0.0120

0.0150

0.0200

0.0250

0.0300

0.0400

0.0500

0.0650

0.0800

0.1000

0.1500

0.2000

0.3000

0.4000

0.6000

$3.0419321=1$
$2.8480014=1$
$2.5069739=1$
$2.2251098=1$
$1.9977543=1$
$1.8198569=1$
$1.6860987=1$
$1.5908754=1$
$1.5283452=1$
$1.4926286=1$
$1.4781321=1$
$1.4934424=1$
$1.5749775=1$
$1.7484042=1$
$1.9046323=1$
$2.0305480=1$
$2.2057135=1$
$2.3093429=1$
$2.3827820=1$
$2.3964154=1$
$2.3618394=1$
$2.1767009=1$
$2.0056920=1$
$1.8997239=1$
$1.9961416=1$
$2.3142495=1$

4. 1917770

4. 1176716

3. 9684550

3. 8166625

3.6616770

3. 5041091

$3 \cdot 3458545$

3.1898294

3. 0393747

2. 8975471

2.7665476

2. 5406583

2.2859974

2.0186003

1.8611261

1.7610593

1.6476932

1. 5931762

1. 5656101

1. 5746972

1. 6214021

1.8395658

2. 1344167

2. 7503673

3. 2484455

3. 9068399

$\begin{array}{ll}1.5702963 & 1.4000780 \\ 1.5697963 & 1.3948535 \\ 1.5687963 & 1.3845147 \\ 1.5677963 & 1.3743220 \\ 1.5667963 & 1.3642736 \\ 1.5657963 & 1.3543670 \\ 1.5647963 & 1.3445989 \\ 1.5637963 & 1.3349661 \\ 1.5627963 & 1.3254647 \\ 1.5617963 & 1.3160909 \\ 1.5607963 & 1.3068406 \\ 1.5587963 & 1.2886954 \\ 1.5557963 & 1.2623103 \\ 1.5507963 & 1.2203378 \\ 1.5457963 & 1.1805854 \\ 1.5407963 & 1.1428039 \\ 1.5307963 & 1.0724503 \\ 1.5207963 & 1.0082107 \\ 1.5057963 & 9.2176798 \\ 1.4907963 & 8.4566114 \\ 1.4707963 & 7.5787071 \\ 1.4207963 & 5.9120543 \\ 1.3707963 & 4.7678510 \\ 1.2707963 & 3.3479623 \\ 1.1707963 & 2.5187906 \\ 9.7079630-1 & 1.5902204\end{array}$

Table 67. Effective reflection coefficient (amplitude, $\left|C_{j}\right|$, and phase, Arg $C_{j}$ ) for the special ray configuration of the Norton type 301 atmosphere, applicable to the propagation of waves between the D-region $\left(h_{3}=70\right.$ kilometers) or the $E$-region $\left(h_{3}=90\right.$ kilometers $)$ of the ionosphere and the earth. 


$$
\begin{array}{ll}
\omega / \omega_{r}=1.0000 & f=42.826552 \mathrm{kilocycles} \\
\phi_{1}=60 \text { degrees } & \sigma=0.005 \mathrm{mhos} / \mathrm{meter} \\
& \epsilon_{2}=15
\end{array}
$$

$\begin{array}{ll}\Psi & \left|C_{2}\right| \\ \text { radians } & \\ & \\ 0.0005 & 5.5993240=1 \\ 0.0010 & 5.4283210=1 \\ 0.0020 & 5.1038375=1 \\ 0.0030 & 4.8024110=1 \\ 0.0040 & 4.5235505=1 \\ 0.0050 & 4.2666636=1 \\ 0.0060 & 4.0310833=1 \\ 0.0070 & 3.8161080=1 \\ 0.0080 & 3.6210055=1 \\ 0.0090 & 3.4450217=1 \\ 0.0100 & 3.2873893=1 \\ 0.0120 & 3.0239864=1 \\ 0.0150 & 2.7457582=1 \\ 0.0200 & 2.5308301=1 \\ 0.0250 & 2.5214389=1 \\ 0.0300 & 2.6168096=1 \\ 0.0400 & 2.8996513=1 \\ 0.0500 & 3.1660899=1 \\ 0.0650 & 3.4593202=1 \\ 0.0800 & 3.6369754=1 \\ 0.1000 & 3.7439585=1 \\ 0.1500 & 3.6454886=1 \\ 0.2000 & 3.3200310=1 \\ 0.3000 & 2.5670268=1 \\ 0.4000 & 1.9543700=1 \\ 0.6000 & 1.2395280=1 \\ & \end{array}$

$\operatorname{Arg~C}_{2}$

$\mathrm{h}_{3}=70$ kilometers

3.1606849
3.1278453
3.0620418
2.9958860
2.9291749
2.8617314
2.7934147
2.7241262
2.6538201
2.5825067
2.5102624
2.3636138
2.1422186
1.7929726
1.4996847
$1.2723434=$
$9.7081496=1$
$7.9149715=1$
$6.3327400=1$
$5.4051762=1$
$4.6675562=1$
$3.9072395=1$
$3.8046942=1$
$4.4252644=1$
$5.6566036=1$
$8.8718383=1$

$\mathrm{h}_{3}=90$ kilometers

$\begin{array}{ll}0.0005 & 5.2105255=1 \\ 0.0010 & 5.0533610=1 \\ 0.0020 & 4.7552425=1 \\ 0.0030 & 4.4784546=1 \\ 0.0040 & 4.2225354=1 \\ 0.0050 & 3.9869195=1 \\ 0.0060 & 3.7709788=1 \\ 0.0070 & 3.5740454=1 \\ 0.0080 & 3.3954268=1 \\ 0.0090 & 3.2343990=1 \\ 0.0100 & 3.0902339=1 \\ 0.0120 & 2.8494385=1 \\ 0.0150 & 2.5949775=1 \\ 0.0200 & 2.3969351=1 \\ 0.0250 & 2.3853479=1 \\ 0.0300 & 2.4695382=1 \\ 0.0400 & 2.7250440=1 \\ 0.0500 & 2.9694173=1 \\ 0.0650 & 3.2430779=1 \\ 0.0800 & 3.4137715=1 \\ 0.1000 & 3.5240860=1 \\ 0.1500 & 3.4618829=1 \\ 0.2000 & 3.1799246=1 \\ 0.3000 & 2.4923513=1 \\ 0.4000 & 1.9160526=1 \\ 0.6000 & 1.2289413=1\end{array}$

${ }^{\top} j$

$\mathrm{d} / \mathrm{j}$

miles

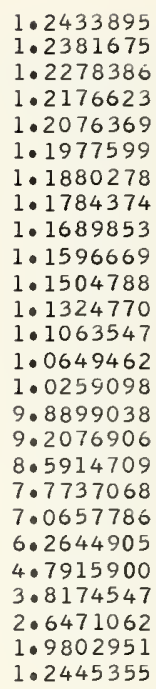
3
3
3
3
3
3
3
3
3
3
3
3
3
3
3
2
2
2
2
2
2
2
2
2
2

1.1707963

$9.7079630-1$

$\begin{array}{lll}3.1605307 & 1.5702963 & 1.4000780 \\ 3.1275248 & 1.5697963 & 1.3948535 \\ 3.0614167 & 1.5687963 & 1.3845147 \\ 2.9950047 & 1.5677963 & 1.3743220 \\ 2.9280983 & 1.5667963 & 1.3642736 \\ 2.8605352 & 1.5657963 & 1.3543670 \\ 2.7921888 & 1.5647963 & 1.3445989 \\ 2.7229764 & 1.5637963 & 1.3349661 \\ 2.6528656 & 1.5627963 & 1.3254647 \\ 2.5818813 & 1.5617963 & 1.3160909 \\ 2.5101081 & 1.5607963 & 1.3068406 \\ 2.3648470 & 1.5587963 & 1.2886954 \\ 2.1465552 & 1.5557963 & 1.2623103 \\ 1.8038188 & 1.5507963 & 1.2203379 \\ 1.5160876 & 1.5457963 & 1.1805854 \\ 1.2921384- & 1.5407963 & 1.1428039 \\ 9.9293489-1 & 1.5307963 & 1.0724503 \\ 8.1360066-1 & 1.5207963 & 1.0082107 \\ 6.5444406-1 & 1.5057963 & 9.2176798 \\ 5.6067864-1 & 1.4907963 & 8.4566114 \\ 4.8574801-1 & 1.4707963 & 7.5787071 \\ 4.0758536-1 & 1.4207963 & 5.9120543 \\ 3.9593828-1 & 1.3707963 & 4.7678510 \\ 4.5623193-1 & 1.2707963 & 3.3479623 \\ 5.7774838-1 & 1.1707963 & 2.5187906 \\ 8.9481412-1 & 9.7079630-1 & 1.5902204\end{array}$

Table 68. Effective reflection coefficient (amplitude, $\left.\mid C_{j}\right)$, and phase, Arg $C_{j}$ ) for the special ray configuration of the Norton type 301 atmosphere, applicable to the propagation of waves between the D-region $\left(h_{3}=70\right.$ kilometers $)$ or the $E$-region $\left(h_{3}=90\right.$ kilometers $)$ of the ionosphere and the earth. 


$$
\begin{array}{ll}
\omega / \omega_{r}=2.0000 & f=85.653105 \mathrm{kilocycles} \\
\phi_{1}=60 \text { degrees } & \sigma=0.005 \mathrm{mhos} / \mathrm{meter} \\
& \epsilon_{2}=15
\end{array}
$$

$\Psi$

radians

$\left|\mathrm{C}_{2}\right|$
$\operatorname{Arg~C}_{2}$

$h_{3}=70$ kilometers

0.0005

0.0010

0.0020

0.0030

0.0040

0.0050

$0.006 \mathrm{C}$

0.0070

0.0080

0.0090

0.0100

0.0120

0.0150

0.0200

0.0250

0.0300

0.0400

0.0500

0.0550

0.0300

0.1000

0.1500

0.2000

0.3000

0.4000

0.6000

0.0005

0.0010

0.0020

0.0030

0.0040

0.0050

0.0060

0.0070

0.0080

0.0090

0.0100

0.0120

0.0150

0.0200

0.0250

0.0300

0.0400

0.0500

0.0650

0.0800

0.1000

0.1500

0.2000

0.3000

0.4000

0.6000

2.7766235
2.7531825
2.7062763
2.6592763
2.6121120
2.5647192
2.5170415
2.4690297
2.4206450
2.3718599
2.3226584
2.2230095
2.0708168
1.8142854
1.5654421
1.3379411
$9.7379513-1$
$7.2120633-1$
$4.7669254-1$
$3.2054341-1$
$1.8077521-1$
$6.2547461-1$
6.1198005
5.9212018
5.7648999
5.5740401

$\mathrm{h}_{3}=90$ kilometers

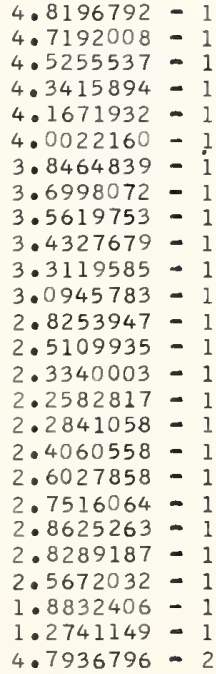

$5.2311740=1$
$5.1199835=1$
$4.9056081=1$
$4.7018439=1$
$4.5085709=1$
$4.3256319=1$
$4.1528494=1$
$3.9900139=1$
$3.8369066=1$
$3.6932976=1$
$3.5589448=1$
$3.3170107=1$
$3.0171357=1$
$2.6669408=1$
$2.4709771=1$
$2.3892141=1$
$2.4245791=1$
$2.5649696=1$
$2.7850137=1$
$2.9467974=1$
$3.0616013=1$
$3.0029549=1$
$2.7031133=1$
$1.9585461=1$
$1.3151052=1$
$4.9168725-2$

2.7168098
2.6932124
2.6460186
2.5987680
2.5513974
2.5038486
2.4560718
2.4080250
2.3596774
2.3110070
2.2620060
$2 \cdot 1630359$
2.0126183
1.7610192
1.5187501
1.2979367
$9.4351678-1$
$6.9557810-1$
$4 \cdot 5355964-1$
$2.9831071-1$
$1.5940414-1$
6.2360174
6.1039487
5.9101026
5.7572481
5.5736467

$\boldsymbol{T}_{\mathbf{j}} \quad \mathrm{d} / \mathbf{j}$

miles

Table 69. Effective reflection coefficient (amplitude, $\left|C_{j}\right|$, and phase, $A r g C_{j}$ ) for the special ray configuration of the Norton type 301 atmosphere, applicable to the propagation of waves between the $D$-region $\left(h_{3}=70\right.$ kilometers) or the $E$-region $\left(h_{3}=90\right.$ kilometers $)$ of the

\begin{tabular}{|c|c|}
\hline $\begin{array}{l}1.5702963 \\
1.5697963\end{array}$ & $\begin{array}{l}1.4000780 \\
1.3948535\end{array}$ \\
\hline $\begin{array}{l}1.5697963 \\
1.5687963\end{array}$ & $1 \cdot 3845147$ \\
\hline 1.5677963 & 1.3743220 \\
\hline 1.5667963 & 1.3642736 \\
\hline 1.5657963 & 1.3543670 \\
\hline 1.5647963 & 1.3445989 \\
\hline 1.5637963 & 1.3349661 \\
\hline 1.5627963 & 1.3254647 \\
\hline 1.5617963 & 1.3160909 \\
\hline 1.5607963 & 1.3068406 \\
\hline 1.5587963 & 1.2886954 \\
\hline 1.5557963 & 1.2623103 \\
\hline 1.5507963 & 1.2203378 \\
\hline 1.5457963 & 1.1805854 \\
\hline 1.5407963 & 1.1428039 \\
\hline 1.5307963 & 1.0724503 \\
\hline 1.5207963 & 1.0082107 \\
\hline .5057963 & 9.2176798 \\
\hline .4907963 & 8.4566114 \\
\hline .4707963 & 7.5787071 \\
\hline 1.4207963 & 5.9120543 \\
\hline .3707963 & 4.7678510 \\
\hline .2707963 & 3.3479623 \\
\hline .1707963 & 2.5187906 \\
\hline & 1.5902204 \\
\hline
\end{tabular}
ionosphere and the earth.

1.2433895 $1.2381675 \quad 3$ $1.2278386 \quad 3$ $1.2176623 \quad 3$ 1.1977599 1.1880278 $1.1784374 \quad 3$ $1.1689853 \quad 3$ 1.1596669 1.1324770 1.1063547 1.06494623 $1.0259098 \quad 3$ 9.8899038 2 7.7737068 7.06577862 $6.2644905 \quad 2$ 4.7915900 2 3.8174547 2 $2.6471062 \quad 2$ 1.9802951
1.2445355

1.1707963 $9.7079630-1$ 2






$$
\begin{array}{ll}
\omega / \omega_{x}=5.0000 & f=214.13276 \text { kilocycles } \\
\phi_{1}=60 \text { degrees } & \sigma=0.005 \text { mhos } / \text { meter } \\
& \epsilon_{2}=15
\end{array}
$$

$\Psi$

radians

$\left|\mathrm{C}_{2}\right|$

$3.8497352=1$

3.5672916 - 1

3.3163991 - 1

$3.0956570-1$

2.9034240 - 1

$2.7379016=$

2.5971985 - 1

$2.4793455=1$

$2.3823354=1$

$2.3041327-1$

$2.1960841=1$

$2.1264160=1$

$2.1540090=1$

$2.2552456-1$

$2.3709818-1$

2.5721888 - 1

$2.7112674=$

2.8205366 - 1

$2.8439289=1$

2.7876623 -

2.4256992 - 1

1.9645660 - 1

$1.1312075=1$

$5.3964585-2$

$7.2967105-3$

0.4000

0.6000

0.0005

0.0010

0.0020

0.0030

0.0040

0.0050

0.0060

0.0070

0.0080

0.0090

0.0100

0.0120

0.0150

0.0200

0.0250

0.0300

0.0400

0.0500

0.0650

0.0800

0.1000

0.1500

0.2000

0.3000

0.4000

0.6000
Arg $C_{2}$

$\mathrm{h}_{3}=70$ kilometers

2.2122308
2.1631139
2.0649651
1.9666905
1.8681295
1.7692834
1.6703228
1.5715861
1.4735553
1.3768188
1.2820179
$1.1007403=$
$8.5688508-1$
$5.3621693=1$
$3.0817107=1$
$1.4454802-1$
6.2125827
6.0759966
5.9381822
5.8381241
5.7306589
5.5043075
5.2881673
4.8366937
4.3795450
3.9832254

1.5702963

1.5697963

1.5687963

1.5677963

1.5667963

1.5657963

1.5647963

1.5637963

1.5627963

1.5617963

1.5607963

1.5587963

1.5557963

1. 5507963

1.5457963

1. 5407963

1. 5307963

1.5207963

1.5057963

1.4907963

1.4707963

1.4207963

1. 3707963

1.2707963

1.1707963

$9.7079630-1$

$\mathrm{h}_{3}=90$ kilometers

$3.5684011=1$
$3.4384606=1$
$3.1997347=1$
$2.9883523=1$
$2.8029917=1$
$2.6421025=1$
$2.5040009=1$
$2.3869224=1$
$2.2890569=1$
$2.2085704=1$
$2.1436555=1$
$2.0535173=1$
$1.9935284=1$
$2.0104680=1$
$2.0887657=1$
$2.1807846=1$
$2.3442140=1$
$2.4596375=1$
$2.5530274=1$
$2.5758657=1$
$2.5325627=1$
$2.2278798=1$
$1.8227461=1$
$1.0615337=1$
$5.0697230=2$
$6.9430885=3$

2.0577634

2.0079813

1.9088077

1.8100008

1. 7114981

1.6133801

1.5158729

1.4193316

1.3242111

1.2310199

1. 1402879

9.6812364 - 1

7.3817178 -

$4.3544915-1$

$2.1769202-1$

5.9460610 -

6. 1319576

5.9971233

5.8611284

5.7630738

5.6587592

5.4411320

5.2328732

4.7933691

4.3504330

3.9847346
1. 5702963

1.5697963
1.5687963

1.5677963

1.5667963

1.5657963

1.5647963

1.5637963

1. 5627963

1. 5617963

1.5607963

1.5587963

1.5557963

1.5507963

1.5457963

1. 5407963

1.5307963

1.5207963

1.5057963

1.4907963

1.4707963

1.4207963

1. 3707963

1. 2707963

1. 1707963

$9.7079630-1$ $\mathrm{d} / \mathrm{j}$

miles

Table 70. Effective reflection coefficient (amplitude, $\left|C_{j}\right|$, and phase, Arg $C_{j}$ ) for the special ray configuration of the Norton type 301 atmosphere, applicable to the propagation of waves between the D-region $\left(h_{3}=70\right.$ kilometers $)$ or the E-region $\left(h_{3}=90\right.$ kilometers $)$ of the ionosphere and the earth.

$1.4000780 \quad 3$ $1.3948535 \quad 3$ $1.3845147 \quad 3$

$1.3743220 \quad 3$

$1.3642736 \quad 3$

$1.3543670 \quad 3$

1.3349661

$1.3254647 \quad 3$

1.31609093

$1.3068406 \quad 3$

$1.2886954 \quad 3$

$1.2203378 \quad 3$

1. $1805854 \quad 3$

1.14280393

$1.0724503 \quad 3$

1.00821073

$9.2176798 \quad 2$

8.45661142

$7.5787071-2$

5.91205432

4. $7678510 \quad 2$

$3.3479623 \quad 2$

2.51879062

1. 59022042 


$$
\begin{array}{ll}
\omega / \omega_{r}=0.0100 & f=0.66622256 \mathrm{kilocycles} \\
\phi_{1}=60 \text { degrees } & \sigma=0.005 \text { mhos } / \text { meter } \\
\epsilon_{2}=15
\end{array}
$$

$\Psi$

radians $\left|c_{2}\right|$

1. $3711855=$

0.0005

0.0010

0.0020

0.0030

0.0040

0.0050

0.0060

0.0070

0.0080

0.0090

0.0100

0.0120

0.0150

0.0200

0.0250

0.0300

0.0400

0.0500

0.0650

0.0800

0.1000

0.1500

0.2000

0.3000

0.4000

0.6000

0.0005

0.0010

$0.002 \mathrm{C}$

0.0030

0.0040

0.0050

0.0060

0.0070

0.0080

0.0090

0.0100

0.0120

0.0150

0.0200

0.0250

0.0300

0.0400

0.0500

0.0650

0.0800

0.1000

0.1500

0.2000

0.3000

0.4000

0.6000
Arg C,

$h_{3}=70$ kilometers

1.0344734
$7.4802207-1$
$1.0934717-1$
5.8262211
5.4585190
5.2340700
5.0890900
4.9889747
4.9159977
4.8605621
4.8171031
4.7536028
4.6928147
4.6371014
4.6093062
4.5962778
4.5948012
4.6124545
4.6593566
4.7205031
4.8126514
5.0451042
5.2402733
5.5077214
5.8420973
5.6686286

$h_{3}=90$ kilometers

$1.9878443=1$
$1.5558197=1$
$1.1199725=1$
$1.0786645=1$
$1.1944944=1$
$1.3382346=1$
$1.4713172=1$
$1.5864061=1$
$1.6843814-1$
$1.7678540=1$
$1.8394226=1$
$1.9552477-1$
$2.0822926=1$
$2.2233174=1$
$2.3180368-1$
$2.3887238-1$
$2.4950262=1$
$2.5808668-1$
$2.6986756=1$
$2.8169534=1$
$2.9839132-1$
$3.4481613-1$
$3.9397820-1$
$4.8469119-1$
$5.5691655-1$
$6.5394305-1$
1

$\tau_{j}$

$\mathrm{d} / \mathrm{j}$

miles

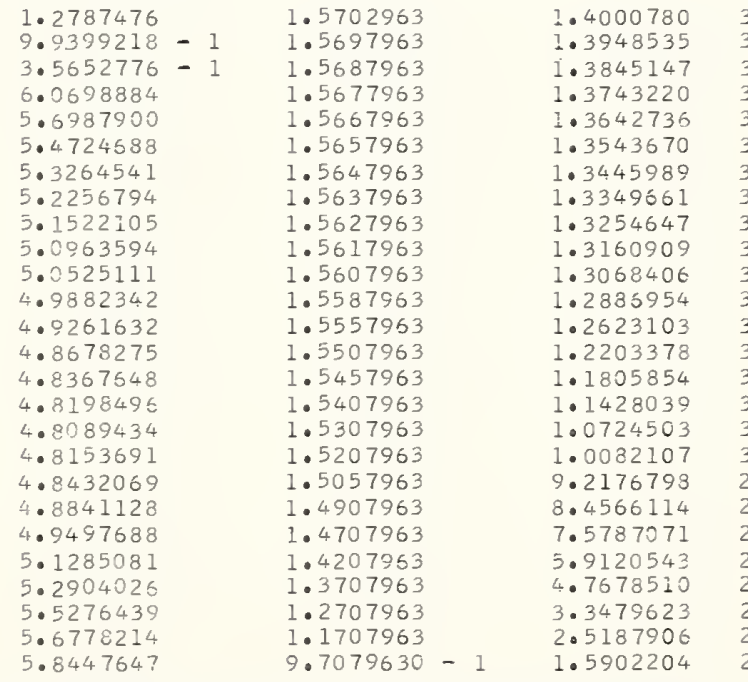

Table 71. Effective reflection coefficient (amplitude, $\left|C_{j}\right|$, and phase, $A r g C_{j}$ ) for the special

\begin{tabular}{|c|c|c|}
\hline 1.5702963 & & 1.2433895 \\
\hline 1.5697963 & & 1.2381675 \\
\hline I.5687963 & & 1.2278336 \\
\hline .5677963 & & 1.2176623 \\
\hline 1.5667963 & & 1.2076369 \\
\hline 1.5657963 & & 1.1977599 \\
\hline 1.5647963 & & 1.1880278 \\
\hline 1.5637963 & & 1.1784374 \\
\hline I. 5627963 & & 1. 1689853 \\
\hline 1.5617963 & & I. 1596659 \\
\hline 1. 5607963 & & 1.1504788 \\
\hline 1.5587963 & & 1.1324770 \\
\hline 1.5557963 & & 1.1063547 \\
\hline 1.5507963 & & 1.0649462 \\
\hline 1. 5457963 & & 1.0259098 \\
\hline 1. 5407963 & & 0.8899038 \\
\hline 1.5307963 & & .2076906 \\
\hline 1.5207963 & & 8.5914709 \\
\hline 1.5057963 & & 7.7737068 \\
\hline 1.4907963 & & 7.0657786 \\
\hline 1.4707953 & & 5.2644905 \\
\hline 1.420796 & & 4.7915000 \\
\hline 1.370796 & & 3.8174547 \\
\hline 1. 270796 & & 2.6471062 \\
\hline 9.70796 & -1 & 1.9802951 \\
\hline . 17 & & I. 24453 \\
\hline
\end{tabular}
ray configuration of the Norton type 301 atmosphere, applicable to the propagation of waves between the $\mathrm{D}$-region $\left(\mathrm{h}_{3}=70\right.$ kilometers $)$ or the $E$-region $\left(h_{3}=90\right.$ kilometers $)$ of the ionosphere and the earth. 


$$
\begin{array}{ll}
\omega / \omega_{\mathbf{r}}=0.0200 & f=1.332425 \mathrm{kilocycles} \\
\phi_{1}=60 \text { degrees } & \sigma=0.005 \mathrm{mhos} / \mathrm{mete}
\end{array}
$$

$\Psi$

radians

$\left|c_{2}\right|$
$\mathrm{Arg} \mathrm{C}_{2}$

$\mathrm{h}_{3}=70$ kilometers

$1.4219680=1$
$1.1907815=1$
$8.9076515=2$
$7.6914815=2$
$7.6399865=2$
$8.1396935=2$
$8.8177900=2$
$9.5104225=2$
$1.0157948=1$
$1.0743541=1$
$1.1266726=1$
$1.2148203=1$
$1.3154750=1$
$1.4303706=1$
$1.5079185=1$
$1.5648733=1$
$1.6468600=1$
$1.7086945=1$
$1.7887017=1$
$1.8674971=1$
$1.9818683=1$
$2.3340784=1$
$2.7566166=1$
$3.6329430=1$
$4.3977834=1$
$5.4990375=1$

$5.4990375=1$

$2.5114971-1$
$4 \cdot 7495870-2$
5.8867163
5.4278503
5.0376529
$4 \cdot 7520662$
$4 \cdot 5518948$
4.4092555
4.3041677
4.2241356
$4 \cdot 1614232$
4.0699780
3.9826796
3.9026206
3.8622407
3.8426874
3.8380895
3.8603992
3.9230618
4.0068847
4.1358552
4.4706363
4.7579581
5.1538568
5.3904612
5.6429410

$\mathrm{h}_{3}=90$ kilorneters

$5.9305017-1$
$3.8985458-1$
6.2255666
5.7566269
5.3566005
5.0658330
4.8637370
4.7206081
4.6155491
4.5356924
4.4731486
4.3818521
4.2942015
4.2122673
4.1687739
4.1451142
4.1298900
4.1390021
4.1785040
4.2369265
4.3315039
4.5927064
4.8320343
5.1832178
5.4038991
5.6467978

${ }_{j}$

$\mathrm{d} / \mathrm{j}$

miles
0.0005
0.0010
0.0020
0.0030
0.0040
0.0050
0.0060
0.0070
0.0080
0.0090
0.0100
0.0120
0.0150
0.0200
0.0250
0.0300
0.0400
0.0500
0.0650
0.0800
0.1000
0.1500
0.2000
0.3000
0.4000
0.6000

0.0005

0.0010

0.0020

0.0030

0.0040

0.0050

0.0060

0.0070

0.0080

0.0090

0.0100

0.0120

0.0150

0.0200

0.0250

0.0300

0.0400

0.0500

0.0650

0.0800

0.1000

0.1500

0.2000

0.3000

0.4000

0.6000

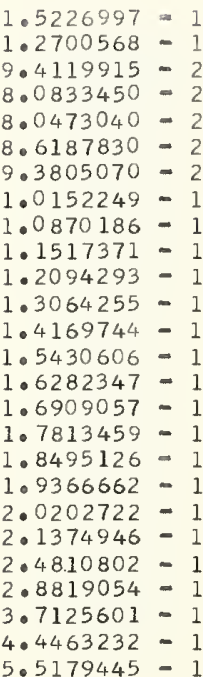

1. 5702963

1. 5697963

1. 5687963

1. 5677963

1.5667963

1.5657963

1. 5647963

1.5637963

1.5627963

1.5617963

1.5607963

1.5587963

1.5557963

1.5507963

1.5457963

1. 5407963

1.5307963

1.5207963

1.5057963

1.4907963

1.4707963

1.4207963

1.3707963

1.2707963

1.1707963

$9.7079630-1$

1.2433895

1.2381675

1.2278386

1. 2176623

1.2076369

1. 1977599

1. 1880278

1.1784374

1. 1689853

1.1596669

1.1504788

1. 1324770

1. 1063547

1.0649462

1.0259098

9.8899038

9.2076906

8.5914709

7.7737068

7.0657786

6.2644905

4. 7915900

3.8174547

2.6471062

1.9802951

1. 2445355

1. 5702963

1.5697963

1. 5687963

1.5677963

1.5667963

1.5657963

1. 5647963

1.5637963

1.5627963

1. 5617963

1.5607963

1.5587963

1.5557963

1.5507963

1.5457963

1. 5407963

1.5307963

1.5207963

1.5057963

1.4907963

1.4707963

1.4207963

1.3707963

1. 2707963

1.1707963

9.7079630 -
1.4000780 1.3948535 1.3845147 l. 3743220 1.3642736 1.3543670 1. 3445989 1.3349661 1.3254647 1.3160909 1.3068406 1. 2886954 1.2623103 1.2203378 1. 1805854 1.1428039 1. 0724503 1. 0082107 9.2176798 8.4566114 7.5787071 5.9120543 4. 7678510 3.3479623 2.5187906

1.5902204

Table 72. Effective reflection coefficient (amplitude, $\left|C_{j}\right|$, and phase, $A r g C_{j}$ ) for the special ray configuration of the Norton type 301 atmosphere, applicable to the propagation of waves between the $D$-region $\left(h_{3}=70\right.$ kilometers) or the $E$-region $\left(h_{3}=90\right.$ kilometers $)$ of the ionosphere and the earth.

$-146-$ 


$$
\begin{array}{ll}
\omega / \omega_{r}=0.0500 & f=3.3311126 \text { kilocycles } \\
\phi_{1}=60 \text { degrees } & \sigma=0.005 \text { mhos } / \text { meter } \\
& \epsilon_{2}=15
\end{array}
$$

$\Psi$

radians
Arg $\mathrm{C}_{2}$

$h_{3}=70$ kilometers

$1.6796985=1$
$1.5097986=1$
$1.2416579=1$
$1.0594163=1$
$9.4954290=2$
$8.9644855=2$
$8.8376075=2$
$8.9689995=2$
$9.2437400=2$
$9.6037170=2$
$9.9891895=2$
$1.0759706=1$
$1.1787187=1$
$1.3092018=1$
$1.4005764=1$
$1.4659038=1$
$1.5491390=1$
$1.5959757=1$
$1.6319544=1$
$1.6490021=1$
$1.6642850=1$
$1.7416998=1$
$1.9096918=1$
$2.4233513=1$
$3.0031622=1$
$4.0152933=1$

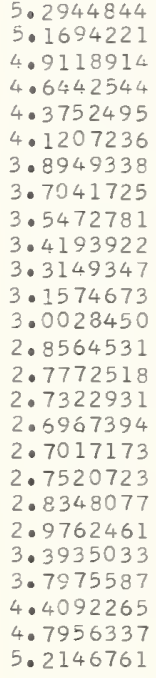

$\mathrm{h}_{3}=90$ kilometers

$$
\begin{aligned}
& 5.6137001 \\
& 5.4844672 \\
& 5.2172481 \\
& 4.9395092 \\
& 4.6630592 \\
& 4.4055269 \\
& 4.1810322 \\
& 3.9940932 \\
& 3.8418419 \\
& 3.7184442 \\
& 3.6179190 \\
& 3.4664547 \\
& 3.3172330 \\
& 3.1745474 \\
& 3.0958281 \\
& 3.0496522 \\
& 3.0086463 \\
& 3.0054403 \\
& 3.0398678 \\
& 3.1034919 \\
& 3.2164132 \\
& 3.5611251 \\
& 3.9076645 \\
& 4.4564677 \\
& 4.8177978 \\
& 5.2210711
\end{aligned}
$$

$T_{j}$

$\mathrm{d} / \mathrm{j}$

miles

1.5702963 1.5697963

1.5687963

1. 5677963

1.5667963

1.5657963

1. 5547963

1.5637963

1. 5527963

1.5617963

1.5507963

1.5587963

1. 5557963

1.5507963

1.5457963

1.5407963

1. 5307963

1. 5207963

1. 5057963

1.4007963

1.4707963

1.4207963

1. 3707963

1,2707963

1. 1707963

$9.7079630-1$

I. 2433895 1.2381675 1. 2278386 1. 2176623 1. 2076369 1. 1977599 1. 1880278 1. 1784374 1. 1689853 1. 1596669 I. 1504788 1. 1324770 1. 1063547 1. 0649462 1.0259098 9.8899038 9.2076906 8. 5914709 7.7737068 7.0657786 6.2644905 4.7915900 3.8174547 2.6471062 1. 9802951 $1 \cdot 2445355$

$\begin{array}{lll}1.5702963 & 1.4000780 & 3 \\ 1.5697963 & 1.3948535 & 3 \\ 1.5687963 & 1.3845147 & 3 \\ 1.5677963 & 1.3743220 & 3 \\ 1.5667963 & 1.3642736 & 3 \\ 1.5557963 & 1.3543670 & 3 \\ 1.5647963 & 1.3445989 & 3 \\ 1.5637963 & 1.3349661 & 3 \\ 1.5627963 & 1.3254647 & 3 \\ 1.5617963 & 1.3160909 & 3 \\ 1.5507963 & 1.3068406 & 3 \\ 1.5587963 & 1.2886954 & 3 \\ 1.5557963 & 1.2523103 & 3 \\ 1.5507963 & 1.2203378 & 3 \\ 1.5457963 & 1.1805854 & 3 \\ 1.5407963 & 1.1428039 & 3 \\ 1.5307963 & 1.0724503 & 3 \\ 1.5207963 & 1.0082107 & 3 \\ 1.5057963 & 9.2176798 & 2 \\ 1.4907963 & 8.4565114 & 2 \\ 1.4707963 & 7.5787071 & 2 \\ 1.4207963 & 5.9120543 & 2 \\ 1.3707963 & 4.7678510 & 2 \\ 1.2707963 & 3.3479623 & 2 \\ 1.1707963 & 2.5187906 & 2 \\ 9.7079630 & 1.5902204 & 2 \\ 1 & & \end{array}$

Table 73. Effective reflection coefficient (amplitude, $\left|C_{j}\right|$, and phase, $\operatorname{Arg} C_{j}$ ) for the special ray configuration of the Norton type 301 atmosphere, applicable to the propagation of waves between the $D$-region $\left(h_{3}=70\right.$ kilometers) or the $E$-region $\left(h_{3}=90\right.$ kilometers $)$ of the ionosphere and the earth. 


$$
\begin{array}{ll}
\omega / \omega_{\mathrm{r}}=0.1000 & \mathrm{f}=6.6622252 \mathrm{kilocycles} \\
\phi_{1}=60 \text { degrees } & \sigma=0.005 \mathrm{mhos} / \mathrm{meter}
\end{array}
$$

$\Psi$

radians

$\left|c_{2}\right|$
$\mathrm{Arg} \mathrm{C}_{2}$

$\mathrm{h}_{3}=70$ kilometers

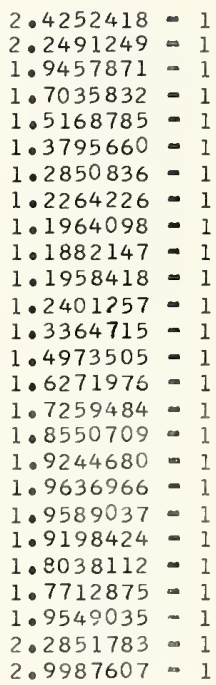

0.4000
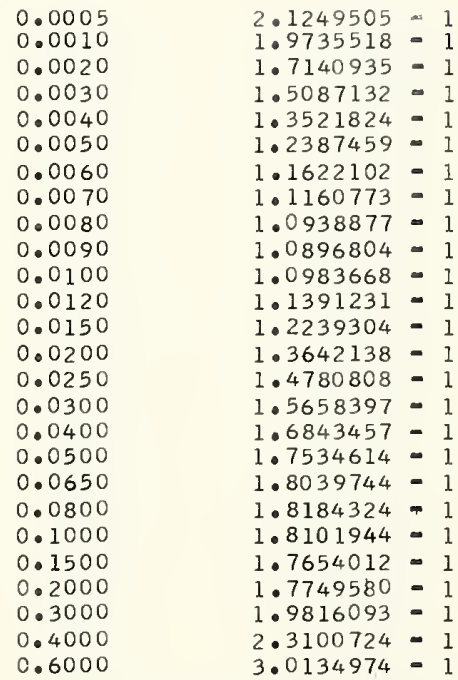

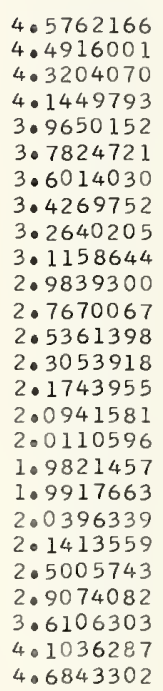

$4 \cdot 7918875$
$4 \cdot 7052266$
$4 \cdot 5300919$
$4 \cdot 3513533$
$4 \cdot 1693342$
$3 \cdot 9865645$
$3 \cdot 8073415$
$3 \cdot 6365975$
$3 \cdot 4785312$
$3 \cdot 3357260$
$3 \cdot 2089921$
$3 \cdot 0007763$
2.7781732
$2 \cdot 5532083$
2.4236832
2.3432139
$2 \cdot 2575145$
$2 \cdot 2245356$
$2 \cdot 2263041$
$2 \cdot 2646404$
$2 \cdot 3514847$
$2 \cdot 6663366$
$3 \cdot 0278924$
$3 \cdot 6689175$
$4 \cdot 1330966$
$4 \cdot 6935287$

$\boldsymbol{T}_{j}$

$\mathrm{d} / \mathrm{j}$

miles

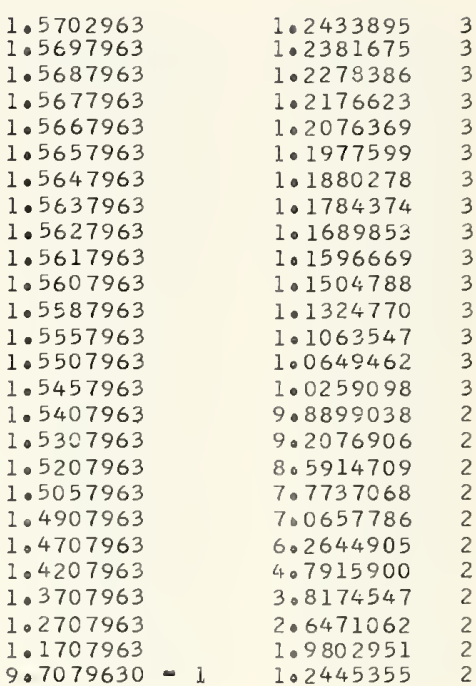

1.5702963

1.4000780

1. 5687963

1.5677963

1.5657963

1.5657963

1.5647963

1.5637963

1.5627963

1.5617963

1. 5607963

1.5587963

1.5557963

1.5507963

1.5457963

1.5407963

1.5307963

1. 5207963

1.5057963

1.4907963

1.4707963

1.4207963

1.3707963

1. 2707963

1.1707963

1.3948535

1.3845147

1.3743220

1.3642736

1.3543670 1.3445989 1.3349661

1.3254647 1.3160909 1.3068406 1. 2886954 1.2623103 1.2203378 1. 1805854 1. 1428039 1.0724503 1.0082107 9.2176798 8.4566114 7.5787071 5.9120543 4. 7678510 3.3479623 2. 5187906 $9.7079630=1 \quad 1.5902204$

Table 74. Effective reflection coefficient (amplitude, $\left|C_{j}\right|$, and phase, $\operatorname{Arg}_{C_{j}}$ ) for the special ray configuration of the Norton type 301 atmosphere, applicable to the propag;ation of waves between the $D$-region $\left(h_{3}=70\right.$ kilometers) or the E-region $\left(h_{3}=90\right.$ kilometers) of the ionosphere and the earth. 


$$
\begin{array}{ll}
\omega / \omega_{r}=0.2000 & f=13.324450 \text { kilocycles } \\
\phi_{1}=60 \text { degrees } & \sigma=0.005 \mathrm{mhos} / \text { meter } \\
\epsilon_{2}=15
\end{array}
$$

$\Psi$

radians

0.0005

0.0010

0.0020

0.0030

0.0040

0.0050

0.0060

0.0070

0.0080

0.0090

0.0100

0.0120

0.0150

0.0200

0.0250

0.0300

0.0400

0.0500

0.0650

0.0800

0.1000

0.1500

0.2000

0.3000

0.4000

0.6000

$\begin{array}{ll}0.0005 & 3.0822604=1 \\ 0.0010 & 2.9229433=1 \\ 0.0020 & 2.6347606=1 \\ 0.0030 & 2.3856739=1 \\ 0.0040 & 2.1734565=1 \\ 0.0050 & 1.9956236=1 \\ 0.0060 & 1.8495583=1 \\ 0.0070 & 1.7325357=1 \\ 0.0080 & 1.6417091=1 \\ 0.0090 & 1.5741077=1 \\ 0.0100 & 1.5266955=1 \\ 0.0120 & 1.4804146=1 \\ 0.0150 & 1.4923407=1 \\ 0.0200 & 1.6068893=1 \\ 0.0250 & 1.7443971=1 \\ 0.0300 & 1.8686084=1 \\ 0.0400 & 2.0568376=1 \\ 0.0500 & 2.1777709=1 \\ 0.0650 & 2.2744707=1 \\ 0.0800 & 2.3066431=1 \\ 0.1000 & 2.2908752=1 \\ 0.1500 & 2.1345813=1 \\ 0.2000 & 1.9783557=1 \\ 0.3000 & 1.8837023=1 \\ 0.4000 & 1.9825983=1 \\ 0.6000 & 2.3012053=1\end{array}$

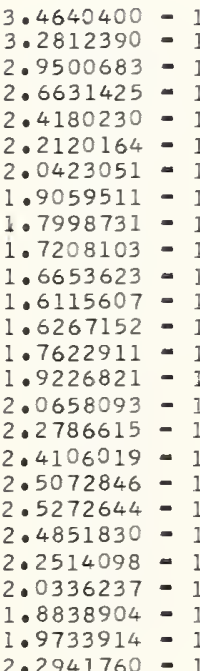

Arg C 2

$h_{3}=70$ kilometers

4. 0720714

4.0133695

3.8953859

3.7757778

3.6538120

3.5292049

3.4022195

3.2737197

3. 1451184

3. 0182170

2.8949490

2.6560848

2.3808906

2. 0540237

1.8533757

1.7245302

1.5775799

1.5052302

1.4639252

1.4668554

1. 5112238

1. 7368469

2.0479456

2.7010751

3. 2230497

3.8999101

$\mathrm{h}_{3}=90$ kilometers

4. 2064644

4.1470849

4. 0278970

3. 9073898

3.7849715

3.6605034

3.5343705

3.4074999

3.2812808

3. 1573981

3.0375811

2. 8159795

2.5399262

2. 2209669

2.0226141

1. 8938362

1. 7449491

1. 6698601

1.6239455

1.6218772

1.6590802

1.8646132

2.1527855

2.7612019

3.2556088

3.9114774
1. 5702963

1. 5697963

$1.5687963^{\circ}$

1.5677963

1.5667963

1.5657963

1.5647963

1.5637963

1.5627963

1.5617963

1.5607963

1. 5587963

1.5557963

1.5507963

1.5457963

1. 5407963

1. 5307963

1.5207963

1. 5057963

1.4907963

1.4707963

1. 4207963

1.3707963

1.2707963

1.1707963

9.7079630 -

1.2433895

1.2381675

1.2278386

1. 2176623

1.2076359

1.1977599

1.1880278

1.1784374

1.1689853

1.1595669

1.1504788

1. 1324770

1.1063547

1.0649462

1.0259098

9.8899038

9.2076906

8.5914709

7.7737068

7.0657786

6.2644905

4. 7915900

3.8174547

2.6471062

1. 9802951

1.2445355

1.5702963

1.5697963

1.5687963

1.5677963

1.5667963

1.5657963

1.5647963

1. 5637963

1.5627963

1.5617963

1.5607963

1.5587963

1.5557963

1. 5507963

1.5457963

1. 5407963

1.5307963

1. 5207963

1.5057963

1.4907963

1.4707963

1.4207963

1.3707963

1.2707963

1. 1707963

$9.7079530=1$
1.4000780

1.3948535

1. 3845147

1. 3743220

1. 3642736

1. 3543670

1.3445989

1. 3349661

1. 3254647

1. 3160909

1. 3068406

1. 2886954

1. 2623103

i. 2203378

1. 1805854

1. 1428039

1. 0724503

1. 0082107

9.2176798

8.4566114

7.5787071

5. 9120543

4. 7678510

3.3479623

2. 5187906

1. 5902204

Table 75. Effective reflection coefficient (amplitude, $\left|C_{j}\right|$, and phase, Arg $C_{j}$ ) for the special ray configuration of the Norton type 301 atmosphere, applicable to the propagation of waves between the $\mathrm{D}$-region $\left(\mathrm{h}_{3}=70\right.$ kilometers) or the $\mathrm{E}$-region $\left(\mathrm{h}_{3}=90\right.$ kilometers $)$ of the ionosphere and the earth. 


$$
\begin{array}{ll}
\omega / \omega_{\mathbf{r}}=1.0000 & \mathrm{f}=66.622252 \mathrm{kilocycles} \\
\phi_{1}=60 \text { degrees } & \sigma=0.005 \mathrm{mhos} / \mathrm{meter} \\
& \epsilon_{2}=15
\end{array}
$$

$\Psi$

radians

$$
\left|c_{2}\right|
$$

Arg $\mathrm{C}_{2}$

$\mathrm{h}_{3}=70$ kilometers

$\begin{array}{ll}0.0005 & 5.6338640=1 \\ 0.0010 & 5.4952975=1 \\ 0.0020 & 5.2294315=1 \\ 0.0030 & 4.9784626=1 \\ 0.0040 & 4.7421757=1 \\ 0.0050 & 4.5203084=1 \\ 0.0060 & 4.3125467=1 \\ 0.0070 & 4.1185602=1 \\ 0.0080 & 3.9379909=1 \\ 0.0090 & 3.7704674=1 \\ 0.0100 & 3.6156137=1 \\ 0.0120 & 3.3423567=1 \\ 0.0150 & 3.0175294=1 \\ 0.0200 & 2.6736559=1 \\ 0.0250 & 2.5234792=1 \\ 0.0300 & 2.5036103=1 \\ 0.0400 & 2.6540443=1 \\ 0.0500 & 2.8732890=1 \\ 0.0650 & 3.1613205=1 \\ 0.0800 & 3.3599426=1 \\ 0.1000 & 3.5030593=1 \\ 0.1500 & 3.4826421=1 \\ 0.2000 & 3.2096464=1 \\ 0.3000 & 2.5147576=1 \\ 0.4000 & 1.9287864=1 \\ 0.6000 & 1.2308834=1 \\ 0 & \end{array}$

3.1672019
$3 \cdot 1408844$
3.0881950
3.0353344
2.9821916
2.9286655
2.8746664
2.8201191
2.7649669
2.7091710
2.6527179
2.5379025
2.3618662
2.0671806
1.7906500
1.5510096
1.1973777
$9.7210563-1$
$7.6853569-1$
$6.4814197-1$
$5.5150913-1$
$4.4686706-1$
$4.2311612-1$
$4.7190319-1$
$5.8769606-1$
$8.9953086-1$
5

\begin{tabular}{|c|c|c|}
\hline $\begin{array}{l}0.0005 \\
0.0010\end{array}$ & $\begin{array}{l}5.2422760 \\
5.1149145\end{array}$ & 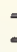 \\
\hline 0.0020 & 4.8706207 & 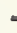 \\
\hline 0.0030 & 4.6401136 & 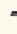 \\
\hline 0.0040 & 4.4231940 & 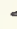 \\
\hline 0.0050 & 4.2196045 & 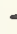 \\
\hline 0.0060 & 4.0290538 & 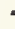 \\
\hline 0.0070 & 3.8512246 & \\
\hline 0.0080 & 3.6857826 & \\
\hline 0.0090 & 3.5323691 & . \\
\hline 0.0100 & 3.3906306 & \\
\hline 0.0120 & 3.1406813 & \\
\hline 0.0150 & 2.8437909 & 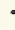 \\
\hline 0.0200 & 2.5293064 & \\
\hline 0.0250 & 2.3907984 & \\
\hline 0.0300 & 2.3705526 & 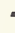 \\
\hline 0.0400 & 2.5043286 & 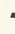 \\
\hline 0.0500 & 2.7038712 & 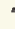 \\
\hline 0.0650 & 2.9706798 & \\
\hline 0.0800 & 3.1591674 & \\
\hline 0.1000 & 3.3014199 & \\
\hline 0.1500 & 3.3096648 & \\
\hline 0.2000 & 3.0758626 & \\
\hline 0.3000 & 2.4424929 & \\
\hline 0.4000 & 1.8914204 & \\
\hline 0.6000 & 1.2204191 & \\
\hline
\end{tabular}

$\mathrm{d} / \mathrm{j}$

miles $h_{3}=90$ kilometers
1. 5702963 1.5697963

1.5677963

1.5667963

1.5657963

1.5647963

1. 5637963

1. 5627963

1.5617963

1. 5607963

1.5587963

1.5557963

1.5507963

1. 5457963

1.5407963

1.5307963

1. 5207963

1.5057963

1.4907963

1.4707963

1.4207963

1.3707963

1.2707963

1.1707963

9.7079630 - 1
1. 5687963

1.2433895
1.2381675
1.2278386
1.2176623
1.2076369
1.1977599
1.1880278
1.1784374
1.1689853
1.1596669
1.1504788
1.1324770
1.1063547
1.0649462
1.0259098
9.8899038
9.2076906
8.5914709
7.7737068
7.0657736
6.2644905
4.7915900
3.8174547
2.6471062
1.9802951
1.2445355

1. 5702963

1. 5697963

1. 5687963

1.5677963

1. 5667963

1.5657963

1. 5647963

1. 5637963

1.5627963

1.5617963

1. 5607963

1.5587963

1. 5557963

1.5507963

1. 5457963

1. 5407963

1.5307963

1. 5207963

1.5057963

1.4907963

1.4707963

1.4207963

_.3707963

1.2707963

1.1707963

$9.7079630-1$
1.4000780

1.3948535

1.3845147

1.3743220

1.3642736

1.3543670

1.3445989

1.3349661

1.3254647

1.3160909

1.3068406

1. 2886954

1. 2623103

1.2203378

1.1805854

1. 1428039

1.0724503

1. 0082107

9.2176798

8.4566114

7.5787071

5.9120543

$4 \cdot 7678510$

3.3479623

2.5187906

1. 5902204

Table 76. Effective reflection coefficient (amplitude, $\left|C_{j}\right|$, and phase, Arg $C_{j}$ ) for the special ray configuration of the Norton type 301 atmosphere, applicable to the propagation of waves between the $D$-region $\left(h_{3}=70\right.$ kilometers $)$ or the E-region $\left(h_{3}=90\right.$ kilometers $)$ of the ionosphere and the earth. 


$$
\begin{array}{ll}
\omega / \omega_{r}=2.0000 & f=133.24450 \text { kilocycles } \\
\phi_{1}=60 \text { degrees } & \sigma=0.005 \text { mhos } / \text { meter } \\
\epsilon_{2}=15
\end{array}
$$

$\Psi$

radians

$\left|\mathrm{C}_{2}\right|$
$\operatorname{Arg~C}_{2}$

$\mathrm{h}_{3}=70$ kilometers

$5.2534630=1$
$5.1635950=1$
$4.9889825=1$
$4.8211805=1$
$4.6601429=1$
$4.5058039=1$
$4.3580922=1$
$4.2169116=1$
$4.0821654=1$
$3.9537475=1$
$3.8315438=1$
$3.6053078=1$
$3.3095003=1$
$2.9237477=1$
$2.6564809=1$
$2.4880004=1$
$2.3639974=1$
$2.4008976=1$
$2.5523543=1$
$2.6999410=1$
$2.8283780=1$
$2.8351922=1$
$2.5904313=1$
$1.9111705=1$
$1.2975026=1$
$4.9016160=2$

2.7812819

2. 7624967

2. 7249139

2.6872818

2.6495631

2.6117215

2. 5737255

2. 5355444

2.4971514

2.4585248

2.4196461

2. 3410820

2. 2211759

2.0167299

1. 8103870

1.6090262

1.2487830

9.6662851 - 1

6.7233777 - 1

$4.7879072-1$

$3.0586134-1$

$5.4410240-2$

6.1830170

5. 9655401

5.7988260

5. 5917134

$\mathrm{h}_{3}=90$ kilometers

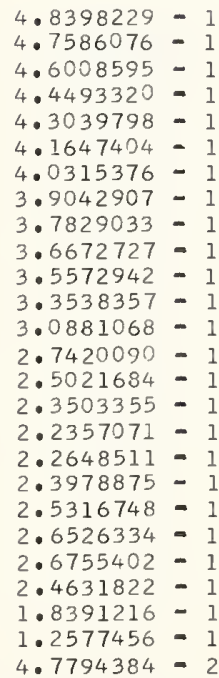

2.7214999
2.7025878
2.5647670
2.6259197
2.5890121
2.5510115
2.5128889
2.4746175
2.4361751
2.3975422
2.3587050
2.2803800
2.1612723
1.9594631
1.7573310
1.5612451
1.2115926
$9.3685758-1$
$6.4798389-1$
$4.5660404-1$
$2.8509924-1$
$3.6348920-2$
6.1676374
5.9545896
5.7911028
5.5910805

$T_{j}$

$\mathrm{d} / \mathrm{j}$

miles

\subsection{3}

1.5697963

1. 5687963

1.5677963

1.5667963

1. 5657963

1.5647963

1. 5637963

1. 5627963

1.5617963

1. 5607963

1. 5587963

1.5557963

1.5507963

1. 5457963

1.5407963

1. 5307963

1. 5207963

1.5057963

1.4907963

1.4707963

1.4207963

1.3707963

1.2707963

1.1707963

9.7079630

- 1

1.2433895 1.2381675 1. 2273386 1. 2176623 1.2076369 1.1977599 1. 1880278 1. 1784374 1. 1689853 1. 1596669 1. 1504788 1.1324770 1.1063547 1.0649462 1.0259098 9.8899038 9.2076906 8. 5914709 7.7737068 7.0657786 6.2644905 4. 7915900 3.8174547 2.6471062 1. 9802951 1.2445355

1. 5702963

1.5697963

1.5687963

1.5677963

1.5667963

1. 5657963

1. 5647963

1. 5637963

1.5627963

1. 5617963

1.5607963

1. 5587963

1.5557963

1.5507963

1. 5457963

1.5407963

1.5307963

1. 5207963

1.5057963

1.4907963

1.4707963

1.4207963

1.3707963

1. 2707963

1.1707963

$9.7079630-1$

$\begin{array}{ll}1.4000780 & 3 \\ 1.3948535 & 3 \\ 1.3845147 & 3 \\ 1.3743220 & 3 \\ 1.3642736 & 3 \\ 1.3543670 & 3 \\ 1.3445989 & 3 \\ 1.3349661 & 3 \\ 1.3254647 & 3 \\ 1.3160909 & 3 \\ 1.3068406 & 3 \\ 1.2886954 & 3 \\ 1.2623103 & 3 \\ 1.2203378 & 3 \\ 1.1805854 & 3 \\ 1.1428039 & 3 \\ 1.0724503 & 3 \\ 1.0082107 & 3 \\ 9.2176798 & 2 \\ 8.4566114 & 2 \\ 7.5787071 & 2 \\ 5.9120543 & 2 \\ 4.7678510 & 2 \\ 3.3479623 & 2 \\ 2.5187906 & 2 \\ 1.5902204 & 2\end{array}$

0.6000

Table 77. Effective reflection coefficient (amplitude, $\left|C_{j}\right|$, and phase, $A_{r g} C_{j}$ ) for the special ray configuration of the Norton type 301 atmosphere, applicable to the propagation of waves between the $D$-region $\left(h_{3}=70\right.$ kilometers $)$ or the E-region $\left(h_{3}=90\right.$ kilometers $)$ of the ionosphere and the earth. 


$$
\begin{array}{ll}
\omega / \omega_{r}=5.0000 & f=333.11126 \text { kilocycles } \\
\phi_{1}=60 \text { degrees } & \sigma=0.005 \text { mhos } / \text { meter } \\
& \epsilon_{2}=15
\end{array}
$$

$\begin{array}{lllll}\Psi & \left|C_{2}\right| & A_{g} C_{2} & \tau_{j} & \mathrm{~d} / \mathrm{j} \\ \text { radians } & & \text { miles }\end{array}$

$h_{3}=70$ kilometers

$\begin{array}{ll}0.0005 & 4.1227652=1 \\ 0.0010 & 4.0812721=1 \\ 0.0020 & 3.9997864=1 \\ 0.0030 & 3.9202975=1 \\ 0.0040 & 3.8428099=1 \\ 0.0050 & 3.7673249=1 \\ 0.0060 & 3.6938349=1 \\ 0.0070 & 3.6223368=1 \\ 0.0080 & 3.5528155=1 \\ 0.0090 & 3.4852640=1 \\ 0.0100 & 3.4196661=1 \\ 0.0120 & 3.2942707=1 \\ 0.0150 & 3.1204030=1 \\ 0.0200 & 2.8670874=1 \\ 0.0250 & 2.6565413=1 \\ 0.0300 & 2.4850581=1 \\ 0.0400 & 2.2425892=1 \\ 0.0500 & 2.1045057=1 \\ 0.0650 & 2.0196595=1 \\ 0.0800 & 2.0047761=1 \\ 0.1000 & 2.0083352=1 \\ 0.1500 & 1.9196652=1 \\ 0.2000 & 1.6828378=1 \\ 0.3000 & 1.0742949=1 \\ 0.4000 & 5.3519045=2 \\ 0.6000 & 7.1349755=3 \\ & \end{array}$

2.2489936
2.2365453
2.2116334
2.186944
2.1617221
2.1367101
2.1116525
2.0865435
2.0613785
2.0361532
2.0108630
1.9600816
1.8833821
1.7541991
1.6237492
1.4929546
$1.2357851-$
$9.9438218-1$
$6.7958145-1$
$4.2551660-1$
$1.6237022-1$
5.9941619
5.6611392
5.0751307
4.5232841
4.0425153

$h_{3}=90$ kilometers

2.0950794
2.0824392
2.0571616
2.0318823
2.0065979
1.9813020
1.9559905
1.9306599
1.9053089
1.8799313
1.8545285
1.8036395
1.7271004
1.5991333
1.4711724
1.3441427
1.0976767
$8.6920867-1$
$5.7277141-1$
$3.3257822-1$
$8.1512850-2$
5.9284061
5.6033268
5.0275851
4.4903564
4.0433322

\begin{tabular}{|c|c|c|}
\hline 1.5702963 & & 1.2433895 \\
\hline 1.5697963 & & 1.2381675 \\
\hline 1.5687963 & & 1.2278386 \\
\hline 1.5677963 & & 1.2176623 \\
\hline 1.5667963 & & 1.2076369 \\
\hline 1.5657963 & & 1.1977599 \\
\hline 1.5647963 & & 1.1880278 \\
\hline 1.5637963 & & d. 1784374 \\
\hline 1.5627963 & & 1.1689853 \\
\hline 1.5617963 & & 1.1596669 \\
\hline 1.560 .7963 & & 1.1504788 \\
\hline 1.5587963 & & 1.1324770 \\
\hline 1.5557963 & & 1.1063547 \\
\hline 1.5507963 & & 1.0649462 \\
\hline 1.5457963 & & 1.0259098 \\
\hline 1.5407963 & & 9.8899038 \\
\hline 1.5307963 & & 9.2076906 \\
\hline 1.5207963 & & 8.5914709 \\
\hline 1.5057963 & & 7.7737068 \\
\hline 1.4907963 & & 7.0657786 \\
\hline 1.4707963 & & 6.2644905 \\
\hline 1.4207963 & & 4.7915900 \\
\hline 1.3707963 & & 3.8174547 \\
\hline & & 2.6471062 \\
\hline 1707963 & & 1.9802951 \\
\hline 7079630 & -1 & 1.2445355 \\
\hline
\end{tabular}

4.0433322

\begin{tabular}{|c|c|}
\hline $\begin{array}{l}1.5702963 \\
1.5697963\end{array}$ & $\begin{array}{l}1.4000780 \\
1.3948535\end{array}$ \\
\hline 1.5687963 & 1.3845147 \\
\hline 1.5677963 & 1.3743220 \\
\hline 1.5667963 & 1.364 .2736 \\
\hline 1.5657963 & 1.3543670 \\
\hline 1.5647963 & 1.3445989 \\
\hline 1.5637963 & 1.3349661 \\
\hline 1.5627963 & 1.3254647 \\
\hline 1.5617963 & 1.3160909 \\
\hline 1.5607963 & $1 \cdot 3068406$ \\
\hline 1.5587963 & 1.2886954 \\
\hline 1.5557 .963 & 1.2623103 \\
\hline 1.5507963 & 1.2203378 \\
\hline 1.5457963 & 1.1805854 \\
\hline 1.5407963 & 1.1428039 \\
\hline 1.5307963 & 1.0724503 \\
\hline 1.5207963 & 1.0082107 \\
\hline 1.5057963 & 9.2176798 \\
\hline 1.4907963 & 8.4566114 \\
\hline 1.4707963 & 7.5787071 \\
\hline $\begin{array}{l}1.4207963 \\
1.3707963\end{array}$ & $\begin{array}{l}5.9120543 \\
4.7678510\end{array}$ \\
\hline 1.2707963 & 3.3479623 \\
\hline $\begin{array}{l}707963 \\
079630\end{array}$ & 2.5187906 \\
\hline 079630 & 1.5902204 \\
\hline
\end{tabular}

1.5902204

$\begin{array}{ll}0.0005 & 3.6698621=1 \\ 0.0010 & 3.6346538=1 \\ 0.0020 & 3.5655491=1 \\ 0.0030 & 3.4981948=1 \\ 0.0040 & 3.4325961=1 \\ 0.0050 & 3.3687450=1 \\ 0.0060 & 3.3066346=1 \\ 0.0070 & 3.2462600=1 \\ 0.0080 & 3.1876137=1 \\ 0.0090 & 3.1306718=1 \\ 0.0100 & 3.0754307=1 \\ 0.0120 & 2.9699781=1 \\ 0.0150 & 2.8240847=1 \\ 0.0200 & 2.6122559=1 \\ 0.0250 & 2.4368699=1 \\ 0.0300 & 2.2944375=1 \\ 0.0400 & 2.0932342=1 \\ 0.0500 & 1.9777548=1 \\ 0.0650 & 1.9040611=1 \\ 0.0800 & 1.8876914=1 \\ 0.1000 & 1.8859939=1 \\ 0.1500 & 1.8010230=1 \\ 0.2000 & 1.5843136=1 \\ 0.3000 & 1.0151502=1 \\ 0.4000 & 5.0366720=2 \\ 0.6000 & 6.7885665=3\end{array}$

Table 78. Effective reflection coefficient (amplitude, $\left|C_{j}\right|$, and phase, $A_{r g} C_{j}$ ) for the special ray configuration of the Norton type 301 atmosphere, applicable to the propagation of waves between the $D$-region $\left(h_{3}=70\right.$ kilometers $)$ or the E-region $\left(h_{3}=90\right.$ kilometers $)$ of the ionosphere and the earth. 


$$
\begin{array}{ll}
\omega / \omega_{r}=0.3002 & f=20 \text { kilocycles } \\
\phi_{1}=60 \text { degrees } & \sigma=0.005 \text { mhos } / \text { meter } \\
& \epsilon_{2}=15
\end{array}
$$

$\Psi$

radians

$\left|C_{3}\right| \quad \quad \quad A r g C_{3}$

$\mathrm{h}_{3}=70$ kilometers

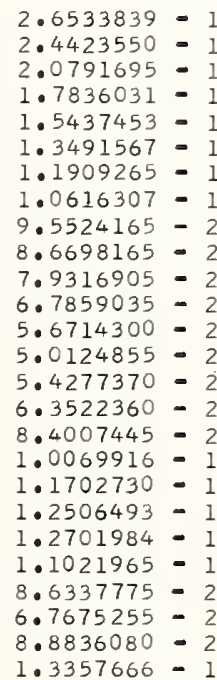

1.3357666 -

4.0944117
3.9957923
3.7995427
3.6046185
3.4113240
3.2202185
3.0319715
2.8471914
2.6662863
2.4893828
2.3163054
1.9798163
1.4914007
$7.2650433-1$
$1.1698126-1$
5.9860773
5.5242364
5.2915025
5.1188826
5.0474156
5.0336982
5.2125956
5.5870417
$4.3732266-1$
1.2957395
2.0662384

$\mathrm{h}_{3}=90$ kilometers

0.0005

0.0010

0.0030

0.0040

0.0050

0.0060

0.0070

0.0080

0.0090

0.0100

0.0120

0.0150

0.0200

0.0250

0.0300

0.0400

0.0500

0.0650

0.0800

0.1000

0.1500

0.2000

0.3000

0.4000

0.6000
4.2141574

4.1128204

3.9117065

3. 7131161

3. 5178678

3. 3269423

3. 1412767

2.9615642

2. 7881357

2.6209366

2.4595479

2.1513493

1. 7129397

1.0169364

$4.1282141-1$

6.253394

5.7464935

5.4925743

5.3047898

5. 2249542

5. 2040001

5. 3746971

5.7501638

$5.5363598-1$

1.3410109

2.0779513 $\top_{j}$

$\mathrm{d} / \mathrm{j}$

miles
1.2433895 1.2381675 1.2278386 1.2176623 1.2076369 1.1977599 1.1880278 1.1784374 1.1689853 1.1596669 1.1504788 1.1324770 1. 1063547 1.0649462 1.0259098 9.8899038 9.2076906 8.5914709 7.7737068 7.0657786 6.2644905 4.7915900 3.8174547 2.5471062 1.9802951 1.2445355
1.1707963

9.7079630

Table 79. Effective reflection coefficient (amplitude, $\left|C_{j}\right|$, and phase, Arg $C_{j}$ ) for the special ray configuration of the Norton type 301 atmosphere, applicable to the propagation of waves between the $D$-region $\left(h_{3}=70\right.$ kilometers) or the E-region $\left(h_{3}=90\right.$ kilometers $)$ of the ionosphere and the earth.

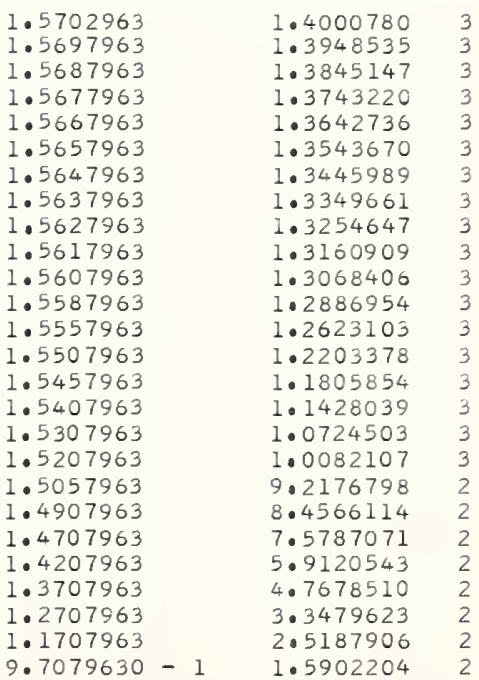




$$
\begin{array}{ll}
\omega / \omega_{r}=0.467 & f=20 \text { kilocycles } \\
\phi_{1}=60 \text { degrees } & \sigma=0.005 \text { mhos } / \text { meter } \\
\epsilon_{2}=15
\end{array}
$$

$\Psi$

radians

$$
\begin{aligned}
& 0.0005 \\
& 0.0010 \\
& 0.0020 \\
& 0.0030 \\
& 0.0040 \\
& 0.0050 \\
& 0.0060 \\
& 0.0070 \\
& 0.0080 \\
& 0.0090 \\
& 0.0100 \\
& 0.0120 \\
& 0.0150 \\
& 0.0200 \\
& 0.0250 \\
& 0.0300 \\
& 0.0400 \\
& 0.0500 \\
& 0.0650 \\
& 0.0800 \\
& 0.1000 \\
& 0.1500 \\
& 0.2000 \\
& 0.3000 \\
& 0.4000 \\
& 0.6000
\end{aligned}
$$

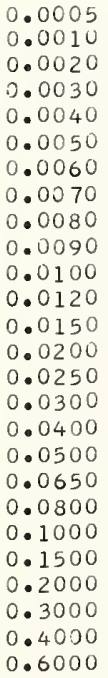

$\left|c_{3}\right|$

$\operatorname{Arg~C} 3$

$\mathrm{h}_{3}=70$ kilometers

$3.3105805-1$
$3.0418877-1$
$2.5791258-1$
$2.2022719-1$
$1.8964558-1$
$1.6486441-1$
$1.4476924-1$
$1.2842861-1$
$1.1508220-1$
$1.0412347-1$
$9.5081540-2$
$8.1417295-2$
$6.9033890-2$
$6.3947640-2$
$7.1477150-2$
$8.4287885-2$
$1.1113089-1$
$1.3293297-1$
$1.5490362-1$
$1.6672762-1$
$1.7165388-1$
$1.5553399-1$
$1.2590440-1$
$7.9906975-2$
$7.4525715-2$
$1.0359002-1$

$2.9067103-1$
$2.6785701-1$

$2.2865567-1$

$1.9682375-1$

$1.7104638-1$

$1.5017178-1$

$1.3321889-1$

$1.1937311-1$

$1.0797559-1$

$9.8507365-2$

$9.0570920-2$

$7.8189375-2$

$6.5982870-2$

$5.8277345-2$

$6.2162970-2$

$7.1923220-2$

$9.4560885-2$

$1.1375483-1$

$1.3369593-1$

$1.4500216-1$

$1.5069101=1$

$1.3950218=1$

$1.1527603-1$

$7.7264615-2$

$7.5270530-2$

$1.0410651-1$

$3 \cdot 7385426$
$3 \cdot 6403700$
$3 \cdot 4445981$
$3 \cdot 2493977$
$3 \cdot 0548432$
$2 \cdot 8613045$
$2 \cdot 6693353$
$2 \cdot 4795155$
$2 \cdot 2923205$
$2 \cdot 1080141$
$1 \cdot 9266262$
$1 \cdot 5718434$
$1 \cdot 0570634$
$2 \cdot 7653615-1$
5.9697101
5.5756118
5.1287872
4.8949125
$4 \cdot 7100034$
$4 \cdot 6198712$
$4 \cdot 5742419$
$4 \cdot 6411040$
$4 \cdot 8491373$
$5 \cdot 5796316$
$1 \cdot 8457793-1$
$1 \cdot 0972028$

$\mathrm{h}_{3}=90$ kilometers

$3 \cdot 8117892$
$3 \cdot 7117515$
$3 \cdot 5127430$
$3 \cdot 3153004$
$3 \cdot 1198998$
$2 \cdot 9272590$
$2 \cdot 7381619$
$2 \cdot 5532824$
$2 \cdot 3730408$
$2 \cdot 1975423$
$2 \cdot 0265699$
$1 \cdot 6962389$
$1 \cdot 2212522$
$4 \cdot 8188268-1$
$6 \cdot 1666944$
$5 \cdot 7499736$
$5 \cdot 2740952$
$5 \cdot 0271135$
$4 \cdot 8325157$
$4 \cdot 7366115$
$4 \cdot 6854111$
$4 \cdot 7433711$
$4 \cdot 9484911$
$5 \cdot 6771868$
$2 \cdot 4089864-1$
$1 \cdot 1096194$
4

1.5702963

1.5697963

1. 5687963

1.5677963

1. 5667963

1. 5657963

1. 5647963

1.5637963

1.5627963

1.5617963

1. 5607963

1. 5587963

1.5557963

1. 5507963

1. 5457963

1.5407963

1. 5307963

1.5207963

1.5057963

1.4907963

1.4707963

1.4207963

1.3707963

1.2707963

1. 1707963

9.7079630 - 1 $\mathrm{d} / \mathrm{j}$

miles

Table 80. Effective reflection coefficient (amplitude, $\left|C_{j}\right|$, and phase, $A r g C_{j}$ ) for the special ray configuration of the Norton type 301 atmosphere, applicable to the propagation of waves between the D-region $\left(h_{3}=70\right.$ kilometers) or the E-region $\left(h_{3}=90\right.$ kilometers $)$ of the ionosphere and the earth. 


$$
\begin{array}{ll}
\omega / \omega_{r}=0.1501 & f=10 \text { kilocycles } \\
\phi_{1}=60 \text { degrees } & \sigma=0.005 \text { mhos } / \text { meter }
\end{array}
$$

$\begin{array}{lllll}\Psi & \left|C_{3}\right| & A^{I} C_{3} & T_{j} & \mathrm{~d} / \mathrm{j} \\ \text { radians } & & \mathrm{miles}\end{array}$

$\begin{array}{ll}0.0005 & 1.6228006=1 \\ 0.0010 & 1.4514668=1 \\ 0.0020 & 1.1778682=1 \\ 0.0030 & 9.7718310=2 \\ 0.0040 & 8.2972850=2 \\ 0.0050 & 7.1993985=2 \\ 0.0060 & 6.3624200=2 \\ 0.0070 & 5.7054230=2 \\ 0.0080 & 5.1751710=2 \\ 0.0090 & 4.7386852=2 \\ 0.0100 & 4.3768659=2 \\ 0.0120 & 3.8418232=2 \\ 0.0150 & 3.4656528=2 \\ 0.0200 & 3.7449695=2 \\ 0.0250 & 4.5465415=2 \\ 0.0300 & 5.4077500=2 \\ 0.0400 & 6.8199370=2 \\ 0.0500 & 7.7536040=2 \\ 0.0650 & 8.4703985=2 \\ 0.0800 & 8.6381855=2 \\ 0.1000 & 8.3624795=2 \\ 0.1500 & 6.8812590=2 \\ 0.2000 & 6.1227615=2 \\ 0.3000 & 8.4131040=2 \\ 0.4000 & 1.1992628=1 \\ 0.6000 & 1.7344026=1 \\ 0.750 & \end{array}$

\begin{tabular}{|c|c|c|c|}
\hline \multirow{2}{*}{\multicolumn{2}{|c|}{$\begin{array}{l}4.6882584 \\
4.5435164\end{array}$}} & 1.5702963 & $1 \cdot 2433895$ \\
\hline & & 1.5697963 & 1.2381675 \\
\hline \multicolumn{2}{|l|}{4.2567709} & 1.5687963 & $1 \cdot 2278386$ \\
\hline \multicolumn{2}{|l|}{3.9759417} & 1.5677963 & 1.2176623 \\
\hline \multicolumn{2}{|l|}{3.7045096} & 1.5667963 & 1.2076369 \\
\hline \multicolumn{2}{|l|}{3.4455440} & 1.5657963 & 1.1977599 \\
\hline \multicolumn{2}{|l|}{3.2006177} & 1.5647963 & $1 \cdot 1880278$ \\
\hline \multicolumn{2}{|l|}{2.9694737} & 1.5637963 & $1 \cdot 1784374$ \\
\hline \multicolumn{2}{|l|}{2.7504015} & 1.5627963 & $1 \cdot 1689853$ \\
\hline \multicolumn{2}{|l|}{2.5408980} & 1.5617963 & 1.1596669 \\
\hline \multicolumn{2}{|l|}{2.3383299} & 1.5607963 & $1 \cdot 1504788$ \\
\hline \multicolumn{2}{|l|}{1.9459693} & 1.5587963 & $1 \cdot 1324770$ \\
\hline \multicolumn{2}{|c|}{$1 \cdot 3850976$} & 1.5557963 & 1.1063547 \\
\hline 6.3110825 & -1 & 1.5507963 & 1.0649462 \\
\hline 1.6809511 & -1 & 1.5457963 & 1.0259098 \\
\hline 6.1763280 & & 1.5407963 & 9.8895038 \\
\hline 5.8877241 & & 1.5307963 & 9.2076906 \\
\hline 5.7550752 & & 1.5207963 & 8.5914709 \\
\hline 5.6841667 & & 1.5057963 & 7.7737068 \\
\hline 5.6947519 & & 1.4907963 & 7.0657786 \\
\hline 5.7849057 & & 1.4707963 & 6.2644905 \\
\hline 6.2594258 & & 1.4207963 & 4.7915900 \\
\hline 6.8253659 & -1 & 1.3707963 & 3.8174547 \\
\hline 1.8811428 & & 1.2707963 & 2.6471062 \\
\hline 2.5246325 & & 1.1707963 & 1.9802951 \\
\hline 3.2331951 & & $9.7079630-1$ & 1.2445355 \\
\hline
\end{tabular}

$\mathrm{h}_{3}=70$ kilometers

$\mathrm{h}_{3}=90$ kilometers

$\begin{array}{ll}0.0005 & 1.3164471=1 \\ 0.0010 & 1.1845800=1 \\ 0.0020 & 9.7787820=2 \\ 0.0030 & 8.3027290=2 \\ 0.0040 & 7.2433350=2 \\ 0.0050 & 6.4650005=2 \\ 0.0060 & 5.8705335=2 \\ 0.0070 & 5.3954105=2 \\ 0.0080 & 4.9996202=2 \\ 0.0090 & 4.6599058=2 \\ 0.0100 & 4.3636829=2 \\ 0.0120 & 3.8808194=2 \\ 0.0150 & 3.4156723=2 \\ 0.0200 & 3.2953088=2 \\ 0.0250 & 3.7113899-2 \\ 0.0300 & 4.3001810=2 \\ 0.0400 & 5.3933450=2 \\ 0.0500 & 6.1783215=2 \\ 0.0650 & 6.8489210=2 \\ 0.0800 & 7.0962840-2 \\ 0.1000 & 7.0339730-2 \\ 0.1500 & 6.2712245-2 \\ 0.2000 & 6.1446980-2 \\ 0.3000 & 8.7553295=2 \\ 0.4000 & 1.2229357-1 \\ 0.6000 & 1.7430823=1 \\ & \end{array}$

4.8944173
4.7411516
4.4387954
4.1467159
3.0705775
3.6141515
3.3784109
3.1619431
2.9619418
2.7751252
2.5983130
2.2644028
1.7916985
1.0973961
$6.0439875-1$
$2.9200201-1$
6.2421189
6.0880873
6.0008259
6.0024613
6.0856745
$2.6384927-1$
$9.1553214-1$
1.9657904
2.5608673
3.2451086

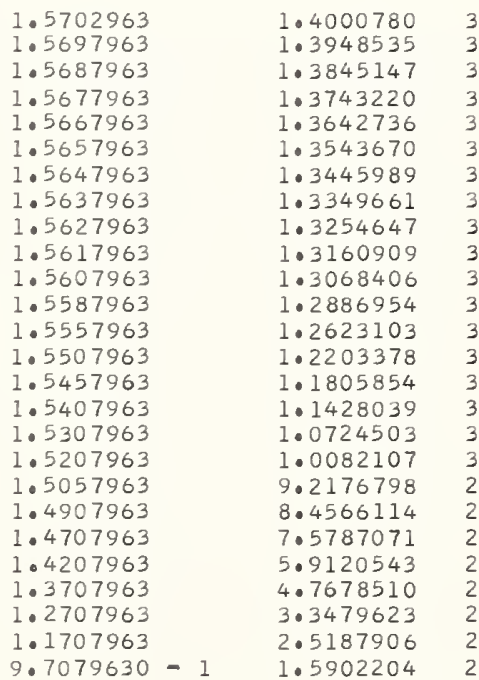

Table 81. Effective reflection coefficient (amplitude, $\left|C_{j}\right|$, and phase, $A_{r} C_{j}$ ) for the special ray configuration of the Norton type 301 atmosphere, applicable to the propagation of waves between the D-region ( $h_{3}=70$ kilometers) or the E-region $\left(h_{3}=90\right.$ kilometers $)$ of the ionosphere and the earth.

$$
-155-
$$




$$
\begin{array}{ll}
\omega / \omega_{\mathbf{r}}=0.2335 & \mathbf{f}=10 \mathrm{kilocycles} \\
\phi_{1}=60 \text { degrees } & \sigma=0.005 \mathrm{mhos} / \mathrm{meter} \\
& \epsilon_{2}=15
\end{array}
$$

$\begin{array}{ll}\Psi & \left|C_{3}\right| \\ \text { radians } & \\ & \\ 0.0005 & 2.2075288=1 \\ 0.0010 & 1.9692076=1 \\ 0.0020 & 1.5852795=1 \\ 0.0030 & 1.3000166=1 \\ 0.0040 & 1.0879071=1 \\ 0.0050 & 9.2874055=2 \\ 0.0060 & 8.0732115=2 \\ 0.0070 & 7.1281355=2 \\ 0.0080 & 6.3790930=2 \\ 0.0090 & 5.7795690=2 \\ 0.0100 & 1.9636390=1 \\ 0.0120 & 5.3018220=2 \\ 0.0150 & 4.3647100=2 \\ 0.0200 & 5.1058660=2 \\ 0.0250 & 6.3819980=2 \\ 0.0300 & 7.6372305=2 \\ 0.0400 & 9.6326950=2 \\ 0.0500 & 1.0953439=1 \\ 0.0650 & 1.2008514=1 \\ 0.0800 & 1.2318513=1 \\ 0.1000 & 1.2025056=1 \\ 0.1500 & 9.8049960=2 \\ 0.2000 & 7.5616750=2 \\ 0.3000 & 7.1249920=2 \\ 0.4000 & 1.0055132=1 \\ 0.6000 & 1.4973615=1 \\ & \end{array}$

$\mathrm{Arg} \mathrm{C}_{3}$

$\mathrm{h}_{3}=70$ kilometers

4.2666521
4.1263458
3.8481268
3.5741051
3.3063000
3.0467928
2.7968163
2.5562934
2.3239379
2.0977675
4.1288421
1.8757837
$8.2748152-1$
$7.0775960-2$
5.9203837
5.6637933
5.3864858
5.2500295
5.1610109
5.1440883
5.1855220
5.4818215
5.9868373
$9.8241232-1$
1.7690915
2.5132455

$\mathrm{h}_{3}=90$ kilometers

0.0005

0.0010

0.0020

0.0030

0.0040

$0.005 \mathrm{C}$

0.0060

0.0070

0.0080

0.0090

0.0100

0.0120

0.0150

0.0200

0.0250

0.0300

0.0400

0.0500

0.0650

0.0800

0.1000

0.1500

0.2000

0.3000

0.4000

0.6000

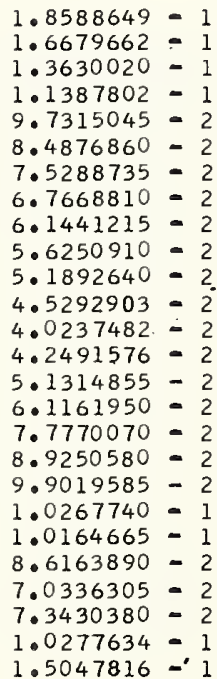

4. 4134308

4.2682492

3.9819036

3. 7033934

3.4361052

3.1826713

2.7196437

2. 5072897

2.1081301

1. 7271372

1. 1763770

$4.1478475-1$

6.2195015

5.9329245

5.6268416

5.4774997

5.3781149

5.3546874

5.3902167

5.6812929

6.1856657

1. 0903498

1. 8201795

2. 5250446
2.9440963

2. 3043666
1. 5702963

1.5697963

1. 5687963

1.5677963

1.5667963

1.5657963

1.5647963

1.5637963

1.5627963

1. 5617963

1.5697963

1. 5607963

1.5557963

1.5507963

1. 5457963

1. 5407963

1.5307963

1.5207963

1.5057963

1.4907963

1.4707963

1. 4207963

1.3707963

1.2707963

1. 1707963

9.7079630 - $d / j$

miles
Table 82. Effective reflection coefficient (amplitude, $\left|C_{j}\right|$, and phase, $A r g C_{j}$ ) for the special ray configuration of the Norton type 301 atmosphere, applicable to the propagation of waves between the D-region $\left(h_{3}=70\right.$ kilometers) or the E-region $\left(h_{3}=90\right.$ kilometers $)$ of the ionosphere and the earth.

$\begin{array}{lll}1.5702963 & 1.4000780 & 3 \\ 1.5697963 & 1.3948535 & 3 \\ 1.5687963 & 1.3845147 & 3 \\ 1.5677963 & 1.3743220 & 3 \\ 1.5667963 & 1.3642736 & 3 \\ 1.5657963 & 1.3543670 & 3 \\ 1.5647963 & 1.3445989 & 3 \\ 1.5637963 & 1.3349661 & 3 \\ 1.5627963 & 1.3254647 & 3 \\ 1.5617963 & 1.3160909 & 3 \\ 1.5607963 & 1.3068406 & 3 \\ 1.5587963 & 1.2886954 & 3 \\ 1.5557963 & 1.2623103 & 3 \\ 1.5507963 & 1.2203378 & 3 \\ 1.5457963 & 1.1805854 & 3 \\ 1.5407963 & 1.1428039 & 3 \\ 1.5307963 & 1.0724503 & 3 \\ 1.5207963 & 1.0082107 & 3 \\ 1.5057963 & 9.2176798 & 2 \\ 1.4907963 & 8.4566114 & 2 \\ 1.4707963 & 7.5787071 & 2 \\ 1.4207963 & 5.9120543 & 2 \\ 1.3707963 & 4.7678510 & 2 \\ 1.2707963 & 3.3479623 & 2 \\ 1.1707963 & 2.5187906 & 2 \\ 9.7079630 & 1.5902204 & 2\end{array}$

1.2433895

1.2278386

1.2176623

1.2076369

1.1977599

1. 1880278

1.1784374

1. 1689853

1. 1596669

$1 \cdot 1504788$

l. 1324770

1. 1063547

1.0649462

1. 0259098

9.8899038

.

8.5914709

. 7737068

4. 7915900

3.8174547

2.6471062

1.9802951

1.2445355

.




$$
\begin{array}{ll}
\omega / \omega_{r}=0.0100 & f=0.42826552 \mathrm{kilocycles} \\
\phi_{1}=60 \text { degrees } & \sigma=0.005 \mathrm{mhos} / \mathrm{meter} \\
& \epsilon_{2}=15
\end{array}
$$

$\begin{array}{lllll}\Psi & \left|C_{3}\right| & A_{\text {rgdians }} C_{3} & T_{j} & \mathrm{~d} / \mathrm{j} \\ \text { miles }\end{array}$

radians

$\mathrm{h}_{3}=70$ kilometers

0.0005

0.0010

0.0020

0.0030

0.0040

0.0050

0.0060

0.0070

0.0080

0.0090

0.0100

0.0120

0.0150

0.0200

0.0250

0.0300

0.0400

0.0500

0.0650

0.0800

0.1000

0.1500

0.2000

0.3000

0.4000

0.6000

$5.7724405=2$
$3.0240559=2$
$1.4735341=2$
$2.2256838=2$
$3.1779552=2$
$4.0313949=2$
$4.7664937=2$
$5.3951070=2$
$5.9339535=2$
$6.3987125=2$
$6.8026240=2$
$7.4688485=2$
$8.2202770=2$
$9.0849165=2$
$9.6887240=2$
$1.0155558=1$
$1.0892845=1$
$1.1525679=1$
$1.2447902=1$
$1.3423650=1$
$1.4861379=1$
$1.9108081=1$
$2.3898933=1$
$3.3373521=1$
$5.2918225=1$
$4.1434070=1$

2. 9264599

2. 2490910

4. $1799113-1$

5.5156561

4. 9933146

4. 6947925

4.4992305

4.3607820

4.2575884

4. 177774 ?

4. 1143020

4. 0200851

$3.928171 \div$

3. $842135 \%$

3.7980247

3. 776324 \%

3.7703379

3.7934108

3.8589193

3.9459998

4.078491€

4.4164054

4.7034508

5. 1011708

5.6040300

5. 3424940

\section{5702963}

1.5697963

1. 5687963

1. 5677963

1.5667963

1. 5657963

1.5647963

1.5637963

1.5627963

1. 5617963

1.5607963

1. 5587963

1.5557963

1.5507963

1. 5457963

1. 5407963

1.5307963

1.5207963

1. 5057963

1.4907963

1.4707953

1. 4207963

1.3707963

1.2707963

$9.7079630-1$

miles

$\mathrm{h}_{3}=90$ kilometers

0.0005

0.0010

0.0030

0.0040

0.0050

0.0060

0.0070

0.0080

0.0090

0.0100

0.0120

0.0150

0.0200

0.0250

0.0300

0.0400

0.0500

0.0650

0.0800

0.1000

0.1500

0.2000

0.3000

0.4000

0.6000

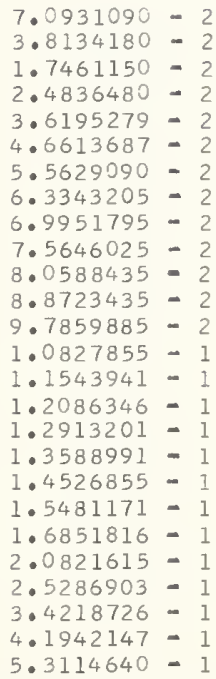

3. 2771282

2.6133767

8.8602405 - 1

5. 9968765

5.3407829

5.0328962

4. 8344071

4.6950224

4. 5915588

4. 5116885

4. 4481921

4.3538034

4.2611230

4. 1724989

4. 1244448

4.0976840

4. 0788455

4.0862513

4. 1248687

4. 1832415

4.2780212

1. 1707963

1.2433895 1.2381675

$1 \cdot 2278386$

I. 2176623

1.2076369

1. 1977599

1.1880278

1.1784374

1. 1689853

1.1596669

1.1504788

$1 \cdot 1324770$

1.1063547

1.0649462

1.0259098

9.8899038

9.2076906

8.5914709

7.7737068

7.0657786

6.2544905

4. 7915900

3.8174547

2.6471052

1.9802951

1.2445355

4. 5389411

4. 7777212

5. 1309992

$5 \cdot 3563296$

1. 5702963

1. 5697963

1.5687963

1.5677963

1. 5667963

1. 5657963

1. 5647953

1. 5637963

1.5627963

1.5617963

1.5607963

1.5587963

1. 5557063

1. 5507963

1.5457963

1.5407963

1. 5307963

1. 5207963

?. 5057963

1. 4907963

1.4707963

1.4207963

1. 3707963

1.2707963

1. 1707963

$9.7079630-1$

?. 4000780

1. 3948535

1. 3845147

I. 3743220

1. 3642736

1. 3543670

1. 3445989

1. 3349661

1. 3254647

1. 3160909

1. 3068406

1. 2886954

1.2623103

1.2203378

1. 1805854

1. 1428039

1.0724503

1. 0082107

9. 2176798

8.4566114

7.5787071

5.9120543

4.7678510

3.3479623

2. 5187906

1.5902204

Table 83. Effective reflection coefficient (amplitude, $\left|C_{j}\right|$, and phase, Arg $C_{j}$ ) for the special ray configuration of the Norton type 301 atmosphere, applicable to the propagation of waves between the $\mathrm{D}$-region $\left(\mathrm{h}_{3}=70\right.$ kilometers $)$ or the $\mathrm{E}$-region $\left(\mathrm{h}_{3}=90\right.$ kilometers $)$ of the ionosphere and the earth. 


$$
\begin{array}{ll}
\omega / \omega_{r}=0.0200 & f=0.85653105 \mathrm{kilocycles} \\
\phi_{1}=60 \text { degrees } & \sigma=0.005 \mathrm{mhos} / \mathrm{meter} \\
& \epsilon_{2}=15
\end{array}
$$

$\begin{array}{lllll}\Psi & \left|\mathrm{C}_{3}\right| & \mathrm{Arg} \mathrm{C}_{3} & \mathrm{~T}_{\mathrm{j}} & \mathrm{d} / \mathrm{j} \\ \text { radians } & & & \text { miles }\end{array}$

$\begin{array}{ll}0.0005 & \\ 0.0010 & 2.0571558=2 \\ 0.0020 & 1.1399656=2 \\ 0.0030 & 1.6033557=2 \\ 0.0040 & 2.1177146=2 \\ 0.0050 & 2.5517911=2 \\ 0.0060 & 2.9308608=2 \\ 0.0070 & 3.2688659=2 \\ 0.0080 & 3.5724492=2 \\ 0.0090 & 3.8458474=2 \\ 0.0100 & 4.0925423=2 \\ 0.0120 & 4.5179792=2 \\ 0.0150 & 5.0241110=2 \\ 0.0200 & 5.6348955=2 \\ 0.0250 & 6.0715940=2 \\ 0.0300 & 6.4082765=2 \\ 0.0400 & 6.9243330=2 \\ 0.0500 & 7.3442075=2 \\ 0.0650 & 7.9262510=2 \\ 0.0800 & 8.5272920=2 \\ 0.1000 & 9.4180390=2 \\ 0.1500 & 1.2182600=1 \\ 0.2000 & 1.5560650=1 \\ 0.3000 & 2.3021533=1 \\ 0.4000 & 3.0146380=1 \\ 0.6000 & 4.1429748=1 \\ 0 & \end{array}$

$$
\mathrm{h}_{3}=70 \text { kilometers }
$$

1.8765219

1.3490791

6.0052561

4. 9070541

4.3803008

4.0409291

3.7969187

3. 6128653

3. 4697540

3.3558483

3. 1235621

2. 9842727

2. 8510229

2.7805312

2. 7437225

2. 7260814

2. 7515549

2.8352228

2. 9506544

3. 1292724

3.5927296

3.9936807

4.5596926

4. 9075306

5. 2862168

$h_{3}=90$ kilometers

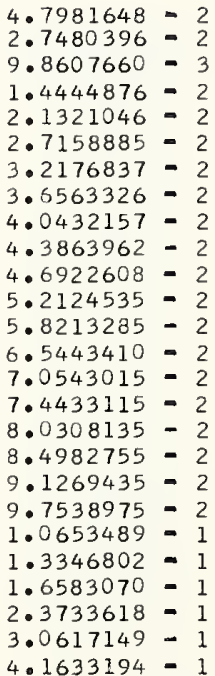

3. 2634331

1.5702963
1.5697963
1.5687963
1.5677963
1.5667963
1.5657963
1.5647963
1.5637963
1.5627963
1.5617963
1.5607963
1.5587963
1.5557963
1.5507963
1.5457963
1.5407963
1.5307963
1.5207963
1.5057963
1.4907963
1.4707963
1.4207963
1.3707963
1.2707963
1.1707963
$9.7079630-1$

1.2433895

1. 2381675

1. 2278386

1. 2176623

1.2076369

1. 1977599

1. 1880278

1. 1784374

1. 1689853

1. 1596669

1. 1504788

1. 1324770

1. 1063547

1.0649462

1.0259098

9.8899038

9.2076906

8.5914709

7. 7737068

7.0657786

6.2644905

4. 7915900

3.8174547

2. 6471062

1.9802951

\begin{tabular}{|c|c|}
\hline $\begin{array}{l}1.5702963 \\
1.5697963\end{array}$ & $\begin{array}{l}1.4000780 \\
1.3948535\end{array}$ \\
\hline I. 5687963 & 1.3845147 \\
\hline 1.5677963 & 1.3743220 \\
\hline 1.5667963 & I. 3642736 \\
\hline 1.5657963 & 1.3543670 \\
\hline 1.5647963 & 1.3445989 \\
\hline 1.5637963 & 1.3349661 \\
\hline 1.5627963 & 1.3254647 \\
\hline 1.5617963 & 2.3160909 \\
\hline 1.5607963 & 1.3068406 \\
\hline 1.5587963 & 1.2886954 \\
\hline 1.5557963 & 1.2623103 \\
\hline 1.5507963 & 1.2203378 \\
\hline 1.5457963 & 1.1805854 \\
\hline 1.5407963 & 1. 1428039 \\
\hline 1.5307963 & 1.0724503 \\
\hline 1.5207963 & 2.0082107 \\
\hline 1.5057963 & 9.2176798 \\
\hline 1.4907963 & 8.4566114 \\
\hline 1.4707963 & 7.5787071 \\
\hline $\begin{array}{l}1.4207963 \\
1.3707963\end{array}$ & 5.9120543 \\
\hline 1.2707963 & 3.3479623 \\
\hline 1.1707963 & 2.5187906 \\
\hline .7079630 & 1.5902204 \\
\hline
\end{tabular}

1. 2445355

$2 \cdot 4124663$
1.9546788
$4 \cdot 2793498-1$
$5 \cdot 2931982$
$4 \cdot 7306496$
$4 \cdot 3971443$
$4 \cdot 1649056$
$3 \cdot 9923360$
$3 \cdot 8590926$
$3 \cdot 7533379$
$3 \cdot 6675573$
$3 \cdot 5374368$
$3 \cdot 4068523$
$3 \cdot 2794002$
$3 \cdot 2089034$
$3 \cdot 1687156$
$3 \cdot 1380221$
$3 \cdot 1449812$
$3 \cdot 1948708$
$3 \cdot 2731490$
$3 \cdot 4021855$
$3 \cdot 7629301$
$4 \cdot 0983470$
$4 \cdot 6024813$
$4 \cdot 9275030$
$5 \cdot 2920560$

0.0005
0.0010
0.0020
0.0030
0.0040
0.0050
0.0060
0.0070
0.0080
0.0090
0.0100
0.0120
0.0150
0.0200
0.0250
0.0300
0.0400
0.0500
0.0650
0.0800
0.1000
0.1500
0.2000
0.3000
0.4000
0.6000

Table 84. Effective reflection coefficient (amplitude, $\left|C_{j}\right|$, and phase, Arg $C_{j}$ ) for the special ray configuration of the Norton type 301 atmosphere, applicable to the propagation of waves between the D-region $\left(h_{3}=70\right.$ kilometers) or the E-region $\left(h_{3}=90\right.$ kilometers $)$ of the ionosphere and the earth. 


$$
\begin{aligned}
& \omega / \omega_{r}=0.0500 \\
& \phi_{1}=60 \text { degrees }
\end{aligned}
$$

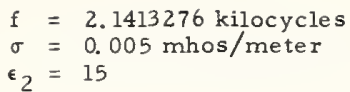

$\epsilon_{2}=15$

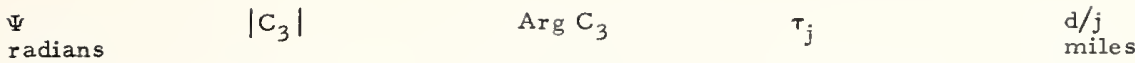

$\begin{array}{ll}0.0005 & 5.3577440=2 \\ 0.0010 & 4.1794694=2 \\ 0.0020 & 3.0994575=2 \\ 0.0030 & 2.8365686=2 \\ 0.0040 & 2.7733636=2 \\ 0.0050 & 2.7345105=2 \\ 0.0060 & 2.7016864=2 \\ 0.0070 & 2.6858374=2 \\ 0.0080 & 2.6957332=2 \\ 0.0090 & 2.7335400=2 \\ 0.0100 & 2.7965703=2 \\ 0.0120 & 2.9776218=2 \\ 0.0150 & 3.3129787=2 \\ 0.0200 & 3.8617008=2 \\ 0.0250 & 4.3119494=2 \\ 0.0300 & 4.6626720=2 \\ 0.0400 & 5.1443020=2 \\ 0.0500 & 5.4391705=2 \\ 0.0650 & 5.7018405=2 \\ 0.0800 & 5.8813615=2 \\ 0.1000 & 6.1372865=2 \\ 0.1500 & 7.3297200=2 \\ 0.2000 & 9.283115=2 \\ 0.3000 & 1.4067446=1 \\ 0.4000 & 1.8932734=1 \\ 0.6000 & 2.7502932=1\end{array}$

$h_{3}=70$ kilometers

6.1268185
5.7316167
4.9418864
4.3050712
3.8365001
3.4719474
3.1681564
2.9044132
2.6717785
2.4663981
2.2860578
1.9919643
1.6812595
1.3762909
1.2102263
1.1153998
1.0359845
1.0359435
1.1166596
1.2560850
1.4940349
2.1532024
2.7206677
3.5110758
4.0151450
4.5986316

$\begin{array}{ll}1.5702963 & 1.2433895 \\ 1.5697963 & 1.2381675 \\ 1.5687963 & 1.2278386 \\ 1.5677963 & 1.2176623 \\ 1.5667963 & 1.2076369 \\ 1.5657963 & 1.1977599 \\ 1.5647963 & 1.1880278 \\ 1.5637963 & 1.1784374 \\ 1.5627963 & 1.1689853 \\ 1.5617963 & 1.1596669 \\ 1.5607963 & 1.1504788 \\ 1.5587963 & 1.1324770 \\ 1.5557963 & 1.1063547 \\ 1.5507963 & 1.0649462 \\ 1.5457963 & 1.0259098 \\ 1.5407963 & 9.8899038 \\ 1.5307963 & 9.2076906 \\ 1.5207963 & 8.5914709 \\ 1.5057963 & 7.7737068 \\ 1.4907963 & 7.0657786 \\ 1.4707963 & 6.2644905 \\ 1.4207963 & 4.7915900 \\ 1.3707963 & 3.8174547 \\ 1.2707963 & 2.6471062 \\ 1.1707963 & 1.9802951 \\ 9.7079630-1 & 1.2445355\end{array}$

1.2433895

1675

1.2176623

-.2076369

.1977599

1.1880278

1.1689853

1. 1596669

1.1324770

1. 1063547

.8899038

9.2076906

.

.0657786

2644905

3.8174547

1. 9802951

4.5986316

9.7079630

. 2445355

$\mathrm{h}_{3}=90$ kilometers

$\begin{array}{ll}0.0005 & 4.1829063=2 \\ 0.0010 & 3.1026629=2 \\ 0.0020 & 2.3288486=2 \\ 0.0030 & 2.4161717=2 \\ 0.0040 & 2.6060784=2 \\ 0.0050 & 2.7420453=2 \\ 0.0060 & 2.8326669=2 \\ 0.0070 & 2.9023566=2 \\ 0.0080 & 2.9674665=2 \\ 0.0090 & 3.0361196=2 \\ 0.0100 & 3.1111387=2 \\ 0.0120 & 3.2789851=2 \\ 0.0150 & 3.5526899=2 \\ 0.0200 & 3.9900925=2 \\ 0.0250 & 4.3595490=2 \\ 0.0300 & 4.6606349=2 \\ 0.0400 & 5.1103235=2 \\ 0.0500 & 5.4311755=2 \\ 0.0650 & 5.7921125=2 \\ 0.0800 & 6.1004585=2 \\ 0.1000 & 6.5257165=2 \\ 0.1500 & 7.9553575=2 \\ 0.2000 & 9.9125825=2 \\ 0.3000 & 1.4537201=1 \\ 0.4000 & 1.9264909=1 \\ 0.6000 & 2.7658486=1\end{array}$

$3.7604797-1$
6.2136555
5.2542123
4.5450379
4.0844393
3.7492016
3.4807531
3.2544826
3.0590808
2.8885938
2.7392569
2.4932155
2.2242537
1.9443525
1.7819674
1.6836456
1.5897836
1.5706292
1.6164225
1.7143818
1.8899827
2.3985386
2.8673846
3.5716121
$4 \cdot 0448696$
4.6079185

$\begin{array}{ll}1.5702963 & 1.4000780 \\ 1.5697963 & 1.3948535 \\ 1.5687963 & 1.3845147 \\ 1.5677963 & 1.3743220 \\ 1.5667963 & 1.3642736 \\ 1.5657963 & 1.3543670 \\ 1.5647963 & 1.3445989 \\ 1.5637963 & 1.3349661 \\ 1.5627963 & 1.3254647 \\ 1.5617963 & 1.3160909 \\ 1.5607963 & 1.3068406 \\ 1.5587963 & 1.2886954 \\ 1.5557963 & 1.2623103 \\ 1.5507963 & 1.2203378 \\ 1.5457963 & 1.1805854 \\ 1.5407963 & 1.1428039 \\ 1.5307963 & 1.0724503 \\ 1.5207963 & 1.0082107 \\ 1.5057963 & 9.2176798 \\ 1.4907963 & 8.4566114 \\ 1.4707963 & 7.5787071 \\ 1.4207963 & 5.9120543 \\ 1.3707963 & 4.7578510 \\ 1.2707963 & 3.3479623 \\ 1.1707963 & 2.5187906 \\ 0.7079630-1 & 1.5902204\end{array}$

Table 85. Effective reflection coefficient (amplitude, $\left|C_{j}\right|$, and phase, $\operatorname{Arg}_{\mathbf{j}} \mathrm{C}_{j}$ ) for the special ray configuration of the Norton type 301 atmosphere, applicable to the propagation of waves between the $D$-region ( $h_{3}=70$ kilometers) or the $E$-region ( $h_{3}=90$ kilometers) of the ionosphere and the earth. 


$$
\begin{aligned}
& \omega / \omega_{r}=0.1000 \\
& \phi_{1}=60 \text { degrees }
\end{aligned}
$$

$f=4.2826552$ kilocycles

$\sigma=0.005 \mathrm{mhos} / \mathrm{meter}$

$\epsilon_{2}=15$

$\begin{array}{ll}\Psi & \left|C_{3}\right| \\ \text { radians } & \\ & \\ 0.0005 & 1.0939798=1 \\ 0.0010 & 9.3006410=2 \\ 0.0020 & 7.0577590=2 \\ 0.0030 & 5.7264900=2 \\ 0.0040 & 4.8970776=2 \\ 0.0050 & 4.3281351=2 \\ 0.0060 & 3.9003009=2 \\ 0.0070 & 3.5637365=2 \\ 0.0080 & 3.3019205=2 \\ 0.0090 & 3.1115958=2 \\ 0.0100 & 2.9924340=2 \\ 0.0120 & 2.9535976=2 \\ 0.0150 & 3.2581251=2 \\ 0.0200 & 4.1138951=2 \\ 0.0250 & 4.9385559=2 \\ 0.0300 & 5.6098585=2 \\ 0.0400 & 6.5263775=2 \\ 0.0500 & 7.0290150=2 \\ 0.0650 & 7.3010590=2 \\ 0.0300 & 7.2352115=2 \\ 0.1000 & 6.9025265=2 \\ 0.1500 & 6.1630070=2 \\ 0.2000 & 6.6902850=2 \\ 0.3000 & 1.0247114=1 \\ 0.4000 & 1.4131163=1 \\ 0.6000 & 2.0282913=1 \\ & \end{array}$

$\operatorname{Arg~C} 3$

$\mathrm{h}_{3}=70$ kilometers

5.0819352

4.8483638

4. 3904668

3. 9628733

3. 5800840

3.2419598

2.9390547

2.6602331

2.3968661

2. 1443585

I. 9022466

1.4609770

9.4846181 -

$4.4576183-1$

$1.8208202-1$

$2.9221000-2$

6.1594122

6.1055279

6. 1170366

6.1953789

$8.7419790-2$

$7.5647269-1$

1.5054542

2. 5246320

3. 1106500

3. 8007835

$\mathrm{h}_{3}=90$ kilometers

0.0005
0.0010
0.0020
0.0030
0.0040
0.0050
0.0060
0.0070
0.0080
0.0090
0.0100
0.0120
0.0150
0.0200
0.0250
0.0300
0.0400
0.0500
0.0650
0.0800
0.1000
0.1500
0.2000
0.3000
0.4000
0.6000

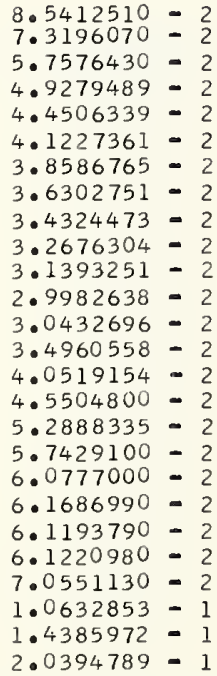

$\tau_{j}$

$d / j$

miles

1.2433895

I. 5702963

1.5697963

1.5687963

1.5677963

I. 5667963

1.5657963

1.5647963

1. 5537963

1.5627963

1.5617963

1.5607963

1.5587963

1.5557963

l. 5507963

1.5457963

1. 5407963

1. 5307963

1.5207963

1.5057963

1. 4907963

1.4707963

1.4207963

1.3707963

1. 2707963

1.1707963

$9.7079630-1$

1. 2381675

1. 2278386

1. 2176623

1.2076369

1. 1377599

1.1880278

1. 1784374

1. 1589853

1. 1596669

1. 1504788

1. 1324770

l. 1063547

1.0649462

1.0259098

9. 8899038

9.2076906

8.5914709

7.7737068

7.0657786

6. 2644905

4. 7915900

3. 8174547

2. 6471062

1.9802951

1. 2445355

1.5702963

1.5697963

1. 5687963

1.5677963

I. 5667963

1. 5657963

1.5647963

1. 5537963

1. 5627963

1.5617963

1.5607963

1. 5587963

1. 5557963

1.5507963

1. 5457963

1. 5407963

1. 5307963

1. 5207963

1.5057963

1.4907963

1. 4707963

1.4207963

1.3707963

1. 2707963

1.1707963

9.7079630 $\begin{array}{ll}1.4000780 & 3 \\ 1.3948535 & 3 \\ 1.3845147 & 3 \\ 1.3743220 & 3 \\ 1.3642736 & 3 \\ 1.3543670 & 3 \\ 1.3445989 & 3 \\ 1.3349661 & 3 \\ 1.3254647 & 3 \\ 1.3160909 & 3 \\ 1.3068406 & 3 \\ 1.2836954 & 3 \\ 1.2623103 & 3 \\ 1.2203378 & 3 \\ 1.1805854 & 3 \\ 1.1428039 & 3 \\ 1.0724503 & 3 \\ 1.0082107 & 3 \\ 9.2176798 & 2 \\ 8.4566114 & 2 \\ 7.5787071 & 2 \\ 5.9120543 & 2 \\ 4.7678510 & 2 \\ 3.3479623 & 2 \\ 2.5187906 & 2 \\ 1.5902204 & 2\end{array}$

Table 86. Effective reflection coefficient (amplitude, $\left|C_{j}\right|$, and phase, Arg $C_{j}$ ) for the special ray configuration of the Norton type 301 atmosphere, applicable to the propagation of waves between the $D$-region $\left(h_{3}=70\right.$ kilometers) or the $E$-region $\left(h_{3}=90\right.$ kilometers) of the ionosphere and the earth. 


$$
\begin{array}{ll}
\omega / \omega_{r}=0.2000 & f=8.5653105 \mathrm{kilocycles} \\
\phi_{1}=60 \text { degrees } & \sigma=0.005 \mathrm{mhos} / \mathrm{meter} \\
\epsilon_{2}=15
\end{array}
$$

$\Psi$

radians

$$
\left|c_{3}\right|
$$

$\operatorname{Arg~} C_{3}$

$\mathrm{h}_{3}=70$ kilometers

$\begin{array}{ll}0.0005 & 1.9767811=1 \\ 0.0010 & 1.7499697=1 \\ 0.0020 & 1.3918679=1 \\ 0.0030 & 1.1329832=1 \\ 0.0040 & 9.4513940=2 \\ 0.0050 & 8.0675120=2 \\ 0.0060 & 7.0230505=2 \\ 0.0070 & 6.2135035=2 \\ 0.0080 & 5.5730585=2 \\ 0.0090 & 5.0631330=2 \\ 0.0100 & 4.6629469=2 \\ 0.0120 & 4.1552305=2 \\ 0.0150 & 4.0397150=2 \\ 0.0200 & 4.9177749=2 \\ 0.0250 & 6.1643480=2 \\ 0.0300 & 7.3265295=2 \\ 0.0400 & 9.1063275=2 \\ 0.0500 & 1.0239375=1 \\ 0.0650 & 1.1087743=1 \\ 0.0300 & 1.1267009=1 \\ 0.1000 & 1.0887717=1 \\ 0.1500 & 8.7496895=2 \\ 0.2000 & 6.8975015=2 \\ 0.3000 & 7.5022445=2 \\ 0.4000 & 1.076092=1 \\ 0.6000 & 1.5845083=1\end{array}$

0.0005

0.0010

0.0020

0.0030

0.0040

0.0050

0.0060

0.0070

0.0080

0.0090

0.0100

0.0120

0.0150

0.0200

0.0250

0.0300

0.0400

0.0500

0.0650

0.0800

0.1000

0.1500

0.2000

0.3000

0.4000

0.6000

4.3947470
4.2420216
3.9397085
3.6434600
3.3563900
3.0811636
2.8187766
2.5682674
2.3272425
2.0928018
1.8625071
1.4116723
$7.9331255-1$
$7.5692480-2$
5.9635380
5.7314034
5.4822954
5.3629333
5.2925349
5.2915335
5.3539521
5.7129127
$1.4861060=2$
1.3127044
2.0445580
2.7744678

$\mathrm{h}_{3}=90$ kilometers

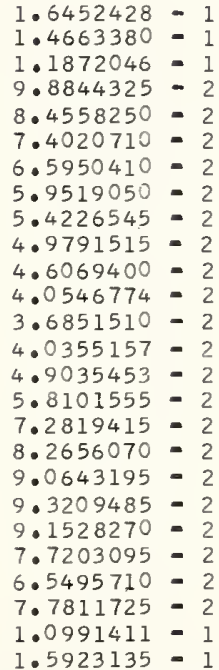

4.5599772
4.4009445
4.0881007
3.7862127
3.5001283
3.2328903
2.9848217
2.7539274
2.5368787
2.3299880
2.1298876
1.7410522
1.1848045
$4.5009334-1$
$7.7102300-3$
5.0289176
5.7509615
5.6183973
5.5373056
5.5297254
5.5864745
5.9413413
$2.3397864-1$
1.4122312
2.0835654
2.7863319

${ }^{\top} \mathbf{j}$

$\mathrm{d} / \mathrm{j}$

miles

\author{
1.5702963
}

1.5697963

1. 5687963

1.5677963

1.5667963

1. 5657963

1. 5647963

1. 5537963

1. 5627963

1.5617963

1. 5607963

1.5587963

1. 5557963

1. 5507963

1.5457963

1.5407963

1. 5307963

1. 5207963

1.5057963

I. 4907963

1.4707963

1.4207963

1.3707963

1. 2707963

1.1707963

$9.7079630-1$

1.2433895

1.2381675

1. 2278386

1. 2176623

1.2076369

1. 1977599

1. 1880278

1.1784374

1.1589853

1. 1595669

1. 1504788

1. 1324770

1.1063547

1.0649462

1.0259098

9. 8899038

9.2075906

8.5914709

7.7737068

7.0657786

6.2644905

4. 7915900

3.8174547

2.6471062

1.9802951

1.2445355
1. 5702953

1.5697963

1.5687963

1. 5677963

1.5667963

1. 5657963

1.5647963

1.5637963

1.5627963

1.5617963

1. 5607963

1. 5587963

1.5557963

1.5507963

1. 5457963

1. 5407963

1.5307963

1. 5207963

1.5057963

1.4907963

1.4707963

1.4207963

1. 3707963

1. 2707963

1.1707963

9.7079630
1.4000780 1. 3948535 1. 3845147 1. 3743220 1. 3642736 1.3543670 1. 3445989 1.3349661 1. 3254647 1.3160909 1. 3068406 1. 2886954 1. 2623103 1. 2203378 1.1805854 1. 1428039 1.0724503 1.0082107 9.2176798 8.4566114 7.5787071 5.9120543 4.7678510 3.3479623 2. 5187906 1.5902204

Table 87. Effective reflection coefficient (amplitude, $\left|C_{j}\right|$, and phase, $\operatorname{Arg} C_{j}$ ) for the special ray configuration of the Norton type 301 atmosphere, applicable to the propagation of waves between the D-region ( $h_{3}=70$ kilometers) or the E-region ( $h_{3}=90$ kilometers) of the ionosphere and the earth.

-161 - 


$$
\begin{array}{ll}
\omega / \omega_{r}=1.0000 & f=42.826552 \mathrm{kilocycles} \\
\phi_{1}=60 \text { degrees } & \sigma=0.005 \mathrm{mhos} / \mathrm{meter} \\
& \epsilon_{2}=15
\end{array}
$$

$\begin{array}{lllll}\Psi & \left|C_{3}\right| & \operatorname{Arg~C}_{3} & \tau_{j} & \mathrm{~d} / \mathrm{j} \\ \text { radians } & & \text { miles }\end{array}$

0.0005

0.0010

0.0020

0.0030

0.0040

0.0050

0.0060

0.0070

0.0080

0.0090

0.0100

0.0120

0.0150

0.0200

0.0250

0.0300

0.0400

0.0500

0.0650

0.0800

0.1000

0.1500

0.2000

0.3000

0.4000

0.6000

$\mathrm{h}_{3}=70$ kilometers

$4.1942849=1$
$3.9529888=1$
$3.5165510=1$
$3.1360878=1$
$2.8052399=1$
$2.5180952=1$
$2.2692237=1$
$2.0537119=1$
$1.8671495=1$
$1.7056178=1$
$1.5656752=1$
$1.3389802=1$
$1.0986991=1$
$8.7203385=2$
$7.8858990=2$
$8.0348495=2$
$9.8431850=2$
$1.2135857=1$
$1.5091786=1$
$1.7168085=1$
$1.8743915=1$
$1.8906496=1$
$1.6558531=1$
$1.0647098=1$
$6.2226705=2$
$3.2371695=2$

3.1097667
3.0430099
2.9096995
2.7764913
2.6432470
2.5099004
2.3764583
2.2429859
2.1096004
1.9764475
1.8436872
1.5799254
1.1901938
$5.5993440-1$
6.2531973
5.7450868
5.0449023
4.6441789
4.3061566
4.1121143
3.9564343
3.7799795
3.7273014
3.7829845
4.0192296
4.9844634

1. 5702963

1. 5697963

1. 5687963

1. 5677963

1. 5667963

1.5657963

1. 5647963

1. 5537963

1. 5627963

1. 5617963

1. 5607963

1. 5587963

1.5557963

1.5507963

1. 5457963

1.5407963

1. 5307963

1. 5207963

1.5057963

1.4907963

1.4707963

1.4207963

1.3707963

1. 2707963

1. 1707963

$9.7079630-1$

$\begin{array}{ll}1.2433895 & 3 \\ 1.2381675 & 3 \\ 1.2278386 & 3 \\ 1.2176623 & 3 \\ 1.2076359 & 3 \\ 1.1977599 & 3 \\ 1.1880278 & 3 \\ 1.1784374 & 3 \\ 1.1689853 & 3 \\ 1.1596669 & 3 \\ 1.1504788 & 3 \\ 1.1324770 & 3 \\ 1.1063547 & 3 \\ 1.0649452 & 3 \\ 1.0259098 & 3 \\ 9.8899038 & 2 \\ 9.2076906 & 2 \\ 8.5914709 & 2 \\ 7.7737068 & 2 \\ 7.0657786 & 2 \\ 6.2644905 & 2 \\ 4.7915900 & 2 \\ 3.8174547 & 2 \\ 2.6471062 & 2 \\ 1.9802951 & 2 \\ 1.2445355 & 2\end{array}$

$\mathrm{h}_{3}=90$ kilometers

$\begin{array}{ll}0.0005 & 3.7779152=1 \\ 0.0010 & 3.5661017=1 \\ 0.0020 & 3.1831926=1 \\ 0.0030 & 2.8495851=1 \\ 0.0040 & 2.5595782=1 \\ 0.0050 & 2.3078622=1 \\ 0.0060 & 2.0895727=1 \\ 0.0070 & 1.9003078=1 \\ 0.0080 & 1.7361293=1 \\ 0.0090 & 1.5935438=1 \\ 0.0100 & 1.4695006=1 \\ 0.0120 & 1.2668309=1 \\ 0.0150 & 1.0474078=1 \\ 0.0200 & 8.2842525=2 \\ 0.0250 & 7.3266245=2 \\ 0.0300 & 7.2707485=2 \\ 0.0400 & 8.6951580=2 \\ 0.0500 & 1.0711684=1 \\ 0.0650 & 1.3400553=1 \\ 0.0800 & 1.5338770=1 \\ 0.1000 & 1.6871944=1 \\ 0.1500 & 1.7291347=1 \\ 0.2000 & 1.5338450=1 \\ 0.3000 & 1.0046891=1 \\ 0.4000 & 5.9678600=2 \\ 0.6000 & 3.2277877=2\end{array}$

3.0958356
3.0283745
2.8938449
2.7597430
2.6260205
2.4926994
2.3598668
2.2276552
2.0962279
1.9657556
1.8363932
1.5814535
$1.2089782-1$
$6.1104300-1$
$4 \cdot 3447860-2$
5.8212773
5.1004250
4.6843722
4.3362706
$4 \cdot 1379975$
3.9795637
3.8003226
3.7470084
3.8054730
4.0488868
5.0054970

1.5702963

1. 5697963

1.5687963

1.5677963

1.5667963

1. 5657963

1. 5647963

1. 5637963

1. 5627963

1.5617963

1.5607963

1. 5587963

1. 5557963

1.5507963

1. 5457963

1. 5407963

1. 5307963

1. 5207963

1.5057963

1.4907963

1.4707963

1.4207963

1.3707963

1. 2707963

1. 1707963

$9.7079630=1$

$\begin{array}{ll}1.4000780 & 3 \\ 1.3948535 & 3 \\ 1.3845147 & 3 \\ 1.3743220 & 3 \\ 1.3642736 & 3 \\ 1.3543670 & 3 \\ 1.3445989 & 3 \\ 1.3349661 & 3 \\ 1.3254647 & 3 \\ 1.3160909 & 3 \\ 1.3068406 & 3 \\ 1.2886954 & 3 \\ 1.2623103 & 3 \\ 1.2203378 & 3 \\ 1.1805854 & 3 \\ 1.1428039 & 3 \\ 1.0724503 & 3 \\ 1.0082107 & 3 \\ 9.2176798 & 2 \\ 8.4566114 & 2 \\ 7.5787071 & 2 \\ 5.9120543 & 2 \\ 4.7678510 & 2 \\ 3.3479623 & 2 \\ 2.5187906 & 2 \\ 1.5902204 & 2\end{array}$

Table 88. Effective reflection coefficient (amplitude, $\left|C_{j}\right|$, and phase, Arg $C_{j}$ ) for the special ray configuration of the Norton type 301 atmosphere, applicable to the propagation of waves between the D-region ( $h_{3}=70$ kilometers) or the E-region $\left(h_{3}=90\right.$ kilometers $)$ of the ionosphere and the earth. 


$$
\begin{array}{ll}
\omega / \omega_{r}=2.0000 & f=85.653105 \text { kilocycles } \\
\phi_{1}=60 \text { degrees } & \sigma=0.005 \text { mhos } / \text { meter } \\
& \epsilon_{2}=15
\end{array}
$$

$\Psi$

radians

$\left|c_{3}\right|$
Arg $\mathrm{C}_{3}$

$h_{3}=70$ kilometers

$\begin{array}{ll}0.0005 & 3.8513132=1 \\ 0.0010 & 3.7003195=1 \\ 0.0020 & 3.4188076=1 \\ 0.0030 & 3.1628449=1 \\ 0.0040 & 2.9303775=1 \\ 0.0050 & 2.7194299=1 \\ 0.0060 & 2.5281286=1 \\ 0.0070 & 2.3546957=1 \\ 0.0080 & 2.1974727=1 \\ 0.0090 & 2.0549157=1 \\ 0.0100 & 1.9255942=1 \\ 0.0120 & 1.7015157=1 \\ 0.0150 & 1.4354006=1 \\ 0.0200 & 1.1236362=1 \\ 0.0250 & 9.1961665=2 \\ 0.0300 & 7.8854910=2 \\ 0.0400 & 6.8912240=2 \\ 0.0500 & 7.3775035=2 \\ 0.0650 & 9.1011240=2 \\ 0.0800 & 1.0769930=1 \\ 0.1000 & 1.2304266=1 \\ 0.1500 & 1.3079672=1 \\ 0.2000 & 1.1493317=1 \\ 0.3000 & 6.8821845=2 \\ 0.4000 & 3.2871175=2 \\ 0.6000 & 1.9658547=3 \\ 0.030 & \end{array}$

0.0005

0.0010

0.0020

0.0030

0.0040

0.0050

0.0060

0.0070

0.0080

0.0090

0.0100

0.0120

0.0150

0.0200

0.0250

0.0300

0.0400

0.0500

0.0650

0.0800

0.1000

0.1500

0.2000

0.3000

0.4000

0.6000

2.5174572
2.4697962
2.3748096
2.2802421
2.1350700
2.0922898
1.0989112
1.9059550
1.8134567
1.7214525
1.6299893
1.4488486
1.1821102
$7.5109509-1$
$3.3313944-$
5.2056383
5.4252150
4.8011588
4.2092988
3.3615894
3.5695170
3.1528423
2.8869061
2.4804511
2.1354035
2.0815994

$\mathrm{h}_{3}=90$ kilometers
1.5697963

I. 5687963

1.5677963

.5667963

I. 5657963

1.5647963

1. 5637963

1.5627963

1.5617963

I. 5607963

?. 5587963

1. 5557963

I. 5507963

1.5457953

1.5407963

1.5307963

1. 5207963

1.5057963

1.4907963

1.4707963

1.4207963

1.3707963

1. 2707963

1.1707963

9.7079630

1. 2433895

1.2381675

1. 227838 á

1.2176623

1.2076369

1. 197759 9

I. 1880278

1.1784374

1.1689853

.1596669

1.1504788

1.1324770

1.1063547

$1.0549 \div 62$

1.0259098

.8899038

9.2076906

8.5914709

7.7737058

7.0657786

6.2644905

4.7915900

3.8174547

2.6471062

1.9802951

1.2445355

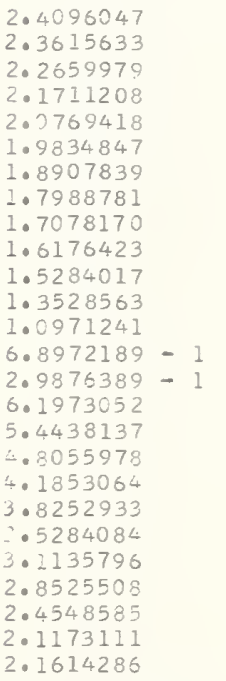

$\begin{array}{ll}1.5702963 & 1.4000780 \\ 1.5697963 & 1.3948535 \\ 1.5687963 & 1.3845147 \\ 1.5677963 & 1.3743220 \\ 1.5667963 & 1.3642736 \\ 1.5657963 & 1.3543670 \\ 1.5647963 & 1.3445989 \\ 1.5637963 & 1.3349661 \\ 1.5627963 & 1.3254647 \\ 1.5617963 & 1.3160909 \\ 1.5507963 & 1.3068406 \\ 1.5587963 & 1.2896954 \\ 1.5557963 & 1.2523103 \\ 1.5507963 & 1.2203378 \\ 1.5457963 & 1.1805854 \\ 1.5407963 & 1.1428039 \\ 1.5307963 & 1.0724503 \\ 1.5207963 & 1.0082107 \\ 1.5057963 & 9.2176798 \\ 1.4907963 & 8.4566114 \\ 1.4707963 & 7.5787071 \\ 1.4207963 & 5.9120543 \\ 1.3707963 & 4.7678510 \\ 1.2707963 & 3.3479623 \\ 1.1707963 & 2.5187906 \\ 9.7079630 & 1.5902204\end{array}$

Table 89. Effective reflection coefficient (amplitude, $\left|C_{j}\right|$, and phase, $A_{r g} C_{j}$ ) for the special ray configuration of the Norton type 301 atmosphere, applicable to the propagation of waves between the $D$-region $\left(h_{3}=70\right.$ kilometers) or the E-region ( $h_{3}=90$ kilometers) of the ionosphere and the earth.

$$
-163-
$$




$$
\begin{array}{ll}
\omega / \omega_{r}=5.0000 & \mathbf{f}=214.13276 \mathrm{kilocycles} \\
\phi_{1}=60 \text { degrees } & \sigma=0.005 \text { mhos } / \text { meter } \\
\varepsilon_{2}=15
\end{array}
$$

$\Psi$

0.0005
0.0010
0.0020
0.0030
0.0040
0.0050
0.0060
0.0070
0.0080
0.0090
0.0100
0.0120
0.0150
0.0200
0.0250
0.0300
0.0400
0.0500
0.0650
0.0800
0.1000
0.1500
0.2000
0.3000
0.4000
0.6000

0.0005

0.0010

0.0020

0.0030

0.0040

0.0050

0.0060

0.0070

0.0080

0.0090

0.0100

0.0120

0.0150

0.0200

0.0250

0.0300

0.0400

0.0500

0.0650

0.0800

0.1000

0.1500

0.2000

0.3000

0.4000

0.6000
$\left|C_{3}\right| \quad \operatorname{Arg~C}_{3}$

$\mathrm{h}_{3}=70$ kilometers

$2.6688918=1$
$2.5034714=1$
$2.2159275=1$
$1.9771831=1$
$1.7777613=1$
$1.6096847=1$
$1.4664545=1$
$1.3429243=1$
$1.2351024=1$
$1.1399626=1$
$1.0552484=1$
$9.1096850=2$
$7.4454025=2$
$5.8016595=2$
$5.4404630=2$
$5.9569220=2$
$7.7691395=2$
$9.4038995=2$
$1.1028204=1$
$1.1817582=1$
$1.1976124=1$
$1.0118899=1$
$7.3448990=2$
$2.9081786=2$
$7.3702130=3$
$1.4738737=4$

1.5751495
1.4768820
1.2853715
1.1010169
$9.2421576-1$
$7.5512660-1$
$5.9362186=1$
$4.3930191-1$
$2.9153035=1$
$1.4949550=1$
$1.2262270-2$
6.0313743
5.6466356
4.9945526
4.3647319
3.8692901
3.2832157
2.9687680
2.6851509
2.4927163
2.2931911
1.8816427
1.4868180
$6.3547405=1$
5.9932758
3.5081427

$h_{3}=90$ kilometers

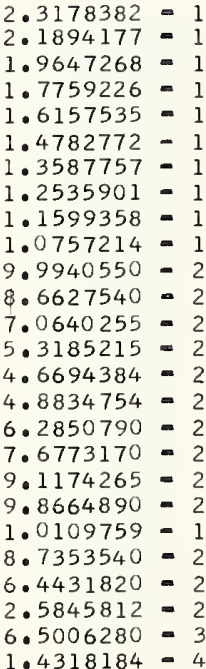

$\tau_{j}$

$\mathrm{d} / \mathrm{j}$

miles

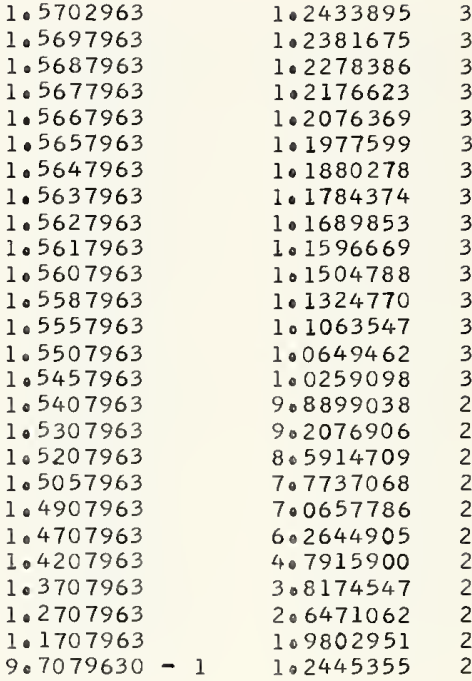

1.5702963

1.5697963

1.5687963

1.5677963

1.5667963

1.5657963

1.5647963

1.5637963

1.5627963

1.5617963

1.5607963

1.5587963

1.5557963

1.5507963

1.5457963

1.5407963

1. 5307963

1.5207963

1.5057963

1.4907963

1.4707963

1.4207963

$1 \cdot 3707963$

1.2707963

1. 1707963

$9.7079630-1$

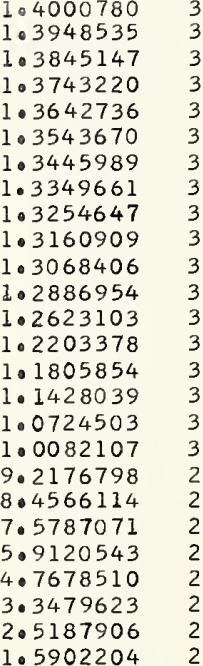

Table 90. Effective reflection coefficient (amplitude, $\left|\mathrm{C}_{j}\right|$, and phase, $\mathrm{Arg} \mathrm{C}_{\mathrm{j}}$ ) for the special ray configuration of the Norton type 301 atmosphere, applicable to the propagation of waves between the D-region $\left(h_{3}=70\right.$ kilometers $)$ or the $E-r e g i o n\left(h_{3}=90\right.$ kilometers $)$ of the ionosphere and the earth. 


$$
\begin{array}{ll}
\omega / \omega_{\mathrm{r}}=0.0100 & \mathrm{f}=0.66622256 \mathrm{kilocycles} \\
\phi_{1}=60 \text { degrees } & \sigma=0.005 \mathrm{mhos} / \mathrm{meter} \\
& \epsilon_{2}=15
\end{array}
$$

$\Psi$

radians $\left|c_{3}\right|$

Arg $\mathrm{C}_{3}$

$h_{3}=70$ kilometers

0.0005

0.0010

0.0020

0.0030

0.0040

0.0050

0.0060

0.0070

0.0080

0.0090

0.0100

0.0120

0.0150

0.0200

0.0250

0.0300

0.0400

0.0500

0.0650

0.0800

0.1000

0.1500

0.2000

0.3000

0.4000

$0.600 \mathrm{C}$

0.0005

0.0010

$0.002 \mathrm{C}$

0.0030

0.0040

0.0050

0.0060

0.0070

0.0080

0.0090

0.0100

0.0120

0.0150

0.0200

0.0250

0.0300

0.0400

0.0500

0.0650

0.0800

0.1000

0.1500

0.2000

0.3000

0.4000

0.6000

$3 \cdot 0510871$
$2 \cdot 5310487$
$1 \cdot 1952232$
$6 \cdot 0835106$
$5 \cdot 3770562$
$4 \cdot 9905805$
$4 \cdot 7421805$
$4 \cdot 5676632$
$4 \cdot 4380276$
$4 \cdot 3379129$
$4 \cdot 2583277$
$4 \cdot 1401003$
$4 \cdot 0242933$
$3 \cdot 9143941$
$3 \cdot 8559364$
$3 \cdot 8246438$
$3 \cdot 8066188$
$3 \cdot 8224358$
$3 \cdot 8812236$
$3 \cdot 9640972$
$4 \cdot 0929499$
$4 \cdot 4260577$
$4 \cdot 7107406$
$5 \cdot 1061260$
$5 \cdot 6066791$
$5 \cdot 3462881$

$h_{3}=90$ kilometers

$6.5777425=2$
$3.8922467=2$
$1.6214498=2$
$1.6892888=2$
$2.4267300=2$
$3.1866282=2$
$3.8802200=2$
$4.4971690=2$
$5.0420380=2$
$5.5231110=2$
$5.9490760=2$
$6.6665265=2$
$7.4954520=2$
$8.4693115=2$
$9.1560560=2$
$9.6858945=2$
$1.0510343=1$
$1.1199139=1$
$1.2173873=1$
$1.3181427=1$
$1.4644492=1$
$1.8917724=1$
$2.3717183=1$
$3.3200360=1$
$5.2773590=1$
$4.1270083=1$

$8.0531770=2$

$4.8523991-2$

$2.0524326=2$

$1.9001374=2$

$2.7182395=2$

$3.6298982-2$

$4.4758840=2$

$5.2318040=2$

$5.9000720=2$

$6.4899435=2$

$7.0117965=2$

$7.8892220=2$

$8.8992210=2$

$1.0076531=1$

$1.0895410=1$

$1.1515999-1$

$1.2451421=1$

$1.3197485=1$

$1.4202258-1$

$1.5198133=1$

$1.6603214=1$

$2.0612961=1$

$2.5094024-1$

$3.4041043=1$

$4.1776108=1$

$5.2969465=1$

$3 \cdot 4016496$
$2 \cdot 8863068$
$1 \cdot 6334352$
$2 \cdot 3404261-1$
$5 \cdot 7470439$
$5 \cdot 3377348$
$5 \cdot 0810728$
$4 \cdot 9032647$
$4 \cdot 7722274$
$4 \cdot 6714825$
$4 \cdot 5915758$
$4 \cdot 4729497$
$4 \cdot 3563577$
$4 \cdot 2440052$
$4 \cdot 1817412$
$4 \cdot 1454973$
$4 \cdot 1247771$
$4 \cdot 1150306$
$4 \cdot 1470279$
$4 \cdot 2012562$
$4 \cdot 2924451$
$4 \cdot 5485989$
$4 \cdot 7850196$
$5 \cdot 1359579$
$5 \cdot 3601251$
$5 \cdot 6107129$
4.67

1. 5702963

1. 5697963

I. 5687963

1. 5677963

1. 5667963

1.5657963

1. 5647963

1.5637963

1.5627963

1. 5617963

1. 5607963

I. 5587963

I. 5557963

1.5507963

. 5457963

1. 5407963

1. 5307963

1.5207963

1. 5057963

I. 4907963

1.4707963

1. 4207963

1. 3707963

1. 2707963

1. 1707963

$9.7079630-1$ $\mathrm{d} / \mathrm{j}$

miles

Table 91. Effective reflection coefficient (amplitude, $\left|C_{j}\right|$, and phase, Arg $C_{j}$ ) for the special ray configuration of the Norton type 301 atmosphere, applicable to the propagation of waves between the D-region ( $h_{3}=70$ kilometers) or the E-region ( $b_{3}=90$ kilometers $)$ of the ionosphere and the earth.

1.2433895 1.2381675 1. 2278386 1.2176623 1.2076369 $1 \cdot 1977599$ $1 \cdot 1880278$ 1.1784374 I. 1689853 I. 1596669 $2 \cdot 1504788$ 1. 1324770 I. 1063547 1.0649462 1. 0259098 9.8899038 9.2076906 8.5914709 7. 7737068 7.0657786 $6 \cdot 2644905$ 4. 7915900 3.8174547 2.6471062 1.980295 1. 2445355

1.4000780 1.3948535

1. 3845147

1.3743220 1. 3642736

1.3543670

1. 3445989 1. 3349661 1. 3254647 1. 3160909 1.3068406 1.2886954 1. 2623103 1. 2203378 I. 1805854 1. 1428039 1. 0724503 1.0082107 9.2176798 8. 4566114 7.5787071 5.9120543 4. 7678510 3.3479623 2. 5187906 1. 5902204 


$$
\begin{array}{ll}
\omega / \omega_{\mathbf{r}}=0.0200 & \mathrm{f}=1.332425 \text { kilocycles } \\
\phi_{1}=60 \text { degrees } & \sigma=0.005 \text { mhos } / \text { meter } \\
& \epsilon_{2}=15
\end{array}
$$

$\Psi$

radians

$\begin{array}{ll}0.0005 & 4.5248830=2 \\ 0.0010 & 2.8847643=2 \\ 0.0020 & 1.2627423=2 \\ 0.0030 & 1.2779985=2 \\ 0.0040 & 1.7170239=2 \\ 0.0050 & 2.1218687=2 \\ 0.0060 & 2.4747229=2 \\ 0.0070 & 2.7900227=2 \\ 0.0080 & 3.0766138=2 \\ 0.0090 & 3.3391132=2 \\ 0.0100 & 3.5803169=2 \\ 0.0120 & 4.0068931=2 \\ 0.0150 & 4.5320980=2 \\ 0.0200 & 5.1892655=2 \\ 0.0250 & 5.6714475=2 \\ 0.0300 & 6.0472985=2 \\ 0.0400 & 6.6231860=2 \\ 0.0500 & 7.0846155=2 \\ 0.0650 & 7.7076675=2 \\ 0.0800 & 8.3341975=2 \\ 0.1000 & 9.2449600=2 \\ 0.1500 & 1.2026449=1 \\ 0.2000 & 1.5403942=1 \\ 0.3000 & 2.2856666=1 \\ 0.4000 & 2.9978495=1 \\ 0.6000 & 4.1268579=1\end{array}$

$\operatorname{Arg~C} 3$

$\mathrm{h}_{3}=70$ kilometers
$T_{j}$

1.5702963

1.5697963

1. 5687963

1.5677963

1.5667963

1. 5657963

1.5647963

1.5637963

1. 5627963

1.5617963

1.5607963

1. 5587963

1. 5557963

1. 5507963

1.5457963

1.5407963

1.5307963

1. 5207963

1.5057963

1. 4907963

1.4 .707963

1.4207963

1.3707963

1.2707963

1. 1707963

$9.7079630-1$ $\mathrm{d} / \mathrm{j}$

miles

1.2433895

1. 2381675

1. 2278386

1.2176623

1.2076369

1. 1977599

1. 1880278

101784374

1. 1689853

1. 1596669

1. 1504788

1. 1324770

1. 1063547

1. 0649462

1.0259098

9. 8899038

9.2076906

8.5914709

7. 7737068

7.0657786

6.2644905

4. 7915900

3.8174547

2.6471062

1.9802951

1.2445355

$h_{3}=90$ kilometers

0.0005

0.0010

0.0020

0.0030

0.0040

0.0050

0.0060

0.0070

0.0080

0.0090

0.0100

0.0120

0.0150

0.0200

0.0250

0.0300

0.0400

0.0500

0.0650

0.0800

0.1000

0.1500

0.2000

0.3000

0.4000

0.6000

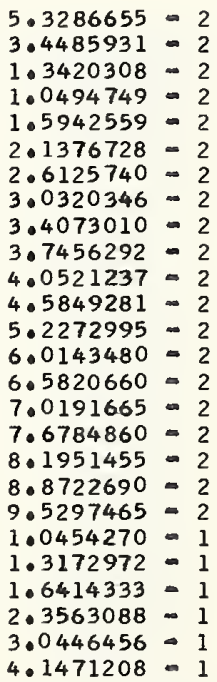

2.4969245

2.1452983

1. 1895169

5.9549224

5. 1425291

4. 7274843

4.4512621

$4 \cdot 2471683$

4.0888146

3.9622547

3. 8589141

3.7008608

3. 5404771

3.3815096

3. 2913557

3. 2377694

3.1899984

3. 1865250

3. 2266492

3.2987580

3. 4224237

3.7760928

4. 1081369

4.6091154

4. 9326305

5. 2957012

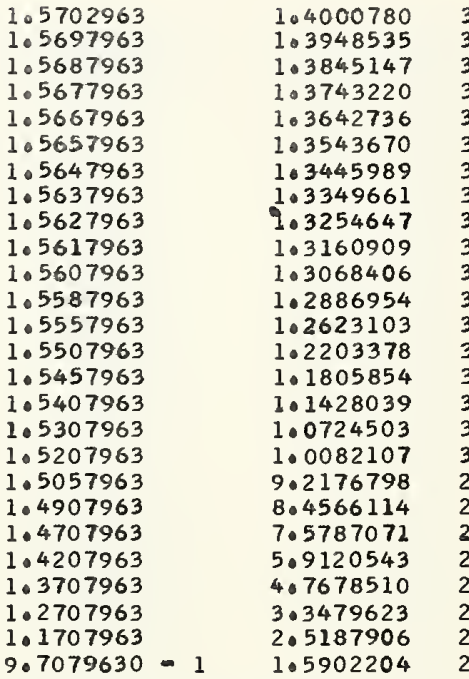

Table 92. Effective reflection coefficient (amplitude, $\left|C_{j}\right|$, and phase, $A r g C_{j}$ ) for the special ray configuration of the Norton type 301 atmosphere, applicable to the propagation of waves between the $\mathrm{D}$-region $\left(\mathrm{h}_{3}=70\right.$ kilometers) or the $\mathrm{E}$-region $\left(\mathrm{h}_{3}=90\right.$ kilometers $)$ of the ionosphere and the earth. 


$$
\begin{array}{ll}
\omega / \omega_{r}=0.0500 & f=3.3311126 \mathrm{kilocycles} \\
\phi_{1}=60 \text { degrees } & \sigma=0.005 \mathrm{mhos} / \text { meter } \\
& \epsilon_{2}=15
\end{array}
$$

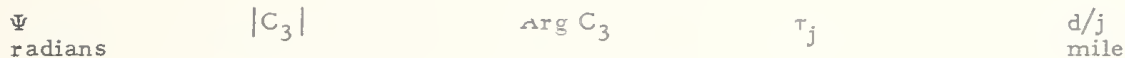

$\mathrm{h}_{3}=70$ kilometers

$\begin{array}{ll}0.0005 & 5.6602185=2 \\ 0.0010 & 4.5816115=2 \\ 0.0020 & 3.3752791=2 \\ 0.0030 & 2.9424262=2 \\ 0.0040 & 2.8167683=2 \\ 0.0050 & 2.7726474=2 \\ 0.0060 & 2.7414671=2 \\ 0.0070 & 2.7132253=2 \\ 0.0080 & 2.6929329=2 \\ 0.0090 & 2.686555=2 \\ 0.0100 & 2.6974993=2 \\ 0.0120 & 2.7720246=2 \\ 0.0150 & 2.9826112=2 \\ 0.0200 & 3.4322372=2 \\ 0.0250 & 3.8615289=2 \\ 0.0300 & 4.2233627=2 \\ 0.0400 & 4.7549512=2 \\ 0.0500 & 5.1027100=2 \\ 0.0650 & 5.4317310=2 \\ 0.0800 & 5.6613640=2 \\ 0.1000 & 5.9642045=2 \\ 0.1500 & 7.2096950=2 \\ 0.2000 & 9.1700315=2 \\ 0.3000 & 1.3935147=1 \\ 0.4000 & 1.8781455=1 \\ 0.6000 & 2.7335982=1\end{array}$

0.2023865
5.8907445
5.2448623
4.6604737
4.1966458
3.8333597
3.5360970
3.2814230
3.0562511
2.8537972
2.6706839
2.3560729
1.9965130
1.6158444
1.3980017
1.2686529
1.1477355
1.1240628
1.1838014
1.3103010
1.5367293
2.1788720
2.7375299
3.5207699
4.0223013
4.6038604

1.5702963

1.5697963

2. 5687963

1.5677963

1.5667963

3. 5657963

1.5647963

1.5637963

1.5627963

1.5617963

1.5607963

1.5587963

1.5557963

1.5507963

1.5457963

?. 5407963

1.5307963

i. 5207963

1.5057963

1.4907963

1.4707963

1.4207963

1.3707963

1.2707963

1.1707963

$9.7079630-1$

1. 2433895 1.2381675 1. 2278385 1.2176623 1.2076369 1. 1977599 1.1880278 1.1784374 1.1689853 1.1596669 1.1504788 1.1324770 1. 1063547 1.0649462 1.0259098 9.8899038 9.2076906 8.5914709 7.7737068 7.0657786 6.2644905 4.7915900 3.8174547 2.6471062 1.9802951

4.6038604

$$
10244535
$$

$\mathrm{h}_{3}=90$ kilometers

0.0005
0.0010
0.0020
0.0030
0.0040
0.0050
0.0060
0.0070
0.0080
0.0090
0.0100
0.0120
0.0150
0.0200
0.0250
0.0300
0.0400
0.0500
0.0650
0.0800
0.1000
0.1500
0.2000
0.3000
0.4000
0.6000

$4.4660367=2$
$3.4642047=2$
$2.4635486=2$
$2.3224547=2$
$2.4570666=2$
$2.6072186=2$
$2.7203856=2$
$2.8013075=2$
$2.8633238=2$
$2.9172558=2$
$2.9699774=2$
$3.0844472=2$
$3.2858113=2$
$3.6551680=2$
$4.0047055=2$
$4.3094492=2$
$4.7907071=2$
$5.1484390=2$
$5.5571725=2$
$5.9019250=2$
$6.3610930=2$
$7.8286495=2$
$9.7905035=2$
$1.4398823=1$
$1.9110126=1$
$2.7500358=1$

$4.5702092-1$
$1.1445666-1$
5.6228756
4.9275693
4.4345362
4.0813790
3.8069382
3.5796100
3.3837784
3.2113310
3.0577069
2.7965085
2.4956445
2.1627569
1.9596830
1.8318636
1.6998847
1.5577133
1.6822946
1.7669077
1.9306879
2.4228231
2.8836537
3.5814231
4.0520192
4.6131504

1.5702963

1.5697963

1. 5687963

1.5677963

1.5667963

1.5657963

1.5647963

1. 5637963

1.5627963

1.5617963

1. 5607963

1. 5587963

1.5557963

1.5507963

1.5457963

1.5407963

1. 5307963

1.5207963

1.5057963

1.4907963

1.4707963

1.4207963

1. 3707963

1. 2707963

1.1707963

$9.7079630-1$

$\begin{array}{ll}1.4000780 & 3 \\ 1.3948535 & 3 \\ 1.3845147 & 3 \\ 1.3743220 & 3 \\ 1.3642736 & 3 \\ 1.3543670 & 3 \\ 1.3445989 & 3 \\ 1.3349661 & 3 \\ 1.3254647 & 3 \\ 1.3160909 & 3 \\ 1.3068406 & 3 \\ 1.2886954 & 3 \\ 1.2623103 & 3 \\ 1.2203378 & 3 \\ 1.1805854 & 3 \\ 1.1428039 & 3 \\ 1.0724503 & 3 \\ 1.0082107 & 3 \\ 9.2176798 & 2 \\ 8.4566114 & 2 \\ 7.5787071 & 2 \\ 5.9120543 & 2 \\ 4.7678510 & 2 \\ 3.3479623 & 2 \\ 2.5187906 & 2 \\ 1.5902204 & 2\end{array}$

Table 93. Effective reflection coefficient (amplitude, $\left|C_{j}\right|$, and phase, Arg $C_{j}$ ) for the special ray configuration of the Norton type 301 atmosphere, applicable to the propagation of waves between the D-region $\left(h_{3}=70\right.$ kilometers $)$ or the E-region $\left(h_{3}=90\right.$ kilometers $)$ of the ionosphere and the parth. 


$$
\begin{array}{ll}
\omega / \omega_{r}=0.1000 & f=6.6622252 \mathrm{kilocycles} \\
\phi_{1}=60 \text { degrees } & \sigma=0.005 \mathrm{mhos} / \mathrm{meter} \\
& \epsilon_{2}=15
\end{array}
$$

$\begin{array}{lllll}\Psi & \left|C_{3}\right| & \mathrm{ArgC}_{3} & \mathrm{~T}_{j} & \mathrm{~d} / \mathrm{j} \\ \text { radians } & & & \mathrm{miles}\end{array}$

radians

0.0005
0.0010
0.0020
0.0030
0.0040
0.0050
0.0060
0.0070
0.0080
0.0090
0.0100
0.0120
0.0150
0.0200
0.0250
0.0300
0.0400
0.0500
0.0650
0.0800
0.1000
0.1500
0.2000
0.3000
0.4000
0.6000

0.0005

0.0010

0.0020

0.0030

0.0040

0.0050

0.0060

0.0070

0.0080

0.0090

0.0100

0.0120

0.0150

0.0200

0.0250

0.0300

0.0400

0.0500

0.0650

0.0800

0.1000

0.1500

0.2000

0.3000

0.4000

0.6000 $\mathrm{h}_{3}=70$ kilometers

$1.1316033=1$
$9.9012465=2$
$7.8084830=2$
$6.4346985=2$
$5.5227850=2$
$4.8901384=2$
$4.4217553=2$
$4.0528992=2$
$3.7508233=2$
$3.5009392=2$
$3.2980000=2$
$3.0293131=2$
$2.9522317=2$
$3.4049821=2$
$4.0862643=2$
$4.7301313=2$
$5.7056950=2$
$6.3026575=2$
$6.7092615=2$
$6.7547420=2$
$6.5377770=2$
$5.9782795=2$
$6.5831900=2$
$1.0148242=1$
$1.4005731=1$
$2.0124475=1$

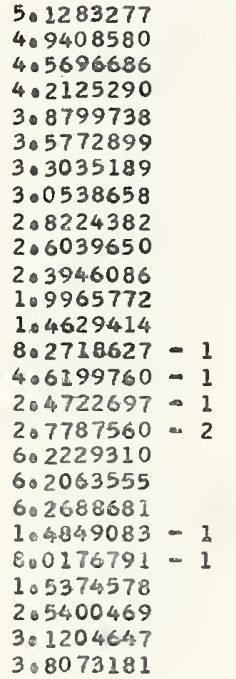

1.5702963

1.5697963

1.5687963

1.5677963

1.5667963

1.5657963

1.5647963

1. 5637963

1.5627963

1.5617963

1.5607963

1.5587963

1.5557963

1.5507963

105457963

1.5407963

1.5307963

1. 5207963

1.5057963

1.4907963

1.04707963

1.4207953

1.3707963

1.2707953

101707963

$907079630=1$

$\begin{array}{ll}1.2433895 & 3 \\ 1.2381675 & 3 \\ 1.2278386 & 3 \\ 102176623 & 3 \\ 102076369 & 3 \\ 1.1977599 & 3 \\ 1.1880278 & 3 \\ 101784374 & 3 \\ 101689853 & 3 \\ 101596669 & 3 \\ 101504788 & 3 \\ 1.1324770 & 3 \\ 1.1063547 & 3 \\ 1.0649462 & 3 \\ 100259098 & 3 \\ 908899038 & 2 \\ 902076906 & 2 \\ 3.5914709 & 2 \\ 707737068 & 2 \\ 7.0657786 & 2 \\ 6.2644905 & 2 \\ 407915900 & 2 \\ 308174547 & 2 \\ 206471062 & 2 \\ 109802951 & 2 \\ 1.2445355 & 2\end{array}$

$\mathrm{h}_{3}=90$ kilometers

5.4109377
5.2056095
407996344
404175828
400755975
307777155
305188499
3.2907522
300855626
208970324
$207206250=$
$203933642=$
1.9529125
$103725572=$
$909087005=1$
$705026359=1$
$409415308=1$
$308496538=1$
$304925340=1$
$309825664=1$
$504378793=1$
$1.1204944=$
107381185
206123410
3.1546232
308188617

$\begin{array}{ll}1.5702963 & 1.4000780 \\ 1.5697963 & 1.3948535 \\ 1.5687963 & 1.3845147 \\ 1.5677963 & 1.3743220 \\ 1.5667963 & 1.3642736 \\ 1.5657963 & 1.3543670 \\ 1.5647963 & 1.3445989 \\ 1.5637963 & 1.334961 \\ 1.5627963 & 1.3254647 \\ 1.5617963 & 1.3160909 \\ 1.5607963 & 1.3068406 \\ 1.5587963 & 1.2886954 \\ 1.5557963 & 1.2623103 \\ 1.5507963 & 1.2203378 \\ 1.5457963 & 1.1805854 \\ 1.5407963 & 1.1428039 \\ 1.5307963 & 1.0724503 \\ 1.5207963 & 1.0082107 \\ 1.5057963 & 9.2176798 \\ 1.5907963 & 8.4566114 \\ 1.4707963 & 7.5787071 \\ 1.4207963 & 5.9120543 \\ 1.3707963 & 4.7678510 \\ 1.2707963 & 3.3479623 \\ 1.1707963 & 2.5187906 \\ 9.7079630 & 1.5902204\end{array}$

Table 94. Effective reflection coefficient (amplitude, $\left|C_{j}\right|$, and phase, $A r g C_{j}$ ) for the special ray configuration of the Norton type 301 atmosphere, applicable to the propagation of waves between the $\mathrm{D}$-region $\left(\mathrm{h}_{3}=70\right.$ kilometers) or the $E$-region $\left(\mathrm{h}_{3}=90\right.$ kilometers $)$ of the ionosphere and the earth. 


$$
\begin{array}{ll}
\omega / \omega_{T}=0.2000 & f=13.324450 \text { kilocycles } \\
\phi_{1}=60 \text { degrees } & \sigma=0.005 \text { mhos } / \text { meter } \\
\epsilon_{2}=15
\end{array}
$$

$\Psi$

radians

0.0005

0.0020

0.0030

0.0040

0.0050

0.0060

0.0070

0.0080

0.0090

0.0100

0.0120

0.0150

0.0200

0.0250

0.0300

0.0400

0.0500

0.0650

0.0800

0.1000

0.1500

0.2000

0.3000

0.4000

0.6000

0.0005

0.0010

0.0020

0.0030

0.0040

0.0050

0.0060

0.0070

0.0080

0.0090

0.0100

0.0120

0.0150

0.0200

0.0250

0.0300

0.0400

0.0500

0.0650

0.0800

0.1000

0.1500

0.2000

0.3000

0.4000

0.6000

$$
\left|c_{3}\right|
$$

Arg $\mathrm{C}_{3}$

$h_{3}=70$ kilometers

$2.0260933=1$
$1.835595=1$
$1.5200754=1$
$1.2765579=1$
$1.0888510=1$
$9.4348410=2$
$8.2970340=2$
$7.3926835=2$
$6.6612735=2$
$6.0601805=2$
$5.5607035=2$
$4.8000980=2$
$4.1465947=2$
$4.1258418=2$
$4.8854146=2$
$5.8504060=2$
$7.5800000=2$
$8.8119385=2$
$9.8614275=2$
$1.0232382=1$
$1.0067636=1$
$8.2904815=2$
$6.6487070=2$
$7.4195865=2$
$1.0666899=1$
$1.5703302=1$

4. 3024865

4. 0590610

3. 8188703

3. 5833638

3.3542055

3. 1327367

2.9195804

2. 7145219

2. 5166300

2. 3245229

1.9518587

1.4106238

$6.1933278-1$

8.4215010 - 2

6.0391413

5.6887347

5. 5194969

5.4089674

5.3853219

5.4296958

$5 \cdot 7688585$

$6.4878910=$

1.3426337

2.0607500

2.7832887

$h_{3}=90$ kilometers

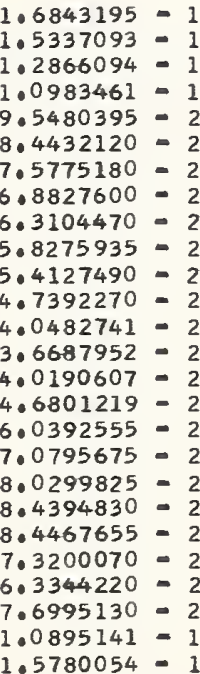

\section{5916611}

4.4638572

4. 2111696

3. 9642417

3.7257083

3.4979273

3. 2823483

3.0792638

2. 8879263

2.7068783

2. 5342826

2. 2069891

1. 7397849

1.0164131

$4.5850002-1$

9.1778410 - 2

5.9785965

5.7883631

5.6622910

5.6300817

5.6676751

6.0018781

2.8623306 - 1

1.4410126

2.0994561

2. 7951215 $\tau_{j}$

$\mathrm{d} / \mathrm{j}$

miles

1.2433895

1.5702963

1.5697963

1. 5687963

1.5677963

1. 5667963

1.5657963

1.5647963

1.5637963

1.5627963

1. 5617963

1. 5607963

1.5587963

1. 5557963

1. 5507963

1.5457963

1. 5407963

1. 5307963

1.5207963

1.5057963

1.4907963

1.4707963

1.4207963

1. 3707963

1.2707963

1. 1707963

1. 2381675

1.2278386

1.2176623

1.2076369

1.1977599

1. 1880278

1.1784374

I. 1589853

1. 1596669

1. 1504788

1.1324770

1.1063547

1.0649462

1.0259098

9.8999038

9.2076906

8.5914709

7.7737068

7.0657786

6.2644905

4.7915900

3. 8174547

2. 5471062

1.9802951

1.2445355

9.7079630

1. 5702963

1. 5597963

1. 5687963

1.5577963

1. 5567963

1.5557963

1.5547963

1.5637963

1.5627963

1.5617963

1. 5607963

I. 5587963

2. 5557963

1. 5507963

1.5457963

1.5407963

1. 5307963

1.5207963

1.5057963

1.4907963

1.4707963

1.4207963

1.3707963

1.2707963

1.1707963

$9.7079630-1$
1. 4000780

1.3948535

1. 3845147

1.3743220

1.3642736

1.3543670

1.3445989

1. 3349661

1.3254647

1. 3160909

1.3068406

1.2886954

1.2623103

1. 2203378

1.1805854

1.1428039

1.0724503

1.0082107

9.2176798

8.4566114

7.5787071

5.9120543

4.7678510

3.3479623

2.5187906

1. 5902204

Table 95. Effective reflection coefficient (amplitude, $\left|C_{j}\right|$, and phase, Arg $C_{j}$ ) for the specia ray configuration of the Norton type 301 atmosphere, applicable to the propagation of waves between the D-region ( $h_{3}=70$ kilometers) or the E-region ( $h_{3}=90$ kilometers) of the ionosphere and the earth. 


$$
\begin{array}{ll}
\omega / \omega_{\mathbf{r}}=1.0000 & \mathrm{f}=66.622252 \text { kilocycles } \\
\phi_{1}=60 \text { degrees } & \sigma^{-}=0.005 \mathrm{mhos} / \text { meter } \\
\epsilon_{2}=15
\end{array}
$$

$\begin{array}{lllll}\Psi & \left|\mathrm{C}_{3}\right| & \mathrm{Arg} \mathrm{C}_{3} & \mathrm{~T}_{\mathrm{j}} & \mathrm{d} / \mathrm{j} \\ \text { radians } & & \text { miles }\end{array}$

$\mathrm{h}_{3}=70$ kilometers

$\begin{array}{ll}0.0005 & 4.2439736=1 \\ 0.0010 & 4.0465671=1 \\ 0.0020 & 3.6821745=1 \\ 0.0030 & 3.3554395=1 \\ 0.0040 & 3.0629927=1 \\ 0.0050 & 2.8016309=1 \\ 0.0060 & 2.5683308=1 \\ 0.0070 & 2.3602782=1 \\ 0.0080 & 2.1748635=1 \\ 0.0090 & 2.0096844=1 \\ 0.0100 & 1.8625523=1 \\ 0.0120 & 1.6146595=1 \\ 0.0150 & 1.3345499=1 \\ 0.0200 & 1.0346125=1 \\ 0.0250 & 8.6802580=2 \\ 0.0300 & 7.9156625=2 \\ 0.0400 & 8.1962555=2 \\ 0.0500 & 9.6704015=2 \\ 0.0650 & 1.2203124=1 \\ 0.0800 & 1.4274791=1 \\ 0.1000 & 1.6076632=1 \\ 0.1500 & 1.6993552=1 \\ 0.2000 & 1.5250862=1 \\ 0.3000 & 1.0050493=1 \\ 0.4000 & 5.9653825=2 \\ 0.6000 & 3.2037566=2\end{array}$

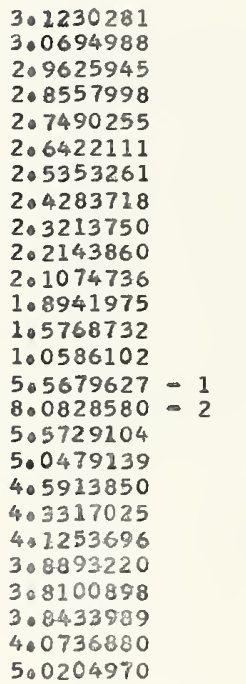

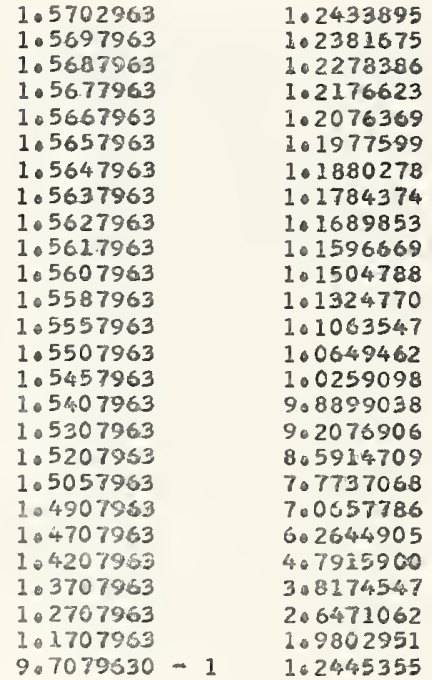

$h_{3}=90$ kilometers

$\begin{array}{ll}0.0005 & 3.8215421=1 \\ 0.0010 & 3.6482359=1 \\ 0.0020 & 3.3284732=1 \\ 0.0030 & 3.0419090=1 \\ 0.0040 & 2.7855226=1 \\ 0.0050 & 2.5564333=1 \\ 0.0060 & 2.3519322=1 \\ 0.0070 & 2.1694944=1 \\ 0.0080 & 2.0067831=1 \\ 0.0090 & 1.8616482=1 \\ 0.0100 & 1.7321368=1 \\ 0.0120 & 1.5130684=1 \\ 0.0150 & 1.2629644=1 \\ 0.0200 & 9.8764850=2 \\ 0.0250 & 8.2500605=2 \\ 0.0300 & 7.3997790=2 \\ 0.0400 & 7.3683180=2 \\ 0.0500 & 8.5618345=2 \\ 0.0650 & 1.0811837=1 \\ 0.0800 & 1.2722157=1 \\ 0.1000 & 1.4442391=1 \\ 0.1500 & 1.5524619=1 \\ 0.2000 & 1.4117148=1 \\ 0.3000 & 9.4820375=2 \\ 0.4000 & 5.7239865=2 \\ 0.6000 & 3.1954208=2\end{array}$

\begin{tabular}{|c|c|}
\hline $\begin{array}{l}1.5702903 \\
1.5697963\end{array}$ & $\begin{array}{l}1.4000760 \\
1.3948535\end{array}$ \\
\hline 1.5687963 & 103845147 \\
\hline 1.5677963 & 1.3743220 \\
\hline I. 5667963 & 1.3642736 \\
\hline 1.5657963 & 1.3543670 \\
\hline 1.5647963 & 1.3445989 \\
\hline 1.5637963 & $1 \cdot 3349661$ \\
\hline 1.5627963 & 1.3254647 \\
\hline 1.5617963 & 1.3160909 \\
\hline 1.5607963 & 1.3068406 \\
\hline 1.5587963 & 1.2886954 \\
\hline 1.5557963 & 1.2623103 \\
\hline 1.5507963 & 1.2203378 \\
\hline 105457963 & 1.1805854 \\
\hline 1.5407963 & 1.1428039 \\
\hline 1.5307963 & 1.0724503 \\
\hline 1.5207963 & 1.0082107 \\
\hline 1.5057963 & 9.2176798 \\
\hline 1.4907963 & 8.4566114 \\
\hline 1.4707963 & 7.5787071 \\
\hline 1.4207963 & 5.9120543 \\
\hline 1.3707963 & 4.7678510 \\
\hline 1.2707963 & $3 \cdot 3479623$ \\
\hline 1. 1707963 & 2.5187906 \\
\hline 9.7079630 & $1 \cdot 5902204$ \\
\hline
\end{tabular}

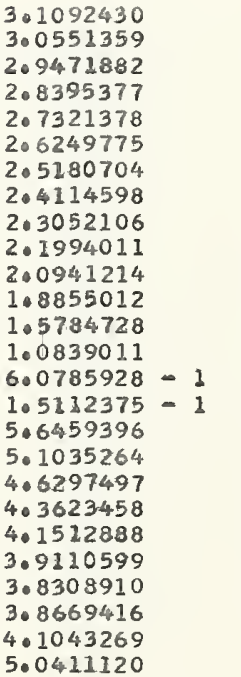

Table 96. Effective reflection coefficient (amplitude, $\left|C_{j}\right|$, and phase, Arg $C_{j}$ ) for the special ray configuration of the Norton type 301 atmosphere, applicable to the propagation of waves between the $\mathrm{D}$-region ( $\mathrm{h}_{3}=70$ Kilometers) or the E-region $\left(\mathrm{h}_{3}=90\right.$ kilometers) of the ionosphere and the earth. 


$$
\begin{array}{ll}
\omega / \omega_{r}=2.0000 & f=133.24450 \text { kilocycles } \\
\phi_{1}=60 \text { degrees } & \sigma=0.005 \text { mhos } / \text { meter } \\
\epsilon_{2}=15
\end{array}
$$

$\Psi$

radians $\left|c_{3}\right|$

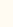

$\mathrm{Arg} \mathrm{C}_{3}$

$h_{3}=70$ kilometers

$3.8819880=1$
$3.7591308=1$
$3.5268024=1$
$3 \cdot 3114182=1$
$3.1119118=1$
$2.9272381=1$
$2.7563956=1$
$2 \cdot 5984095=1$
$2.4523548=1$
$2.3173496=1$
$2.1925577=1$
$1.9705063=1$
$1.6958431=1$
$1.3558399=1$
$1.1177398=1$
$9.4776675=2$
$7.4506100=2$
$6.7634660=2$
$7.3023840=2$
$8.4991070=2$
$9.9353170=2$
$1.1200356=1$
$1.0183619=1$
$6.3045390=2$
$3.0509712=2$
$1.8703981=3$

1.5702963 1.5697963 1.5687963 1.5677963

1.5667963

1. 5657963

1.5647963

1.5637963

1.5627963

1. 5617963

1. 5607963

1. 5587963

1. 5557963

1. 5507963

1. 5457963

1. 5407963

1. 5307963

1.5207963

1.5057963

1.4907963

1.4707963

1.4207963

1.3707963

1. 2707963

1. 1707963

$9.7079630-1$ $\mathrm{d} / \mathrm{j}$

miles
1.2433895 1.2381675 1. 2278386 1.2176623 1. 2076369 1. 1977599 1. 1880278 1. 1784374 1. 1689853 1.1596669 1. 1504788 1. 1324770 1. 1063547 1.0649462 1.0259098 9.8899038 9.2076906 8. 5914709 7.7737068 7.0657786 6.2644905 4.7915900 3.8174547 2.6471062 1. 9802951 1. 2445355

$\mathrm{h}_{3}=90$ kilometers

$\begin{array}{ll}0.0005 & 3.4635264=1 \\ 0.0010 & 3.3583501=1 \\ 0.0020 & 3.1594610=1 \\ 0.0030 & 2.9750597=1 \\ 0.0040 & 2.8042072=1 \\ 0.0050 & 2.6459865=1 \\ 0.0060 & 2.4995233=1 \\ 0.0070 & 2.3639435=1 \\ 0.0080 & 2.2384647=1 \\ 0.0090 & 2.1223078=1 \\ 0.0100 & 2.0147485=1 \\ 0.0120 & 1.8227272=1 \\ 0.0150 & 1.5834783=1 \\ 0.0200 & 1.2824697=1 \\ 0.0250 & 1.0656622=1 \\ 0.0300 & 9.0526465=2 \\ 0.0400 & 6.9995875=2 \\ 0.0500 & 6.1281450=2 \\ 0.0650 & 6.3692590=2 \\ 0.0800 & 7.3806130=2 \\ 0.1000 & 8.7043590=2 \\ 0.1500 & 1.0037401=1 \\ 0.2000 & 9.2713285=2 \\ 0.3000 & 5.8457085=2 \\ 0.4000 & 2.8457209=2 \\ 0.6000 & 1.6947492=3 \\ & \end{array}$

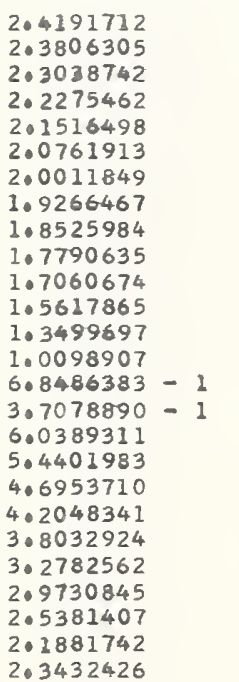

$\begin{array}{ll}1.5702963 & 1.4000780 \\ 1.5697963 & 1.3948535 \\ 1.5687963 & 1.3845147 \\ 1.5677963 & 1.3743220 \\ 1.5667963 & 1.3642736 \\ 1.5657963 & 1.3543670 \\ 1.5647963 & 1.3445989 \\ 1.5637963 & 1.3349661 \\ 1.5627963 & 1.3254647 \\ 1.5617963 & 1.3160909 \\ 1.5607963 & 1.3068406 \\ 1.5587963 & 1.2886954 \\ 1.5557963 & 1.2623103 \\ 1.5507963 & 1.2203378 \\ 1.5457963 & 1.1805854 \\ 1.5407963 & 1.1428039 \\ 1.5307963 & 1.0724503 \\ 1.5207963 & 1.0082107 \\ 1.5057963 & 9.2176798 \\ 1.4907963 & 8.4566114 \\ 1.4707963 & 7.5787071 \\ 1.4207963 & 5.9120543 \\ 1.3707963 & 4.7678510 \\ 1.2707963 & 3.3479623 \\ 1.1707963 & 2.5187906 \\ 9.7079630 & 1.5902204\end{array}$

Table 97. Effective reflection coefficient (amplitude, $\left|C_{j}\right|$, and phase, Arg $C_{j}$ ) for the special ray configuration of the Norton type 301 atmosphere, applicable to the propagation of waves between the $D$-region ( $h_{3}=70$ kilometers) or the E-region ( $h_{3}=90$ kilometers) of the ionosphere and the earth. 


$$
\begin{array}{ll}
\omega / \omega_{\mathrm{r}}=5.0000 & f=333.11126 \mathrm{kilocycles} \\
\phi_{1}=60 \text { degrees } & \sigma=0.005 \mathrm{mhos} / \mathrm{meter}
\end{array}
$$

$\Psi$

radians

$\left|C_{3}\right|$

$\operatorname{Arg~C}_{3}$

$\mathrm{h}_{3}=70$ kilometers

0.0005

0.0020

0.0030

0.0040

0.0050

0.0060

0.0070

0.0080

0.0090

0.0100

0.0120

0.0150

0.0200

0.0250

0.0300

0.0400

0.0500

0.0650

0.0800

0.1000

0.1500

0.2000

0.3000

0.4000

0.6000

0.0005
0.0010
0.0020
0.0030
0.0040
0.0050
0.0060
0.0070
0.0080
0.0090
0.0100
0.0120
0.0150
0.0200
0.0250
0.0300
0.0400
0.0500
0.0650
0.0800
0.1000
0.1500
0.2000
0.3000
0.4000
0.6000

1.6496167
1.6243195
1.5739590
1.5239173
1.4742076
1.4248384
1.3758218
1.3271686
1.2788885
1.2309922
1.1834882
$1.0896996-$
$9.5217951-1$
$7.3177006-1$
$5.2247994-1$
$3.2383681-1$
6.2370746
5.0930386
5.3978728
4.9043436
4.2613832
3.0635788
2.3248020
1.2038181
$1.7203994-1$
3.1177098

$2.8026467=1$ $2.7557242-1$

$2.6650987-1$

$2.5786079=1$

$2.4960785=1$

$2.4173389-1$

$2.3422111-1$

$2.2705309=1$

$2.2021271-1$

$2.1368428=1$

$2.0745196=1$

$1.9581537=1$

$1.8021946=1$

$1.5834456=1$

$1.4048190=1$

$1.0211422=1$

$8.4231870=2$

$6.4566055=2$

$5.1657000-2$

$4.2743070=2$

$3.8290743-2$

$3.3245770=2$

$1.6162227=2$

$4.3527157=3$

$1.2044627=4$

$\mathrm{h}_{3}=90$ kilometers

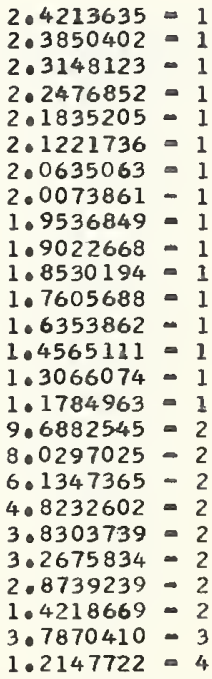

1. 3851331

1.3598830

1. 3097786

1. 2601574

1. 2110331

1.1624115

1.1143019

1.0667123

1.0196537

9.7312455 - 1

$9.2713558-1$

8.3678662 - 1

$7.0535170-1$

$4.9707770-1$

$3.0163179-1$

1.1778072 - 1

6.0614917

5.7477447

5. 2968141

4.8428189

4. 2291196

3.0053424

2. 2508860

1. 1329103

1.1715968 - 1

3.0650052
$1.2561296=1$
${ }^{\top} \mathrm{j}$

$d / j$

miles

1. 5702963

1.5697963

1. 5687963

1.5677963

1. 5667963

1.5657963

1. 5647963

1.5637963

1. 5627963

1.5617963

1.5607963

1.5587963

1.5557963

1. 5507963

1.5457963

1. 5407953

I. 5307963

1.5207963

1.5057963

1.4907963

1.4707963

1.4207963

1.3707963

1. 2707963

1. 1707953

$9.7079630-1$

1.2433895

1.2381675

1. 2278386

1.2176623

1.2076369

1. 1977599

1.1880278

1.1784374

1. 1689853

1. 1596669

1. 1504788

1. 1324770

1.1063547

1.0649462

1.0259098

9.8899038

9.2076906

8.5914709

7.7737068

7.0657786

6.2644905

4.7915900

308174547

206471062

1. 9802951

102445355

3
3
3
3
3
3
3
3
3
3
3
3
2
2
2
2
2

\begin{tabular}{|c|c|}
\hline $\begin{array}{l}1 \cdot 4000780 \\
1 \cdot 3948535\end{array}$ & \\
\hline $\begin{array}{l}1 \cdot 3845147 \\
1 \cdot 3743220 \\
1 \cdot 3642736\end{array}$ & \\
\hline $1 \cdot 3543670$ & \\
\hline 1.3445989 & \\
\hline 1.3349661 & \\
\hline 1.3254647 & \\
\hline 1.3160909 & \\
\hline $1 \cdot 3068406$ & \\
\hline $1 \cdot 2886954$ & \\
\hline 1.2623103 & \\
\hline 1.2203378 & \\
\hline 1.1805854 & \\
\hline 1.1428039 & \\
\hline 1.0724503 & \\
\hline 1.0082107 & \\
\hline 9.2176798 & \\
\hline 8.4566114 & \\
\hline 7.5787071 & \\
\hline 5.9120543 & \\
\hline $\begin{array}{l}4.7678510 \\
3.3479623\end{array}$ & \\
\hline $\begin{array}{l}3.3479623 \\
2.5187906\end{array}$ & \\
\hline $1 \cdot 5902204$ & \\
\hline
\end{tabular}

1.5702963
1.5697963
1.5687963
1.5677963
1.5667963
1.5657963
1.5647963
1.5637963
1.5627963
1.5617963
1.5607963
1.5587963
1.5557963
1.5507963
1.5457963
1.5407963
1.5307963
1.5207963
1.5057963
1.4907963
1.4707963
1.4207963
1.3707963
1.2707963
1.1707963
$9.7079630-1$

Table 98. Effective reflection coefficient (amplitude, $\left|C_{j}\right|$, and phase, Arg $C_{j}$ ) for the special ray configuration of the Norton type 301 atmosphere, applicable to the propagation of waves between the $\mathrm{D}$-region $\left(\mathrm{h}_{3}=70\right.$ kilometers $)$ or the E-region $\left(\mathrm{h}_{3}=90\right.$ kilometers $)$ of the ionosphere and the earth. 


$$
\begin{array}{ll}
\omega / \omega_{r}=0.3002 & f=20 \text { kilocycles } \\
\phi_{1}=60 \text { degrees } & \sigma=0.005 \text { mhos } / \text { meter } \\
\epsilon_{2}=15
\end{array}
$$

$\Psi$

radians

0.0005

0.0010

0.0020

0.0030

0.0040

0.0050

0.0060

0.0070

0.0080

0.0090

0.0100

0.0120

0.0150

0.0200

0.0250

0.0300

0.0400

0.0500

0.0650

0.0800

0.1000

0.1500

0.2000

0.3000

0.4000

0.6000

0.0005

0.0010

0.0020

0.0030

0.0040

0.0050

0.0060

0.0070

0.0080

0.0090

0.0100

0.0120

0.0150

0.0200

0.0250

0.0300

0.0400

0.0500

0.0650

0.0800

0.1000

0.1500

0.2000

0.3000

0.4000

0.6000 $\left|c_{4}\right|$

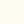

$$
\operatorname{Arg~C} 4
$$

$\mathrm{h}_{3}=70$ kilometers

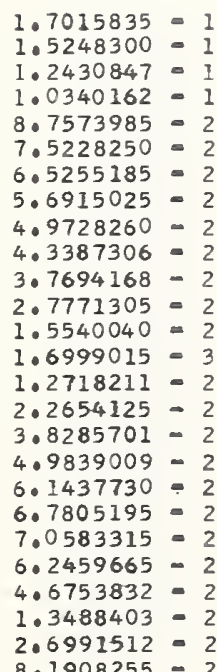

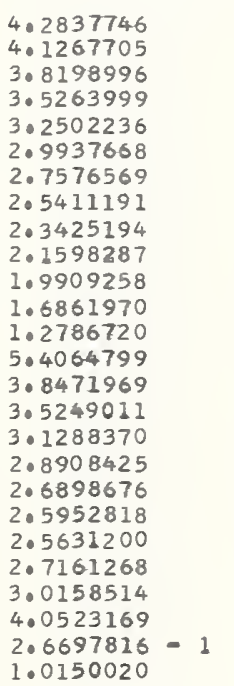

1. 5702963

1. 5697963

1. 5687963

1.5677963

1.5667963

1. 5657963

1. 5647963

1. 5637963

1. 5627963

1. 5617963

1.5607963

1. 5587963

1. 5557963

1. 5507963

I. 5457963

1. 5407963

1. 5307963

1.5207963

1. 5057963

1.4907963

1.4707963

1. 4207963

1.3707963

1.2707963

1. 1707963

9.7079630 - 1

1. 2433895 1.2381675

1.2278386

1. 2176623

1. 2076369

1. 1977599

1. 1880278

I. 1784374

1. 1689853

1. 2596669

1.2504788

1. 1324770

1. 1063547

1.0649462

1.0259098

9.8899038

9.2076906

8. 5914709

7.7737068

7.0657786

6.2644905

4.7915900

3.8174547

2. 6472062

1. 9802951

1. 2445355

$\mathrm{h}_{3}=90$ kilometers

$1.3631884=1$

$1.2343616=1$

$1.0324822-1$

$8.8514870-2$

$7.7391690=2$

$6.8595605-2$

$6.1293000=2$

$5.4970735=2$

$4.9323257=2$

$4.4171773=2$

$3.9410844=2$

$3.0826170=2$

$1.9819460-2$

$5.3191050-3$

$6.5547230=3$

$1.5716483=2$

$2.9953863=2$

$4.0301302=2$

$5.0706825=2$

$5.6642175=2$

$5.9726330=2$

$5.4105125=2$

$4.0683982=2$

$1.0755333=2$

$2.9381311=2$

$8.2823660=2$

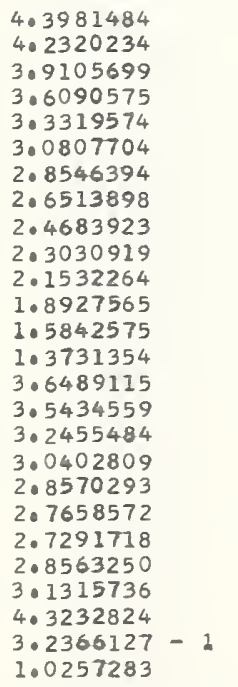

1.5702963

1.5697963

1.5687963

1.5677963

1. 5667963

I. 5657963

1. 5647963

1. 5627963

I. 5617963

1. 5607963

1. 5587963

I. 5557263

I. 5507963

1. 5457963

1. 5407963

1. 5307963

1.5207963

1.5057963

1.4707963

1.4207963

1. 3707963

1. 2707963

1. 1707963

$9.7079630-1$
1.5637963

1.4907963

Table 99. Effective reflection coefficient (amplitude, $\left|C_{i}\right|$, and phase, Arg $C_{j}$ ) for the special ray configuration of the Norton type 301 atmosphere, applicable to the propagation of waves between the $D$-region ( $h_{3}=70$ kilometers) or the $E$-region $\left(h_{3}=90\right.$ kilometers $)$ of the ionosphere and the earth.

1.40007803 1.3845147 1.3743220 $1.3642736 \quad 3$ 1.3543670 1.3349661 3 $1.3254647 \quad 3$ $1.3160909 \quad 3$ 1.2886954 1.2623103 1. $2203378 \quad 3$ 1.1805854 .1428039 1.0082107 9.2776798 8.45661142 7.5787071 4.7678510 2 3.3479623 2.5187906 1. 5902204 


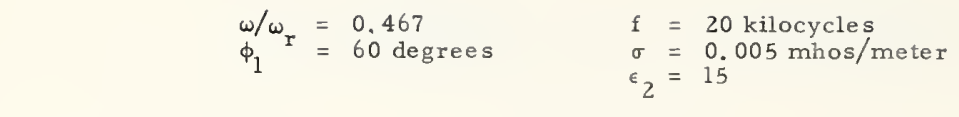

$\Psi$ radians

$$
\left|\mathrm{C}_{4}\right|
$$$$
\operatorname{Arg~C}_{4}
$$

$\mathrm{h}_{3}=70$ kilometers

0.0005

0.0010

0.0020

0.0030

0.0040

0.0050

0.0060

0.0070

0.0080

0.0090

0.0100

0.0120

0.0150

0.0200

0.0250

0.0300

0.0400

0.0500

0.0650

0.0800

0.1000

0.1500

0.2000

0.3000

0.4000

0.6000

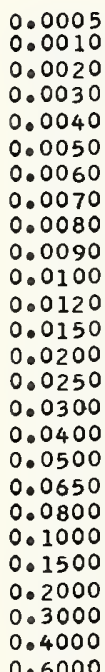

0.0005

0.0020

0.0030

0.0040

0.0060

0.0070

0.0080

- 100

0.0120

0.0150

0.0200

0.0250
0.0300

0.0400

0.0500

080

0.1000

0.1500

0.2000

0.4000

0.6000

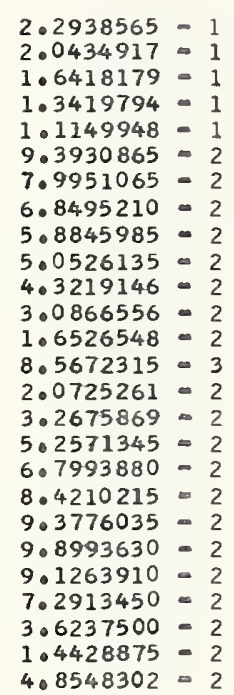

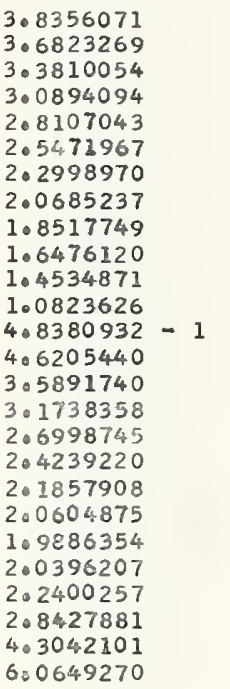

1. 5702963

1.5697963

1. 5687963

1.5677963

1.5667963

I. 5657963

1.5647963

1.5637963

1. 5627963

1.5617963

1.5607963

1.5587963

1.5557963

1. 5507963

1.5457963

1. 5407963

1. 5307963

1.5207963

1. 5057963

1.4907963

1.4707963

1.4207963

1.3707963

1.2707963

1. 1707963

9.7079630 a 1

$h_{3}=90$ kilometers

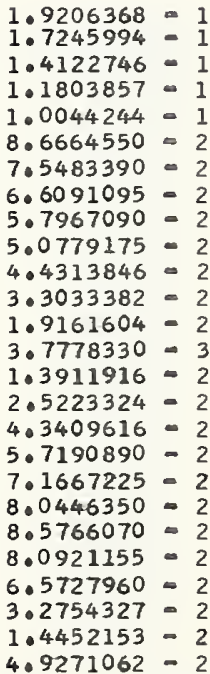

$3 \cdot 9019780$
$3 \cdot 7431059$
$3 \cdot 4330434$
$3 \cdot 1371435$
$2 \cdot 8592182$
$2 \cdot 6013621$
$2 \cdot 3638688$
$2 \cdot 1456595$
$1 \cdot 9448598$
$1 \cdot 7592422$
$1 \cdot 5864917$
$1 \cdot 2703228$
$8 \cdot 2875460-1$
$5 \cdot 4475438$
$3 \cdot 6258497$
$3 \cdot 2364108$
$2 \cdot 8012797$
$2 \cdot 5422609$
$2 \cdot 3132828$
$2 \cdot 1891213$
$2 \cdot 1137448$
$2 \cdot 1480967$
$2 \cdot 3314275$
$2 \cdot 9262162$
$4 \cdot 4823672$
$6 \cdot 0780633$

1.5702963

1.5697963

1.5687963

1. 5677963

1.5667963

1. 5657963

1.5647963

1.5637963

1.5627963

1.5617963

1. 5607963

1. 5587963

1.5557963

1. 5507963

1. 5457963

1. 5407963

1.5307963

1.5207963

1.5057963

1.4907963

1. 4707963

1.4207963

1.3707963

1.2707963

1. 1707963

$9.7079630=1$ $\mathrm{d} / \mathrm{j}$

miles

\begin{tabular}{|c|c|}
\hline $\begin{array}{l}1.2433895 \\
1 \cdot 2381675\end{array}$ & \\
\hline $1 \cdot 2278386$ & \\
\hline 1.2176623 & \\
\hline 1.2076369 & \\
\hline 1.1977599 & \\
\hline 1.1880278 & \\
\hline $1 \cdot 1784374$ & \\
\hline 1.1689853 & \\
\hline 1.1596669 & \\
\hline 1.1504788 & \\
\hline 1.1324770 & \\
\hline 1.1063547 & \\
\hline 1.0649462 & \\
\hline 1.0259098 & \\
\hline 908899038 & \\
\hline $9: 2076906$ & \\
\hline 8.5914709 & \\
\hline 7.7737068 & \\
\hline 7.0657786 & \\
\hline 6.2644905 & \\
\hline 407915900 & \\
\hline 308174547 & \\
\hline 2.6471063 & \\
\hline 802953 & \\
\hline 1.2445355 & \\
\hline
\end{tabular}

$\begin{array}{ll}1.4000780 & 3 \\ 1.3948535 & 3 \\ 1.3845147 & 3 \\ 1.3743220 & 3 \\ 1.3642736 & 3 \\ 1.3543670 & 3 \\ 1.3445989 & 3 \\ 1.3349661 & 3 \\ 1.3254647 & 3 \\ 1.3160909 & 3 \\ 1.3068406 & 3 \\ 1.2886954 & 3 \\ 1.2623103 & 3 \\ 1.2203378 & 3 \\ 1.1805854 & 3 \\ 1.1428039 & 3 \\ 1.0724503 & 3 \\ 1.0082107 & 3 \\ 9.2176798 & 2 \\ 8.4566114 & 2 \\ 7.5787071 & 2 \\ 5.9120543 & 2 \\ 4.7678510 & 2 \\ 3.3479623 & 2 \\ 2.5187906 & 2 \\ 1.5902204 & 2 \\ & \end{array}$

Table 100. Effective reflection coefficient (amplitude, $\left|C_{j}\right|$, and phase, Arg $C_{j}$ ) for the special ray configuration of the Norton type 301 atmosphere, applicable to the propagation of waves between the D-region ( $h_{3}=70$ kilometers) or the E-region ( $h_{3}=90$ kilometers) of the ionosphere and the earth. 


$$
\begin{array}{ll}
\omega / \omega_{r}=0.1501 & f=10 \text { kilocycles } \\
\phi_{1}=60 \text { degrees } & \sigma=0.005 \text { mhos } / \text { meter } \\
& \epsilon_{2}=15
\end{array}
$$

$\begin{array}{lllll}\Psi & \left|\mathrm{C}_{4}\right| & \mathrm{Arg} \mathrm{C} & \mathrm{T}_{4} & \mathrm{~d} / \mathrm{j} \\ \text { radians } & \mathrm{j}_{4} & \mathrm{miles}\end{array}$

$\mathrm{h}_{3}=70$ kilometers

$\begin{array}{ll}0.0005 & 8.3861665=2 \\ 0.0010 & 7.3415975=2 \\ 0.0020 & 5.9203790=2 \\ 0.0030 & 5.0318510=2 \\ 0.0040 & 4.4000708=2 \\ 0.0050 & 3.8857803=2 \\ 0.0060 & 3.4287113=2 \\ 0.0070 & 3.0058383=2 \\ 0.0080 & 2.6093846=2 \\ 0.0090 & 2.2370158=2 \\ 0.0100 & 1.8880038=2 \\ 0.0120 & 1.2587075=2 \\ 0.0150 & 5.0486285=3 \\ 0.0200 & 7.6335575=3 \\ 0.0250 & 1.5692721=2 \\ 0.0300 & 2.2300891=2 \\ 0.0400 & 3.2031162=2 \\ 0.0500 & 3.8383816=2 \\ 0.0650 & 4.3604007=2 \\ 0.0800 & 4.5337506=2 \\ 0.1000 & 4.4249349=2 \\ 0.1500 & 3.3203307=2 \\ 0.2000 & 1.7351603=2 \\ 0.3000 & 2.5443186=2 \\ 0.4000 & 6.6562880=2 \\ 0.6000 & 1.2926273=1\end{array}$

$4 \cdot 9926785$
$4 \cdot 7450460$
$4 \cdot 2785345$
$3 \cdot 8689704$
$3 \cdot 5221467$
$3 \cdot 2312971$
$2 \cdot 9863105$
$2 \cdot 7783021$
$2 \cdot 6007452$
$2 \cdot 4493993$
$2 \cdot 3220980$
$2 \cdot 1428928$
$2 \cdot 2472908$
$3 \cdot 9019658$
$3 \cdot 8562842$
$3 \cdot 7230049$
3.5190909
$3 \cdot 4004324$
$3 \cdot 3247298$
$3 \cdot 3248028$
$3 \cdot 3961015$
$3 \cdot 7601358$
$4 \cdot 3209310$
$1 \cdot 1114474$
1.7319183
$2 \cdot 3762388$

1. 5702963

1.5697963

1. 5687963

1.5677963

1.5667963

1.5657963

1. 5647963

1. 5637963

1.5627963

1.5617963

1.5607963

1. 5587963

1. 5557963

1.5507963

1. 5457963

1.5407963

1.5307963

1.5207963

1.5057963

1.4907963

1.4707963

1.4207963

1.3707963

1.2707963

1. 1707963

$9.7079630-1$

1.2433895

1.2381675

1.2278386

$1 \cdot 2176623$

1. 2076369

1. 1977599

1.1880278

1.1784374

1. 1689853

1.1596669

1. 1504788

$1 \cdot 1324770$

1.1063547

1. 0649462

1. 0259098

9.8899038

9.2076906

8. 5914709

7. 7737068

7.0657786

6.2644905

4. 7915900

3.8174547

2.6471062

1. 9802951

1.2445355

$h_{3}=90$ kilometers

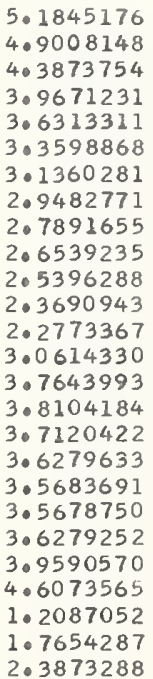

1.5702963

1. 5697963

1.5687963

1. 5677963

1.5667963

1.5657963

1.5647963

1. 5637963

1. 5627963

1. 5617963

1. 5607963

1. 5587963

1.5557963

1. 5507963

1.5457963

1. 5407963

1. 5307963

1. 5207963

1.5057963

1.4907963

1.4707963

1.4207963

1. 3707963

1.2707963

1.1707963

\begin{tabular}{|c|c|}
\hline 1.4000780 & \\
\hline 1.3948535 & \\
\hline 1.3845147 & \\
\hline 1.3743220 & \\
\hline 1.3642736 & \\
\hline 1.3543670 & \\
\hline 1.3445989 & \\
\hline 1.3349661 & \\
\hline 1.3254647 & \\
\hline 1.3160909 & \\
\hline 1.3068406 & \\
\hline 1.2886954 & \\
\hline 1.2623103 & \\
\hline 1.2203378 & \\
\hline 1.1805854 & \\
\hline 1.1428039 & \\
\hline 1.0724503 & \\
\hline 1.0082107 & \\
\hline 9.2176798 & \\
\hline 8.4566114 & \\
\hline 7.5787071 & \\
\hline 5.9120543 & \\
\hline 4.7678510 & \\
\hline 3.3479623 & \\
\hline 2.5187906 & \\
\hline 590 & \\
\hline
\end{tabular}

$9.7079630-1$
3
3
3
3
3
3
3
3
3
3
3
3
3
3
2
2
2
2
2
2
2
2
2
2
2

Table 101. Effective reflection coefficient (amplitude, $\left|C_{j}\right|$, and phase, Arg $C_{j}$ ) for the special ray configuration of the Norton type 301 atmosphere, applicable to the propagation of waves between the $\mathrm{D}$-region $\left(\mathrm{h}_{3}=70\right.$ kilometers) or the $\mathrm{E}$-region $\left(\mathrm{h}_{3}=90\right.$ kilometers $)$ of the ionosphere and the earth. 


$$
\begin{array}{ll}
\omega / \omega_{\mathrm{r}}=0.2335 & \mathrm{f}=10 \mathrm{kilocycles} \\
\phi_{1}=60 \text { degrees } & \sigma=0.005 \text { mhos } / \text { meter } \\
\epsilon_{2}=15
\end{array}
$$

$\begin{array}{lllll}\Psi & \left|\mathrm{C}_{4}\right| & \mathrm{Arg} \mathrm{C}_{4} & \tau_{j} & \mathrm{~d} / \mathrm{j} \\ \text { radians } & & & \mathrm{miles}\end{array}$

$\begin{array}{ll} & \\ 0.0005 & 1.3205560=1 \\ 0.0010 & 1.1427645=1 \\ 0.0020 & 8.8633670=2 \\ 0.0030 & 7.1580725=2 \\ 0.0040 & 5.9377970=2 \\ 0.0050 & 4.9894260=2 \\ 0.0060 & 4.2014122=2 \\ 0.0070 & 3.5175657=2 \\ 0.0080 & 2.9091193=2 \\ 0.0090 & 2.3601632=2 \\ 0.0100 & 1.1383254=1 \\ 0.0120 & 1.8607462=2 \\ 0.0150 & 1.2051170=3 \\ 0.0200 & 1.5115592=2 \\ 0.0250 & 2.6042267=2 \\ 0.0300 & 3.4909505=2 \\ 0.0400 & 4.8265887=2 \\ 0.0500 & 5.7303755=2 \\ 0.0650 & 6.5139280=2 \\ 0.0800 & 6.8221960=2 \\ 0.1000 & 6.7648290=2 \\ 0.1500 & 5.5344060=2 \\ 0.2000 & 3.8284954=2 \\ 0.3000 & 8.0738200=3 \\ 0.4000 & 4.0836882=2 \\ 0.6000 & 1.0130729=1 \\ 0 & \end{array}$

$\mathrm{h}_{3}=70$ kilometers

4.4809915
402549415
3.8237672
3.4321290
3.0865520
207856199
205238468
202949524
2.0934274
1.9150367
4.2572214
1.7569336
3.7088890
307716317
304915069
3.2829260
3.0108365
208554138
207416618
2.7104085
207431481
3.0127904
304002096
504545285
$8.6796131=1$
105457766

\subsection{3}

1.5697963

1.5687963

1.5677963

1. 5667963

1. 5657963

1.5647963

1.5637963

1.5627963

105617963

1.5697963

1.5607963

1.5557963

1. 5507963

1.5457963

1. 5407963

1.5307963

1.5207963

1.5057963

1.4907963

1.4707963

1.4207963

1. 3707963

1.2707963

1.1707963

$9.7079630=1$

102433885

2. 2381675

1. 2278386

1.2176623

1.2076369

201977599

1.1880278

1.1784374

101689853

101596669

101504788

1. 1324770

1.1063547

1.0649462

1. 0259098

908899028

9.2076906

805974709

707737068

700657760

602644905

407915900

308174547

206471062

109802951

102445355

$\mathrm{h}_{3}=90$ kilometers

0.0005
0.0010
0.0020
0.0030
0.0040
0.0050
0.0060
0.0070
0.0080
0.0090
0.0100
0.0120
0.0150
0.0200
0.0250
0.0300
0.0400
0.0500
0.0650
0.0800
0.1000
0.1500
0.2000
0.3000
0.4000
0.6000

$1.0257405=1$
$9.0489705=2$
$7.3724145=2$
$6.2829215=2$
$5.4810560=2$
$4.8182111=2$
$4.2296387=2$
$3.6895863=2$
$3.1882925=2$
$2.7218528=2$
$2.2881869=2$
$1.5132592=2$
$5.8849050=3$
$9.0038015=3$
$1.8648008=2$
$2.6537495=2$
$3.8287618=2$
$4.6216101=2$
$5.3277980=2$
$5.6404150=2$
$5.6640395=2$
$4.7261568=2$
$3.2318354=2$
$9.3123920=3$
$4.3523065=2$
$1.0229642=1$

4.6174596
4.3745778
3.9217997
3.5267524
3.1915608
2.9087782
2.6689222
2.4638875
2.2877272
2.1365474
2.0083369
1.8238945
1.9079204
3.6263626
3.5612571
304173823
3.1958190
3.0591022
2.9533125
2.9197620
2.9423245
3.1756579
3.5370008
5.9558002
$9.0894939-1$
1.5565078

1.5702963

1.5697963

1. 5687963

1. 5677963

1.5667963

1.5657963

1.564 .7963

1.5637963

1.5627963

1. 5617963

1.5607963

1.5587963

1.5557963

1.5507963

1.5457963

1.5407963

1.5307963

1. 5207963

1.5057963

1.4907963

104707963

1.4207963

1. 3707963

1. 2707963

1.1707963

$9.7079630-1$

$\begin{array}{ll}1.4000780 & 3 \\ 1.3948535 & 3 \\ 1.3845147 & 3 \\ 1.3743220 & 3 \\ 1.3642736 & 3 \\ 1.3543670 & 3 \\ 1.3445989 & 3 \\ 103349651 & 3 \\ 103254647 & 3 \\ 1.3160909 & 3 \\ 1.3068406 & 3 \\ 1.2886954 & 3 \\ 1.2623103 & 3 \\ 102203378 & 3 \\ 1.1805854 & 3 \\ 1.1428039 & 3 \\ 1.0724503 & 3 \\ 1.0082107 & 3 \\ 9.2176798 & 2 \\ 8.4566114 & 2 \\ 7.5787071 & 2 \\ 5.9120543 & 2 \\ 407678520 & 2 \\ 3.3479623 & 2 \\ 205187906 & 2 \\ 1.5902204 & 2\end{array}$

Table 102. Effective reflection coefficient (amplitude, $\left|C_{j}\right|$, and phase, Arg $\left.C_{j}\right\rangle$ for the specia ray configuration of the Norton type 301 atmosphere, applicable to the propagation of waves between the D-region $\left(h_{3}=70\right.$ kilometers) or the $E$-region $\left(h_{3}=90\right.$ kilometers $)$ of the ionosphere and the earth. 


$$
\begin{array}{ll}
\omega / \omega_{r}=0.0100 & f=0.42826552 \text { kilocycles } \\
\phi_{1}=60 \text { degrees } & \sigma=0.005 \mathrm{mhos} / \text { meter } \\
& \epsilon_{2}=1.5
\end{array}
$$

$\Psi$

radians

0.0010

0.0020

0.0030

0.0040

0.0050

0.0060

0.0070

0.0080

0.0090

0.0100

0.0120

0.0150

0.0200

0.0250

0.0300

0.0400

0.0500

0.0650

0.0800

0.1000

0.1500

0.2000

0.3000

0.4000

0.6000

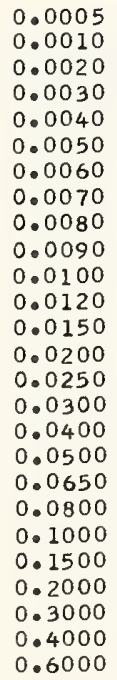

$\left|C_{4}\right|$

$\operatorname{Arg~C} 4$

$\mathrm{h}_{3}=70$ kilometers

$2.3485875-$ $1.1767889=2$ $2.0672752=3$

$1.2123268=2$ $1.6305349-2$

$1.9831230=2$ $2.2871460-2$ $2.5528761=2$ $2.7873303-2$ $2.9957538-2$ $3.3502085=2$ $3.7676043=2$ $4.2734230=2$ 4.6445716 - 2 4.9426320 $5.4340800=2$ $5.8745565-2$ 6.5389240 $7.2601355=2$ $8.3441250=2$ 1. 1652770 $1.5565972=1$ 2.3872679 4.3197481 $3.1506726=1$

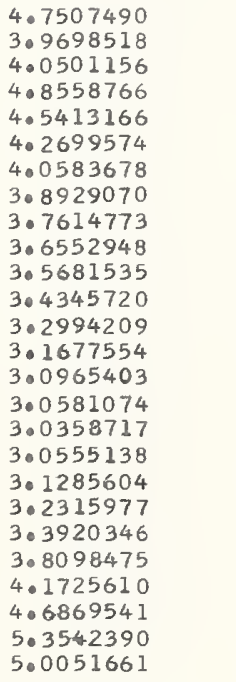

1.5702963

1.5697963

1.5687963

I. 5677963

1.5667963

1.5657963

1.5647963

1.5637963

1.5627963

1.5617963

I. 5607963

1.5587963

1.5557963

1. 5507963

1. 5457963

I. 5407963

1.5307963

1. 5207963

1.5057963

1.4907963

1.4707963

1.4207963

1.3707963

1. 2707963

9.7079630 - 1

1. 1707963

$\mathrm{h}_{3}=90$ kilometers

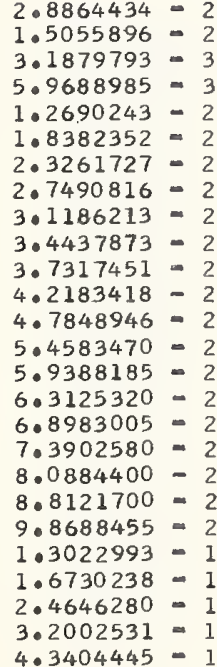

$5 \cdot 1880007$
$4 \cdot 3002723$
3.5210068
$5 \cdot 1779135$
$4 \cdot 8871223$
4.6212755
$4 \cdot 4160533$
$4 \cdot 2569738$
$4 \cdot 1313178$
$4 \cdot 0301253$
3.9471936
3.8200359
3.6908116
3.5628960
3.4909041
3.4488528
3.4138122
3.4157469
3.4569541
3.5254245
3.6402494
$3 \cdot 9645833$
4.2678806
$4 \cdot 7261295$
5.0235585
5.3596597

$\mathrm{d} / \mathrm{j}$

\begin{tabular}{|c|c|}
\hline I. 5702963 & 1.4000780 \\
\hline 1.5697963 & 1. 3948535 \\
\hline 1.5687963 & 1.3845147 \\
\hline 1.5677963 & 1.3743220 \\
\hline I. 5667963 & 1.3642736 \\
\hline I. 5657963 & 1.3543670 \\
\hline I. 5647963 & 1.3445989 \\
\hline 1.5637963 & 1.3349661 \\
\hline I. 5627963 & 1. 3254647 \\
\hline 1.5617963 & 1.3160909 \\
\hline I. 5607963 & 1.3068406 \\
\hline I. 5587963 & I. 2886954 \\
\hline I. 5557963 & 1.2623103 \\
\hline 1. 5507963 & I. 2203378 \\
\hline I. 5457963 & I. 1805854 \\
\hline 1. 5407963 & 1. 1428039 \\
\hline 1. 5307963 & 1.0724503 \\
\hline 1.5207963 & 1.0082107 \\
\hline 1.5057963 & 9.2176798 \\
\hline 1.4907963 & 8.4566114 \\
\hline 1.4707963 & 7.5787071 \\
\hline 1.4207963 & 509120543 \\
\hline 1.3707963 & 407678510 \\
\hline 1. 2707963 & 3.3479623 \\
\hline 1.1707963 & 2.5187906 \\
\hline 9.7079630 & 1.5902204 \\
\hline
\end{tabular}

miles

Table 103. Effective reflection coefficient (amplitude, $\left|C_{j}\right|$, and phase, Arg $C_{j}$ ) for the special ray configuration of the Norton type 301 atmosphere, applicable to the propagation of waves between the D-region $\left(h_{3}=70\right.$ kilometers $)$ or the E-region $\left(h_{3}=90\right.$ kilometers $)$ of the ionosphere and the earth. 


$$
\begin{array}{ll}
\omega / \omega_{\mathbf{r}}=0.0200 & \mathrm{f}=0.85653105 \text { kilocycles } \\
\phi_{1}=60 \text { degrees } & \sigma=0.005 \text { mhos } / \text { meter } \\
& \epsilon_{2}=15
\end{array}
$$

$\Psi$

radians

$\left|C_{4}\right|$

$\operatorname{Arg~} C_{4}$

$\mathrm{h}_{3}=70$ kilometers

0.0005

0.0010

0.0020

0.0030

0.0040

0.0050

0.0060

0.0070

0.0080

0.0090

0.0100

0.0120

0.0150

0.0200

0.0250

0.0300

0.0400

0.0500

0.0650

0.0800

0.1000

0.1500

0.2000

0.3000

0.4000

0.6000

0.0005

0.0010

0.0020

0.0030

0.0040

0.0050

0.0060

0.0070

0.0080

0.0090

0.0100

0.0120

0.0150

0.0200

0.0250

0.0300

0.0400

0.0500

0.0650

0.0800

0.1000

0.1500

0.2000

0.3000

0.4000

\begin{tabular}{|c|}
\hline $\begin{array}{l}3.6800940 \\
3.4474469\end{array}$ \\
\hline $\begin{array}{l}4.1886537 \\
4 \cdot 2310526\end{array}$ \\
\hline 3.9828581 \\
\hline $\begin{array}{l}3.7343863 \\
3.5124693\end{array}$ \\
\hline 3.3171783 \\
\hline 3.1454201 \\
\hline 2.9941702 \\
\hline 2.8608322 \\
\hline $\begin{array}{l}2.6392185 \\
2.3935006\end{array}$ \\
\hline 2.1342514 \\
\hline 1.9849403 \\
\hline 1.8985691 \\
\hline 1.8309925 \\
\hline 1.84 .15839 \\
\hline 1.9334306 \\
\hline 2.0726397 \\
\hline 2.2891151 \\
\hline 2.8363307 \\
\hline 30,3033545 \\
\hline 3.9797092 \\
\hline $\begin{array}{l}404147227 \\
4.9078158\end{array}$ \\
\hline
\end{tabular}

0.6000
1.5702963

1. 5697963

1. 5687963

1. 5677963

1.5667963

1. 5657963

1. 5647963

1.5637963

1. 5627963

1.5617963

1. 5607963

1. 5587963

1. 5557963

1. 5507963

1. 5457963

1.5407963

1.5307963

1. 5207963

1.5057963

1.4907963

1. 4707963

1.4207963

1.3707963

1.2707963

1.1707963

$9.7079630-1$

$\mathrm{h}_{3}=90$ kilometers

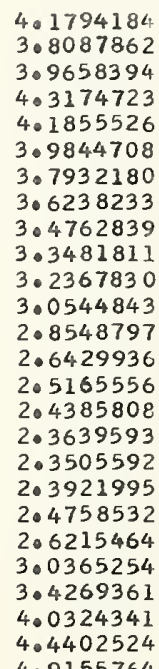

1.5702963

I. 5697963

1.5687963

1. 5677963

I. 5667963

1.5657963

I. 5647963

1. 5637963

1.5627963

1.5617963

1.5607963

1. 5587963

1.5557963

1.5507963

1. 5457963

1.5407963

1.5307963

1. 5207963

1. 5057963

1. 4907963

1.4707963

1.4207963

1.3707963

I. 2707963

1. 1707963

$9.7079630-1$ $\mathrm{d} / \mathrm{j}$

miles

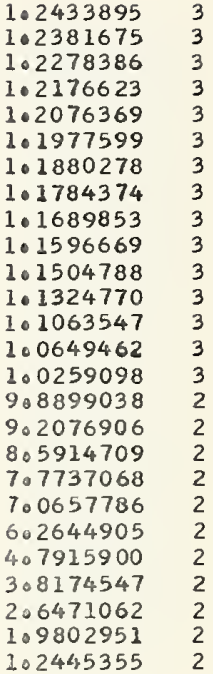

1.4000780 1. 3948535 1.3845147 1.3743220 1.3642736 1.3543670 1.3445989 1.3349661 1. 3254647 1. 3160909 1.3068406 I. 2886954 1. 2623103 1.2203378 1. 1805854 1. 1428039 1.0724503 1. 0082107 9.2176798 8.4566114 7.5787071 5.9120543 407678510 3.3479623 2.5187906 1. 5902204

Table 104. Effective reflection coefficient (amplitude, $\left|C_{j}\right|$, and phase, Arg $C_{j}$ ) for the special ray configuration of the Norton type 301 atmosphere, applicable to the propagation of waves between the D-region $\left(h_{3}=70\right.$ kilometers) or the E-region ( $h_{3}=90$ kilometers) of the ionosphere and the earth. 


$$
\begin{array}{ll}
\omega / \omega_{r}=0.0500 & f=2.1413276 \text { kilocycles } \\
\phi_{1}=60 \text { degrees } & \sigma=0.005 \text { mhos } / \text { meter } \\
& \epsilon_{2}=15
\end{array}
$$

$\Psi$
radians

0.0005

0.0010

0.0020

0.0030

0.0040

0.0050

0.0060

0.0070

$0.008 \mathrm{C}$

0.0090

0.0100

0.0120

0.0150

0.0200

$0.025 \mathrm{C}$

0.0300

0.0400

0.0500

0.0650

0.0800

0.1000

0.1500

0.2000

0.3000

0.4000

0.6000

0.0005

0.0010

0.0020

0.0030

0.0040

0.0050

0.0060

0.0070

0.0080

0.0090

0.0100

0.0120

0.0150

0.0200

0.0250

0.0300

0.0400

0.0500

0.0650

0.0800

0.1000

0.1500

0.2000

0.3000

0.4000

0.6000
$\left|\mathrm{C}_{4}\right|$

$\operatorname{Arg~} \mathrm{C}_{4}$

$h_{3}=70$ kilometers

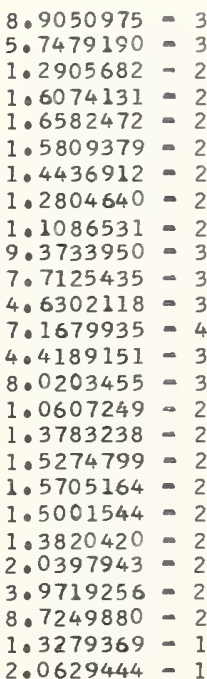

2.0629444 -

$9.4974760=3$

$3.0913258=3$

$1.0734970=2$

$1.5326484=2$

$1.7183154-2$

$1.7571912-2$

$1.7165699=2$

$1.6331589-2$

$1.5277291=2$

$1.4123842-2$

$1.2943039-2$

$1.0655397-2$

$7.6748075-3$

$4.4285419-3$

$3.9399651-3$

$5.1417895-3$

$7.7166550=3$

$9.4454605=3$

$1.0999477=2$

$1.2177263=2$

$1.4403958-2$

$2.6802526-2$

$4.6276466-2$

$9.1838330-2$

$1.3579478=1$

$2.0765066=1$

3.4029313

4. 2606492

4.0036570

3. 7515579

3.5350320

3.3506818

3.1919634

3.0529403

2. 9287354

2. 8153509

2.6080738

2. 3044217

1.6350984

$7.5541111=1$

2.3458859 -

6. 1894820

6. 1468044

6.2686491

2.2656579 - 1

6.3367025 -

1. 5260373

2. 1019925

2.8503135

3.3512530

$3.987602 \epsilon$

$8.9368665-1$
5.6092541
4.3838669
3.9227357
3.6010566
3.3518347
3.1510211
2.9852913
2.8458643
2.7263825
2.6218556
2.4391685
1.9058595
5.3678586
5.2361955
5.1696583
5.1273448
5.1571590
5.2939319
5.5270273
5.9929377
1.1316603
1.9298924
2.7862443
3.3190113
3.9765533

1.5702963

1.5697963

1. 5687963

1.5677963

1.5667963

1.5657963

1. 5647963

1.5637963

1. 5627963

1.5617963

1. 5607963

1.5587963

1.5557963

1. 5507963

1. 5457963

1. 5407963

1. 5307963

1.5207963

1. 5057963

1.4907963

1.4707963

1.4207963

1. 3707963

1.2707963

1.1707963

9.7079630 -

$h_{3}=90$ kilometers
1. 5702963

1.5697963

1. 5687963

1.5677963

1.5667963

1.5657963

I. 5647963

1. 5637963

1. 5627963

1. 5617963

1. 5607963

1.5587963

1.5557963

1. 5507963

1.5457963

1. 5407963

1.5307963

1. 5207963

1.5057963

1.4907963

1.4707963

1.4207963

1.3707963

1. 2707963

1. 1707963

$9.7079630-1$ 1. 5902204
1. 2433895

1.2381675

1. 2278386

1.2176623

1.2076369

1. 1977599

1. 1880278

1. 1784374

1. 1689853

1.1596669

1. 1504788

1.1324770

1. 1063547

1.0649462

1.0259098

9.8899038

9.2076906

8.5914709

7.7737068

7.0657786

6.2644905

4.7915900

3. 8174547

2.6471062

1.9802951

1. 2445355

3

1.4000780 1.3948535 1. 3845147

1. 3743220

1. 3642736 1. 3543670

1. 3445989

1. 3349661 1. 3254647

1. 3160909

1.3068406 1. 2886954

1. 2623103

1. 2203378

1. 1805854

1. 1428039

1. 0724503 1. 0082107 9.2176798 8.4566114 7.5787071 5.9120543 4.7678510 3.3479523

2. 5187906

Table 105. Effective reflection coefficient (amplitude, $\left|C_{j}\right|$, and phase, $A r g C_{j}$ ) for the special ray configuration of the Norton type 301 atmosphere, applicable to the propagation of waves between the $\mathrm{D}$-region ( $h_{3}=70$ kilometers) or the $E$-region $\left(h_{3}=90\right.$ kilometers $)$ of the ionosphere and the earth. 


$$
\begin{array}{ll}
\omega / \omega_{r}=0.1000 & f=4.2826552 \text { kilocycles } \\
\phi_{1}=60 \text { degrees } & \sigma=0.005 \text { mhos } / \text { meter } \\
& \epsilon_{2}=15
\end{array}
$$

$\Psi$

radians

$\left|\mathrm{C}_{4}\right|$

$\operatorname{Arg~C}_{4}$

$\mathrm{h}_{3}=70$ kilometers

0.0005
0.0010
0.0020
0.0030
0.0040
0.0050
0.0060
0.0070
0.0080
0.0090
0.0100
0.0120
0.0150
0.0200
0.0250
0.0300
0.0400
0.0500
0.0650
0.0800
0.1000
0.1500
0.2000
0.3000
0.4000
0.6000

0.0005

0.0010

0.0020

0.0030

0.0040

0.0050

0.0060

0.0070

0.0080

0.0090

0.0100

0.0120

0.0150

0.0200

0.0250

0.0300

0.0400

0.0500

0.0650

0.0800

0.1000

0.1500

0.2000

0.3000

0.4000

0.6000
5.4241739
4.9847927
4.2548386
3.7391643
3.3665608
3.0837031
2.8629707
2.6909145
2.5625591
2.4804095
2.4579913
2.7817235
3.9743208
4.1127290
4.0149538
3.9270328
3.8167643
3.7713159
3.7787877
3.8445616
3.9918276
4.5926219
6.2295753
1.7806194
2.3466992
3.0152572

$\mathrm{h}_{3}=90$ kilometers

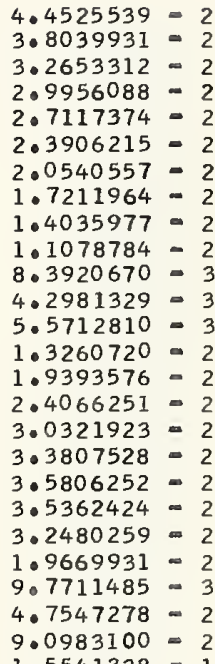

$1.5541338=1$

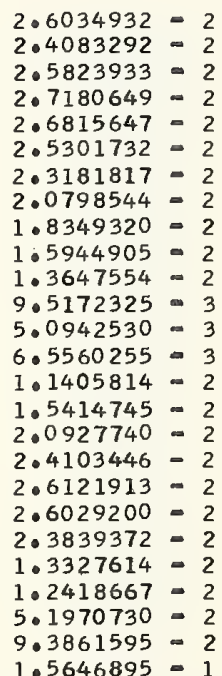

${ }^{\top}{ }_{j}$

$\mathrm{d} / \mathrm{j}$

miles $\begin{array}{ll}1.2433895 & 3 \\ 1.2381675 & 3 \\ 1.2278386 & 3 \\ 1.2176623 & 3 \\ 1.2076369 & 3 \\ 1.1977599 & 3 \\ 1.1880278 & 3 \\ 1.1784374 & 3 \\ 1.1689853 & 3 \\ 1.1596669 & 3 \\ 1.1504788 & 3 \\ 1.1324770 & 3 \\ 1.1063547 & 3 \\ 1.0649462 & 3 \\ 1.0259098 & 3 \\ 908899038 & 2 \\ 9.2076906 & 2 \\ 8.5914709 & 2 \\ 7.7737068 & 2 \\ 7.0657786 & 2 \\ 6.2644905 & 2 \\ 4.7915900 & 2 \\ 3.8174547 & 2 \\ 206471062 & 2 \\ 109802951 & 2 \\ 1.2445355 & 2\end{array}$

1.4000780 1.3948535

1. 3845147 1.3743220 1.3642736 1.3543670 1. 3445989 1.3349661 I. 3254647 1.3160909 1.3068406 1. 2886954 1.2623103 1.2203378 1. 1805854 1. 1428039 1.0724503 1.0082107 9.2176798 8.4566114 7. 5787071 5.9120543 4. 7678510 3.3479623 2. 5187906 1.5902204

\begin{tabular}{l}
3 \\
3 \\
3 \\
3 \\
3 \\
3 \\
3 \\
3 \\
3 \\
3 \\
3 \\
3 \\
3 \\
3 \\
3 \\
2 \\
2 \\
2 \\
2 \\
2 \\
2 \\
2 \\
2 \\
2 \\
2 \\
2 \\
3 \\
2 \\
2 \\
2 \\
3 \\
3 \\
3 \\
3 \\
3 \\
3 \\
3 \\
3 \\
3 \\
3 \\
3 \\
3 \\
3 \\
3 \\
3 \\
2 \\
2 \\
\hline
\end{tabular}

Table 106. Effective reflection coefficient (amplitude, $\left|C_{j}\right|$, and phase, $A r g C_{j}$ ) for the special ray configuration of the Norton type 301 atmosphere, applicable to the propagation of waves between the $D$-region $\left(h_{3}=70\right.$ kilometers $)$ or the $E$-region $\left(h_{3}=90\right.$ kilometers $)$ of the ionosphere and the earth. 


$$
\begin{array}{ll}
\omega / \omega_{r}=0.2000 & f=8.5653105 \text { kilocycles } \\
\phi_{1}=60 \text { degrees } & \tau=0.005 \text { mhos } / \text { meter } \\
& \epsilon_{2}=15
\end{array}
$$

$\Psi$

radians

0.0005

0.0010

0.0020

0.0030

0.0040

0.0050

0.0060

0.0070

0.0080

0.0090

0.0100

0.0120

0.0150

0.0200

0.0250

0.0300

0.0400

0.0500

0.0650

0.0800

0.1000

0.1500

0.2000

0.3000

0.4000

0.6000

0.0005

0.0010

0.0020

0.0030

0.0040

0.0050

0.0060

0.0070

0.0080

0.0090

0.0100

0.0120

0.0150

0.0200

0.0250

0.0300

0.0400

0.0500

0.0650

0.0800

0.1000

0.1500

0.2000

0.3000

0.4000

0.6000 $\left|c_{4}\right|$

Arg $\mathrm{C}_{4}$

$h_{3}=70$ kilometers

1.1292827 -

$9.7184710=2$

$7.5304795-2$

$6.1224015-2$

$5.1154435-2$

$4.3156484-2$

$3.6333815-2$

$3.0287498-2$

$2.4832528-2$

$1.9870224-2$

$1.5337355=2$

$7.3987890-3$

$3.2867359-3$

$1.5857959=2$

$2.5842953=2$

$3.3863215-2$

$4.5692244-2$

$5.3439810=2$

$5.9811360-2$

$6.1933980-2$

$6.0673720=2$

4. $8181619-2$

$3.1333610-2$

$1.1625166=2$

\begin{tabular}{|c|c|}
\hline $\begin{array}{l}4.6321797 \\
4.3827714\end{array}$ & \\
\hline 3.9126996 & \\
\hline 3.4963794 & \\
\hline 3.1392647 & \\
\hline 2.8360189 & \\
\hline 2.5778143 & \\
\hline 2.3565572 & \\
\hline 2.1662817 & \\
\hline 2.0034956 & \\
\hline 1.8677484 & \\
\hline 1.7119042 & \\
\hline 3.7349857 & \\
\hline 3.7753131 & \\
\hline 3.5395817 & \\
\hline 3.3571959 & \\
\hline $\begin{array}{l}3.1188123 \\
2.9857359\end{array}$ & \\
\hline 2.8960487 & \\
\hline 2.8834988 & \\
\hline 2.9372426 & \\
\hline 3.2500474 & \\
\hline 3.6811209 & \\
\hline 1.9302138 & - \\
\hline $\begin{array}{l}1.1917083 \\
1.8502564\end{array}$ & \\
\hline & \\
\hline
\end{tabular}

$4.9923999-2$

$1.1204565-1$ $\mathrm{h}_{3}$

$8.5447785-2$

$7.5313735-2$

$6.2092920-2$

$5.3876235=2$

$4.7696060-2$

$4.2317913-2$

$3.7323404-2$

$3.2604781-2$

$2.8150958=2$

$2.3971132-2$

$2.0072577-2$

$1.3134888-2$

$5.4144750-3$

$9.7097960-3$

$1.8342922-2$

$2.5404956=2$

$3.5767697-2$

$4.2575776=2$

$4.8383622-2$

$5.0673230-2$

$5.0264305-2$

$4.0462634-2$

$2.5476785-2$

$1.4975066-2$

$5.2703885=2$

1. $1305154=1$
3
1. 5702963

1. 5697963

1. 5687963

1.5677963

1. 5667963

1. 5657963

1.5647963

1.5637963

1.5627963

1.5617963

1. 5607963

1.5587963

1.5557963

1. 5507963

1.5457963

1.5407963

1.5307963

1.5207963

1.5057963

1.4907963

1.4707963

1.4207963

1.3707963

1.2707963

1. 1707963

$9.7079630=1$

1.5702963

1. 5697963

1.5687963

1.5677963

1.5667963

1.5657963

1. 5647963

1. 5637963

1. 5627963

1. 5617963

1. 5607963

1.5587963

1.5557963

1.5507963

1.5457963

1. 5407963

1. 5307963

1.5207963

1.5057963

1. 4907963

1.4707963

1. 4207963

1.3707963

1.2707963

1. 1707963

9.7079630 -
d/J

miles $\begin{array}{ll}1 \cdot 2433895 & 3 \\ 1 \cdot 2381675 & 3 \\ 1 \cdot 2278386 & 3 \\ 1 \cdot 2176623 & 3 \\ 1 \cdot 2076369 & 3 \\ 1 \cdot 1977599 & 3 \\ 1 \cdot 1880278 & 3 \\ 1 \cdot 1784374 & 3 \\ 1 \cdot 1689853 & 3 \\ 1 \cdot 1596669 & 3 \\ 1 \cdot 1504788 & 3 \\ 1 \cdot 1324770 & 3 \\ 1 \cdot 1063547 & 3 \\ 1 \cdot 0649462 & 3 \\ 1 \cdot 0259098 & 3 \\ 9 \cdot 8899038 & 2 \\ 9.2076906 & 2 \\ 8 \cdot 5914709 & 2 \\ 7 \cdot 7737068 & 2 \\ 7 \cdot 0657786 & 2 \\ 6.2644905 & 2 \\ 4 \cdot 7915900 & 2 \\ 3.8174547 & 2 \\ 2.6471062 & 2 \\ 1.9802951 & 2 \\ 1.2445355 & 2\end{array}$

1.4000780

1. 3948535

1. 3845147

1.3743220

1. 3642736

1. 3543670

1.3445989

1.3349661

1.3254647

1.3160909

1.3068406

1. 2886954

1.2623103

1.2203378

1. 1805854

1. 1428039

1. 0724503

1. 0082107

9.2176798

8.4566114

7.5787071

5. 9120543

4. 7678510

3.3479623

2. 5187906

1. 5902204

Table 107. Effective reflection coefficient (amplitude, $\left|C_{j}\right|$, and phase, Arg $C_{j}$ ) for the special ray configuration of the Norton type 301 atmosphere, applicable to the propagation of waves between the $\mathrm{D}$-region $\left(\mathrm{h}_{3}=70\right.$ kilometers $)$ or the $\mathrm{E}$-region $\left(\mathrm{h}_{3}=90\right.$ kilometers $)$ of the ionosphere and the earth. 


$$
\begin{array}{ll}
\omega / \omega_{r}=1.0000 & f=42.826552 \mathrm{kilocycles} \\
\phi_{1}=60 \text { degrees } & \sigma=0.005 \mathrm{mhos} / \mathrm{meter} \\
& \epsilon_{2}=15
\end{array}
$$

$\Psi$

radians

$\left|\mathrm{C}_{4}\right|$

0.0005

0.0010

0.0020

0.0030

0.0040

0.0050

0.0060

0.0070

0.0080

0.0090

0.0100

0.0120

0.0150

0.0200

0.0250

0.0300

0.0400

0.0500

0.0650

0.0800

0.1000

0.1500

0.2000

0.3000

0.4000

0.6000

0.0005

0.0010

0.0020

0.0030

0.0040

0.0050

0.0060

0.0070

0.0080

0.0090

0.0100

0.0120

0.0150

0.0200

0.0250

0.0300

0.0400

0.0500

0.0650

0.0800

0.1000

0.1500

0.2000

0.3000

0.4000

0.6000
Arg $\mathrm{C}_{4}$

$\mathrm{h}_{3}=70$ kilometers

$3.1717276=1$
$2.9184308=1$
$2.4809554=1$
$2.1216081=1$
$1.8257527=1$
$1.5811025=1$
$1.3775028=1$
$1.2066864=1$
$1.0620138=1$
$9.3821520=2$
$8.3115945=2$
$6.5514090=2$
$4.5694612=2$
$2.3807747=2$
$1.4765032=2$
$1.9886235=2$
$3.8779154=2$
$5.6031455=2$
$7.7109895=2$
$9.2512275=2$
$1.0518964=1$
$1.0971709=1$
$9.5364635=2$
$5.8318035=2$
$3.0974986=2$
$6.2541615=3$

3.0274102

2.9252094

2. 7225505

2. 5226401

2. 3260684

2. 1334601

1. 9453760

1. 7622427

1.5842933

1.4115350

1.2437429

$9.2103176=1$

4.5569394 . 1

5.8974238

$4 \cdot 6470393$

3. 5259224

2.5833339

2.1118289

1.6885618

104289990

1.2121143

9.5762098 - 1

$8.7476708-1$

$8.9779247-1$

1.0424277

2. 1154720

$\mathrm{h}_{3}=90$ kilometers

2.9905061
2.8867122
2.6816395
2.4805610
2.2842847
2.0935453
1.9089399
1.7308423
1.5593831
1.3944581
1.2357398
$9.3471086-1$
$5.1186565-1$
6.0729970
4.9332929
3.5583482
2.5717509
2.1166448
1.7066061
1.4523623
1.2378199
$9.8265833-1$
$8.9743194=1$
$9.1642418-1$
1.0604554
2.1717862

$2.7707776=1$
$2.5594659=1$
$2.1946025=1$
$1.8946775=1$
$1.6471362=1$
$1.4415150=1$
$1.2692483=1$
$1.1234453=1$
$9.9864310=2$
$8.9056225=2$
$7.9589020=2$
$6.3715985=2$
$4.5248882=2$
$2.3515646=2$
$1.1575827=2$
$1.4586768=2$
$3.2552956=2$
$4.8471705=2$
$6.7547630=2$
$8.1498225=2$
$9.3268970=2$
$9.8949650=2$
$8.7360770=2$
$5.4614640=2$
$2.9321612=2$
$6.0991285=3$

$\tau_{\mathrm{j}}$

$d / j$

miles

\begin{tabular}{|c|c|}
\hline .5702963 & 1.4000780 \\
\hline 1.5697963 & 1.3948535 \\
\hline 1.5687963 & 1.3845147 \\
\hline 1.5677963 & 1.3743220 \\
\hline .5667963 & 1.3642736 \\
\hline .5657963 & $1 \cdot 3543670$ \\
\hline .5647963 & 1.3445989 \\
\hline 5637963 & 1.3349661 \\
\hline 627963 & 1.3254647 \\
\hline 5617963 & 1.3160909 \\
\hline .5607963 & $1 \cdot 3068406$ \\
\hline .5587963 & 1.2886954 \\
\hline .5557963 & 1.2623103 \\
\hline .5507963 & 1.2203378 \\
\hline .5457963 & 1.1805854 \\
\hline .5407963 & 1.1428039 \\
\hline .5307963 & 1.0724503 \\
\hline .5207963 & 1.0082107 \\
\hline .5057963 & 9.2176798 \\
\hline .4907963 & 8.4566114 \\
\hline 1.4707963 & 7.5787071 \\
\hline 1.4207963 & 5.9120543 \\
\hline .3707963 & 4.7678510 \\
\hline 2707963 & 3.3479623 \\
\hline 1707963 & 2.5187906 \\
\hline 7079630 & 1.5902204 \\
\hline
\end{tabular}

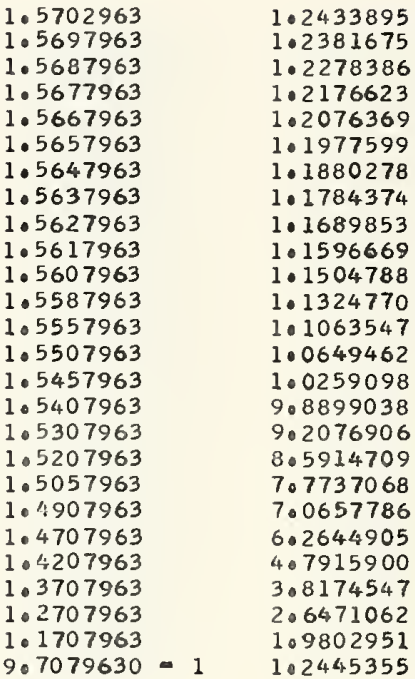

9.7079630

1. 5902204

Table 108. Effective reflection coefficient (amplitude, $\left|C_{j}\right|$, and phase, $\operatorname{Arg} C_{j}$ ) for the special ray configuration of the Norton type 301 atmosphere, applicable to the propagation of waves between the $\mathrm{D}$-region $\left(\mathrm{h}_{3}=70\right.$ kilometers) or the E-region $\left(\mathrm{h}_{3}=90\right.$ kilometers $)$ of the ionosphere and the earth. 


$$
\begin{array}{ll}
\omega / \omega_{r}=2.0000 & f=85.653105 \text { kilocycles } \\
\phi_{1}=60 \text { degrees } & \sigma=0.005 \text { mhos } / \text { meter } \\
& \epsilon_{2}=15
\end{array}
$$

$\Psi$

radians

0.0005

0.0010

0.0020

0.0030

0.0040

0.0050

0.0060

0.0070

0.0080

0.0090

0.0100

0.0120

0.0150

0.0200

0.0250

0.0300

0.0400

0.0500

0.0650

0.0800

0.1000

0.1500

0.2000

0.3000

0.4000

0.6000

0.0005

0.0010

0.0020

0.0030

0.0040

0.0050

0.0060

0.0070

0.0080

0.0090

0.0100

0.0120

0.0150

0.0200

0.0250

0.0300

0.0400

0.0500

0.0650

0.0800

0.1000

0.1500

0.2000

0.3000

0.4000

0.6000
$\left|C_{4}\right|$

$\operatorname{Arg~C} 4$

$\mathrm{h}_{3}=70$ kilometers

$2.9075192=1$
$2.7541564=1$
$2.4765191=1$
$2.2333293=1$
$2.0198678=1$
$1.8319711=1$
$1.6660010=1$
$1.5187991=1$
$1.3876535=1$
$1.2702480=1$
$1.1646175=1$
$9.8230630=2$
$7.6344975=2$
$4.9444403=2$
$3.0158236=2$
$1.6296748=2$
$1.2269573=2$
$2.5466971=2$
$4.1661732=2$
$5.3507990=2$
$6.3873900=2$
$6.9889925=2$
$6.0581990=2$
$3.4181154=2$
$1.5916617=2$
$2.0291360=3$

2. 2195112

2. 1474747

2. 0051242

1. 8652570

1. 7280952

1. 5938419

1.4626727

1. 3347138

1. 2100522

1.0887252

$9.7071615-1$

7.4434654 - 1

4.2671962 - 1

6.2228437

5.7450401

5. 1553838

2. 9153454

2.0585350

1.4665849

1.0880167

$7.3604163=1$

6. 1379677

5. 6434115

5. 2336416

4.4404484 $\mathrm{h}_{3}=$

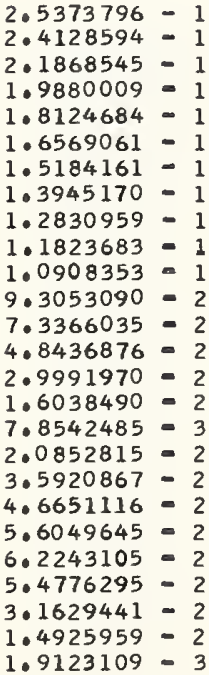

$1.9706333=1$

${ }^{\boldsymbol{\top}} \mathbf{j}$

$\mathrm{d} / \mathrm{j}$

miles

1. 5702963

1.5697963

1.5687963

1.5677963

1.5667963

1.5657963

1.5647963

1. 5637963

1. 5627963

1. 5617963

1.5607963

1.5587963

1.5557963

1. 5507963

1. 5457963

1. 5407963

1.5307963

1.5207963

1.5057963

1.4907963

1. 4707963

1.4207963

1.3707963

1.2707963

1.1707963

$9.7079630-1$

1. 2433895

1. 2381675

1.2278386

1.2176623

1.2076369

1.1977599

1. 1880278

1. 1784374

1. 1689853

1. 1596669

1. 1504788

1.1324770

1. 1063547

1.0649462

1.0259098

9.8899038

9.2076906

8. 5914709

7. 7737068

7.0657786

6.2644905

4. 7915900

3.8174547

2.6471062

1.9802951

1.2445355

3
3
3
3
3
3
3
3
3
3
3
3
3
3
3
2
2
2
2
2
2
2
2
2
2
2

2.0559971
1.9838621
1.8419390
1.7033537
1.5683243
1.4370280
1.3095839
1.1860650
1.0664846
$9.5080485-1$
$8.3894213-1$
$6.2614809-1$
$3.3159871-1$
6.1743844
5.7646233
5.3088467
2.7909513
1.9135053
1.3677716
1.0106851
$6.7309326=1$
$1.4974424-1$
6.0988992
5.6142479
5.2098906
4.4214909

1. 5702963

1. 5697963

1. 5687963

1. 5677963

1.5667963

1.5657963

1.5647963

1. 5637963

1. 5627963

1. 5617963

1. 5607963

1.5587963

1. 5557963

1. 5507963

1. 5457963

1.5407963

1. 5307963

1.5207963

1.5057963

1.4907963

1.4707963

1.4207963

1. 3707963

1. 2707963

1.1707963

$9.7079630-1$

Table 109. Effective reflection coefficient (amplitude, $\left|C_{j}\right|$, and phase, Arg $C_{j}$ ) for the special ray configuration of the Norton type 301 atmosphere, applicable to the propagation of waves between the D-region ( $h_{3}=70$ kilometers) or the E-region $\left(h_{3}=90\right.$ kilometers) of the ionosphere and the earth. 


$$
\begin{array}{ll}
\omega / \omega_{\mathrm{T}}=5.0000 & \mathrm{f}=214.13276 \mathrm{kilocycles} \\
\phi_{1}=60 \text { degrees } & \sigma=0.005 \mathrm{mhos} / \mathrm{meter}
\end{array}
$$

$\Psi$

radians

0.0005
0.0010
0.0020
0.0030
0.0040
0.0050
0.0060
0.0070
0.0080
0.0090
0.0100
0.0120
0.0150
0.0200
0.0250
0.0300
0.0400
0.0500
0.0650
0.0800
0.1000
0.1500
0.2000
0.3000
0.4000
0.6000

0.0005

0.0010

0.0020

0.0030

0.0040

0.0050

0.0060

0.0070

0.0080

0.0090

0.0100

0.0120

0.0150

0.0200

0.0250

0.0300

0.0400

0.0500

0.0650

0.0800

0.1000

0.1500

0.2000

0.3000

0.4000

0.6000
$\left|\mathrm{C}_{4}\right|$

$\operatorname{Arg~C} 4$

$\mathrm{h}_{3}=70$ kilometers

$1.9409431=1$
$1.799735=1$
$1.5567633=1$
$1.3541429=1$
$1.1813613=1$
$1.0313726=1$
$8.9943535=2$
$7.8229015=2$
$6.7760110=2$
$5.8362005=2$
$4.9897089=2$
$3.5333391=2$
$1.8153967=2$
$2.4033724=3$
$1.5978848=2$
$2.6145865=2$
$4.0549318=2$
$5.0334860=2$
$5.9451210=2$
$6.3712570=2$
$6.4127970=2$
$5.1824430=2$
$3.5211175=2$
$1.2163748=2$
$2.8687685=3$
$6.6012400=5$

1.6830667 $1.5762028-1$ $1.3867013=1$ $1.2225533=1$ $1.0780241-1$ $9.4936565=2$ $8.3404115=2$ $7.3023705=2$ $6.3656640-2$ $5.5189995=2$ $4.7527940=2$ $3.4290126=2$ $1.8630745=2$ $1.0499163=3$ $1.2220839-2$ $2.1151759=2$ $3.3411397=2$ $4.1480957=2$ 4.8920362 - 2 $5.2493475-2$ $5.3118910-2$ 4.3814998 - 2 $3.0360057-2$ $1.0725615=2$ $2.5378834=3$ $6.0444880=5$

$8.8979055=1$
$7.5272322=1$
$4.9529085=1$
$2.5942137=1$
$4.3574020=2$
6.1289704
5.9471670
5.7793566
5.6237050
5.4785699
5.3424728
5.0920541
4.7489414
1.7486554
$9.7942981=1$
$6.7419775=1$
$2.3703032-1$
6.2110209
5.8792993
5.6340883
5.3747570
408558521
403863364
3.4370676
2.4848666
1.5025103

$\mathrm{h}_{3}=$

$3.9866727=1$

$1.5661661-1$

6. 2201217

6.0204454

5.8383980

5.6718017

5. 5187401

5.3775816

5. 2469552

5.1257600

4.9084452

4.6407188

5.5389258

$604032276-1$

401307066 - 1

2. $7657480-2$

6.0233266

5. 7080942

5.4728463

5.2236464

4.7254659

4. 2726336

3. 3478143

2.4239069

1.4952684 $\tau_{j}$

$\mathrm{d} / \mathrm{j}$

miles

1. 5702963

1.5697963

1.5687963

1.5677963

1.5667963

1.5657963

1.5647963

1.5637963

1.5627963

1.5617963

1. 5607963

1.5587963

1.5557963

1. 5507963

1. 5457963

1.5407963

1. 5307963

1.5207963

1.5057963

1.4907963

1.4707963

1.4207963

1.3707963

1. 2707963

1.1707963

9.7079630 - 1

1.2433895 1. 2381675

]. 2278386

1.2176623

?.2076369

1. 1977599

1. 1880278

1. 1784374

$1 \cdot 1689853$

1. 1596669

1.1504788

1. 1324770

1.1063547

1.0649462

1.0259098

9.8899038

9.2076906

8.5914709

7.7737068

7.0657786

6.2644905

4. 7915900

3.8174547

2.6471062

1.9802951

1.2445355

3
3
3
3
3
3
3
3
3
3
3
3
3
3
3
2
2
2
2
2
2
2
2
2
2
2

1. 5702963

1.5697963

1.5687963

1.5677963

1.5667963

1.5657963

1.5647963

1.5637963

1.5627963

1. 5617963

1.5607963

1.5587963

1.5557963

1.5507963

1.5457 .963

1.5407963

1.5307963

1.5207963

1.5057963

1.4907963

1.4707963

1.4207963

1.3707963

1. 2707963

1.1707963

$9.7079630-1$

$\begin{array}{ll}1.4000780 & 3 \\ 1.3943535 & 3 \\ 1.3845147 & 3 \\ 1.3743220 & 3 \\ 1.3642736 & 3 \\ 1.3543670 & 3 \\ 1.3445989 & 3 \\ 1.3349661 & 3 \\ 1.3254647 & 3 \\ 1.3160909 & 3 \\ 1.3068406 & 3 \\ 1.2886954 & 3 \\ 1.2623103 & 3 \\ 1.2203378 & 3 \\ 1.1805854 & 3 \\ 1.1428039 & 3 \\ 1.0724503 & 3 \\ 1.0082107 & 3 \\ 9.2176798 & 2 \\ 8.4566114 & 2 \\ 7.5787071 & 2 \\ 5.9120543 & 2 \\ 4.7678510 & 2 \\ 3.3479623 & 2 \\ 2.5187906 & 2 \\ 1.5902204 & 2\end{array}$

Table 110. Effective reflection coefficient (amplitude, $\left|C_{j}\right|$, and phase, Arg $C_{j}$ ) for the special ray configuration of the Norton type 301 atmosphere, applicable to the propagation of waves between the $\mathrm{D}$-region $\left(\mathrm{h}_{3}=70\right.$ kilometers) or the $\mathrm{E}$-region $\left(\mathrm{h}_{3}=90\right.$ kilometers) of the ionosphere and the earth. 


$$
\begin{array}{ll}
\omega / \omega_{r}=0.0100 & f=0.66622256 \text { kilocycles } \\
\phi_{1}=60 \text { degrees } & \sigma=0.005 \mathrm{mhos} / \text { meter } \\
& \epsilon_{2}=15
\end{array}
$$

$\Psi$

radians

0.0005

0.0010

0.0020

0.0030

0.0040

0.0050

0.0060

0.0070

0.0080

0.0090

0.0100

0.0120

0.0150

0.0200

0.0250

0.0300

0.0400

0.0500

0.0650

0.0800

0.1000

0.1500

0.2000

0.3000

0.4000

0.6000

0.0005

0.0010

0.0020

0.0030

0.0040

0.0050

0.0060

0.0070

0.0080

0.0090

0.0100

0.0120

0.0150

0.0200

0.0250

0.0300

0.0400

0.0500

0.0650

0.0800

0.1000

0.1500

0.2000

0.3000

0.4000

0.6000 $\left|c_{4}\right|$

Arg $\mathrm{C}_{4}$

$\mathrm{h}_{3}=70$ kilometers

$2.7325776=2$

$1.5352570=2$

$4.5280269-3$

$3.4361167-3$

$8.1466675=3$

1. $2164858-2$

$1.5574772=2$

$1.8538501=2$

$2.1158112=2$

$2.3499264=2$

$2.5607814-2$

$2.9258309=2$

$3.3663396=2$

$3.9137964-2$

$4.3216968=2$

$4.6499907=2$

$5.1857825=2$

$5.6556650=2$

$6.3473625-2$

$7.0840440=2$

$8.1783350=2$

$1.1489821=1$

$1.5395700=1$

$2.3689498=1$

$4.3019633=1$

$3.1320155=1$

4.9328293

4. 2470796

3. 5025757

4.8754099

4. 7940809

4.5392123

4. 3172575

4. 1353181

3.9865923

3. 8640251

3. 7619452

3.6029909

3.4391045

3.2756589

3. 1840708

3. 1315598

3. 0912000

3.0996927

3.1622758

$3 \cdot 2587123$

3.4134367

3. 8238249

4. 1830319

4.6940957

5. 3581401

5. 0106809

$\mathrm{h}_{3}$

$3.3666778=$

$1.9105429=2$

$7.0068275=3$

$1.8760599=3$

$7.4514245-3$

$1.2745123=2$

$1.7375637=2$

2. $1462169-2$

$2.5097337=2$

$2.8350695=2$

$3.1276903=2$

$3.6320423=2$

$4.2346279=2$

$4.9700207=2$

$5.5037865=2$

$5.9208875=2$

$6.5701530=2$

$7.1046395=2$

$7.8435595=2$

$8.5919595-2$

$9.6676550=2$

$1.2838040=1$

$1.6545698=1$

$2.4456721=1$

$3.1812881=$ =

$4.3225726=1$
5. 3853581

4. 6240321

3.6076364

4. 7721179

5.1286456

4.6672663

4.4901833

4.3465491

4.2288443

3. 9794175

3. 8228131

3. 6649440

3.5738440

3. 5186095

3. 4666101

3.4581166

3.4895346

3. 5518175

3. 6612608

3. 9784926

4. 2783519

4. 7332798

5.0290774

5.3635616
4.8849544

4. 1311628
$T_{j}$

d $/ j$

miles

1. 5702963

1.5697963

1.5687963

1.5677963

1. 5667963

1.5657963

1.5647963

1. 5637963

1.5627963

1. 5617963

1. 5607963

1. 5587963

1. 5557963

1.5507963

1. 5457963

1. 5407963

1. 5307963

1. 5207963

1. 5057963

1.4907963

1.4707963

1.4207963

1.3707963

1. 2707963

$9.7079630-1$

1. 1707963

1. 2433895

1. 2381675

1. 2278386

1. 2176623

1. 2076369

1. 1977599

1.1880278

1. 1784374

1.1689853

1. 1596669

1. 1504788

1. 1324770

1.1063547

1.0649462

1.0259098

9.8899038

9.2076906

8. 5914709

7.7737068

7.0657786

6.2644905

4. 7915900

3.8174547

2. 6471062

1. 9802951

1. 2445355

1. 5702363

1.5697963

1. 5687963

1. 5677963

1.5667963

1. 5657963

1.5647963

1. 5637963

1.5627963

1. 5617963

1. 5607963

1. 5587963

1. 5557963

1. 5507963

1.5457963

1. 5407963

1. 5307963

1. 5207963

1.5057963

1.4907963

1.4707963

1.4207963

1.3707963

1.2707963

1. 1707963

$9.7079630=1$
1.4000780

1. 3845147

1. 3743220

1. 3642736

1. 3543670

I. 3445989

1. 3349661

1. 3254647

1.3160909

1. 3068406

1. 2886954

1. 2623103

1. 2203378

1. 1805854

1. 1428039

1. 0724503

1.0082107

9.2176798

8.4566114

7.5787071

5.9120543

4.7678510

3.3479623

2. 5187906

1. 5902204
L. 3948535
3
3
3
3
3
3
3
3
3
3
3
3
3
3
3
2
2
2
2
2
2
2
2
2
2
2

3
3
3
3
3
3
3
3
3
3
3
3
3
3
3
3
3
3
2
2
2
2
2
2
2
2

Table 111. Effective reflection coefficient (amplitude, $\left|C_{j}\right|$, and phase, Arg $C_{j}$ ) for the special ray configuration of the Norton type 301 atmosphere, applicable to the propagation of waves between the D-region ( $h_{3}=70$ kilometers) or the E-region $\left(h_{3}=90\right.$ kilometers $)$ of the ionosphere and the earth. 
$\Psi$

radians
$\left|C_{4}\right|$

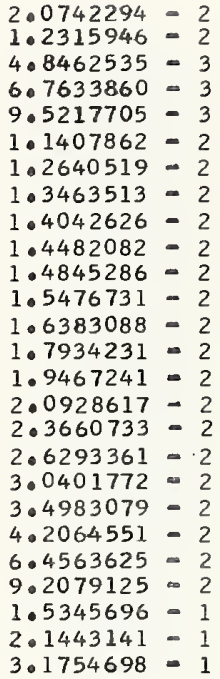

$\operatorname{Arg~C}$

$\mathrm{h}_{3}=70$ kilometers

$3 \cdot 7588510$
$3 \cdot 5003536$
$3 \cdot 8053291$
$4 \cdot 3013522$
$4 \cdot 1845825$
$3 \cdot 9809545$
$3 \cdot 7801828$
$3 \cdot 5961127$
$3 \cdot 4298245$
$3 \cdot 2799691$
$3 \cdot 1448923$
$2 \cdot 9131380$
$2 \cdot 6434133$
$2 \cdot 3410885$
$2 \cdot 1561697$
$2 \cdot 0425882$
1.9381140
1.9254881
$1 \cdot 9953821$
$2 \cdot 1206821$
$2 \cdot 3251392$
$2 \cdot 8572433$
$3 \cdot 3178256$
$3 \cdot 9890086$
$4 \cdot 4219054$
$4 \cdot 9130485$

1.5702963

1. 5697963

105687963

1.5677963

1.5667963

1.5657963

1.5647963

1.5637963

1. 5627963

1.5617963

1.5607963

1. 5587963

1.5557963

1. 5507963

1. 5457963

1.5407963

1.5307963

1.5207963

1.5057963

1.4907963

1.4707963

1.4207963

1.3707963

1.2707963

101707963

$9.7079630=1$

$\mathrm{h}_{3}=90$ kilometers

0.0005

0.0010

0.0020

0.0030

0.0040

0.0050

0.0060

0.0070

0.0080

0.0090

0.0100

0.0120

0.0150

0.0200

0.0250

0.0300

0.0400

0.0500

0.0650

0.0800

0.1000

0.1500

0.2000

0.3000

0.4000

0.6000

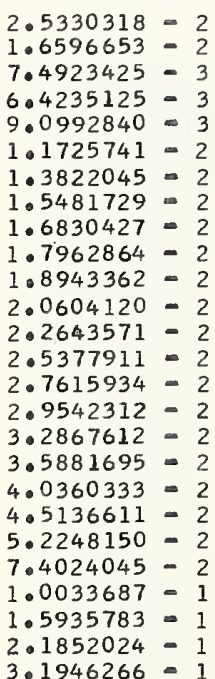

4.2771389
3.9287041
3.7412202
4.2104189
4.3073260
4.1841177
4.0230771
3.8656360
3.7212567
3.5914538
3.4754976
3.2797338
3.0567569
2.8095823
2.6558449
2.5571937
2.4541595
2.4224616
2.4464539
2.5187580
2.6545120
3.0565829
3.4411668
4.0417234
4.4474418
4.9208116

1.5702963

1.5697963

105687963

1.5677963

1.5667963

1.05657963

1.5647963

1.5637963

1.5627963

1.5617963

1.5607963

1.5587963

1.5557963

1.5507963

1.5457963

1.5407963

1.5307963

1.5207963

1.5057963

1.4907963

1.4707963

1.4207963

1.3707963

1.2707963

1.1707963

$9.7079630-1$ $d / j$

miles

2.2433895 1.2381675

1.2278386

1.2176623

1.2076369

1.1977599

1.1880278

1.1784374

1.1689853

1. 1596669

1.1504788

1.1324770

1. 1063547

1. 0649462

1.0259098

9.8899038

9.2076906

8.5914 .709

7.7737068

7.0657786

6.2644905

4. 7915900

3. 8174547

2.6471062

109802951

1.2445355

3
3
3
3
3
3
3
3
3
3
3
3
3
3
3
2
2
2
2
2
2
2
2
2
2
2

Table 112. Effective reflection coefficient (amplitude, $\left|C_{j}\right|$, and phase, $A_{r} C_{j}$ ) for the special ray configuration of the Norton type 301 atmosphere, applicable to the propagation of waves between the $\mathrm{D}-\mathrm{region}\left(\mathrm{h}_{3}=70\right.$ kilometers) or the $\mathrm{E}-\mathrm{region}\left(\mathrm{h}_{3}=90\right.$ kilometers $)$ of the ionosphere and the earth.

$\begin{array}{ll}1.4000730 & 3 \\ 1.3948535 & 3 \\ 1.3845147 & 3 \\ 1.3743220 & 3 \\ 1.3642736 & 3 \\ 1.3543670 & 3 \\ 1.3445989 & 3 \\ 1.3349661 & 3 \\ 1.3254647 & 3 \\ 1.3160909 & 3 \\ 1.3068406 & 3 \\ 1.2886954 & 3 \\ 1.2623103 & 3 \\ 1.2203378 & 3 \\ 1.1805854 & 3 \\ 1.1428039 & 3 \\ 1.0724503 & 3 \\ 1.0082107 & 3 \\ 9.2176798 & 2 \\ 8.4566114 & 2 \\ 7.5787071 & 2 \\ 5.9120543 & 2 \\ 407678510 & 2 \\ 3.3479623 & 2 \\ 2.5187906 & 2 \\ 1.5902204 & 2\end{array}$




$$
\begin{array}{ll}
\omega / \omega_{r}=0.0500 & f=3.3311126 \mathrm{kilocycles} \\
\phi_{1}=60 \text { degrees } & \sigma=0.005 \mathrm{mhos} / \text { meter } \\
& \epsilon_{2}=15
\end{array}
$$

$\Psi$

radians

$\left|\mathrm{C}_{4}\right|$

$\operatorname{Arg~C} 4$

$h_{3}=70$ kilometers

0.0005
0.0010

0.0020

0.0030

0.0040

0.0050

0.0060

0.0070

0.0080

0.0090

0.0100

0.0120

0.0150

0.0200

0.0250

0.0300

0.0400

0.0500

0.0650

0.0800

0.1000

0.1500

0.2000

0.3000

0.4000

0.6000

0.0005

0.0010

0.0020

0.0030

0.0040

0.0050

0.0060

0.0070

0.0080

0.0090

0.0100

0.0120

0.0150

0.0200

0.0250

0.0300

0.0400

0.0500

0.0650

0.0800

0.1000

0.1500

0.2000

0.3000

0.4000

0.6000

1.0800711
3.2259800
$4 \cdot 6645207$
4.1706090
3.8483571
3.5989054
3.3953068
3.2248245
3.0796681
2.9544202
2.8450481
2.6617673
2.4378703
6.2020944
5.4110705
5.3037120
5.2291856
5.2432850
5.3695892
5.6027825
6.0804198
1.1967949
1.9622656
2.8012506
3.3291791
3.9837077

$h_{3}=90$ kilometers

$1.0869451=2$ $5.4587875-3$

$1.0430211=2$

I. $4640221=2$

$1.6336806-2$

$1.6580305=2$

$1.6019476=2$

$1.5020563-2$

$1.3792251=2$

$1.2455755=2$

$1.1082621-2$

$8.3795815-3$

$4.6766575-3$

$5.1350870-4$

$4.1774532=3$

$7.0026400-3$

$1.0667298-2$

1.2582626 - 2

$1.3519997-2$

$1.3218346-2$

$1.2514781=2$

$2.0001573-2$

$3.9310257=2$

$8.6341465=2$

$1.3146697=1$

$2.0453566-1$

$1.1596296-2$ $4.4542575-3$

$7.7283100-3$

$1.3042216=2$

$1.5880514-2$

$1.7191471=2$

$1.7575788=2$

$1.7393701-2$

$1.6865758-2$

$1.6130489-2$

$1.5276671-2$

$1.3425264-2$

$1.0694319=2$

$6.9561780-3$

$4.6476233-3$

$4.0527770-3$

$7.5201230-3$

$9.4517675-3$

$1.1015522-2$

$1.3669378-2$

$2.6445209=2$

$4.5778565-2$

$9.0857460=2$

$1.3442397=$

$2.0587630=1$
$5.6920560-3$

2.4431732
2.7202418
4.3031924
4.1632156
3.9489298
3.7496971
3.5737259
3.4192492
3.2828891
3.1613462
3.0517257
2.8588258
2.6079241
2.1973128
1.6612061
1.0063614
$2.8025179=1$
$1.0968795-1$
$1.6539937-1$
$3.7643870-1$
$7.5116962-1$
1.5803019
2.1316447
2.8650158
3.3613649
3.9947582

1.5702963

1.5697963

1. 5687963

1. 5677963

1. 5667963

1. 5657963

1. 5647963

1. 5637963

1. 5627963

1.5617963

1. 5607963

1. 5587963

1.5557963

1. 5507963

1. 5457963

1.5407963

1.5307963

1. 5207963

1.5057963

1.4907963

1.4707963

1.4207963

1. 3707963

1. 2707963

1. 1707963

$9.7079630=1$ $\mathrm{d} / \mathrm{j}$

miles

$\begin{array}{ll}1.2433895 & 3 \\ 1.2381675 & 3 \\ 1.2278386 & 3 \\ 1.2176623 & 3 \\ 1.2076369 & 3 \\ 1.1977599 & 3 \\ 1.1880278 & 3 \\ 1.1784374 & 3 \\ 1.1689853 & 3 \\ 1.1596669 & 3 \\ 1.1504788 & 3 \\ 1.1324770 & 3 \\ 1.1063547 & 3 \\ 1.0649462 & 3 \\ 1.0259098 & 3 \\ 9.8899038 & 2 \\ 9.2076906 & 2 \\ 8.5914709 & 2 \\ 7.7737058 & 2 \\ 7.0657786 & 2 \\ 6.2644905 & 2 \\ 4.7915900 & 2 \\ 3.8174547 & 2 \\ 2.6471062 & 2 \\ 1.9802951 & 2 \\ 1.2445355 & 2 \\ 1 & \end{array}$

1.4000780

1. 3948535

1.3845147

1.3743220

1. 3642736

1.3543670

1. 3445989

1. 3349661

1. 3254647

1. 3160909

1. 3068406

1. 2886954

1.2623103

1. 2203378

1. 1805854

1. 1428039

1. 0724503

1.0082107

9.2176798

8.4566114

7.5787071

5.9120543

4. 7678510

3. 3479623

2.5187906

1. 5902204
Table 113. Effective reflection coefficient (amplitude, $\left|C_{j}\right|$, and phase, Arg $C_{j}$ ) for the special ray configuration of the Norton type 301 atmosphere, applicable to the propagation of waves between the D-region $\left(h_{3}=70\right.$ kilometers) or the E-region $\left(h_{3}=90\right.$ kilometers $)$ of the ionosphere and the earth. 


$$
\begin{array}{ll}
\omega / \omega_{\mathrm{r}}=0.1000 & \mathrm{f}=6.6622252 \mathrm{kilocycles} \\
\phi_{1}=60 \text { degrees } & \sigma=0.005 \mathrm{mhos} / \mathrm{meter} \\
& \epsilon_{2}=15
\end{array}
$$

$\Psi$

radians

0.0005

0.0010

0.0020

0.0030

0.0040

0.0050

0.0060

0.0070

0.0080

0.0090

0.0100

0.0120

0.0150

0.0200

0.0250

0.0300

0.0400

0.0500

0.0650

0.0800

0.1000

0.1500

0.2000

0.3000

0.4000

0.6000

0.0005

0.0010

0.0020

0.0030

0.0040

0.0050

0.0060

0.0070

0.0080

0.0090

0.0100

0.0120

0.0150

0.0200

0.0250

0.0300

0.0400

0.0500

0.0650

0.0800

0.1000

0.1500

0.2000

0.3000

0.4000

0.6000 $\left|c_{4}\right|$

$\operatorname{Arg} C_{4}$

\begin{tabular}{|c|c|}
\hline $\begin{array}{l}4.6346140 \\
4.0137580\end{array}$ & $=$ \\
\hline $\begin{array}{l}3.4114764 \\
3.1496930\end{array}$ & - \\
\hline 2.9399246 & - \\
\hline 2.7087949 & \\
\hline 2.4534002 & \\
\hline 2.1854488 & - \\
\hline 1.9160166 & \\
\hline 1.6528313 & \\
\hline 1.4007763 & \\
\hline 9.4199230 & \\
\hline 4.3239978 & \\
\hline 7.0690325 & \\
\hline 1.3039658 & \\
\hline 1.7901872 & \\
\hline 2.4751039 & \\
\hline 2.8862904 & \\
\hline 3.1697675 & \\
\hline 3.1932745 & \\
\hline 2.9741142 & - \\
\hline 1.7947624 & - \\
\hline 9.1136080 & \\
\hline 4.7293728 & \\
\hline 9.0147690 & \\
\hline 59 & \\
\hline
\end{tabular}

$h_{3}=70$ kilometers

5. 1551806

4. 5160621

4.0238946

3.6527068

3. 3640463

3. 1324200

2. 9429750

2.7872670

2. 6608492

2. 5622850

2. 4595569

2. 7816362

4.0739115

$4 \cdot 1158304$

400455341

3. 9256500

3. 8643024

3. 8518741

3.9027467

4.0354846

$4 \cdot 6194490$

$7.2573590=$

1. 8088131

2. 3622876

3.0246275

$\mathrm{h}_{3}=90$ kilometers

$2.6968962-2$

$2.4399687-2$

$2.4899863-2$

$2.6589511-2$

$2.7228727=2$

$2.6803953=2$

$2.5648112-2$

$2.4052352-2$

$2.2219772=2$

$2.0282706=2$

$1.8324794-2$

$1.4536670-2$

$9.5293965-3$

$4.4652402-3$

$6.4104390-3$

$1.0181699-2$

$1.6076117-2$

$1.9763131=2$

$2.2472031-2$

$2.2937527-2$

$2.1317600-2$

$1.1694422-.2$

$1.2244753-2$

$5.1654480-2$

$9.2980090-2$

$1.5489843=1$
5. 8050342

5. 3294475

4. 5815739

400991409

3. 7619471

3. 5046202

3. 2978314

3. 1270325

2.9841373

2. 8643099

2. 7645824

$2 \cdot 6197074$

$2 \cdot 5455625$

3. 1210804

3. 9952377

4. 1663533

4. 1742072

4. 1510419

4. 1564177

4.2093824

4.3407044

7. $1195880-1$

1.8814202

2. 3951147
5.0520060

3. 0361727
$T_{j}$

$\mathrm{d} / \mathrm{j}$

miles

Table 114. Effective reflection coefficient (amplitude, $\left|C_{j}\right|$, and phase, $\mathrm{Arg}_{\mathrm{r}} \mathrm{C}_{j}$ ) for the special ray configuration of the Nortan type 301 atmosphere, applicable to the propagation of waves between the D-region $\left(h_{3}=70\right.$ kilometers) or the E-region $\left(h_{3}=90\right.$ kilometers $)$ of the

ionosphere and the earth.

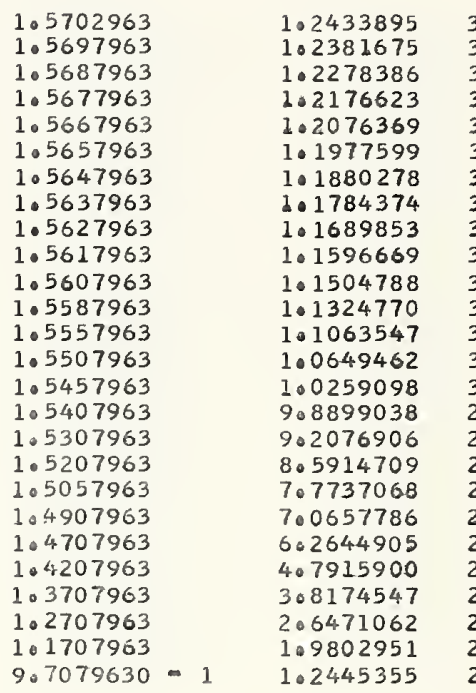

1.5702963

1.5697963

1.5687963

1.5677963

1.5667963

1. 5657963

1.5647963

1. 5637963

1.5627963

1.5617963

1. 5607963

1.5587963

1.5557963

1. 5507963

1.5457963

1. 5407963

1. 5307963

. 5207963

1. 5057963

1.4907963

1.4707963

1.4207963

1. 3707963

1.1707963

1.4000780

1. 3845147

1.3743220

$1 \cdot 3642736$

1.3543670

1.3445989

1.3349661

1.3254647

1. 3160909

1.3068406

1. 2886954

1. 2623103

1. 2203378

1. 1805854

1. 1428039

1.0724503

1.0082107

9.2176798

8.4566114

7. 5787071

5.9120543

4. 7678510

3.3479623

2. 5187906

1. 5902204 


$$
\begin{array}{ll}
\omega / \omega_{r}=0.2000 & f=13.324450 \text { kilocycles } \\
\phi_{1}=60 \text { degrees } & \sigma=0.005 \text { mhos } / \text { meter } \\
\epsilon_{2}=15
\end{array}
$$

$\Psi$

radians

0.0005
0.0010
0.0020
0.0030
0.0040
0.0050
0.0060
0.0070
0.0080
0.0090
0.0100
0.0120
0.0150
0.0200
0.0250
0.0300
0.0400
0.0500
0.0650
0.0800
0.1000
0.1500
0.2000
0.3000
0.4000
0.6000

0.0005

0.0020

0.0030

0.0040

0.0050

0.0060

0.0070

0.0080

0.0090

0.0100

0.0120

0.0150

0.0200

0.0250

0.0300

0.0400

0.0500

0.0650

0.0800

0.1000

0.1500

0.2000

0.3000

0.4000

0.6000
0.0010 $\left|c_{4}\right|$

Arg C 4

$\mathrm{h}_{3}=70$ kilometers

$1.1655163=1$
$1.0294902=1$
$8.2746325=2$
$6.8912455=2$
$5.8882995=2$
$5.1064985=2$
$4.4553905=2$
$3.8865912=2$
$3.3749175=2$
$2.9070590=2$
$2.4755428=2$
$1.7041698=2$
$7.3798450=3$
$5.9299035=3$
$1.5695704=2$
$2.3690619=2$
$3.5801975=2$
$4.4147774=2$
$5.1661540=2$
$5.4943915=2$
$5.5044700=2$
$4.4823518=2$
$2.9132130=2$
$1.1724844=2$
$4.9728627=2$
$1.1082665=1$

$1.1082665=$

4.6823692
4.4808393
4.0935270
3.7369757
3.4178409
3.1366650
2.8901690
2.6737597
2.4830070
2.3142685
2.1648665
1.9187929
1.7178722
3.8871879
3.7734202
3.5867181
3.3145519
3.1506040
3.0263739
2.9893383
3.0197744
3.2967979
3.7064486
$3.1138897-1$
1.2206050
1.8641436

$\mathrm{h}_{3}=90$ kilometers

$8.7847425=2$

$7.8965280-2$

$6.6481390-2$

$5.8370550=2$

$5.2478830-2$

$4.7636535-2$

$4.3292625=2$

$3.9219177-2$

$3.5336375-2$

$3.1624823=2$

$2.8085578-2$

$2.1543526-2$

$1.3112039=2$

$4.4145045-3$

$9.6166185-3$

$1.6502214=2$

$2.7210491-2$

$3.4607161-2$

$4.1404620=2$

$4.4641784=2$

$4.5322688=2$

$3.7364536=2$

$2.3373145-2$

$1.5200190-2$

$5.2464920=2$

$1.1181406=1$

3. 2512514

2.6523052
4.8389635

4.6182588

4. 2030241

3. 8356688

3. 5200612

3.0215483

2.8237883

2.5029646

2. 3729031

2. 1645785

1. 9884963

2. 6939671

3.6503966

3. 6468060

3. 4761784

3.3459957

3. 2386086

3.2026414

3. 2241916

3.4648187

3. 8582953

5. $2679571-1$

1.2566140

1. 8749039
1. 5702963

1. 5697963

1.5687963

1.5677963

1.5667963

1.5657963

1.5647963

1.5637963

1.5627963

I. 5617963

1.5557963

1. 5507963

1.5457963

1. 5407963

1.5307963

1.5207963

1.5057963

1.4907963

1.4707963

1.4207963

1.2707963

1.1707963

$9.7079630-1$

1.2433895 1.2381675

1. 2278386

1.2176623

1.2076369

1.1977599

1. 1880278

1.1784374

1. 1689853

1. 1596669

I. 1504788

1. 1324770

1.1063547

1.0649462

1.0259098

9.8899038

9.2076906

8.5914709

7. 7737068

7.0657786

6.2644905

4.7915900

3.8174547

2.6471062

1.9802951

1. 2445355

1.5702963

1.5697963

1.5687963

1.5677963

I. 5657963

1.5647963

1.5637963

1.5627963

1.5617963

1.5607963

1. 5587963

1.5557963

1. 5507963

I. 5457963

1. 5407963

1. 5307963

1. 5207963

1.5057963

1.4907963

1.4707963

1.4207963

1.3707963

1. 2707963

1. 1707963

9.7079630 - 1
1.4000780

1.3948535

1. 3845147

1.3743220

1.3642736

1.3543670

1.3445989

1.3349661

1.3254647

1.3160909

1.3068406

1.2886954

1.2623103

1.2203378

1.1805854

1. 1428039

1.0724503

1. 0082107

9.2176798

8.4566114

7.5787071

5.9120543

4. 7678510

3.3479623

2. 5187906

1. 5902204
1. 5607963

1.5587963

1.3707963

1.5667963

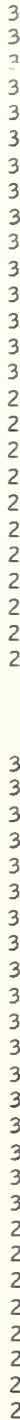

Table 115. Effective reflection coefficient (amplitude, $\left|C_{j}\right|$, and phase, $\operatorname{Arg} C_{j}$ ) for the special ray configuration of the Norton type 301 atmosphere, applicable to the propagation of waves between the D-region $\left(h_{3}=70\right.$ kilometers $)$ or the E-region $\left(h_{3}=90\right.$ kilometers $)$ of the ionosphere and the earth. 


$$
\begin{array}{ll}
\omega / \omega_{r}=1.0000 & f=66.622252 \text { kilocycles } \\
\phi_{1}=60 \text { degrees } & \sigma=0.005 \text { mhos } / \text { meter } \\
& \epsilon_{2}=15
\end{array}
$$

$\Psi$

radians

0.0005
0.0010
0.0020
0.0030
0.0040
0.0050
0.0060
0.0070
0.0080
0.0090
0.0100
0.0120
0.0150
0.0200
0.0250
0.0300
0.0400
0.0500
0.0650
0.0800
0.1000
0.1500
0.2000
0.3000
0.4000
0.6000

0.0005

0.0010

0.0020

0.0030

0.0040

0.0050

0.0060

0.0070

0.0080

0.0090

0.0100

0.0120

0.0150

0.0200

0.0250

0.0300

0.0400

0.0500

0.0650

0.0800

0.1000

0.1500

0.2000

0.3000

0.4000

0.6000 $\left|c_{4}\right|$

$\operatorname{Arg~C} 4$

$\mathrm{h}_{3}=70$ kilometers

$3.2248785=1$
$3.0156985=1$
$2.6437971=1$
$2.3262601=1$
$2.0549163=1$
$1.8226138=1$
$1.6231476=1$
$1.4511977=1$
$1.3022391=1$
$1.1724587=1$
$1.0586766=1$
$8.6901990=2$
$6.5226070=2$
$4.0111525=2$
$2.3589660=2$
$1.5029184=2$
$2.3091968=2$
$3.7923947=2$
$5.7210315=2$
$7.2223720=2$
$8.5880010=2$
$9.5509755=2$
$8.5910445=2$
$5.4340000=2$
$2.9206674=2$
$5.9323140=3$

3.0477568
2.9657134
2.8026916
2.6412923
2.4817995
2.3245328
2.1698124
2.0179401
1.8691622
1.7236552
1.5815083
1.3071844
$9.1771302-1$
$2.9742364-1$
5.8964334
4.9313452
3.2437794
2.5767566
2.0395245
1.7147973
1.4411816
1.1098954
$9.8790233-1$
$9.6996605-1$
1.0934935
2.1834488

$\mathrm{h}_{3}=90$ kilometer $\mathrm{s}$

3.0111938
2.9278186
2.7626001
2.5997402
2.4396533
2.2827498
2.1294104
1.9799556
1.8346116
1.6935145
1.5566959
1.2955422
$9.3154595-1$
$3.7136205-1$
6.0718840
5.2312610
3.2409412
2.5647401
2.0459591
1.7308934
1.4626281
1.1331216
1.0093696
$9.8784773-1$
1.1112329
2.2417016

1. 5702963

1. 5697963

1. 5687963

1.5677963

1. 5667963

1. 5657963

1. 5647963

1. 5637963

1. 5627963

1.5617963

1. 5607963

1. 5587963

1.5557963

1. 5507963

1. 5457963

1.5407963

1. 5307963

1. 5207963

1. 5057963

1.4907963

1.4707963

1.4207963

1.3707963

1. 2707963

1.1707953

$907079630-1$

1. 5702963

1.5697963

1. 5637963

1. 5677963

1. 5667963

1.05657963

1.5647963

1.5637963

1.5627963

1.5617963

1.5607963

1.5587963

1.5557963

1. 5507963

1.5457963

1. 5407963

1.5307963

1. 5207963

1. 5057963

1.4907963

1.4707963

1.4207963

1. 3707963

1. 2707963

1. 1707963

$9.7079630-1$

$\begin{array}{ll}1.2433895 & 3 \\ 1.2381675 & 3 \\ 1.2278386 & 3 \\ 1.2176623 & 3 \\ 1.2076369 & 3 \\ 1.1977599 & 3 \\ 1.1880278 & 3 \\ 1.1784374 & 3 \\ 1.1689853 & 3 \\ 1.1596669 & 3 \\ 1.1504788 & 3 \\ 1.1324770 & 3 \\ 1.1063547 & 3 \\ 1.0649462 & 3 \\ 1.0259098 & 3 \\ 9.8899038 & 2 \\ 9.2076906 & 2 \\ 8.5914709 & 2 \\ 7.7737068 & 2 \\ 7.0657786 & 2 \\ 6.2644905 & 2 \\ 407915900 & 2 \\ 3.8174547 & 2 \\ 2.6471062 & 2 \\ 1.9802951 & 2 \\ 1.2445355 & 2\end{array}$

$d / j$

miles

$\begin{array}{ll}1.4000780 & 3 \\ 1.3948535 & 3 \\ 1.3845147 & 3 \\ 1.3743220 & 3 \\ 1.3642736 & 3 \\ 1.3543670 & 3 \\ 1.3445989 & 3 \\ 1.3349661 & 3 \\ 1.3254647 & 3 \\ 1.3160909 & 3 \\ 1.3068406 & 3 \\ 1.2886954 & 3 \\ 1.2623103 & 3 \\ 1.2203378 & 3 \\ 1.1805854 & 3 \\ 1.1428039 & 3 \\ 1.0724503 & 3 \\ 1.0082107 & 3 \\ 9.2176798 & 2 \\ 8.4566114 & 2 \\ 7.5787071 & 2 \\ 5.9120543 & 2 \\ 4.7678510 & 2 \\ 3.3479623 & 2 \\ 2.5187906 & 2 \\ 1.5902204 & 2\end{array}$

Table 116. Effective reflection coefficient (amplitude, $\left|C_{j}\right|$, and phase, Arg $C_{j}$ ) for the special ray configuration of the Norton type 301 atmosphere, applicable to the propagation of waves between the D-region ( $h_{3}=70$ kilometers) or the E-region $\left(h_{3}=90\right.$ kilometers $)$ of the ionosphere and the earth. 


$$
\begin{array}{ll}
\omega / \omega_{\mathbf{r}}=2.0000 & f=133.24450 \mathrm{kilocycles} \\
\phi_{1}=60 \text { degrees } & \sigma=0.005 \mathrm{mhos} / \mathrm{meter} \\
\epsilon_{2}=15
\end{array}
$$

$\begin{array}{lllll}\Psi & \left|\mathrm{C}_{4}\right| & \mathrm{Arg} \mathrm{C}_{4} & \tau_{j} & \mathrm{~d} / \mathrm{j} \\ \text { radians } & & \mathrm{milles}\end{array}$

$\mathrm{h}_{3}=70$ kilometers

$\begin{array}{ll}0.0005 & 2.9390474=1 \\ 0.0010 & 2.8135028=1 \\ 0.0020 & 2.5817278=1 \\ 0.0030 & 2.3733962=1 \\ 0.0040 & 2.1859386=1 \\ 0.0050 & 2.0170156=1 \\ 0.0060 & 1.8645180=1 \\ 0.0070 & 1.7265454=1 \\ 0.0080 & 1.6014028=1 \\ 0.0090 & 1.4875847=1 \\ 0.0100 & 1.3837597=1 \\ 0.0120 & 1.2015467=1 \\ 0.0150 & 9.7826470=2 \\ 0.0200 & 6.9777160=2 \\ 0.0250 & 4.9102153=2 \\ 0.0300 & 3.3244403=2 \\ 0.0400 & 1.2001792=2 \\ 0.0500 & 1.1656553=2 \\ 0.0650 & 2.6755526=2 \\ 0.0800 & 3.8535920=2 \\ 0.1000 & 4.9349917=2 \\ 0.1500 & 5.8691135=2 \\ 0.2000 & 5.3203965=2 \\ 0.3000 & 3.1535555=2 \\ 0.4000 & 1.5057656=2 \\ 0.6000 & 1.9448005=3\end{array}$

2.2338879
2.1760292
2.0613769
1.9482436
1.8367459
1.7269962
1.6191034
1.5131545
1.4092285
1.3073860
1.2076630
$1.0146247-1$
$7.4075734-1$
$3.2147098-1$
6.2184722
5.8407975
4.8223441
2.8813367
1.9183363
1.4560974
1.0403782
$4.0877797-1$
$1.5844260-2$
5.7495621
5.3071616
4.4683727

1.5702963

1. 5697963

1. 5687963

1. 5677963

1. 5667963

1. 5657963

1. 5647963

I. 5637963

1. 5627963

I. 5617963

I. 5607963

1.5587963

1.5557963

1. 5507963

1. 5457963

1. 5407963

1. 5307963

1. 5207963

I.5057963

1.4907963

1.4707963

I. 4207963

1. 3707963

1. 2707963

1. 1707963

$9.7079630-1$

1.2433895

1.2381675

1. 2278386

1. 2176623

1.2076369

1.1977599

1. 1880278

1. 1784374

1. 1689853

1.1596669

1. 1504788

1.1324770

1. 1063547

1.0649462

1.0259098

9.8899038

9.2076906

8.5914709

7.7737068

7.0657786

6.2644905

4.7915900

3.8174547

2.6471062

1.9802951

1. 2445355

$\mathrm{h}_{3}=90$ kilometers

0.0005
0.0010
0.0020
0.0030
0.0040
0.0050
0.0060
0.0070
0.0080
0.0090
0.0100
0.0120
0.0150
0.0200
0.0250
0.0300
0.0400
0.0500
0.0650
0.0800
0.1000
0.1500
0.2000
0.3000
0.4000
0.6000
2.0704173

2.0124303

1. 8979229

$1.785478 \%$

1.6752251

1. 5672661

1.4616904

1.3585685

1. 2579458

1. 1598444

1.0642638

$8.8057058-1$

6. $2287465-1$

2.3511525 -

6.1701972

5.8433641

5. 0699471

2. 7518408

1. 7834107

1.3576480

9.6585768 - 1

$3.5762962-1$

6.2579563

5. 7191423

5.2822960

4.4483991
1.5702963

1.5697963

I. 5687963

1.5677963

I. 5667963

1. 5657963

1.5647963

1.5637963

1. 5627963

1. 5617963

1.5607963

I. 5587963

1.5557963

I. 5507963

1.5457963

1. 5407963

1. 5307963

i. 5207963

1. 5057963

1.4907963

1.4707963

1.4207963

1.3707963

1. 2707963

1.1707963

$9.7079630=1$ $\begin{array}{ll}1.4000780 & 3 \\ 1.3948535 & 3 \\ 1.3845147 & 3 \\ 1.3743220 & 3 \\ 1.3642736 & 3 \\ 1.3543670 & 3 \\ 1.3445989 & 3 \\ 1.3349661 & 3 \\ 1.3254647 & 3 \\ 1.3160909 & 3 \\ 1.3068406 & 3 \\ 1.2886954 & 3 \\ 1.2623103 & 3 \\ 1.2203378 & 3 \\ 1.1805854 & 3 \\ 1.1428039 & 3 \\ 1.0724503 & 3 \\ 1.0082107 & 3 \\ 9.2176798 & 2 \\ 8.4566114 & 2 \\ 7.5787071 & 2 \\ 5.9120543 & 2 \\ 4.7678510 & 2 \\ 3.3479623 & 2 \\ 2.5187906 & 2 \\ 1.5902204 & 2\end{array}$
3
3
3
3
3
3
3
3
3
3
3
3
3
3
3
2
2
2
2
2
2
2
2
2
2
2

Table 117. Effective reflection coefficient (amplitude, $\left|C_{j}\right|$, and phase, Arg $C_{j}$ ) for the special ray configuration of the Norton type 301 atmosphere, applicable to the propagation of waves between the D-region ( $h_{3}=70$ kilometers) or the E-region ( $h_{3}=90$ kilometers) of the ionosphere and the earth. 


$$
\begin{array}{ll}
\omega / \omega_{\mathbf{r}}=5.0000 & f=333.11126 \text { kilocycles } \\
\phi_{1}=60 \text { degrees } & \sigma=0.005 \text { mhos } / \text { meter } \\
& \epsilon_{2}=15
\end{array}
$$

$\Psi$

radians

0.0010

0.0020

0.0030

0.0040

0.0050

0.0060

0.0070

0.0080

0.0090

0.0100

0.0120

0.0150

0.0200

0.0250

0.0300

0.0400

0.0500

0.0650

0.0800

0.1000

0.1500

0.2000

0.3000

0.4000

0.6000

0.0005

0.0010

0.0020

0.0030

0.0040

0.0050

0.0060

0.0070

0.0080

0.0090

0.0100

0.0120

0.0150

0.0200

0.0250

0.0300

0.0400

0.0500

0.0650

0.0800

0.1000

0.1500

0.2000

0.3000

0.4000

0.6000
$\left|\mathrm{C}_{4}\right|$

$\operatorname{Arg~C} 4$

$\mathrm{h}_{3}=70$ kilometers

$2.0565230=$ $2.0157257-1$

$1.9374755-1$

1.8633887 - 1

$1.7931459=1$

I. $7264481-I$

$1.6630135=1$

$1.6025939-1$

$1.5449547-1$

$1.4898903-1$

$1.4372072=1$

$1.3383099-1$

$1.2039973=1$

$1.0102847=1$

$8.4598955-2$

$7.0480330=2$

4. $7620161-2$

$3.0253844-2$

$1.1584061-2$

$1.3788795-3$

1. $1534486-2$

$2.0750594=2$

$1.8938349-2$

$8.7776735-3$

$2.3430928-3$

$5.5694900 \approx 5$

$9.9585851-1$
$9.5962166-1$
$8.8812963-1$
$8.1795409-1$
$7.4910561-1$
$6.8158839-1$
$6.1540119-1$
$5.5053775-1$
$4.8698773-1$
$4.2473724-1$
$3.6376582-1$
$2.4558268-1$
$7.7328460-2$
6.1022415
5.8684164
5.6555004
5.2808288
4.9612761
4.5825850
$2.2975636-1$
$4.7764362-1$
5.9375121
5.2377286
3.9868555
2.8277043
1.6404501

$h_{3}=90$ kilometers

$6.3006286-1$
$5.9539056-1$
$5.2719508-1$
$4.6051758-1$
$3.9534660-1$
$3.3165155-1$
$2.6940714-1$
$2.0858690-1$
$1.4916028-1$
$9.1084530-2$
$3.4332340-2$
6.2078371
6.0523173
5.8147485
5.6006635
5.4065766
5.0681622
4.7867076
4.4905642
5.2262117
$1.7745991-1$
5.7668973
5.1021089
3.8845314
2.7577122
1.6304243

1.5702963

1.5697963

I. 5687963

I. 5677963

1.5667963

1.5657963

1. 5647963

1.5637963

I. 5627963

1.5617963

I. 5607963

I. 5587963

1. 5557963

1.5507963

1. 5457963

1. 5407963

I. 5307963

1.5207963

1. 5057963

1.4907963

1.4707963

1.4207963

1.3707963

1.2707963

1. 1707963

$9.7079630-1$ $\mathrm{d} / \mathrm{j}$

miles

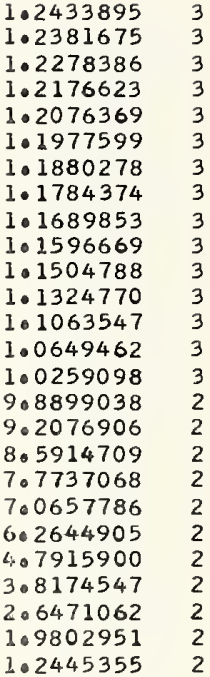

$1.4000780 \quad 3$

$1.3948535 \quad 3$

1. $3845147 \quad 3$

$1.3743220 \quad 3$

$1 \cdot 3642736 \quad 3$

$1.3543670 \quad 3$

1. $3445989 \quad 3$

$1.3349661 \quad 3$

$1.3254647 \quad 3$

1.31609093

$1.3068406 \quad 3$

1. $2886954 \quad 3$

$1.2623103 \quad 3$

1. $2203378 \quad 3$

1. $1805854 \quad 3$

1. 14280393

$1.0724503 \quad 3$

1. 00821073

$9.2176798 \quad 2$

$8.4566114 \quad 2$

$7.5787071 \quad 2$

$5.9120543 \quad 2$

4. $7678510 \quad 2$

$3.3479623 \quad 2$

$2.5187906 \quad 2$

I. $5902204 \quad 2$

Table 118. Effective reflection coefficient (amplitude, $\left.\mid C_{j}\right)$, and phase, Arg $C_{j}$ ) for the special ray configuration of the Norton type 301 atmosphere, applicable to the propagation of waves between the D-region ( $h_{3}=70$ kilometers) or the E-region $\left(h_{3}=90\right.$ kilometers) of the ionosphere and the earth. 


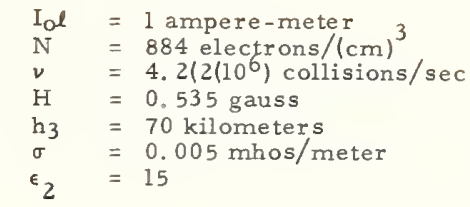

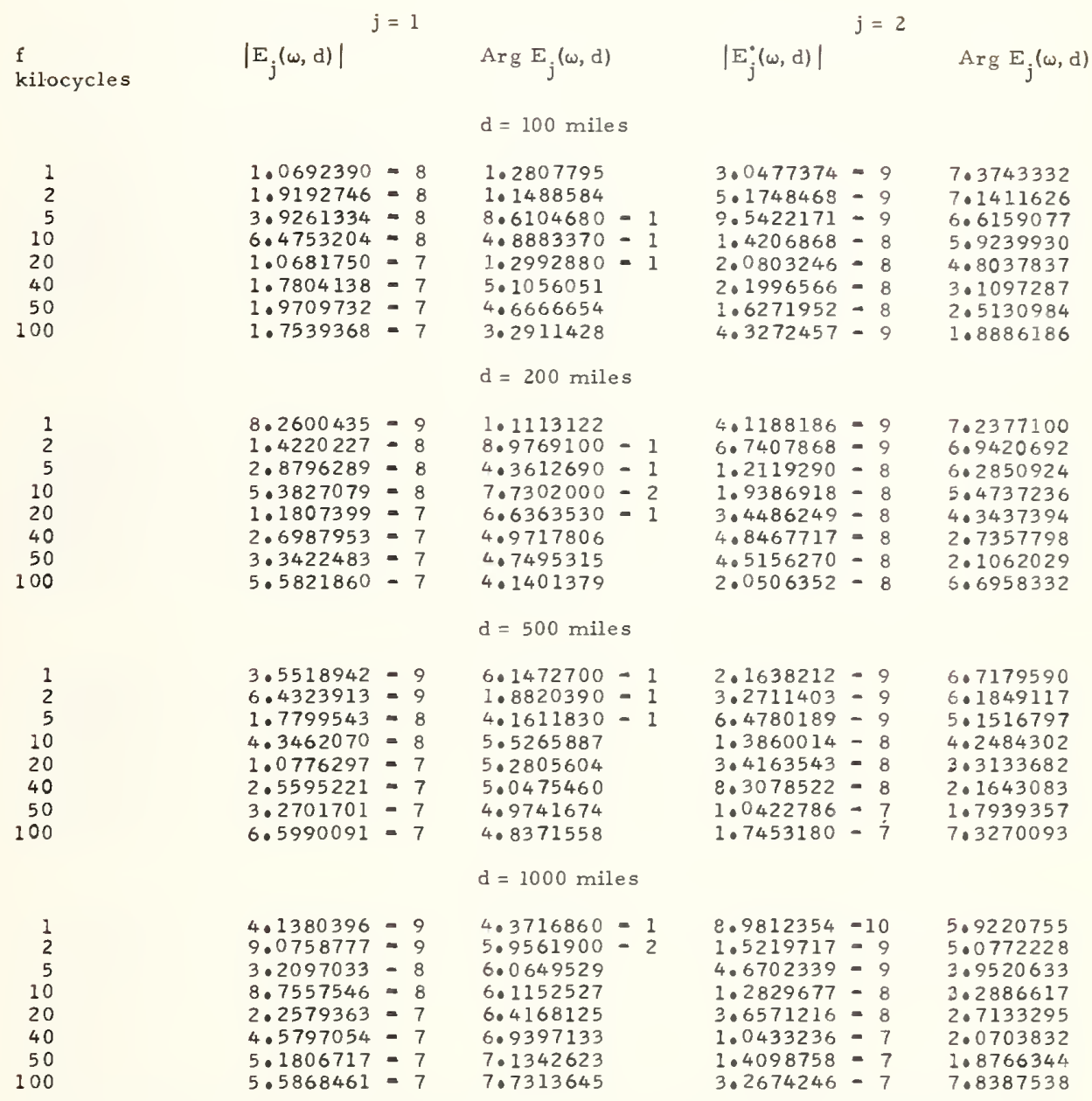

Table 119. Very low frequency and low frequency part of sky-wave transfer characteristic (amplitude, $\left|E_{j}(\omega, d)\right|$, and phase, $\left.\operatorname{Arg} E_{j}(\omega, d)\right)$ as suming the geometrical-optical and quasilongitudinal-Fresnel approximations, for various distances, d, electron density, N, collision frequency, $v$ and earth's magnetic field strength, $H$.

$-193-$ 


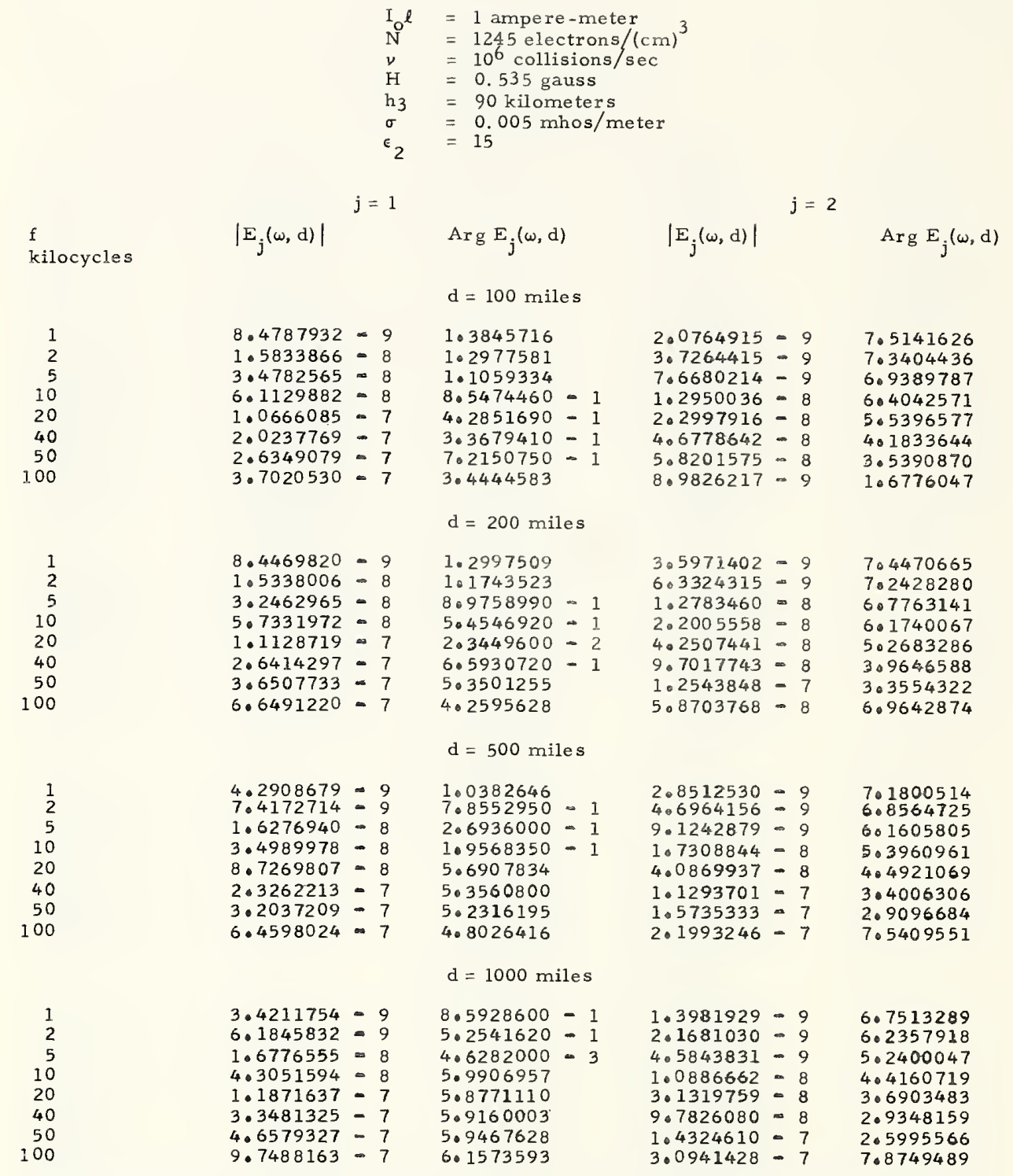

Table 120. Very low frequency and low frequency part of sky-wave transfer characteristic (amplitude, $\left|E_{j}(\omega, d)\right|$, and phase, $\left.\operatorname{Arg} E_{j}(\omega, d)\right)$ assuming the geometrical-optical and quasilongitudinal-Fresnel approximations, for various distances, d, electron density, N, collision frequency, $\nu$ and earth's magnetic field strength, $H$. $-194-$ 


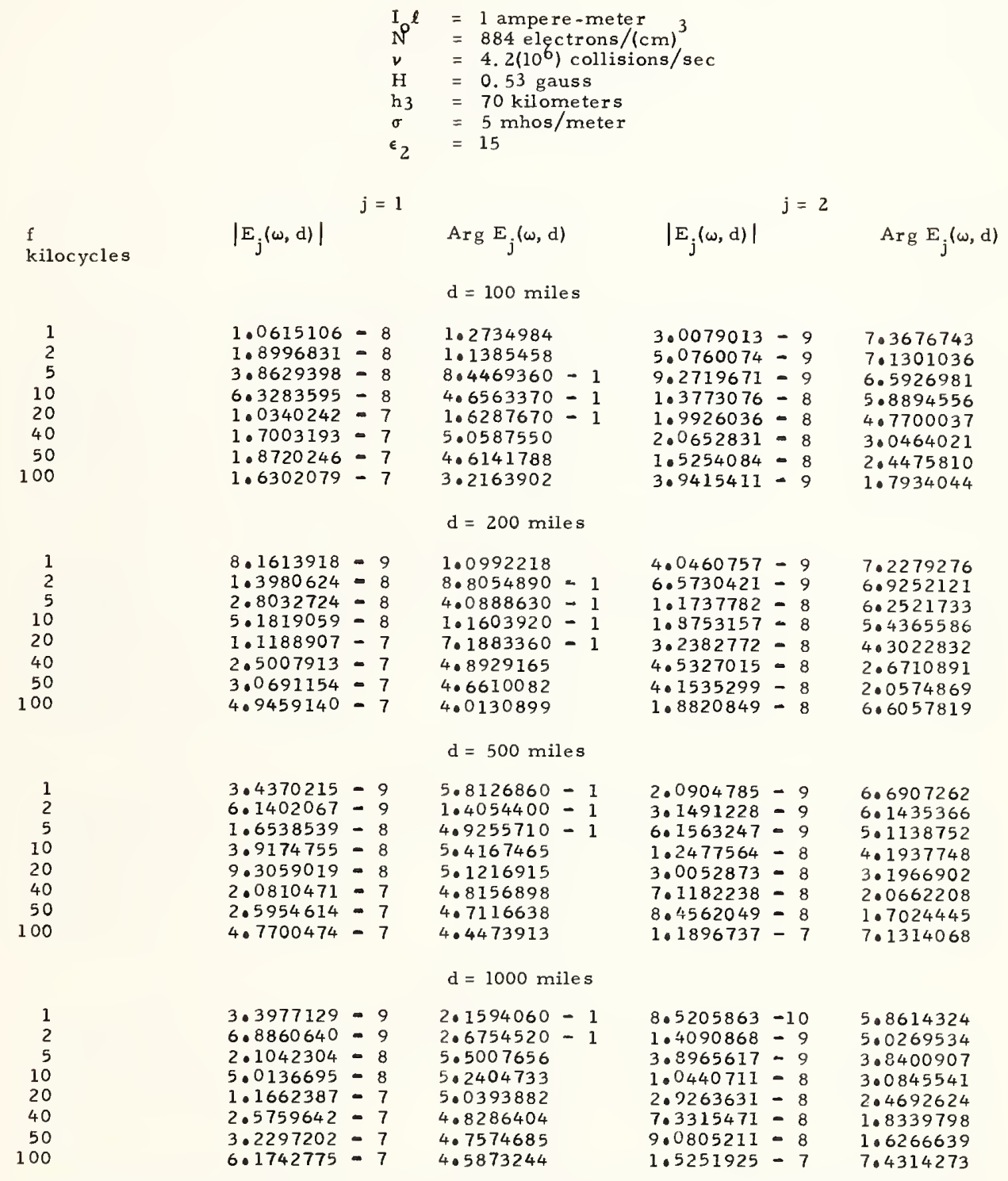

Table 12l. Very low frequency and low frequency part of sky-wave transfer characteristic (amplitude, $\left|E_{j}(\omega, d)\right|$, and phase, $\left.\operatorname{Arg} E_{j}(\omega, d)\right)$ assuming the geometrical-optical and quasilongitudinal-Fresnel approximations, for various distances, d, electron density, $N$, collision frequency, $v$ and earth's magnetic field strength, $H$, 


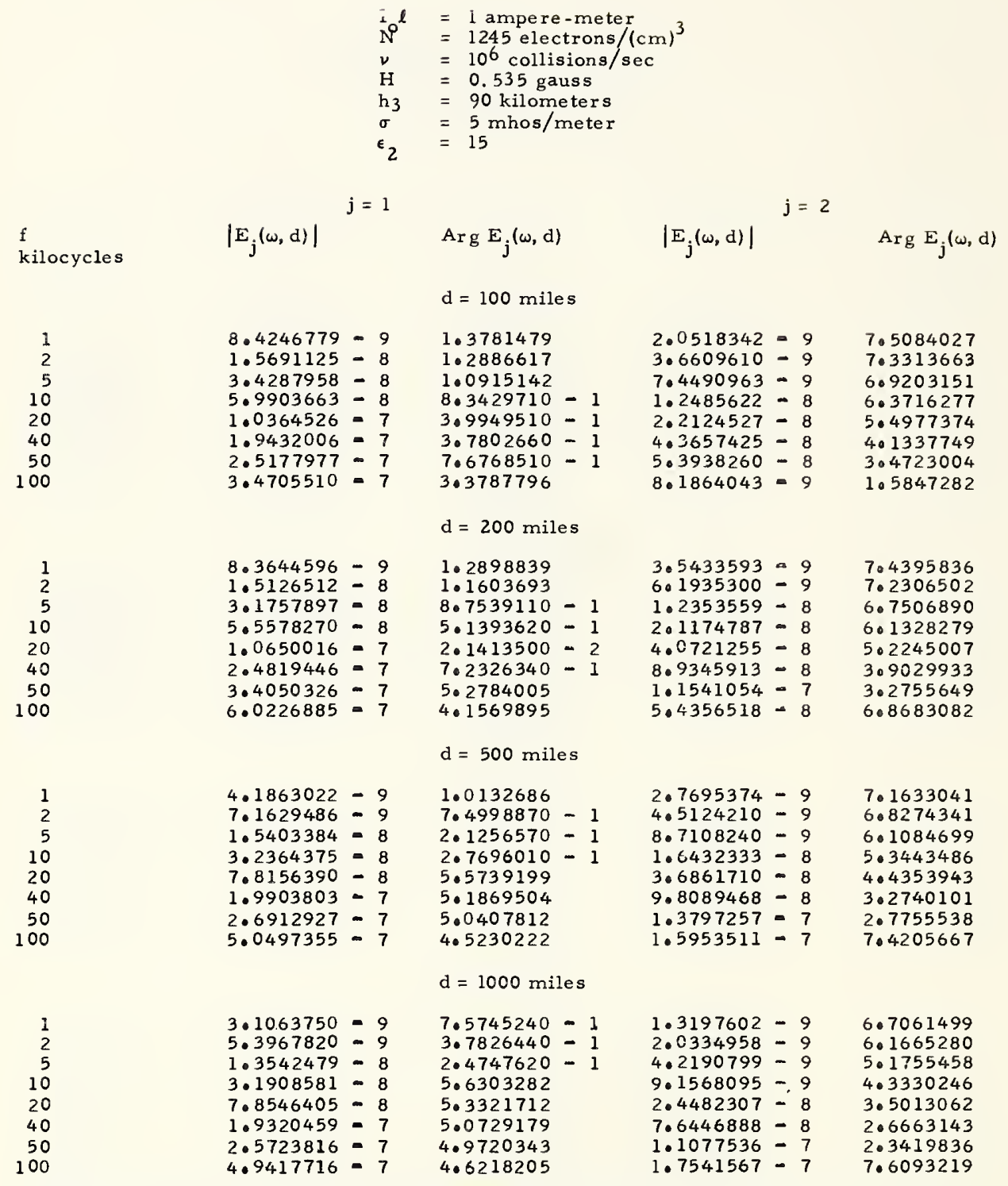

Table 122. Very low frequency and low frequency part of sky-wave transfer characteristic (amplitude, $\left|E_{j}(\omega, d)\right|$, and phase, $\left.\operatorname{Arg~} E_{j}(\omega, d)\right)$ assuming the geometrical-optical and quasilongitudinal-Fresnel approximations, for various distances, d, electron density, $N$, collision frequency, $v$ and earth's magnetic field strength, $H$.

$-196-$ 
d

miles

100

200

500

1000 a - a

seconds

$\alpha_{j}$

$\mathrm{h}_{3}=70$ kilometers

$\mathrm{j}=1$

$1.9845215=4$

1. $1693188=4$

$5.7792319-5$

1.0192435

$4.6361986=5 \quad 2.5826105$

$\widehat{D}_{j}$

meters

2.2040960 3.5591320 8.2199360 1.6232416

$$
\mathrm{j}=2
$$

$h_{3}=90$ kilometers $\mathrm{j}=1$

$$
j=2
$$

100
200
500
1000

$\begin{array}{ll}8.1837688=4 & 1.0161502 \\ 5.9810433=4 & 1.0210649 \\ 3.1058500=4 & 1.0533450 \\ 1.8207517=4 & 1.1771578\end{array}$

4. 0619720 5.0111760

Table 123. Skywave delay, $\widehat{a}$ - a, the geometrical-optical ray divergence convergence coefficient, $\propto_{j}$, and ray length, $\bar{D}_{j}$, for various distances, $d$. 

U. S. DEPARTMENT OF COMMERCE

Sinclair Weeks, Secretary

NATIONAL BUREAU OF STANDARDS

A. V. Astin, Director

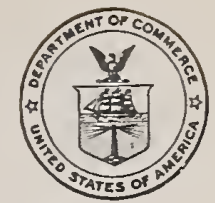

\section{THE NATIONAL BUREAU OF STANDARDS}

The scope of the scientific program of the National Bureau of Standards at laboratory centers in Washington, D. C., and Boulder, Colorado, is given in the following outline:

Washington, D.C.

Electricity and Electronics. Resistance and Reactance. Electron Devices. Electrical Instruments. Magnetic Measurements. Dielectrics. Engineering Electronics. Electronic Instrumentation. Electrochemistry.

Optics and Metrology. Photometry and Colorimetry. Optical Instruments. Photographic Technology. Length. Engineering Metrology.

Heat. Temperature Physics. Thermodynamics. Cryogenic Physics. Rheology. Engine Fuels. Free Radicals.

Atomic and Radiation Physics. Spectroscopy. Radiometry. Mass Spectrometry. Solid State Physics. Electron Physics. Atomic Physics. Neutron Physics. Nuclear Physics. Radioactivity. X-rays. Betatron. Nucleonic Instrumentation. Radiological Equipment. AEC Radiation Instruments.

Chemistry. Organic Coatings. Surface Chemistry. Organic Chemistry. Analytical Chemistry. Inorganic Chemistry. Electrodeposition. Molecular Structure and Properties. Physical Chemistry. Thermochemistry. Spectrochemistry. Pure Substances.

Mechanics. Sound. Mechanical Instruments. Fluid Mechanics. Engineering Mechanics. Mass and Scale. Capacity, Density, and Fluid Meters. Combustion Controls.

Organic and Fibrous Materials. Rubber. Textiles. Paper. Leather. Testing and Specifications. Polymer Structure. Plastics. Dental Research.

Metallurgy. Thermal Metallurgy. Chemical Metallurgy. Mechanical Metallurgy. Corrosion. Metal Physics.

Mineral Products. Engineering Ceramics. Glass. Refractories. Enameled Metals. Concreting Materials. Constitution and Microstructure.

Building Technology. Structural Engineering. Fire Protection. Air Conditioning, Heating, and Refrigeration. Floor, Roof, and Wall Coverings. Codes and Safety Standards. Heat Transfer. Applied Mathematics. Numerical Analysis. Computation. Statistical Engineering. Mathematical Physics.

Data Processing Systems. SEAC Engineering Group. Components and Techniques. Digital Circuitry. Digital Systems. Analogue Systems. Application Engineering.

- Office of Basic Instrumentation

- Office of Weights and Measures

Boulder, Colorado

BOULDER LABORATORIES

F. W. Brown, Director

Cryogenic Engineering. Cryogenic Equipment. Cryogenic Processes. Properties of Materials. Gas Liquefaction.

Radio Propagation Physics. Upper Atmosphere Research. Ionosphere Research. Regular Propagation Services. Sun-Earth Relationships. VHF Research. Ionospheric Communications Systems.

Radio Propagation Engineering. Data Reduction Instrumentation. Modulation Systems. Navigation Systems. Radio Noise. Tropospheric Measurements. Tropospheric Analysis. Radio Systems Application Engineering.

Radio Standards. High Frequency Electrical Standards. Radio Broadcast Service. High Frequency Impedance Standards. Electronic Calibration Center. Microwave Physics. Microwave Circuit Standards. 


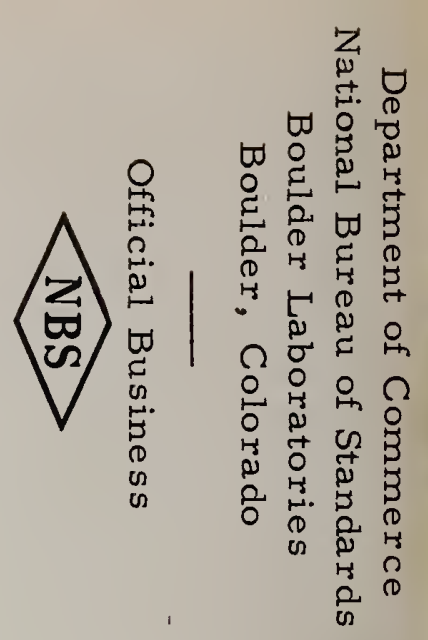

$C$

$\Omega$

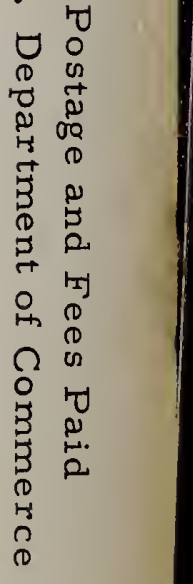

